\title{
Mechanism and Kinetics of Catalyzed Chain Growth
}

\author{
Dissertation
}

zur Erlangung des mathematisch-naturwissenschaftlichen Doktorgrades

"Doctor rerum naturalium"

der Georg-August-Universität Göttingen

im Promotionsprogramm Catalysis for Sustainable Synthesis (CaSuS)

vorgelegt von

Sebastian Primpke

aus Warburg

Göttingen, 2014 


\section{Betreuungsausschuss}

Prof. Dr. Philipp Vana, MBA Institut für Physikalische Chemie, Georg-August-Universität Göttingen

Prof. Dr. Franc Meyer Institut für Anorganische Chemie, Georg-August-Universität Göttingen

Prof. Dr. Michael Buback Institut für Physikalische Chemie, Georg-August-Universität Göttingen

\section{Mitglieder der Prüfungskommission}

\section{Referent}

Prof. Dr. Philipp Vana, MBA Institut für Physikalische Chemie, Georg-August-Universität Göttingen

\section{Koreferent}

Prof. Dr. Franc Meyer Institut für Anorganische Chemie, Georg-August-Universität Göttingen

\section{Weitere Mitglieder der Prüfungskommission}

Prof. Dr. Michael Buback Institut für Physikalische Chemie, Georg-August-Universität Göttingen

Prof. Dr. Burkhard Geil Institut für Physikalische Chemie, Georg-August-Universität Göttingen

Prof. Dr. Dietmar Stalke Institut für Anorgansiche Chemie, Georg-August-Universität Göttingen

Jun.-Prof. Dr. Ricardo Mata Institut für Physikalische Chemie, Georg-August-Universität Göttingen

Tag der mündlichen Prüfung: 17.12.14 
Meiner Familie 



\section{Contents}

$\begin{array}{ll}\text { Abstract } & 1\end{array}$

1 Introduction 3

2 Theoretical Background $\quad 7$

2.1 Polymer Characterization . . . . . . . . . . . . . . . 7

2.2 Conventional Polymerization Techniques . . . . . . . . . . . . . . . . 9

2.2.1 Conventional Radical Polymerization . . . . . . . . . . . . . . . 9

2.2.2 Ziegler-Natta (ZN) Polymerization . . . . . . . . . . . . . . . . . . 12

2.2.3 Polymerization in Presence of Methylaluminoxane (MAO) . . . . . 14

2.2 .4 Monomer Addition . . . . . . . . . . . . . . . . . 16

2.2 .5 Termination Reactions . . . . . . . . . . . . . . . . . . 17

2.2 .6 Mechanistic Studies . . . . . . . . . . . . . . . . . . . . 18

2.3 Living/Controlled Polymerization Techniques . . . . . . . . . . . . . . 20

2.3.1 Reversible-Deactivation Radical Polymerization (RDRP) . . . . . . 21

2.3.2 Coordinative Chain Transfer Polymerization . . . . . . . . . . . . 22

2.3 .3 Catalyzed Chain Growth . . . . . . . . . . . . . . . . . . . 24

2.3 .4 Mechanistic Studies of CCTP . . . . . . . . . . . . . . . . . 25

2.3 .5 Nickel Catalyzed Chain Growth (Ni-CCG) . . . . . . . . . . . . . . 27

2.4 Methods . . . . . . . . . . . . . . . . . . . . . . 28

2.4.1 Infrared (IR) and Ultraviolet-Visible (UV/Vis) spectroscopy . . . . 28

2.4 .2 Nuclear Magnetic Resonance (NMR) spectroscopy . . . . . . . . . . . 30

2.4 .3 Size Exclusion Chromatography (SEC) . . . . . . . . . . . . . 32

2.4 .4 Modeling via PREDICI ${ }^{\mathrm{TM}} \ldots \ldots \ldots \ldots$. . . . . . . . . 34

\begin{tabular}{ll|l}
3 & Objective & 35
\end{tabular}

4 Monomer Free Kinetic Models for Catalyzed Chain Growth 37

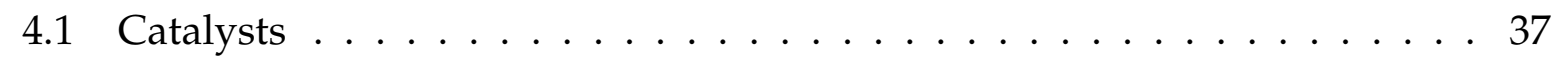

4.2 Magnesium based Co-catalysts . . . . . . . . . . . . . 38 
4.3 The Model System $\mathrm{Cp}_{2}^{*} \mathrm{ZrCl}_{2}$ and BzMgBz . . . . . . . . . . . . . . . . . 40

$4.3 .1 \quad$ Kinetic NMR Experiments . . . . . . . . . . . . . . . . . . . . 41

$4.3 .2 \quad \mathrm{UV} /$ Vis Studies on $\mathrm{Cp}_{2}^{*} \mathrm{ZrCl}_{2}$ and $\mathrm{BzMgBz} \ldots \ldots \ldots . . \ldots 56$

4.3.3 NMR Study on $\mathrm{Cp}_{2}^{*} \mathrm{ZrCl}_{2}, \mathrm{BzMgBz}$ and di- $n$-butyl ether (DBE) . . 62

$4.3 .4 \mathrm{UV} /$ Vis Studies on $\mathrm{Cp}_{2}^{*} \mathrm{ZrCl}_{2}, \mathrm{BzMgBz}$ and DBE . . . . . . . . . . . 65

4.4 NMR and UV/Vis Studies on $\mathrm{Cp}_{2}^{*} \mathrm{ZrCl}_{2}$ with Dialkyl Magnesium Com-

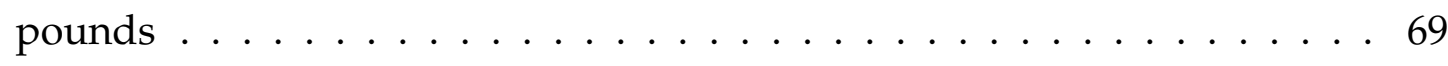

$4.5 \mathrm{UV} /$ Vis Studies on $\mathrm{Cp}_{2}^{*} \mathrm{NdCl}_{2} \mathrm{Li}\left(\mathrm{OEt}_{2}\right)_{2}$ and BzMgBz $\ldots \ldots \ldots$. . . . . 75

4.5 .1 Conclusion . . . . . . . . . . . . . 81

5 Modeling Catalyzed Chain Growth of Styrene-d8 with $\mathrm{Cp}_{2}^{*} \mathrm{ZrCl}_{2}$ and $\mathbf{B z M g B z} 83$

5.1 NMR Studies of the Model System $\mathrm{Cp}_{2}^{*} \mathrm{ZrCl}_{2}$ and $\mathrm{BzMgBz}$ with Styrene-d8 83

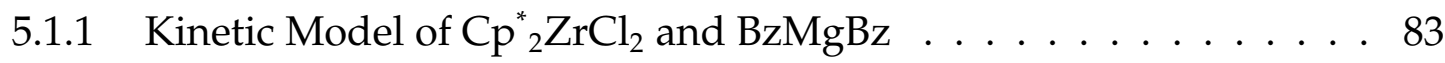

5.1.2 Modeling of CCG with $\mathrm{Cp}_{2}^{*} \mathrm{ZrCl}_{2}, \mathrm{BzMgBz}$ and Styrene-d8 . . . . . 100

5.1.3 Simulation of Influences on CCG of $\mathrm{Cp}_{2}^{*} \mathrm{ZrCl}_{2}$ and $\mathrm{BzMgBz}$ with

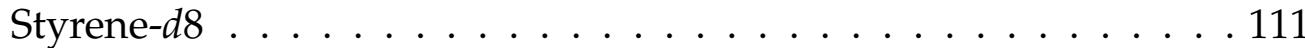

$5.1 .4 \quad$ Further NMR Polymerization Studies . . . . . . . . . . . . . . . . 125

5.1 .5 Conclusion . . . . . . . . . . . . . . . . 133

6 Modeling and Simulation of CCG-Systems $\mathbf{1 3 5}$

6.1 Polymerization of Styrene with $\mathrm{Cp}_{2}^{*} \mathrm{ZrCl}_{2}$ and $\mathrm{BzMgBz} \ldots$. . . . . . 135

6.1 .1 Polymerization in Absence of DBE . . . . . . . . . . . . . . . 135

6.1 .2 Polymerization in Presence of DBE . . . . . . . . . . . . . . . . . . 145

6.2 Polymerization of Styrene with $\mathrm{Cp}^{*}{ }_{2} \mathrm{ZrCl}_{2}$ and Dialykl Magnesium . . . 148

6.3 Polymerization of Styrene with $\mathrm{Cp}^{*}{ }_{2} \mathrm{NdCl}_{2} \mathrm{Li}\left(\mathrm{OEt}_{2}\right)$ and $\mathrm{BzMgBz}$. . . . . . 152

6.4 Polymerization of Styrene with $\mathrm{Cp}_{2}{ }_{2} \mathrm{NdCl}_{2} \mathrm{Li}\left(\mathrm{OEt}_{2}\right)$ and BuMgOct $\ldots$. . . . 162

6.4 .1 Conclusion . . . . . . . . . . . . . . . . . . . . . . . 169

6.5 Polymerization of Ethylene with $\mathrm{Cp}_{2}{ }_{2} \mathrm{NdCl}_{2} \mathrm{Li}\left(\mathrm{OEt}_{2}\right)$ and BuOctMg $\ldots 172$

6.5.1 Simulation of CCG polymerization performed in the "Büchi ecoclave $075^{\prime \prime} \ldots \ldots \ldots \ldots \ldots \ldots \ldots \ldots$. . . . . . . . . . . . . . . . . . . .

6.5.2 Simulation of CCG Polymerization performed at CPE in Lyon . . . 178

7 Additional Polymerizations and End-group Modification of Polyethylene 181

7.1 Polymerization of Ethylene with $\mathrm{Cp}_{2}^{*} \mathrm{NdCl}_{2} \mathrm{Li}\left(\mathrm{OEt}_{2}\right)_{2}$ and $\mathrm{BzMgBz}$ in Presence of 1,4-dioxane . . . . . . . . . . . . . . 181

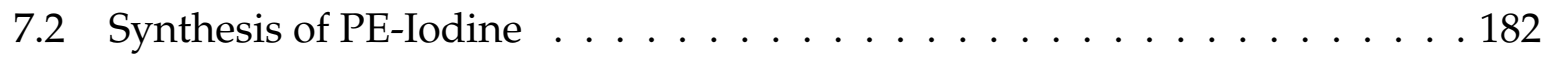

$7.3 \quad$ PE-b-P3HT $\ldots \ldots \ldots \ldots \ldots \ldots$

7.4 Conclusion . . . . . . . . . . . . . . . . . . . 188

8 Concluding Remarks and Outlook 
9 Experimental 195

9.1 General . . . . . . . . . . . . . . . . . . . . . . . . . 195

9.1 .1 Materials and Chemicals . . . . . . . . . . . . . . . . . 195

9.1 .2 Determination of Conversion . . . . . . . . . . . . . . . . . 195

9.1 .3 Size Exclusion Chromatography (SEC) . . . . . . . . . . . . . . . 196

9.1 .4 Nuclear Magnetic Resonance (NMR) . . . . . . . . . . . . . . . . . . 197

9.1 .5 Data Handling of Kinetic NMR . . . . . . . . . . . . . . . . . . . 197

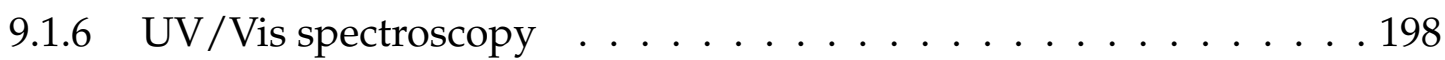

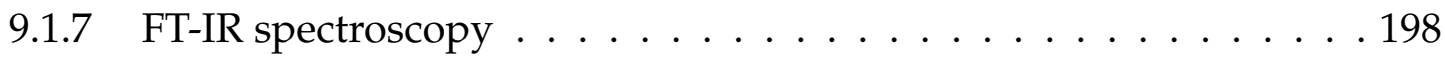

9.1 .8 PE-reactor . . . . . . . . . . . . . . . . . . . . . 199

9.1 .9 Differential scanning calorimetry (DSC) . . . . . . . . . . . . . . . 202

9.1 .10 Mass Spectrometry . . . . . . . . . . . . . . . . . . . . . . . . . . . . . . . . . . . . . . . . . . . . .

9.2 Synthesis . . . . . . . . . . . . . . . . . . . . . . . . 203

9.2 .1 Catalysts . . . . . . . . . . . . . . . . . . . . . . . . . . . . . . . . . . . . . . .

9.2 .2 Co-catalysts . . . . . . . . . . . . . . . . . . 203

9.3 General Procedures . . . . . . . . . . . . . . . . . . . . . . . . . 204

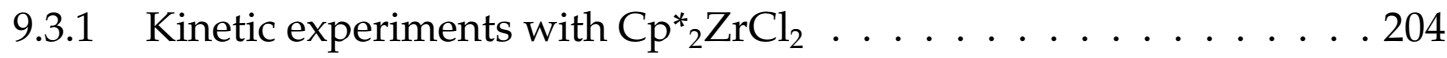

9.3.2 Kinetic experiments with $\mathrm{Cp}^{*}{ }_{2} \mathrm{NdCl}_{2} \mathrm{Li}\left(\mathrm{OEt}_{2}\right)_{2} \ldots$. . . . . . . . . . . 207

9.3.3 Catalyzed chain growth polymerization in the reactor . . . . . . . . 209

9.3.4 Polymer modification reactions . . . . . . . . . . . . . . 210

\begin{tabular}{ll}
\hline A Appendix & 213
\end{tabular}

A.1 Figures . . . . . . . . . . . . . . . . . . . . . . . . . . . . . . . . . . . . . . . . . . . . . . . . . . .

A.2 Reaction Rate Equations . . . . . . . . . . . . . . . . . . . . . 217

A.3 PREDICI Models . . . . . . . . . . . . . . . . . . . . . . 218

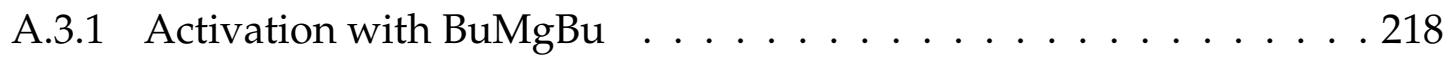

A.3.2 $\quad$ Polymerization with $\mathbf{A}$ and BzMgBz with Reactivation of $\mathbf{G}$. . . . 218

A.3.3 $\quad$ Extended Model for Polymerization with A and BuMgOct . . . . . 222

A.3.4 Polymerization of Styrene with I and BzMgBz . . . . . . . . . . . . 226

A.3.5 Model for Polymerization of I and BuMgOct in the Reactor . . . . 228

\begin{tabular}{ll}
\hline Abbreviations & 231
\end{tabular}

\begin{tabular}{ll}
\hline References & 237
\end{tabular}

\begin{tabular}{ll}
\hline Acknowledgments & 255
\end{tabular} 



\section{Abstract}

In this work, the activation and polymerization mechanism of Catalyzed Chain Growth (CCG) polymerization of ethylene and styrene was studied. On the basis of two metallocene catalysts $\left(\mathrm{Cp}_{2}{ }_{2} \mathrm{ZrCl}_{2}\right.$ and $\left.\mathrm{Cp}_{2}{ }_{2} \mathrm{NdCl}_{2} \mathrm{Li}\left(\mathrm{OEt}_{2}\right)_{2}\right)$ and dibenzyl magnesium (BzMgBz) and $n$-butyloctyl magnesium (BuMgOct) as co-catalysts the mechanism was determined via a model reaction and afterwards transferred to a typical CCG system. The model system consisting of $\mathrm{Cp}_{2}{ }_{2} \mathrm{ZrCl}_{2}$ and $\mathrm{BzMgBz}$ cannot perform $\beta$-hydride elimination and was first studied via Nuclear Magnetic Resonance (NMR). A mechanistic scheme was proposed and kinetic coefficients were successfully derived via modeling in PREDICI ${ }^{\mathrm{TM}}$. Data analysis was simplified due to direct calculation of the coefficients from NMR data. The analytical toolbox was extended to Ultra-Violet/Visible (UV/Vis) spectroscopic studies. Based on this method the influence of di-n-butyl ether (DBE) on the catalyst/co-catalyst system was studied and a strong lowering of rate coefficients was found.

The co-catalyst was switched to BuMgOct, where $\beta$-hydride elimination of the alkyl chains was found to be fast at $70{ }^{\circ} \mathrm{C}$ and dominating the activation mechanism. This hinders the system to perform ethylene polymerization.

The UV/Vis method was applied to the reaction of $\mathrm{Cp}^{*}{ }_{2} \mathrm{NdCl}_{2} \mathrm{Li}\left(\mathrm{OEt}_{2}\right)_{2}$ with $\mathrm{BzMgBz}$. Equilibrium constants between a monoalkylated complex and the benzyl brigded bimetallic $\mathrm{Nd}-\mathrm{Mg}$ complex were determined in dependence of temperature. The reaction turned out to be strongly dependent on the presence of other coordinating molecules like DBE.

The activation mechanism of $\mathrm{Cp}^{*}{ }_{2} \mathrm{ZrCl}_{2}$ and $\mathrm{BzMgBz}$ was successfully utilized for modeling the polymerization of styrene- $d 8$ by NMR studies. It was found that the basic steps of the exchange reactions are similar to those of activation. The model was successfully extended to the introduction of polymeric species, and kinetic parameters were derived. The process of monomer addition was found to be chain-length dependent and the influence of the single rate coefficients on the reaction were screened. It was found that a low catalyst precursor concentration can lead to a rate enhancement. Addition of tetrahydrofurane was found to be disadvantageous for the polymerization reaction. BuMgOct as co-catalyst leads to a process where the termination reaction of the alkyl 
chains is the main catalyst activation mechanism. Chain growth occurs starting from the hydride catalyst formed during termination. $\beta$-hydride elimination of the polystyrene chain is much slower than for alkyl chains.

Polymerization of styrene was successfully studied via UV/Vis analysis and a gravimetric- and Infra-Red (IR)-determination of the conversion. The catalysts $\mathrm{Cp}_{2}{ }_{2} \mathrm{ZrCl}_{2}$ and $\mathrm{Cp}_{2}{ }_{2} \mathrm{NdCl}_{2} \mathrm{Li}\left(\mathrm{OEt}_{2}\right)_{2}$ were activated with either BzMgBz or BuMgOct. The influence of DBE on each system was investigated individually. Modeling based on the proposed model was successful. The system of $\mathrm{Cp}^{*}{ }_{2} \mathrm{ZrCl}_{2}$ and $\mathrm{BuMgOct}$ indicated an additional activation pathway. For the system $\mathrm{Cp}_{2}{ }_{2} \mathrm{NdCl}_{2} \mathrm{Li}\left(\mathrm{OEt}_{2}\right)_{2}$ with $\mathrm{BzMgBz}$ it was found that the termination reaction via $\beta$-hyrdide elimination and reformation of the active catalyst is the main reaction pathway.

The kinetic model was successfully applied to the polymerization of ethylene in a batch reactor and the polymerization was modeled successfully.

As part of a cooperation with Dr. Florian Ehlers and Jan Schwellenbach a new method for the production of PE- $b$-P3HT block copolymers was found. The formed polymer has combined properties regarding the solubility and viscosity of polyethylene and poly-3-hexyl-thiophene. 


\section{Introduction}

The word "polymer" is a combination of the greek word $\pi o \lambda v ́ s$ (polus) for many and $\mu \epsilon ́ p o \varsigma$ (meros) for parts describing molecules that consist of many linked equal building units. These building units are called monomers and can be simple organic molecules like ethylene or complex structures like functionalized styrene derivatives. The first systematic research in this area by $\mathrm{H}$. Staudinger and J. Fritschi ${ }^{[1,2]}$ opened the door for a broad field of studies afterwards. These studies led to commercial production of materials like e.g. polyethylene (PE) and Nylon ${ }^{[3]}$ and became important in industry. Nowadays polymer based products are commonly used in a wide range of every day application; e.g. as packaging materials (LD-PE and HD-PE), building materials (Styropor $^{\odot}$, Styrodur, PUR), coatings (cars, paints, teflon $^{\odot}$ ), fabrics (polyesters, teflon ${ }^{\odot}$ ) and in car components and tires. The production of plastics in Europe was around $57 \mathrm{Mt}$ of a world wide $288 \mathrm{Mt}$ production in $2013^{4}$, which is still increasing. In Europe almost $40 \%$ of the applied polymers is used for packing materials followed by building and construction $(20 \%)$ and the broad field of customer products, healthcare and safety equipment (22\%). ${ }^{4]}$

There are different types of polymers used in industry and end-consumer applications. They need different production techniques based on one of two main principles, the stepgrowth polymerization or the chain-growth polymerization. Important representatives for the first principle are polycondensation and polyaddition.

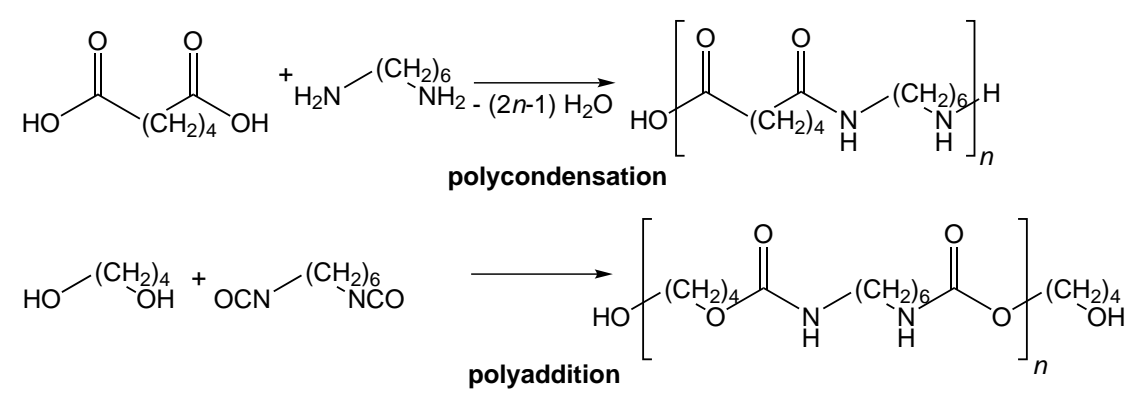

Scheme 1.1: Example for a polycondensation and a polyaddition reaction

Via polycondensation materials like $\mathrm{Nylon}^{\odot}$ and polyesters are produced in industrial scale. A principle disadvantage of polycondensation is the formation of a byproduct, 
which has to be removed from the reaction mixture (see Scheme 1.1 polycondensation). In contrast polyaddition reactions, which are used to produce e.g. polyurethanes produce no side product, but need highly reactive functional groups (see Scheme 1.1 polyaddition). Both processes must be driven with exact stoichiometry to yield high molecular weights.

The chain-growth polymerization (see Scheme 1.2), has the advantage that no exact stoichiometry is needed. The reaction is typically started by a chain initiator leading to first monomer addition. The reactive group is transferred to the monomer, which is then able to add additional monomer until a termination event occurs.

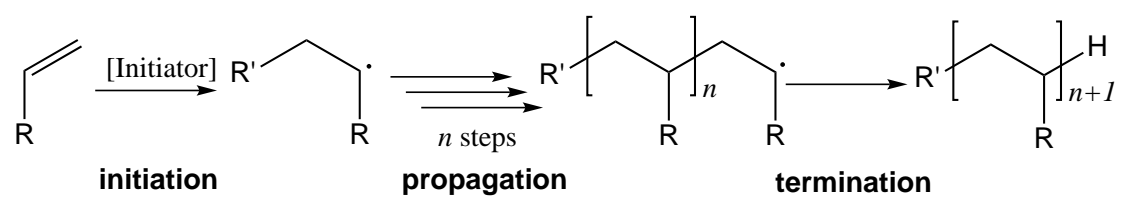

Scheme 1.2: Example for a radical initiated chain growth reaction. The reaction starts via initiation, here from a radical starter, leading to a reactive group capable of adding monomer into the chain without being consumed until it reacts via a termination reaction step.

Typical chain growth reactions are anionic, cationic, radical and coordination polymerization. Of these processes the radical and coordination polymerization are the most widely applied processes in industry due to their high efficiency and low costs. The produced polymers like PE show varying properties according to the applied production method, e.g. high density polyethylene (HD-PE) is only accessible via coordination polymerization while radical polymerization yields low density polyethylene (LD-PE). The main difference is found in their polymer architecture and the defined synthesis of the different macromolecular structures is an important goal in polymer science (see Figure 1.1).

Topology has an important impact on materials. The targeted synthesis of different topologies is interesting for special purpose materials. Linear topology is achieved most easily way, e.g. via radical polymerization or coordination polymerization via ZieglerNatta $(\mathrm{ZN})$ catalysts, as the most common reaction product. Star-shaped polymers have higher synthetic demand and are available via several polymerization techniques. ${ }^{[5-7]}$ Physical polymer networks are produced by introduction of a multifunctional monomer, a crosslinker. ${ }^{[3]}$ Branched polymers are typically formed during the high pressure polymerization of ethylene leading to Low-Density Polyethylene (LDPE) ${ }^{[3]}$ or are achieved by special methods as hyperbranched or dendrimeric polymers. ${ }^{[8]}$ Another characterizing property of polymer materials is their microstructure (Figure 1.1). Atactic polymers show a random distribution within their constitution between neighboring units. It is the typical structure of products made by radical poly- 


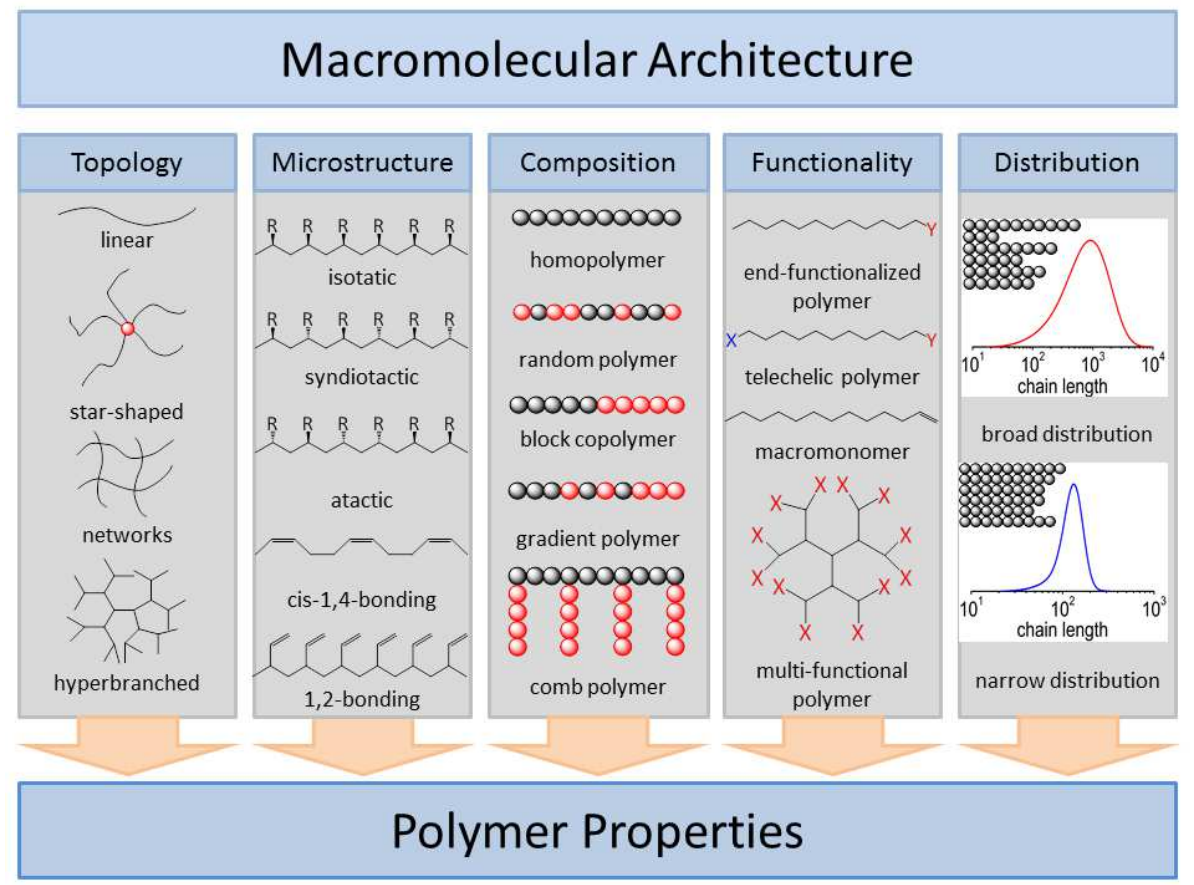

Figure 1.1: Overview of different polymer architectures defining polymer properties.

merization processes. The polymers are amorphous and have a glass-like behavior. In the case of syndiotatic polymer the constitution alternates between two monomer building units and the synthesis of such structures is more challenging. A typical example is the propylene polymerization with single side transition metal catalysts ${ }^{[9}$. In the isotactic case all monomer units show the same constitution along the polymer chain. These are also available by $\mathrm{ZN}$-polymerization ${ }^{[10,11]}$ and show high crystallinity. The other microstructures are based on 1,3-butadiene and are synthesized by specialized polymerization approaches. [12,13]

Homopolymers consists of only of one kind of monomer, while the application of two or more monomers yields statistical copolymers. In gradient polymers the concentration of the second monomer unit increases while the one of the first decreases along the chain. Block copolymers $(\mathrm{BCP})^{[14-16]}$ are produced by polymerizing one monomer after another and are available by a series of methods like living, coordination polymerization and reversible-deactivation radical polymerization (RDRP) techniques. In addition these techniques allow the production of end-functionalized polymers, telechelic polymers (polymers bearing functionalities on both ends) or macromonomers. Polymers made by uncontrolled methods often have a broad molar mass distribution (MMD) i.e. a rather undefined chain length distribution is gained, while controlled processes yield only a small spectrum of different chain-lengths and therefore a narrow MMD.

To produced these polymers in industrial scale the polymerization has to work safely in 
multi ton scale at low costs. Further, it is often not possible to build a completely new setup for production but it is necessary to work on existing plants. Kinetic research is an important field to reduce costs. It allows the screening of existing reactor designs to find the optimum conditions before actually applying the process at the production plant. One of the most powerful tools for kinetic studies is the program package PREDICI ${ }^{\mathrm{TM}}$ which is commonly applied for radical polymerization. A broad spectrum of different measurement techniques is available and are applied for modeling and simulation of experiments. ${ }^{[17]}$ The determined kinetic coefficients are introduced via defined reaction steps into a model setup which can take external events into account. Outgoing from the results of simulation the most successful process can be run in a test plant or the recipe can be modified to gain the desired properties of the product. In coordination chemistry mainly quench flow studies and spectroscopic studies build the basis for kinetic research the application of PREDICI ${ }^{\mathrm{TM}}$ is only known for one example in literature. ${ }^{[18]}$ Therefore, it is of interest to describe such systems via PREDICI ${ }^{\mathrm{TM}}$.

Out of this variety of polymerization techniques the process known as CatalyzedChain-Growth (CCG) polymerization ${ }^{[19]}$ is interesting because the proposed mechanism is similar to the already well researched RAFT-process. ${ }^{201}$ This method is most promising for the controlled polymerization of ethylene by a neodymium based catalyst $\mathrm{Cp}_{2}{ }_{2} \mathrm{Nd}_{2} \mathrm{Cl}_{2} \mathrm{Li}\left(\mathrm{OEt}_{2}\right)_{2}$ in presence of a co-catalyst based on dialkyl magnesium derivatives $^{[21,22]}$ (see Scheme 1.2).

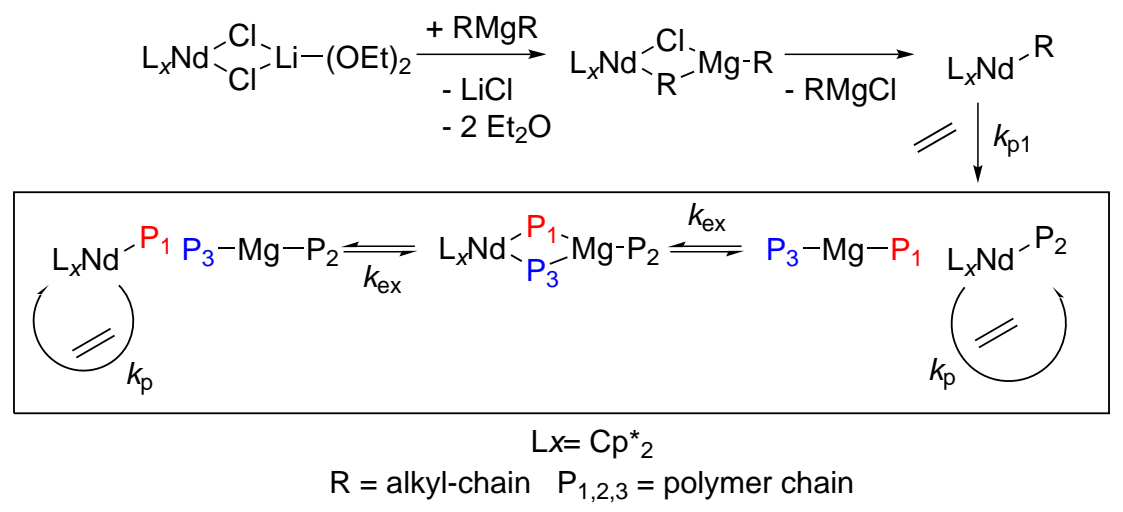

Figure 1.2: Basic principle of catalyzed chain growth polymerization based on $\mathrm{Cp}_{2}{ }_{2} \mathrm{Nd}_{2} \mathrm{Cl}_{2} \mathrm{Li}\left(\mathrm{OEt}_{2}\right)_{2}$

This reaction is widely used to produce functionalized polyethylene ${ }^{[23]}$ while the reaction mechanism and rate coefficients are unknown. In this work the mechanistic and kinetic studies were performed to setup a reaction model. Modeling of experiments is performed with the program package PREDICI ${ }^{\mathrm{TM}}$ for reactions within a NMR-tube, UV/Vis cuvettes and a typical polymerization reactor. Chosen polymers produced during these measurements were end-group functionalized with iodine and a second polymer block based on poly-3-hexylthiophene (P3HT). 


\section{Theoretical Background}

Nowadays polymers show a wide range of mechanical, structural or physical properties by the synthesis of tailored polymers. One powerful tool is the Ziegler-Natta (ZN) polymerization. Recently, with the method of catalyzed chain growth (CCG) a straightforward method of producing functionalized polyethylene (PE) was found. After a short introduction into polymer characterization the main aspect in this chapter will be polymerization techniques, especially ZN-catalysis, the CCG-process and their respective kinetic studies.

\subsection{Polymer Characterization}

Polymers consist of a distribution of molecule chains with different length, the molar mass distribution (MMD). The Size Exclusion Chromatography (SEC) is one of the most common used methods to characterize the MMD and provides information about the number average molecular mass $\left(\bar{M}_{\mathrm{n}}\right)$ and mass averaged molecular mass $\left(\bar{M}_{\mathrm{w}}\right)$. The number average molecular mass is defined as the fraction of the sum of polymer chains with the chain length $i N_{i}$ and molecular weight $M_{i}$ and the overall number of polymer chains.

$$
\bar{M}_{\mathrm{n}}=\sum x_{i} N_{i}=\frac{\sum N_{i} M_{i}}{\sum N_{i}}
$$

The mass averaged molar mass is defined as the fraction of the sum of all polymer chains with the chain length $i N_{i}$ and the square of molecular weight $M_{i}$ over the overall sum of polymer chains with the chain length $i N_{i}$ and molecular weight $M_{i}$.

$$
\bar{M}_{\mathrm{w}}=\sum w_{i} N_{i}=\frac{\sum N_{i} M_{i}^{2}}{\sum N_{i} M_{i}}
$$


Both values are characteristic for a polymer and are applied to quantify the broadness of a MMD. Another charaterisitc value is the molar mass at the peak maximum $M_{p}$ that is necessary for the calibration of a SEC setup. In Figure 2.1 a comparison of the different values within a MMD is shown.

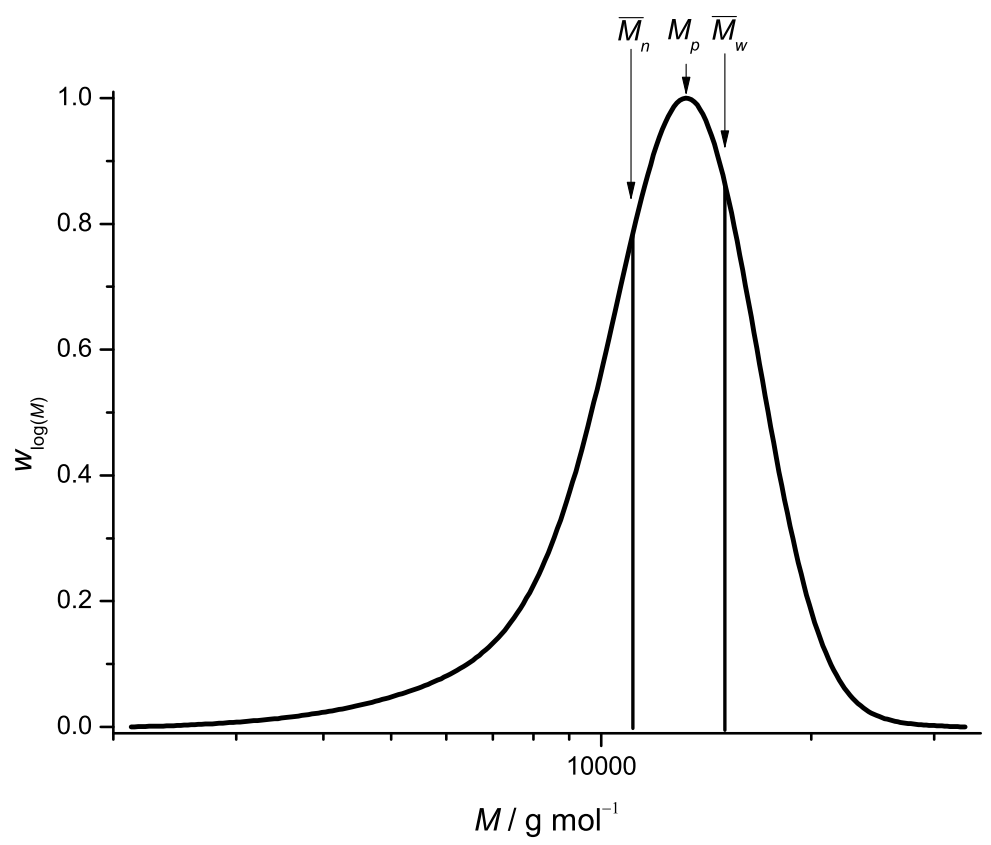

Figure 2.1: Example for $\bar{M}_{\mathrm{n}}, M_{\mathrm{p}}$ and $\bar{M}_{\mathrm{w}}$ in a MWD.

$\bar{M}_{\mathrm{w}}$ has always a higher value than $\bar{M}_{\mathrm{n}}$ and the quotient of both is defined as the dispersity index $Ð$ (see eq. 2.3).

$$
Đ=\frac{\bar{M}_{\mathrm{w}}}{\bar{M}_{\mathrm{n}}}
$$

Typical values for the dispersity e.g. in the conventional radical polymerization are in the range of 1.5 to 2 while well controlled polymerizations yield a narrow MMD with values around 1.05 to 1.1 . A value of 1 is only possible for chains with identical molar mass like a well defined bio-molecule, e.g. a protein. For technical processes a dispersity index of 1 is not possible, because every known polymerization process produces polymers with an assortment of chain-lengths. 
A second way to express the dispersity is via the degree of polymerization $\bar{P}_{\mathrm{n}}$, the average number of monomer units in a polymer (eq. 2.4 and 2.5).

$$
\begin{gathered}
\bar{P}_{\mathrm{n}}=\bar{M}_{\mathrm{n}} / M_{\text {Mono }} \\
Đ=1+\frac{1}{\bar{P}_{\mathrm{n}}}
\end{gathered}
$$

For analysis of the microstructure of polymers nuclear magnetic resonances (NMR) measurements are applied most frequently. ${ }^{[24-26]}$ They allow to determine the different constitutions within a polymer caused by different couplings with the neighbor hydrogen atoms, which can be analyzed quantitatively. This leads to the determination of isotatic or syndiotatic fractions in a polymer and thus of the efficiency of a catalyst in stereo controlled synthesis.

The physical behavior of polymers has a large influence on their use in industry. End consumer products are often not only based on a single polymer. A composition of polymers, additives and/or filler materials are commonly used to increase product stability and duration and to reduce costs.

Different methods are able to determine mechanical properties like the Young modulus either via dynamic mechanical analysis or tensile testing. Physical properties like glass-transition temperature, melting point and degree of crystallinity are measured via differential scanning calorimetry (DSC) and the thermal duration by thermal gravimetric analysis. For the production of polymers with the desired properties several polymerization techniques were developed allowing the utilization of a broad spectrum of monomers and access to desired polymer structures. These techniques are described in the following section.

\subsection{Conventional Polymerization Techniques}

\subsubsection{Conventional Radical Polymerization}

One of the most applied techniques for industrial polymerization is the conventional radical polymerization (CRP). It is a well researched field, with a broad scope of kinetic studies and experimental setups present. ${ }^{[17]}$ Its major advantage is the applicability to a broad spectrum of monomers like acrylates, methacrylates, vinyl acetates, vinyl amides and styrene derivatives. $\alpha$-olefins can also be applied but due to weak radical stabilization high pressures and temperatures are needed. For example, the LDPE 
produced by conventional radical polymerization shows high branching caused by radical transfer steps.

The CRP is based on three major reaction steps. The initiation consists of two substeps. At first the initiator decomposes with the rate coefficient $k_{\mathrm{d}}$ and the initiator efficiency $f$ forming reactive radicals (2.6). Afterwards the first monomer addition to an initiator radical with the rate coefficient $k_{\mathrm{i}}(2.7)$ takes place. The initiator decay is the rate determining step during the process.

$$
\begin{gathered}
\operatorname{lni}_{2} \stackrel{k_{\mathrm{d}} \cdot f}{\longrightarrow} 2 \operatorname{lni} . \\
\operatorname{Ini} \cdot+\mathrm{M} \stackrel{k_{\mathrm{i}}}{\longrightarrow} \mathrm{R}_{1} .
\end{gathered}
$$

Chain-growth is performed during the propagation step by the addition of monomer to a polymeric radical while the radical function is retained after reaction (2.8). This enables polymer-growth as long as monomer is present and the radical functions are not depleted.

$$
\mathrm{R}_{\mathrm{n}} \cdot+\mathrm{M} \stackrel{k_{\mathrm{p}}}{\longrightarrow} \mathrm{R}_{\mathrm{n}+1}
$$

Chain transfer occurs by addition of e.g. a thiol-functionalized reactant or a solvent molecule. The radical function is transferred from the growing chain to a another monomer (2.9). The growing chain is terminated and a new chain is started. Chaintransfer agents (CTA) are applied to decrease the molar mass during CRP.

$$
\mathrm{R}_{\mathrm{n}} \cdot+\mathrm{CTA} \stackrel{k_{\mathrm{tr}}}{\longrightarrow} \mathrm{P}_{\mathrm{n}}+\mathrm{R}_{1} .
$$

During the polymerization process the radical concentration increases, together with the chance that two polymeric radicals meet. This leads to termination either via combination or disproportionation. Combination of two the polymer chains forms a chain with the combined molar mass (2.10). Disproportion leads to hydrogen abstraction at one polymer chain yielding one saturated and one unsaturated polymer chain (2.11).

$$
\begin{aligned}
& \mathrm{R}_{\mathrm{n}}+\mathrm{R}_{\mathrm{m}} \cdot \stackrel{k_{\mathrm{t}, \mathrm{c}}}{\longrightarrow} \mathrm{P}_{\mathrm{n}+\mathrm{m}} \\
& \mathrm{R}_{\mathrm{n}} \cdot+\mathrm{R}_{\mathrm{m}} \cdot \stackrel{k_{\mathrm{t}, \mathrm{d}}}{\longrightarrow} \mathrm{P}_{\mathrm{n}}+\mathrm{P}_{\mathrm{m}}
\end{aligned}
$$

Initiation of this process is typical achieved by a radical starter like azo-compounds or peroxides, but styrene based monomers can undergo a self-initiation process. 
Self-initiation of Styrene In styrene polymerization the monomer can act as initiator at elevated temperatures by a self-initiation mechanism (see Scheme 2.1).

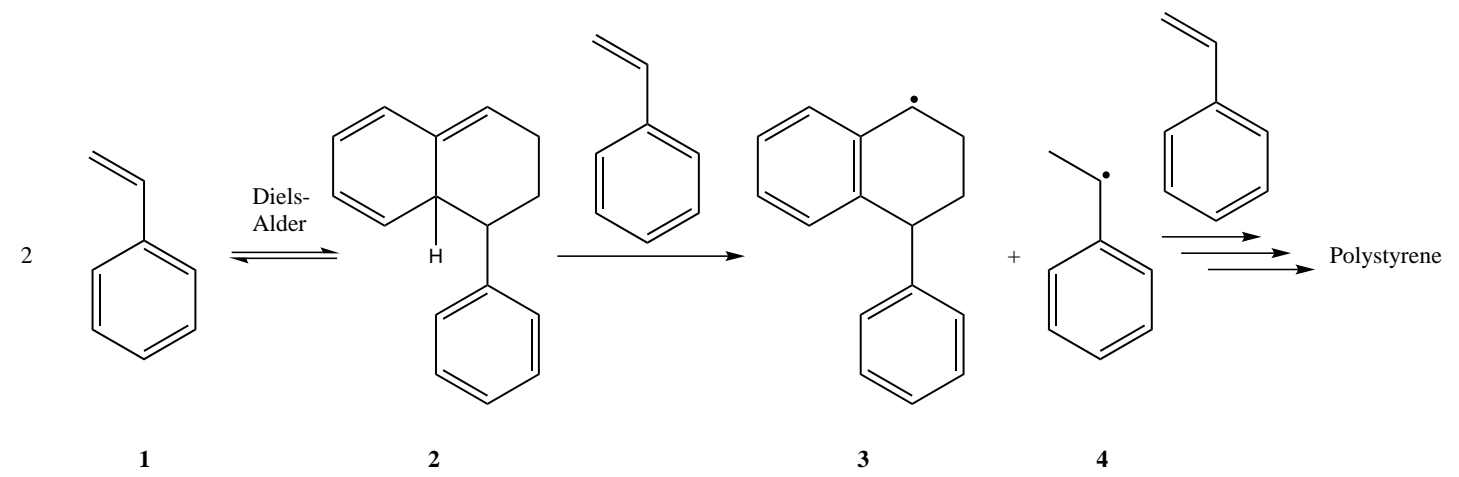

Scheme 2.1: Self-initiation of styrene via dimerization and radical proton abstraction. Adapted with permission from S. Bogaert et al. ${ }^{[21]}$ Copyright 2000 WILEY-VCH Verlag GmbH, D-69451 Weinheim

Two monomer molecules $\mathbf{1}$ undergo a Diels-Alder reaction and form molecule 2, which has several reaction pathways yielding different products. ${ }^{\mid 21,27,28}$ To act as initiator the hydrogen atom from the ring is abstracted following a radical reaction pathway. Both molecules formed 3,4 are able to start the polymerization. The produced chains show a mass difference of two hydrogen atoms.

Dialkyl Magnesium as CTA for Radical Polymerization S. Bogaert et al. ${ }^{[21]}$ showed that magnesium derivatives can act as a CTA during self-initiated polymerization of styrene without lowering the reaction rate and proposed a mechanism for the exchange reaction (see Scheme 2.2).

$$
\begin{aligned}
& \mathrm{R}-\mathrm{Mg}-\mathrm{R}^{\prime}+\mathrm{P}-(\mathrm{St})_{\mathrm{n}}{ }^{\circ} \rightleftharpoons \mathrm{P}-(\mathrm{St})_{\mathrm{n}^{-}}-\mathrm{Mg}-\mathrm{R}^{\prime}+\mathrm{R}^{\cdot} \\
& \mathrm{P}-(\mathrm{St})_{\mathrm{n}}-\mathrm{Mg}-\mathrm{R}^{\prime}+\mathrm{P}-(\mathrm{St})_{\mathrm{m}}^{\cdot} \rightleftharpoons \mathrm{P}-(\mathrm{St})_{\mathrm{n}}-\mathrm{Mg}-\mathrm{P}(\mathrm{St})_{\mathrm{m}}+\mathrm{R}^{\prime} \\
& \mathrm{P}-(\mathrm{St})_{\mathrm{m}}-\mathrm{Mg}-\mathrm{R}^{\prime}+\mathrm{P}-(\mathrm{St})_{\mathrm{n}}^{\cdot} \rightleftharpoons \mathrm{P}-(\mathrm{St})_{\mathrm{n}^{-}}-\mathrm{Mg}-\mathrm{R}^{\prime}+\mathrm{P}-(\mathrm{St})_{\mathrm{m}}^{\cdot}
\end{aligned}
$$

Scheme 2.2: Transfer reaction scheme for magnesium derivatives. Adapted with permission from $S$. Bogaert et al. ${ }^{21]}$ Copyright 2000 WILEY-VCH Verlag GmbH, D-69451 Weinheim

The reactive polymer chain is transferred to the magnesium moiety and a reactive radical is set free, which can add monomer or start termination. The same process is possible when one or two polystyrene units are bound to the magnesium. The process was screened for a spectrum of organometallic compounds like $\mathrm{AlR}_{3}, \mathrm{AlR}_{2} \mathrm{H}, \mathrm{InR}_{3}, \mathrm{ZnR}_{2}$ and $\mathrm{CdR}_{2} \cdot{ }^{29,30]}$ 
Metallocene Catalyst as CTA for Radical Polymerization Metallocene catalyst are as known as a CTA in CRP. ${ }^{31-36]}$ The reaction mechanisms involved need an external radical starter or reducing agent like zinc transferring one electron to the catalyst. These species can add react with a second often oxygen containing molecule (MMA or a ethylene oxide group) forming the initiating species.

\subsubsection{Ziegler-Natta (ZN) Polymerization}

The Ziegler-Natta polymerization was first reported in 1955 by K. Ziegler ${ }^{[37 / 38]}$ for ethylene while G. Natta ${ }^{[10,11]}$ applied a similar catalyst on higher $\alpha$-olefins. For their work, both were granted the Nobel prize in chemistry in 1963. ${ }^{[39.40 \mid}$ The main advantage in comparison to radical polymerization lays in the application of low temperatures and pressure. The catalyst systems are mainly based on the combination of a main group metal (I-III) alkyl or alkyl halogenide with transition metal halogenide, hydroxide, -oxide or alkoxide (IV-VIII). ${ }^{[3]}$ An example for this type of reaction is shown in Scheme 2.3.

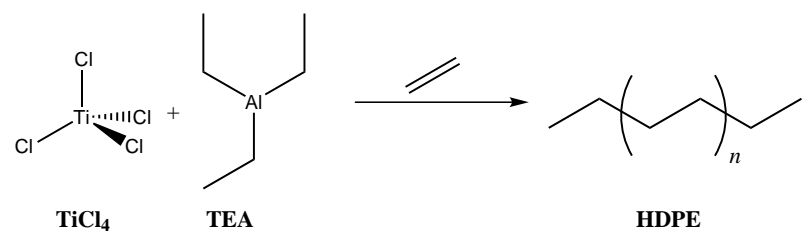

Scheme 2.3: Example for a ZN-catalyst system producing HDPE using titantetrachloride $\left(\mathrm{TiCl}_{4}\right)$ and triethyl aluminium (TEA).

The transition metal catalyst, here $\mathrm{TiCl}_{4}$ is insoluble in the applied solvents thus direct observation of the activation process is impossible. To gain insight into the activation process studies with $\mathrm{Cp}_{2} \mathrm{TiCl}_{2}$ and different alkyl aluminium compounds were performed. ${ }^{[41-44]}$ The coordination and monomer insertion into the Ti-C bond follows the mechanism of monomer addition reported by Cossée and Arlman. ${ }^{45-501}$ The combination of all these studies led to the mechanism of the alkylation of the $\mathrm{TiCl}_{3}$ surface by the alkyl aluminium compound and the process of monomer addition described in the following (see Scheme 2.4 on the facing page). [3]

The catalyst surface (5) is unsaturated and triethyl aluminium coordinates to an open reaction site (6). During disassociation of the aluminium reactant a chloride ligand is exchanged by an alkyl chain (7). This complex is able to coordinate the monomer to an open reaction site (8), thus the double bond is activated for insertion into the Ti-C bond (9). After insertion (10) a reactive site for syndiotatic polymer is formed. If the rearrangement of the polymer chain to (11) is faster than monomer addition isotactic 

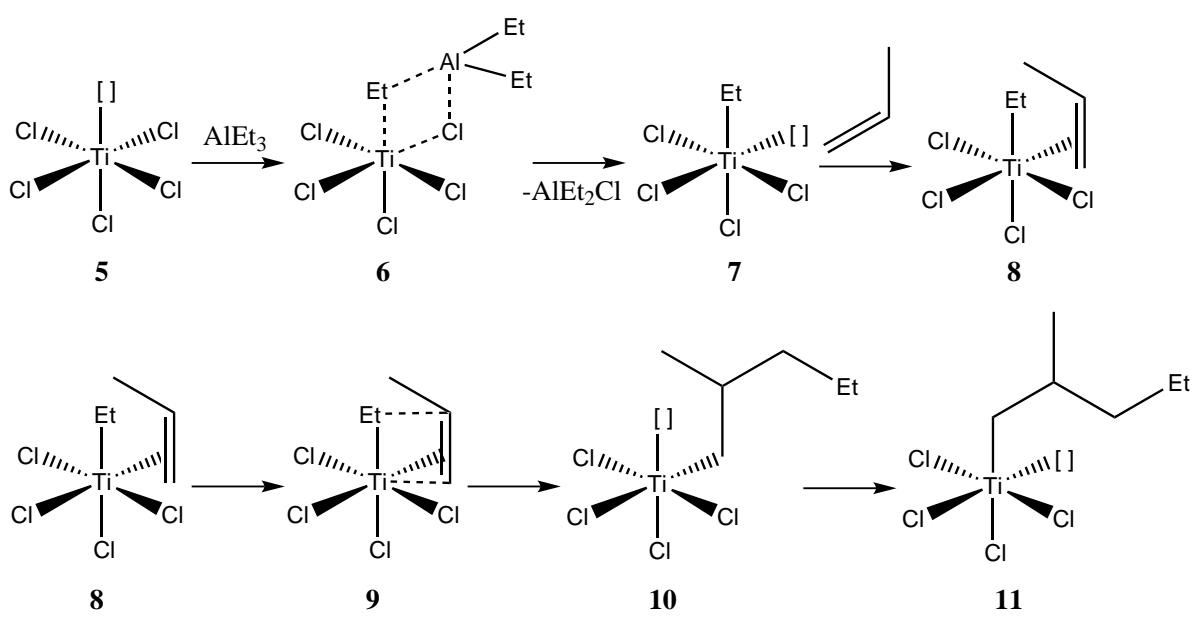

Scheme 2.4: Activation of a heterogenus $\mathrm{TiCl}_{3}$ surface with triethyl aluminium and addition of monomer. ${ }^{[3]}$

polymers are formed. The formed polymer chains can now either terminate via $\beta$ hydride elimination yielding a macromonomer and a hydride complex (12) or produce a polymeric aluminium species and the ethyl complex (7) due to chain transfer to TEA (see Scheme 2.5).

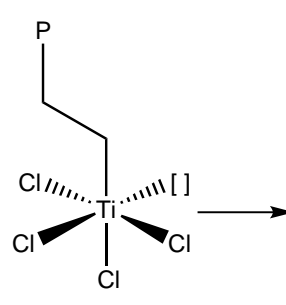

11

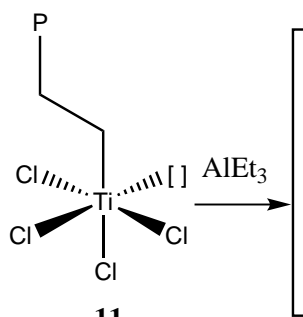

11

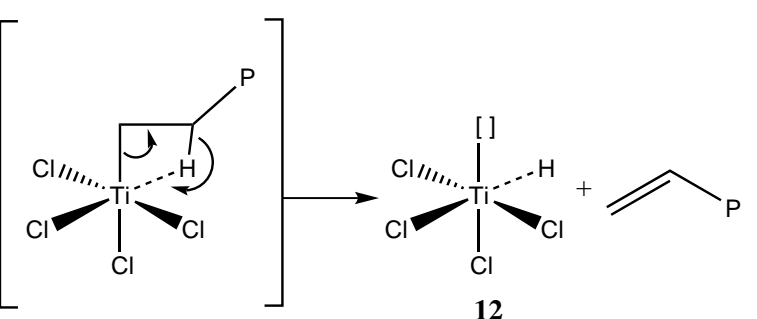

b-hydride elimination

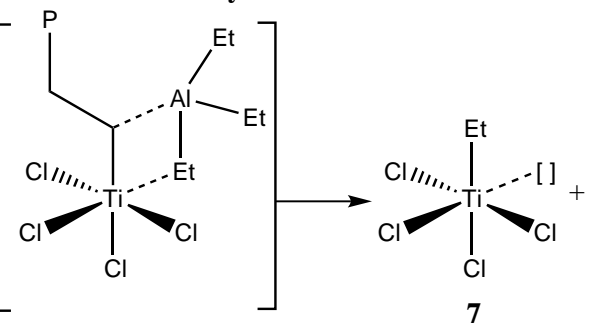

chain transfer to aluminium

Scheme 2.5: Chain growth termination via $\beta$-hydride elimination or chain transfer to triethyl aluminium. [3]

Based on the early studies ${ }^{[41-44]}$ the activation mechanism was investigated more precisely using different kinds of homogenous $\mathrm{ZN}$ catalysts and alkyl halogen aluminium compounds where performed. Various types of intermediate species and structures were discussed and characterized via different methods. ${ }^{[5152]}$ Henrici-Olivé and S. Olivé ${ }^{[53,54]}$ discussed an octahedral coordination sphere on the basis of EPR measure- 
ments. G. Fink ${ }^{[55,56]}$ found tetrahedral coordination by application of enriched ${ }^{13} \mathrm{C}-\mathrm{NMR}$ measurements and fully discussed a polymerization system for the quenched-flow polymerization of $\mathrm{Cp}_{2} \mathrm{TiEtCl}$ as catalyst with $\mathrm{AlEtCl}_{2}$ as co-catalyst ${ }^{[57-60]}$. By application of the methyl analoga a different behavior was found which led to the introduction of the Intermittent Chain Growth model. ${ }^{61]}$ It was found to be a suitable model in later mechanistic studies (see Scheme 2.6). ${ }^{\sqrt{62}}$ The titanium starting compound (Ti) is in equi-

$$
\begin{aligned}
& \mathrm{Ti}+1 / 2 \mathrm{Al}_{2} \stackrel{\mathrm{K}_{1}}{\rightleftharpoons} \text { Cat } \\
& \mathrm{Cat}+\mathrm{Al}_{2} \stackrel{\mathrm{K}_{2}}{\rightleftharpoons} \mathrm{Cat}^{*}
\end{aligned}
$$

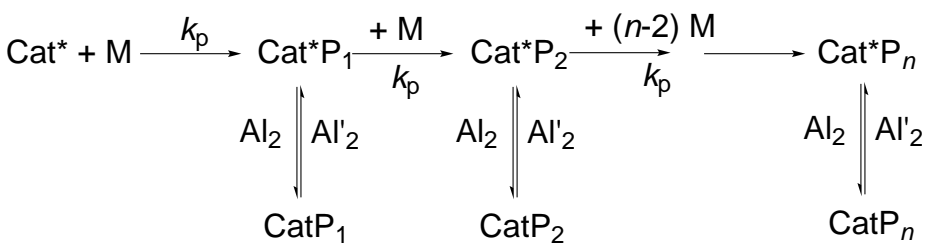

Scheme 2.6: Intermittent Chain Growth Model based on a titanium based catalyst $\left(\mathrm{Cp}_{2} \mathrm{TiRCl}, \mathrm{R}=\right.$ alkyl or $\mathrm{Cl})$ and an alkyl chloro aluminium compound $\left(\mathrm{AlR}_{m} \mathrm{Cl}_{3-m}\right)_{2}$. ${ }^{6163]}$

librium with the catalyst precursor (Cat) by association of an alkyl chloro aluminium $\left(\mathrm{AlR}_{m} \mathrm{Cl}_{3-m}\right)_{2}$ compound. By reaction with another a aluminium compound, the catalytic active species is formed (Cat*). Monomer adds to the complex (Cat* $\left.\mathbf{P}_{\mathbf{1}}\right)$ which either adds more monomer ( $\mathbf{C} \mathbf{a t}^{*} \mathbf{P}_{\mathbf{2}}$ ) or is deactivated in an equilibrium reaction by association/dissociation of the aluminium compound. This model allows chain growth while the catalyst can be deactivated at each state of polymerization.

The $\mathrm{ZN}$ polymerization is today applied mainly on support materials like e.g. $\mathrm{MgCl}_{2}$. The influence of the support material ${ }^{[64]}$, additives ${ }^{[65,66]}$ and the kinetics ${ }^{[67,68]}$ are a broad field of research. $69-73]$

\subsubsection{Polymerization in Presence of Methylaluminoxane (MAO)}

This mechanism was the basis for further studies with new types of activators applied after the discovery of methylaluminoxane (MAO), which was developed by Sinn and Kaminsky ${ }^{[74-76]}$ as an activator for alkylated metallocene catalysts. ${ }^{[75]}$ It led to an increase of activity and scope of possible catalysts. ${ }^{\text {[77-85] }}$ The structure of MAO is still not fully known [86-88], due to its complex nature. It consists of several oligomeric species in equilibrium and is discussed based on the structure motive (AlMeO) $)_{n}$. It is nowadays the most common activator for polymerization in industrial scale (5 million tons polymer in 2010). 88,89 


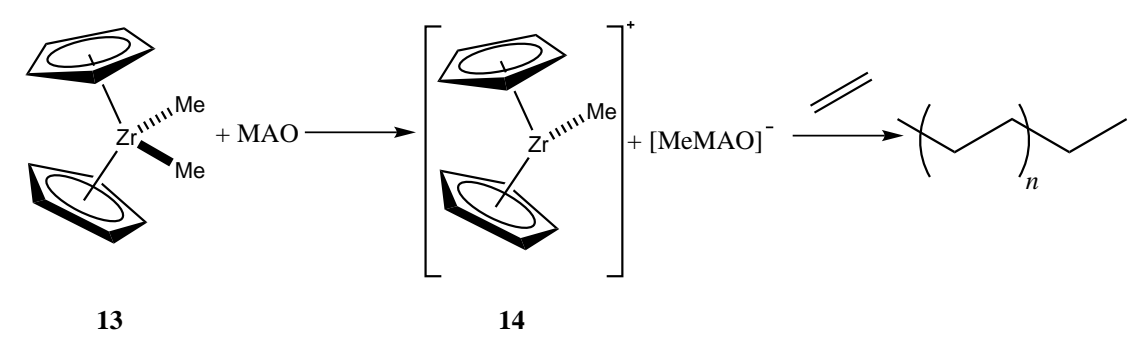

Scheme 2.7: Activation of bis(cyclopentadienyl)dimethylzirconium with methylaluminoxane (MAO) and polymerization.

Starting from an alkylated catalyst, bis(cyclopentadienyl)dimethylzirconium $\left(\mathrm{Cp}_{2} \mathrm{ZrMe}_{2}\right.$, see Scheme 2.7 13) and MAO, the cleavage of one methyl group from the $\mathrm{Zr}$ complex yields a separated anion and a positively charged complex (14). ${ }^{[63]}$ This complex is now very active for monomer addition. Several types of alkyl aluminoxanes are available ${ }^{[88,90-92]}$ while MAO is the most applied one. New types of activators as e.g. salts of weak anions and highly reactive cations for the abstraction of an alkylor hydride moiety were developed. ${ }^{\text {[1] }}$ Typical cations are the trityl cation $\mathrm{Ph}_{3} \mathrm{C}^{+}$and ammonium cations based on structure motive $\mathrm{HNRR}_{2}{ }^{+}$while anions are mainly based on the perflouroaryl borates and aluminates. ${ }^{[93,94]}$ In spectroscopic studies the system of choice is often $\left[\mathrm{Ph}_{3} \mathrm{C}\right]^{+}\left[\mathrm{B}\left(\mathrm{C}_{6} \mathrm{~F}_{5}\right)_{4}\right]^{-}$.

The ansa-metallocenes developed by H.H. Brintzinger and coworkers ${ }^{[5596]}$ extended the spectrum of possible ligands and thus provided the synthesis of highly reactive catalysts. ${ }^{1961}$ The activation mechanisms and intermediates are broadly researched ${ }^{\text {[97-991 }}$ and for metallocene catalysts the application of NMR ${ }^{[100-111]}$, FT-IR-[112[113] and UV/Visspectroscopy ${ }^{[114-127]}$ as well as quenched-flow studies ${ }^{[128]}$ and quantum mechanical calculations ${ }^{[129,130]}$ suggest the formation of several complex species. These species formed with MAO show that one methyl group is transferred and ion pairs are formed. These ionpairs can either be weak coordinated (15) or solvent separated (16, see Scheme 2.8 on the following page. ${ }^{[46}$

In the presence of trimethyl aluminium $\mathrm{AlMe}_{3}$ a third complex (17) is the found, allowing the chain transfer from the catalyst center to the aluminium. ${ }^{[131]}$ When using $\left[\mathrm{Ph}_{3} \mathrm{C}\right]^{+}\left[\mathrm{B}\left(\mathrm{C}_{6} \mathrm{~F}_{5}\right)_{4}\right]^{-}$the structures are similar (18-20) but the extracted methyl group reacts irreversible with the trityl cation. The anion is either strongly or weakly coordinated and in presence of $\mathrm{AlMe}_{3}$ a complex similar to MAO is formed. The application of MAO and other activators led to several kinetic studies. ${ }^{46,991}$ Furthermore, current developments lead to the synthesis of different polymer topologies ${ }^{[132] 133]}$ and functional polymers. [134-138] 


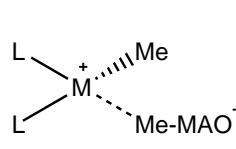

15

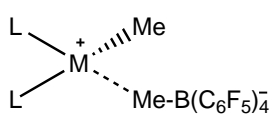

18

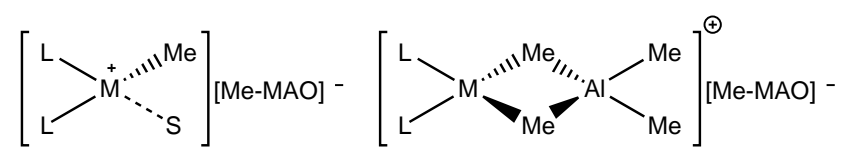

16<smiles></smiles>

19
17

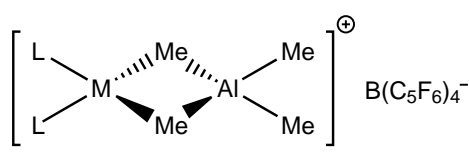

20

Scheme 2.8: Ion pairs formed via activation by MAO. Adapted from Bryliakov et al. .46] $^{[4 i t h ~ p e r m i s-~}$ sion from Elsevier.

\subsubsection{Monomer Addition}

The mechanism of Cossée and Arlman ${ }^{[45-50,139]}$ (cf. 2.2.2 on page 12) is basis for a more detailed discussion on the exact mechanism of monomer addition at the metal center (see Scheme 2.9). ${ }^{140]}$ The main difference between the four types are additional intermediate species, which involve the participation of hydrogen atoms. These $\alpha$-agostic contributions may lead to kinetic isotope effects. The Cossée-Arlman mechanism describes the addition of monomer to the complex $\mathbf{2 1}$ forming the monomer coordinated complex 22. This complex passes through into the transition state $\mathbf{2 3}$ by migration of an alkyl-chain to the coordinated monomer. Under inversion of the configuration the chain propagation is performed.

This model was followed by a second mechanism described by Rooney and Green ${ }^{[140-142]}$ which starts with a oxidative 1,2-hydrogen shift from the polymer chain of complex 25 forming complex 26. Monomer can coordinate to the complex yielding (27) from which a metalla-cyclobutane complex $\mathbf{2 8}$ is yielded. This complex performs chain propagation by a reductive elimination step (29).

The third model ${ }^{[140,143-146]}$ is a combination of the previous shown ones. Opposed to the second mechanism an $\alpha$-hydrogen atom from the polymer chain forms a hydrogen bond towards the zirconium atom (30), which allows monomer addition in a fixed conformation (31). With the hydrogen bond still present, a four ring based intermediate (32) similar to the Cossée-Arlman mechanism is formed. The chain propagates while the hydrogen is still facing towards the metal center (34).

The forth mechanism shows monomer coordination without interference from a hydrogen coordination (35 to 36 ), while in the transition state it lead to a favored conformation as a directing group (37). This directing functionality is still present after bond formation (38) and only discoordination of the hydrogen the open reaction side is accessible again (39). 

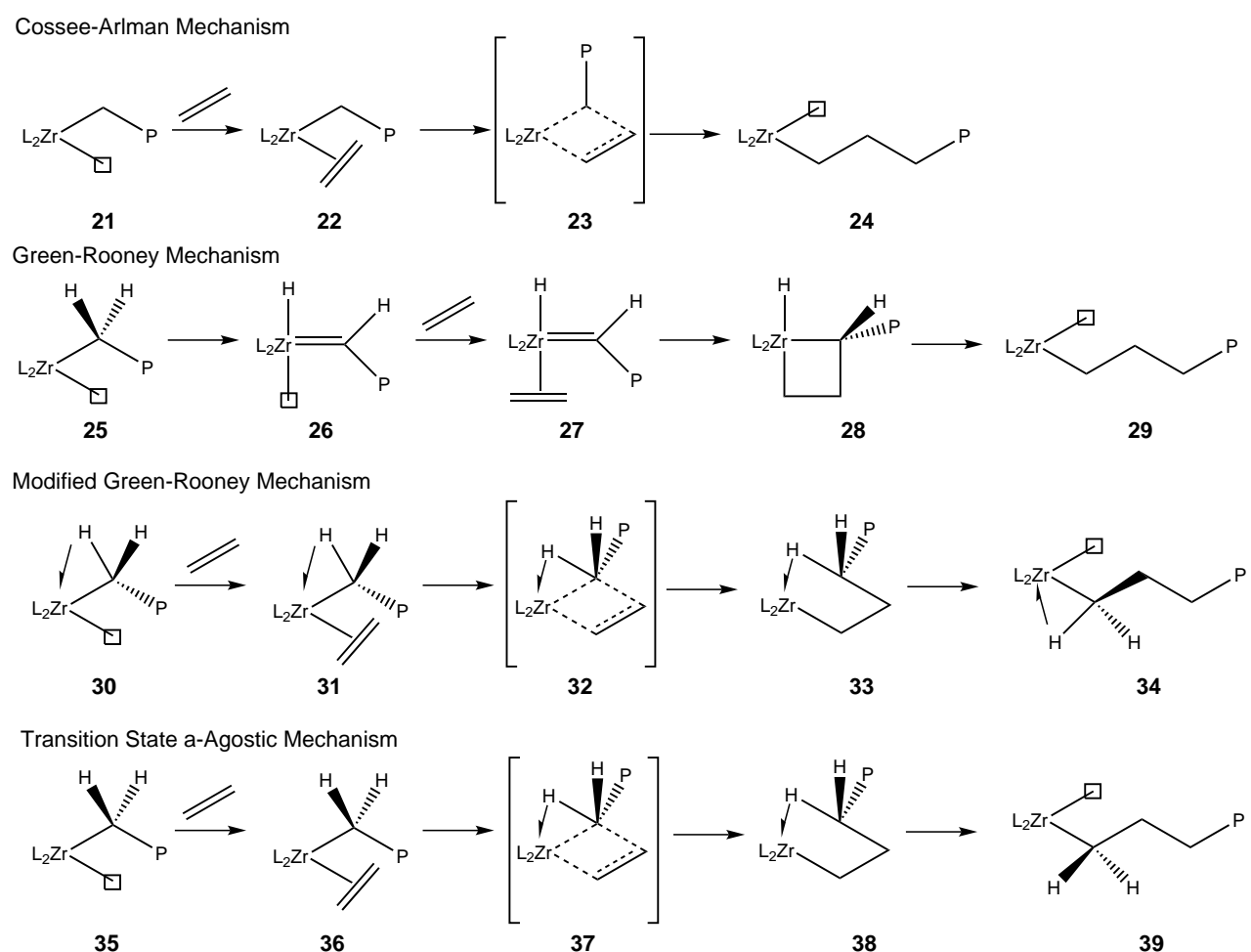

Scheme 2.9: Different monomer addition mechanism after Cossée-Arlmann mechanism, GreenRooney mechanism, Modified Green-Rooney mechanism and Transition State $\alpha$-Agostic Mechanism. Adapted with permission from Grubbs et al. ${ }^{[140]}$ Copyright 1996 Amercian Chemical Society.

The forth mechanism only shows a $\alpha$-agostic hydrogen interaction at the intermediate state. The mechanism 3 and 4 are quite similar to the early Cossée-Arlman mechanism. These mechanisms can show kinetic isotope effects. Several examples are known in literature. ${ }^{[147-150]}$ Typical values of $k_{\mathrm{H}} / k_{\mathrm{D}}$ are around of 1.3 especially for polymerization reactions. ${ }^{[140]}$

\subsubsection{Termination Reactions}

For the different catalysts system the main termination reaction is the $\beta$-hydride elimination (see Scheme 2.10, 40 to 42). ${ }^{[18]}$ Other mechanisms can be performed as well on the catalyst if monomer is coordinated.

The second mechanism describes the process which follows after monomer coordination (44). A $\beta$-hydrogen atom from the polymer chain is transferred to the monomer (45) yielding an unsaturated polymer chain and an active catalyst with an ethyl moiety (46). The third process transfers one $\alpha$-hydrogen from the coordinated monomer to the 


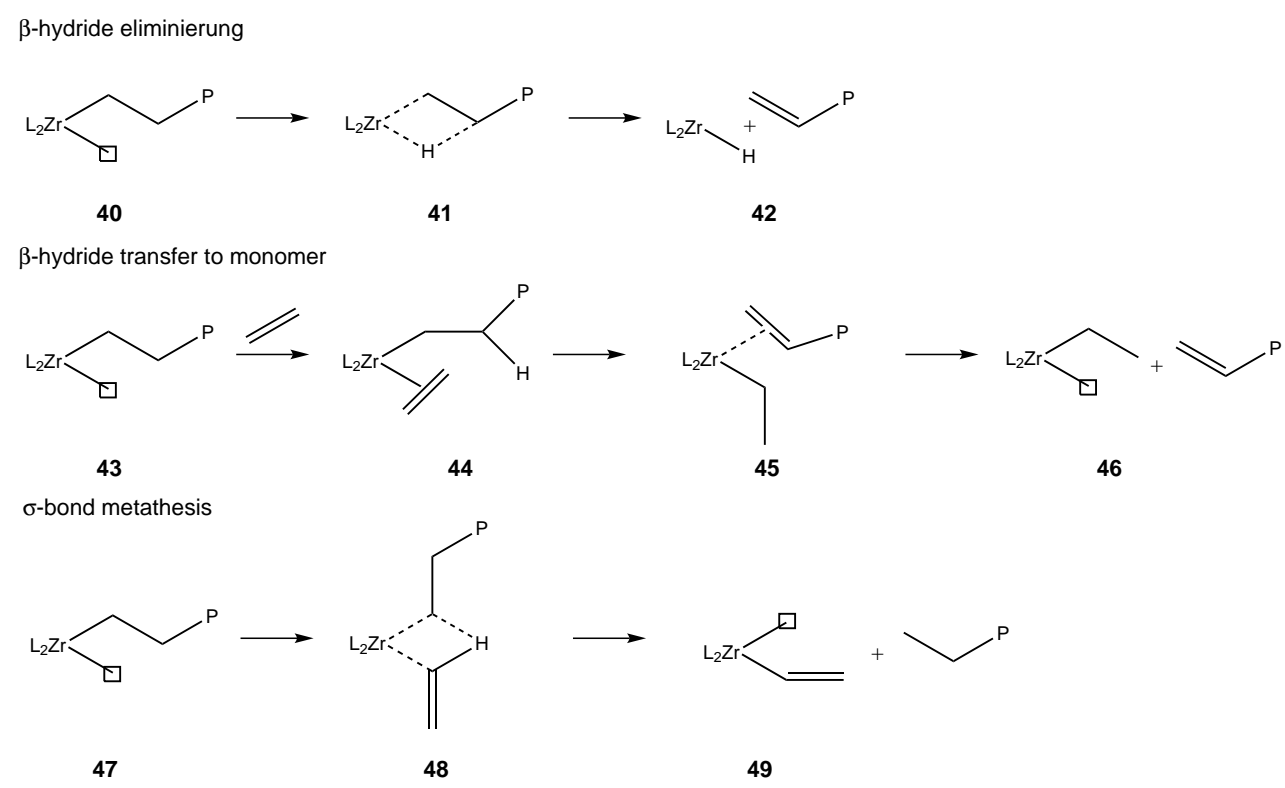

Scheme 2.10: The different termination reactions for a catalyst center via a) $\beta$-hydride elimination; $b$ ) $\beta$-hydride transfer to monomer and c) $\sigma$-bond metathesis.

polymer chain by an metathesis reaction (48) yielding an saturated polymer chain and ethylene moiety coordinated to the catalyticallly active complex (49). ${ }^{[151,152]}$

\subsubsection{Mechanistic Studies}

Additional to the broad analysis of the different species occurring during activation the overall mechanistic research is targeted by several methods for kinetic research. The different systems ${ }^{[153,154]}$ have only one monomer ${ }^{[63,124,155-162]}$ present or were studied via copolymerization. ${ }^{[163-167]}$ Kinetic data can be as well distinguished via copolymer analysis. ${ }^{[163,164]}$ Out of these studies two processes could be found which lead to interesting insights. Different studies were performed using quenched-flow and IRstudies of 1-hexene polymerization with $\left[\mathrm{rac}-\left(\mathrm{C}_{2} \mathrm{H}_{4}(1 \text {-idenyl })_{2}\right) \mathrm{ZrMe}\right]\left[\mathrm{MeB}\left(\mathrm{C}_{6} \mathrm{~F}_{5}\right)_{3}\right] \mathbf{5 0}$ (see Scheme 2.11 on the facing page). $157-159,168]$

Complex 50 was generated in situ and it was found that the complex is in equilibrium with the solvent separated complex 51. The first monomer addition to complex $\mathbf{5 2}$ has a lower reaction rate $k_{\mathrm{i}}$ than the rate of propagation $k_{\mathrm{p}}$ with longer alkyl chains coordinated (53). The process is stopped by $\beta$-hydride elimination with the rate coefficient $k_{\mathrm{t}}$ and yields the hydride complex 54 and a vinyl terminated polymer. The process was recently successfully kinetically modeled ${ }^{[169]}$ with a closer look at the MMD gained during the process. 


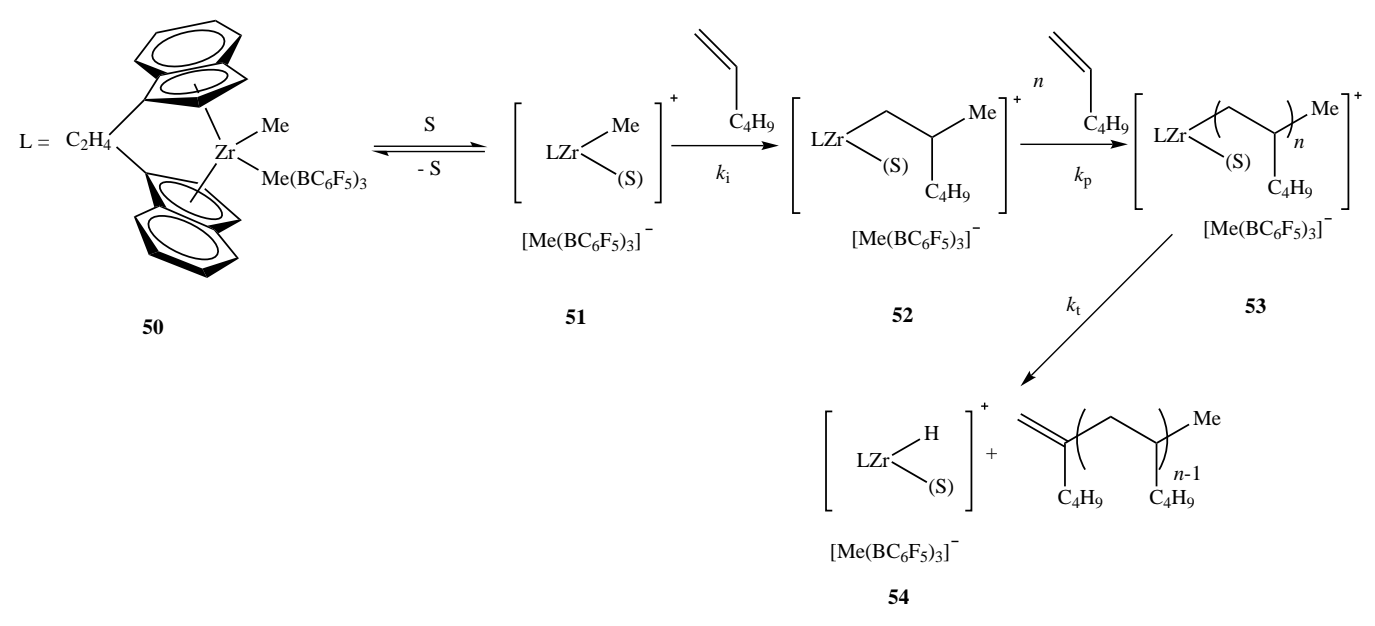

Scheme 2.11: Catalytic species during 1-hexene polymerization with $\left[\right.$ rac- $\left(\mathrm{C}_{2} \mathrm{H}_{4}(1-\right.$ idenyl $\left.\left.)_{2}\right) \mathrm{ZrMe}\right]\left[\mathrm{MeB}\left(\mathrm{C}_{6} \mathrm{~F}_{5}\right)_{3}\right]$ (50). ${ }^{[158]}$

The process was investigated for 1-propene polymerization with different activators by Song et al. ${ }^{63]}$ and their proposed mechanism for catalyst activation and monomer insertion process is depicted in Scheme 2.12 .

The catalyst precursor 55 reacts with a trityl-cation based activator. Depending on the alkyl chain on the catalyst two reaction products are possible (green). In case of a methyl group a bimetallic zirconium complex with a bridged methyl moiety (56) is found. With a more bulky ligand complex $\mathbf{5 7}$ is formed. The methylated variant of complex $\mathbf{5 7}$ is in equilibrium with complex 58 by association/dissociation of an additional molecule of $55 .{ }^{[170]}$ By addition of $\mathrm{AlMe}_{3}$ an equilibrium between complex 57 and 58 is found. These processes cause inhibition of the polymerization process. It is started by reversible monomer coordination to form complex 59, the starting point for monomer insertion into the $\mathrm{Zr}-\mathrm{C}$ bond. On this complex the monomer can perform either the favored 1,2-insertion yielding complex $\mathbf{6 1}$ or the disfavored 2,1-insertion towards complex $\mathbf{6 0}$, which shows a much lower reaction rate for the next monomer insertion. Complex $61 \mathrm{can}$ either add additional monomer, coordinate the anion $\mathrm{X}^{-}$or terminate the polymerization via $\beta$-hydride elimination. The termination product complex $\mathbf{6 2}$ is highly reactive to add monomer and forms a complex similar to 57 . As shown before the first monomer insertion has a different rate coefficient. In both cases no exchange towards a CTA has been investigated. 


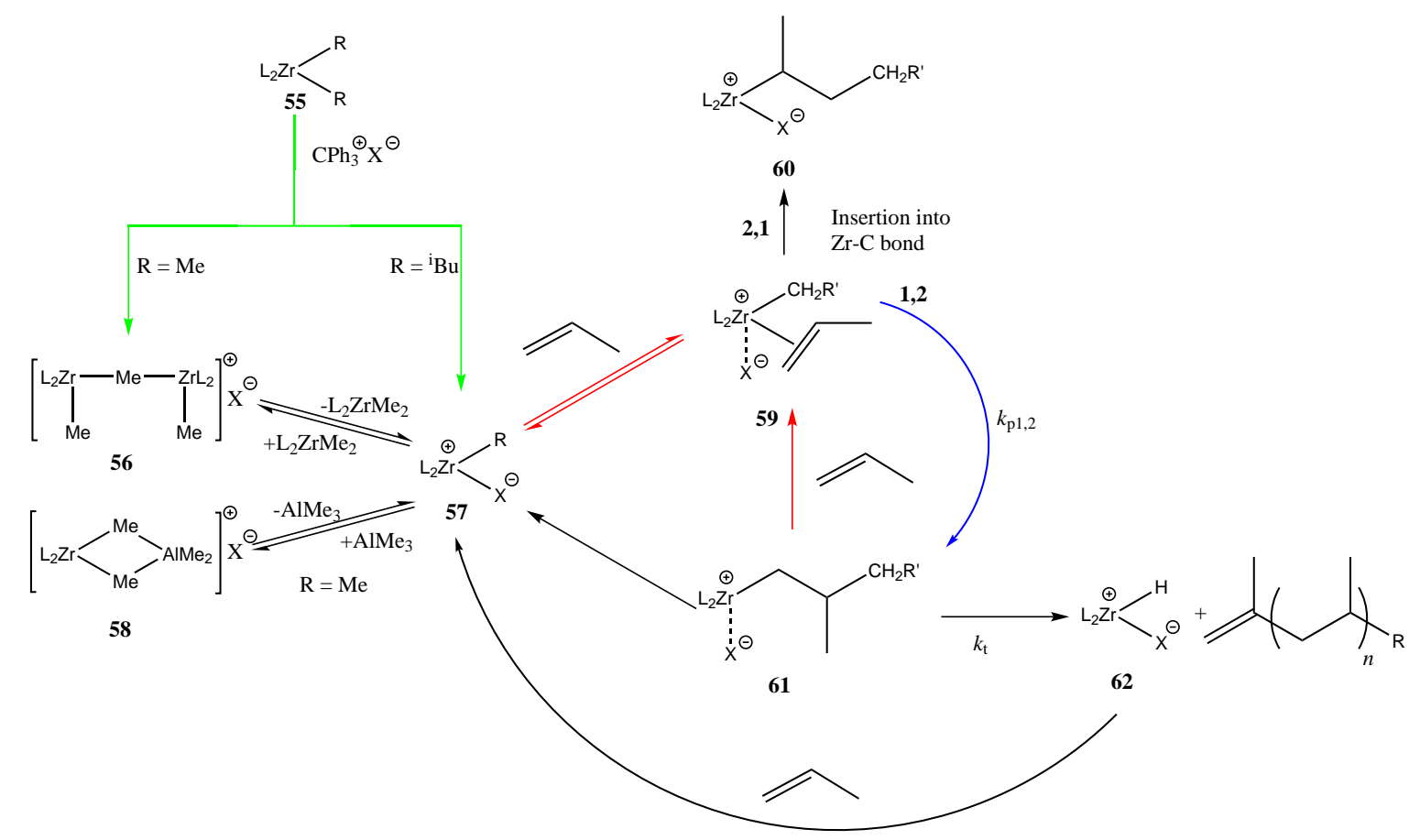

Scheme 2.12: Activation and insertion mechanism of propylene for $r a c-\mathrm{Me}_{2} \mathrm{Si}\left(1\right.$-indenyl) ${ }_{2} \mathrm{ZrMe}_{2}$ activated by trityl-cation based activators. See text for details. Adapted with permission from Song et al. ${ }^{[63]}$ Copyright 2003 American Chemical Society.

\subsection{Living/Controlled Polymerization Techniques}

In the last decades controlled and/or living polymerization processes were in the main focus of research. The principle was defined by M. Szwarc in $1956^{[171]}$ on the basis of the anionic polymerization. During this process, the growing polymer chains face neither termination nor transfer. Anionic polymerization is the perfect living system, but it has a high synthetic demand concerning purification and reaction performance, due to the high reactivity of the formed anions. After initiation the formed anion shows a high stability over several days and the addition of a second monomer is possible $e^{[171]}$ making it is easy to produce BCPs. Current developments in this field have been recently summarized in a review by A. Hirao et al. 6

Catalytic systems are also able to follow the principle of living polymerization ${ }^{[172-174]}$ while only one chain per catalyst is growing. ${ }^{[174]}$ To fulfill the category of (quasi) living polymerizations, a system is required to match the following conditions: ${ }^{[3,175]}$

- The system has neither termination nor transfer reactions.

- The chain growth must start equally at all chains and show a constant propagation rate 
- Narrow MMD with dispersity close to 1.

- Linear increase of $\bar{M}_{\mathrm{n}}$ with conversion

- Predictability of $\bar{P}_{\mathrm{n}}$ as a function of the monomer/initiator ratio.

This definition is valid for a wide range of processes from all kinds of polymerizations, which are then either labeled "living" or "controlled". For each major principle of the chain growth mechanism such reactions are known. The principles for radical polymerization and one method for coordination polymerization will be discussed in the following. Living cationic polymerization was reported by Miyamoto et al. ${ }^{[176]}$ and the process was reviewed recently. ${ }^{[177]}$

\subsubsection{Reversible-Deactivation Radical Polymerization (RDRP)}

Controlled polymerization processes were found also in the field of radical polymerization. Via the addition of a control agent (CA) (see Scheme 2.13) the radical function on a polymer chain (64) is reversibly transferred or deactivated (63). Termination reactions to dead polymer (65) and transfer reactions can still occur. During this process the radical concentration may be lowered to a small amount, making the chance of such a reaction negligibly small.

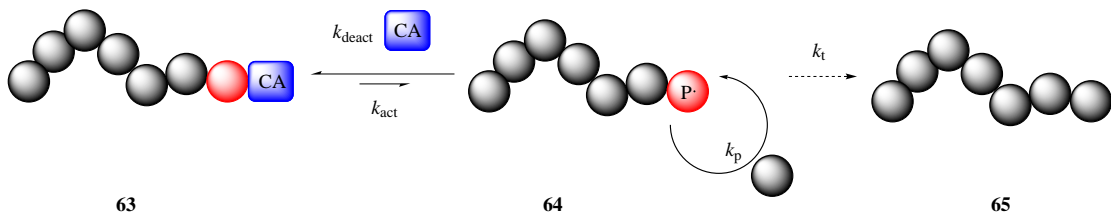

Scheme 2.13: Main principle of controlled radical polymerization. A control agent (CA) reversibly deactivates the polymer radical $\mathrm{P}$ yielding an dormant species while chance of termination is lowered.

Several methods are known ${ }^{[178-185]}$ and the most prominent examples following a similar mechanism are ATRP [182,183] and Nitroxide-mediated polymerization (NMP) ${ }^{[184}$. The scope of these reactions have been recently summarized. [186,187]

The Reversible Addition-Fragmentation Chain Transfer Polymerization (RAFT) is also a powerful method to produce polymer with a narrow molar mass distribution. ${ }^{[180,181]} \mathrm{A}$ RAFT-agent is added to a CRP reaction as CTA and in contrast to the shown principle before it is only decreasing the rate of polymerization slightly by shuttling the growing 


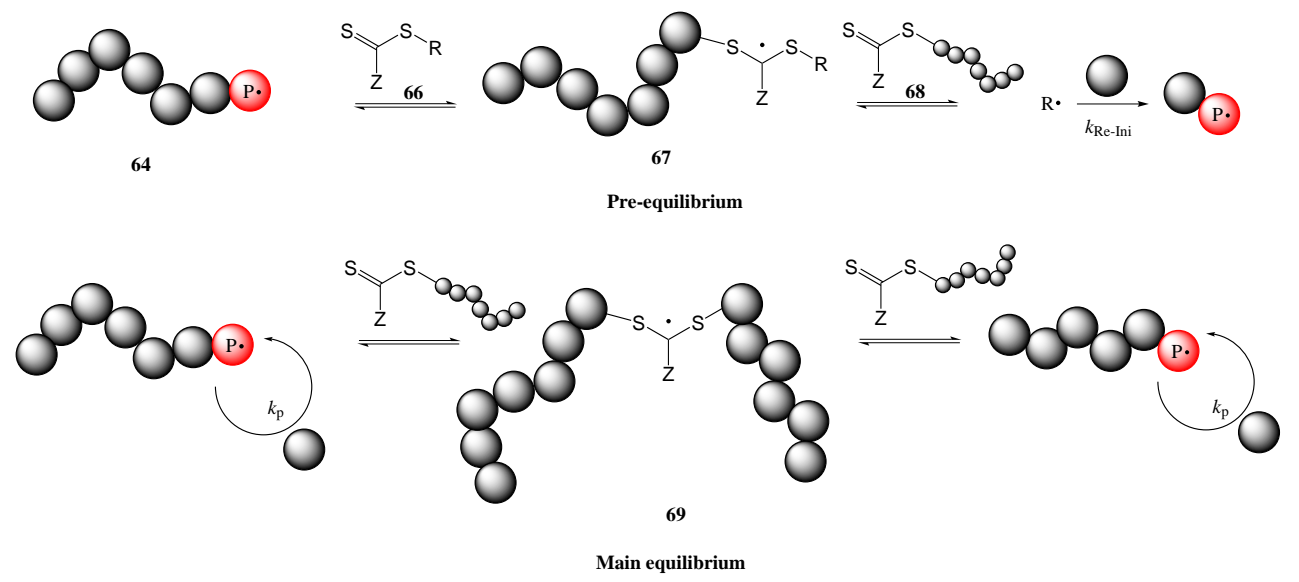

Scheme 2.14: Pre-and main equilibrium of the RAFT process which is occur additional to CRP.

polymer chain via an intermediate species (see Scheme 2.14).

The reaction mechanisms involves two stages. First the radical functionalized polymer chain 64 reacts with the RAFT-agent precursor (66) yielding the intermediate 67 with the radical stabilized on the carbon atom of the dithioester moiety. This intermediate either reacts back or sets free the polymeric RAFT agent $\mathbf{6 8}$ and the re-initiating group $(\mathrm{R})$, which starts chain growth of another polymer chain. This process must be fast, otherwise the MMD gets broadened and the polymerization cannot be performed in a controlled manner. In the main equilibrium two polymer chains are shuttled by formation of an intermediate with two polymer chains (69). Monomer addition is possible only in short time intervals. Several sulfur based functionalities can act as a RAFT-agent like dithiobenzoates, trithiocarbonates, dithiocarbamates and xanthates. ${ }^{[188]}$ All have in common that at least one " $\mathrm{S}=\mathrm{C}-\mathrm{SR}$ " moiety is found. The lifetime of the intermediate is influenced by the stabilizing group (Z). The RAFT-process is well investigated ${ }^{[7,188}$ and simulation models are available. ${ }^{[189]}$ Besides, it is most similar to the process of catalyzed chain growth found in coordination polymerization in presence of a CTA. ${ }^{201}$ This broad understanding lead to new kind of polymers ${ }^{[190-192]}$ and applications ${ }^{[7]}$, e.g. in stimulated emission depletion microscopy (STED) for staining of BCP-phases ${ }^{[193]}$, in polymer networks ${ }^{[194]}$ and for new nano structural approach based on gold nanoparticles. ${ }^{[195,196]}$

\subsubsection{Coordinative Chain Transfer Polymerization}

In principle the Coordinative Chain Transfer Polymerization (CCTP) is based on the application of a transition metal catalyst and a main group metal chain transfer agent (CTA). The basic reactions are shown in Scheme 2.15 on the next page.

The growing polymer chain is exchanged from the active catalyst site $\mathbf{7 0}$ to the dor- 


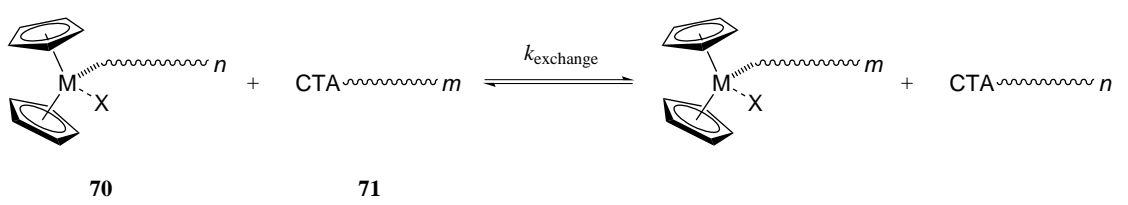

Scheme 2.15: Basic principle of Coordinative Chain Transfer Polymerization (CCTP) via chain transfer between a metallocene complex and a CTA.

mant CTA 71 via degenerative chain transfer. The process is considered as living, if termination via $\beta$-hydride elimination is negligibly small. A broad spectrum of systems is known ${ }^{[197-199]}$ as well as mechanistic ${ }^{[200]}$ and kinetic studies. CCTP is the basis for the synthesis of different functional polymers. ${ }^{[201-203]}$ Outgoing from this approach two major concepts were found. First, if all chains on the CTA get activated quickly, the polymer seems to grow on the main group metal ${ }^{[151 / 199]}$ which is then named catalyzed chain growth (see below) similar to the "Aufbaureaktion". 204]

Secondly, CCTP is the basis for the main principle of chain-shuttling polymerization shown by Arriola et al. ${ }^{[205]}$ In this setup two types of catalyst were used, one e.g. only

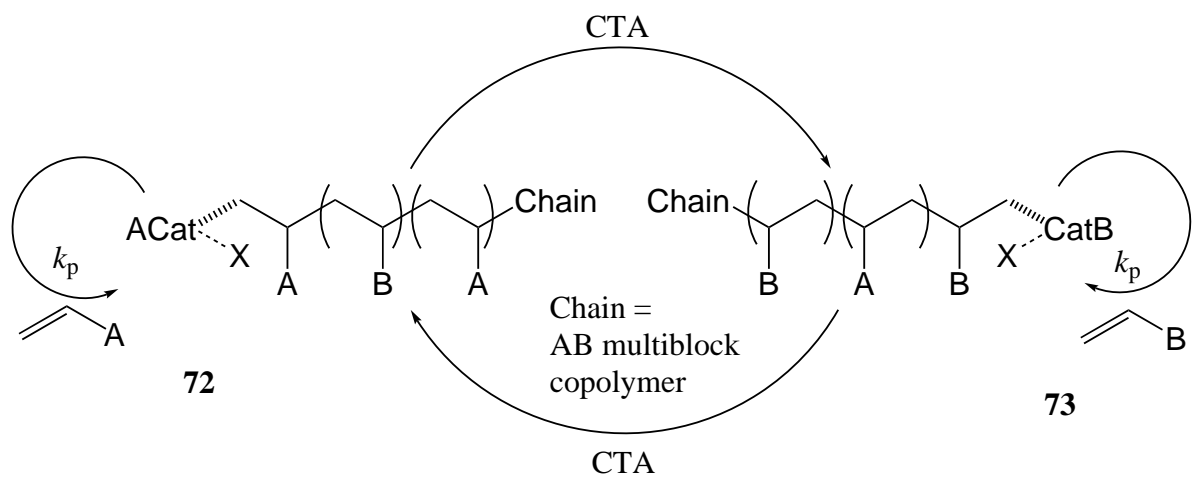

Figure 2.2: Chain-shuttling between catalyst 72 and catalyst 73 . Each monomer can only be inserted at the corresponding catalyst yielding mircostructured multiblock copolymers.

propagating higher $\alpha$-olefins (72) while second catalyst (73) can build in only ethylene. The growing polymer chain then is transferred via a CTA, often $\mathrm{ZnEt}_{2}{ }^{[206]}$, from one active side to the other producing micro structured multiblock copolymers. ${ }^{205,207]}$ The process was recently kinetically analyzed by Zhang et al. ${ }^{[208-210]}$ and additional modeled by Mohammadi et al. ${ }^{[211]}$ via a Monte Carlo approach. Further studies were performed via Deuterium labeling. ${ }^{[212]}$ 


\subsubsection{Catalyzed Chain Growth}

The process of CCG was established by V. C. Gibson ${ }^{[19]}$ basing on the polymerization of ethylene with bis(imido)pyridyl iron ${ }^{[213]}$ and diethyl zinc. ${ }^{[151,214,215]}$ The main principle is based on the reversible chain transfer between a bifunctional organometallic reagent 75 and the catalytic active species $\mathbf{7 4}$ of a lanthanide or transition metal complex (see Scheme 2.16).

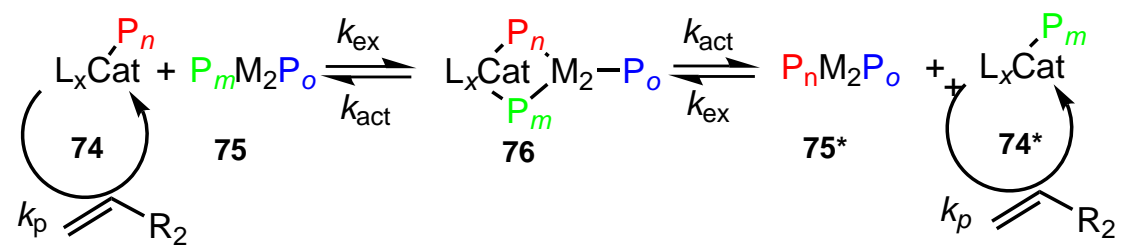

Scheme 2.16: Basic principle of CCG with a bifunctional main group CTA (75).

After full activation of all alkyl chains on the CTA, the polymer chains are exchanged via association/dissociation to complex 74 forming a bimetallic complex $\mathbf{7 6}$. The fast chain shuttling yields uniform chain growth for all polymer chains. It is important that under the applied conditions polymer chains undergo neither propagation nor termination while coordinated to the CTA, yielding a dormant species. The process was found suitable for chromium [216-219], iron [151,215], lanthanide ${ }^{[220-222]}$ and hafnium [223,224] based catalysts. For bis(imido)pyridyl iron a CTA screening based on $\mathrm{Al}(\mathrm{R})_{3}, \mathrm{Ga}(\mathrm{R})_{3}$, $\mathrm{Mg}(\mathrm{R})_{2}$ and $\mathrm{Zn}(\mathrm{R})_{2}$ was performed. ${ }^{[151]}$ It was found that $\mathrm{ZnEt}_{2}$ is the most active CTA, while $\mathrm{ZnMe}_{2}$ and $\mathrm{Zn}^{i} \mathrm{Pr}_{2}$ were also suitable. Based on these results a catalyst screening with $\mathrm{ZnEt}_{2}$ was performed ${ }^{[215]}$ resulting in only a few working combinations.

After polymerization all polymer chains are functionalized with a carbon-metal bond. This functionality can easily be converted as shown in Figure 2.3 on the next page. 23,225] Starting from the metal-carbon bond the polyethylene can be transferred by addition of elemental iodine ${ }^{[23,225]}$, sulfur ${ }^{[226]}$ or dry air ${ }^{[23]}$. The most common reaction is the addition of iodine. The product can easily be transformed using substitution reactions to e.g. an azide moiety ${ }^{[225]}$ allowing the azide-alkine cycloaddition reaction ${ }^{[227,228]}$ which is broadly applicated in polymer science. ${ }^{[22]}$ Based on this reaction the full range of functionalities can be introduced (see Figure 2.3). 


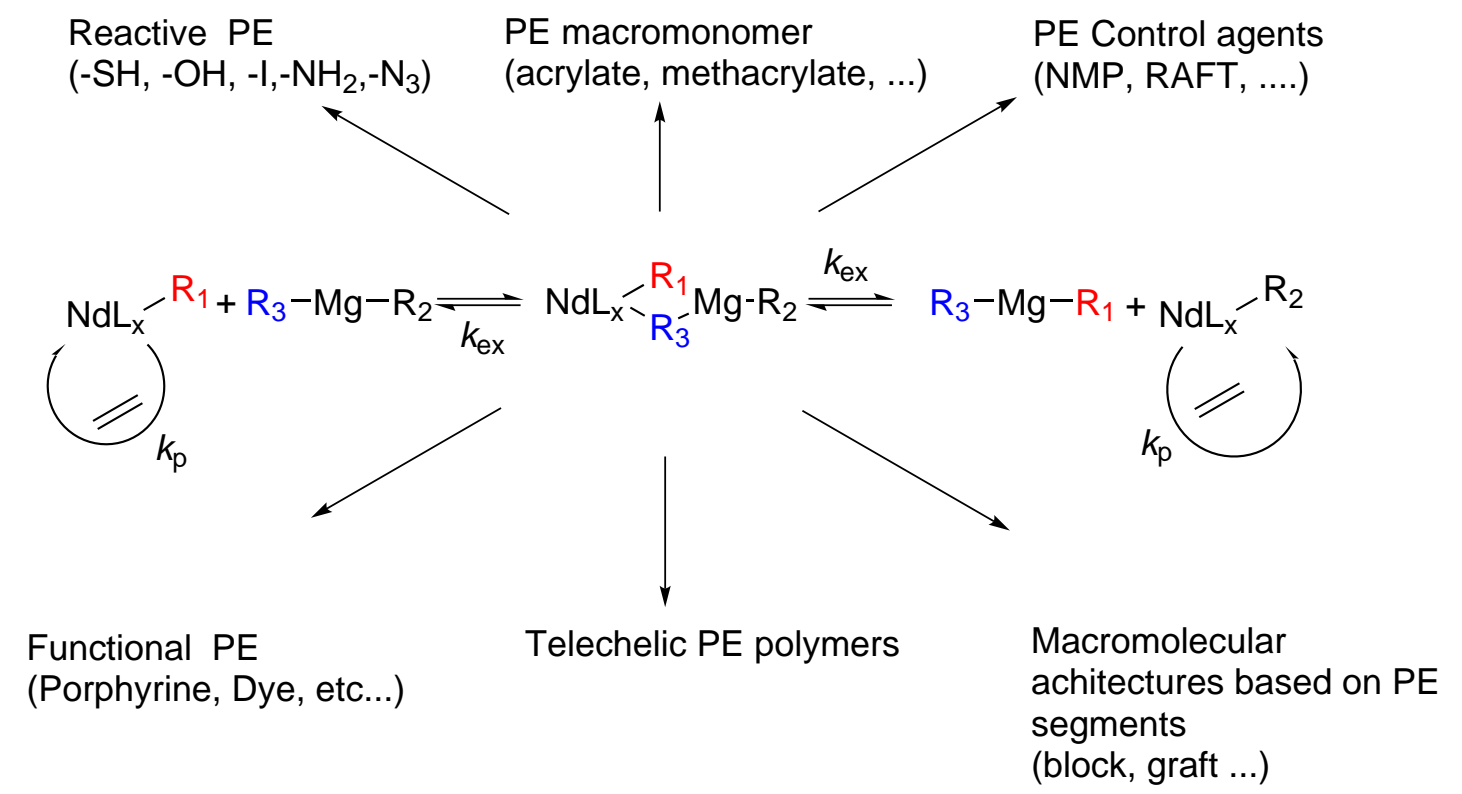

Figure 2.3: Variety of modifications available via CCG polymerization and post-polymerization synthesis. Adapted from Mazzolini et. al. ${ }^{[23]}$ with permission of The Royal Society of Chemistry.

\subsubsection{Mechanistic Studies of CCTP}

Recently two working groups published reaction mechanisms and kinetic studies for the reactions following the concept of CCTP. The first was proposed by F. Rouholahnejad et al. ${ }^{[230}$ based on $\mathrm{Cp}^{*}{ }_{2} \mathrm{ZrCl}_{2}$ activated by $\mathrm{MAO}$ with TMA as a result of previous observations. ${ }^{231,232]}$

In the model (see Scheme 2.17 on the following page) several reaction pathways are introduced. Beginning with the activation process of the catalyst precursor 77 by MAO to form the active complex $\mathbf{7 8}$ which adds monomer in a first addition process to complex 79. After that the polymer chain is introduced via intermediate $\mathbf{8 0}$ into $\mathrm{Zr}-\mathrm{C}$ bond into the polymeric active complex $\mathbf{8 1}$. This complex can now undergo reversible chain transfer with a trialkylated aluminium compound to complex $\mathbf{8 2}$ or irreversible chain transfer with MAO to complex 83. After monomer coordination $\mathbf{8 4}$ the chain growth is achieved. Due to experimental results the termination reactions via $\beta$-hydride transfer to monomer 85 and $\beta$-elimination 86 were excluded because no chains with an even number of carbon atoms were yielded.

In the second work, J. Camara et al. ${ }^{[131]}$ reported the introduction of $\alpha$-olefins into the carbon-aluminium bond (see Scheme 2.18$)$ for the catalyst $\left[\right.$ rac- $\left(\mathrm{C}_{2} \mathrm{H}_{4}(1 \text {-idenyl })_{2}\right) \mathrm{Zr}(\mu$ $\left.\mathrm{Me})_{2} \mathrm{AlMe}_{2}\right]\left[\mathrm{B}\left(\mathrm{C}_{6} \mathrm{~F}_{5}\right)_{4}\right]$, $\left.\left[\mathrm{Cp}_{2}\right) \mathrm{Zr}(\mu-\mathrm{Me})_{2} \mathrm{AlMe}_{2}\right]\left[\mathrm{B}\left(\mathrm{C}_{6} \mathrm{~F}_{5}\right)_{4}\right]$ and $\left.\left[\mathrm{Me}_{2} \mathrm{C}(\mathrm{Cp})_{2}\right) \mathrm{Zr}(\mu-\mathrm{Me})_{2} \mathrm{AlMe}_{2}\right]\left[\mathrm{B}\left(\mathrm{C}_{6} \mathrm{~F}_{5}\right)_{4}\right]$.

The catalyst is in a dormant species (87) which is in equilibrium with the catalytic active 


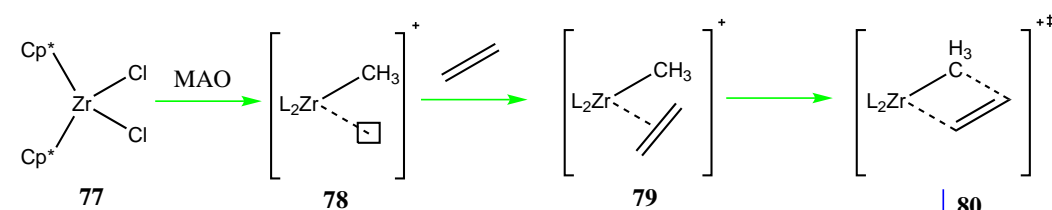

78

80

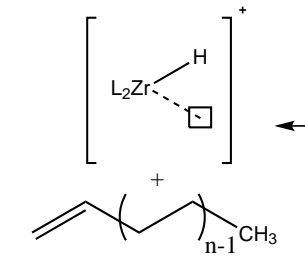

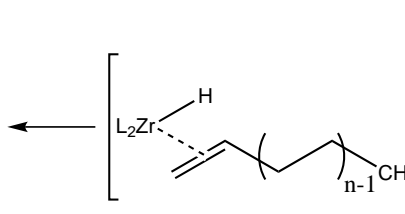

86<smiles>C1CCCCC1</smiles>

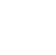

even chains

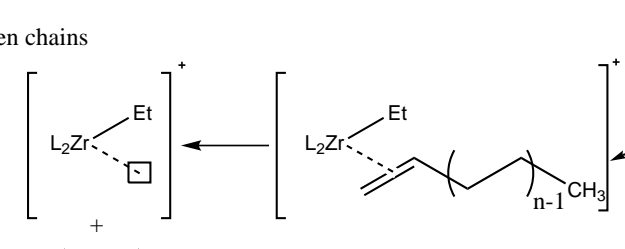<smiles>C=CCCCC(C)C</smiles><smiles>CCCC(C)C(Cl)CCC</smiles>

81

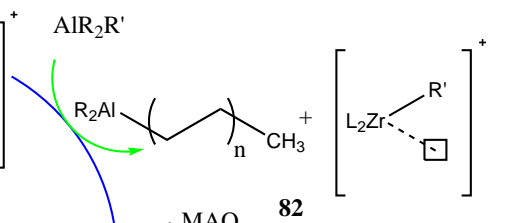
uneven chains<smiles>CC#CC(COC)C(C)OCCC(C)C</smiles>

85

84

83

Scheme 2.17: Reaction mechanism for narrowly distributed polyethylene by activation of $\mathrm{Cp}_{2}{ }_{2} \mathrm{ZrCl}_{2}$ by MAO and chain transfer to aluminium by TMA proposed by F. Rouholahnejad et al. ${ }^{[230]}$ For details see text. Adapted with permission from F. Rouholahnejad et al. ${ }^{[230]}$ Copyright 2009 American Chemical Society.

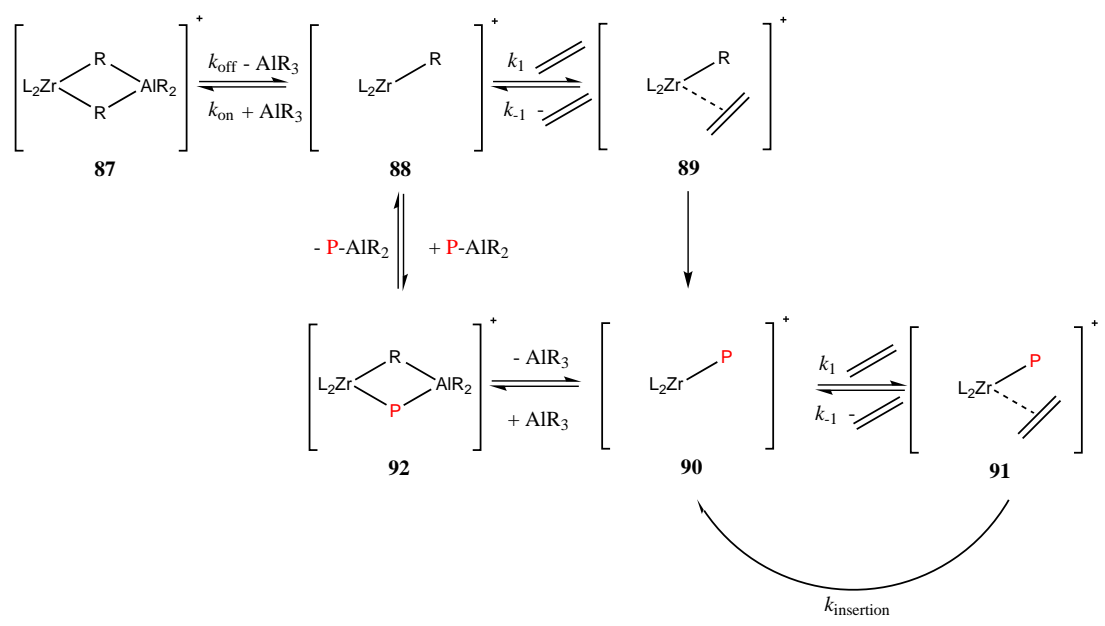

Scheme 2.18: Reaction mechanism of carboalumination. For details see text. Adapted with permission from J. Camara et al. ${ }^{[131]}$ Copyright 2011 Amercian Chemical Society. 
form 88 by association and dissociation of $\mathrm{AlMe}_{3}$. The complex 88 is in equilibrium with species 89 by the coordination and discoordination of monomer while the insertion into the $\mathrm{Zr}-\mathrm{C}$ bond is an additional reaction step. The formed complex $\mathbf{9 0}$ is able to add monomer for further chain growth (91) or can react with $\mathrm{AlMe}_{3}$ forming complex 92 which on the one hand may dissociate $\mathrm{AlMe}_{3}$ and react back to 90. On the other hand the polymer chain undergoes chain transfer back to $\mathbf{8 8}$, and a polymeric aluminium compound is formed. In comparison to the first model, no MAO is present, which excludes one transfer step. The kinetics of this model have been described as a Michealis-Menten like kinetics as found for enzymes, where the $\mathrm{AlMe}_{3}$ acts as an inhibitor and $\mathbf{8 8}$ must add a substrate (monomer) before polymerization can occur.

\subsubsection{Nickel Catalyzed Chain Growth (Ni-CCG)}

The method of Nickel Catalyzed Chain Growth or Kumada catalyzed transfer polymerization is a versatile tool to produce conductive polymers. It was found independently by Yokozawa ${ }^{[233]}$ and McCullough ${ }^{[234]}$ in 2004 and is an easy way to produce conjugated polyarenes $^{[235-237]}$. The polymerization reaction ${ }^{[235,238]}$ of 2,5-dibromo-3-hexylthiophene is depicted in Scheme 2.19. 2,5-dibromo-3-hexylthiophene 93 is transferred via metalhalogen exchange into a Grignard reactant (94) and the Nickel catalyst, here $\mathrm{Ni}(\mathrm{dppp}) \mathrm{Cl}_{2}$, undergoes a transmetalation with two Grignard type monomers. One thiophene moiety is inserted into the $\mathrm{Ni}-\mathrm{C}$ bond (complex 95) and starts the polymerization reaction where further monomer is introduced by a reaction cycle, starting with transmetalation (96), reductive elimination of the polymer chain (complex 97) and an intramolecular oxidative addition (complex 98). The resulting polymer 98 is capped with the Nickel-catalyst allowing in situ endgroup modification via the reaction with another kind of Grignard reactant like an allyl-Grignard (see 99) or via protonolysis (see 100). Several different kinds of polymer types are known and the reaction is not limited to the thiophene back bone. ${ }^{[239,240]} \mathrm{A}$ wide range of different block copolymers is also available. ${ }^{[241+244]}$ 


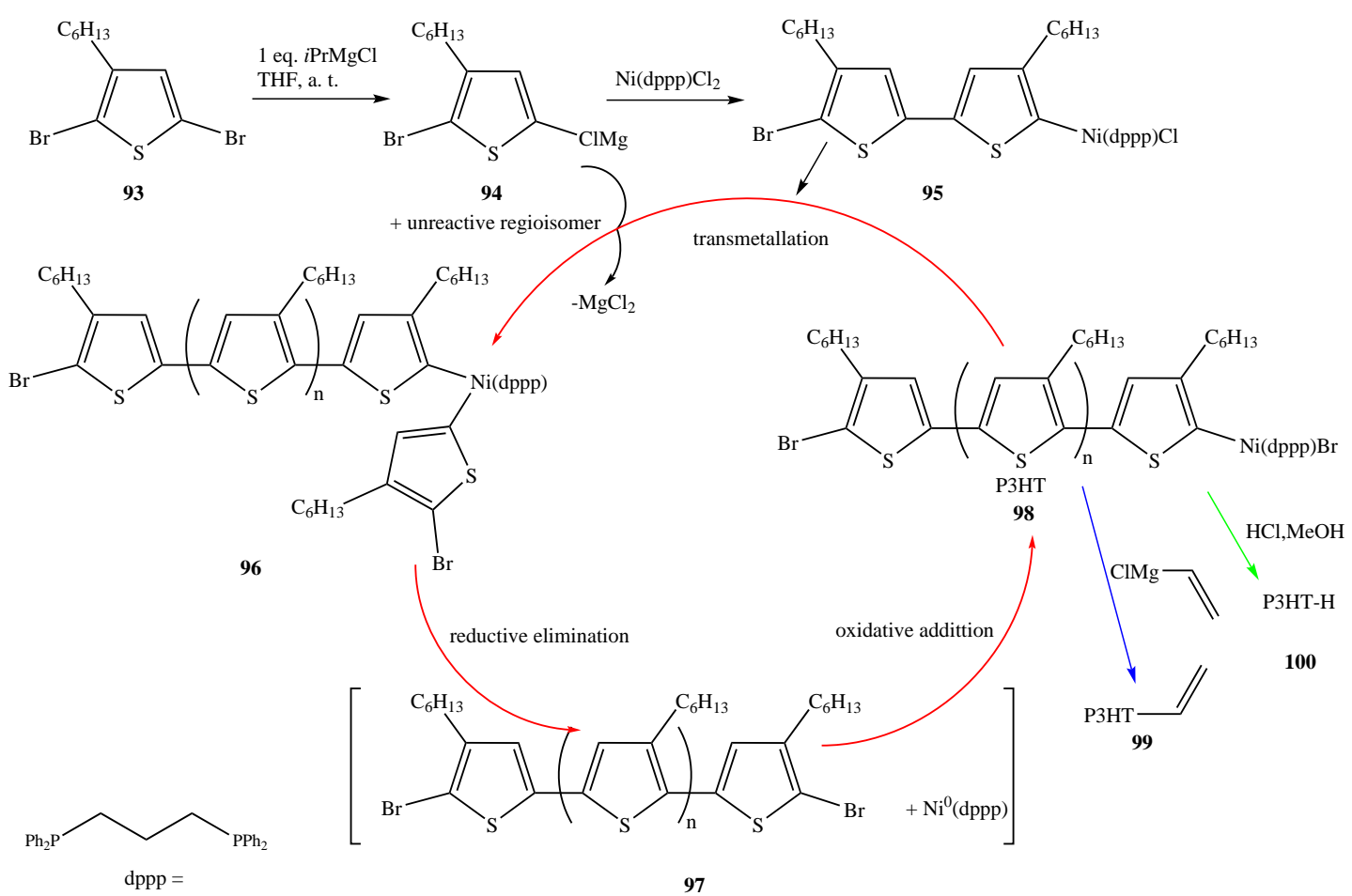

Scheme 2.19: Ni-CCG polymerization of 2,5-dibromo-3-hexyl-thiophene with $\mathrm{Ni}(\mathrm{dppp}) \mathrm{Cl}_{2}$ as catalyst. [235]

\subsection{Methods}

\subsubsection{Infrared (IR) and Ultraviolet-Visible (UV/Vis) spectroscopy}

IR- and UV/Vis-spectroscopy are based on the absorption of electromagnetic radiation of different wavelengths. The initial intensity $I_{0}$ is reduced to $I$ following the Lambert-Beer law on the way through the sample:

$$
\begin{aligned}
\operatorname{Tr} a . & =\frac{I}{I_{0}} \\
I & =I_{0} 10^{-\epsilon c d} \\
\log \frac{I}{I_{0}} & =-\epsilon c d
\end{aligned}
$$

The transmission (Tra., eq. 2.12) is defined as the quotients $I$ and $I_{0}$. The transmission can be calculated by the molar absorption coefficient $\epsilon$ in dependence of the concentration $c$ and the thickness $d$ of the sample (2.13).

Equation 2.13 can be transformed into equation 2.14. Introducing the absorbance $(A$, 
2.15) the equation can be transferred into equation 2.16, which can be easily used for calculations of concentrations when the other parameters are known.

$$
\begin{aligned}
& A=\log \frac{I_{0}}{I}=-\log \frac{I}{I_{0}} \\
& A=\epsilon c d
\end{aligned}
$$

FT-IR Spectroscopy In Fourier-Transformation (FT)-IR-spectroscopy the vibrational energies of molecules were measured. These can be the vibrations of chemical bonds as well as higher molecule vibrations. Important in this case is the occurrence of a transition dipol moment $\mu_{l, u}$ between the states the lower and upper state. The vibrational states in molecules are commonly approximated as an anharmonic oscillator. This model allows the dissociation of the atoms at high potential energy and the occurrence of different kinds of absorption bands, which mark transitions from the ground state to higher vibrational states. Such overtones were chosen for the determination of the conversion of vinyl monomers ${ }^{[245-248]}$, because these bands are well separated. They allow the application of high concentrations of monomer and long measuring cells.

The advantage of FT-IR is that a full spectrum is collected in short time interval as a interferogram. The absorbance of the single wavelength is extracted afterwards mathematically via the process of Fourier-Transformation. All these calculations were directly performed by the acquisition software and result direct in a full IR-spectrum. 249]

UV-Vis Spectroscopy In metal complexes different kinds of transition can be measured with UV-Vis spectroscopy. There are the $d$ - $d$-, charge-transfer- and $\pi * \leftarrow \pi$ or $\pi * \leftarrow n$ - transitions.

$\boldsymbol{d}$ - $\boldsymbol{d}$ transition Complexes of d-transition metals often show absorbance in the visible region, which is caused by the splitting of the $d$ oribtals in the ligand field. In Figure 2.4 the ligand-field splitting for an octahedral and a tetrahedral coordination sphere, most often found for transition metals metals, is shown.

In an octahedral sphere the $d$ orbitals splits into two $e_{\mathrm{g}}$ orbitals with higher energy and three $t_{2 g}$ orbitals with lower energy. An electron with the energy $\left(\Delta_{\mathrm{O}}\right)$ would undergo a $d$ - $d$ transition within the visible region, but it is symmetry forbidden. Still the transition can be weakly observed, because the asymmetric vibrations lower the symmetry. The tetrahedral transition is not symmetry forbidden, but the ligand-field splitting is only 


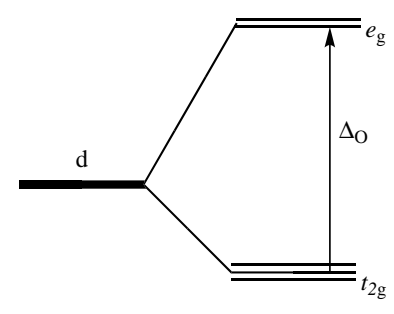

Octahedral

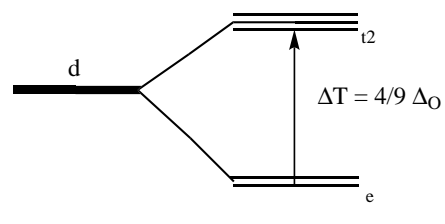

Tetrahedral

Figure 2.4: Schematic overview on the ligand-field splitting of d-orbitals in an octahedral and a tetrahedral coordination sphere

$4 / 9 \Delta_{O}$ and the absorption bands are found in the near-IR region. These transitions are widely used for kinetic studies for ATRP polymerization. [250]

Charge-Transfer transitions Charge-Transfer transition occur when an electron is transferred either from a ligand to the metal (LMCT) or from the metal to the ligand (MLCT). They have a high transition dipole moment and therefore an intense absorption.

$\pi * \leftarrow \pi$ or $\pi * \leftarrow n$ - transitions Besides of metal-complexes carbon double bonds also show absorption of UV-light, where the electron is transferred from a bonding $\pi$ orbital to an antibonding $\pi *$ orbital. A single double bond is typical adsorbing at $180 \mathrm{~nm}$, but conjugated systems of several double bonds can reach the visible region. The carbon-oxygen bond of a carbonyl group can undergo a transition as well, where an electron from a non-bonding orbital $n$, is transferred to an antibonding $\pi *$ orbital. Such transitions symmetry-forbidden yielding only in a weak adsorption. Because most organic solvents, monomers and polymers show these kinds of this absorptions the area of measurement in the UV region may be limited.

\subsubsection{Nuclear Magnetic Resonance (NMR) spectroscopy}

All atoms have a nuclear spin which characterized by the spin quantum number $I$. It can take values of $0,1 / 2,1,3 / 2 \ldots$, which depends on whether the number of protons and neutrons are even or uneven. When an external magnetic field is applied (see Figure 2.5) the magnetic moment of the spin is oriented relative to the axis direction of the magnetic field and $2 I+1$ orientations are possible. For hydrogen $(I=1 / 2)$ two orientations result. The energy difference between these states can be calculated by the Larmor frequency 

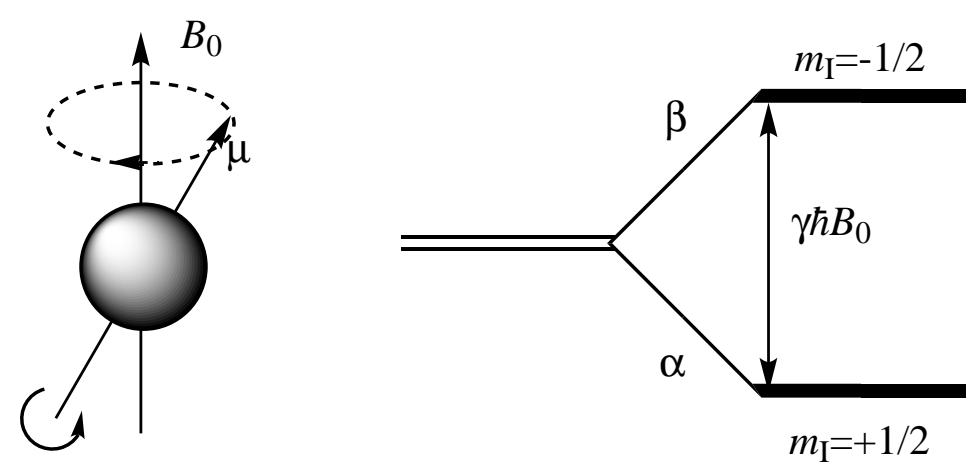

Figure 2.5: Magnetic moment $\mu$ of a nucleus in an external magnetic field $B_{0}$, causing an splitting ...

(Equation 2.17), where $\gamma$ is the magnetogyric ratio for the measured nucleus and $B_{0}$ the external magnetic field.

$$
\begin{array}{ll}
\Delta E=h \nu_{L} & \nu_{L}=\frac{\gamma B_{0}}{2 \pi} \\
\Delta E & =h \nu_{L}=\gamma \hbar B_{0}=h \nu
\end{array}
$$

The energy difference of the splitting is direct correlated to $\gamma$ and $B_{0}$ (Equation 2.18). E. $\mathrm{g}$. at a magnetic field of $4.7 \mathrm{~T}$ the Larmor frequency is $200 \mathrm{MHz}$ for a hydrogen atom.

A modern NMR-spectrometer works with superconducting magnets, which yield strong external magnetic fields, and high frequencies can be applied. The frequency is directly correlated with the resolution and the signal to noise ratio. The magnetic field is held constant and the resonance frequencies of the different atoms are measured using Fourier-Transformation-methods. This method permits short measuring times with high resolutions. To identify the different atoms all spectra are measured against a reference standard $\left(\mathrm{SiMe}_{4}, \nu_{r e f}\right)$ and the different signals are characterized by the chemical shift (Equation 2.19).

$$
\delta=\frac{\nu-\nu_{r e f}}{\nu_{r e f}} \times 10^{6} \mathrm{ppm}
$$

The chemical shift is influenced by neighbor-atoms and additional electric effects from e.g. aromatic-rings, triple and double bonds. Typical signals can be found from -1 to $12 \mathrm{ppm}$ for ${ }^{1} \mathrm{H}$ protons. The preparation of NMR-experiments within the spectrometer is far more complex then shown here and the procedures within a spectrometer are of importance signal quality and the results. For more details see reference ${ }^{[251]}$. 


\subsubsection{Size Exclusion Chromatography (SEC)}

The principle of size exclusion chromatography ${ }^{[252]}$ is the separation of the differently sized polymer chains by a porous column material. The column material is a crosslinked polymer with different pore sizes. The choice of material depends on the applied temperatures and eluents. The polymer chain diffuses in and out of the pores, long chains only in the large sized ones, small ones into almost all leading to separation and two characteristics of a SEC-setup. The upper and lower exclusion volumes state which polymers are too big and too small to be separated. Only for sizes in-between a useful characterization is possible. The important separation factor is the hydrodynamic volume, which makes a calibration necessary, due to the fact that e.g. PS has a different retention time compared with PMMA of the same chain length. The typical calibration is performed with narrowly distributed polymers over a range of several orders of magnitude in chain-length. Further the signals undergo a band broadening, which can be quantified ${ }^{[253]}$. Typical detectors are the refractive-index (RI) and IR-detectors for concentration measurements and UV/Vis-detector for functional group detection. To gain absolute data a setup can be equipped with a multi-angle laser light scattering (MALLS)-detector and/or an online viscometer. Both methods allow the determination of molar mass by an universal calibration.

While working with polymers which have a calibration on a SEC-setup no further data management is necessary but once this is not the case an universal calibration can be performed. The intrinsic viscosity $[\eta]$ is related with the hydrodynamic volume $\left(V_{h}\right)$ of a polymer chain by the Einstein-Simha law (Equation 2.20) by constant $C$. For linear polymers with the root-mean square radius of their gyration $\left.<s^{2}\right\rangle^{1 / 2}$ by the Flory-Fox equation (Equation 2.21) with the molar mass $(M)$ and constant $\Phi$.

$$
\begin{aligned}
& {[\eta]=C\left(\frac{V_{h}}{M}\right)} \\
& {[\eta]=\Phi\left(\frac{<s^{2}>^{3 / 2}}{M}\right)}
\end{aligned}
$$

While using the same setup the molar mass could be calculated as shown in equation 2.22 for two different polymers. The Mark-Houwink (MH) equation with the Mark-Houwink coefficients $K_{M H}$ and $\alpha_{M H}$ is applied (eq. 2.23) for a better description of relation between $[\eta]$ and molar mass. Combining equation 2.22 and 2.23 allows the determination of the 
molar mass of second polymer via a calibration of the first (eq. 2.24). The conversion via the $\mathrm{MH}$ equation into the $[\eta]$ yield the universal calibration.

$$
\begin{aligned}
{[\eta]_{1} K_{1} } & =[\rho]_{2} K_{2} \\
{[\eta] } & =K_{\mathrm{MH}} M^{\alpha_{\mathrm{MH}}} \\
\log M_{2} & =\frac{1}{1+\alpha_{\mathrm{MH}, 2}} \log \frac{K_{\mathrm{MH}, 1}}{K_{\mathrm{MH}, 2}}+\frac{1+\alpha_{\mathrm{MH}, 1}}{1+\alpha_{\mathrm{MH}, 2}} \log M_{1}
\end{aligned}
$$

If $\mathrm{MH}$ coefficients are not available it is possible to gain an universal calibration using a online viscometer. One kind of online viscometers uses a setup similar to a Wheatstone bridge (see Figure 2.6).

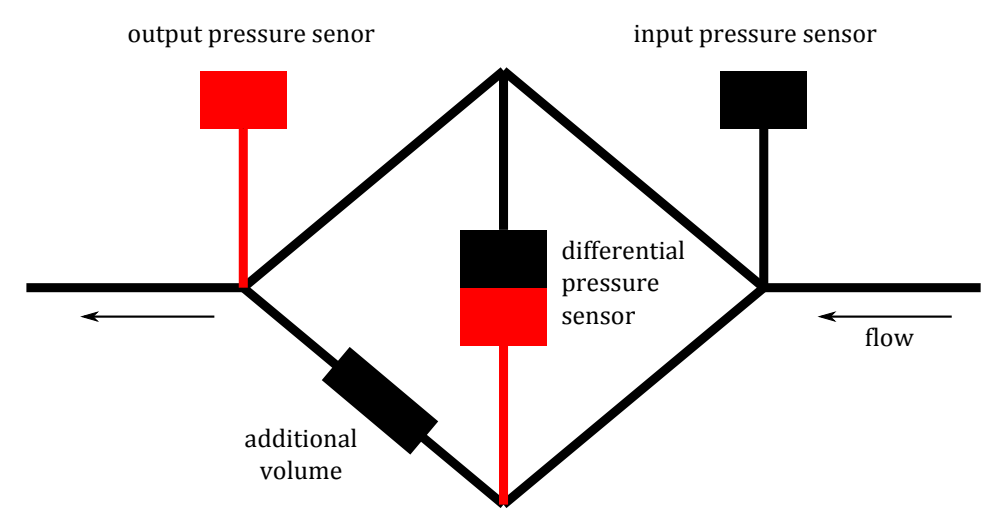

Figure 2.6: Schematic viscometer for SEC-online measurement

The pressure is measured before the sample flows into the setup and at the end of the setup. In between the flow is splitt into two streams, each going trough two defined measuring capillaries. At the connection points the pressure sensors are applied to determine the pressure difference of the upper and lower setup. While on the upper side the pressure of the dissolved polymer $\Delta p$ is measured it is the pressure of the mobile phase $\left(\Delta p_{s o l}\right)$ on the down side due to dilution by the additional volume of the solved polymer. The intrinsic viscosity $[\eta]$ can be calculated if the concentration $c$ is small as: 252

$$
[\eta]=\frac{\ln \left(\frac{\Delta p}{\Delta p_{s o l}}\right)}{c}
$$

For a universal calibration the product of the molar mass $M$ and $[\eta]$ is measured for a series of narrow distributed polymers and a calibration curve is determined, allowing the application of equations 2.20 and 2.21 . 


\subsubsection{Modeling via PREDICl ${ }^{\mathrm{TM}}$}

The simulation and modeling of reactions and polymerization processes is important to science and industry. With a good reaction model at hand and kinetic coefficients and thermodynamic data determined by different methods, reactions can be accurately described without starting a real reaction. This permits the prediction of the resulting product, security problems and an online reactor control. For e.g. CRP the broad spectrum of kinetic data is used to simulate the results of polymerization and effects of solvent before the reaction is applied in a plant. For polymerization reactions the molecular mass distribution is of great importance for the resulting product and its properties. This leads to a complex situation for the simulation, because every polymer chain must be considered. Furthermore, the chain length of the polymer can influence the reaction system, which should be taken into account as well.

A fast method for simulation is necessary, and the program PREDICI ${ }^{\mathrm{TM}}$ is one of the most commonly used program packages in $\mathrm{CRP}^{[254]}$ and RDRP ${ }^{[18,189,250,255-263]}$ simulation. It offers the fast implementation of different reaction steps by an internal catalog of predefined reaction sets, the parameter estimation via an implemented tool and the variation of coefficients. The introduced model is solved numercially via discrete Galerkin h-p methods ${ }^{[254264]}$ and is able to take chain-length dependent reaction steps into account. 


\section{Objective}

The analysis of reaction mechanisms is important for a detailed understanding on a reaction system with look on possible applications. The broad understanding in the RAFT process led to a wide range of new applications and functional polymers. [193-196] In recent studies in kinetic research for catalytic polymerization often work with major simplifications or approximations which makes their direct conversion into a model for CCG unsuitable. Additionally, the most applied neodymium system needs no additional activator like MAO, which is present within the typical studies. Compared to this system the activation is similar to typical $\mathrm{ZN}$ catalysts which are activated by Lewis acids.

Diorganyl magnesium derivatives are ideal candidates for such approaches. On the one hand they show high polymerization activity when applied by neodymium based catalysts. On the other hand they are commercially available or very easy to synthesize. The system was already found to allow a broad spectrum of possible end group modifications. ${ }^{[23]}$

For kinetic determination of the process PREDICI ${ }^{\mathrm{TM}}$ is chosen, because of its broad application in the similar RAFT-process in RDRP. Methods to describe polymer exchange reactions as well different deactivation pathways have already been reported in literature. ${ }^{189,259-263]}$ Similar exchange reactions are to be expected for CCG systems. ${ }^{201}$ In this work six major objectives were planned:

1. The detailed determination of the activation mechanism in absence of monomer by a model system based on $\mathrm{Cp}^{*}{ }_{2} \mathrm{ZrCl}_{2}$ and dibenzyl magnesium in NMR spectroscopy and the transfer to UV/Vis spectroscopy for later analysis of paramagnetic systems.

2. The detailed analysis of styrene- $d 8$ polymerization in NMR spectroscopy to gain insights in the reaction mechanism with monomer present and for extraction of kinetic information of the occurring processes.

3. Transfer of the mechanistic model to polymerization of styrene analyzed via $\mathrm{UV} / \mathrm{Vis}$ spectroscopy and modeling of the overall process. 
4. Transfer of the determined model to the polymerization of styrene performed in presence of $\mathrm{Cp}_{2}{ }_{2} \mathrm{Nd}_{2} \mathrm{Cl}_{2} \mathrm{Li}\left(\mathrm{OEt}_{2}\right)_{2}$ as catalyst and BuMgOct (BOMAG) as cocatalyst monitored via UV/Vis spectroscopy.

5. Modeling and Simulation of ethylene polymerization in batch reactors with $\mathrm{Cp}_{2}{ }_{2} \mathrm{Nd}_{2} \mathrm{Cl}_{2} \mathrm{Li}\left(\mathrm{OEt}_{2}\right)_{2}$ as catalyst and BuMgOct (BOMAG) as cocatalyst .

6. Synthesis of functional polymers based on polyethylene produced by CCG.

The first point is important to understand the basic reaction steps occurring during the process, which shall be transferred to the polymerization. The main objective is the successful modeling of the CCG process in batch reactors. 


\section{Monomer Free Kinetic Models for Catalyzed Chain Growth}

For research of CCG process several catalyst/co-catalyst systems were studied to establish different experimental methods. For this thesis in situ studies which provide the most detailed data of occurring species and monomer conversion were chosen. The most used polymerization system containing $\mathrm{Cp}_{2}{ }_{2} \mathrm{NdCl}_{2} \mathrm{Li}\left(\mathrm{OEt}_{2}\right)_{2}$ has the disadvantage of being paramagnetic which leads to problems in characterization in NMR experiments. In order to gain information about formed complexes during the polymerization process a suitable non-paramagnetic system, i. e. $\mathrm{Cp}_{2}{ }_{2} \mathrm{ZrCl}_{2}$, which can be well characterized by NMR was researched. This zirconium catalyst precursor is commercial available and a broad spectrum of literature data is available. In addition the complex has the same set of ligands as the neodymium catalyst and ligand effects can be excluded. The model system is able to polymerize styrene in the presence of different magnesium derivatives (see section 6.1 on page 135) and outgoing from the results the catalyst systems with $\mathrm{Cp}_{2}{ }_{2} \mathrm{NdCl}_{2} \mathrm{Li}\left(\mathrm{OEt}_{2}\right)_{2}$ were studied.

\subsection{Catalysts}
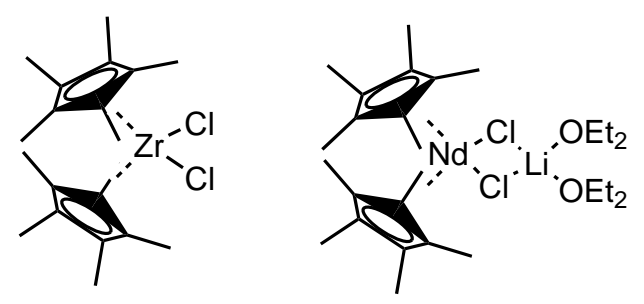

$\mathrm{Cp}_{2}{ }_{2} \mathrm{ZrCl}_{2}$

$\mathrm{Cp}_{2}{ }_{2} \mathrm{NdCl}_{2} \mathrm{Li}(\mathrm{OEt})_{2}$

Figure 4.1: Catalysts applied for establishment of kinetic models in monomer free CCG activation and CCG polymerization. 
Two catalysts were studied (see Figure 4.1) of which $\mathrm{Cp}_{2}{ }_{2} \mathrm{ZrCl}_{2}$ is commercially available and $\mathrm{Cp}_{2}{ }_{2} \mathrm{NdCl}_{2} \mathrm{Li}\left(\mathrm{OEt}_{2}\right)_{2}$ was synthesized. The process is a literature known ${ }^{[265]}$ one step procedure and has been slightly modified yielding a blue powder characterized by NMR and ESI-MS (see Scheme 4.1). The catalyst is highly oxygen and moisture sensitive and was therefore stored and handled in a glove box with dry and oxygen free solvents.

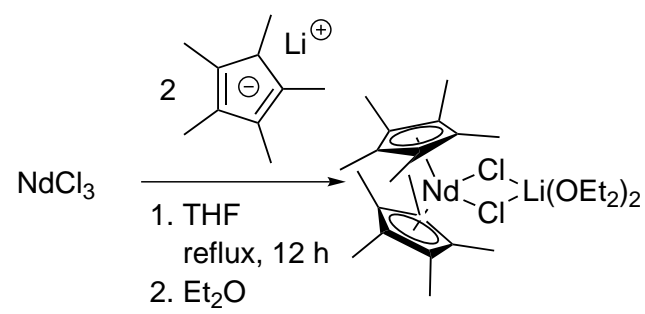

Scheme 4.1: Synthesis of $\mathrm{Cp}_{2}{ }_{2} \mathrm{NdCl}_{2} \mathrm{Li}\left(\mathrm{OEt}_{2}\right)_{2}$ from $\mathrm{NdCl}_{3}$ and freshly prepared $\mathrm{LiCp}$ in THF under reflux conditions and work up in $\mathrm{Et}_{2} \mathrm{O}$.

\subsection{Magnesium based Co-catalysts}

The common applied BOMAG ( $n$-butyl-n-octyl-magnesium, BuMgOct) is commercially available in $1 \mathrm{~kg}$ scale while di- $n$-butyl magnesium $(\mathrm{BuMgBu})$ is available but additives like TEA are added, depending on the supplier. BzMgBz was chosen as starting cocatalyst to setup a kinetic model as it could be well characterized by NMR and additional spectroscopic methods. It allows insight into the formed species by a CTA and the formed complexes cannot undergo $\beta$-hydride elimination. BzMgBz was preferred to either to dimethyl magnesium or more sterical hindered magnesium derivatives like di$t$-butyl magnesium. Both possibilities have been excluded because the methyl catalysts have the tendency to form a bimetallic zirconium complex depending on the activator ${ }^{[99]}$ and the steric demand of di-t-butyl magnesium is too high. The synthesis of BzMgBz is straight forward (see experimental part 9.2.2.1 on page 203) and the steric demand is similar to styrene due to the phenyl group, allowing comparable results during studies with styrene as monomer present. The most successful synthesis ${ }^{[26]}$ of BzMgBz was performed by application of fresh made benzyl magnesium bromide and the addition of 1,4-dioxane (see Scheme 4.2). Synthesis was not successful from commercial available solutions of benzyl magnesium chloride in different ethers.

The thermal stability of BzMgBz was studied by UV/Vis experiments. It was found that at temperatures above $90^{\circ} \mathrm{C}$ a side reaction occurs which is assumed to be the coupling 
<smiles>O=[W]c1cccc(CCNCc2ccccc2)c1</smiles>

Scheme 4.2: Synthesis of dibenzyl magnesium (BzMgBz) outgoing from benzyl bromide and magnesium by the formation of benzyl magnesium bromide in diethyl ether and precipitation of magnesium chloride by addition of 1,4-dioxane.

of two benzyl group towards various reaction products. A long term study revealed that full conversion is gained after two days (see Scheme 4.2). The determination of the reaction order was not possible. Because most measurements shown later were performed at $400 \mathrm{~nm}$ it was the wavelength of choice.

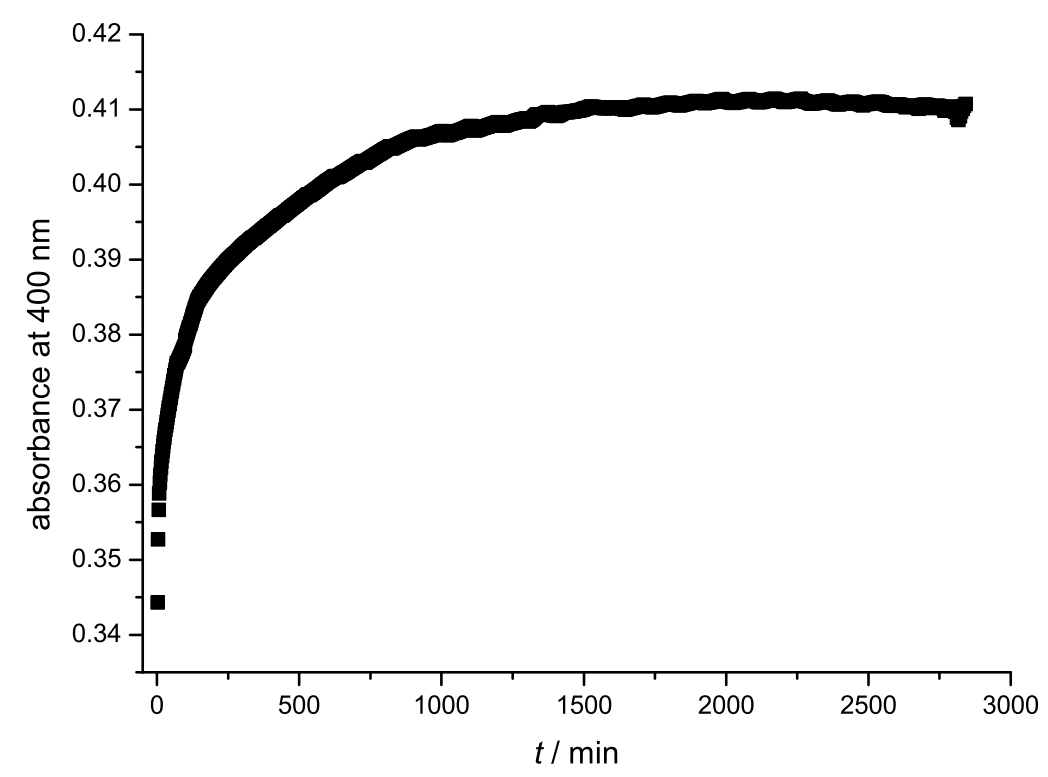

Figure 4.2: Time-resolved UV/Vis measurement $c(\mathrm{BzMgBz})=44 \mathrm{mmoll}^{-1}$ in toluene at $90^{\circ} \mathrm{C}$.

The reaction was further investigated by NMR and two new signals occur with proceeding time, one in the area for Mg-alkyl signals and one in the area for $\mathrm{CH}_{3}-\mathrm{Ph}$. The signal of 1,2-diphenyl ethane (BzBz) is increasing. Small amounts of $\mathrm{BzBz}$ are found beforehand, because it is a side product formed during synthesis.

The reaction seems to be slower compared to the reaction studied by UV/Vis spectroscopy. The concentration is higher in the NMR study and magnesium compounds are known to form clusters ${ }^{[267268]}$, which seems to mainly influence the reaction rate. The overall reaction rate for side product formation is notably high at $90^{\circ} \mathrm{C}$ and it is con- 


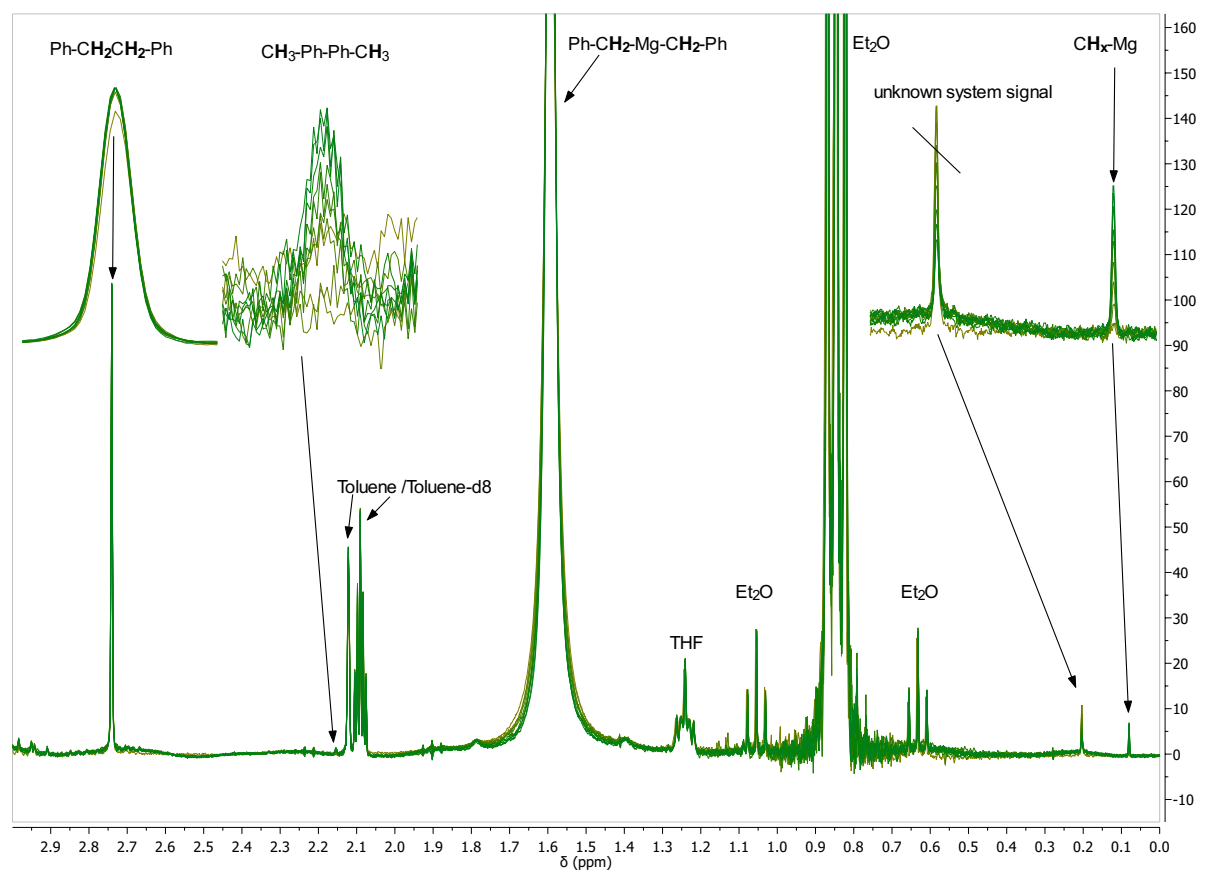

Figure 4.3: Determined species of self-reaction of BzMgBz via time-resolved NMR measurement $c(\mathrm{BzMgBz})=44 \mathrm{mmol} \mathrm{L}^{-1}$ in toluene- $d_{8}$ at $90^{\circ} \mathrm{C}$ with only every tenth measurement shown for clarity reasons (short - short reaction time, green - long reaction time).

sidered negligibly small at $80^{\circ} \mathrm{C}$ and below. In the NMR studies shown later only very small amounts of these could be detected at high reaction times which is in agreement with the approximation.

In all cases residual amounts of diethyl ether are found after synthesis, which cannot be removed completely (strong signal in Figure 4.3). The ether is coordinated to the magnesium, causing good solubility in toluene. It is additional lowering the amount of higher structures e.g. found in retarded anionic polymerization. ${ }^{267,268}$ The influence of ether on different reactions is discussed for the catalyst/co-catalyst systems as well.

\subsection{The Model System $\mathrm{Cp}_{2}^{*} \mathrm{ZrCl}_{2}$ and $\mathrm{BzMgBz}$}

In contrast to neodymium and iron-based catalysts, the chosen $\mathrm{Cp}_{2}{ }_{2} \mathrm{ZrCl}_{2}(\mathrm{~A}) / \mathrm{BzMgBz}$ model system is non-paramagnetic. Initialization is a critical phase during polymerization in CCG. If the chains from the CTA are not transferred fast and the first monomer addition is slow the MMD will be broadened or the process goes over into a CCTP like polymerization. Results of this section were already published in literature [269]. Copyright 2014 WILEY-VCH Verlag GmbH \& Co. KGaA, Weinheim. 


\subsubsection{Kinetic NMR Experiments}

Analysis based on time-resolved NMR measurements was applied for kinetic studies. During these experiments the reaction cannot be stirred within the NMR tube. The reactants can move only via diffusion. The experiments were performed in toluene- $d_{8}$ for best comparability of the results with non NMR experiments in the conventional solvent. The process found is leading to several species which could be clearly characterized. The assignments to formed species are shown in Figure 4.4. The measurement data at the Zr:Mg ratio 1:20 was provided by Adrian Deinert within his bachelor thesis.

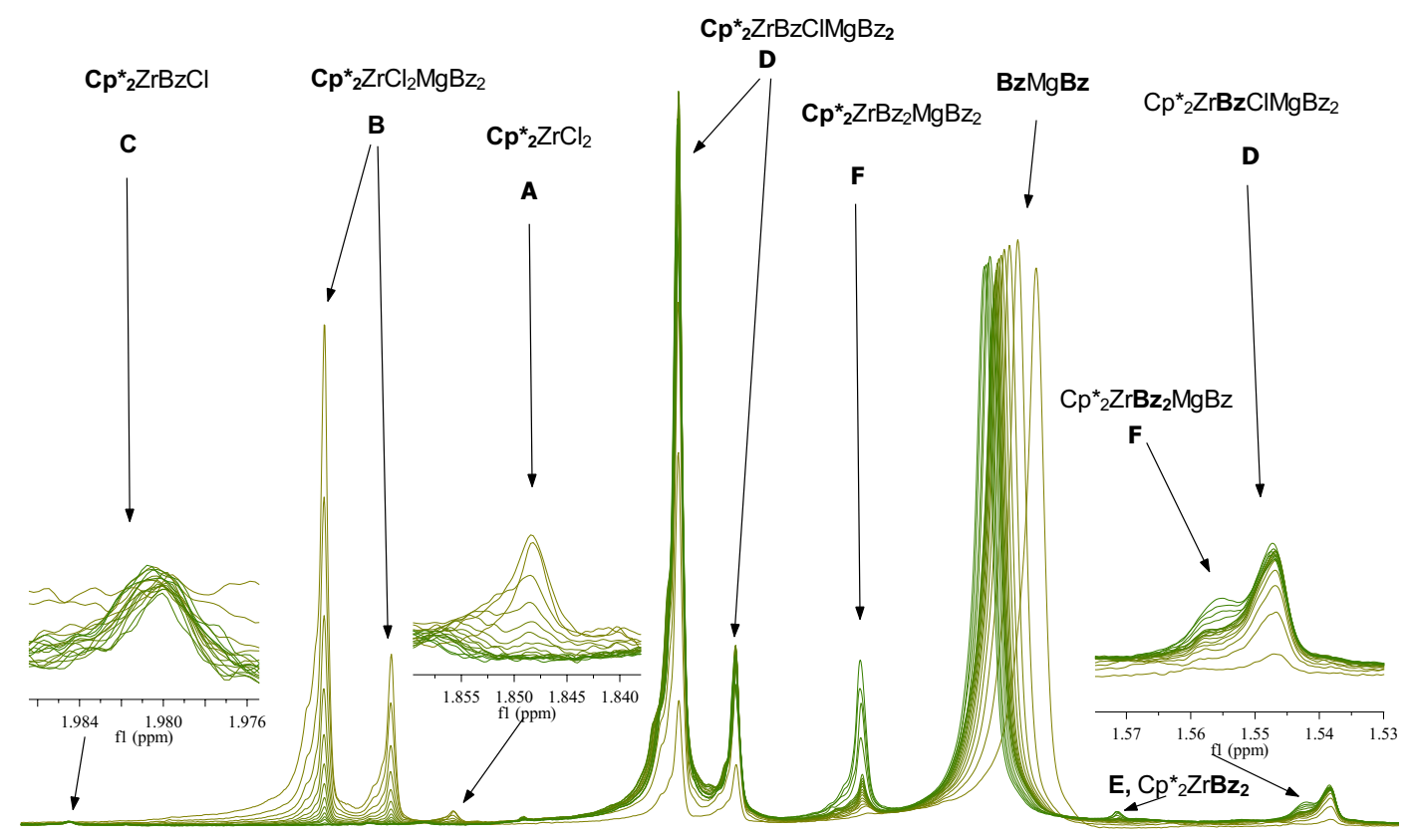

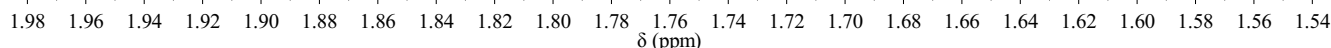

Figure 4.4: Determined species via time-resolved NMR measurement of monomer-free CCG model system using $\mathrm{Cp}^{*}{ }_{2} \mathrm{ZrCl}_{2}$ (A) and $\mathrm{BzMgBz}$ at $80^{\circ} \mathrm{C}, c\left(\mathrm{Cp}^{*} \mathrm{ZrCl}_{2}\right)=4.6 \mathrm{mmol} \mathrm{L}^{-1}$ $c(\mathrm{BzMgBz})=44 \mathrm{mmol} \mathrm{L}^{-1}$ in toluene- $d_{8}$ with only every tenth measurement shown for clarity reasons (yellow - short reaction time, green - long reaction time).

The catalyst precursor $\left(\mathrm{Cp}_{2}{ }_{2} \mathrm{ZrCl}_{2}, \mathbf{A}\right)$ has the signal at $1.85 \mathrm{ppm}$ which is rapidly transferred into an aduct of $\mathbf{A}$ and $\mathrm{BzMgBz}\left(\mathbf{C p}^{*}{ }_{2} \mathrm{ZrCl}_{2} \mathrm{MgBz}_{2}, \mathbf{B}\right)$ yielding a splitted signals at $1.89 \mathrm{ppm}$ and $1.87 \mathrm{ppm}$. It is considered as a bimetallic $\mathrm{Zr}-\mathrm{Mg}$-complex with the chloride atoms as bridge atoms. The splitting may be caused by the presence of two or more kinds of magnesium clusters. ${ }^{[267]}$ With increasing time a signal at $1.98 \mathrm{ppm}$ $\left(\mathrm{Cp}_{2}{ }_{2} \mathrm{ZrBz}(\mathrm{Cl}), \mathrm{C}\right)$ appears and is nearly stable during measurement. It is shifted strongly to lower field compared to $\mathbf{A}$ and $\mathbf{B}$. It is assumed that it belongs to a beny- 
zlated complex in which the chloride is not directly or weakly coordinated to the zirconium. It yields a positively charged catalyst because the $\mathrm{BzMgBz}$ acts as an activator. This is in agreement with literature data from complexes of $\left[\mathrm{Cp}_{2}{ }_{2} \mathrm{ZrR}\right]^{+}$cations. ${ }^{\text {[1,270,271] }}$ Two new signals emerge at $1.77 \mathrm{ppm}$ and $1.75 \mathrm{ppm}$ which correspond to the bridged mono-benzylated complex $\left(\mathbf{C p}_{2}{ }_{2} \mathrm{ZrBzClMgBz}_{2}, \mathbf{D}\right)$ and their combined integrals increase similarly to the signal at $1.55 \mathrm{ppm}$. This signal is the benzyl moiety bridged between zirconium and magnesium $\left(\mathrm{Cp}_{2}{ }_{2} \mathrm{ZrBzClMgBz}, 2\right.$ D). At higher reaction times the signals of the bridged di-benzylated complex appear at $1.70 \mathrm{ppm}\left(\mathbf{C p}^{*}{ }_{2} \mathrm{ZrBz}_{2} \mathrm{MgBz}_{2}, \mathbf{F}\right)$ for the ligand and at $1.56 \mathrm{ppm}$ for the benzyl groups $\left.\mathrm{Cp}_{2}{ }_{2} \mathrm{ZrBz}_{2} \mathrm{MgBz}_{2}, \mathbf{F}\right)$. The signals for the bridged mono- and di-benzylated complex are in agreement with literature ${ }^{[272]}$ for the ligand signals while the bridged benzyl moieties are slightly shifted to higher field. At 1.62 ppm a signal similar to the benzyl moieties of the unbridged and fully alkylated complex $\left(\mathrm{Cp}_{2}{ }_{2} \mathrm{ZrBz}_{2}, \mathrm{E}\right)$ are found while the ligand signals were not observed due to the low concentration. ${ }^{272,273]}$ The signal of dibenzyl magnesium $(\mathbf{B z M g B z})$ is found as a broad peak at $1.66 \mathrm{ppm}^{[274]}$ which shifts slightly to lower field with increasing reaction time. In Figure 4.5 this effect could be shown via a plot of the peak maximum against reaction time.

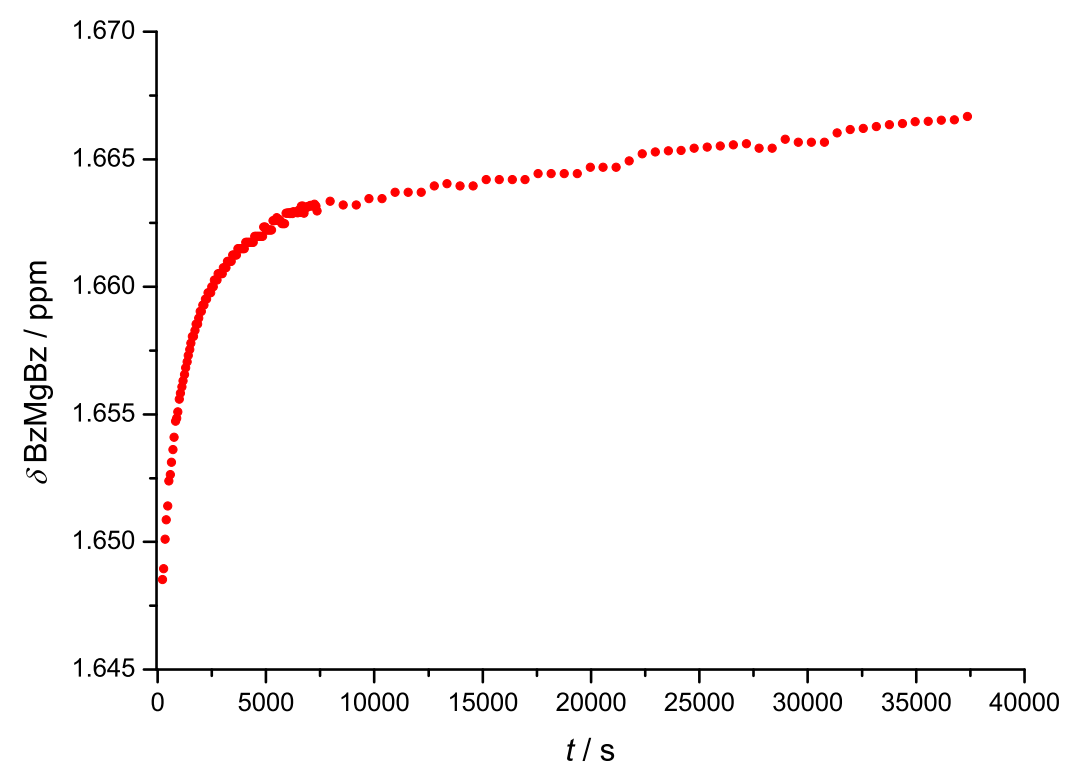

Figure 4.5: Alteration of the chemical shift of $\mathrm{BzMgBz}$ in monomer-free CCG activation at $80{ }^{\circ} \mathrm{C}$.

In comparison with the formation of $\mathbf{B}$ and $\mathbf{D}$ (see Figure 4.7 on page 45 ) the signal shift is correlated to the proceeding reaction. The small part of $\mathrm{BzMgBz}$ in the coordination sphere of the complexes is exchanged by molecules from solution. While the peaks are not broadened, the observation shows that the reaction is faster than the time scale of 
the NMR-experiment.

For the determination of concentrations the signals were integrated and the NMR raw data was normalized with the solvent signals of toluene. The exact procedure is described in the experimental section (see experimental part 9.1 .5 on page 197). One example of the measurements is shown in Figure 4.6 on the next page. Beginning from the catalyst precursor $\mathbf{A}$ complex $\mathbf{B}$ was formed rapidly and the concentration of $\mathbf{A}$ is detectable for around $2500 \mathrm{~s}$. With proceeding reaction time complex $\mathbf{B}$ is transferred slowly into complex $\mathbf{D}$ and complex $\mathbf{F}$ and both signals occur at the same time. The formation of $\mathbf{C}$ is detectable shortly afterwards with an almost stable concentration during the measurement. It is assumed that this complex is the catalytic active species for polymerization. The concentration of $\mathrm{BzMgBz}$ is measured simultaneously and the consumption is in direct correlation to formation of $\mathbf{D}$ while the formation of complex $\mathbf{F}$ is overlaid by side effects (see Figure 4.7 on page 45 ).

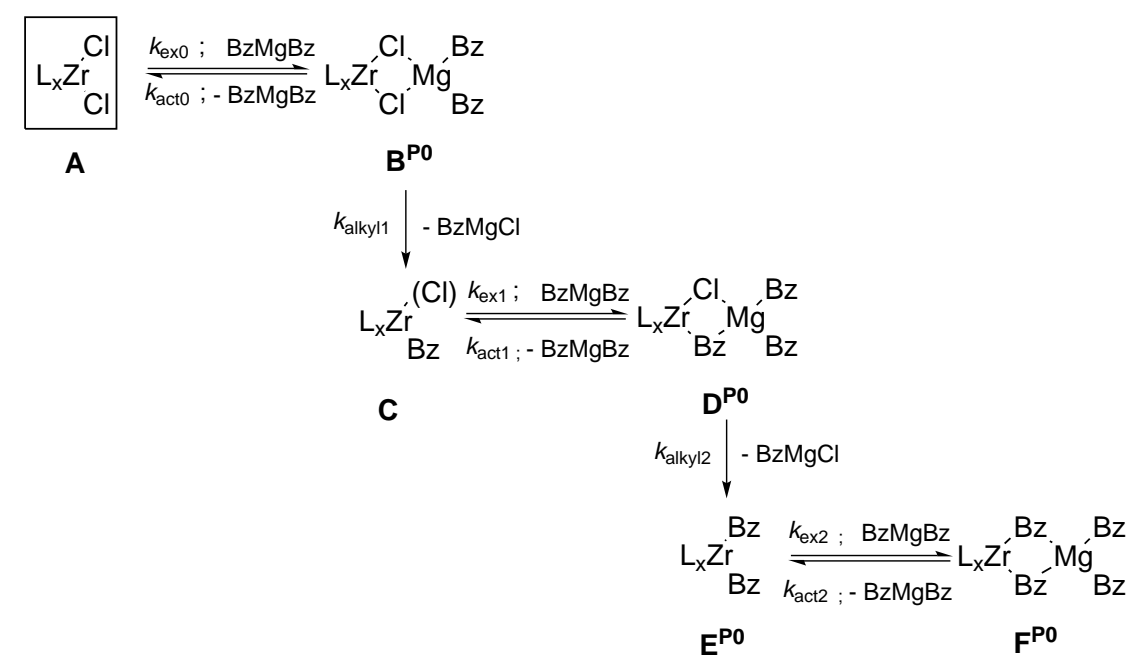

Scheme 4.3: Kinetic Scheme of the process of monomer free CCG activation outgoing from complex $\mathbf{A}$ by BzMgBz via the formation of complex $\mathbf{C}$ and $\mathbf{D}^{\mathbf{P 0}}$ and further reaction to complex $\mathbf{F}^{\mathbf{P 0}}$. Complex $\mathrm{C}$ is assumed to be catalytic active. The suffix $\mathrm{P}$ states the amount of polymer chains present in the complex, in this case $x=0$.

The combination of these results leads to the kinetic Scheme 4.3 for monomer-free catalyst activation in CCG. In the beginning, the catalyst precursor $\mathbf{A}$ is in equilibrium with species $\mathbf{B}^{\mathbf{P 0}}$ (The suffix $\mathrm{P}$ stands for the amount of polymer chain present in the complex, here $\mathrm{x}=0$ ) via reversible association and dissociation of BzMgBz. Outgoing from species $\mathbf{B}^{\mathbf{P 0}}$ a second reaction pathway leads to species $\mathbf{C}$ by intramolecular alkyl-chloride exchange followed by dissociation of a Grignard reactant. Via reversible association and dissociation of $\mathrm{BzMgBz}$ species $\mathrm{C}$ is in equilibrium with species $\mathbf{D}^{\mathrm{P} 0}$. By a second intramolecular alkyl-chloride exchange at complex $\mathbf{D}^{\mathrm{P0}}$ and dissociation complex $\mathbf{E}^{\mathrm{P0}}$ 


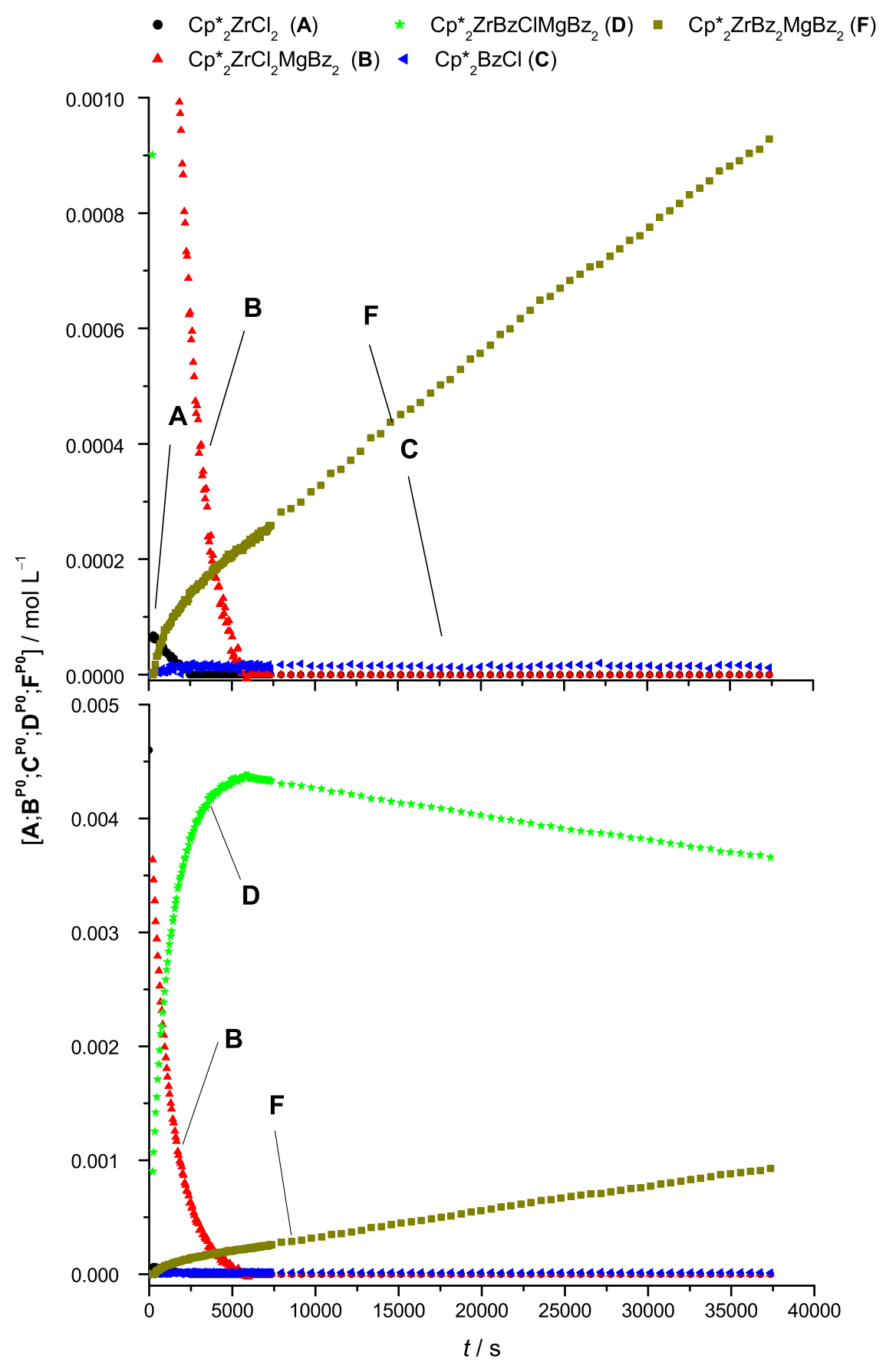

Figure 4.6: Concentration vs. time profiles of the different complex species for monomer-free CCG activation at $80^{\circ} \mathrm{C}$ with $c\left(\mathrm{Cp}_{2}^{*} \mathrm{ZrCl}_{2}\right)=4.6 \mathrm{mmoll}^{-1} c(\mathrm{BzMgBz})=44 \mathrm{mmol}^{-1}$ in toluene- $d 8$. 


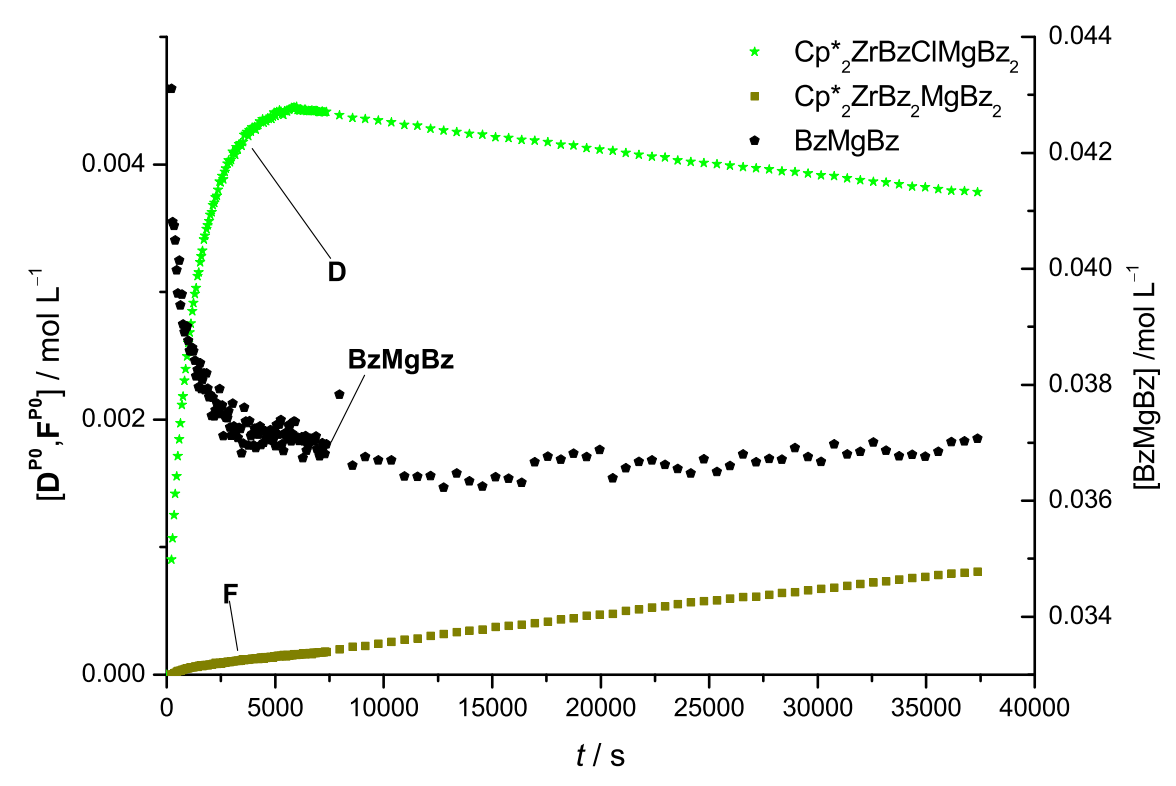

Figure 4.7: Comparsion of concentration time profiles of complex $\mathbf{D}$ and $\mathbf{F}$ with $\mathrm{BzMgBz}$ measured at $80^{\circ} \mathrm{C}$ in toluene- $d 8$.

and a further Grignard reactant are formed. Species $\mathbf{E}$ is in equilibrium with species $\mathbf{F}^{\mathrm{P0}}$ by association and dissociation of $\mathrm{BzMgBz}$ which is found as the final product of the reaction processes in NMR.

For further investigations of the kinetics the different reaction rate equations were described by classical reaction time laws (see eq. 4.1 to 4.6 ).

$$
\begin{aligned}
& \frac{\mathrm{d}[\mathbf{A}]}{\mathrm{d} t}=-k_{\mathrm{ex} 0}[\mathbf{A}][\mathbf{B z M g B z}]+k_{\text {act0 }}\left[\mathbf{B}^{\mathbf{P} 0}\right] \\
& \frac{\mathrm{d}\left[\mathbf{B}^{\mathbf{P} 0}\right]}{\mathrm{d} t}=k_{\text {ex0 }}[\mathbf{A}][\mathbf{B z M g B z}]-k_{\text {act0 }}\left[\mathbf{B}^{\mathbf{P 0}}\right]-k_{\text {alkyl1 }}\left[\mathbf{B}^{\mathbf{P 0}}\right] \\
& \frac{\mathrm{d}[\mathbf{C}]}{\mathrm{d} t}=-k_{\text {ex1 }}[\mathbf{C}][\mathbf{B z M g B z}]+k_{\text {act } 1}\left[\mathbf{D}^{\mathbf{P} 0}\right]+k_{\text {alkyl1 }}\left[\mathbf{B}^{\mathbf{P 0}}\right] \\
& \frac{\mathrm{d}\left[\mathbf{D}^{\mathbf{P} 0}\right]}{\mathrm{d} t}=k_{\mathrm{ex} 1}[\mathbf{C}][\mathbf{B z M g B z}]-k_{\mathrm{act} 1}\left[\mathbf{D}^{\mathbf{P} \mathbf{0}}\right]-k_{\mathrm{alky12}}\left[\mathbf{D}^{\mathbf{P} \mathbf{0}}\right] \\
& \frac{\mathrm{d}\left[\mathbf{E}^{\mathbf{P} \mathbf{0}}\right]}{\mathrm{d} t}=-k_{\mathrm{ex} 2}\left[\mathbf{E}^{\mathbf{P} \mathbf{0}}\right][\mathbf{B z M g B z}]+k_{\text {act2 }}\left[\mathbf{F}^{\mathbf{P} 0}\right]+k_{\text {alkyl2 }}\left[\mathbf{D}^{\mathbf{P} \mathbf{0}}\right] \\
& \frac{\mathrm{d}\left[\mathbf{F}^{\mathrm{P} 0}\right]}{\mathrm{d} t}=k_{\mathrm{ex} 2}\left[\mathbf{E}^{\mathrm{P} \mathbf{0}}\right][\mathbf{B z M g B z}]-k_{\mathrm{act} 2}\left[\mathbf{F}^{\mathrm{P} \mathbf{0}}\right]
\end{aligned}
$$

Due to the complex assembly of equilibria and following reactions an analytical solution was not possible. Thus numerical methods were the ones of choice. The program 
package PREDICI ${ }^{\mathrm{TM}}$ was chosen to allow an easy transfer of the results to a model system containing polymer information (see section 2.4 .4 on page 34 for more details to this program package). For simulation with PREDICI ${ }^{\mathrm{TM}}$ a kinetic model has to be declared. In the following the input reactions in PREDICI ${ }^{\mathrm{TM}}$ nomenclature are present:

$$
\begin{aligned}
& \mathbf{A}+\mathrm{BzMgBz} \stackrel{k_{\mathrm{ex0}}}{\longrightarrow} \mathbf{B}^{\mathbf{P 0}} \\
& \mathbf{B}^{\mathrm{P} 0 \stackrel{k_{\text {act0 }}}{\longrightarrow}} \mathbf{A}+\mathrm{BzMgBz} \\
& \mathbf{B}^{\mathbf{P 0}} \stackrel{k_{\text {alkyl }}}{\longrightarrow} \mathrm{C}+\mathrm{BzMgCl} \\
& \mathrm{C}+\mathrm{BzMgBz} \stackrel{k_{\mathrm{ex} 1}}{\longrightarrow} \mathrm{D}^{\mathrm{P} 0} \\
& \mathrm{D}^{\mathrm{P0}} \stackrel{k_{\text {act }}}{\longrightarrow} \mathrm{C}+\mathrm{BzMgBz} \\
& \mathbf{D}^{\mathbf{P 0}} \stackrel{k_{\text {alkyl2 }}}{\longrightarrow} \mathbf{E}^{\mathbf{P 0}}+\mathrm{BzMgCl} \\
& \mathbf{E}^{\mathrm{P} 0}+\mathrm{BzMgBz} \stackrel{k_{\mathrm{ex} 2}}{\longrightarrow} \mathbf{F}^{\mathrm{P0}} \\
& \mathrm{F}^{\mathrm{P} 0} \stackrel{k_{\text {act2 }}}{\longrightarrow} \mathrm{E}^{\mathrm{P} 0}+\mathrm{BzMgBz} \\
& \mathrm{BzMgCl}+\mathrm{BzMgCl} \stackrel{k_{\text {schlenk }}}{\longrightarrow} \mathrm{BzMgBz}+\mathrm{ClMgCl}
\end{aligned}
$$

In all cases simple "elemental" reaction steps were applied and the reaction 4.15 of benzyl magnesium chloride was additionally declared because during the reaction a white solid precipitates which is magnesium chloride. The formation of dibenzyl magnesium is yielded by the Schlenk equilibrium of two Grignard species formed during reaction 4.9 and 4.12. The reaction is quantitatively due to the participation of $\mathrm{MgCl}_{2}$.

The NMR measurements allowed the time dependent concentration determination of six from the seven occurring species. With this comprehensive kinetic data the rate coefficients are available and can be estimated by modeling on the basis of the model scheme. The results of the parameter estimation tool of PREDICI ${ }^{\mathrm{TM}}$ are shown in Table 4.1.

In Figure 4.8 the simulated time evolution of the species concentrations by PREDICI ${ }^{\mathrm{TM}}$ parameter estimation are shown together with the experimental data. The resulting fits are in agreement with experimental data and point towards the validity of the mechanistic scheme as well as for the reliability of the estimated kinetic coefficients. While $k_{\text {alykl1 }}$ and $k_{\text {alykl1 }}$ were estimated independently the values of $k_{\text {ex }}$ and $k_{\text {act }}$ depend on each other. Thus, determination of individual values is impossible. Simulation for different reaction rates with constant ratio indicated no influence on the concentration profiles for the applied variety. Therefore the values of $k_{\text {act }}$ were fixed to the value 
Table 4.1 Parameters estimated by PREDCI of momomer free CCG activation modeling at $80^{\circ} \mathrm{C}$, $c\left(\mathbf{C p}^{*}{ }_{2} \mathrm{ZrCl}_{2}\right)=0.0046 \mathrm{~mol} \mathrm{~L}^{-1}, c(\mathrm{BzMgBz})=0.044 \mathrm{~mol} \mathrm{~L}^{-1}$ in toluene- $d_{8}$ with fixed $k_{\text {act }}$ values at $1 \mathrm{~s}^{-1}$.

\begin{tabular}{c|r|l} 
coefficient & value & unit \\
\hline$k_{\mathrm{ex} 0}$ & 972 & $\mathrm{~L} \mathrm{~mol}^{-1} \mathrm{~s}^{-1}$ \\
$k_{\mathrm{ex} 1}$ & 19808 & $\mathrm{~L} \mathrm{~mol}^{-1} \mathrm{~s}^{-1}$ \\
$k_{\mathrm{ex} 2}$ & 7994 & $\mathrm{~L} \mathrm{~mol}^{-1} \mathrm{~s}^{-1}$ \\
$k_{\text {alkyl1 }}$ & 1.21 & $10^{-3} \mathrm{~s}^{-1}$ \\
$k_{\text {alkyl2 }}$ & 6.96 & $10^{-6} \mathrm{~s}^{-1}$ \\
$k_{\text {Schlenk }}$ & 1000 & $\mathrm{~L} \mathrm{~mol}^{-1} \mathrm{~s}^{-1}$
\end{tabular}
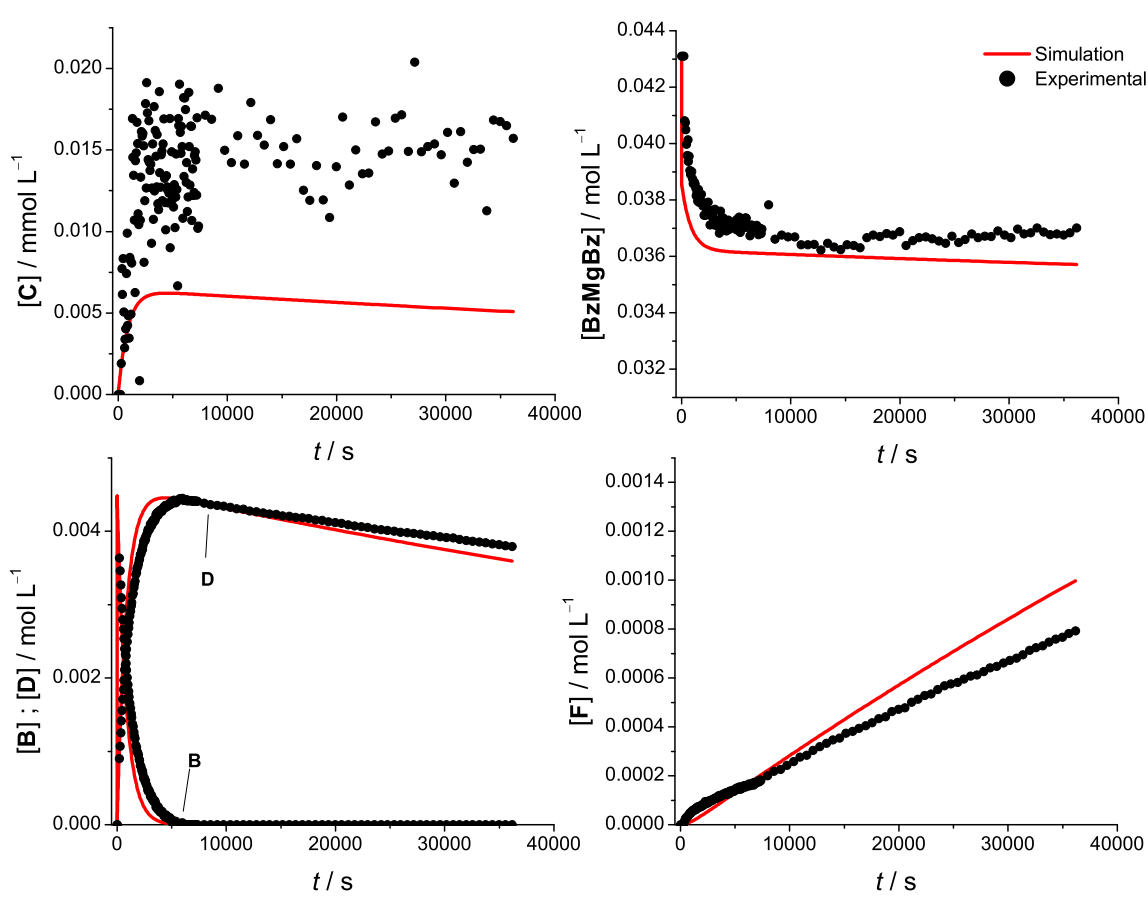

Figure 4.8: Simulatied and experimental concentration vs. time profiles with the input data of Table 4.1. 
of $1 \mathrm{~s}^{-1}$ for PREDICI ${ }^{\mathrm{TM}}$ simulation describing the ratio as an equilibrium constant by modeling $k_{\mathrm{ex}}$. Within the variety the reaction rates are only dependent on the applied ratio. The exact values are of importance in presence of monomer (see Chapter 5.1 .3 on page 111). The reaction rate coefficient for the Schlenk equilibrium was chosen fast to represent the participation process of $\mathrm{MgCl}_{2}$ while regeneration of $\mathrm{BzMgBz}$. The value of $1000 \mathrm{~L} \mathrm{~mol}^{-1} \mathrm{~s}^{-1}$ is not considered of high significance.

Based on these results the mechanistic scheme and the simulation results were reviewed for simplifications and optimization of the determination of kinetic coefficients. The PREDICI $^{\mathrm{TM}}$ parameter optimization tool was found not to be the optimal method to gain the best suitable kinetic data for the chosen scheme. The data of Table 4.1.indicates that the association/dissociation processes are faster by several orders of magnitude than the ligand exchange (alkylation) reactions and it can be considered that $\mathbf{A}$ and $\mathbf{B}^{\mathbf{P 0}}$ as well as $\mathbf{C}$ and $\mathbf{D}^{\mathbf{P 0}}$ are in equilibrium in the relevant time scale for the ligand exchange. This allows that equation 4.1 may be rewritten to define the equilibrium constant $K_{\mathrm{Zr}, 1}$ (see eq. 4.16).

$$
\frac{k_{\text {ex } 0}}{k_{\text {act } 0}}=\frac{\left[\mathbf{B}^{\mathrm{P} 0}\right]}{[\mathbf{A}][\mathbf{B z M g B z}]}=K_{\mathrm{Zr}, 1}
$$

To verify this approximation equation 4.16 was applied to the simulation data (see Figure 4.9 a) upper part). After fast establishment of equilibrium the concentration ratio of $\left[\mathbf{B}^{\mathrm{P} 0}\right] /[\mathbf{A}][\mathbf{B z M g B z}]$ has a constant value equivalent to the input ratio $k_{\text {exo }} / k_{\text {act0. }}$. A slight influence of the simulation process is found while the process is independent from the ligand exchange reaction. This situation can be transferred simultaneously to the reaction of $\mathbf{C}$ with $\mathrm{BzMgBz}$ to $\mathbf{D}^{\mathrm{P} 0}$ (Equation 4.9) and it is characterized by the equilibrium constant $K_{\mathrm{Zr}, 2}$ (see Equation

$$
\frac{k_{\mathrm{ex} 1}}{k_{\mathrm{act} 1}}=\frac{\left[\mathbf{D}^{\mathrm{P} 0}\right]}{[\mathbf{C}][\mathbf{B z M g B z}]}=K_{\mathrm{Zr}, 2}
$$

The calculated ratio of $k_{\text {ex } 1} / k_{\text {act1 }}$ from the species concentrations ratio $\left[\mathbf{D}^{\mathrm{P} 0}\right] /[\mathbf{C}][\mathbf{B z M g B z}]$ reaches fast an equilibrium value (see Figure 4.9 a) lower part). To further validate these approximations it was cross-checked with the experimental NMRdata. The determined concentration ratios of $\left[\mathbf{B}^{\mathbf{P 0}}\right] /[\mathbf{A}][\mathbf{B z M g B z}]$ and $\left[\mathbf{D}^{\mathrm{P0}}\right] /[\mathbf{C}][\mathbf{B z M g B z}]$ are shown in Figure $4.9 \mathrm{~b}$ ). The results support the approximation and the kinetics can be simplified by the application of equilibrium reactions of $\mathbf{A}$ and $\mathrm{C}$ with $\mathrm{BzMgBz}$ and the description with the equilibrium constants $K_{1, Z \mathrm{r}}$ and $K_{2, \mathrm{Zr}}$. The equilibrium reaction of $\mathbf{A}$ with $\mathrm{BzMgBz}$ (Equation 4.16) can be inserted into equation 


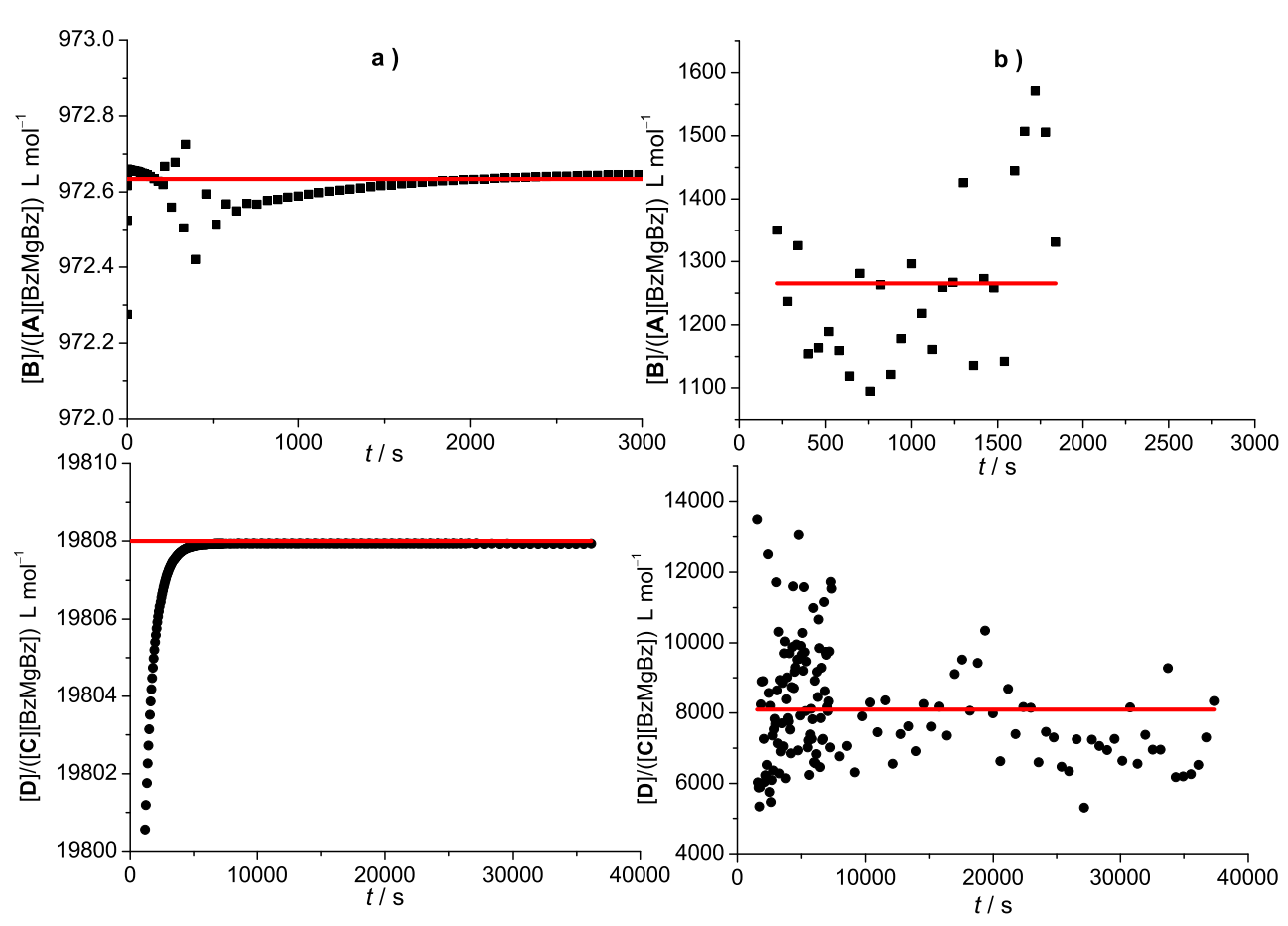

Figure 4.9: Simulated (a)) and experimental (b)) results for determination of $K_{\mathrm{Zr}, 1}$ with equation 4.16 and $K_{\mathrm{Zr}, 2}$ with equation 4.17 with the input data of Table 4.1 for monomer-free CCG activation. The red lines show the constant ratio of concentrations.

4.2. The reaction order is simplified to a pseudo-first-order reaction with respect to the concentration of $\mathbf{B}^{\mathbf{P 0}}$ only reduced by the alkylation reaction (see Equation 4.18 ).

$$
\frac{\mathrm{d}\left[\mathbf{B}^{\mathbf{P} 0}\right]}{\mathrm{d} t}=-k_{\mathrm{alkyl} 1}\left[\mathbf{B}^{\mathbf{P 0}}\right]
$$

And for the reaction of $\mathbf{C}$ with BzMgBz equation 4.17 is inserted into equation 4.4 giving a pseudo first-order reaction for the consumption of $\mathbf{D}^{\mathbf{P 0}}$ by the alkylation reaction (see Equation 4.19).

$$
\frac{\mathrm{d}\left[\mathbf{D}^{\mathbf{P} 0}\right]}{\mathrm{d} t}=-k_{\mathrm{alkyl1}}\left[\mathbf{D}^{\mathbf{P} 0}\right]
$$

Both equations are cross-checked with first-order reaction plots for the concentrations of $\mathbf{B}^{\mathbf{P 0}}$ and $\mathrm{D}^{\mathrm{P0}}$ (see Figure $\left.4.10, \mathbf{a}\right)$ ). In both cases it can be seen that a nearby perfect linear fit is achieved. It supports the validity of the approximations discussed previously. In comparison with the input data of simulation the determined rate coefficients $k_{\text {alkyl1 }}=$ $1.17 \times 10^{-3} \mathrm{~s}^{-1}$ and $k_{\text {alkyl2 }}=6.88 \times 10^{-6} \mathrm{~s}^{-1}$ deduced from the slopes of the linear plots are ca. $3 \%$ smaller. This is in agreement with the confidence interval of the estimated data. It can be concluded that the approximations found by simulation allow the simple deduction of $k_{\text {alkyl1 }}$ and $k_{\text {alkyl2 }}$ via first-order plots of the experimental concentration profiles of species $\mathbf{B}^{\mathbf{P 0}}$ and $\mathbf{D}^{\mathbf{P 0}}$ and of $K_{\mathrm{Zr}, 1}$ and $K_{\mathrm{Zr}, 2}$ calculated with experimental concentrations 


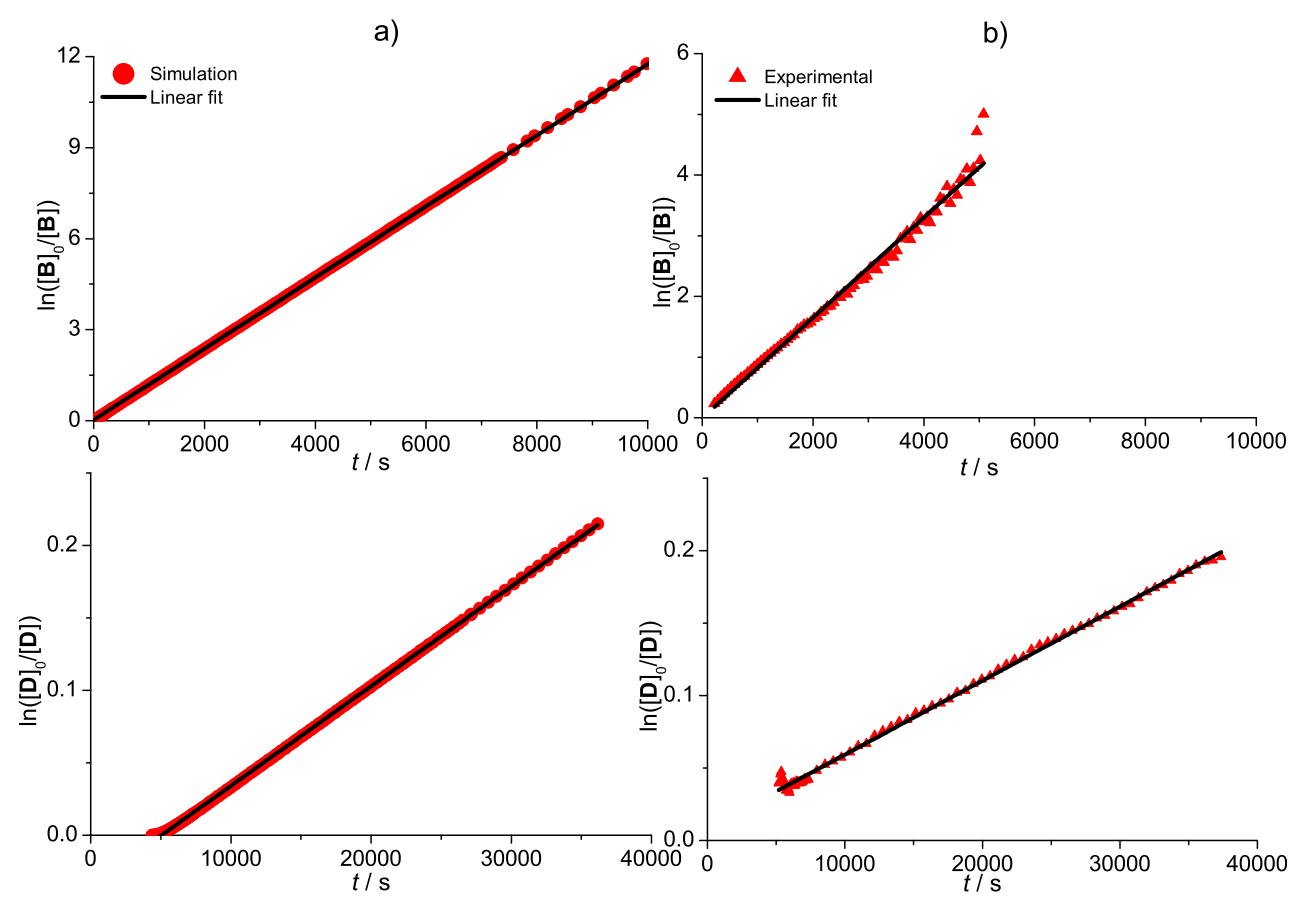

Figure 4.10: Simulatated (a)) and experimental (b)) results for determination of $K_{\mathrm{Zr}, 1}$ (Equation 4.16) and $K_{\mathrm{Zr}, 2}$ (Equation 4.17) with the input data of 4.1 .

inserted into (Equation 4.16) and (Equation 4.17). In Figure 4.9, b) and 4.10, b) the results from the experimental data are shown. Despite the higher scattering the achieved plots show good agreement with the simulated plots and the derived values are shown in Table 4.2 .

Table 4.2 Kinetic parameters derived from experimental NMR data of momomer-free CCG activation at $80{ }^{\circ} \mathrm{C}, c\left(\mathbf{C p}^{*}{ }_{2} \mathrm{ZrCl}_{2}\right)=0.0046 \mathrm{~mol} \mathrm{~L}^{-1}, c(\mathrm{BzMgBz})=0.044 \mathrm{~mol} \mathrm{~L}^{-1}$ in toluene- $d_{8}$ with Equation 4.16 to 4.19 .

\begin{tabular}{c|r|l} 
coefficients & value & unit \\
\hline$K_{1, \mathrm{Zr}}$ & 1.2 & $10^{3} \mathrm{~mol} \mathrm{~L}^{-1}$ \\
$K_{2, \mathrm{Zr}}$ & 8.0 & $10^{3} \mathrm{~mol} \mathrm{~L}^{-1}$ \\
$k_{\text {alkyl1 }}$ & 8.77 & $10^{-4} \mathrm{~s}^{-1}$ \\
$k_{\text {alkyl2 }}$ & 5.41 & $10^{-6} \mathrm{~s}^{-1}$
\end{tabular}

High stability of the zirconium-complex magnesium-compound adduct $\mathbf{B}$ and $\mathbf{D}$ are indicated by the equilibrium constant with values in the order of $10^{3} \mathrm{~mol} \mathrm{~L}^{-1}$ to $10^{4} \mathrm{~mol} \mathrm{~L}^{-1}$. The individual rate coefficients are not deducible in this kind of experiment as no monomer is present. The determination of the rate coefficients is part of Section 5.1 on page 83 . The reaction rate of the ligand exchange leading to alkylation within the 
bimetallic complex is rather slow (see data for $k_{\text {alkyl }}$ in Table 4.2) which indicates that the process of yet known mechanism is energetically hardly favored. The second benzyl transfer is even slower which can be explained by larger steric constraints within the complex. After the first benzyl transfer the steric demand is higher in comparison to chloride and it reduces the possibility of a successful second exchange reaction. This result is consistent with the higher lifetime of $\mathbf{D}^{\mathrm{P} 0}$ above the time scale of the NMR experiment indicated by the peak of the bridged benzyl species of $\mathbf{D}^{\mathrm{P} 0}$. The influence of this finding is discussed later for polymerization of styrene.

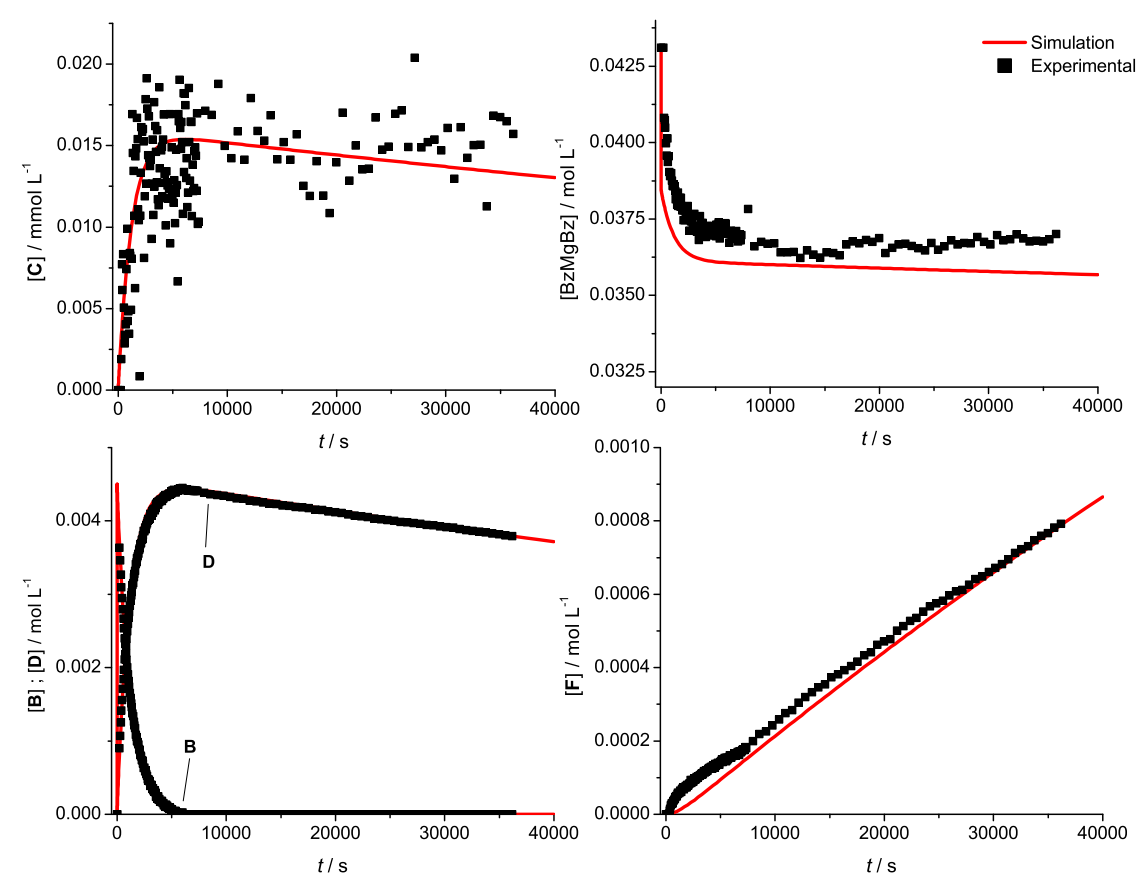

Figure 4.11: Simulated and experimental concentration vs. time profiles for monomer free CCG activation with the input data of Table 4.2 .

The match between simulation and experiment is improved by application of the kinetic parameters derived via pseudo-first order kinetic treatment of the NMR data in the simulation (see 4.11). For the equilibrium constants the ratio of $k_{\mathrm{ex}} / k_{\mathrm{act}}$ was introduced into simulation by assuming the value of $1 \mathrm{~s}^{-1}$ for $k_{\text {act }}$. The concentration vs. time profiles of $\mathbf{B}, \mathbf{C}$ and $\mathbf{D}^{\mathrm{P} 0}$ are well described by simulation while the profile of $\mathrm{BzMgBz}$ still is slightly drifted. For $\mathbf{F}^{\mathrm{P0}}$ the slope of the simulation fits excellently but the profile is minimal shifted to a lower value which may be caused by a systematic error. At low concentrations of $\mathbf{F}^{\mathrm{P0}}$ the absolute concentration is determined too high because a small ${ }^{13} \mathrm{C}-{ }^{1} \mathrm{H}$ coupling satellite from the signal of $\mathbf{D}^{\mathrm{P0}}$ is overlaying with the signal of $\mathbf{F}^{\mathrm{P} 0}$. This finding explains the high increase at low reaction times while with proceeding time the influence is lowered to a small ratio.

The shown approach was performed for further analysis of two catalyst/co-catalyst 
ratios. The equilibrium constants and rate coefficients were determined at three temperatures $\left(60,70\right.$, and $\left.80^{\circ} \mathrm{C}\right)$. In Figure 4.12 and 4.13 the corresponding linear plots for $\mathrm{Zr}: \mathrm{Mg}=1: 10$ are shown. For Zr:Mg = 1:20 the results are depicted in Figure 4.14 and 4.15 and the combined data of both ratios which showed no big differences as predicted by the model, is summarized in Table 4.3 .

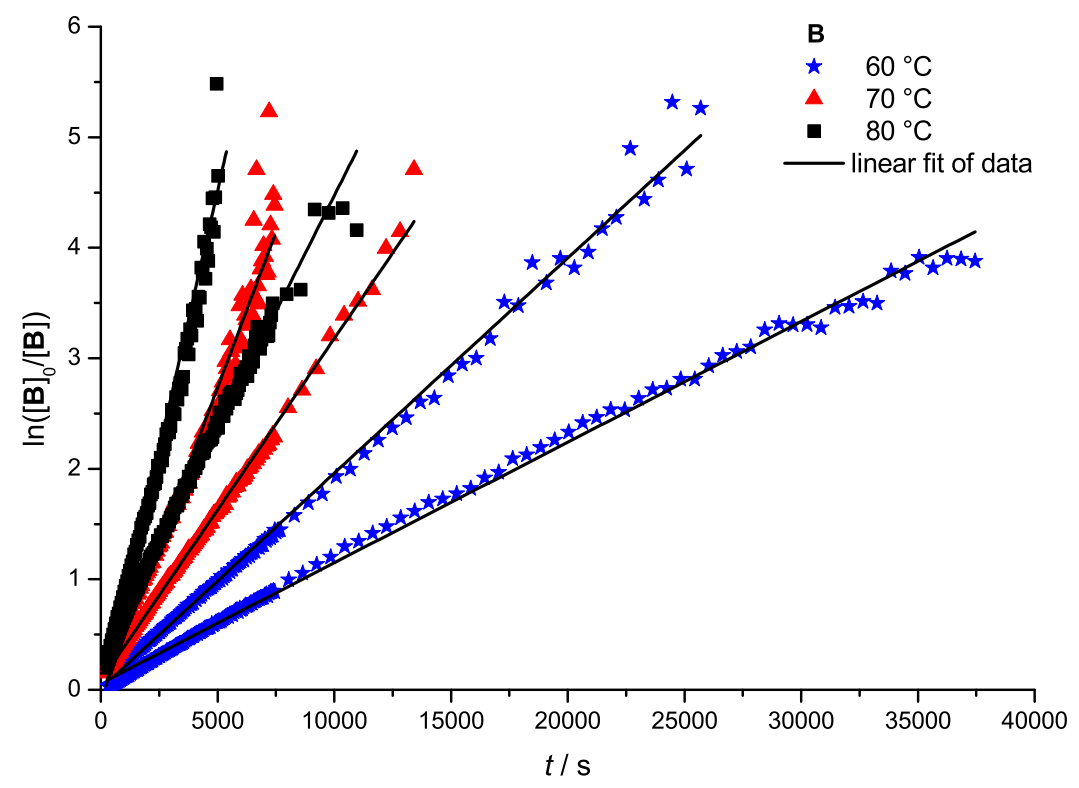

Figure 4.12: First-order rate plots of complex $B$ for monomer-free CCG activation with $\mathrm{Zr}: \mathrm{Mg}=1: 10.60^{\circ} \mathrm{C}$ (blue star) $c\left(\mathrm{Cp}_{2}{ }_{2} \mathrm{ZrCl}_{2}\right)=0.0046 \mathrm{~mol} \mathrm{~L}^{-1}, c(\mathrm{BzMgBz})=0.043 \mathrm{~mol} \mathrm{~L}^{-1} ; 70^{\circ} \mathrm{C}$ (red triangle) $c\left(\mathrm{Cp}_{2}^{*} \mathrm{ZrCl}_{2}\right)=0.0046 \mathrm{~mol} \mathrm{~L}^{-1}, c(\mathrm{BzMgBz})=0.042 \mathrm{~mol} \mathrm{~L}^{-1} ; 80^{\circ} \mathrm{C}$ (black square) $c\left(\mathrm{Cp}_{2}{ }_{2} \mathrm{ZrCl}_{2}\right)=0.0046 \mathrm{~mol} \mathrm{~L}^{-1}, c(\mathrm{BzMgBz})=0.041 \mathrm{~mol} \mathrm{~L}^{-1}$. The measurements were performed twice and the lines show the best linear fit.

Table 4.3 Kinetic parameters of momomer-free CCG activation at various temperatures and two catalyst/co-catalyst ratios, $c\left(\mathrm{Cp}_{2}{ }_{2} \mathrm{ZrCl}_{2}\right)=0.0046 \mathrm{~mol} \mathrm{~L}^{-1}, c(\mathrm{BzMgBz})=0.044 \mathrm{~mol} \mathrm{~L}^{-1}$; $c\left(\mathrm{Cp}_{2}{ }_{2} \mathrm{ZrCl}_{2}\right)=0.0023 \mathrm{~mol} \mathrm{~L}^{-1}, c(\mathrm{BzMgBz})=0.046 \mathrm{~mol} \mathrm{~L}^{-1}$ in toluene- $d_{8}$ derived by NMR data application of Equation 4.16 to 4.19 .

\begin{tabular}{c|c|c|c|c}
$T /{ }^{\circ} \mathrm{C}$ & $K_{1, \mathrm{Zr}} / 10^{3} \mathrm{~L} \mathrm{~mol}^{-1}$ & $K_{2, \mathrm{Zr}} / 10^{3} \mathrm{~L} \mathrm{~mol}^{-1}$ & $k_{\mathrm{alkyl} 1} / 10^{-4} \mathrm{~s}^{-1}$ & $k_{\mathrm{alkyl} 2} / 10^{-6} \mathrm{~s}^{-1}$ \\
\hline 60 & $2.6 \pm 1.0$ & $7.7 \pm 1.5$ & $1.9 \pm 0.7$ & - \\
70 & $2.3 \pm 0.6$ & $8.9 \pm 2.0$ & $5.8 \pm 2.2$ & $3 \pm 1$ \\
80 & $1.2 \pm 0.2$ & $8.8 \pm 1.2$ & $7.8 \pm 2.2$ & $7 \pm 3$
\end{tabular}

Only for measurements with $\mathrm{Zr}: \mathrm{Mg}$ ratio $1: 20$ at $60^{\circ} \mathrm{C}$ a kink shaped induction period is found which may be the result of the the presence of remaining diethyl ether. A detailed plot depicted in Figure 4.16 indicates two phases of reaction, a slow reaction with a rate 


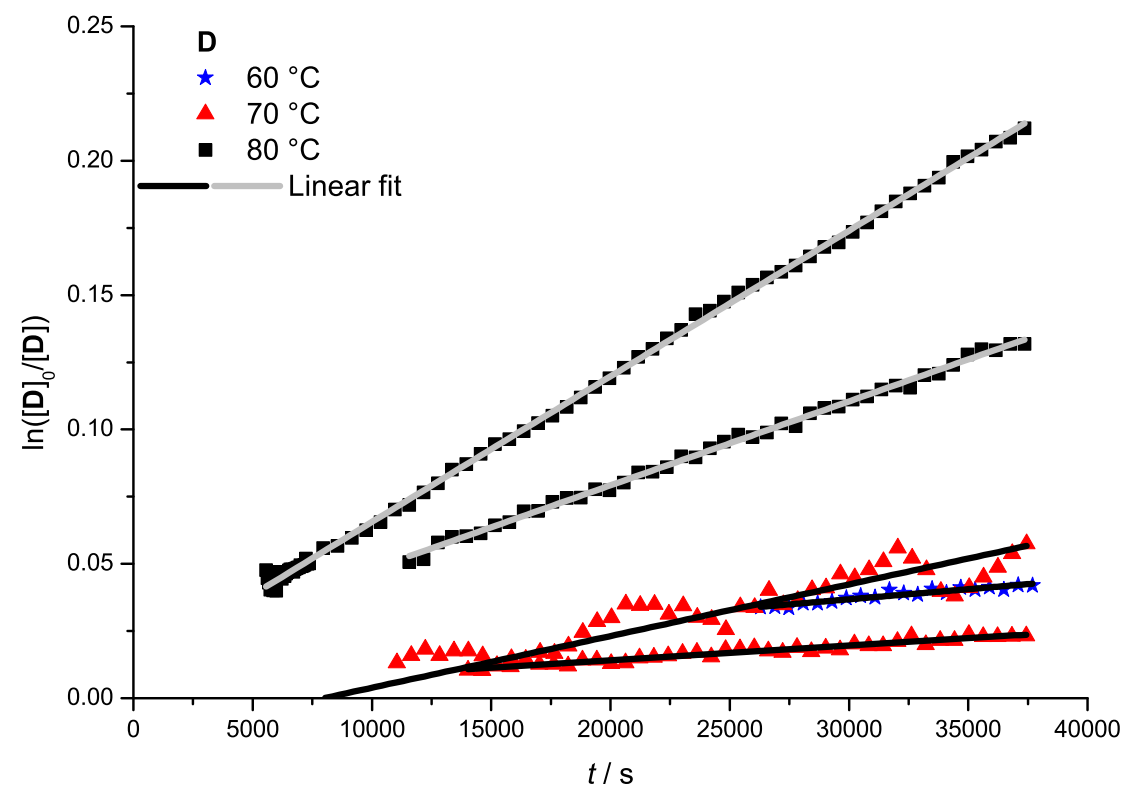

Figure 4.13: First-order rate plots of complex $D$ for monomer-free CCG activation with $\mathrm{Zr}: \mathrm{Mg}=1: 20.60^{\circ} \mathrm{C}$ (blue star) $c\left(\mathrm{Cp}_{2}{ }_{2} \mathrm{ZrCl}_{2}\right)=0.0046 \mathrm{~mol} \mathrm{~L}^{-1}, c(\mathrm{BzMgBz})=0.043 \mathrm{~mol} \mathrm{~L}^{-1} ; 70^{\circ} \mathrm{C}$ (red triangle) $c\left(\mathrm{Cp}_{2}{ }_{2} \mathrm{ZrCl}_{2}\right)=0.0046 \mathrm{~mol} \mathrm{~L}^{-1}, c(\mathrm{BzMgBz})=0.042 \mathrm{~mol} \mathrm{~L}^{-1} ; 80^{\circ} \mathrm{C}$ (black square) $c\left(\mathrm{Cp}_{2}{ }_{2} \mathrm{ZrCl}_{2}\right)=0.0046 \mathrm{~mol} \mathrm{~L}^{-1}, c(\mathrm{BzMgBz})=0.041 \mathrm{~mol} \mathrm{~L}^{-1}$. The measurements were performed twice and the lines show the best linear fit.

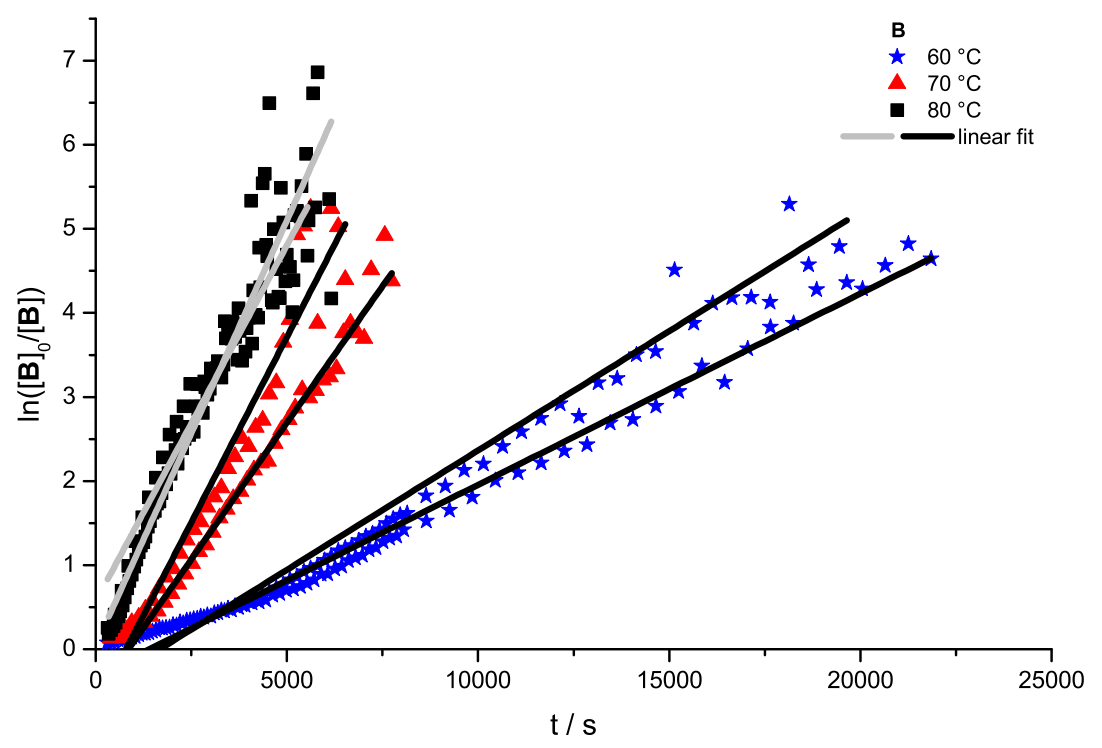

Figure 4.14: First-order rate plots of complex $B$ for monomer-free CCG activation with $\mathrm{Zr}: \mathrm{Mg}=1: 20.60^{\circ} \mathrm{C}$ (blue star) $c\left(\mathrm{Cp}_{2}{ }_{2} \mathrm{ZrCl}_{2}\right)=0.0023 \mathrm{~mol} \mathrm{~L}^{-1}, c(\mathrm{BzMgBz})=0.046 \mathrm{~mol} \mathrm{~L}^{-1} ; 70^{\circ} \mathrm{C}$ (red triangle) $c\left(\mathrm{Cp}_{2}^{*} \mathrm{ZrCl}_{2}\right)=0.0023 \mathrm{~mol} \mathrm{~L}^{-1}, c(\mathrm{BzMgBz})=0.045 \mathrm{~mol} \mathrm{~L}^{-1} ; 80^{\circ} \mathrm{C}$ (black square) $c\left(\mathrm{Cp}_{2}{ }_{2} \mathrm{ZrCl}_{2}\right)=0.0023 \mathrm{~mol} \mathrm{~L}^{-1}, c(\mathrm{BzMgBz})=0.048 \mathrm{~mol} \mathrm{~L}^{-1}$. The measurements were performed twice and the lines show the best linear fit. 


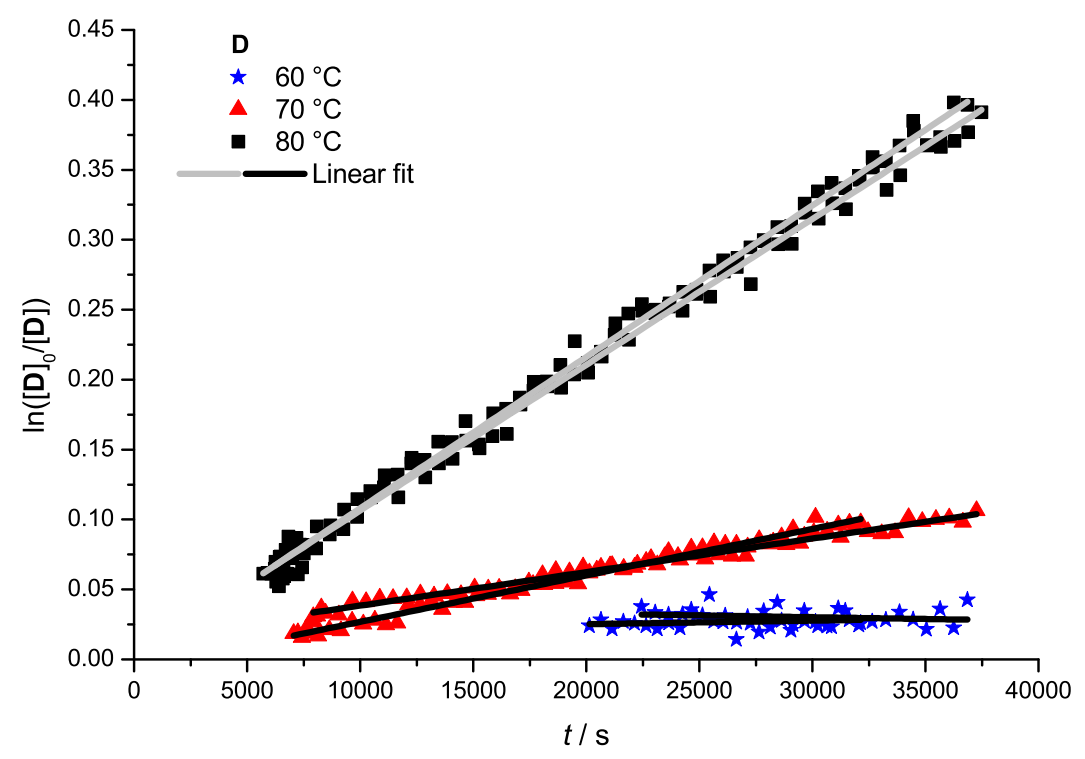

Figure 4.15: First-order rate plots of complex $\mathrm{D}$ for monomer-free CCG activation with $\mathrm{Zr}: \mathrm{Mg}=1: 10.60^{\circ} \mathrm{C}$ (blue star) $c\left(\mathrm{Cp}_{2}{ }_{2} \mathrm{ZrCl}_{2}\right)=0.0023 \mathrm{~mol} \mathrm{~L}^{-1}, c(\mathrm{BzMgBz})=0.046 \mathrm{~mol} \mathrm{~L}^{-1} ; 70^{\circ} \mathrm{C}$ (red triangle) $c\left(\mathrm{Cp}_{2}{ }_{2} \mathrm{ZrCl}_{2}\right)=0.0023 \mathrm{~mol} \mathrm{~L}^{-1}, c(\mathrm{BzMgBz})=0.045 \mathrm{~mol} \mathrm{~L}^{-1} ; 80^{\circ} \mathrm{C}$ (black square) $c\left(\mathrm{Cp}_{2}{ }_{2} \mathrm{ZrCl}_{2}\right)=0.0023 \mathrm{~mol} \mathrm{~L}^{-1}, c(\mathrm{BzMgBz})=0.048 \mathrm{~mol} \mathrm{~L}^{-1}$. The measurements were performed twice and the lines show the best linear fit.

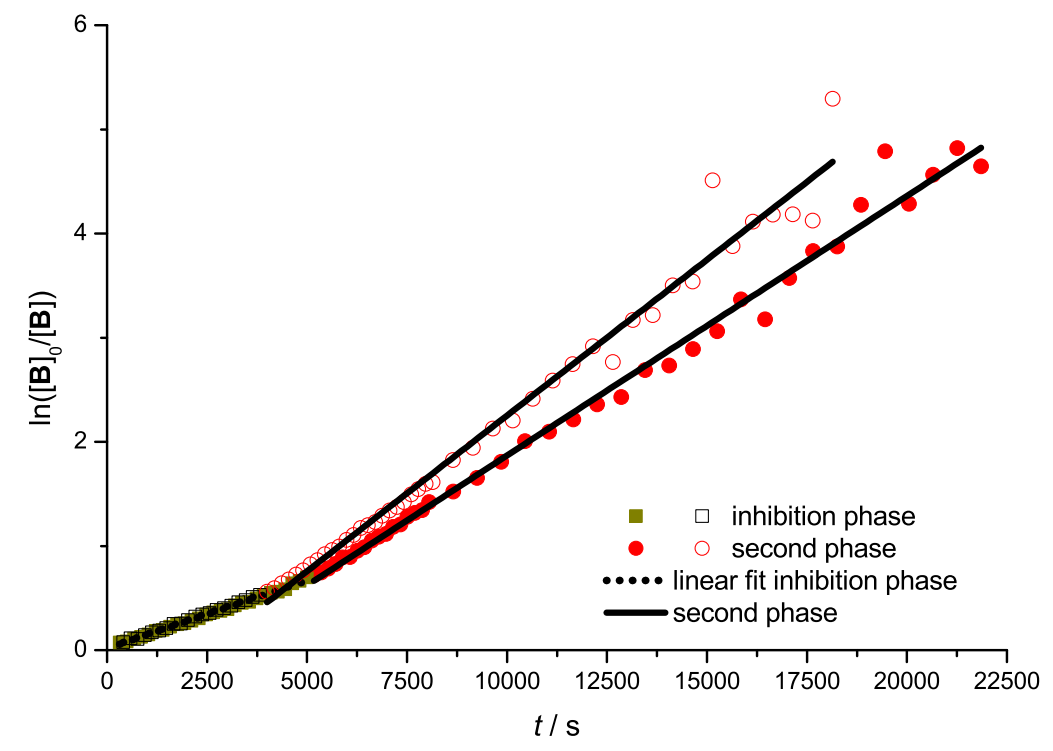

Figure 4.16: Kink shaped inhibtion period for alkylation in monomer-free CCG activation at $60^{\circ} \mathrm{C}$. 
of $1.32(2) \times 10^{-4} \mathrm{~s}^{-1}$ directly followed by a fast phase with a rate of $2.8(3) \times 10^{-6} \mathrm{~s}^{-1}$. The value of first equilibrium constant $K_{1, Z r}$ shows a slight temperature dependence which can be applied to determine thermodynamic data. A plot of $\ln \left(K_{1, Z \mathrm{r}}\right)$ versus $1 / T$ depicted in Figure 4.17 gives a reaction enthalpy of $\Delta H_{\mathrm{R}}=(-45 \pm 15) \mathrm{kJ} \mathrm{mol}^{-1}$ and a reaction entropy of $\Delta S_{\mathrm{R}}=(-69 \pm 45) \mathrm{J} \mathrm{mol}^{-1} \mathrm{~K}^{-1}$. The process is exotherm and entropically disfavored. For the second equilibrium constant $K_{2, \mathrm{Zr}}$, a maximum at $70{ }^{\circ} \mathrm{C}$ appears only slight above the value of $80^{\circ} \mathrm{C}$, i.e. that it is independent of temperature within the error tolerance.

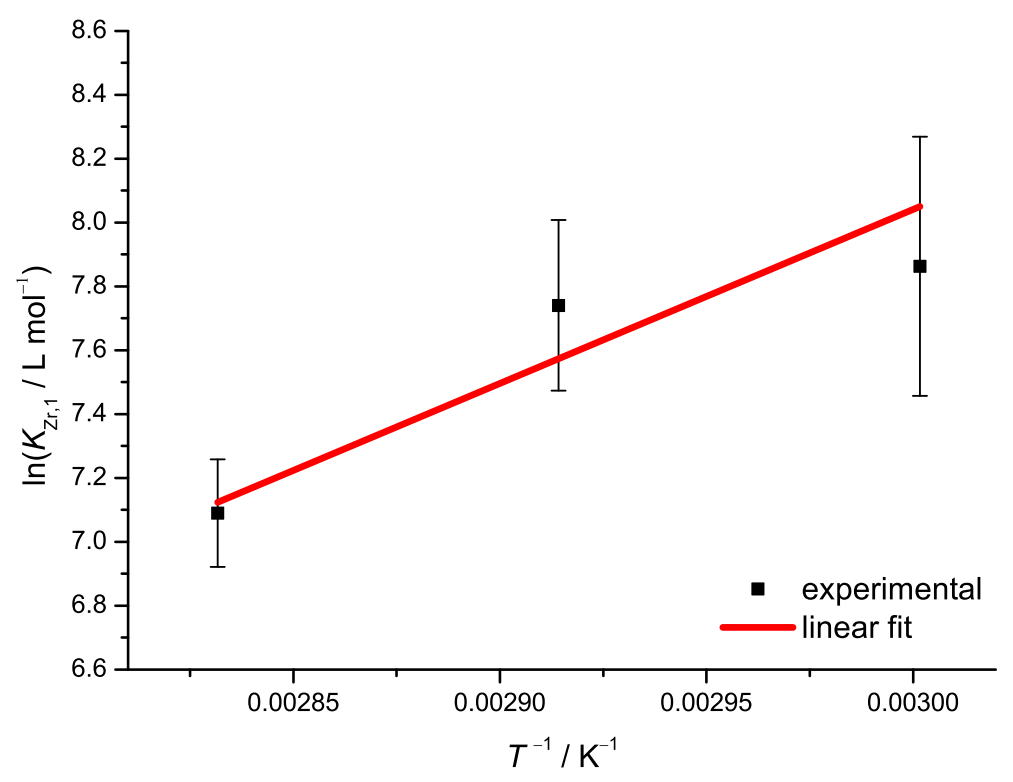

Figure 4.17: Plot of $\ln \left(K_{1, Z \mathrm{r}}\right)$ versus $1 / T$ for the determined equilibrium constant shown in Table 4.3 .

Because of the low concentration of $\mathbf{F}^{\mathrm{P0}}$ it was not possible to calculate $k_{\text {alkyl2 }}$ for $60^{\circ} \mathrm{C}$ and therefore the investigation of temperature influences was not successful. For the rate coefficient $k_{\text {alkyl1 }}$ temperature dependence was found and an activation energy $E_{\mathrm{A}}=\left(68 \pm 23 \mathrm{~kJ} \mathrm{~mol}^{-1}\right)$ was derived from an Arrhenius plot (see Figure 4.18). The process is endotherm. Hence the alkyl-chloride exchange is energetically disfavored which indicates that the chain transfer process on the bimetallic $\mathrm{Zr}-\mathrm{Mg}$ complex has a high reaction barrier. As this process is crucial for the success of the molar mass control it will strongly be influenced by temperature. 


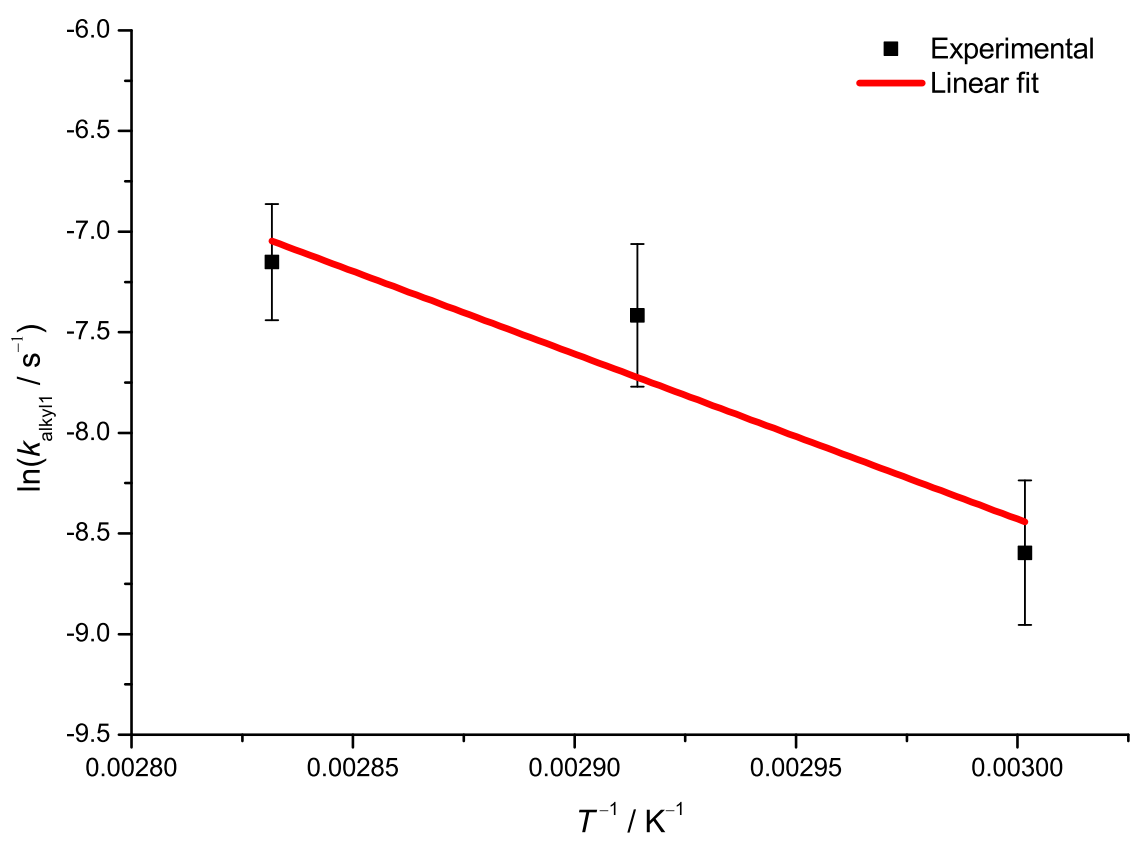

Figure 4.18: Arrhenius plot for $k_{\text {alkyl1 }}$ shown in Table 4.3

\subsubsection{UV/Vis Studies on $\mathrm{Cp}_{2}^{*} \mathrm{ZrCl}_{2}$ and $\mathrm{BzMgBz}$}

While the NMR studies lead to acceptable results in the research on CCG activation further suitable experimental methods for determination of kinetic coefficients were researched to allow higher catalyst/co-catalyst ratios, the presence of additives, nondeuterated solvent and the measurement of para-magnetic substances like the complex $\mathrm{Cp}_{2}{ }_{2} \mathrm{NdCl}_{2} \mathrm{Li}\left(\mathrm{OEt}_{2}\right)_{2}$. For this propose similar reactions were performed in a UV/Visspectrometer. Kinetic determination of the process was performed in the range of $800 \mathrm{~nm}$ to $400 \mathrm{~nm}$ because a strong increase in absorbance is found below $400 \mathrm{~nm}$. If the absorbance is too high, the resulting concentrations have a strong scattering. In Figure 4.19 time-resolved spectra within the range from $800 \mathrm{~nm}$ to $400 \mathrm{~nm}$ are shown.

Within the region from $500 \mathrm{~nm}$ to $400 \mathrm{~nm}$ (see Figure 4.19 a) and $\mathbf{b}$ )) the spectrum changes with proceeding reaction time. During the first 100 minutes of reaction the narrow absorbance at $400 \mathrm{~nm}$ changes and broadens while decreasing. A isosbestic point is found around $412 \mathrm{~nm}$. Absorbance at higher wavelenghts decreases while it increases at higher wavelengths until approx. $500 \mathrm{~nm}$. In combination with the NMR results (see Figure 4.6 on page 44) it can be described as the concentration decrease of species $\mathbf{B}^{\mathbf{P 0}}$ and the formation of species $\mathbf{D}^{\mathrm{P} 0}$.A closer look reveals, that the decrease of absorbance at $400 \mathrm{~nm}$ yields in a minimum. It is assumed that the minimum is reached after full reaction of $\mathbf{B}^{\mathrm{P} 0}$ to $\mathbf{D}^{\mathrm{P} 0}$ (see Figure $4.19 \mathrm{c}$ )). This allows the determination of time dependent con- 
a)

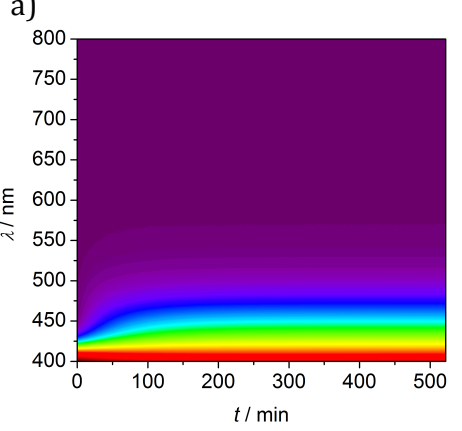

c)

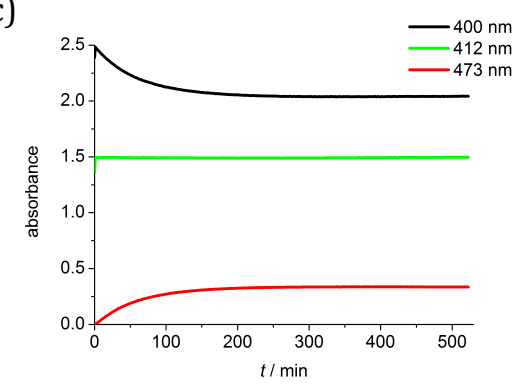

b)
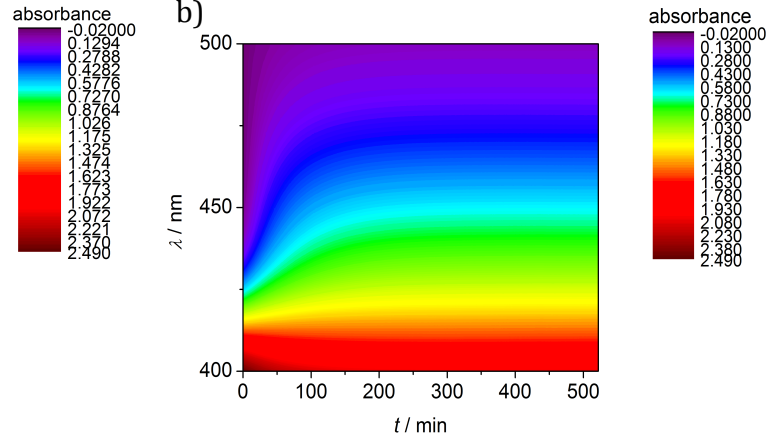

d)

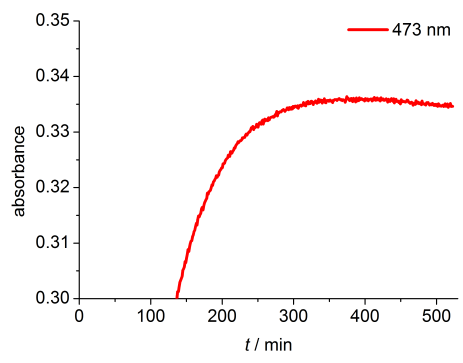

Figure 4.19: a) Diagram of absorbance in dependence of wavelength and time; b) zoom of the region of $400 \mathrm{~nm}$ to $500 \mathrm{~nm}$; c) Extracted time profile of absorbance; d) zoom at the peak of the absorbance at $473 \mathrm{~nm}$. Measured at $80^{\circ} \mathrm{C}, c\left(\mathrm{Cp}_{2}{ }_{2} \mathrm{ZrCl}_{2}\right)=0.0013 \mathrm{~mol} \mathrm{~L}^{-1}, c(\mathrm{BzMgBz})=0.039 \mathrm{~mol} \mathrm{~L}^{-1}$.

sumption of species $\mathbf{B}^{\mathbf{P 0}}$. The highest concentration was found within the first minutes of measurements as the maximum absorbance at $400 \mathrm{~nm}$. The species A shows absorbance at $400 \mathrm{~nm}$ as well, but much weaker as species B. Known from NMR measurements $\mathbf{A}$ is present in small concentration and as previously shown species $\mathbf{A}$ and $\mathbf{B}^{\mathbf{P 0}}$ are in equilibrium with each other. Therefore it is assumed that the absorbance of $\mathbf{A}$ and $\mathbf{B}^{\mathbf{P 0}}$ decrease with the same ratio. From the absorbance at $400 \mathrm{~nm}$ the concentration of $\mathbf{B}^{\mathbf{P 0}}$ can be calculated with Equation 4.20 .

$$
\left[\mathbf{B}^{\mathbf{P 0}}\right]=\frac{A^{400 \mathrm{~nm}}-A_{\min }^{400 \mathrm{~nm}}}{A_{\max }^{400 \mathrm{~nm}}-A_{\min }^{400 \mathrm{~nm}}}[\mathbf{A}]_{0}
$$

During all measurements an isosbestic point is found between $414 \mathrm{~nm}$ to $409 \mathrm{~nm}$, which could be applied to reduce influences of non-reaction nature, e.g. baseline shifts. Further, it marks the area for determination of species $\mathbf{D}^{\mathbf{P 0}}$. A single wavelength was chosen as well with $473 \mathrm{~nm}$ because the absorbance of $\mathbf{B}^{\mathbf{P 0}}$ is near to zero and small changes can be monitored giving the best compromise between signal quality and influence of species $\mathbf{B}^{\mathbf{P 0}}$ (see Figure $4.19 \mathrm{c}$ )). After the conversion of $\mathbf{B}^{\mathbf{P 0}}$ to $\mathbf{D}^{\mathbf{P 0}}$ the signal begins to decrease which is in agreement with the reaction mechanism (see Figure $4.19 \mathrm{~d}$ )) allowing the reaction from $\mathbf{D}^{\mathrm{P} 0}$ to $\mathbf{F}^{\mathrm{P} 0}$ to be monitored. It is found that $\mathbf{F}^{\mathrm{P} 0}$ shows absorbance at $473 \mathrm{~nm}$. Because of the low reaction rate the extinction coefficient of $\mathbf{F}^{\mathrm{P0}}$ was determined from a 
few measurements and was applied for calculation for species $\mathbf{D}^{\mathrm{P0}}$ by Equation 4.21 and Equation 4.22 .

$$
\begin{aligned}
& {\left[\mathbf{D}^{\mathbf{P 0}}\right]=\frac{A^{473 \mathrm{~nm}}-A_{\min }^{473 \mathrm{~nm}}}{\epsilon_{\mathrm{D}^{\mathrm{P0}}}^{473 \mathrm{~nm}} d}} \\
& {\left[\mathbf{D}^{\mathbf{P 0}}\right]=\frac{\frac{A^{473}{ }^{4 m}-A_{\min }^{473}}{d[\mathbf{n}]_{0}}-\epsilon_{\mathrm{F}^{\mathrm{P0}}}^{473 \mathrm{~nm}}}{\left(\epsilon_{\mathrm{D}^{\mathrm{P0}}}^{473 \mathrm{~nm}}-\epsilon_{\mathrm{F}^{\mathrm{P0}}}^{473 \mathrm{~nm}}\right)}[\mathbf{A}]_{0}}
\end{aligned}
$$

Equation 4.21 is valid until the absorbance at $473 \mathrm{~nm}$ reached its maximum. Afterwards the concentration has to be calculated via equation 4.22 applying the different extinction coefficients for $\mathbf{D}^{\mathbf{P 0}}$ to $\mathbf{F}^{\mathbf{P} 0}$. It cannot be excluded that it may cause a higher error within the concentration and influences the kinetic coefficients, but it was not possible to find an unaffected wavelength. Based on Equation 4.21 and $4.22 \mathrm{UV} / \mathrm{Vis}$ measurements were performed for the different concentrations in the range from $60^{\circ} \mathrm{C}$ to $80^{\circ} \mathrm{C}$. The solutions were stirred during measurements to prevent diffusion control. Similar to NMR experiments first-order rate plots for each temperature and concentration were performed and an example for each measurement is shown in figures 4.20 and 4.21 . The results were summarized in Table 4.4 with the combined rate coefficients of all $\mathrm{BzMgBz}$ concentrations because it was found that the alkylation rate is independent from it.

Table 4.4 Kinetic parameters derived from experimental UV/Vis data of momomer free CCG activation of various temperatures, $c\left(\mathrm{Cp}_{2}^{*} \mathrm{ZrCl}_{2}\right)=0.0013 \mathrm{~mol} \mathrm{~L}^{-1}, c(\mathrm{BzMgBz})=0.069 \mathrm{~mol} \mathrm{~L}^{-1}, 0.053 \mathrm{~mol} \mathrm{~L}^{-1}$ and $0.033 \mathrm{~mol} \mathrm{~L}^{-1}$ in toluene by application of Equation 4.18 to 4.19 .

\begin{tabular}{c|c|c}
$T /{ }^{\circ} \mathrm{C}$ & $k_{\mathrm{alkyl} 1} \times 10^{-5} \mathrm{~s}^{-1}$ & $k_{\mathrm{alkyl} 2} \times 10^{-6} \mathrm{~s}^{-1}$ \\
\hline 60 & $19 \pm 7$ & $2 \pm 1$ \\
70 & $65 \pm 27$ & $4 \pm 2$ \\
80 & $118 \pm 86$ & $11 \pm 6$
\end{tabular}

The results show a high scattering for each temperature, especially at $80^{\circ} \mathrm{C}$. For $80^{\circ} \mathrm{C}$ and $70^{\circ} \mathrm{C}$ the plots are well described by a linear fit (see Figure 4.20$)$, but for $60^{\circ} \mathrm{C}(4.20$ c) ) a pronounced induction phase is observed similar to the NMR experiments at the $\mathrm{Zr}: \mathrm{Mg}$ ratio of 1:20. At higher temperatures only short induction phases are found, which are caused by heating up the sample and reorganization of the equilibrium from room temperature to measurement temperature. As an explanation the presence of remaining diethyl ether may affect the rate coefficient. The ether cannot be removed completely during synthesis and even small amounts of ether have an influence on the kinetics of the experiments. To confirm this assumption the effect was discussed in more detail in 

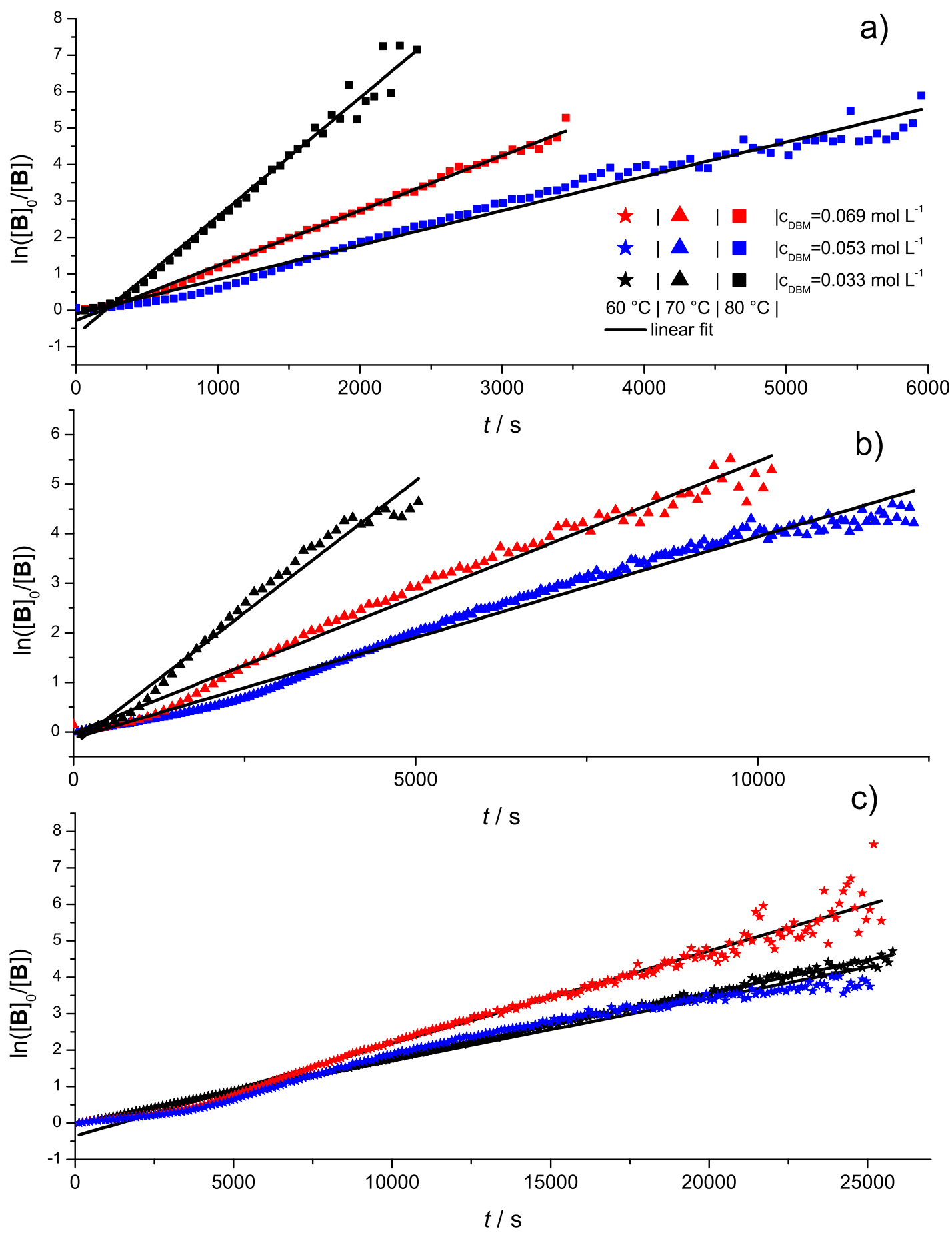

Figure 4.20: First order plots of species $\mathbf{B}^{\mathbf{P 0}}$ determined by UV/Vis analysis with $c\left(\mathrm{Cp}_{2}{ }_{2} \mathrm{ZrCl}_{2}\right)=0.0013 \mathrm{~mol} \mathrm{~L}^{-1}$ using different concentrations of BzMgBz and different temperatures; $80^{\circ} \mathrm{C}(\mathrm{a}) ; 70^{\circ} \mathrm{C}(\mathrm{b}) ; 60^{\circ} \mathrm{C}$; (c) $c(\mathrm{BzMgBz})=0.069 \mathrm{~mol} \mathrm{~L}^{-1}$ (red), $c(\mathrm{BzMgBz})=0.053 \mathrm{~mol} \mathrm{~L}^{-1}$ (blue) and $c(\mathrm{BzMgBz})=0.033 \mathrm{~mol} \mathrm{~L}^{-1}$ (black). The lines are the best linear fits of the measurements. 

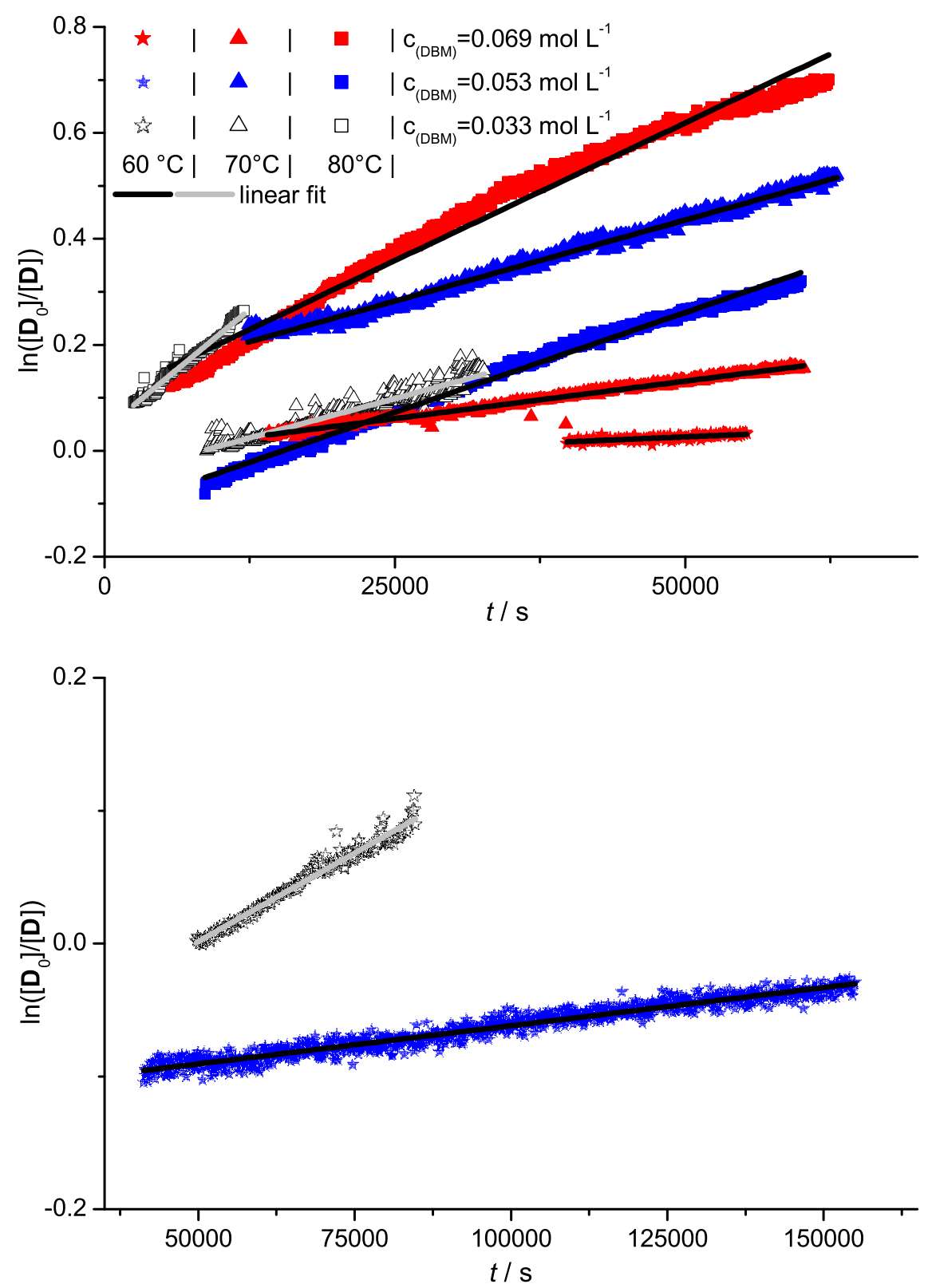

Figure 4.21: First order plots of species $\mathrm{D}^{\mathrm{P0}}$ determined by UV/Vis analysis $c\left(\mathrm{Cp}^{*}{ }_{2} \mathrm{ZrCl}_{2}\right)=0.0013 \mathrm{~mol} \mathrm{~L}^{-1}$ using different concentrations of $\mathrm{BzMgBz}$ and different temperatures; $80^{\circ} \mathrm{C}(\mathrm{a}) ; 70^{\circ} \mathrm{C}(\mathrm{b}) ; 60^{\circ} \mathrm{C}(\mathrm{c}) ; c(\mathrm{BzMgBz})=0.069 \mathrm{~mol} \mathrm{~L}^{-1}(\mathrm{red}), c(\mathrm{BzMgBz})=0.053 \mathrm{~mol} \mathrm{~L}^{-1}$ (blue) and $c(\mathrm{BzMgBz})=0.033 \mathrm{~mol} \mathrm{~L}^{-1}$ (black). The lines are the best linear fits of the measurements. 
Section 4.3 .3 on the following page and 4.3 .4 on page 65 .

The results fit well for $60^{\circ} \mathrm{C}$ and $70^{\circ} \mathrm{C}$ with the NMR data while for $80^{\circ} \mathrm{C}$ a higher value was achieved. The Arrhenius plot is giving a better fit then in NMR experiments (see Figure 4.22 and 4.23 . Activation energy of $E_{\mathrm{A}}=101 \pm 18 \mathrm{~kJ} \mathrm{~mol}^{-1}$ and a pre-exponential factor of $A=1.2 \times 10^{12} \mathrm{~s}^{-1}$ are found. Similar scattering as in NMR experiments is achieved with the $70^{\circ} \mathrm{C}$ value determined above the linear fit, while the overall error is reduced. For $k_{\text {alkyl2 }}$ temperature dependence is found and an Arrhenius plot shows good agreement with the linear fit. Activation energy of $E_{\mathrm{A}}=83 \pm 13 \mathrm{~kJ} \mathrm{~mol}^{-1}$ and a pre-exponential factor of $A=2 \times 10^{7} \mathrm{~s}^{-1}$ were derived.

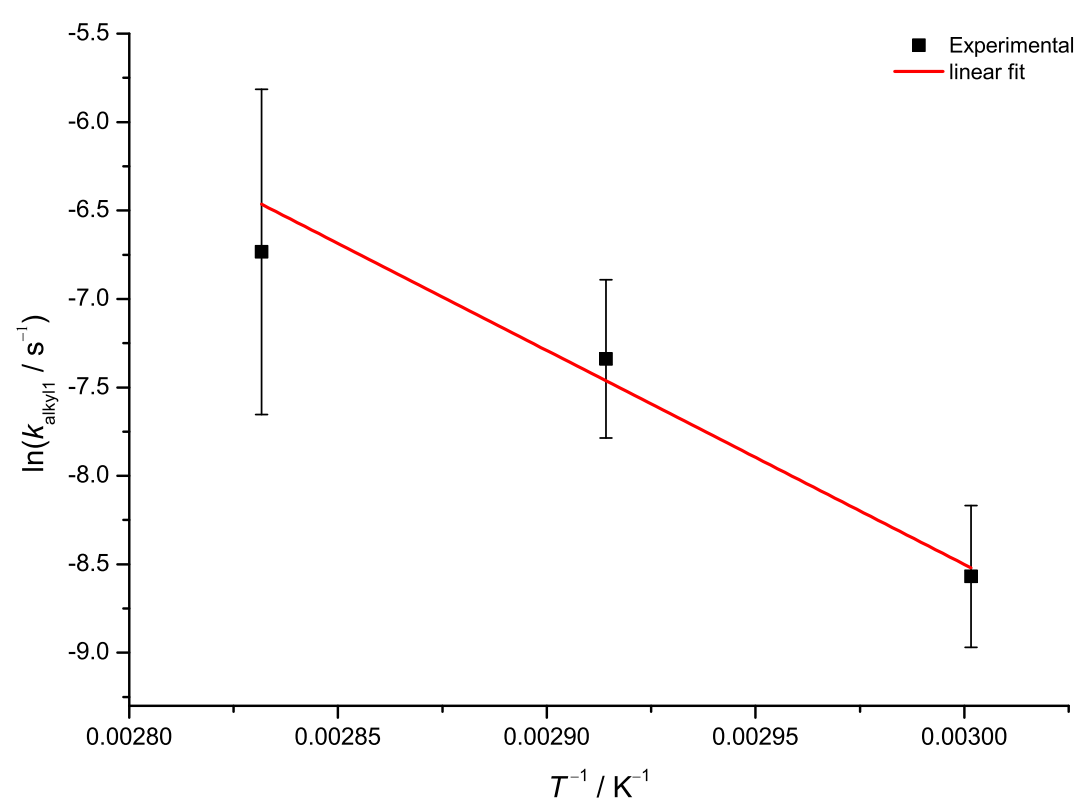

Figure 4.22: Arrhenius plot of $k_{\mathrm{alkyl} 11}$ derived from the kinetic coefficients derived by UV/Vis measurements.

The measurements with UV/Vis are suitable for kinetic research on the model system and can be applied for further studies. The assumption made for the analysis have only a slight influence on the derived kinetic data while higher error values are determined. This allows the application of UV/Vis methods to derive rate coefficients if NMR measurements are not suitable. 


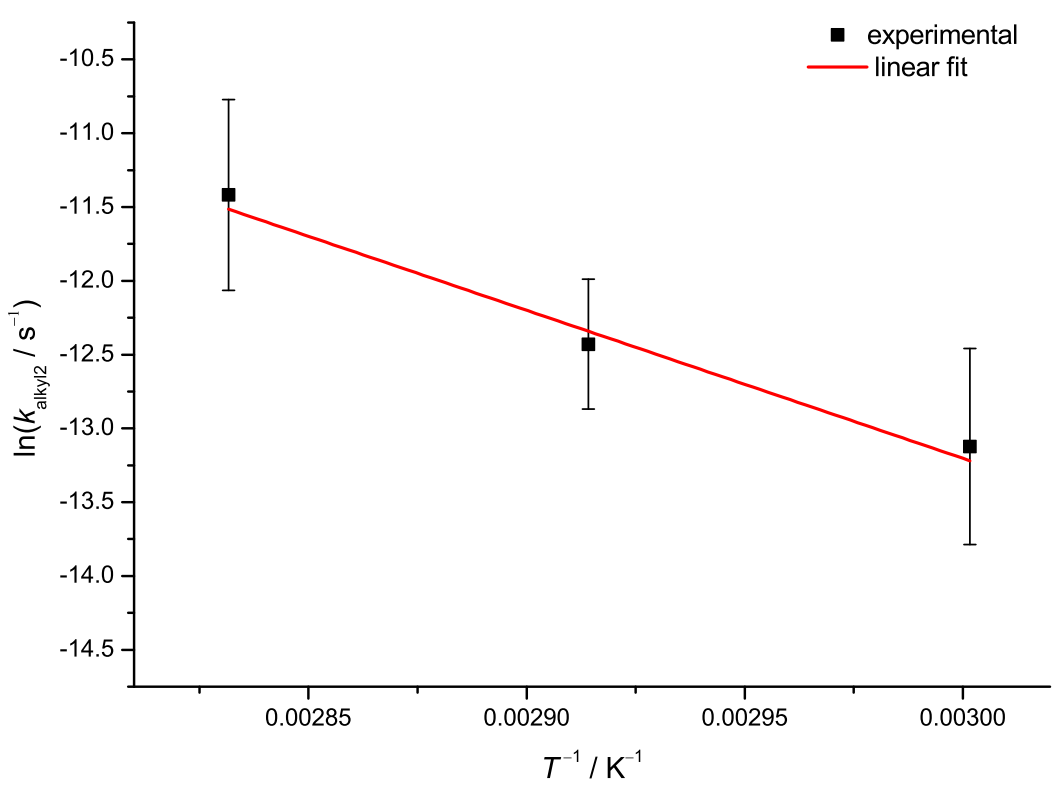

Figure 4.23: Arrhenius plot of $k_{\text {alkyl2 }}$ derived from the kinetic coefficients derived by UV/Vis measurements.

\subsubsection{NMR Study on $\mathrm{Cp}_{2}^{*} \mathrm{ZrCl}_{2}, \mathrm{BzMgBz}$ and di- $n$-butyl ether (DBE)}

In order to investigate the influence of ether present during the measurement a series of further experiments on the one hand via NMR spectroscopy and on the other hand by a broad series of UV/Vis studies were performed. For NMR studies a suitable fully deuterated ether is not available in the desired temperature range of $60^{\circ} \mathrm{C}$ to $80^{\circ} \mathrm{C}$ for measurements at high ether concentrations. This makes the studies with UV/Vis more convenient and allows the use of di- $n$-butyl ether (DBE) which is already applied in CCG. ${ }^{681}$ Via a time resolved NMR measurement it was cross-checked that the same species are formed in presence of DBE (see Figure 4.24).

The stacked spectra show that the similar species are formed as shown in Figure 4.4 on page 41 but the formation of $\mathbf{F}^{\mathbf{P 0}}$ is hindered. On a closer look the concentration vs. time profile for $\mathbf{B}$ and $\mathbf{D}$ shows a dent (see Figure 4.25). The reason for this behavior is not exactly clear. The reaction rates were determined for the full time without any further analysis of the dent (see Figure 4.26 and Table 4.5).

The determined coefficients were introduced into the model (see section 4.3 .1 on page 46) and the comparison of simulation and experiment is shown in Figure 4.27. The model is not able to show the dent which is the mayor difference for the concentration of $\mathbf{B}$ and $\mathbf{D}$ while $\mathbf{B z M g B z}$ and $\mathbf{C}$ are well described. For the concentration of $\mathbf{F}$ a mayor difference is found which is caused one the one hand caused by the discussed overlay 


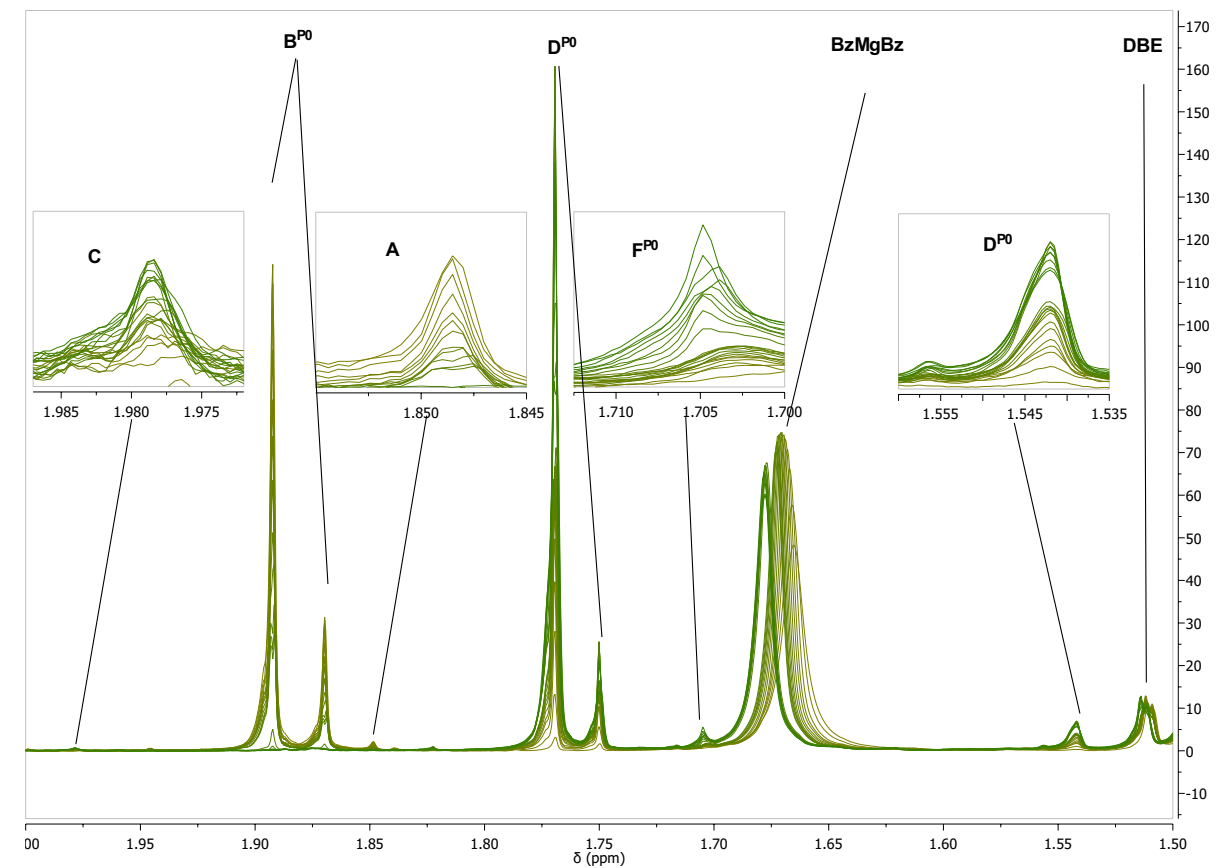

Figure 4.24: Determined species via time-resolved NMR measurement of monomer-free CCG model system using $\mathrm{Cp}^{*}{ }_{2} \mathrm{ZrCl}_{2}$ and $\mathrm{BzMgBz}$ with $\mathrm{DBE}$ at $70^{\circ} \mathrm{C}, c\left(\mathrm{Cp}^{*}{ }_{2} \mathrm{ZrCl}_{2}\right)=4.6 \mathrm{mmoll}^{-1}$ $c(\mathrm{BzMgBz})=46 \mathrm{mmoll}^{-1} c(\mathrm{DBE})=96 \mathrm{mmoll}^{-1}$ in toluene- $\mathrm{d} 8$ with only every tenth measurement shown for clarity reasons (yellow - short reaction time, green - long reaction time).

Table 4.5 Kinetic parameters derived from experimental NMR data of momomer-free CCG activation with DBE present at $70^{\circ} \mathrm{C}, c\left(\mathrm{Cp}_{2}{ }_{2} \mathrm{ZrCl}_{2}\right)=4.6 \mathrm{mmoll}^{-1}, c(\mathrm{BzMgBz})=46 \mathrm{mmoll}^{-1}$, $c(\mathrm{DBE})=96 \mathrm{mmol}^{-1}$ in toluene- $d 8$ by application of Equation 4.16 to 4.19 .

\begin{tabular}{c|r|l} 
coefficients & value & lnit \\
\hline$K_{1, \mathrm{Zr}}$ & 2.6 & $\times 10^{3} \mathrm{~mol} \mathrm{~L}^{-1}$ \\
$K_{2, \mathrm{Zr}}$ & 5.9 & $\times 10^{3} \mathrm{~mol} \mathrm{~L}^{-1}$ \\
$k_{\text {alkyl1 }}$ & 2.6 & $10^{-4} \mathrm{~s}^{-1}$ \\
$k_{\text {alkyl2 }}$ & 6.4 & $10^{-6} \mathrm{~s}^{-1}$
\end{tabular}




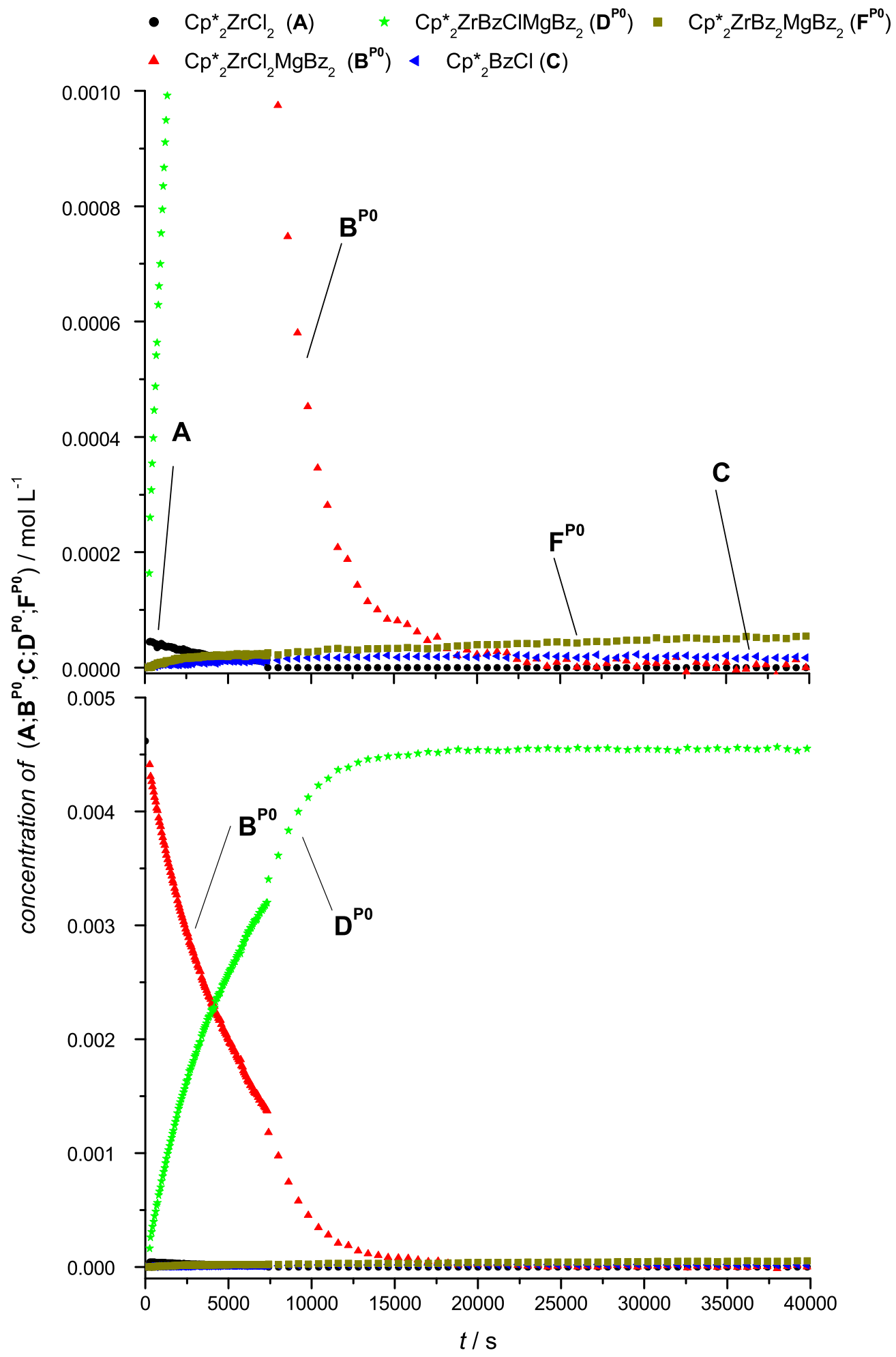

Figure 4.25: Concentration time profile of the formed species in monomer-free CCG activation with DBE present at $70{ }^{\circ} \mathrm{C}, c\left(\mathrm{Cp}_{2}{ }_{2} \mathrm{ZrCl}_{2}\right)=4.6 \mathrm{mmoll}^{-1}, c(\mathrm{BzMgBz})=46 \mathrm{mmol}^{-1}, \mathrm{c}_{\mathrm{DBE}}=96 \mathrm{mmol}^{-1}$ in toluene- $d 8$. 


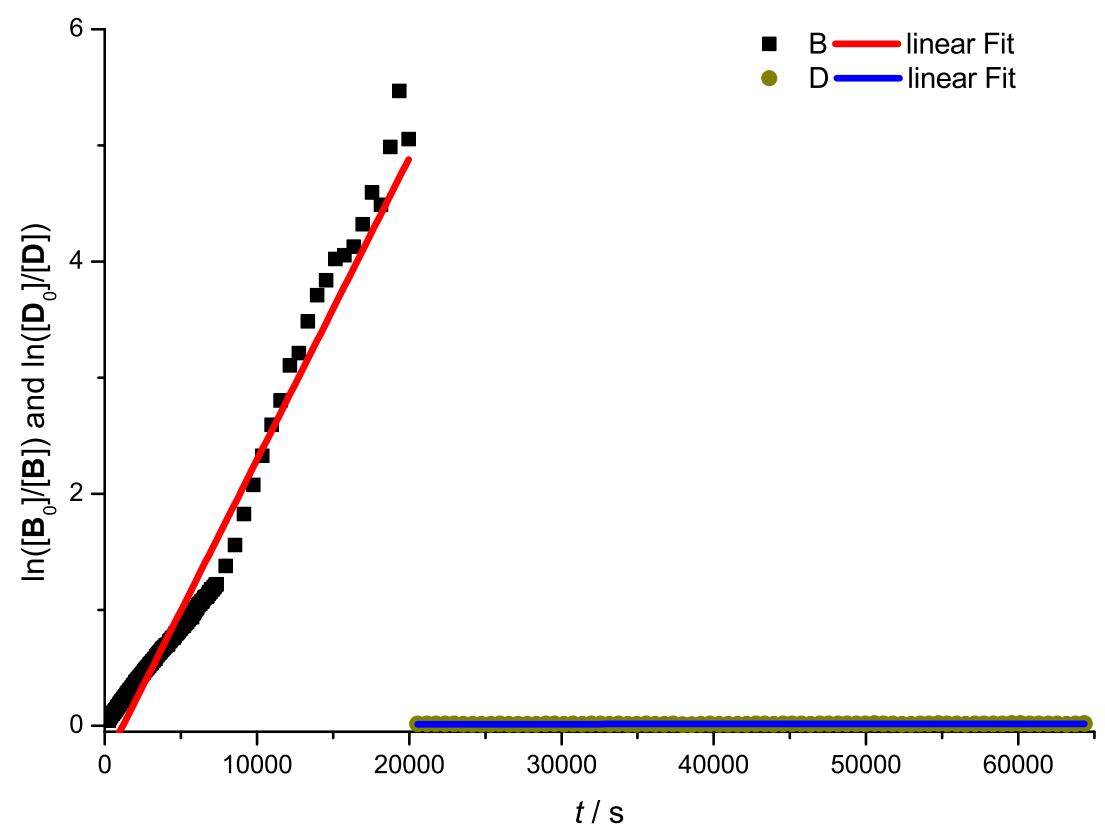

Figure 4.26: First linear plots of species $B$ and $D$ for monomer-free CCG activation with DBE present at $70{ }^{\circ} \mathrm{C}, c\left(\mathrm{Cp}_{2}^{*} \mathrm{ZrCl}_{2}\right)=4.6 \mathrm{mmol}^{-1}, c(\mathrm{BzMgBz})=46 \mathrm{mmoll}^{-1}, c_{\mathrm{DBE}}=96 \mathrm{mmol}^{-1}$ in toluene- $d 8$.

with the ${ }^{13} \mathrm{C}-{ }^{1} \mathrm{H}$ coupling of the signal of $\mathbf{D}$ and on the other hand by the small overall concentration change leading to a lower rate coefficient determined by the first order rate plot.

\subsubsection{UV/Vis Studies on $\mathrm{Cp}_{2}^{*} \mathrm{ZrCl}_{2}, \mathrm{BzMgBz}$ and DBE}

With the NMR results showing that the same species are formed as in absence of DBE further measurements were performed using DBE as co-solvent. Parts of the measurement data were provided by Adrian Deinert within his bachelor thesis. The ether was chosen due to its high boiling point and its application in CCG polymerization for a neodymium/ magnesium system. ${ }^{[68 \mid}$ Five different volume fractions of toluene/DBE ether were chosen from 0.005 to 0.89 (v:v). Measurements at $100 \%$ DBE are not possible because $\mathbf{A}$ is insoluble in ether. The measurements with no DBE added have $0.1 \%$ diethyl ether left. The calculation were performed similar to the UV/Vis experiments shown previously (see section 4.3 .2 on page 58) and results are summarized in Table 4.6.

The results show a strong effect induced by the addition of DBE. It was found that the reaction rate is lowered by one order of magnitude at each temperature by increasing the amount. The difference in the range of $0.1 \%$ to $0.6 \%$ is found to be higher for each 


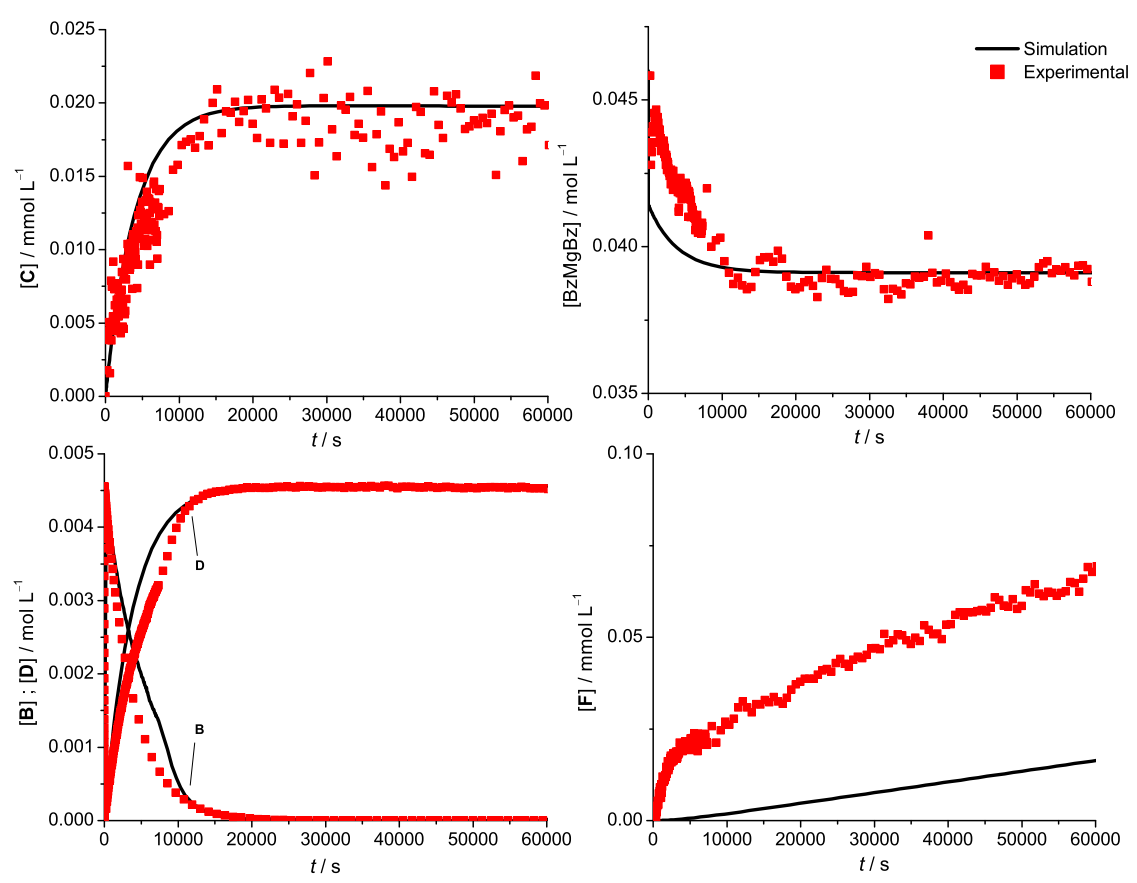

Figure 4.27: Simulation versus experiment for monomer-free CCG activation in presence of DBE with the data of Table 4.5

Table 4.6 Rate coefficient $k_{\text {alkyl1 }}$ derived from experimental UV/Vis data of momomer free CCG activation in presence of di- $n$-butyl ether. $c\left(\mathrm{Cp}_{2}^{*} \mathrm{ZrCl}_{2}\right)=0.0013 \mathrm{~mol} \mathrm{~L}^{-1}, c(\mathrm{BzMgBz})=0.044 \mathrm{~mol} \mathrm{~L}^{-1}$ in toluene/DBE by application of Equation 4.18 to 4.19 .

\begin{tabular}{c|c|c|c|c}
$\varphi_{\text {Ether }}(v: v)$ & $60^{\circ} \mathrm{C}$ & $70^{\circ} \mathrm{C}$ & $80^{\circ} \mathrm{C}$ & unit \\
\hline 0.001 & $19 \pm 7$ & $65 \pm 27$ & $118 \pm 86$ & $10^{-5} \mathrm{~s}^{-1}$ \\
0.006 & $6.3 \pm 2.3$ & $19 \pm 1$ & $45 \pm 7$ & $10^{-5} \mathrm{~s}^{-1}$ \\
0.051 & $4.0 \pm 0.8$ & $11 \pm 1$ & $24 \pm 3$ & $10^{-5} \mathrm{~s}^{-1}$ \\
0.25 & $2.0 \pm 0.1$ & $5.3 \pm 0.8$ & $11 \pm 1$ & $10^{-5} \mathrm{~s}^{-1}$ \\
0.50 & $1.0 \pm 0.1$ & $2.8 \pm 0.5$ & $6.3 \pm 1$ & $10^{-5} \mathrm{~s}^{-1}$ \\
0.89 & $1.1 \pm 0.3$ & $2.1 \pm 0.1$ & $4.5 \pm 0.5$ & $10^{-5} \mathrm{~s}^{-1}$
\end{tabular}


temperature than at $50 \%$ to $89 \%$. In Figure 4.28 a plot of $k_{\text {alkyll }}$ against volume fraction of ether is shown for $80^{\circ} \mathrm{C}$.

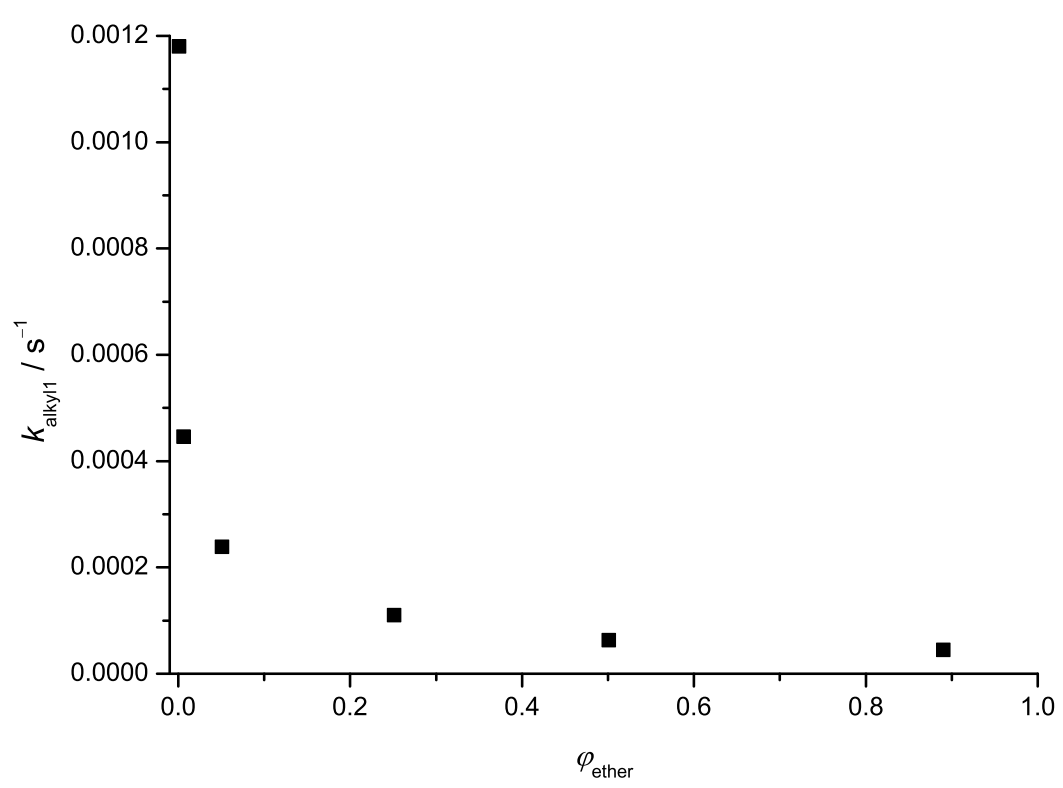

Figure 4.28: Plot of the volume fraction of ether against $k_{\text {alkyl1 }}$ at $80^{\circ} \mathrm{C}$.

The influence of additional ether reveals to be dramatic at low amounts of ether. The addition of $5 \%$ ether lowers the reaction rate nearby to the factor of 3 . It explains the high scattering of the experimental results, because of the fact that at a low ether fraction the kinetics are strongly influenced by only small variations in diethyl ether concentrations. Similar effects are found in cationic polymerization. ${ }^{[275]}$ In addition to normal scattering this leads to higher uncertainty. A double logarithmic plot of $\log (\varphi$ Ether $)$ against $\log \left(k_{\mathrm{alkyl}}\right)$ for each temperature shows linear behavior for each temperature (see Figure 4.29) which makes the calculation of the influence of ether possible.

Equations 4.23 to 4.25 were determined from the data, allowing calculation of $k_{\text {alkyl1 }}$ for each temperature.

$$
\begin{aligned}
& \log \left(k_{\text {alkly } 1,60^{\circ} \mathrm{C}}\right)=-5.01(6)-0.41(4) \log \left(\varphi_{\text {ether }}\right) \\
& \log \left(k_{\text {alkly } 1,70^{\circ} \mathrm{C}}\right)=-4.65(6)-0.47(4) \log \left(\varphi_{\text {ether }}\right) \\
& \log \left(k_{\text {alkly } 1,80^{\circ} \mathrm{C}}\right)=-4.31(5)-0.46(3) \log \left(\varphi_{\text {ether }}\right)
\end{aligned}
$$

The values in brackets determine the accuracy of the last digit of constant and slope. While the $y$-intercepts increase with temperature the slope is not affected in the same manner. In comparison to the lower value of $60^{\circ} \mathrm{C}$ the slope stays constant at higher 


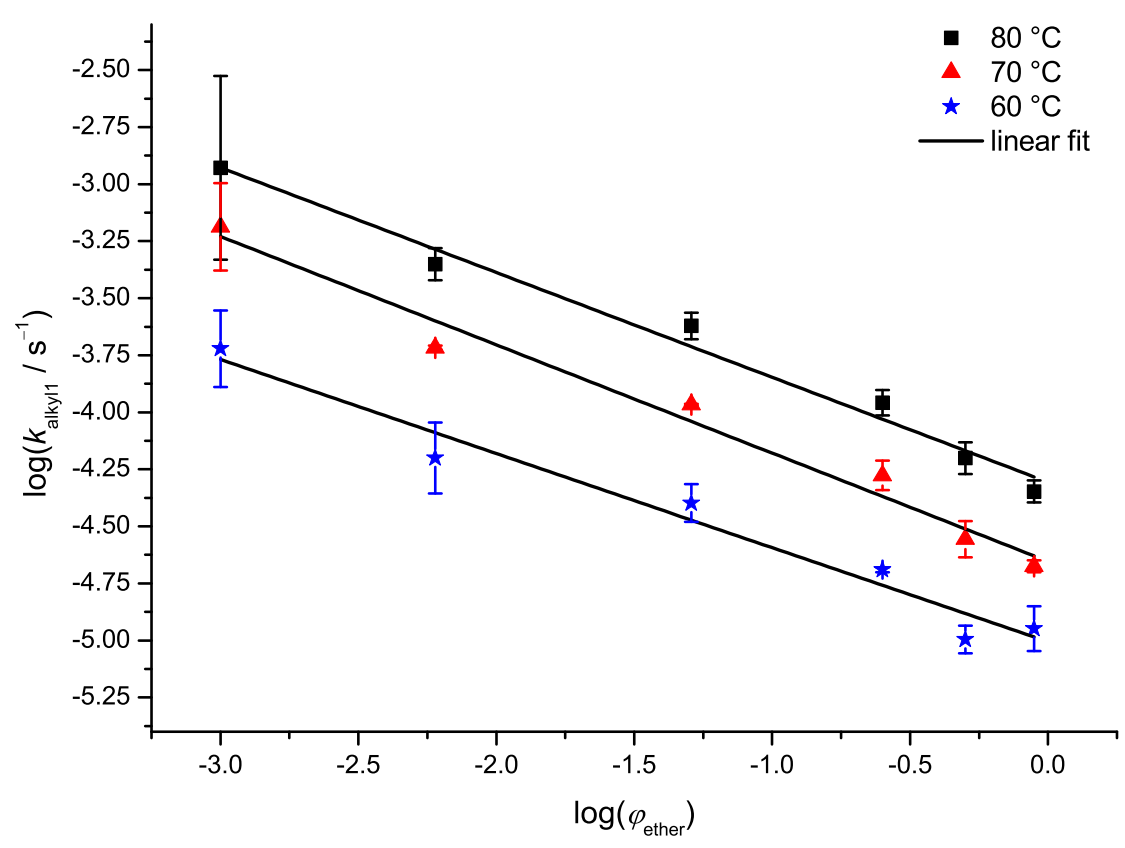

Figure 4.29: Double logarithmic plot of $\log \left(\varphi_{\text {Ether }}\right)$ against $\log \left(k_{\text {alkyl1 }}\right)$ at $60^{\circ} \mathrm{C}($ blue $), 70^{\circ} \mathrm{C}($ red $)$ and $80^{\circ} \mathrm{C}$ (black).

temperature. The individual reaction rates remain temperature dependent and Arrhenius plots for each volume fraction give further information (see Figure 4.30 and Table 4.7).

Table 4.7 Arrhenius plot parameters for different volume fractions of ether-

\begin{tabular}{c|c|c}
$\varphi_{\text {Ether }}$ & $E_{\mathrm{A}} / \mathrm{kJ} \mathrm{mol}^{-1}$ & Pre-exponential factor $/ 10^{9} \mathrm{~s}^{-1}$ \\
\hline 0.001 & $101 \pm 18$ & 1250 \\
0.006 & $87 \pm 5$ & 4 \\
0.051 & $83 \pm 8$ & 5 \\
0.25 & $83 \pm 2$ & 0.2 \\
0.50 & $89 \pm 3$ & 1 \\
0.89 & $74 \pm 9$ & 0.003
\end{tabular}

The activation energy and the pre-exponential factor are nearby constant in the range of $\varphi_{\text {Ether }}=0.5 \%-50 \%$. For lower fractions the effect is dramatic, the pre-exponential factor increases by several orders of magnitude and the activation energy is higher. Vice versa at high fractions the pre-exponential factor decreases by several orders of magnitude and the activation energy is lower. In combination these results show that the addition of DBE changes strongly the reaction rates. Mainly by coordination to magnesium and 


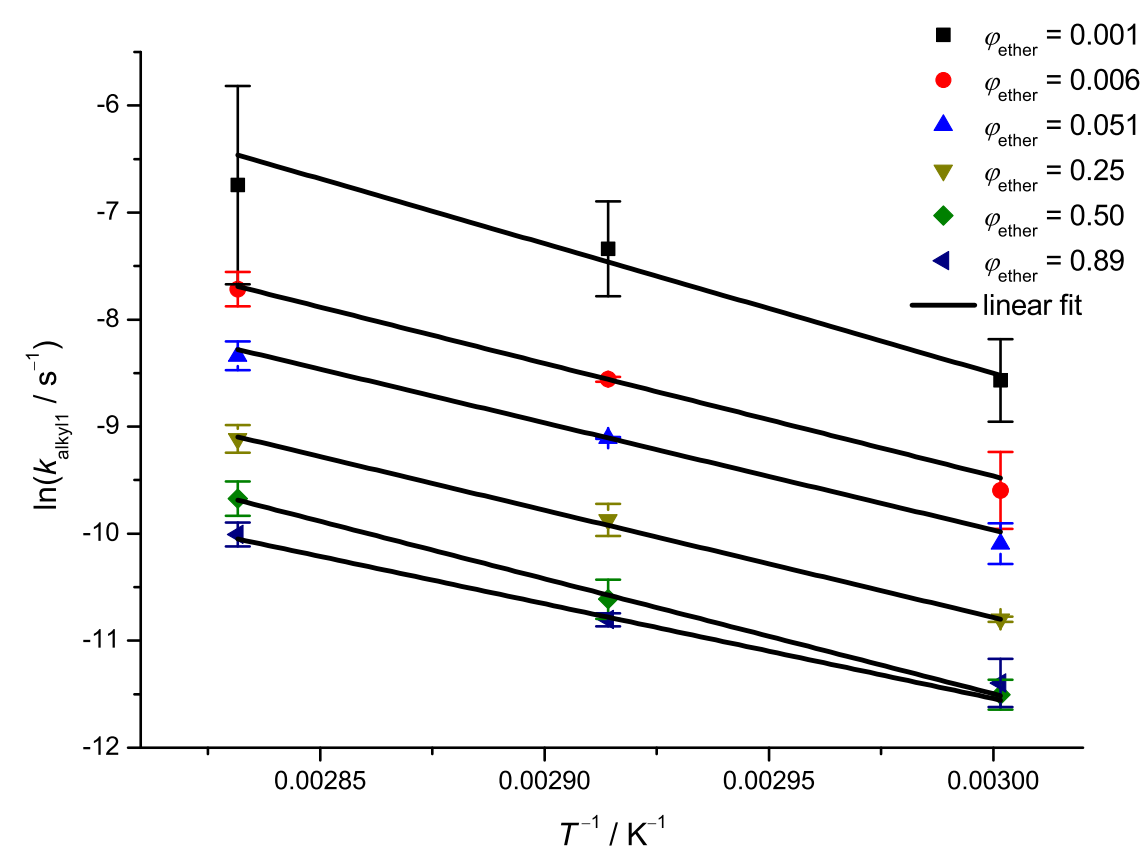

Figure 4.30: Arrhenius plots for the different volume fractions of ether.

zirconium it is hindering the ligand exchange reaction while the NMR-results showed that the equilibrium constant is not effected in the same manner. The lowering in activation energy indicates that the overall chain exchange is energetically favored with ether present while the chance of reaction is statistically reduced in presence of ether.

\subsection{NMR and UV/Vis Studies on $\mathrm{Cp}_{2}^{*} \mathrm{ZrCl}_{2}$ with Dialkyl Magnesium Compounds}

The advantage of the $\beta$-hydride elimination free activation comes in hand with high equilibrium constants and slow alkylation of the catalyst. With the performed experiments previous shown it was possible to investigate the influence of the termination process on the mechanistic scheme by experiments in presence of the typical co-catalyst di- $n$-butyl magnesium (BuMgBu). To determine the different species NMR experiments were performed first with $\mathrm{BuMgBu}$ and $\mathrm{A}$.

Due to the low solubility of BuMgBu in absence of equal amounts of ether the concentration of the magnesium compound was very low. It was impossible to determine the exact $\mathrm{Zr}: \mathrm{Mg}$ ratio but different species could be successful derived in comparison with literature data. At $1.84 \mathrm{ppm}$ the catalyst precursor $\mathbf{A}$ is found which forms the chloride 

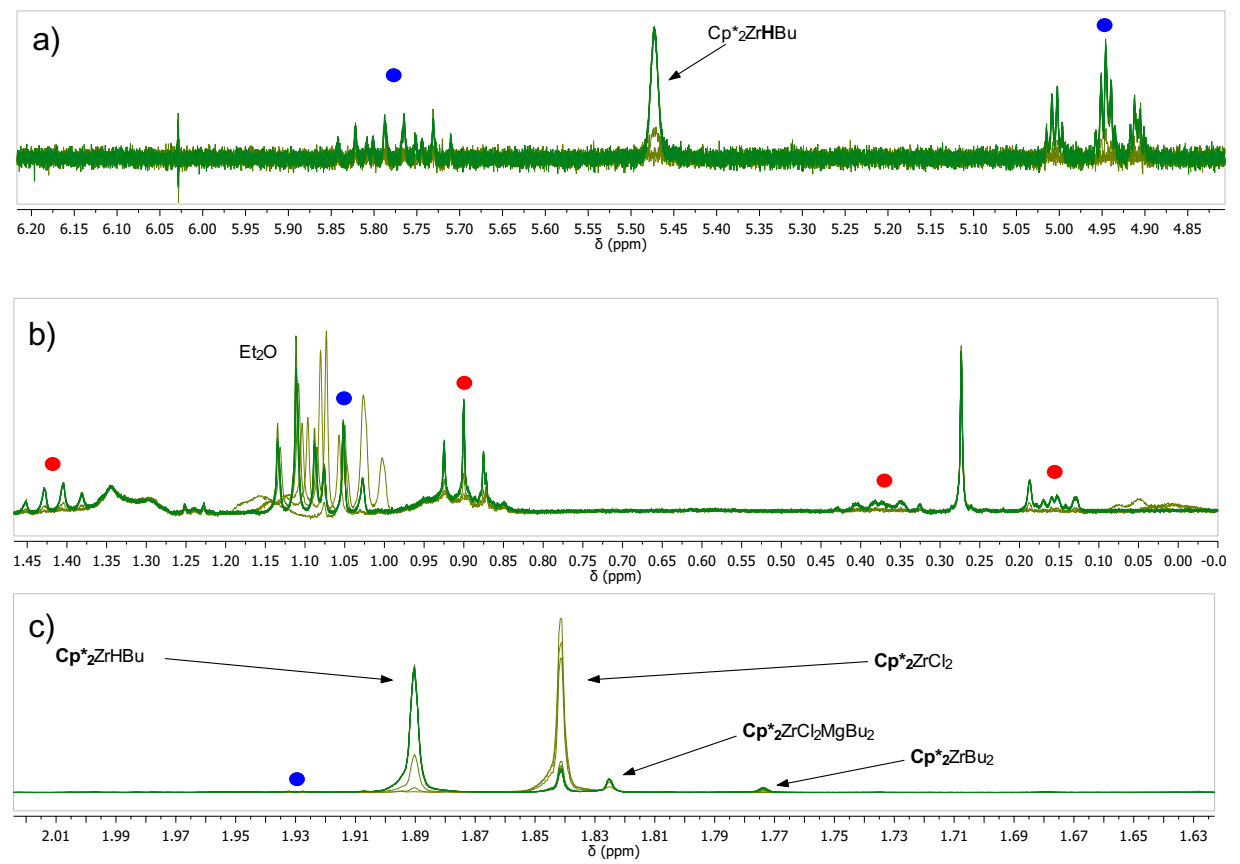

Figure 4.31: Catalyst species and reaction productes derived from NMR by the reaction of $\mathrm{A}$ with a small amount of $\mathrm{BuMgBu}, c\left(\mathrm{Cp}_{2}{ }_{2} \mathrm{ZrCl}_{2}\right)=1 \mathrm{~mol} \mathrm{~L}^{-1}$.

brigded $\mathrm{Zr}-\mathrm{Mg}$ complex (B) with BuMgBU and is found at $1.83 \mathrm{ppm}$ (see Figure 4.31). The complex formations of $\mathbf{C}$ and $\mathbf{D}$ were not detected. At $1.89 \mathrm{ppm}$ a new signal occurs with proceeding time together with three further signals in the alkene region and was identified as ligand signal of the complex $\mathrm{Cp}^{*}{ }_{2} \mathrm{ZrHBu} \mathbf{H}$ which is in agreement with the literature spectra. ${ }^{276,277]}$ The broad signals at $5.77 \mathrm{ppm}$ and $4.95 \mathrm{ppm}$ were identified as butene (blue dots). The signal at $5.48 \mathrm{ppm}$ belongs to the hydride of complex $\mathrm{Cp}_{2}{ }_{2} \mathrm{ZrHBu}$ which is further confirmed by the alkyl signals at $0.16 \mathrm{ppm}$ and $0.37 \mathrm{ppm}$ combined with the further signals (red dots). After the rapid formation of species $\mathbf{H}$ the signal at $1.78 \mathrm{ppm}$ increases with proceeding time. It is assumed to be the bialkylated catalyst $\mathbf{E}$, which is formed by ligand exchange reactions between the complexes $\mathbf{A}, \mathbf{B}$ and $\mathbf{H}$. Similar processes are found for e.g. $\mathrm{Cp}^{*}{ }_{2} \mathrm{ZrHCl}$ with $\mathrm{Cp}^{*}{ }_{2} \mathrm{ZrCl}_{2}$ complexes in literature. ${ }^{[276]}$ The long term stability of complex species $C$ is due to slow dissociation of the complex. A mechanism based on these reactions can be proposed and is shown in Scheme 4.4.

Complex A is transformed into $\mathbf{B}$ which slowly dissociates back or performs the alkylation to $C$ which rapidly undergoes $\beta$-hydride elimination to the high reactive species G. Via metal-ligand exchange between complex $\mathbf{G}$ and BuMgBu complex $\mathbf{H}$ and butyl magnesium chloride are formed. $\mathbf{H}$ is capable to undergo a further ligand exchange with $\mathbf{A}$ forming $\mathbf{C}$ and $\mathbf{G}$. The reaction is auto-catalytic, the conversion of $\mathbf{A}$ is rapidly increased after the first termination event, resulting in a mixture of $\mathbf{G}$ and $\mathbf{B}$. The dis- 


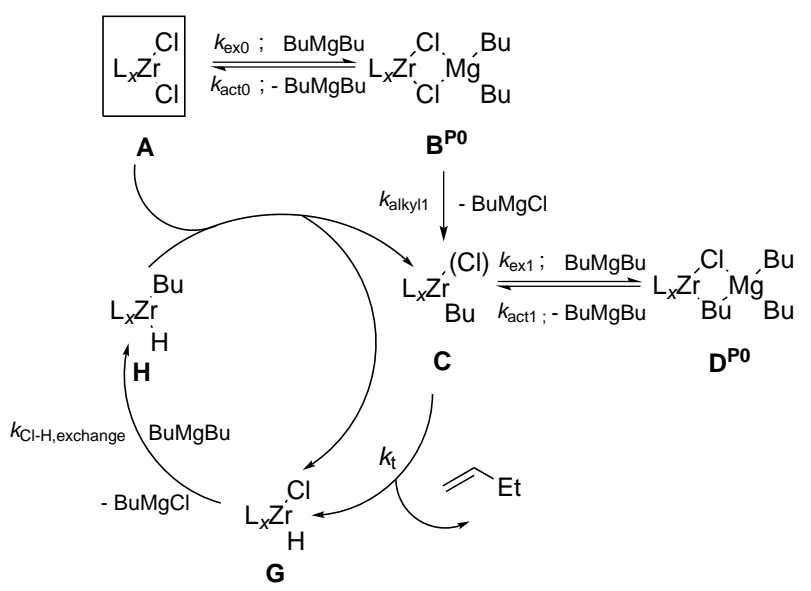

Scheme 4.4: Mechanistic scheme for activation and termination processes of $\mathrm{Cp}^{*}{ }_{2} \mathrm{ZrCl}_{2}$ with BuMgBu via the complex $\mathbf{B}$ and $\beta$-hydride elimination and metal-ligand exchange to complex $\mathbf{H}$.

sociation reaction of $\mathbf{B}$ is slow at ambient temperature resulting in a detectable signal. Complex $\mathbf{H}$ is not stable and is transformed via a side reaction into an undefined reaction product. A detailed analysis was not performed, because the reaction product is not seen during polymerization (see Section 5.1 .4 on page 128 ).

To confirm the scheme the reaction was performed once more with additional diethyl ether added to the BuMgBu suspension. The solubility was increased and it was possible to reproduce the found species in presence of excess magnesium. But due to the higher amounts of co-catalyst the signals of $\mathrm{BuMgBu}$ and $\mathbf{A}$ are overlayed and it was not possible to determine the exact concentrations of the formed species because the signals of $\mathrm{BuMgBu}$ show the same effect as $\mathrm{BzMgBz}$ and the chemical shift is slightly influenced by the termination reaction (see Figure 4.32). That indicates that complex $\mathbf{H}$ is able to form a bimetallic $\mathrm{Zr}-\mathrm{Mg}$ complex, yet no signal for such a complex could be determined. For clarity reasons the additional signals around $0 \mathrm{ppm}$ to $0.4 \mathrm{ppm}$ and $4 \mathrm{ppm}$ to $6 \mathrm{ppm}$ are not shown in this plot. In the appendix (see A.1 on page 214) the full overview is shown.

In comparison to the experiment with too low concentration of $\mathrm{BuMgBu}$ two mayor differences are found by peak height analysis. First the signal of $\mathbf{A}$ and $\mathbf{B}$ are fully consumed and secondly the formation of $\mathbf{E}$ does not occur showing that that at ambient temperature the equilibrium reactions between the different species is only possible when $\mathrm{BuMgBu}$ is absent. The integral of the catalyst species were not determinable. Therefore the peak heights of the complex species were analyzed. The analysis gave information about the occurring processes (see Figure 4.33) which are in agreement with Scheme 4.4. The analysis is influenced by the overlay with the signal of BuMgBu with the complex species. It was reduced to small amount by manual calculation of the peak height with respect to the signal symmetry and signal shifts allowing the qualitative 


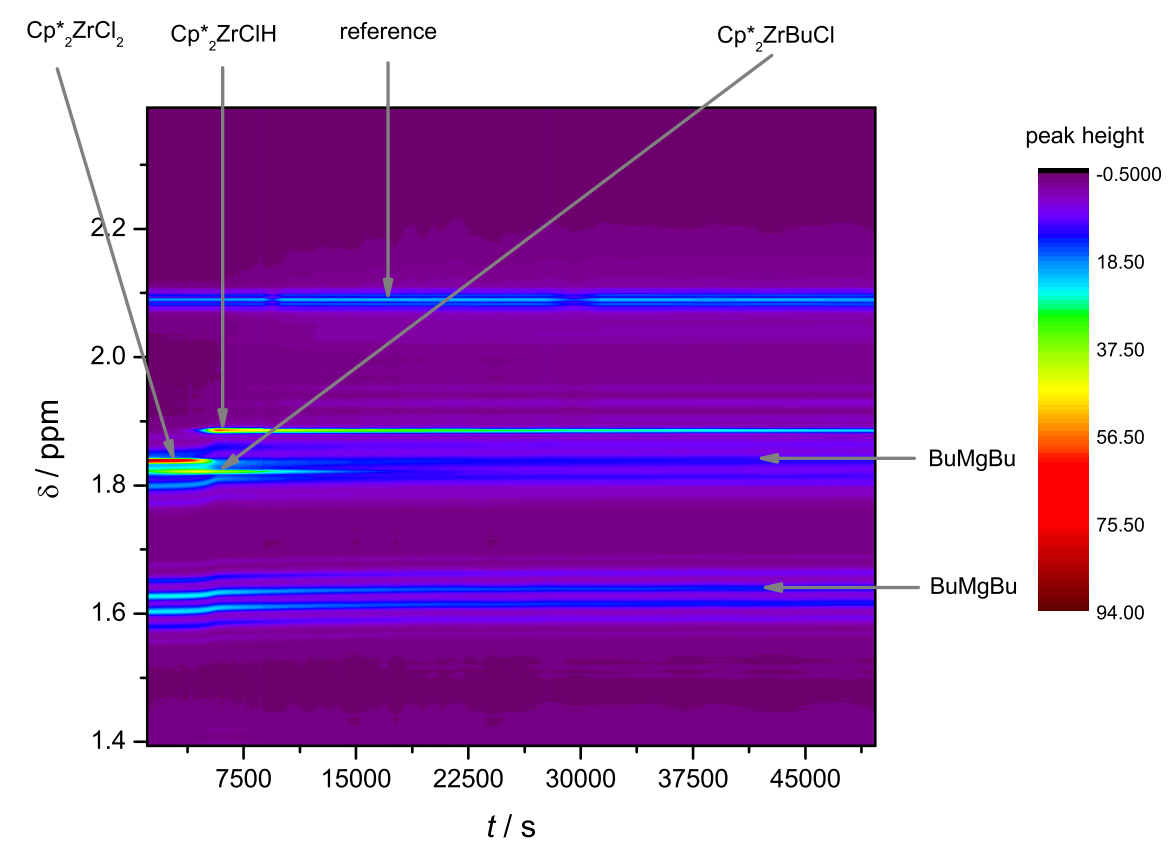

Figure 4.32: Monomer free CCG activation of $\mathrm{A}$ with $\mathrm{BuMgBu}, \mathrm{NMR}$ spectra series within the ligand region of $\mathbf{A}$ at ambient temperature, $c\left(\mathrm{Cp}_{2}^{*} \mathrm{ZrCl}_{2}\right)=0.00385 \mathrm{~mol} \mathrm{~L}^{-1}, c(\mathrm{BuMgBu})=0.059 \mathrm{~mol} \mathrm{~L}^{-1}$ in toluene- $d 8$.

analysis of the species together with the termination reaction product and the $\mathrm{Mg}-\mathrm{C}$ bond signal.

The reaction was modeled into a PREDICI ${ }^{\mathrm{TM}}$ simulation. The reaction leading to $\mathrm{C}$ and the Schlenk equilibrium are the same as previously applied (see 4.7 to $4.11,4.15$ ).

$$
\begin{gathered}
\mathbf{C} \stackrel{k_{\mathrm{t}}}{\longrightarrow} \mathbf{G}+\mathrm{CH}_{2} \mathrm{CHCH}_{2} \mathrm{CH}_{3} \\
\mathbf{G} \stackrel{k_{\mathrm{Cl}, \mathrm{H} \text { exchange }}}{\longrightarrow} \mathbf{H}+\mathrm{BuMgCl} \\
\mathbf{A}+\mathbf{H} \stackrel{k_{\mathrm{Cl}, \mathrm{H} \text { exchange } 2}}{\longrightarrow} \mathrm{C}+\mathbf{G}
\end{gathered}
$$

The simulation is highly sensitive (see Figure 4.34) to values of $k_{\mathrm{ex} 0}$ and $k_{\text {act } 0}$ because they are responsible for the formation of $\mathbf{B}$. The values of $k_{\mathrm{alkyl}}$ and $k_{\mathrm{t}}$ are of importance as well while the whole simulation is only valid for the first 6000 seconds. Afterwards a series of several different side reactions lead to further reactions involving the complexes $\mathbf{B}$ and $\mathbf{H}$. In the experiment it was found that at least three times more butene is formed as predicted by the process after the first termination reaction has occurred. The strong increase around the reference signal is due to complex decompostion as well as the formation of a new alkyl based product, due to the four times higher consumption of $\mathrm{BuMgBu}$ as necessary for this process. In all cases this process is always started after the 


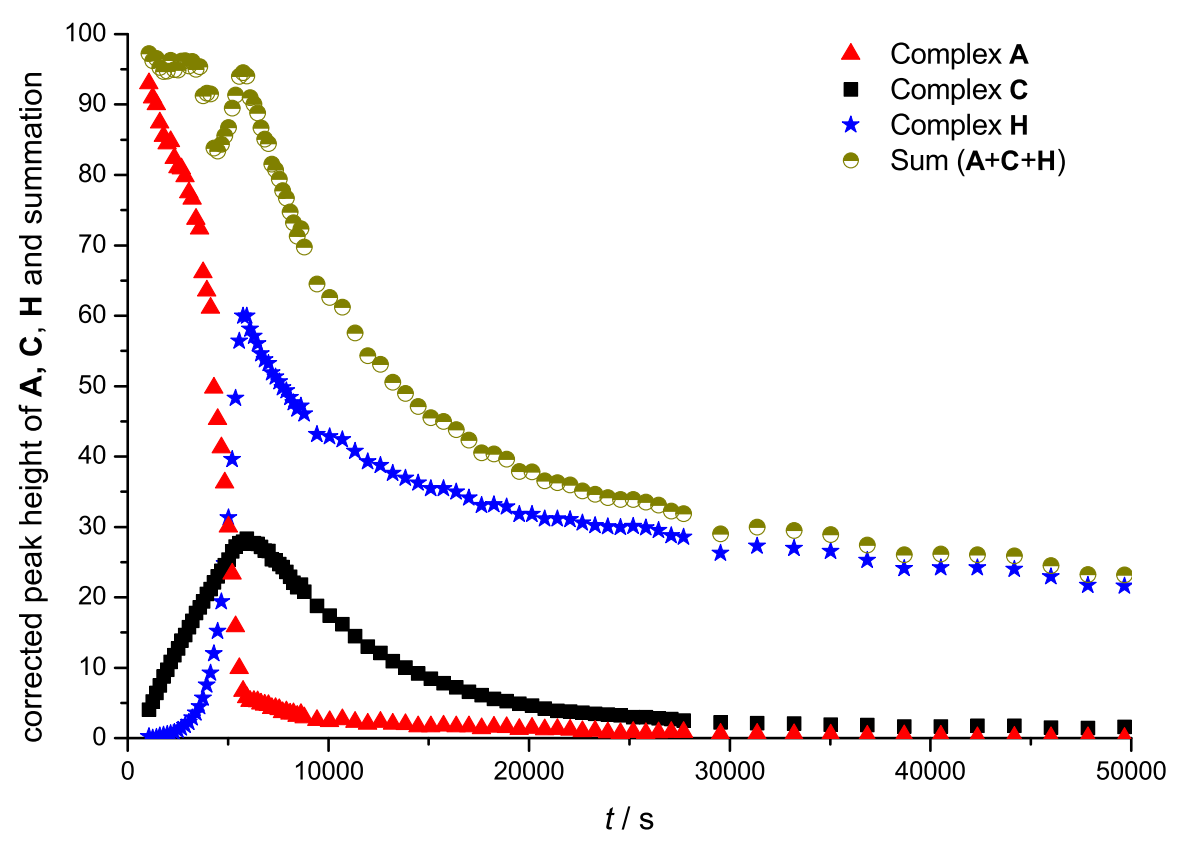

Figure 4.33: Peak height analysis of the complexes $\mathrm{A}, \mathrm{C}$ and $\mathbf{H}$ depicted together with the sum of peak heights. The complexes $\mathbf{A}$ and $\mathbf{B}$ were baseline corrected by subtraction of a constant value of the height of BuMgBu . Measurement at ambient temperature, $c\left(\mathrm{Cp}_{2}{ }_{2} \mathrm{ZrCl}_{2}\right)=0.00385 \mathrm{~mol} \mathrm{~L}^{-1}$, $c_{\mathrm{BuMgBu}}=0.059 \mathrm{~mol} \mathrm{~L}^{-1}$ in toluene- $d_{8}$.

full conversion of $\mathbf{A}$.

The determined fit between experiment and model data is rather low (see Figure 4.34) indicating that the side reaction process occurs even in presence of $\mathbf{A}$. The fit of $\mathbf{A}$ is good but at high reaction times a difference is yielded caused by the overlay of $\mathrm{BuMgBu}$ and $\mathbf{A}$. The concentration of $\mathbf{B}$ is slightly overestimated while the maximum value is in agreement between experiment and simulation. The consumption of $\mathrm{BuMgBu}$ is well simulated. The rate coefficients shown in Table 4.8 are not of high accuracy. The concentration of $\mathbf{H}$ is determined delayed which may be caused by the signal mixing of $\mathbf{H}$ with $\mathrm{BuMgBu}$ and butene signals yielding a virtual higher peak of $\mathbf{H}$.

An NMR experiment at $70^{\circ} \mathrm{C}$ was performed with BuMgOct as co-catalyst. It was preferred because of its better solubility and application in polymerization processes (see section 5.1.4 on page 128). It was not possible to determine the different catalyst species sucessfully. The reaction product of the side reaction seems to be the main product of the monomer-free activation of $\mathbf{A}$ (see Figure 4.35) with BuMgBu and BuMgOct. This result is important as with monomer being present these reaction products do not occur (see section 5.1.4 on page 128 for detailed discussion).

The detailed simulation of the termination process with $\mathbf{A}$ was not further investigated 


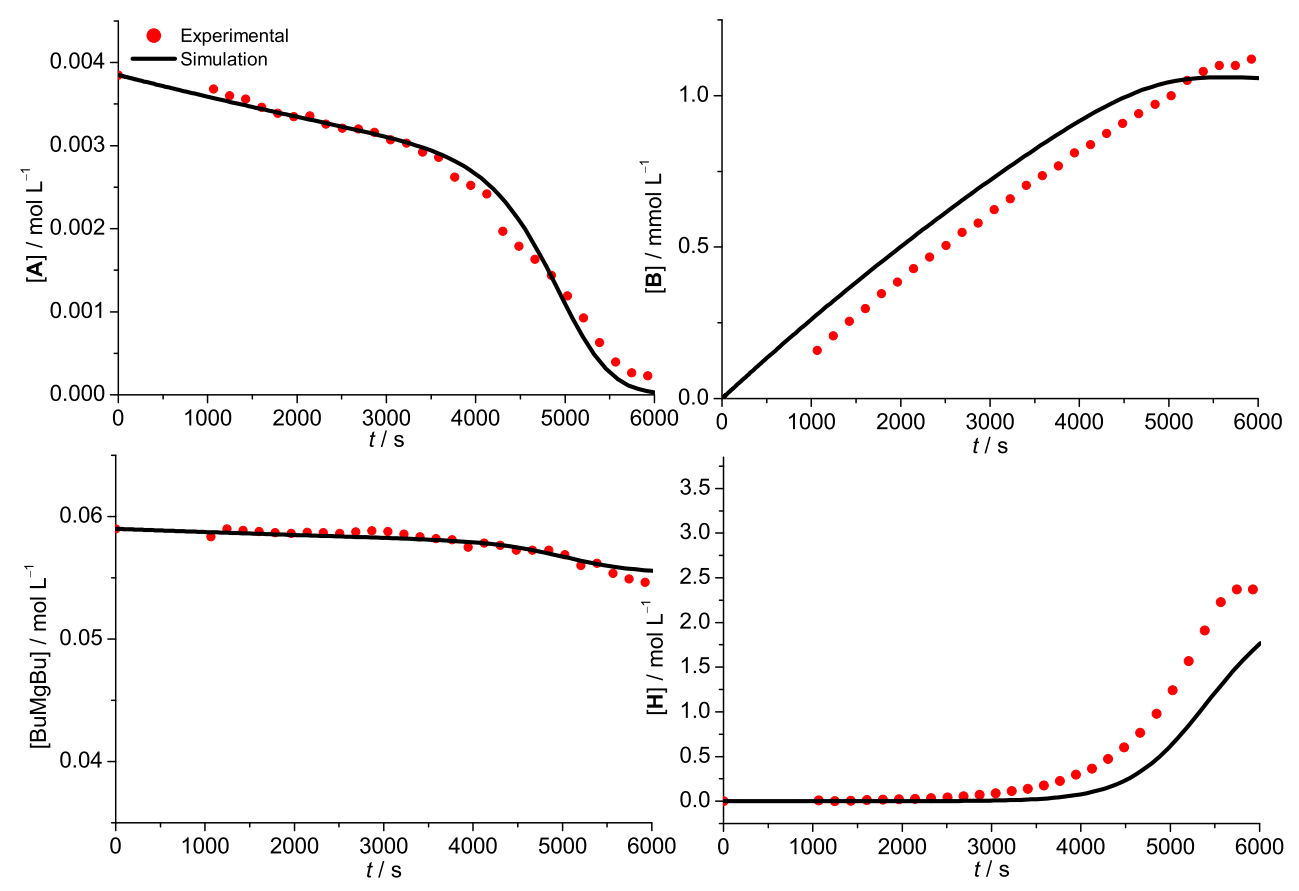

Figure 4.34: Comparsion of concentration vs. time profile of experimental (red dots) and simulated (black lines) data for monomer-free CCG activation in presence of $\beta$-hydride elimination.

Table 4.8 Parameter estimates by PREDCI of momomer-free CCG activation modeling at ambient temperature, $c\left(\mathrm{Cp}_{2}{ }_{2} \mathrm{ZrCl}_{2}\right)=0.00385 \mathrm{~mol} \mathrm{~L}^{-1}, c(\mathrm{BuMgBu})=0.059 \mathrm{~mol} \mathrm{~L}^{-1}$ in toluene- $d 8$.

\begin{tabular}{c|r|l} 
coefficient & value & unit \\
\hline$k_{\text {ex } 0}$ & 1 & $10^{-3} \mathrm{~L} \mathrm{~mol}^{-1} \mathrm{~s}^{-1}$ \\
$k_{\text {act0 }}$ & 1 & $10^{-5} \mathrm{~s}^{-1}$ \\
$k_{\text {ex1,ex2 }}$ & 1 & $10^{-3} \mathrm{~L} \mathrm{~mol}^{-1} \mathrm{~s}^{-1}$ \\
$k_{\text {act1,act2 }}$ & 1 & $10^{-5} \mathrm{~s}^{-1}$ \\
$k_{\text {alkyl1 }}$ & 5 & $10^{-7} \mathrm{~s}^{-1}$ \\
$k_{\mathrm{t}}$ & 1 & $10^{-3} \mathrm{~s}^{-1}$ \\
$k_{\mathrm{Cl}, \mathrm{H} \text { exhange }}$ & 3 & $\mathrm{~s}^{-1}$ \\
$k_{\text {activation }}$ & 3 & $\mathrm{~s}^{-1}$ \\
$k_{\text {Schlenk }}$ & 1000 & $\mathrm{~L} \mathrm{~mol}^{-1} \mathrm{~s}^{-1}$
\end{tabular}




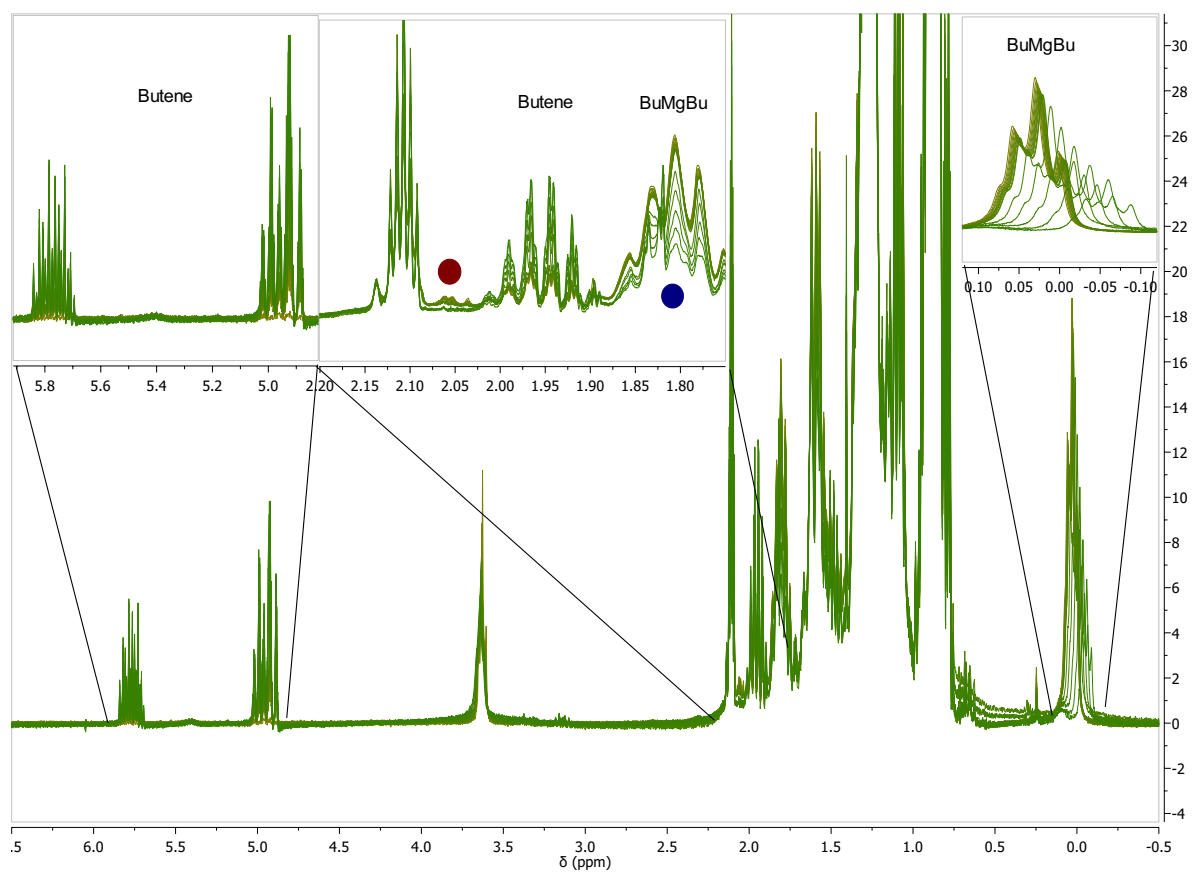

Figure 4.35: Kinetic NMR measurement of $\mathbf{A}$ in presence of BuMgOct $c\left(\mathrm{Cp}_{2}^{*} \mathrm{ZrCl}_{2}\right)=0.0023 \mathrm{~mol} \mathrm{~L}^{-1}$, $c(\mathrm{BuMgOct})=0.08 \mathrm{~mol} \mathrm{~L}^{-1}$ in toluene- $d 8$.

because the different side reactions could not be determined and are not of importance if monomer is present.

\subsection{UV/Vis Studies on $\mathrm{Cp}_{2}^{*} \mathrm{NdCl}_{2} \mathrm{Li}\left(\mathrm{OEt}_{2}\right)_{2}$ and $\mathrm{BzMgBz}$}

The system of $\mathrm{Cp}_{2}{ }_{2} \mathrm{NdCl}_{2} \mathrm{Li}\left(\mathrm{OEt}_{2}\right)_{2}(\mathrm{I})$ and $\mathrm{BzMgBz}$ is not suitable for NMR experiments because the $\mathrm{Nd}$-catalyst is strongly paramagnetic leading to problems for the exact determination of complex species and equilibrium constants. Based on the results on the work with complex $\mathbf{A}$ an activation mechanism for $\mathbf{I}$ towards the catalytic active center is proposed (see Scheme 4.5).

The scheme is slightly adopted because from the catalyst precursor $\mathbf{I ~} \mathrm{LiCl}$ precipitates in toluene solution and complex $\mathbf{J}$ is formed. This complex associates $\mathrm{BzMgBz}$ to form the bimetallic Nd-Mg complex $\mathbf{K}^{\mathrm{P0}}$, which either dissociates back or the chloride is exchanged between the neodymium and the magnesium center and complex $\mathbf{L}$ is formed by dissociation of benzyl magnesium chloride. This is the catalytic active species of the catalyst ${ }^{[21 / 22.221]}$ and is in equilibrium by association/dissociation of $\mathrm{BzMgBz}$ with the bimetallic complex $\mathbf{M}^{\mathbf{P} 0}$, which is similar to $\mathbf{D}^{\mathbf{P 0}}$ but does not undergo a second alkyation 


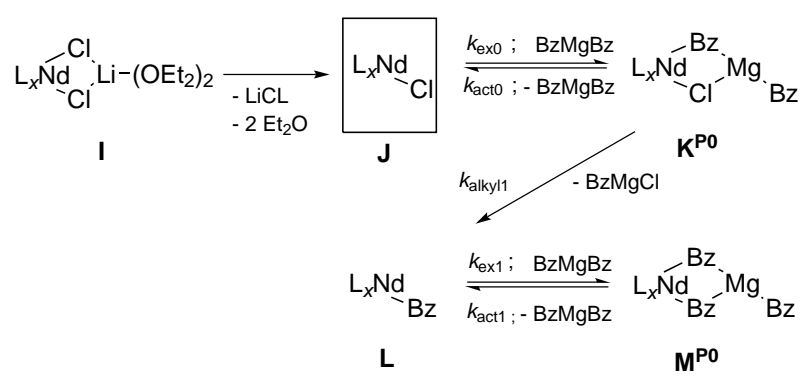

Scheme 4.5: Mechanistic scheme for activation of $\mathrm{Nd}_{2}{ }_{2} \mathrm{ZrCl}_{2} \mathrm{Li}\left(\mathrm{OEt}_{2}\right)_{2}$ (I) with BzMgBz via the complex $\mathbf{K}^{\mathbf{P 0}}$ to complex $\mathbf{L}$ and chain transfer via formation of $\mathbf{M}^{\mathbf{P 0}}$.

step to a dialkylated complex.

In this case UV/Vis spectroscopy is of choice because the formed equilibrium is not able to undergo further reaction allowing the determination of the equilibrium constant. To exclude systematic errors for the calculation the temperature dependence of $\mathrm{BzMgBz}$ absorbance in toluene was researched first for the two applied concentrations. It was found that the solution of $\mathrm{BzMgBz}$ has a temperature-dependent absorption at $400 \mathrm{~nm}$ which is increasing exponential with temperature (see Figure 4.36 and 4.37 ). To determine the influence on further calculations fit functions were determined for the applied concentrations (see Equation 4.29 for $c(B z M g B z)=0.076 \mathrm{~mol} \mathrm{~L}^{-1}$ and Equation 4.30 for $\left.c(\mathrm{BzMgBz})=0.035 \mathrm{~mol} \mathrm{~L}^{-1}\right)$.

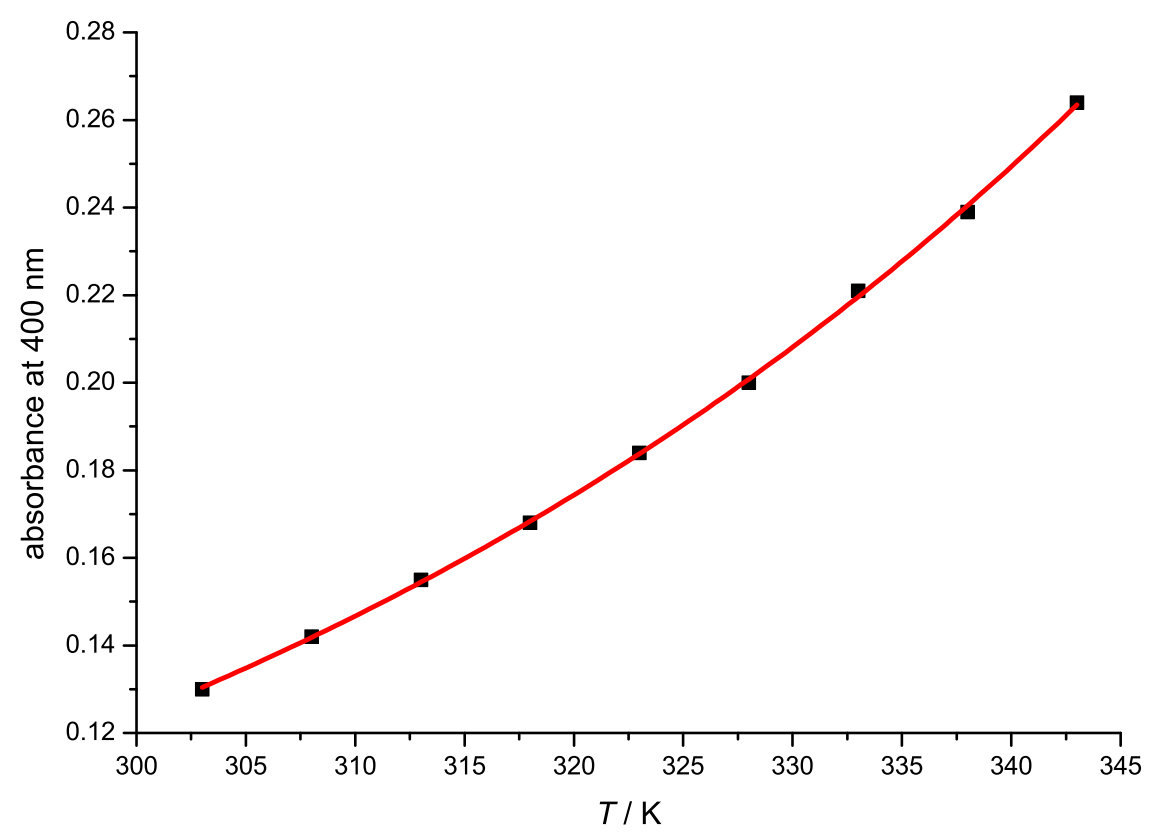

Figure 4.36: Absorbance of $\mathrm{BzMgBz}$ at $400 \mathrm{~nm}$ with an exponential fit (see Equation 4.29) for $c(\mathrm{BzMgBz})=0.076 \mathrm{~mol} \mathrm{~L}^{-1}$. 


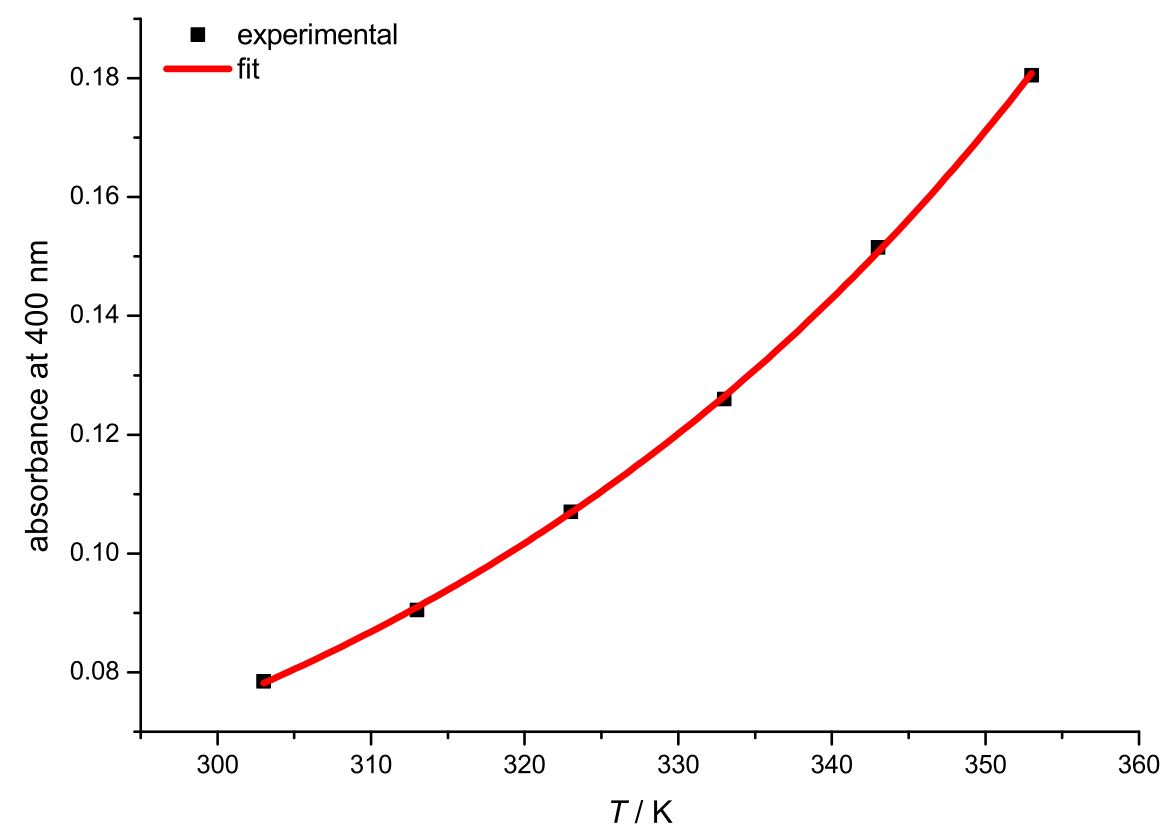

Figure 4.37: Absorbance of $\mathrm{BzMgBz}$ at $400 \mathrm{~nm}$ with an exponential fit (see Equation 4.30) for $c(\mathrm{BzMgBz})=0.035 \mathrm{~mol} \mathrm{~L}^{-1}$.

$$
\begin{aligned}
& A_{\mathrm{BzMgBz}, 400 \mathrm{~nm}, 1}=0.02203+2.50994 \times 10^{-4} \cdot \exp \left(\frac{T / \mathrm{K}}{49.93584}\right) \\
& A_{\mathrm{BzMgBz}, 400 \mathrm{~nm}, 2}=0.02411+8.5365 \times 10^{-5} \cdot \exp \left(\frac{T / \mathrm{K}}{46.97786}\right)
\end{aligned}
$$

The benzylated neodymium complex absorbes at $400 \mathrm{~nm}$ as well, but it was not possible to determine the absorbance in the same manner as for BzMgBz. With a closer look on an example of one measurement the changes at the metal center were visible. The neodymium complex shows a absorption at $599 \mathrm{~nm}$ (see Figure 4.38, red signal). By application of different temperatures in presence of $\mathrm{BzMgBz}$ new signals and shifts in intensity are gained. The signal at $599 \mathrm{~nm}$ decreases with higher temperatures (see Figure 4.39 , b) ) while several peaks at wavelengths above $600 \mathrm{~nm}$ increase in intensity (see Figure 4.39 , c)). The steps in Figure 4.39 represent a temperature change of $10 \mathrm{~K}$ starting at $333 \mathrm{~K}$.

The absorbance at $400 \mathrm{~nm}$ has the best signal to noise ratio compared to the signals around $599 \mathrm{~nm}$ and is best suited for determination of the equilibrium concentration of $\mathbf{M}^{\mathbf{P 0}}$. The measured data was corrected by the BzMgBz absorbance and then afterward analyzed via a Hill1 growth function of the program package Origin8.5 (4.31).

$$
A_{400 \mathrm{~nm}}=A_{\mathbf{I}_{0}, 400 \mathrm{~nm}}+\left(A_{\mathbf{M}^{\mathbf{P 0}}{ }_{\max }, 400 \mathrm{~nm}}-A_{\mathbf{I}_{0}, 400 \mathrm{~nm}}\right) \frac{x^{n}}{k^{n}+x^{n}}
$$




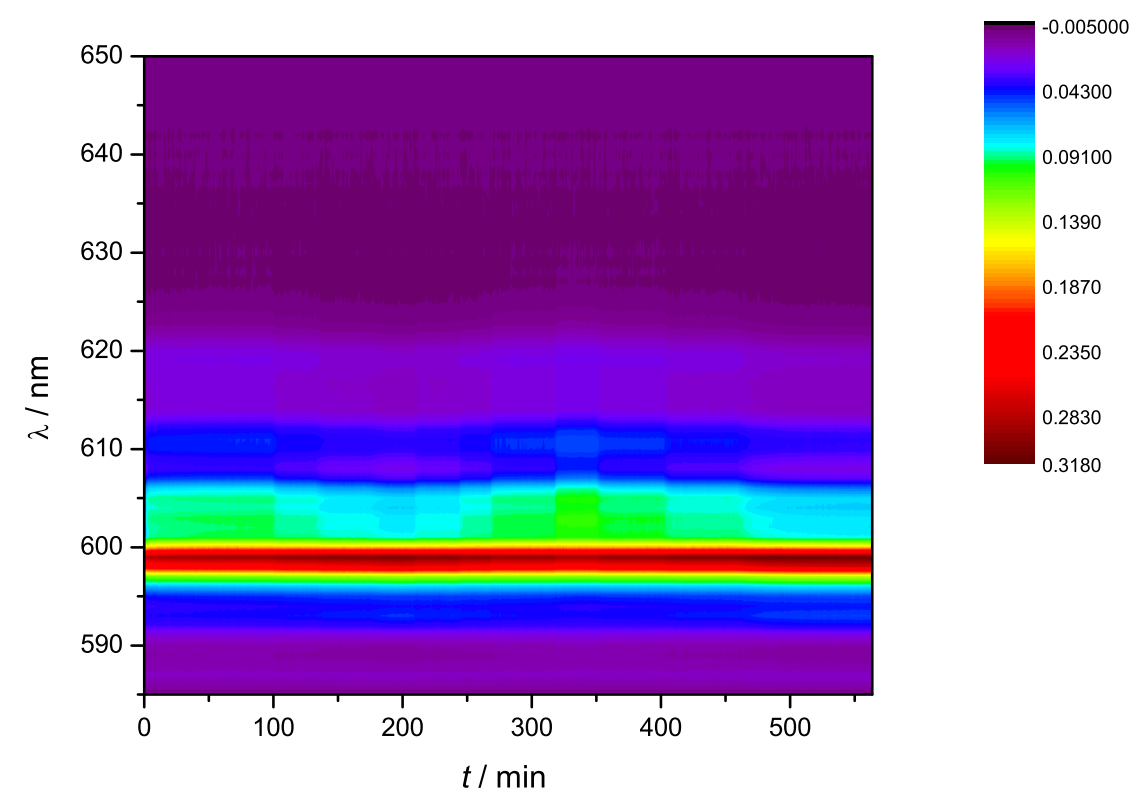

Figure 4.38: Temperature dependent absorbance of the neodymium metal center in presence of BzMgBz with $c(\mathbf{I})=1.59 \times 10^{-3} \mathrm{~mol} \mathrm{~L}^{-1}$ and $c(\mathrm{BzMgBz})=0.035 \mathrm{~mol} \mathrm{~L}^{-1}$.
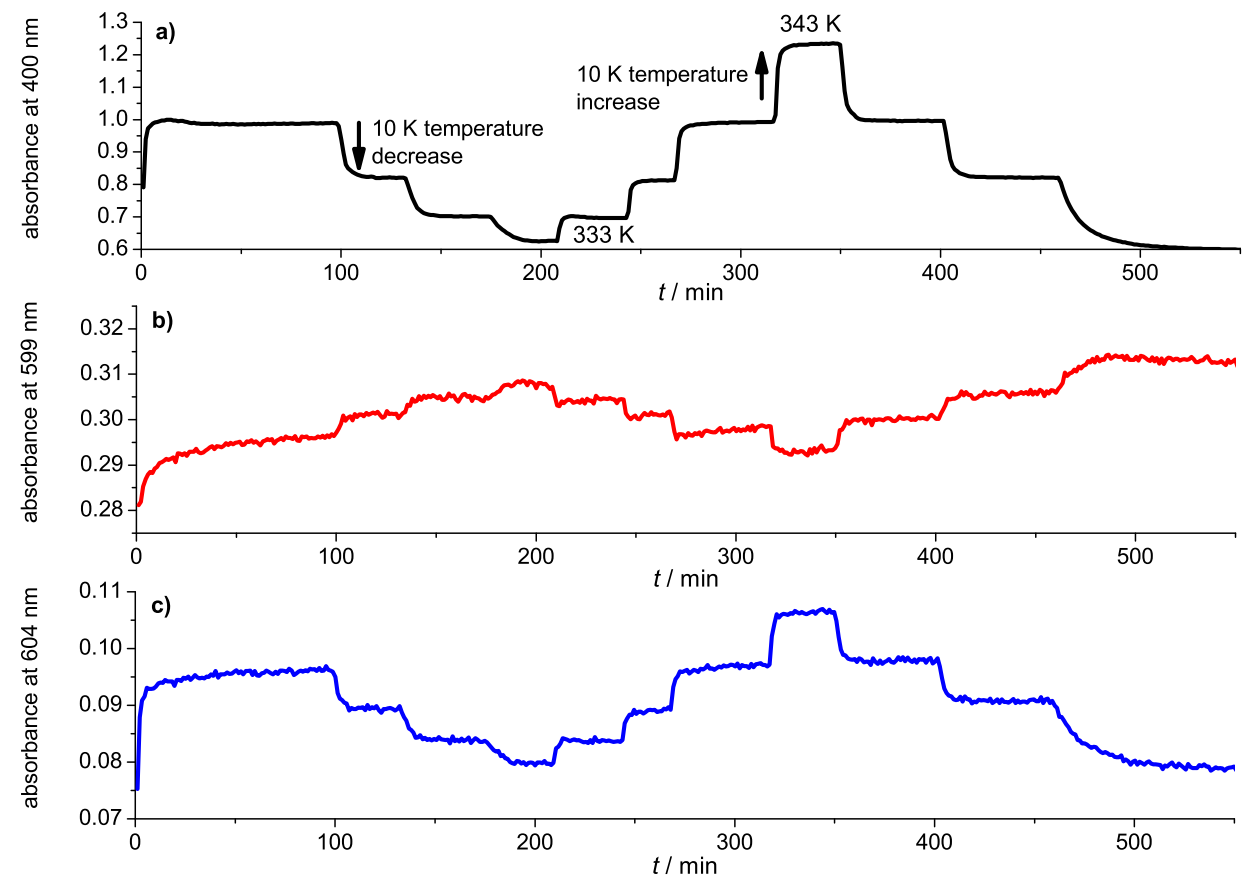

Figure 4.39: Selected absorbance vs. time plots showing temperature dependent absorbance of the neodymium metal center in presence of BzMgBz with $c(\mathbf{I})=1.59 \times 10^{-3} \mathrm{~mol} \mathrm{~L}^{-1}$ and $c(\mathrm{BzMgBz})=0.035 \mathrm{~mol} \mathrm{~L}^{-1}$. 
This equation was chosen because at low concentrations of I a maximum value is

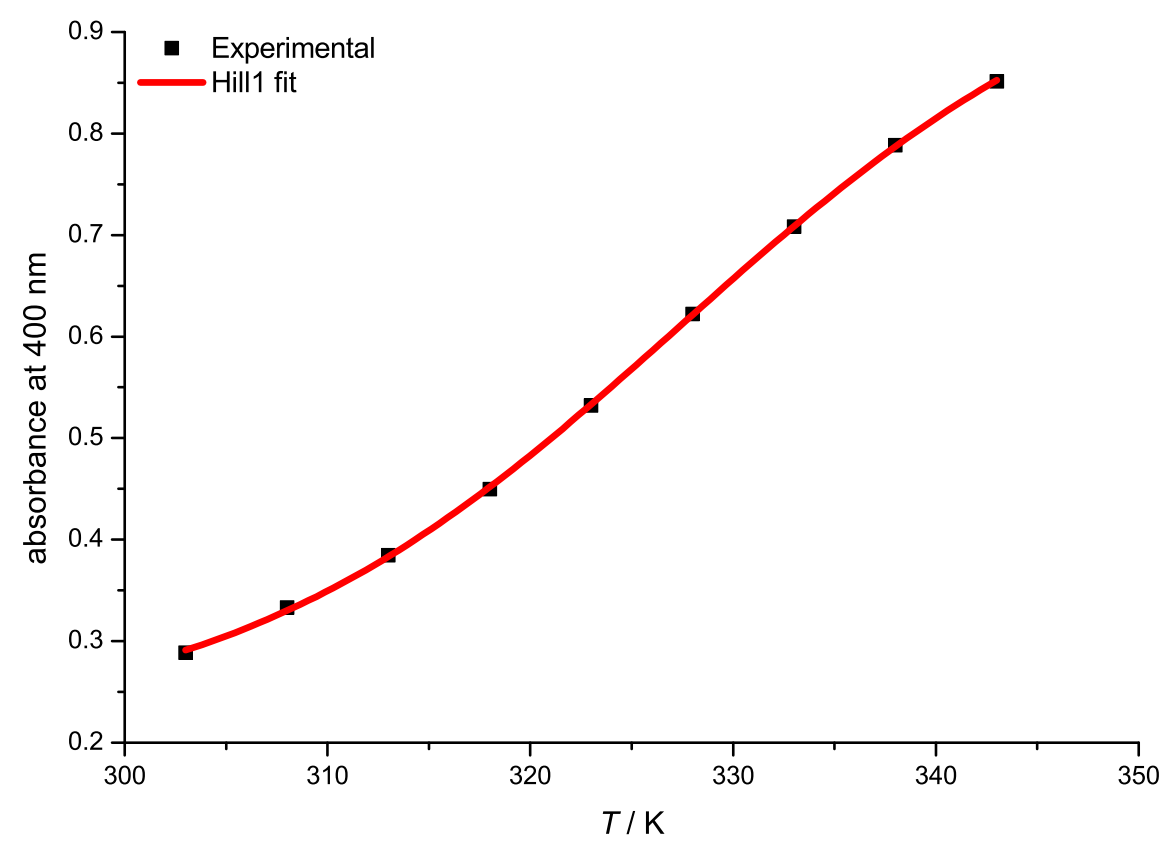

Figure 4.40: Example of Hill1 fit (Origin8.5) for measurement with $c(\mathbf{I})=3.18 \times 10^{-4} \mathrm{~mol} \mathrm{~L}^{-1}$ and $c(\mathrm{BzMgBz})=0.035 \mathrm{~mol} \mathrm{~L}^{-1}$

reached and the increase levels off (see Figure 4.40). It cannot increase further because off full conversion to $\mathbf{M}^{\mathbf{P}}$. The factors $k$ and $n$ are fit parameters that describe the shape of the curve.

$$
c\left(\mathbf{M}^{\mathbf{P 0}}\right)_{T}=\frac{A_{\text {measured, } \mathrm{T}}-A_{\mathrm{BzMgBz}, \mathrm{T}}-A_{0, \mathrm{I}, 400 \mathrm{~nm}}}{\frac{\mathbf{A}_{\text {max }, \mathbf{M}^{\mathbf{P 0}}}}{d \cdot[\mathrm{I}]_{0}}}
$$

This allows to evaluate the concentration of $\mathbf{M}^{\mathrm{P0}}$ (see eq. 4.32) from the absorbance at $400 \mathrm{~nm}$ by the assumption that the thermal dependence of $\epsilon_{\mathbf{M}}$ is negligibly small. It is

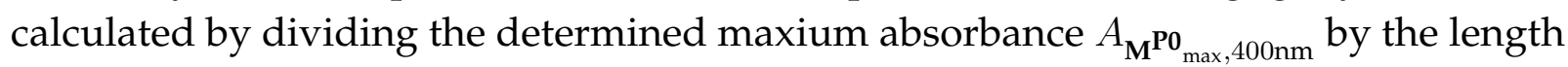
of the cuvette $(d)$ and the starting concentration $[\mathrm{I}]_{0}$. The equilibrium constant $K_{\mathrm{ND}, 1}$ could be calculated by application of Equation 4.33 for each temperature. Values are summarized in Table 4.9 .

$$
K_{N d, 1}=\frac{c\left(\mathbf{M}^{\mathbf{P 0}}\right)}{\left(c(\mathbf{I})_{0}-c\left(\mathbf{M}^{\mathbf{P 0}}\right)\right) \times\left(c(\mathrm{BzMgBz})_{0}-c\left(\mathbf{M}^{\mathbf{P 0}}\right)\right)}
$$

The determined values for $K_{1, \mathrm{ND}}$ are temperature dependent and the plot of $\ln \left(K_{1, \mathrm{ND}}\right)$ 
Table 4.9 Equilibrium constants $K_{\mathrm{Nd}, 1}$ for momomer-free CCG activation at various temperatures and concentrations in toluene.

\begin{tabular}{c|c|c|c|c|c}
$T / \mathrm{K}$ & 343 & 338 & 333 & 328 & 323 \\
\hline$K_{\mathrm{Nd}, 1} / \mathrm{L} \mathrm{mol}^{-1}$ & $42 \pm 25$ & $28 \pm 16$ & $18 \pm 10$ & $12 \pm 6.0$ & $7.3 \pm 3.5$ \\
\hline$T / \mathrm{K}$ & 318 & 313 & 318 & 303 & \\
\hline$K_{\mathrm{Nd}, 1} / \mathrm{L} \mathrm{mol}^{-1}$ & $4.7 \pm 2.1$ & $3.1 \pm 1.3$ & $2.0 \pm 0.8$ & $1.2 \pm 0.5$ &
\end{tabular}

against $T^{-1}$ (see Figure 4.41) gave a reaction enthalpy of $\Delta H_{\mathrm{R}}=(72.4 \pm 0.5) \mathrm{kJ} \mathrm{mol}^{-1}$ and a reaction entropy of $\Delta S_{\mathrm{R}}=(239 \pm 2) \mathrm{J} \mathrm{mol}^{-1} \mathrm{~K}^{-1}$.

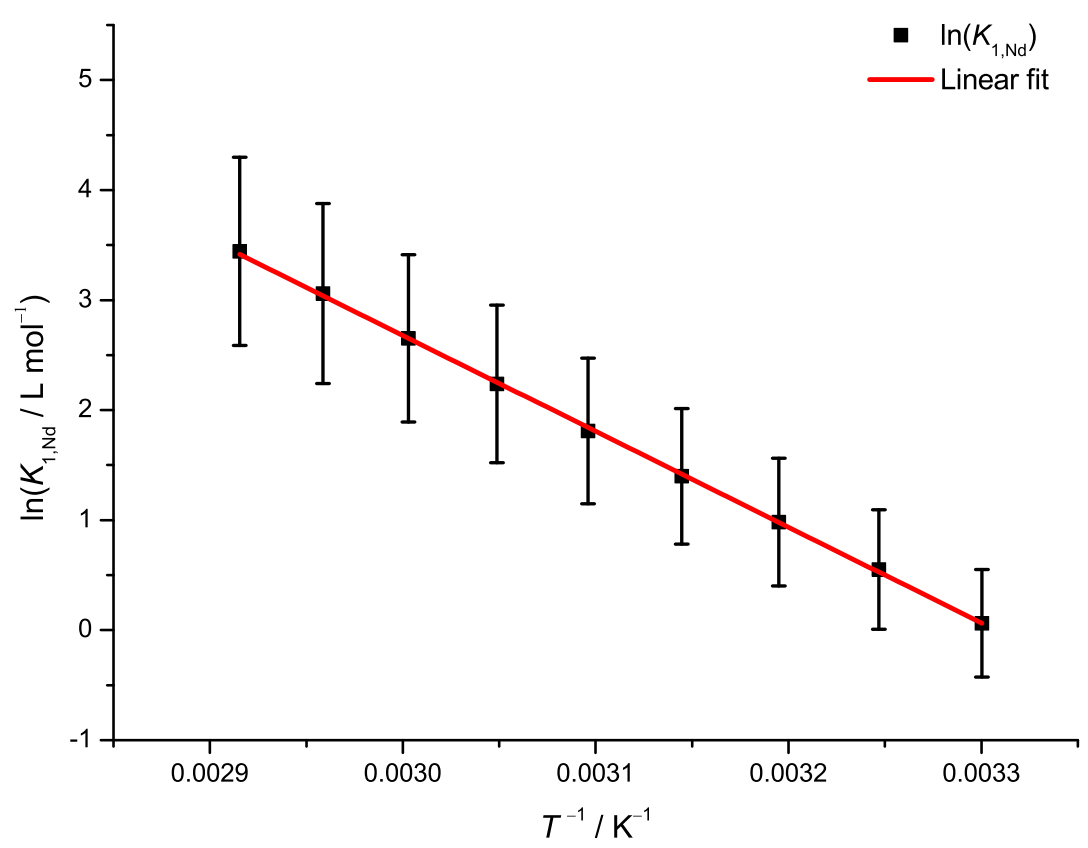

Figure 4.41: Plot of $\ln \left(K_{1, \mathrm{ND}}\right)$ versus $1 / T$ based on Table 4.9

In comparison to the zirconium based system the bimetallic complex is favored at higher temperatures indicating that the quality of molar mass control will increase temperature. In addition the accuracy of the determined values increases with temperature, which is results in a lower absorption coefficient in presence of DBE. The addition of DBE lowers the equilibrium constant (see Figure 4.42) and only small equilibrium constants can be reached at elevated temperatures. This finding indicates that the reaction rate enhancement in CCG polymerization with I and BuMgOct is caused by a lower equilibrium constant (see Table 4.10). For the determined equilibrium constants the reaction enthalpy is calculated and it is found that addition of ether leads to an increase of reaction enthalpy to $\Delta H_{\mathrm{R}}=(198 \pm 7) \mathrm{kJ} \mathrm{mol}^{-1}$ and of reaction entropy to $\Delta S_{\mathrm{R}}=(586 \pm 19) \mathrm{J} \mathrm{mol}^{-1} \mathrm{~K}^{-1}$. 
Because of the much higher value of the reaction entropy the reaction is hindered at low temperatures.

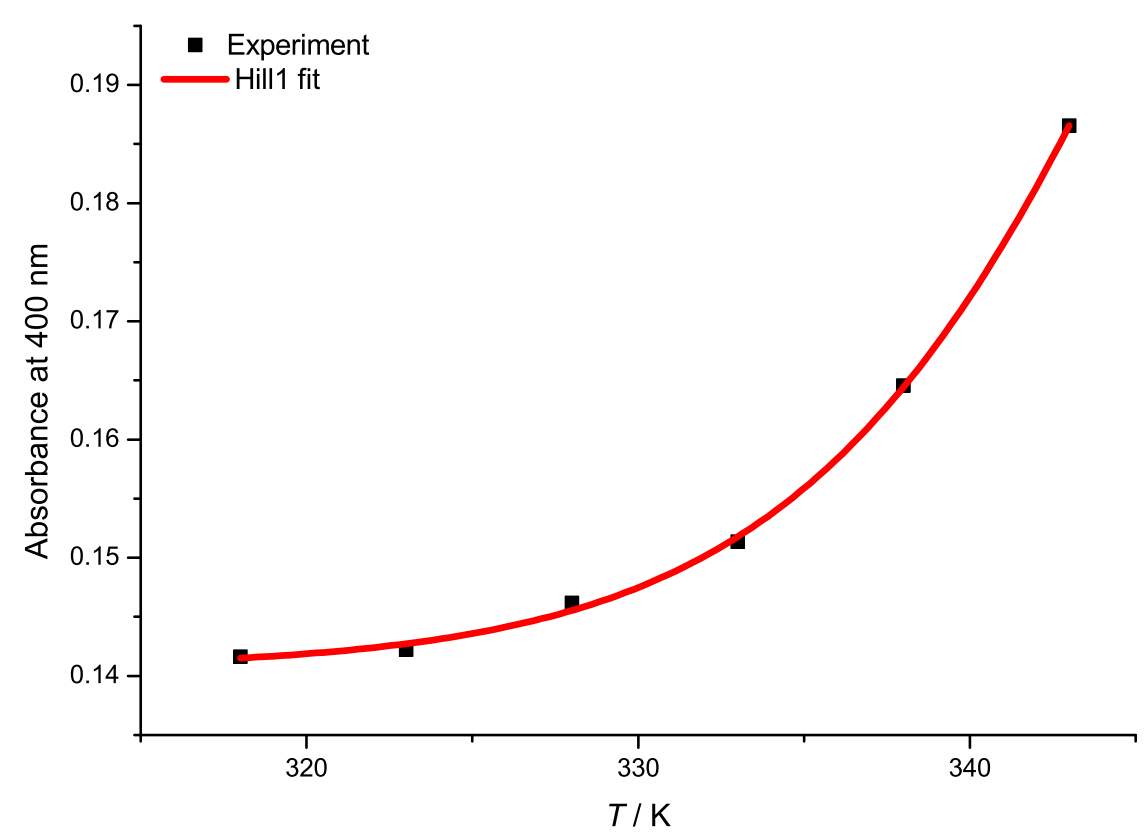

Figure 4.42: Example of Hill1 fit (Origin8.5) for measurement with $c(\mathbf{I})=6.3 \times 10^{-4} \mathrm{~mol} \mathrm{~L}^{-1}$, $c(\mathrm{BzMgBz})=0.073 \mathrm{~mol} \mathrm{~L}^{-1}$ and $c(\mathrm{DBE})=0.23 \mathrm{~mol} \mathrm{~L}^{-1}$

In conclusion it is possible to determine the equilibrium constants and changes at the

Table 4.10 Equilibrium constants $\mathrm{K}_{\mathrm{Nd}, 1}$ for momomer free CCG activation at various temperatures in toluene with $c(\mathbf{I})=6.3 \times 10^{-4} \mathrm{~mol} \mathrm{~L}^{-1}, c(\mathrm{BzMgBz})=0.073 \mathrm{~mol} \mathrm{~L}^{-1}$ and $c(\mathrm{DBE})=0.23 \mathrm{~mol} \mathrm{~L}^{-1}$

\begin{tabular}{c|c|c|c|c|c}
$T / \mathrm{K}$ & 343 & 338 & 333 & 328 & 323 \\
\hline $\mathrm{K}_{\mathrm{Nd}, 1} / \mathrm{L} \mathrm{mol}^{-1}$ & $2.49 \pm 0.01$ & $1.10 \pm 0.02$ & $0.39 \pm 0.07$ & $0.15 \pm 0.9$ & $0.04 \pm 0.02$
\end{tabular}

neodymium center via UV/Vis spectroscopy with a limited accuracy because of the overlay in absorbance for the studied complexes.

\subsubsection{Conclusion}

NMR measurements were successfully applied to set up a kinetic model for the monomer-free activation kinetics in CCG of $\mathbf{A}$ with BzMgBz. A kinetic scheme was proposed and described as a model in PREDICI ${ }^{\mathrm{TM}}$. By simulation it was found that the kinetic coefficients can be directly derived from NMR data by calculation of $K_{1, Z r}$, 


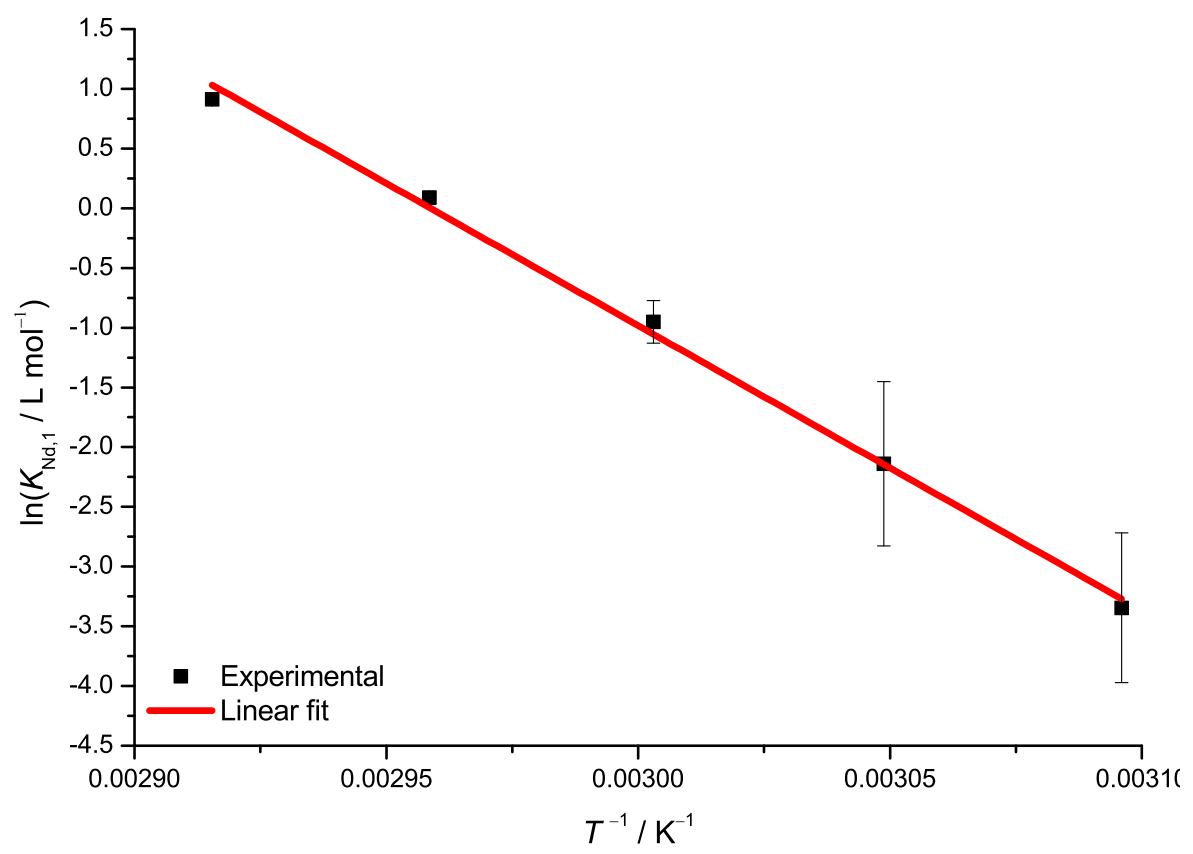

Figure 4.43: Plot of $\ln \left(K_{1, \mathrm{ND}}\right)$ versus $1 / T$ based on Table 4.10

$K_{2, \mathrm{Zr}}, k_{\mathrm{alkyl} 1}$ and $k_{\mathrm{alkyl} 2}$ which resulted in the best fit between simulation and experiment. The coefficients are temperature dependent and analysis showed that the alkylation reactions are endothermic. The exchange reactions are exothermic and have a high reaction barrier. By UV/Vis spectroscopy a second method for determination of $k_{\text {alkyl }}$ and $k_{\text {alkyl2 }}$ was established. The results confirmed the similar to NMR results. For the influence of dibutyl ether on reaction of $\mathbf{A}$ with $\mathrm{BzMgBz}$ it was found that the additional ether induces significant changes to the reaction rate coefficients $k_{\mathrm{alkyl1}}$ and $k_{\mathrm{alkyl2}}$ and activation energies. The rate coefficient $k_{\text {alkyll }}$ is lowered, depending on the temperature by up to two orders of magnitude with increasing ether concentration. The activation energy is lowered while the pre-exponential factor is decreased.

Based on these results the termination reactions occurring in activation with $\mathrm{BuMgBu}$ could be elucidated and simulated while catalyst $\mathbf{A}$ is present. The further reaction products could not be determined and the applied model is limited by this fact.

The equilibrium between I and BzMgBz was successfully researched and an endothermic process was found which was further influenced by the addition of DBE yielding a higher reaction enthalpy that was necessary to form the complex. In comparison to complex $\mathbf{A}$ the behavior in the exchange equilibrium is reversed.

The exact rate coefficients for the equilibrium reaction could not be determined in absence of $\beta$-hydride elimination. In presence of termination the alkylation reaction is a rate limiting step in the case of $k_{\mathrm{ex} 0}$ for the formation of complex $\mathbf{B}$. 


\section{Modeling Catalyzed Chain Growth of Styrene-d8 with $\mathrm{Cp}_{2}^{*} \mathrm{ZrCl}_{2}$ and $\mathrm{BzMgBz}$}

The activation mechanism described above was tested for the polymerization of styrene. Compared to ethylene has styrene the advantage that it is liquid. It can be applied to coordination polymerization with magnesium as co-catalyst. 278,279] Polymerization with different metallocene and half-metallocene complexes are well-known. 35,72,280-286 Furthermore, it is available as fully deuterated monomer making it suitable for NMR studies. Measurements should be carried out below $80^{\circ} \mathrm{C}$ to avoid self-initiation of the monomer (see 2.2.1 on page 10). In the following the model system is investigated at $70^{\circ} \mathrm{C}$, which is close to the CCG-polymerization temperature for ethylene. Parts of this

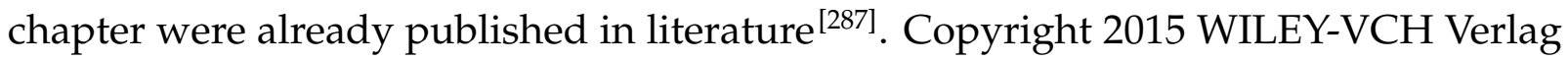
$\mathrm{GmbH} \& \mathrm{Co}$. KGaA, Weinheim.

\subsection{NMR Studies of the Model System $\mathrm{Cp}_{2}^{*} \mathrm{ZrCl}_{2}$ and BzMgBz with Styrene-d8}

\subsubsection{Kinetic Model of $\mathrm{Cp}_{2}{ }_{2} \mathrm{ZrCl}_{2}$ and $\mathrm{BzMgBz}$}

The monomer-free model system was expanded by the addition of styrene- $d 8$ to toluene- $d 8$, thus only slightly changing the solvent environment. The time-resolved NMR spectra (see Figure 5.1) show no new complex species with proceeding time and can be characterized according to the monomer-free system (cf. Figure 4.4 on page 41). One main difference is the occurrence of the polymer chain signals (Figure 5.1, polymer). Therefore it cannot be ruled out that the polymeric complex species signals are overlaid 


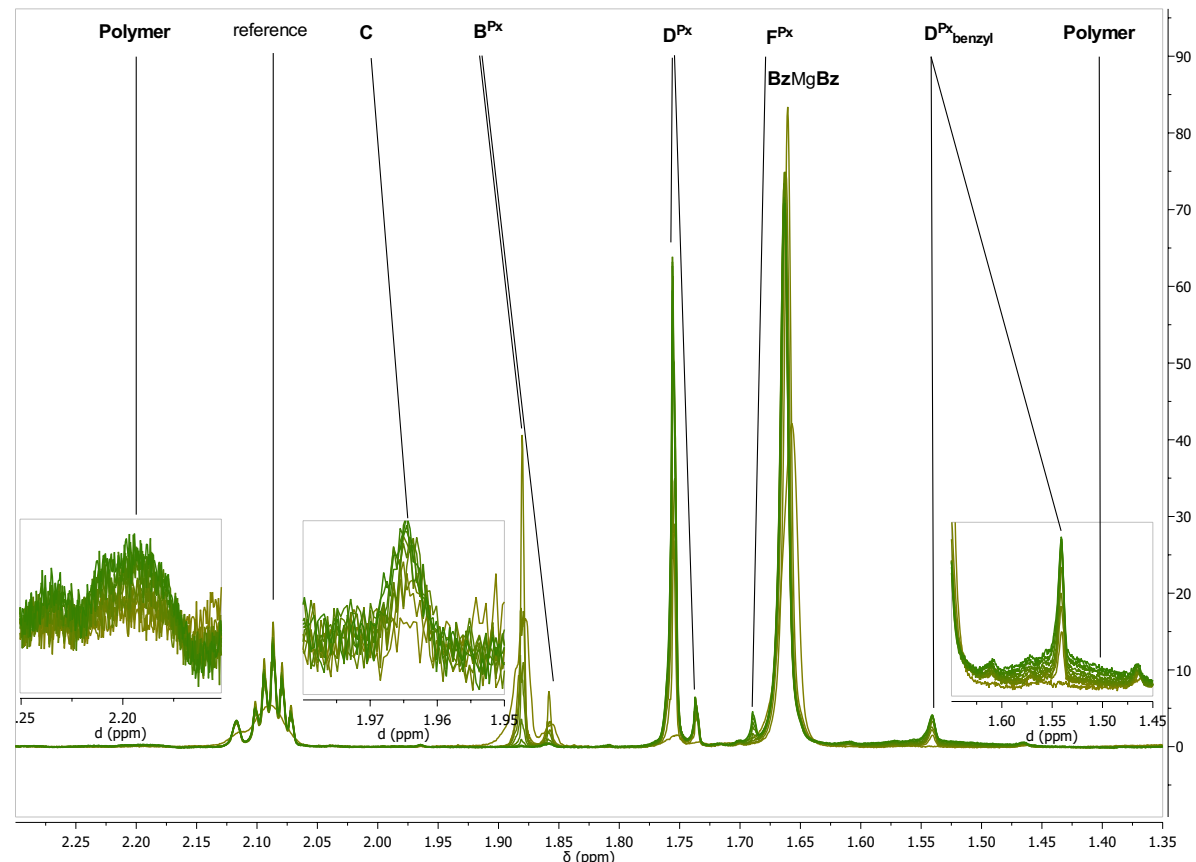

Figure 5.1: Time-resolved NMR-spectra of CCG polymerization of styrene-d8 using $\mathbf{A}$ and $\mathrm{BzMgBz}$ at $70^{\circ} \mathrm{C}, c\left(\mathrm{Cp}_{2}{ }_{2} \mathrm{ZrCl}_{2}\right)=4.6 \mathrm{mmol} \mathrm{L}^{-1}, c(\mathrm{BzMgBz})=92 \mathrm{mmol} \mathrm{L}^{-1}$ in toluene- $d 8 /$ styrene- $d 8$. Only every tenth measurement is shown

by the complexes measured monomer-free. The signals were labeled with PX superscripts, saying that the exact amount of bound polymer chains is unknown. Based on Scheme 4.3 on page 43 the different reactions for the PREDICI ${ }^{\mathrm{TM}}$ model were now described with a monomer and two additional polymeric magnesium species present. Benzyl polystyrenyl magnesium (BzMgP) and dipolystyrenyl magnesium (PMgP) are formed by chain transfer by polymeric complex species. The reaction scheme of $\mathbf{A}$ is extended with the association/dissociation reactions of the $\mathrm{Mg}$ species (see Scheme 5.1). When $\mathbf{A}$ associates BzMgP the generated polymeric species $\mathbf{B}^{\mathbf{P 1}}$ has two possibilities to perform the alkylation process. The rate coefficients for both reactions were assumed to be similar such that for each $1 / 2$ of the alkylation rate coefficient $k_{\text {alkyl1 }}$ is applied. From $\mathbf{B}^{\mathbf{P 1}}$ either the polymeric complex $\mathbf{C}^{\mathbf{P}}$ and the benzyl grignard or the complex $\mathbf{C}$ and the polymeric grignard are formed. The association of PMgP to $\mathbf{A}$ leads to complex $\mathbf{B}^{\mathbf{P 2}}$ were the alkylation reaction forms complex $\mathbf{C}^{\mathbf{P}}$. In all cases reaction rates are assumed to be independent of the chain length and of the amount of involved polymer "PX" chains. These reactions are important at low ratios and with increasing $\mathrm{Zr:Mg}$ ratios these reactions unlikely occur.

The species $\mathbf{C}$ and $\mathbf{C}^{\mathbf{P}}$ should be the catalytic active metal complexes which are able to add monomer (see Scheme 5.2). Such mechanisms are well known in literature ${ }^{[45-50,139,140,288]}$ and catalysts similar to CCG-catalyst were found to perform 


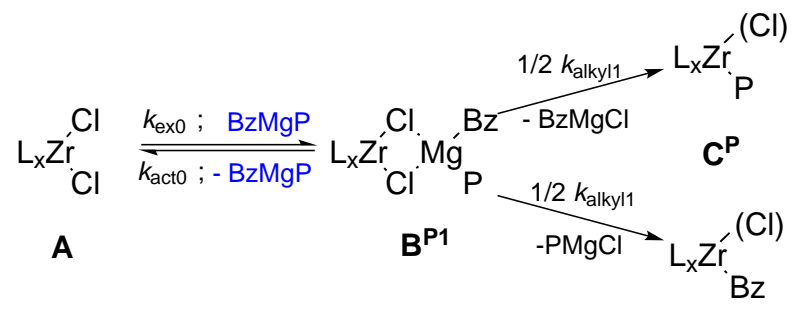

$$
\begin{aligned}
& \text { C }
\end{aligned}
$$

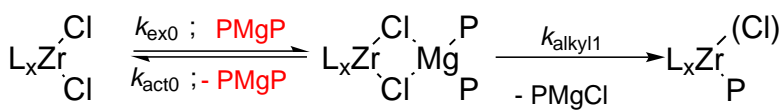

$$
\begin{aligned}
& \begin{array}{lll}
A & B^{\text {P2 }} & C^{P}
\end{array}
\end{aligned}
$$

Scheme 5.1: Activation processes of $\mathbf{A}$ with $\mathrm{BzMgP}$ and $\mathrm{PMgP}$ forming complexes with one $\left(\mathbf{B}^{\mathbf{P} 1}\right)$ and two $\left(\mathbf{B}^{\mathbf{P 2}}\right)$ polymer chains involved.

monomer addition by the Cossée-Arlman process. ${ }^{289}$ The monomer coordinates to the complex at an open or activated reaction side $(\mathbf{C}(\mathbf{M}))$ where it either subsequently discoordinates or is introduced into the zirconium-carbon bond of the benzyl moiety. For the polymeric complex $\mathbf{C}^{\mathrm{P}}$ the mechanism is assumed to be similar. While both active species have the same nature despite the different chain length, they cannot be distinguished in NMR. It was not possible to find the intermediate $\mathbf{C}(\mathbf{M})$ within NMR-spectrum, which is either caused by a low concentration or a short lifetime of the complex. Chain-length dependence (CLD) was excluded for the first simulations but will be discussed later in this chapter in Section 5.1.2 on page 105 in more detail.

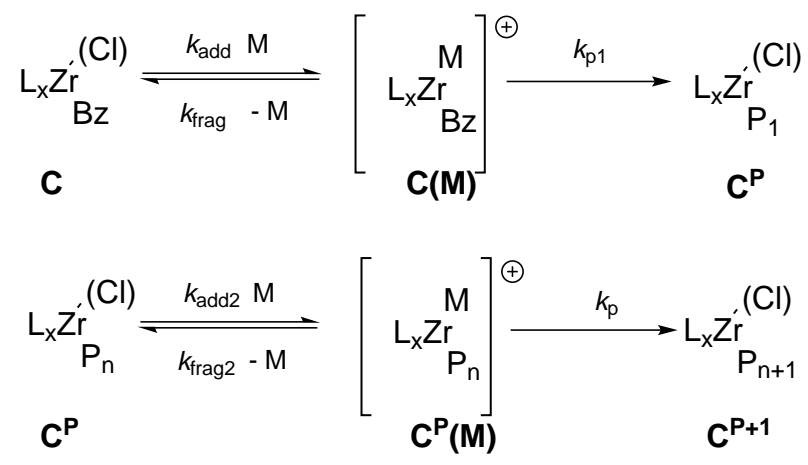

Scheme 5.2: Processes of monomer addition during activation $\mathbf{C}$ and polymerization $\mathbf{C}^{\mathbf{P}}$ at the catalytically active species.

Both complexes are able to associate and dissociate BzMgBz, BzMgP and PMgP. An activation pathway with three different species of $\mathbf{D}^{\mathrm{Px}}$ ( $\mathrm{x}$ refers to the number of polymer chains) was found with up to three polymer chains coordinated to the complex. To show the different states of activation, vertical steps are introduced in Scheme 5.3. Each vertical step represents the exchange of the growing polymer chain with a benzyl moiety 
and vice versa. The reaction setup starts with the association of $\mathrm{BzMgBz}$ to $\mathrm{C}^{\mathbf{P}}$ forming $\mathbf{D}^{\mathbf{P 1}}$ which either dissociates to regenerate $\mathrm{BzMgBz}$ and $\mathbf{C}^{\mathbf{P}}$ or one of the benzyl groups on magnesium undergoes chain transfer with the bridged polymer chain. The exact details for the transfer from magnesium to zirconium at the different $\mathbf{D}^{\mathbf{P x}}$ complexes are yet unknown. Camara et. al. ${ }^{[131]}$ reported a mechanism for intramolecular mechanism with a Zr-based catalyst and $\mathrm{AlMe}_{3}$ which might be possible for magnesium derivates. The associated reactions are only indicated by an equilibrium arrows, for the vertical steps in Scheme 5.3. The first vertical step is performed via dissociation to $\mathrm{C}$ and $\mathrm{BzMgP}$ (blue), a mono polymeric magnesium derivative. The statistical chance of the reaction is included in the rate coefficients while the overall rate is assumed to be independent of the position of the polymer chain. This is a general assumption and applied to all rate coefficients shown in the following. The next vertical step is possible if $\mathbf{C}$ associates PMgP (red) forming the complex $\mathbf{D}^{\mathbf{P 2}}$ which either dissociates back or undergoes chain-transfer between the bridged polymer chain with the benzyl- or polymeric moiety from the magnesium center. By this process BzMgP and $\mathbf{C}^{\mathbf{P}}$ are formed. Complex $\mathbf{D}^{\mathbf{P} 3}$ is exclusively formed by $\mathbf{C}^{\mathbf{P}}$ and $\mathrm{PMgP}$ and it describes the main equilibrium of $\mathrm{CCG}$ where the two polymer chains on magnesium may have the chance to undergo chain transfer with the brigded polymer chain. For all occurring reactions this process is assumed to be independent of the individual chain lengths of the involved polymer chains.

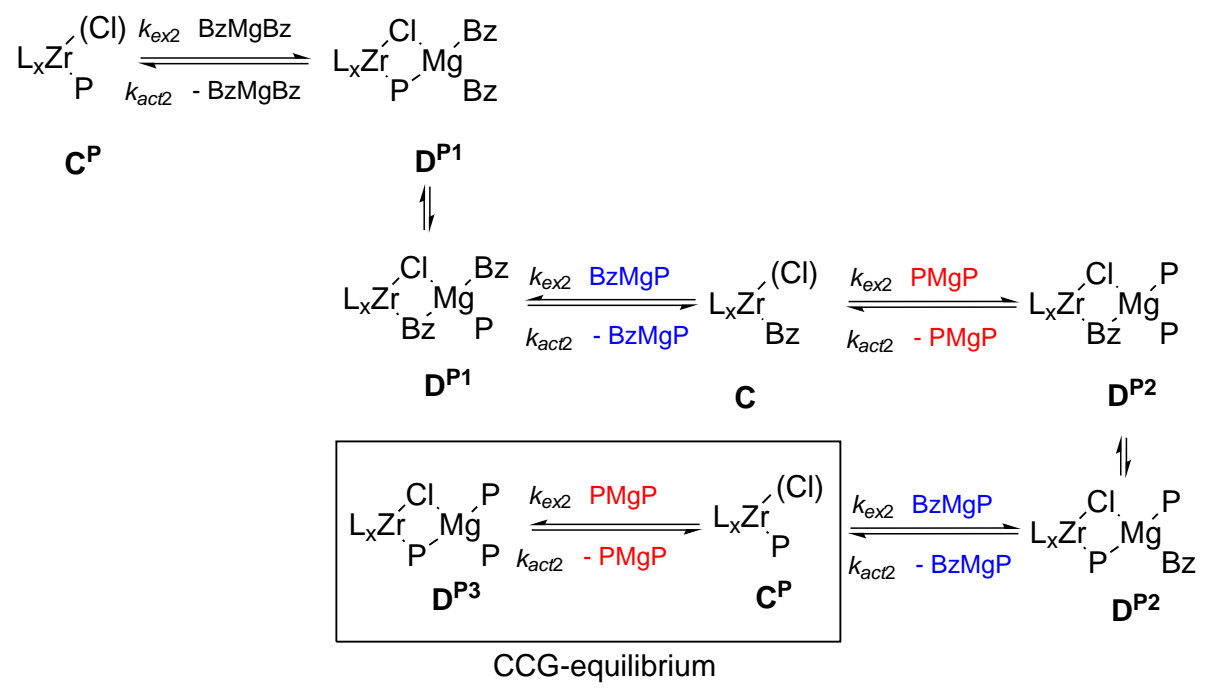

Scheme 5.3: Association/dissociation processes of $\mathbf{C}$ and $\mathbf{C}^{\mathbf{P}}$ with BzMgBz, PSMgBz and PMgP forming complexes with one $\left(\mathbf{D}^{\mathbf{P 1}}\right)$, two $\left(\mathbf{D}^{\mathbf{P 2}}\right)$ and three polymer $\left(\mathbf{D}^{\mathrm{P} 3}\right)$ chains involved.

The $\left(\mathrm{D}^{1,2,3}\right)$ complexes are able to perform the second alkylation reaction by alkyl-chloride exchange (see Scheme 5.4). Like in the case of the non-polymeric system, a complex of the type $\mathbf{E}^{\mathbf{P x}}$ with benzyl- and/or polystyryl- groups is yielded together with either a benzyl- or a polystyryl-grignard as reaction products. 


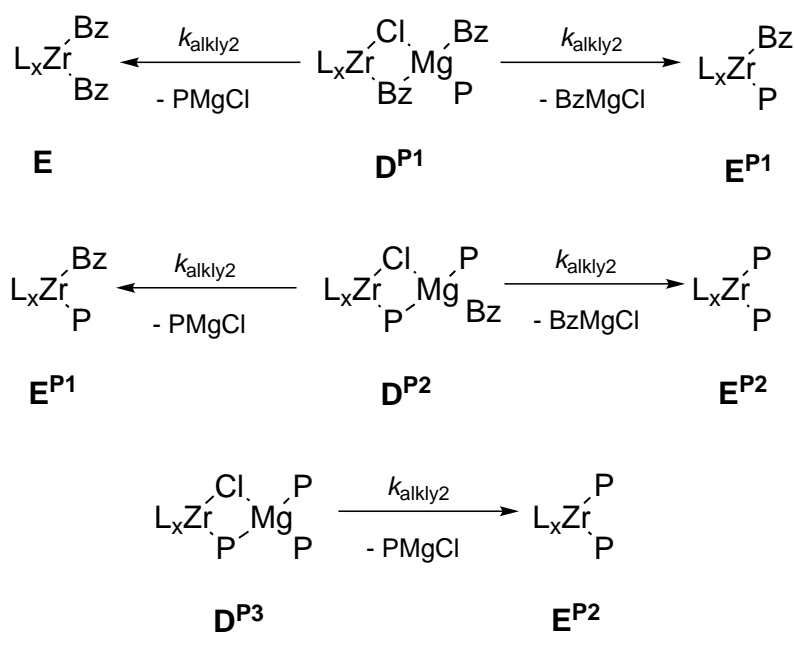

Scheme 5.4: Second alkylation reaction of $\mathbf{D}^{\mathrm{P1} 1,2,3}$ towards $\mathrm{E}^{\mathrm{P} 1}$ and $\mathrm{E}^{\mathrm{P2}}$ via formation of different grignard reactants.

$\mathbf{E}^{\mathbf{P x}}$ can associate either BzMgBz, BzMgP or PMgP and forms $\mathbf{F}^{\mathbf{P x}}$ complexes which can have up to four polymer chains coordinated (see Scheme 5.5). The coordinated polymer chains can undergo chain transfer from magnesium to the bridged positions. Both species are dormant species during polymerization and thus transfer does not affect control of the molar mass.

The complex $\mathbf{C}^{\mathbf{P}}$, which is formed after the first monomer addition, is capable of $\beta$ hydride elimination (cf. section 2.2.5 on page 17). It terminates the growing polymer chain and yields a macromonomer and a chlorohydride zirconium complex $\mathbf{G}$ (see Scheme 5.6). The macromonomer formation cannot be monitored due to the application of a deuterated monomer. Termination cannot be studied properly and will be targeted during by polymerization of non-deuterated styrene polymerization in UV/Vis studies. Therefore the further kinds of termination reactions are not yet introduced and the model is simplified by formation of an unreactive chlorohydride species.

The rate laws of the different species were described by classical reaction kinetics. 


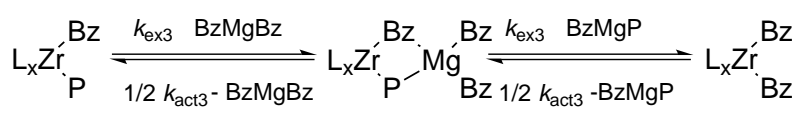

$$
\begin{aligned}
& \begin{array}{lll}
\mathrm{E}^{\mathrm{P} 1} & \mathrm{~F}^{\mathrm{P} 1} & \mathrm{E}^{\mathrm{P0}}
\end{array}
\end{aligned}
$$

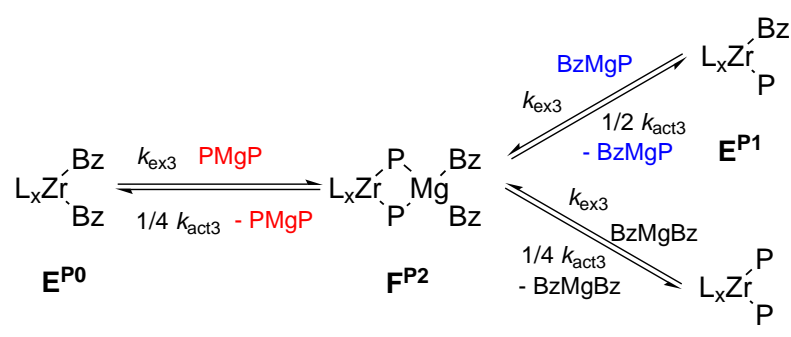

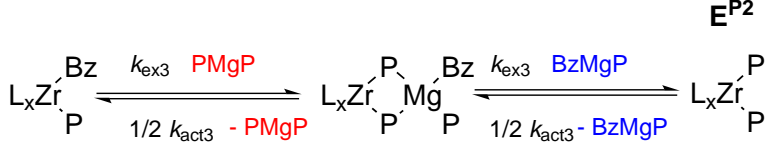

$$
\begin{aligned}
& \mathrm{E}^{\mathrm{P} 1} \quad \mathrm{~F}^{\mathrm{P3}} \quad \mathrm{E}^{\mathrm{P} 2}
\end{aligned}
$$

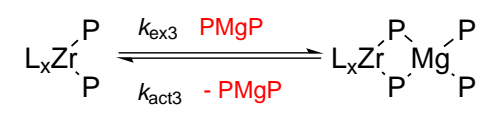

$$
\begin{aligned}
& \mathrm{E}^{\mathrm{P2}} \quad \mathrm{F}^{\mathrm{P} 4}
\end{aligned}
$$

Scheme 5.5: Association/dissociation processes of $\mathbf{E}^{\mathrm{P} 1}$ and $\mathbf{E}^{\mathrm{P} 2}$ with BzMgBz, BzMgP and PMgP forming complexes with one $\left(\mathbf{F}^{\mathbf{P 1}}\right)$, two $\left(\mathbf{F}^{\mathbf{P 2}}\right)$, three $\left(\mathbf{F}^{\mathbf{P} 3}\right)$ and four $\left(\mathbf{F}^{\mathbf{P 4}}\right)$ polymer chains involved.

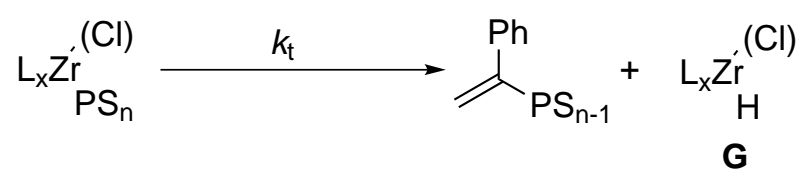

Scheme 5.6: Chain termination via $\beta$-hydride elimination. 
The equations are parted into sections for activation-, monomer addition-, exchange-, deactivation-, termination species and propagation for a better overview.

$$
\begin{aligned}
& \frac{\mathrm{d}[\mathbf{A}]}{\mathrm{d} t}=-k_{\mathrm{ex} 0}[\mathbf{A}][\mathbf{B z M g B z}]+k_{\text {act0 } 0}\left[\mathbf{B}^{\mathbf{P} 0}\right]-k_{\mathrm{ex} 0}[\mathbf{A}][\mathbf{B z M g P}]+k_{\mathrm{act} 0}\left[\mathbf{B}^{\mathbf{P} 1}\right] \\
& -k_{\mathrm{ex0}}[\mathbf{A}][\mathbf{P M g P}]+k_{\mathrm{act} 0}\left[\mathbf{B}^{\mathbf{P 2}}\right] \\
& \frac{\mathrm{d}\left[\mathbf{B}^{\mathbf{P 0}}\right]}{\mathrm{d} t}=k_{\mathrm{ex0}}[\mathbf{A}][\mathbf{B z M g B z}]-k_{\text {act } 0}\left[\mathbf{B}^{\mathbf{P} 0}\right]-k_{\text {alkyl1 }}\left[\mathbf{B}^{\mathbf{P 0}}\right] \\
& \frac{\mathrm{d}\left[\mathbf{B}^{\mathbf{P 1}}\right]}{\mathrm{d} t}=k_{\text {ex } 1}[\mathbf{A}][\mathbf{B z M g P}]-k_{\text {act0 }}\left[\mathbf{B}^{\mathbf{P 1}}\right]-k_{\text {alky11 }}\left[\mathbf{B}^{\mathbf{P 1}}\right] \\
& \frac{\mathrm{d}\left[\mathbf{B}^{\mathbf{P} 2}\right]}{\mathrm{d} t}=k_{\text {ex } 0}[\mathbf{A}][\mathbf{P M g P}]-k_{\text {act0 }}\left[\mathbf{B}^{\mathbf{P} 2}\right]-k_{\text {alkyl1 }}\left[\mathbf{B}^{\mathbf{P} 2}\right]
\end{aligned}
$$

The reaction rates for the catalyst precursor $\mathbf{A}$ and complex $\mathbf{B}^{\mathbf{P 0}, 1,2}(5.1-5.4)$ consist of three similar reactions only discriminated by the number of polymer chains at the magnesium atom. All concerned reaction are either of first or second order.

The complex $\mathbf{C}$ is initially formed from complexes $\mathbf{B}^{\mathrm{P0}, 1,2}$ at short reaction times. With proceeding reaction time the formation outgoing from the complexes $\mathrm{D}^{\mathrm{P} 0,1,2}$ becomes the main formation pathway. The complex is mainly consumed by the addition of monomer $\mathbf{M}$ and by chain propagation on this complex to $\mathbf{C}^{\mathbf{P}}$ and indirectly by second alkylation of the complexes $\mathbf{D}^{\mathrm{P0}, 1,2}$. C interacts with magnesium derivatives bearing none, one or two polystyrene chains. By association of the different magnesium derivatives the complexes $\mathrm{D}^{\mathbf{P 0}, 1,2}$ (see Equation 5.5) are formed.

$$
\begin{aligned}
\frac{\mathrm{d}[\mathbf{C}]}{\mathrm{d} t} & =-k_{\text {ex } 1}[\mathbf{C}][\mathbf{B z M g B z}]+k_{\text {act1 }}\left[\mathbf{D}^{\mathbf{P} 0}\right]+k_{\text {alkyl1 }}\left[\mathbf{B}^{\mathbf{P} 0}\right]-k_{\text {ex2 }}[\mathbf{C}][\mathbf{B z M g P}] \\
& +\frac{2}{3} k_{\text {act2 }}\left[\mathbf{D}^{\mathbf{P} 1}\right]-k_{\text {ex2 }}[\mathbf{C}][\mathbf{P M g P}]+\frac{1}{3} k_{\text {act2 } 2}\left[\mathbf{D}^{\mathbf{P} 2}\right]+\frac{1}{2} k_{\text {alky11 }}\left[\mathbf{B}^{\mathbf{P} 1}\right] \\
& -k_{\text {add }}[\mathbf{C}][\mathbf{M}]+k_{\text {frag }}[\mathbf{C}(\mathbf{M})]
\end{aligned}
$$

The associated polymeric complex $\mathbf{C}^{\mathrm{P}}$ is formed by dissociation of the complexes $\mathbf{D}^{\mathrm{P} 1,2,3}$ species, where the main exchange occurs at later stages of polymerization at $\mathbf{D}^{\mathbf{P 3}}$ (see Equation 5.6 to 5.8). The rate of monomer addition is similar for both complexes and it is possible that this step is chain-length dependent, as well as the insertion of monomer 
into the $\mathrm{Zr}-\mathrm{C}$ bond. In principle PREDICI ${ }^{\mathrm{TM}}$ simulations are capable to take this into account. The equations shown are simplified for clarity reasons.

$$
\begin{aligned}
& \frac{\mathrm{d}\left[\mathbf{C}^{\mathbf{P}}\right]}{\mathrm{d} t}=-k_{\mathrm{ex} 2}\left[\mathbf{C}^{\mathbf{P}}\right][\mathbf{B z M g B z}]+\frac{1}{3} k_{\mathrm{act} 2}\left[\mathbf{D}^{\mathbf{P} 1}\right]+\frac{1}{2} k_{\mathrm{alkyl} 1}\left[\mathbf{B}^{\mathbf{P} 1}\right]-k_{\mathrm{ex} 2}\left[\mathbf{C}^{\mathbf{P}}\right][\mathbf{B z} \mathbf{M g P}] \\
& -k_{\text {ex2 }}\left[\mathbf{C}^{\mathbf{P}}\right][\mathbf{P M g} \mathbf{P}]+\frac{2}{3} k_{\text {act2 }}\left[\mathbf{D}^{\mathbf{P} 2}\right]+k_{\text {alkyl1 }}\left[\mathbf{B}^{\mathbf{P} 2}\right]+\frac{2}{3} k_{\text {act2 }}\left[\mathbf{D}^{\mathbf{P} 2}\right] \\
& -k_{\text {add2 }}\left[\mathbf{C}^{\mathbf{P}}\right][\mathbf{M}]+k_{\text {frag2 }}\left[\mathbf{C}^{\mathbf{P}}(\mathbf{M})\right]+k_{\mathrm{p} 1}[\mathbf{C}(\mathbf{M})]+k_{\mathrm{p}}\left[\mathbf{C}^{\mathbf{P}}(\mathbf{M})\right]-k_{\mathrm{t}}\left[\mathbf{C}^{\mathbf{P}}\right] \\
& \frac{\mathrm{d}[\mathbf{C}(\mathbf{M})]}{\mathrm{d} t}=+k_{\mathrm{add}}[\mathbf{C}][\mathbf{M}]-k_{\mathrm{frag}}[\mathbf{C}(\mathbf{M})]-k_{\mathrm{p} 1}[\mathbf{C}(\mathbf{M})] \\
& \frac{\mathrm{d}\left[\mathbf{C}^{\mathbf{P}}(\mathbf{M})\right]}{\mathrm{d} t}=+k_{\text {add2 }}\left[\mathbf{C}^{\mathbf{P}}\right][\mathbf{M}]-k_{\text {frag2 }}\left[\mathbf{C}^{\mathbf{P}}(\mathbf{M})\right]-k_{\mathrm{p}}\left[\mathbf{C}^{\mathbf{P}}(\mathbf{M})\right]
\end{aligned}
$$

The complex $\mathbf{D}^{\mathbf{P 0}}$ is exclusively formed from species $\mathbf{C}$ and $\mathrm{BzMgBz}$ and can either dissociate or be deactivated to $\mathbf{E}$. Similar reactions are possible for $\mathbf{D}^{\mathbf{P 1}{ }^{2}}$, where they are formed by $\mathbf{C}$ or $\mathbf{C}^{\mathbf{P}}$ with BzMgP and PMgP. $\mathbf{D}^{\mathbf{P} 3}$ is exclusively formed from $\mathbf{C}^{\mathbf{P}}$ and $\mathrm{PMgP}$, which is the state of the main equilibrium in CCG polymerization, after full activation of all Bz groups (see Equation 5.9 to 5.12). All $\mathrm{D}^{\mathrm{P} 1,2,3}$ can be deactivated by the second alkylation step.

$$
\begin{aligned}
& \frac{\mathrm{d}\left[\mathbf{D}^{\mathbf{P} 0}\right]}{\mathrm{d} t}=+k_{\mathrm{ex} 2}[\mathbf{C}][\mathbf{B z M g B z}]-k_{\mathrm{act} 1}\left[\mathbf{D}^{\mathbf{P} 0}\right]-k_{\mathrm{alky} 12}\left[\mathbf{D}^{\mathbf{P} 0}\right] \\
& \frac{\mathrm{d}\left[\mathbf{D}^{\mathbf{P} 1}\right]}{\mathrm{d} t}=+k_{\mathrm{ex} 2}\left[\mathbf{C}^{\mathbf{P}}\right][\mathbf{B z M g B z}]+k_{\mathrm{ex} 2}\left[\mathbf{C}^{\mathbf{P}}\right][\mathbf{B z M g P}]-k_{\mathrm{act} 2}\left[\mathbf{D}^{\mathbf{P} 1}\right]-k_{\mathrm{alkyl} 3}\left[\mathbf{D}^{\mathbf{P} 1}\right] \\
& \frac{\mathrm{d}\left[\mathbf{D}^{\mathbf{P} 2}\right]}{\mathrm{d} t}=+k_{\mathrm{ex} 2}\left[\mathbf{C}^{\mathbf{P}}\right][\mathbf{B z M g P}]+k_{\mathrm{ex} 2}\left[\mathbf{C}^{\mathbf{P}}\right][\mathbf{P M g P}]-k_{\mathrm{act} 2}\left[\mathbf{D}^{\mathbf{P} 1}\right]-k_{\mathrm{alkyl} 3}\left[\mathbf{D}^{\mathbf{P} 2}\right] \\
& \frac{\mathrm{d}\left[\mathbf{D}^{\mathbf{P} 3}\right]}{\mathrm{d} t}=+k_{\mathrm{ex} 2}\left[\mathbf{C}^{\mathbf{P}}\right][\mathbf{P M g P}]-k_{\mathrm{act} 2}\left[\mathbf{D}^{\mathbf{P} 3}\right]-k_{\mathrm{alkyl} 3}\left[\mathbf{D}^{\mathbf{P} 3}\right]
\end{aligned}
$$

The additional exchange reaction have no influence on the formation of the catalytic active species and are shown in the appendix (see A.2 on page 217) The termination can be described with a first order reaction by transformation of $\mathbf{C}^{\mathbf{P}}$ (see Equation 5.13).

$$
\frac{\mathrm{d}[\mathbf{G}]}{\mathrm{d} t}=+k_{\mathrm{t}}\left[\mathbf{C}^{\mathbf{P}}\right]
$$

The monomer concentration is mainly influenced by coordination and discoordination of monomer to the catalytically active center. Higher monomer concentrations can cause 
a preferred formation of $\mathbf{C}^{(\mathbf{P})}(\mathbf{M})$. The propagation step is independent of the monomer concentration (see Equation 5.14).

$$
\frac{\mathrm{d}[\mathbf{M}]}{\mathrm{d} t}=-k_{\text {add }}[\mathbf{C}][\mathbf{M}]+k_{\text {frag }}[\mathbf{C}(\mathbf{M})]-k_{\text {add2 }}\left[\mathbf{C}^{\mathbf{P}}\right][\mathbf{M}]+k_{\text {frag2 }}\left[\mathbf{C}^{\mathbf{P}}(\mathbf{M})\right]
$$

The complexity of equations (5.1 to 5.12) reveal that the interplay between the species is more demanding then without monomer (4.1 to 4.6 on page 45). Modeling of the MMD would be even more complex which needs calculation for each single polymer chain which numerical calculations with PREDICI ${ }^{\mathrm{TM}}$ take into account.

Based on the Schemes 5.2 to 5.6 and Equations (5.1 to 5.46 and A.1 on page 217 to A.8) the kinetic model has been implemented into a PREDICI ${ }^{\mathrm{TM}}$ model which will be discussed in the following beginning with the activation process.

The first steps (5.15 to 5.19) stay the same as shown in section 4.3 .1 on page 46 and describe the formation of the catalytic active species $C$ outgoing from the catalyst precursor $\mathrm{A}$ and the reaction with $\mathrm{BzMgBz}$ to form $\mathrm{D}^{\mathrm{P} 0}$. For all steps "elemental reaction" steps were used in the model.

$$
\begin{aligned}
& \mathrm{A}+\mathrm{BzMgBz} \stackrel{k_{\mathrm{ex} 0}}{\longrightarrow} \mathbf{B}^{\mathbf{P 0}} \\
& \mathbf{B}^{\mathbf{P 0}} \stackrel{k_{\text {act } 0}}{\longrightarrow} \mathbf{A}+\mathrm{BzMgBz} \\
& \mathbf{B}^{\mathbf{P 0}} \stackrel{k_{\text {alkyyl }}}{\longrightarrow} \mathrm{C}+\mathrm{BzMgCl} \\
& \mathrm{C}+\mathrm{BzMgBz} \stackrel{k_{\text {ex } 1}}{\longrightarrow} \mathbf{D}^{\mathbf{P 0}} \\
& \mathbf{D}^{\mathbf{P 0}} \stackrel{k_{\text {act } 1}}{\longrightarrow} \mathrm{C}+\mathrm{BzMgBz}
\end{aligned}
$$

The activation is extended by the reaction of $\mathbf{A}$ with $\mathrm{BzMgP}$ and PMgP on the basis of "Change reactions" steps (5.20 to 5.29). The reaction of $\mathbf{A}$ with BzMgP yields complex $\mathbf{B}^{\mathbf{P 1}}$ (5.20) which either dissociates back (5.21) or performs alkylation. Two reaction pathways are possible for the alkylation, where either the polymer group (5.22) or the benzyl group (5.23) is exchanged. Both processes have the same chance of reaction. The reaction rate of $1 / 2 k_{\text {alkyl1 }}$ is therefore assigned to each process.

The reaction of A with PMgP has the information of two polymer chains involved. Because PREDICI ${ }^{\mathrm{TM}}$ can only store one chain length information per molecule, it is necessary to introduce virtual molecules. These molecules exist to store the chain length information of a polymer chain. In the case of PMgP these virtual molecules are introduced by a second molecule of PMgP. Both have the information of one chain stored and the concentration of this species is virtually doubled. To model processes which 
have these kind of species involved (5.24, see Scheme 5.1) the reaction rate is lowered by the factor $1 / 2$ to compensate the kinetic effect of the doubled concentration of PMgP. The reaction forms $\mathbf{B}^{\mathbf{P 2}}$ where this approach is applied in the same manner. This method is proven valid during the simulation of RAFT-polymerization, where the intermediate species of the process has two polymer chains bound to one molecule (see Scheme 2.14 on page 22). [189]

The described reaction forms an additional virtual molecule help1. It is introduced to transfer the chain length information of a second virtual molecule of PMgP. It performs the reaction with an extremely high chosen reaction rate $k_{\text {help }}$ of $10 \times 10^{10} \mathrm{~L} \mathrm{~mol}^{-1} \mathrm{~s}^{-1}$. In combination these processes lead to the exact description of the occurring process. The first reaction is the rate limiting step (5.24) which is according to the actual stoechiometry. The second virtual reaction (5.25) fast transfers the chain length information in a second virtual molecule of $\mathbf{B}^{\mathbf{P 2}}$. The polystyryl-chloride exchange reaction is described in two steps, first the dissociation reaction by the formation of one molecule of $\mathbf{C}^{\mathbf{P}}$ and the intermediate help2 (5.26). The doubled concentration of $\mathbf{B}^{\mathbf{P 2}}$ is taken into account by application of $1 / 2$ of $k_{\text {alkyl1 }}$ for the reaction rate. By this step one polymer information is transferred to the catalytically active complex and by the following reaction, a further virtual molecule to a polymeric Grignard (5.27). The dissociation reaction is described in the similar manner, where one virtual molecule of $\mathbf{B}^{\mathbf{P 2}}$ is forming a molecule of PMgP containing the chain length information and species help3 (5.28). Because the concentration of $\mathbf{B}^{\mathbf{P 2}}$ is doubled, the rate coefficient is $1 / 2$ of the normal reaction rate $k_{\text {act } 0}$. The intermediate species transforms a second $\mathbf{B}^{\mathbf{P 2}}$ into PMgP (5.29). The chain-length information of these species is labeled with the index 's' within PREDICI ${ }^{\mathrm{TM}}$.

The doubled concentration in PMgP additionally keeps the model simple and models exchange reactions on the magnesium compounds. ${ }^{[267]}$

$$
\begin{aligned}
& \mathbf{A}+\mathrm{BzMgP}_{\mathrm{s}} \stackrel{k_{\mathrm{ex0}}}{\longrightarrow} \mathbf{B}^{\mathbf{P 1}}{ }_{\mathbf{s}} \\
& \mathbf{B}^{\mathbf{P} 1}{ }_{\mathbf{s}} \stackrel{k_{\text {act0 }}}{\longrightarrow} \mathbf{A}+\mathrm{BzMgP}_{\mathrm{s}} \\
& \mathbf{B}^{\mathbf{P} 1}{ }_{\mathbf{s}} \stackrel{k_{\text {alkyl1 }}}{\longrightarrow} \mathbf{C}^{\mathbf{P}}{ }_{\mathbf{s}}+\mathrm{BzMgCl} \\
& \mathbf{B}^{\mathbf{P 1}} \stackrel{k_{\text {alkyl1 }}}{\longrightarrow} \mathbf{C}+\mathrm{PMgCl}_{\mathrm{s}} \\
& \mathbf{A}+\mathrm{PMgP}_{\mathrm{s}} \stackrel{k_{\mathrm{ex} 0}}{\longrightarrow} \mathbf{B}^{\mathbf{P 2}}{ }_{\mathbf{s}}+\text { help1 } \\
& \mathrm{PMgP}_{\mathrm{s}}+\text { help1 } \stackrel{k_{\text {help }}}{\longrightarrow} \mathbf{B}^{\mathbf{P 2}} \mathbf{s} \\
& \mathbf{B}^{\mathbf{P 2}} \stackrel{\frac{1}{2} k_{\mathrm{alkyl}}}{\longrightarrow} \mathbf{C}^{\mathbf{P}}{ }_{\mathbf{s}}+\text { help2 }
\end{aligned}
$$




$$
\begin{gathered}
\mathbf{B}^{\mathbf{P} 2}{ }_{\mathrm{s}}+\text { help } 2 \stackrel{k_{\text {help }}}{\longrightarrow} \mathrm{PMgCl}_{\mathrm{s}} \\
\mathbf{B}^{\mathbf{P} 2}{ }_{\mathbf{s}} \stackrel{\frac{1}{2} k_{\text {act0 }}}{\longrightarrow} \mathrm{PMgP}_{\mathrm{s}}+\text { help3 } \\
\mathbf{B}^{\mathbf{P} 2}{ }_{\mathbf{s}}+\text { help3 } \stackrel{k_{\text {help }}}{\longrightarrow} \mathrm{PMgP}_{\mathrm{s}}+\mathbf{A}
\end{gathered}
$$

The process of monomer addition is defined according to the one proposed by of Cossée and Arlman. ${ }^{[289]} \alpha$-agostic intermediates can easily be described by the rate coefficients (cf. section 2.2.4 on page 16). The process consists of three reaction steps. The monomer coordination (5.30) and discoordination (5.31) were described via "elemental reaction" steps. The monomer is bound to the complex and is reducing the monomer concentration. For the propagating step a "Initiation(decay)" reaction step (5.32) was applied which produces polymer chain with a chain length of 1 . As shown here, all reactions having a polymer chain involved can be identified by the index, such as $\mathbf{C}^{\mathbf{P}}$.

$$
\begin{gathered}
\mathrm{C}+\mathrm{M} \stackrel{k_{\mathrm{add}}}{\longrightarrow} \mathrm{C}(\mathbf{M}) \\
\mathrm{C}(\mathbf{M}) \stackrel{k_{\text {trag }}}{\longrightarrow} \mathrm{C}+\mathrm{M} \\
\mathrm{C}(\mathbf{M}) \stackrel{k_{\mathrm{p} 1}}{\longrightarrow} \mathrm{C}^{\mathrm{P}}{ }_{1}
\end{gathered}
$$

The process of monomer addition for $\mathbf{C}^{\mathrm{P}}>=1$ occurs in the same way. Additionally chain length information is stored. Within PREDICI the chain-growth is introduced virtually at the step of polymer coordination (5.33) with a "Propagation" step, because there is no alternative reaction step available which describes the monomer addition without propagation reaction. The discoordination reaction is introduced via a "Degradation" step of 1 monomer unit (5.34 which lowers the chain length by one. The real chain growth is considered by a "change" reaction or a " $\mathrm{k}(\mathrm{s})$-termination" step for the insertion into the C-Zr bond. The "change" reaction describes the process in absence of a chain-length dependence during chain growth while the " $\mathrm{k}(\mathrm{s})$-termination" is suitable to calculate this effect (5.35).

$$
\begin{gathered}
\mathrm{C}^{\mathrm{P}}{ }_{\mathrm{s}}+\mathrm{M} \stackrel{k_{\mathrm{add}}}{\longrightarrow} \mathrm{C}(\mathbf{M})_{\mathrm{s}+1} \\
\mathrm{C}^{\mathrm{P}}(\mathbf{M})_{\mathrm{s}} \stackrel{k_{\text {frag }}}{\longrightarrow} \mathrm{C}^{\mathrm{P}-1}+\mathrm{M} \\
\mathrm{C}^{\mathrm{P}}(\mathbf{M})_{\mathrm{s}} \stackrel{k_{\mathrm{p}}}{\longrightarrow} \mathrm{C}^{\mathrm{P}}{ }_{\mathrm{s}}
\end{gathered}
$$


To describe the exchange reaction which lead to the formation of $\mathbf{D}^{\mathbf{P 1}}$, "change" reaction steps were applied (5.36 and 5.37). The dissociation of this species can lead either to $\mathrm{C}$ and $\mathrm{BzMgP}(5.38)$ or to $\mathrm{C}^{\mathrm{P}}$ and $\mathrm{BzMgBz}(5.39)$. Two positions for the polymer group are possible for species $\mathbf{D}^{\mathrm{P} 1}$ one located on magnesium and one on zirconium. The first reaction has therefore a statistical probability of $2 / 3$ and the second of $1 / 3 . k_{\text {act } 2}$ is scaled accordingly.

$$
\begin{aligned}
& \mathbf{C}^{\mathbf{P}}{ }_{\mathbf{s}}+\mathrm{BzMgBz} \stackrel{k_{\text {ex2 }}}{\longrightarrow} \mathbf{D}^{\mathbf{P} \mathbf{1}}{ }_{\mathbf{s}} \\
& \mathrm{C}+\mathrm{BzMgP}_{\mathrm{s}} \stackrel{k_{\mathrm{e} \times 2}}{\longrightarrow} \mathrm{D}^{\mathrm{P} 1}{ }_{\mathrm{s}} \\
& \mathbf{D}^{\mathbf{P 1}}{ }_{\mathbf{s}} \stackrel{\frac{2}{3} k_{\text {act2 }}}{\longrightarrow} \mathbf{C}+\mathrm{BzMgP}_{\mathrm{s}} \\
& \mathbf{D}^{\mathbf{P 1}} \stackrel{\frac{1}{3} k_{\mathrm{act} 2}}{\longrightarrow} \mathbf{C}^{\mathbf{P}}{ }_{\mathbf{s}}+\mathrm{BzMgBz}
\end{aligned}
$$

Two polymer chains are involved in the reaction between the polymeric species $\mathbf{C}^{\mathbf{P}}$ and the polymeric BzMgP. To store the information of both chains, the reaction is described via a "d-termination" reaction step (5.40) forming two virtual molecules of $\mathbf{D}^{\mathrm{P2}}$. As shown before the concentration is twice the real concentration. The reaction of species $\mathbf{C}$ and $\mathrm{PMgP}$ is similar to the reaction between $\mathbf{A}$ and PMgP. The previous shown approach by introducing the polymeric help 4 species (5.41) by a "change"-reaction is applied. The second polymer information is transferred by a "d-termination" step with polymeric intermediate species into two $\mathbf{D}^{\mathbf{P 2}}$ species (5.42). The exchange rate coefficient are scaled accordingly to the doubled concentration of PMgP.

$$
\begin{aligned}
& \mathbf{C}^{\mathbf{P}}{ }_{\mathrm{s}}+\mathrm{BzMgP}_{\mathrm{r}} \stackrel{k_{\mathrm{ex} 2}}{\longrightarrow} \mathbf{D}^{\mathbf{P} 2}{ }_{\mathbf{s}}+\mathbf{D}^{\mathbf{P} 2}{ }_{\mathbf{r}} \\
& \mathrm{C}+\mathrm{PMgP}_{\mathrm{s}} \stackrel{k_{\mathrm{act} 2}}{\longrightarrow} \text { help } 4_{\mathrm{s}} \\
& \text { help } 4+\mathrm{PMgP}_{\mathrm{r}} \stackrel{k_{\text {help }}}{\longrightarrow} \mathbf{D}^{\mathbf{P} 1}{ }_{\mathbf{s}}+\mathbf{D}^{\mathbf{P} 1}{ }_{\mathbf{r}}
\end{aligned}
$$

The dissociation of $\mathbf{D}^{\mathbf{P 2}}$ may occur in two different ways: The formation of either $\mathbf{C}^{\mathbf{P}}$ and BzMgP with a probability of $2 / 3(\overline{5.43}-\overline{5.44})$ or $\mathrm{C}$ and PMgP with a probability of $1 / 3$ (5.45-5.46). Both reaction pathways are introduced via "change" reaction steps and the intermediate species help 5 and help 6 are introduced to transfer the polymer information of the second polymer chain. The rate coefficients of $k_{\text {act } 2}$ are scaled by the individual probability and the doubled concentration of $\mathbf{D}^{\mathbf{P 2}}$. 


$$
\begin{aligned}
& \mathbf{D}^{\mathbf{P} 2}{ }_{\mathrm{s}} \stackrel{\frac{1}{2} k_{\text {act2 }}}{\longrightarrow} \mathbf{C}^{\mathbf{P}}{ }_{\mathbf{s}}+\text { help5 } \\
& \mathbf{D}^{\mathbf{P 2}}{ }_{\mathbf{s}}+\text { help } 5 \stackrel{k_{\text {help }}}{\longrightarrow} \mathrm{BzMgP}_{\mathrm{s}} \\
& \mathbf{D}^{\mathbf{P} 2}{ }_{\mathrm{s}} \stackrel{\frac{1}{6} k_{\text {act2 }}}{\longrightarrow} \mathrm{PMgP}_{\mathrm{s}}+\text { help6 } \\
& \mathbf{D}^{\mathbf{P 2}}{ }_{\mathbf{s}}+\text { help } 6 \stackrel{k_{\text {help }}}{\longrightarrow} \mathrm{PMgP}_{\mathrm{s}}+\mathbf{C}
\end{aligned}
$$

Three polymer chains can be exchanged to the active metal center in the CCG main equilibrium between $\mathrm{C}^{\mathrm{P}}$ and PMgP. The association reaction is described via two " $\mathrm{d}$ termination" reaction steps (5.47-5.48). The reaction between $\mathrm{C}^{\mathrm{P}}$ and $\mathrm{PMgP}$ yields one molecule of $\mathbf{D}^{\mathrm{P3}}$ and the polymeric species help 7 which reacts with PMgP forming two additional $\mathbf{D}^{\mathrm{P} 3}$ molecules. The virtual concentration of $\mathbf{D}^{\mathrm{P3}}$ has the threefold value of the real concentration to store all polymer information. For dissociation of $\mathbf{D}^{\mathrm{P3}}$ "change" reaction steps were applied. The first reaction transforms one molecule into $\mathrm{PMgP}$ and the intermediate species help8, which starts the transfer of two additional molecules of $\mathbf{D}^{\mathrm{P3}}$ via help9 into one $\mathrm{PMgP}$ and one $\mathbf{C}^{\mathrm{P}}$ species. The applied rate coefficients are scaled to the twice-fold concentration of $\mathrm{PMgP}$ and the threefold concentration of $\mathbf{D}^{\mathrm{P3}}$.

$$
\begin{aligned}
& \mathbf{C}^{\mathbf{P}}{ }_{\mathbf{s}}+\mathrm{PMgP}_{\mathrm{r}} \stackrel{\frac{1}{2} k_{\mathrm{ex} 2}}{\longrightarrow} \mathbf{D}^{\mathrm{P} 3}{ }_{\mathbf{s}}+\text { help } 7_{\mathrm{r}} \\
& \text { help } 7_{\mathrm{r}}+\mathrm{PMgP}_{\mathrm{r}} \stackrel{k_{\text {help }}}{\longrightarrow} \mathbf{D}^{\mathrm{P} 3}{ }_{\mathbf{s}}+\mathbf{D}^{\mathrm{P} 3}{ }_{\mathrm{r}} \\
& \mathbf{D}^{\mathrm{P} 3}{ }_{\mathrm{s}} \stackrel{\frac{1}{3} k_{\text {act2 }}}{\longrightarrow} \mathrm{PMgP}_{\mathrm{s}}+\text { help8 } \\
& \mathbf{D}^{\mathrm{P} 3}{ }_{\mathrm{s}}+\text { help8 } \stackrel{k_{\text {help }}}{\longrightarrow} \mathrm{PMgP}_{\mathrm{s}}+\text { help9 } \\
& \mathbf{D}^{\mathbf{P 3}}{ }_{\mathbf{s}}+\text { help9 } \stackrel{k_{\text {help }}}{\longrightarrow} \mathbf{C}^{\mathbf{P}}{ }_{\mathbf{s}}
\end{aligned}
$$

A change reaction (5.52) was chosen for the termination via $\beta$-hydride elimination yielding an unreactive polymer chain and a catalyst bearing a hydride moiety $\mathbf{G}$.

$$
\mathrm{C}^{\mathrm{P}}{ }_{\mathrm{s}} \stackrel{k_{\mathrm{t}}}{\longrightarrow} \mathrm{G}+\mathrm{P}_{\text {Dead }, \mathrm{s}}
$$


The Schlenk equilibrium (5.53 to 5.56) was introduced via "change" reaction steps. The reaction between two $\mathrm{PMgCl}$ molecules needs on intermediate species help 26 to perform the formation of $\mathrm{MgCl}_{2}$.

$$
\begin{gathered}
\mathrm{BzMgCl}+\mathrm{BzMgCl} \stackrel{k_{\text {schlenk }}}{\longrightarrow} \mathrm{BzMgBz}+\mathrm{ClMgCl} \\
\mathrm{PMgCl}_{\mathrm{s}}+\mathrm{BzMgCl} \stackrel{k_{\text {schlenk }}}{\longrightarrow} \mathrm{BzMgP}_{\mathrm{s}}+\mathrm{CIMgCl} \\
M g C l \mathrm{PMgCl}+\mathrm{PMgClr} \stackrel{k_{\text {schlenk }}}{\longrightarrow} \mathrm{PMgP}_{\mathrm{s}}+\text { help26 } \\
\text { help26 } \\
\stackrel{k_{\text {help }}}{\longrightarrow} \mathrm{PMgP}_{\mathrm{s}}+\mathrm{CIMgCl}
\end{gathered}
$$

The reactions shown above (5.15 to 5.56) describe the universal model for CCG polymerization and it is also applied on the neodymium system (see section 6.3 on page 152). For the model system shown in the NMR-experiments the reaction set has to be extended. It was found that the zirconium complex can be alkylated a second time by alkyl-chloride exchange. These reaction pathways are shortly introduced in the following. The same approaches as discussed before were applied.

The deactivation reaction from $\mathbf{D}$ to $\mathbf{E}$ is described via an "elemental" reaction step.

$$
\mathbf{D}^{\mathrm{P0}} \stackrel{k_{\text {alkyl2 }}}{\longrightarrow} \mathbf{E}^{\mathbf{P 0}}+\mathrm{BzMgCl}
$$

The complex $\mathbf{D}^{\mathbf{P} 1}$ can perform two possible deactivation pathways. The first forms $\mathbf{E}^{\mathbf{P} 0}$ and a polymeric Gringard $\mathrm{PMgCl}(5.58)$ and the second the polymeric complex $\mathrm{E}^{\mathrm{P} 1}$ and $\mathrm{BzMgCl}$ (5.59). Each reaction pathway is described via a "change" reaction.

$$
\begin{aligned}
& \mathbf{D}^{\mathbf{P} \mathbf{1}_{\mathbf{s}}} \stackrel{\frac{1}{3} k_{\mathrm{alky} 13}}{\longrightarrow} \mathbf{E}^{\mathbf{P} \mathbf{0}}+\mathrm{PMgCl}_{\mathbf{s}} \\
& \mathbf{D}^{\mathbf{P} \mathbf{1}}{ }_{\mathbf{s}} \stackrel{\frac{2}{3} k_{\mathrm{alkyl}}}{\longrightarrow} \mathbf{E}^{\mathbf{P} \mathbf{1}}{ }_{\mathbf{s}}+\mathrm{BzMgCl}
\end{aligned}
$$

The complex $\mathbf{D}^{\mathbf{P 2}}$ can deactivate either by formation of one $\mathbf{E}^{\mathbf{P 1}}$ and $\mathrm{PMgCl}(5.60-5.61)$ with help10 as intermediate species or by $\mathbf{E}^{\mathbf{P 2}}$ and $\mathrm{BzMgCl}(5.62-5.63)$ with help11. For both reaction setups "change" reaction steps were applied and the reaction coefficients scaled according to their individual probability and virtual concentrations of the processes. 


$$
\begin{gathered}
\mathbf{D}^{\mathbf{P 2}}{ }_{\mathbf{s}} \stackrel{\frac{1}{6} k_{\text {alky13 }}}{\longrightarrow} \mathbf{E}^{\mathbf{P 2}}+\text { help10 } \\
\mathbf{D}^{\mathbf{P 2}}{ }_{\mathbf{s}}+\text { help } 10 \stackrel{k_{\text {help }}}{\longrightarrow} \mathbf{E}^{\mathbf{P 2}}+\mathrm{BzMgCl} \\
\mathbf{D}^{\mathbf{P 2}}{ }_{\frac{1}{3} k_{\text {alkyl3 }}}^{\longrightarrow} \mathbf{E}^{\mathbf{P 1}}{ }_{\mathrm{s}}+\text { help11 } \\
\mathbf{D}^{\mathbf{P 2}}{ }_{\mathrm{s}}+\text { help } 11 \stackrel{k_{\text {help }}}{\longrightarrow} \mathrm{PMgCl}_{\mathrm{s}}
\end{gathered}
$$

The deactivation of the species $\mathbf{D}^{\mathrm{P3}}$ is described via three "change" reaction steps in which one virtual molecule of $\mathbf{E}^{\mathrm{P2}}$ and help12 are formed (5.64) as first step. The intermediate species start a reaction cascade to transfer the polymer information of two further molecules into one additional $\mathrm{E}^{\mathrm{P2}}$ species. The $\mathrm{PMgCl}$ species (5.64 5.66) is achieved via help13. The rate coefficients are scaled by the threefold concentration of $\mathbf{D}^{\mathrm{P3}}$.

$$
\begin{gathered}
\mathbf{D}^{\mathrm{P} 3}{ }_{\mathrm{s}} \stackrel{\frac{1}{3} k_{\text {alky12 }}}{\longrightarrow} \mathbf{E}^{\mathrm{P} 2}+\text { help12 } \\
\mathbf{D}^{\mathrm{P} 3}{ }_{\mathrm{s}}+\text { help12 } \stackrel{k_{\text {help }}}{\longrightarrow} \mathbf{E}^{\mathrm{P} 2}+\text { help13 } \\
\mathbf{D}^{\mathrm{P} 3}{ }_{\mathrm{s}}+\text { help } 13 \stackrel{k_{\text {help }}}{\longrightarrow} \mathrm{PMgCl}_{\mathrm{s}}
\end{gathered}
$$

The reaction of $\mathbf{E}^{\mathrm{P0}}$ with $\mathrm{BzMgBz}$ is similar to the monomer free system (5.67-5.68) and is introduced via "elemental" reaction steps.

$$
\begin{aligned}
& \mathbf{E}^{\mathrm{P0}}+\mathrm{BzMgBz} \stackrel{k_{\mathrm{ex} 3}}{\longrightarrow} \mathbf{F}^{\mathrm{P0}} \\
& \mathbf{F}^{\mathrm{P0}} \stackrel{k_{\mathrm{act} 2}}{\longrightarrow} \mathbf{E}^{\mathrm{P0}}+\mathrm{BzMgBz}
\end{aligned}
$$

The equilibrium reactions of $\mathrm{F}^{\mathrm{P1}}$ are introduced via "change" reactions outgoing from $\mathrm{E}$ and $\mathrm{BzMgP}(5.69,5.71)$ or $\mathrm{E}^{\mathrm{P} 1}$ and $\mathrm{BzMgBz}(5.70,5.72)$.

$$
\begin{aligned}
& \mathbf{E}^{\mathbf{P 1}}{ }_{\mathbf{s}}+\mathrm{BzMgBz} \stackrel{k_{\mathrm{ex} 3}}{\longrightarrow} \mathbf{F}^{\mathbf{P 1}} \\
& \mathbf{E}^{\mathrm{P} 0}+\mathrm{BzMgP}_{\mathrm{s}} \stackrel{k_{\mathrm{ex} 3}}{\longrightarrow} \mathbf{E}^{\mathrm{P} \mathbf{1}} \\
& \mathbf{F}^{\mathbf{P} 1} \stackrel{k_{\text {act3 }}}{\longrightarrow} \mathbf{E}^{\mathbf{P} 1}{ }_{\mathbf{s}}+\mathrm{BzMgBz} \\
& \mathbf{F}^{\mathbf{P 1}} \stackrel{k_{\text {act3 }}}{\longrightarrow} \mathbf{E}^{\mathbf{P 0}}+\mathrm{BzMgP}_{\mathrm{s}}
\end{aligned}
$$


$\mathbf{F}^{\mathrm{P2}}$ can be formed via $\mathrm{E}^{\mathrm{P} 1}$ and BzMgP described as "d-termination" reaction step (5.73). From $\mathbf{E}^{\mathrm{P0}}$ and $\mathrm{PMgP}$ the description has two "change" reactions with an intermediate species help14 involved (5.74-5.75). The reaction of $\mathrm{E}^{\mathrm{P2}}$ and $\mathrm{BzMgBz}$ is introduced in an equal manner by help15 (5.76-5.77). The rate coefficients are scaled according to the twofold virtual concentration of $\mathrm{PMgP}$ and $\mathbf{E}^{\mathrm{P} 2}$.

$$
\begin{aligned}
& \mathbf{E}^{\mathbf{P 1}}{ }_{\mathbf{s}}+\mathrm{BzMgP}_{\mathrm{r}} \stackrel{k_{\mathrm{ex} 3}}{\longrightarrow} \mathbf{F}^{\mathbf{P} 2}{ }_{\mathbf{s}}+\mathbf{F}^{\mathbf{P} 2}{ }_{\mathbf{r}} \\
& \mathbf{E}^{\mathrm{P} 0}+\mathrm{PMgP}_{\mathrm{s}} \stackrel{\frac{1}{2} k_{\mathrm{ex}}}{\longrightarrow} \mathbf{F}^{\mathbf{P} 2}{ }_{\mathbf{s}}+\text { help14 } \\
& \text { help14 }+\mathrm{PMgP}_{\mathrm{s}} \stackrel{k_{\text {help }}}{\longrightarrow} \mathbf{F}^{\mathbf{P}^{2}}{ }_{\mathbf{s}} \\
& \mathbf{E}^{\mathbf{P} 2}+\mathrm{BzMgBz} \stackrel{\frac{1}{2} k_{\mathrm{ex}}}{\longrightarrow} \mathbf{F}^{\mathbf{P 2}}{ }_{\mathbf{s}}+\text { help15 } \\
& \text { help15 }+\mathbf{E}^{\mathbf{P 2}} \stackrel{k_{\text {help }}}{\longrightarrow} \mathbf{F}^{\mathbf{P} 2}{ }_{\mathbf{s}}
\end{aligned}
$$

For the dissociation reactions the doubled concentrations of $\mathbf{F}^{\mathrm{P} 2}$ and the probability of the reaction are considered within the implemented kinetic coefficients. All reactions are described as "change" reactions. For $\mathbf{E}^{\mathrm{P1}}$ and BzMgP, the intermediate species help16 is necessary (5.78-5.79). $\mathbf{E}^{\mathbf{P 0}}$ and $\mathrm{PMgP}$ species help17 (5.80-5.81) and $\mathbf{E}^{\mathbf{P 2}}$ and $\mathrm{BzMgBz}$ (5.82-5.83) a species help17 are described in the same way.

$$
\begin{aligned}
& \mathbf{F}^{\mathbf{P 2}} \stackrel{\frac{1}{4} k_{\mathrm{act} 3}}{\longrightarrow} \mathbf{E}^{\mathbf{P 1}}{ }_{\mathbf{s}}+\text { help16 } \\
& \mathbf{F}^{\mathbf{P} 2}{ }_{\mathbf{s}}+\text { help16 } \stackrel{k_{\text {help }}}{\longrightarrow} \mathrm{BzMgP}_{\mathrm{s}} \\
& \mathbf{F}^{\mathbf{P 2}} \stackrel{\frac{1}{8} k_{\text {act3 }}}{\longrightarrow} \mathrm{PMgP}_{\mathrm{s}}+\text { help17 } \\
& \mathbf{F}^{\mathbf{P 2}}{ }_{\mathbf{s}}+\text { help } 17 \stackrel{k_{\text {help }}}{\longrightarrow} \mathrm{PMgP}_{\mathrm{s}}+\mathbf{F}^{\mathbf{P 0}} \\
& \mathbf{F}^{\mathbf{P 2}} \stackrel{\frac{1}{8} k_{\mathrm{act} 3}}{\longrightarrow} \mathbf{E}^{\mathbf{P 2}}{ }_{\mathbf{s}}+\text { help18 } \\
& \mathbf{F}^{\mathbf{P 2}}{ }_{\mathbf{s}}+\text { help18 } \stackrel{k_{\text {help }}}{\longrightarrow} \mathbf{E}^{\mathbf{P 2}}{ }_{\mathbf{s}}+\mathrm{BzMgBz}
\end{aligned}
$$

$\mathbf{F}^{\mathbf{P 3}}$ is formed from $\mathbf{E}^{\mathbf{P} 1}$ and from $\mathrm{PMgP}$ and $\mathbf{E}^{\mathbf{P} 2}$ and $\mathrm{BzMgP}$ which is described via "d-termination reaction" steps with polymeric intermediate species help 19 and help20 (5.84-5.87) to transform all necessary polymer information. The threefold concentration of $\mathbf{F}^{\mathrm{P} 3}$ is considered within the kinetic coefficients. 


$$
\begin{aligned}
& \mathbf{E}^{\mathbf{P} 1}{ }_{\mathbf{s}}+\mathrm{PMgP}_{\mathrm{r}} \stackrel{k_{\mathrm{ex} 3}}{\longrightarrow} \mathbf{F}^{\mathbf{P} 3}{ }_{\mathbf{s}}+\text { help19 } \mathrm{r} \\
& \mathrm{PMgP}_{\mathrm{s}}+\text { help19 } \mathrm{r} \stackrel{k_{\text {help }}}{\longrightarrow} \mathbf{F}^{\mathbf{P 3}}{ }_{\mathbf{s}}+\mathbf{F}^{\mathbf{P 3}}{ }_{\mathbf{r}} \\
& \mathbf{E}^{\mathbf{P 2}}{ }_{\mathbf{s}}+\mathrm{BzMgP}_{\mathrm{r}} \stackrel{k_{\mathrm{ex} 3}}{\longrightarrow} \mathbf{F}^{\mathbf{P} 3}{ }_{\mathbf{s}}+\text { help20 } \mathrm{r} \\
& \mathbf{E}^{\mathbf{P 2}}{ }_{\mathbf{s}}+\text { help } 20_{\mathrm{r}} \stackrel{k_{\text {help }}}{\longrightarrow} \mathbf{F}^{\mathbf{P 3}}{ }_{\mathbf{s}}+\mathbf{F}^{\mathbf{P 3}}{ }_{\mathbf{r}}
\end{aligned}
$$

All dissociation reactions are described via change reaction steps and in each case two intermediate species are necessary. For $\mathbf{E}^{\mathrm{P1}}$ and $\mathrm{PMgP}(5.88-5.90)$ they are help21 and help22 and for $\mathrm{E}^{\mathrm{P2}}$ and $\mathrm{BzMgP}$ (5.91-5.93) help23 and help24.The reactions are described via "change" reaction steps and in each case two intermediate species are necessary. The rate coefficients are scaled according to the threefold concentration and reaction probability.

$$
\begin{gathered}
\mathbf{F}^{\mathbf{P 3}} \stackrel{\frac{1}{6} k_{\text {act3 }}}{\longrightarrow} \text { PMgP }_{\mathbf{s}}+\text { help21 } \\
\mathbf{F}^{\mathbf{P 3}}{ }_{\mathbf{s}}+\text { help } 21 \stackrel{k_{\text {help }}}{\longrightarrow} \mathbf{E}^{\mathbf{P 1}}{ }_{\mathbf{s}}+\text { help22 } \\
\mathbf{F}^{\mathbf{P} 3}+\text { help } 22 \stackrel{k_{\text {help }}}{\longrightarrow} \text { PMgP }_{\mathbf{s}} \\
\mathbf{F}^{\mathbf{P 3}} \stackrel{\frac{1}{6} k_{\text {act3 }}}{\longrightarrow} \mathbf{E}^{\mathbf{P 2}}{ }_{\mathbf{s}}+\text { help } 23 \\
\mathbf{F}^{\mathbf{P 3}}{ }_{\mathbf{s}}+\text { help } 23 \stackrel{k_{\text {help }}}{\longrightarrow} \mathbf{E}^{\mathbf{P 2}}{ }_{\mathbf{s}}+\text { help24 } \\
\mathbf{F}^{\mathbf{P 3}}{ }_{\mathbf{s}}+\text { help } 24 \stackrel{k_{\text {help }}}{\longrightarrow} \text { BzMgP }
\end{gathered}
$$

The reaction of $\mathbf{E}^{\mathbf{P 2}}$ with $\mathrm{PMgP}$ is described using a "d-termination" step. Because the concentration of both species is doubled, the kinetic coefficients are divided by four. Due to the fact that the reaction has to be modelated twice for a full reaction step it is multiplied by the factor of two yielding a scaling factor of $1 / 2$. The same is valid for the reverse reaction introduced via change reactions and the intermediate help 25.

$$
\begin{gathered}
\mathbf{E}^{\mathbf{P} 2}+\mathbf{P M g P}_{\mathbf{r}} \stackrel{\frac{1}{2} k_{\text {ex3 }}}{\longrightarrow} \mathbf{F}^{\mathbf{P 4}}{ }_{\mathbf{s}}+\mathbf{F}^{\mathbf{P 4}}{ }_{\mathbf{r}} \\
\mathbf{F}^{\mathbf{P 4}}{ }_{\mathbf{s}} \stackrel{\frac{1}{2} k_{\text {act3 }}}{\longrightarrow} \operatorname{PMgP}_{\mathbf{s}}+\text { help25 } \\
\mathbf{F}^{\mathbf{P 4}}{ }_{\mathbf{s}}+\text { help } 25 \stackrel{k_{\text {help }}}{\longrightarrow} \mathbf{E}^{\mathbf{P 2}} \mathbf{s}
\end{gathered}
$$




\subsubsection{Modeling of CCG with $\mathrm{Cp}_{2}^{*} \mathrm{ZrCl}_{2}, \mathrm{BzMgBz}$ and Styrene- $d 8$}

The parameter estimation was performed manually because the strong dependencies between the single reactions which would cause the automatic parameter estimation to run into problems. To determine possible influences of the additional monomer on the equilibrium and alkylation reaction, the experiments are analyzed similar to the monomer-free system and compared with the previously determined data shown in chapter 4.3.1. Illustrated in Table 5.1 are the determined parameters. The equilibrium

Table 5.1 Comparison of the kinetic parameter derived via the NMR-experiments at $70^{\circ} \mathrm{C}$ in the absence and in the presence of momomer for CCG activation. The results are the mean values of all experiments. See Table 4.3 on page 52 and 5.4 on page 108 for experimental details.

\begin{tabular}{c|c|c|l} 
coefficients & monomer free & monomer present & unit \\
\hline$K_{1, \mathrm{Zr}}$ & $23 \pm 6$ & $16 \pm 13$ & $10^{2} \mathrm{~mol} \mathrm{~L}^{-1}$ \\
$K_{2, \mathrm{Zr}}$ & $89 \pm 20$ & $54 \pm 31$ & $10^{2} \mathrm{~mol} \mathrm{~L}^{-1}$ \\
$k_{\text {alkyl1 }}$ & $5.8 \pm 2.2$ & $2.7 \pm 0.6$ & $10^{-4} \mathrm{~s}^{-1}$ \\
$k_{\text {alkyl2 }}$ & $3 \pm 1$ & $1.7 \pm 1.0$ & $10^{-6} \mathrm{~s}^{-1}$
\end{tabular}

constants in presence of styrene-d8 are slightly below the monomer-free ones but a higher uncertainty is found as well. While the equilibrium constants in presence of DBE were unaffected, this result is not found in presence of monomer. The alkylation rate coefficient is lowered as well, but the effect is much weaker as in presence of DBE, where similar concentration lead to a decrease by one order of magnitude (cf. Table 4.6 on page 66).

For modeling of the measured experiments the individually determined data was applied. In case that the equilibrium constant could not be determined, the associated values of the monomer-free system were used. In the previous shown experiments the exact rate coefficients for the equilibrium reactions could not be determined and only the ratio was determined (see Table 4.3 on page 52). Additional parameters were introduced and the starting parameters guessed while parameters like $k_{\text {help }}$ were chosen to be very high. To confirm the approximations and the calculations with virtual species the sum of all zirconium complex concentrations was calculated over time. The result is depicted in Figure 5.2. The virtual species with a double, three or fourfold concentration are divided by 2, 3 or 4 to gain the real zirconium concentration. The constant concentration level clearly shows that the assumptions and intermediates applied in the model have no consequences on the summarized zirconium concentration. The small scattering is within the chosen accuracy for processing time.

Starting from the individual equilibrium constants, the parameters were optimized to 


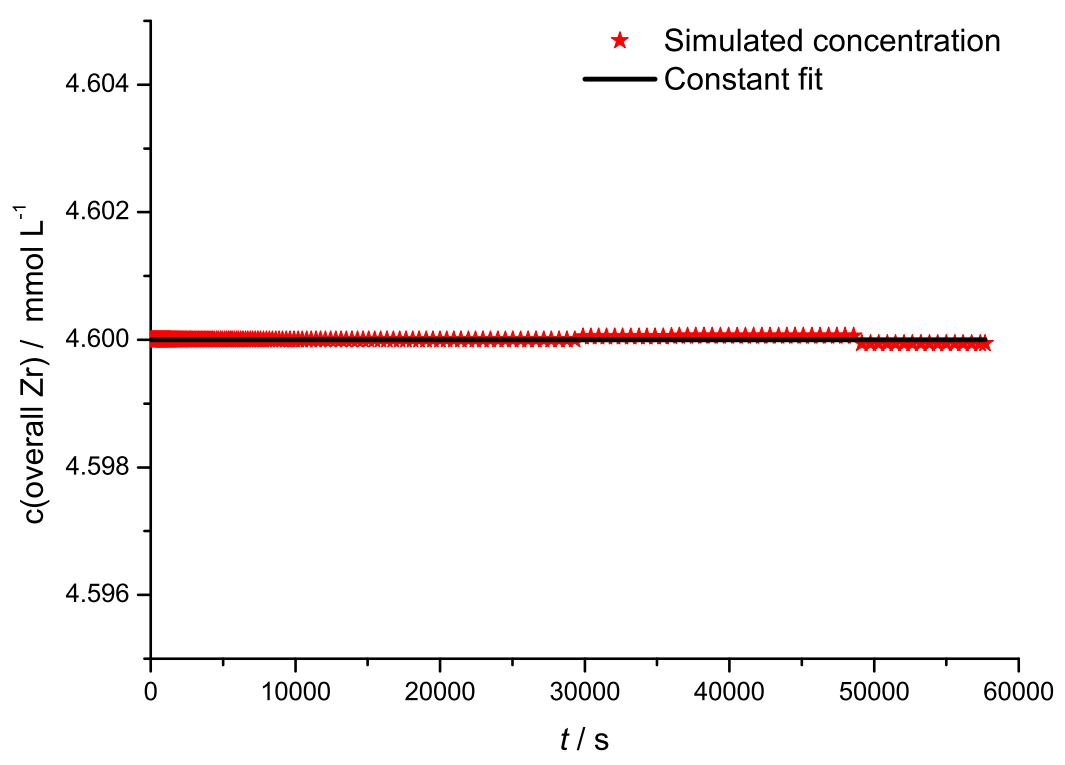

Figure 5.2: Overall zirconium complex concentration for simulation with ${ }^{c} \mathrm{Cp}^{*}\left(2 \mathrm{ZrCl}_{2}\right)=4.6 \mathrm{mmol} \mathrm{L}^{-1}$ $c(\mathrm{BzMgBz})=92 \mathrm{mmol} \mathrm{L}^{-1} c($ styrene- $d 8)=3.5 \mathrm{~mol} \mathrm{~L}^{-1}$ based on the parameters given in Table 5.2.

fit the concentrations of $\mathbf{B}, \mathbf{C}$ and $\mathbf{D}$. The concentration of $\mathbf{B}$ is mainly dependent on the first equilibrium involving $k_{\mathrm{ex} 0}$ and $k_{\text {acto }}$. The concentration profile of $\mathbf{C}$ was found to be significantly influenced by the coefficients $k_{\mathrm{ex} 1}$ and $k_{\mathrm{ex} 2}$. They are responsible for the slow formation of $\mathbf{C}$ in the beginning and the stable concentration with proceeding time. By increasing the order of magnitude of these rate coefficients at a constant ratio $\left(K_{1}\right.$, $K_{2}$ ), the concentration level of $\mathbf{C}$ is unaffected. Only a slight lowering of the magnitude significantly increases the rate of monomer conversion. It was found that for values below 1.5 in dependence of the $\mathrm{BzMgBz}$ concentration the concentration of $\mathrm{C}$ yields a maximum at short reaction time. This theoretical effect was however, not found in the actual experiments monitored by NMR. The formation of species $\mathbf{D}$ is slower than the one of $\mathbf{C}$ via alkylation. In this case, the polymer chain activation is the preferred reaction which leads to an increase in monomer conversion. With increasing magnitude of $k_{\text {ex1 }}$ and $k_{\mathrm{ex} 2}$ values, an increasing inhibition phase is found for monomer conversion and only values between $1.5 \mathrm{~L} \mathrm{~mol}^{-1} \mathrm{~s}^{-1}$ to $2.0 \mathrm{~L} \mathrm{~mol}^{-1} \mathrm{~s}^{-1}$ lead to a good representation of the different experiments (cf. Table 5.4). Especially in the case of the long term experiments these values became significant (iii)). The next step was the optimization of the monomer conversion with regard towards the MMD. $k_{\mathrm{add} 1}, k_{\mathrm{add} 2}, k_{\mathrm{p} 1}$ and $k_{\mathrm{p}}$ are mainly responsible for this process. It was found that the important fit parameters in concern of conversion are $k_{\mathrm{p} 1}$ and $k_{\mathrm{p}}$. The equilibrium constant of the monomer addition should not be over 2.5 otherwise a significantly lower concentration of $\mathbf{D}$ is yielded which could be excluded 
with look on the overall Zr integral determined by NMR (see Figure 5.3). A constant value is achieved which shows only slight scattering in agreement with the reference signal of toluene/toluene- $d 8$. Please note, that the equilibrium of monomer addition is linked with the rate coefficients $k_{\mathrm{p} 1}$ and $k_{\mathrm{p}}$.

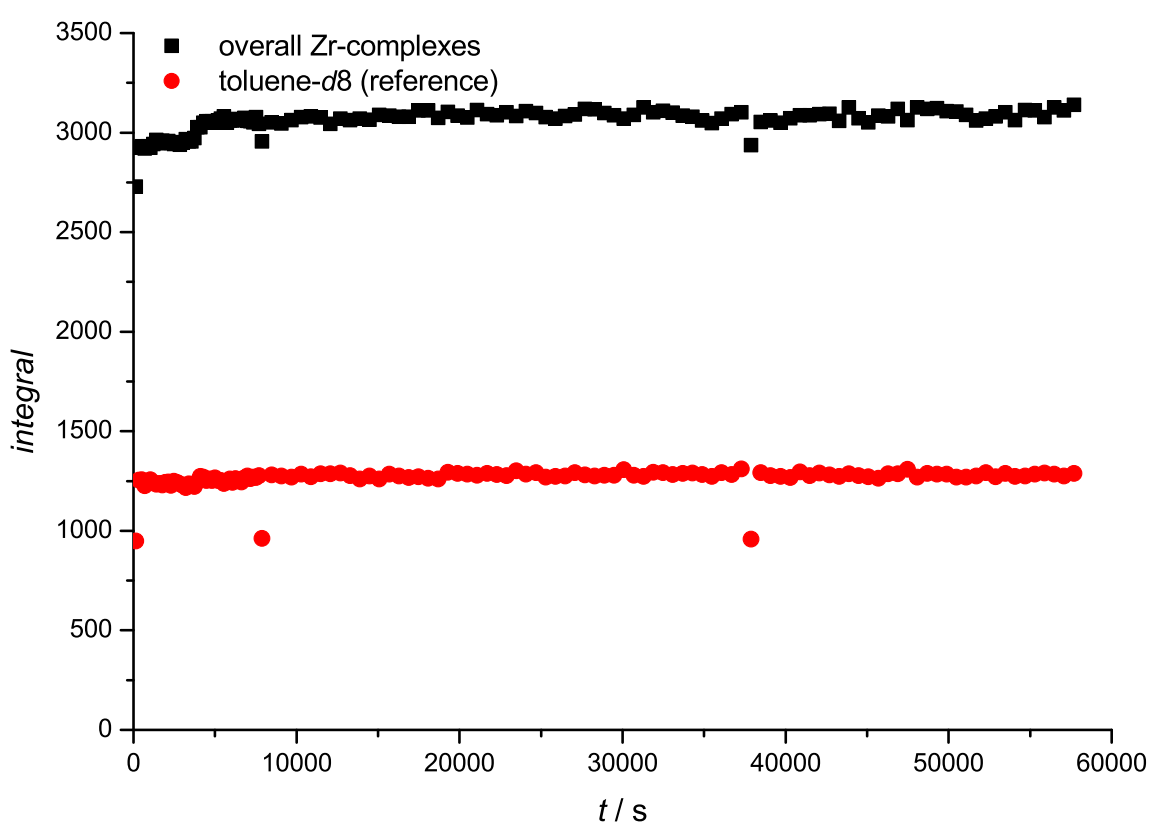

Figure 5.3: Determined combined integral of all the NMR signals of zirconium complexes shown together with the reference integral of toluene/toluene- $d 8$.

The equilibrium constant of the monomer addition was fixed for the modeling of all measurements. This approximation takes into account that the measurement of the monomer coordinated complexes is difficult and often only possible at very low temperatures. 270,271,290,291] The values of $k_{\mathrm{p} 1}$ and $k_{\mathrm{p}}$ were fitted with respect to the maximum of the MMD and the conversion vs. time profile. The difference in $k_{\text {act1 }}$ and $k_{\text {act2 }}$ is the result of the combination of four experiments. The fit of the MMD worked well with equal values for experiment $\mathrm{i}$ and ii where the concentration of $\mathbf{A}$ is high, while it was predicted too broad for the lower concentration of $\mathbf{A}$ in experiment iii and iv (see Table 5.4). Only assuming a difference by one order of magnitude from $k_{\text {act } 1}$ to $k_{\text {act2 }}$ led to similar results between all experiments. The concentration profile of $\mathrm{BzMgBz}$ is influenced by the loss of signal quality during the measurements and an overlay with the signal of species F. Therefore the profile of $\mathrm{BzMgBz}$ was taken into account for the last optimization steps. To deconvolute the overlay, the overall integral was calculated and the peaks analyzed with the program fityk ${ }^{[292]}$ to determine the fraction of $\mathrm{BzMgBz}$ and $\mathbf{F}$ from the overall signal. The profile of BzMgBz suggests only a small consumption at high reaction times. The termination reaction could not be monitored by this method, because 
the resulting double bond in the polymer chain would is fully deuterated. Based on values and a strong kinetic isotope effect found in literature ${ }^{[277} \mathrm{a} k_{\mathrm{t}}$ value of 0 is assumed. An overview of the best fits of measurement i) is depicted in Figure 5.4 and 5.5 and the determined parameters are summarized in Table 5.2 .

The model gives good agreement with the concentration vs. time profiles of the

Table 5.2 Parameters estimated via Predici ${ }^{\mathrm{TM}}$ modeling of CCG polymerization at $70^{\circ} \mathrm{C}$, $\mathrm{c}\left(\mathrm{Cp}_{2}{ }_{2} \mathrm{ZrCl}_{2}\right)=0.0046 \mathrm{~mol} \mathrm{~L}^{-1} c(\mathrm{BzMgBz})=0.092 \mathrm{~mol} \mathrm{~L}^{-1} \mathrm{c}($ styrene- $d 8)=3.5 \mathrm{~mol} \mathrm{~L}^{-1}$ in toluene- $d 8$.

\begin{tabular}{|c|c|c|c|}
\hline coefficients & value & unit & determination \\
\hline$k_{\mathrm{ex} 0}$ & 5.5 & $\mathrm{~L} \mathrm{~mol}^{-1} \mathrm{~s}^{-1}$ & Modeling, $\mathrm{NMR}^{[\mathrm{a}]}$ \\
\hline$k_{\mathrm{ex} 1}$ & 1.8 & $\mathrm{~L} \mathrm{~mol}^{-1} \mathrm{~s}^{-1}$ & Modeling, NMR ${ }^{[\mathrm{a}]}$ \\
\hline$k_{\mathrm{ex} 2}$ & 18 & $\mathrm{~L} \mathrm{~mol}^{-1} \mathrm{~s}^{-1}$ & Modeling, $\mathrm{NMR}^{[\mathrm{a}]}$ \\
\hline$k_{\mathrm{act} 0}$ & 0.01 & $\mathrm{~s}^{-1}$ & Modeling, $\mathrm{NMR}^{[\mathrm{a}]}$ \\
\hline$k_{\text {act1 }}$ & 5.14 & $10^{-4} \mathrm{~s}^{-1}$ & Modeling, $\mathrm{NMR}^{[\mathrm{a}]}$ \\
\hline$k_{\text {act2 }}$ & 5.14 & $10^{-3} \mathrm{~s}^{-1}$ & Modeling, $\mathrm{NMR}^{[\mathrm{a}]}$ \\
\hline$k_{\text {alkyl1 }}$ & 3.1 & $10^{-4} \mathrm{~s}^{-1}$ & NMR \\
\hline$k_{\text {alkyl2 }}, k_{\text {alkyl3 }}$ & 2.6 & $10^{-6} \mathrm{~s}^{-1}$ & NMR \\
\hline$k_{\mathrm{p} 1}$ & $>1.0$ & $10^{-3} \mathrm{~s}^{-1}$ & Modeling \\
\hline$k_{\mathrm{p}}$ & $>0.18$ & $\mathrm{~s}^{-1}$ & Modeling \\
\hline$k_{\text {add1,add2 }} / k_{\text {frag1,frag2 }}$ & $<5 / 2$ & $\mathrm{~L} \mathrm{~mol}^{-1} \mathrm{~s}^{-1}$ & Modeling \\
\hline$k_{\mathrm{ex} 3} / k_{\mathrm{act} 3}$ & 10000 & $\mathrm{~L} \mathrm{~mol}^{-1} \mathrm{~s}^{-1}$ & Approximation ${ }^{[\mathrm{b}]}$ \\
\hline$k_{\text {Schlenk }}$ & 1000 & $\mathrm{~L} \mathrm{~mol}^{-1} \mathrm{~s}^{-1}$ & Approximation ${ }^{[\mathrm{c}]}$ \\
\hline$k_{\text {help }}$ & $10^{10}$ & $\mathrm{~L} \mathrm{~mol}^{-1} \mathrm{~s}^{-1}$ & Approximation $^{[\mathrm{c}]}$ \\
\hline
\end{tabular}

a) Modeling of rate coefficients with a constant ratio determined by NMR.

b) Approximation based on previous experiments (cf. Table 4.3).

c) General approximations (see Text for details).

determined species (cf. Figure 5.4 d, Figure 5.5 a-d) while small differences are found for species $\mathbf{D}$ and $\mathbf{F}$ (see Figure 5.5 c and d). D has a lower concentration than found experimentally while concentration $\mathbf{F}$ is higher which is the result of direct dependence between both complexes. In the experiment, it was impossible to determine the concentration of $\mathbf{F}$ during the first 9000 seconds. While the parameters work well, the MMD differs strongly (Figure 5.4 a)) yielding a remarkably smaller dispersity for the modeled data. For improved comparison with experimental SEC data, the modeled MMD has been band broadened ${ }^{[253]}$ with parameters determined for the applied SEC system (see 


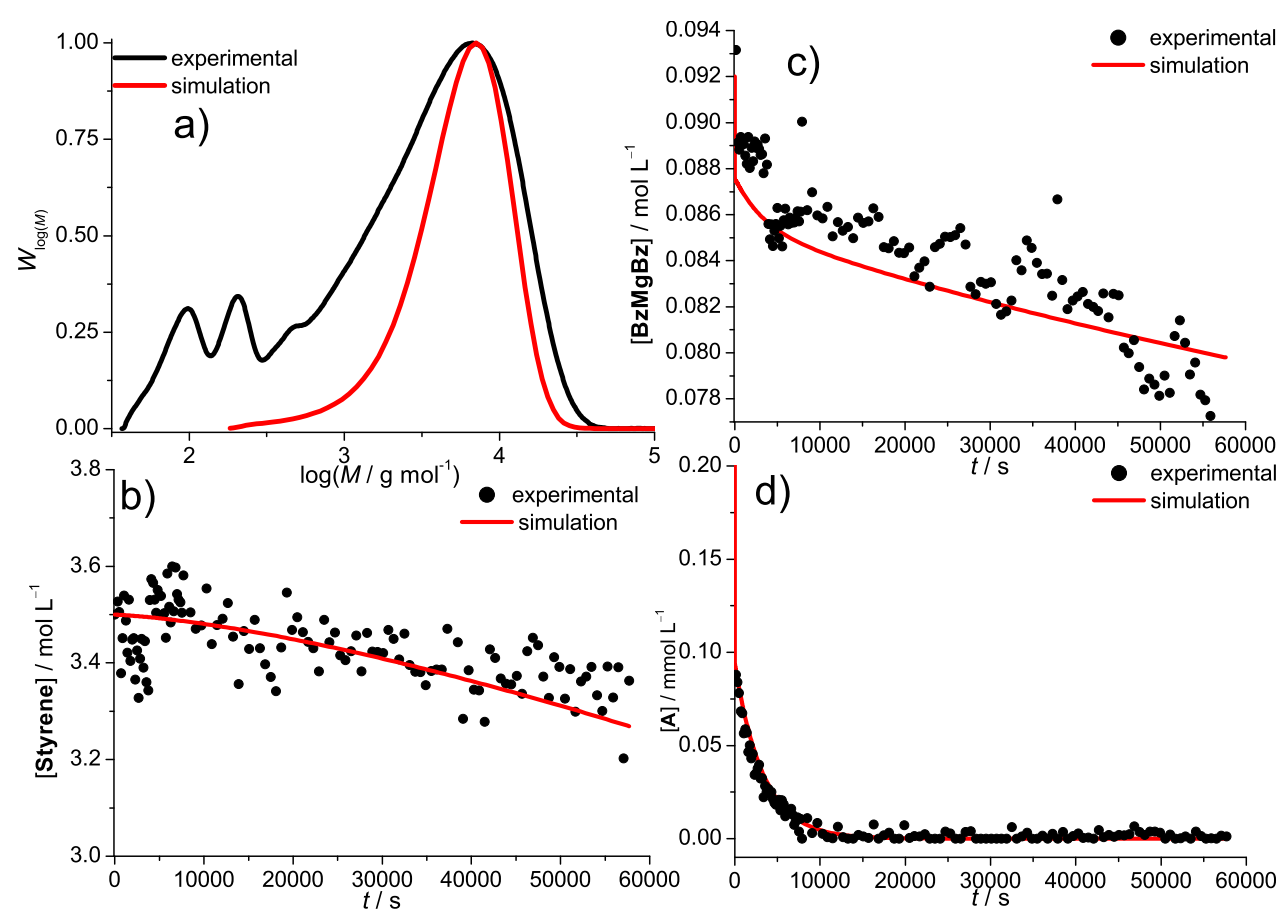

Figure 5.4: Comparison of experiment and simulation with the data shown in Table 5.2 a) MMD distribution; b) BzMgBz concentration vs. time profile; c) Styrene concentration vs. time profile and d) A concentration vs. time profile.
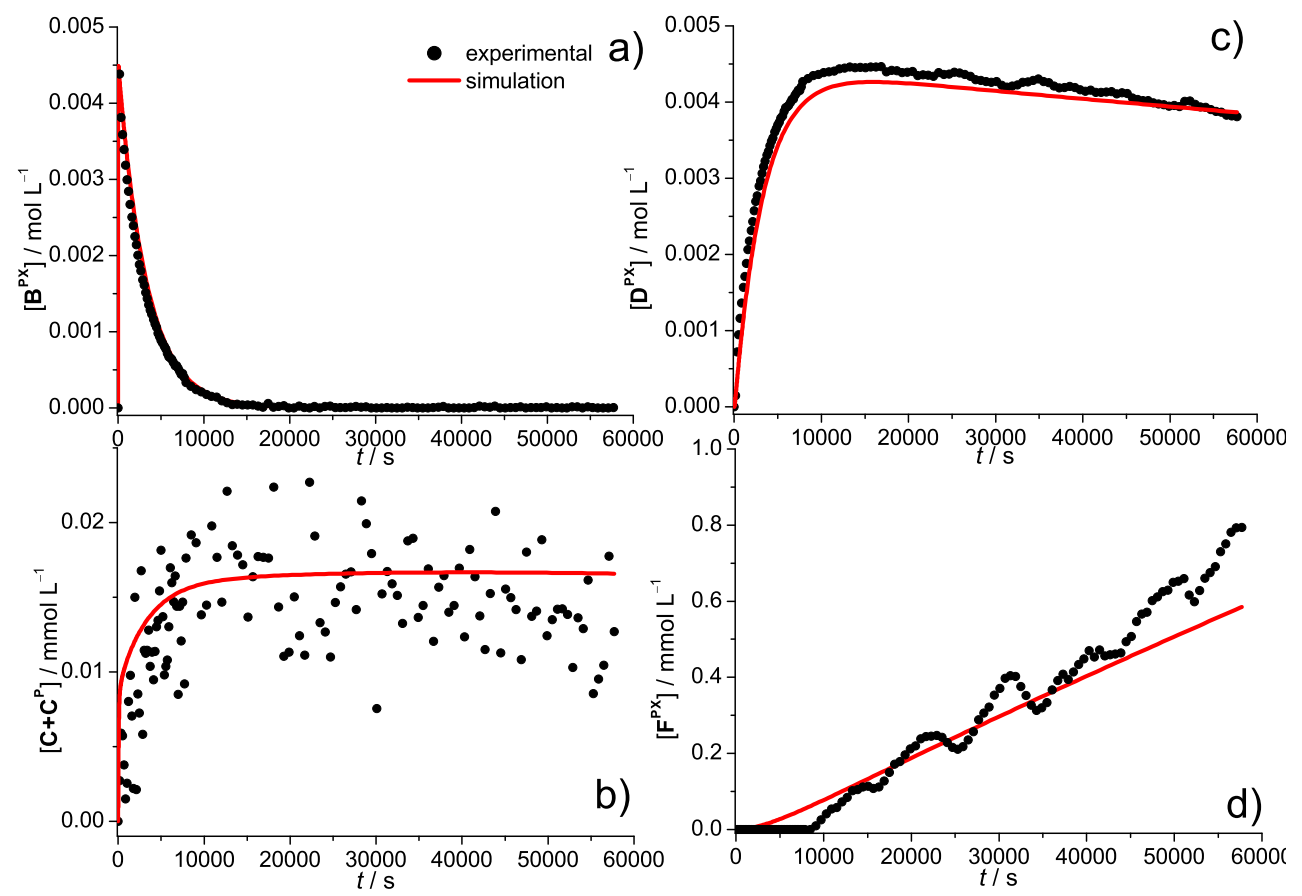

Figure 5.5: Comparison of experiment and simulation with the data shown in Table 5.2, a) $\mathbf{B}^{\mathbf{P}}$ concentration vs. time profile; b) $\mathbf{C}+\mathbf{C}^{\mathbf{P}}$ concentration vs. time profile; c) $\mathbf{D}^{\mathbf{P}}$ concentration vs. time profile and d) $\mathbf{F}^{\mathbf{P}}$ concentration vs. time profile. 
Experimental Section 9.1.3.2 on page 197). It was impossible to model the experimental dispersity and MMD by variation of the kinetic coefficients without running into higher differences on the concentration profiles. On a closer look, the coefficients $k_{\mathrm{p} 1}$ and $k_{\mathrm{p}}$ differ by three orders of magnitude, indicating a chain-length dependence for $k_{\mathrm{p}}$ similar to the previously presented Intermittent model ${ }^{[61,62]}$ and polymerization of 1-hexene with [rac- $\left.\left(\mathrm{C}_{2} \mathrm{H}_{4}(1 \text {-indenyl })_{2}\right) \mathrm{ZrMe}\right]\left[\mathrm{MeB}\left(\mathrm{C}_{6} \mathrm{~F}_{5}\right)_{3}\right]^{[158]}$ (See chapter 2.2.2 on page 14 and 2.2 .6 on page 18 for more details). ${ }^{[9]} \mathrm{A}$ difference was proposed for the first monomer addition, but their calculated MMD-curves show significantly differences from the experimental data, especially for high monomer concentration. Further investigations showed a difference of a factor 400. ${ }^{[159]}$ This effect is similar to the situation already included in the applied model. Based on this information, $k_{\mathrm{p}}$ was assumed to be chain-length dependent for further investigation even beyond the first initiation step. From first modelation it was assumed that a constant coefficient at high chain length is needed. The chain length dependence was simulated using an exponential function (Equation 5.97)

$$
k_{\mathrm{p}, i}=k_{\mathrm{p}, \infty}-k_{\mathrm{p}, \infty} \cdot \exp ^{-(a i)}
$$

This equation allows the description of a broad spectrum of possible shapes of the exponential function by variation of the parameter $a$, while yielding constant $k_{\mathrm{p}, \infty}$ values at high chain length (i). Exponential functions are typically found for CLD coefficients. [293,294] The best fit for the polymerization data is shown in Figure 5.6 and the applied parameters are listed in Table 5.3 .

Table 5.3 Parameter for chain-length dependent simulation of experiment i).

\begin{tabular}{c|c|c}
$k_{\mathrm{p}, \infty} / \mathrm{s}^{-1}$ & $a$ & $k_{\mathrm{p}, i} \times 10^{-3} \mathrm{~s}^{-1}$ \\
\hline 0.31 & 0.028 & 1.75
\end{tabular}

Such chain-length dependence leads to a good agreement for all experimentally derived complexes (see Figure 5.7 and 5.8) and in addition the MMD is also well described. Differences only occur at low molecular mass in the modeled SEC curve. These differences were caused by system signals caused by the inhibitor (SEC eluent). With samples measured in absence of a dissolved polymer, these signals could by verified to be system inherent.

The model is thus capable of describing the polymerization process and may be used to determine the associated rate coefficients. The results of additional NMR-based modeling are summarized in Table 5.4 and the comparison of experiment and simulation for ii) and iv) are shown in the appendix (see A.1 on page 214). 


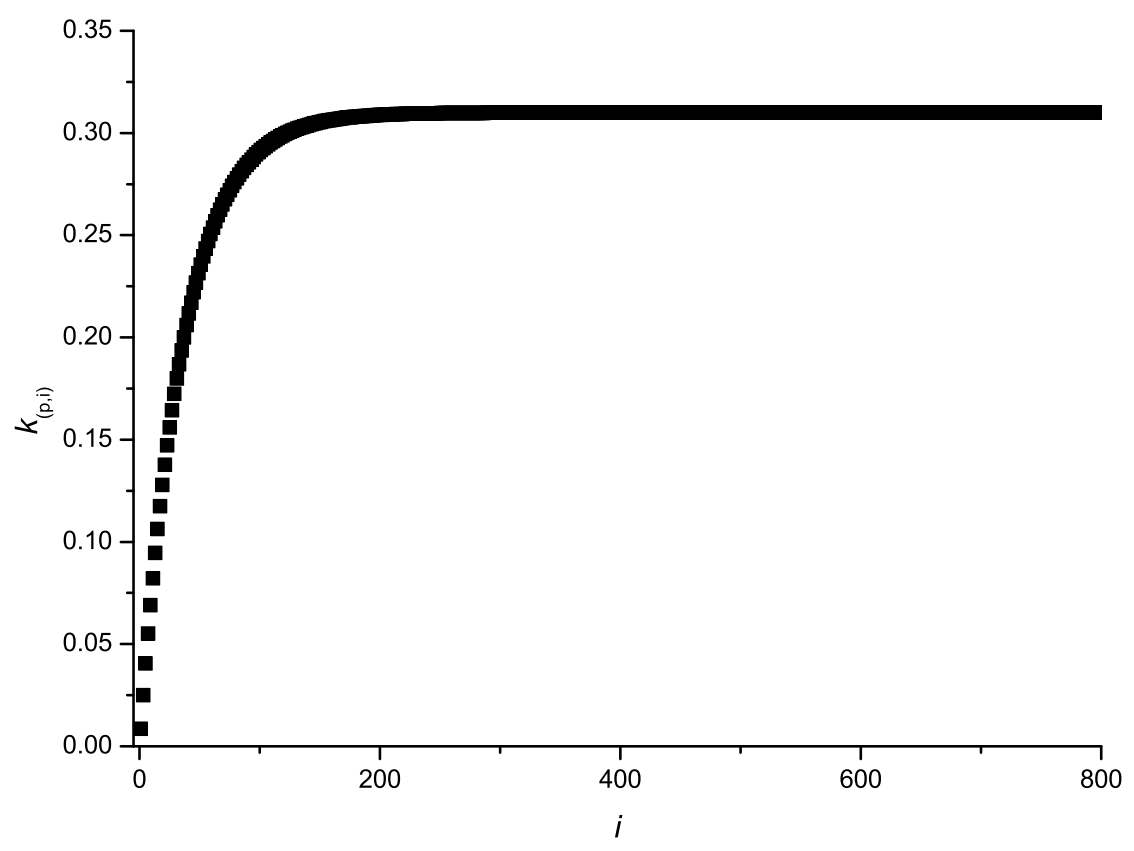

Figure 5.6: Determination of $k_{\mathrm{p}, \mathrm{i}}$ in dependence of the chain length $i$ with $a=0.028$ and $k_{\mathrm{p}, \infty}=0.31 \mathrm{~s}^{-1}$ by Equation (5.97).
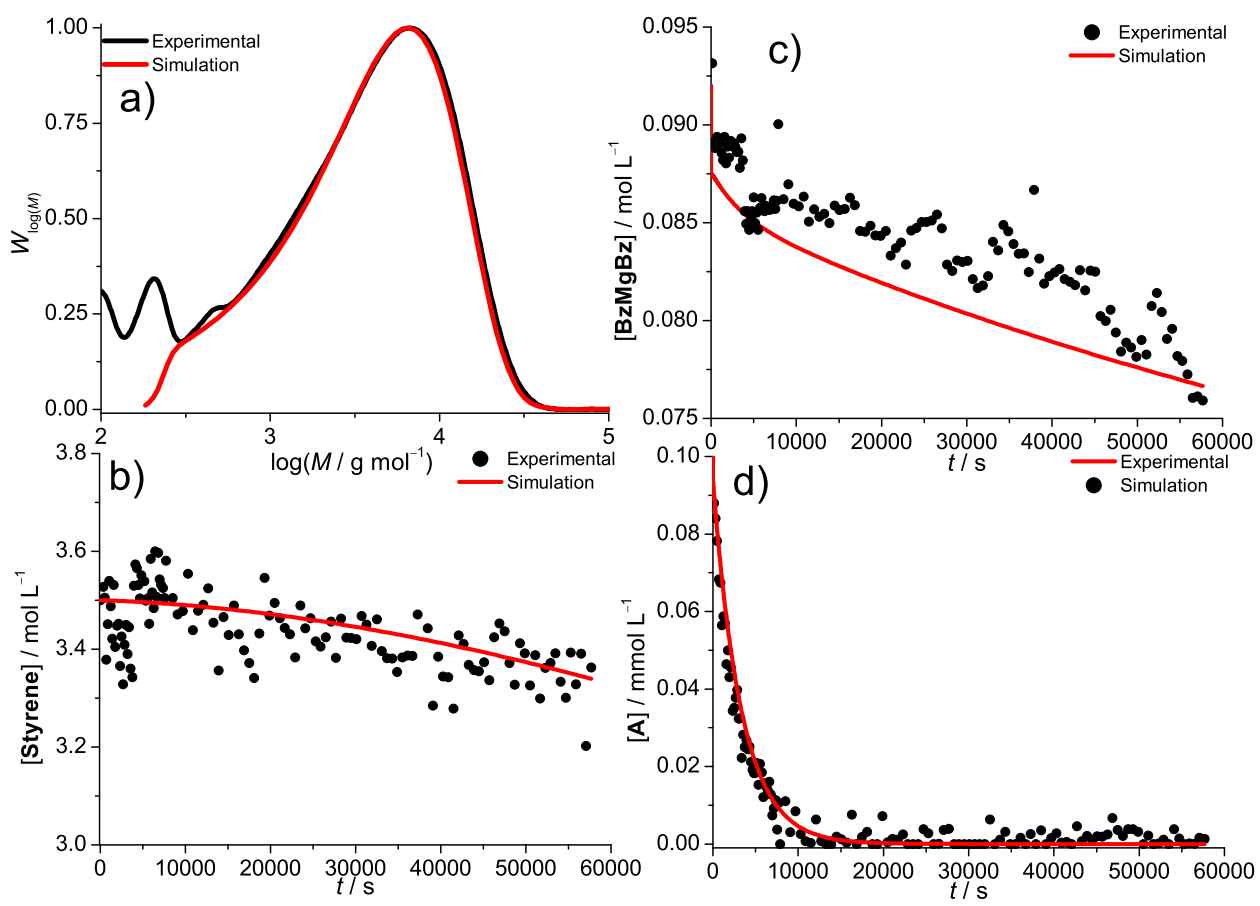

Figure 5.7: Comparison of experiment and simulation with the $k_{\mathrm{p}}$ values from Table 5.3 and the residual coefficients are taken from Table 5.2. a) MMD distribution; b) BzMgBz concentration vs. time profile; c) Styrene concentration vs. time profile and d) A concentration vs. time profile. 


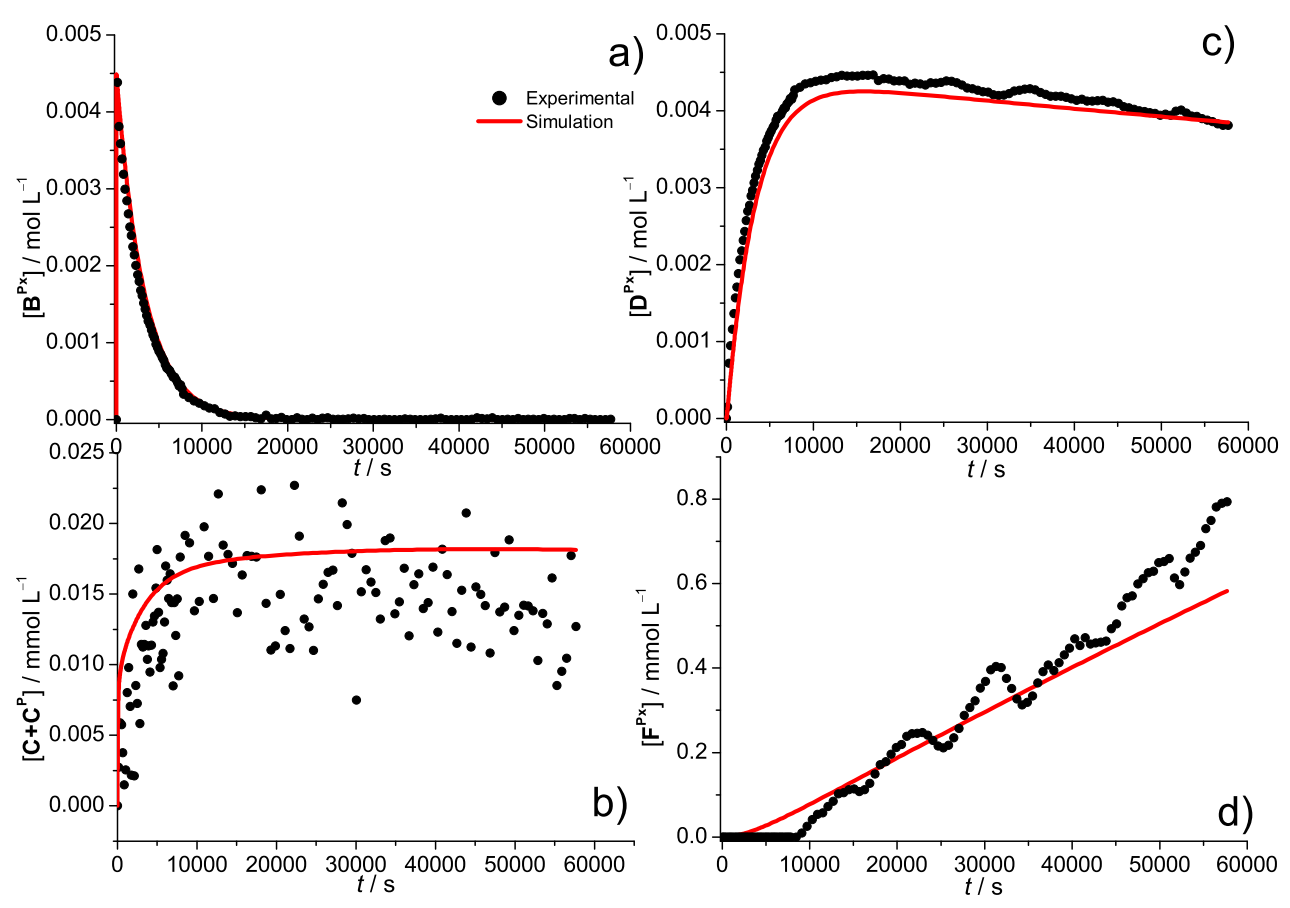

Figure 5.8: Comparison of experiment and simulation with the $k_{\mathrm{p}}$ values from Table 5.3 and the residual coefficients are taken from Table 5.2 a) $\mathbf{B}^{\mathbf{P}}$ concentration vs. time profile; b) $\mathbf{C}+\mathbf{C}^{\mathbf{P}}$ concentration vs. time profile; c) $\mathbf{D}^{\mathbf{P}}$ concentration vs. time profile and d) $\mathbf{F}^{\mathbf{P}}$ concentration vs. time profile.

When using the same starting concentration of $\mathbf{A}$ the experimental results differ only slightly. The equilibrium constants were determined higher for lower starting concentrations which results from the increased uncertainty of the weaker signals of $\mathbf{A}$ and C. In the case of the lower starting concentration the equilibrium constants seem to be dependent of the reaction time or conversion. The monomer addition equilibrium shows a slightly higher constant at lower concentration of $\mathbf{C}$. The fitted $k_{\mathrm{p}}$ and $k_{\mathrm{p} 1}$ values scatter slightly and the parameters $i$ and $i i$ are although determined well. This finding indicates that the main influence on the shape of the time vs. conversion profile and on further concentration profiles is highly dependent of the value of $K_{1, \mathrm{Zr}}$ and $K_{2, \mathrm{Zr}}$. This assumption is supported by the conversion vs. time profile of the long term measurement iii) where a major difference is found going to higher reaction times. The rate of monomer conversion is lowering with proceeding reaction time. The effect is depicted in Figure 5.9 along with the determined values for the equilibrium constant. In the first 60000 seconds the value is constant while it increases with larger conversion. This may have several reasons and the most successful description was to introduce a higher equilibrium constant for $K_{2, \mathrm{ZrP}}$. The results show good agreement with the experimental data (see Figure 5.10 and 5.11) 
Table 5.4 Parameter estimated via Predici ${ }^{\mathrm{TM}}$ modeling of CCG polymerization at $70^{\circ} \mathrm{C}$, (i) $c\left(\mathrm{Cp}^{*}{ }_{2} \mathrm{ZrCl}_{2}\right)=0.0046 \mathrm{~mol} \mathrm{~L}^{-1} c(\mathrm{BzMgBz})=0.092 \mathrm{~mol} \mathrm{~L}^{-1}$, (ii) $c\left(\mathrm{Cp}^{*}{ }_{2} \mathrm{ZrCl}_{2}\right)=0.0046 \mathrm{~mol} \mathrm{~L}^{-1}$ $c(\mathrm{BzMgBz})=0.081 \mathrm{~mol} \mathrm{~L}^{-1}$ (iii) $c\left(\mathrm{Cp}_{2}{ }_{2} \mathrm{ZrCl}_{2}\right)=0.0023 \mathrm{~mol} \mathrm{~L}^{-1} c(\mathrm{BzMgBz})=0.037 \mathrm{~mol} \mathrm{~L}^{-1}$ (iv) $c\left(\mathrm{Cp}_{2}{ }_{2} \mathrm{ZrCl}_{2}\right)=0.0023 \mathrm{~mol} \mathrm{~L}^{-1} c(\mathrm{BzMgBz})=0.040 \mathrm{~mol} \mathrm{~L}^{-1}$ in toluene- $d 8$ and $c($ styrene$d 8)=3.5 \mathrm{~mol} \mathrm{~L}^{-1} \cdot{ }^{*}=$ constant for all experiments.

\begin{tabular}{|c|c|c|c|c|c|c|}
\hline coefficients & (i) & (ii) & (iii) & (vi) & mean value & unit \\
\hline reaction time: & 16 & 17 & 60.2 & 10.4 & mean value & unit \\
\hline$k_{\mathrm{ex} 0}$ & 5.5 & 7.4 & 35 & 20 & $16 \pm 13$ & $\mathrm{~L} \mathrm{~mol}^{-1} \mathrm{~s}^{-1}$ \\
\hline$k_{\mathrm{ex} 1}$ & 1.8 & 1.8 & 1.8 & 1.8 & $1.8^{*}$ & $\mathrm{~L} \mathrm{~mol}^{-1} \mathrm{~s}^{-1}$ \\
\hline$k_{\mathrm{ex} 2}$ & 18 & 18 & $80^{[\mathrm{a}]}$ & 18 & $34 \pm 27$ & $\mathrm{~L} \mathrm{~mol}^{-1} \mathrm{~s}^{-1}$ \\
\hline$k_{\text {act0 }}$ & 0.01 & 0.01 & 0.01 & 0.01 & $0.01^{*}$ & $\mathrm{~s}^{-1}$ \\
\hline$k_{\text {act1 }}$ & 5.1 & 4.3 & 2.0 & 2.4 & $3.5 \pm 1.6$ & $10^{-4} \mathrm{~s}^{-1}$ \\
\hline$k_{\text {act2 }}$ & 5.1 & 4.3 & 2.0 & 2.4 & $3.5 \pm 1.6$ & $10^{-3} \mathrm{~s}^{-1}$ \\
\hline$k_{\mathrm{alkyl} 1}$ & 3.1 & 3.5 & 1.8 & 2.5 & $2.7 \pm 0.6$ & $10^{-4} \mathrm{~s}^{-1}$ \\
\hline$k_{\text {alkyl2 }}, k_{\text {alkyl3 }}$ & 2.6 & 2.7 & 0.7 & 0.8 & $1.7 \pm 1.0$ & $10^{-6} \mathrm{~s}^{-1}$ \\
\hline$k_{\mathrm{p} 1}$ & 1.75 & 1.10 & 3.75 & 1.0 & $>1.9 \pm 1.1$ & $10^{-3} \mathrm{~s}^{-1}$ \\
\hline$k_{\mathrm{p}, \infty}$ & 0.31 & 0.26 & 0.56 & 0.33 & $>0.36 \pm 0.11$ & $\mathrm{~s}^{-1}$ \\
\hline$a$ & 0.028 & 0.031 & 0.026 & 0.12 & $0.05 \pm 0.04$ & \\
\hline$k_{\mathrm{add}} / k_{\mathrm{frag}}$ & & - & - & 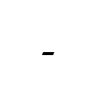 & $<5 / 2$ & $\mathrm{~L} \mathrm{~mol}^{-1} \mathrm{~s}^{-1}$ \\
\hline
\end{tabular}

a) Long term experiment, see Figure 5.9 and Text for details. 


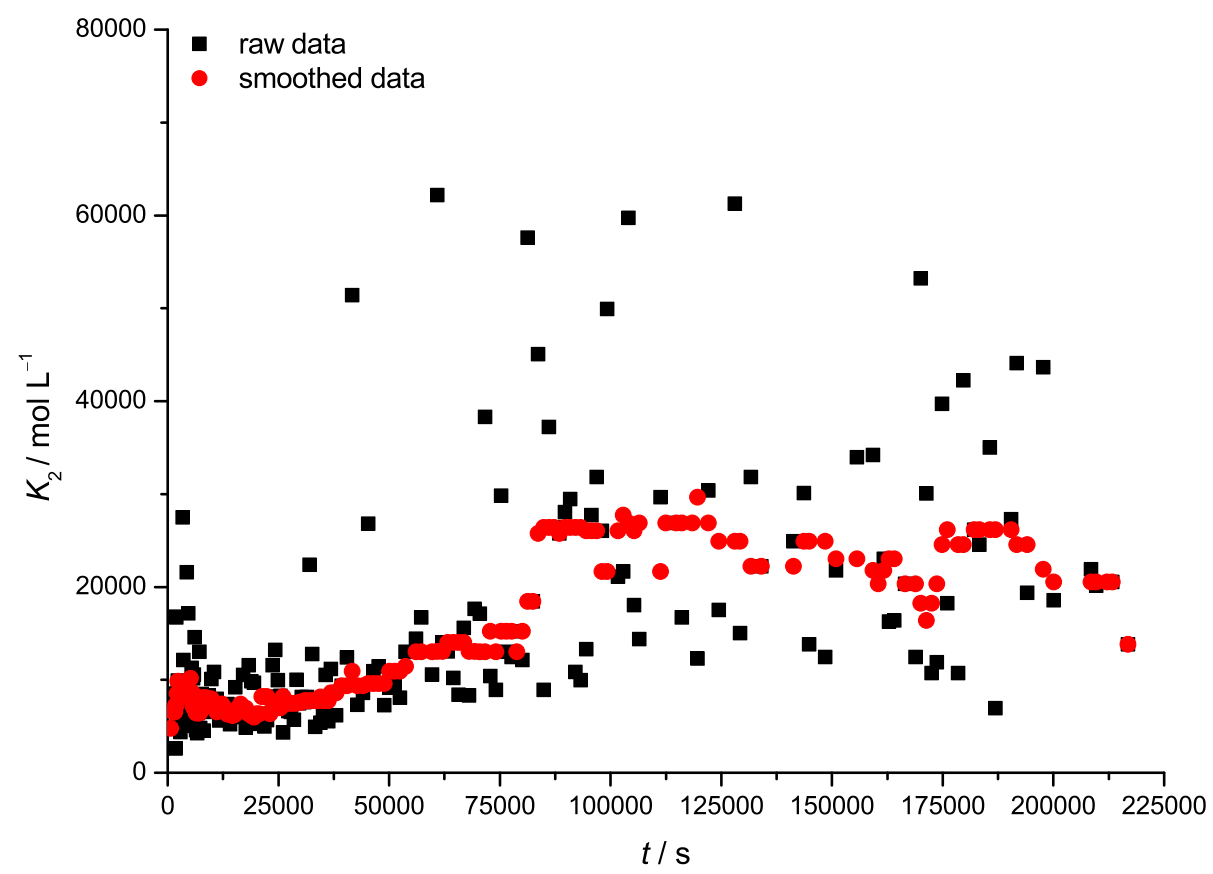

Figure 5.9: Equilibrium constant $K_{\mathrm{Zr}, 2}$ plotted versus the reaction time.

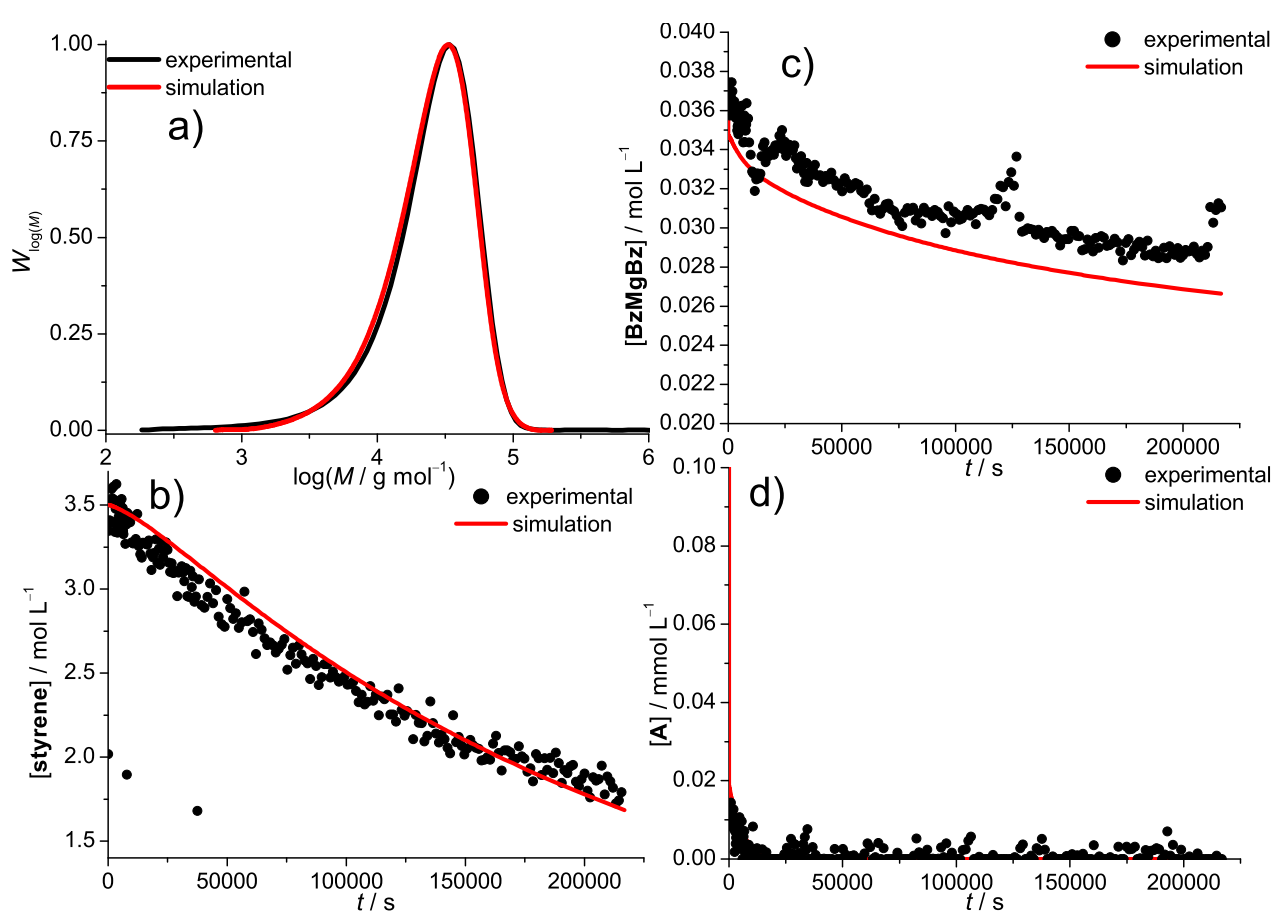

Figure 5.10: Comparison of experiment and simulation of the data shown in Table 5.4, experiment iii). a) MMD distribution; b) BzMgBz concentration vs. time profile; c) Styrene concentration vs. time profile and d) A concentration vs. time profile. 

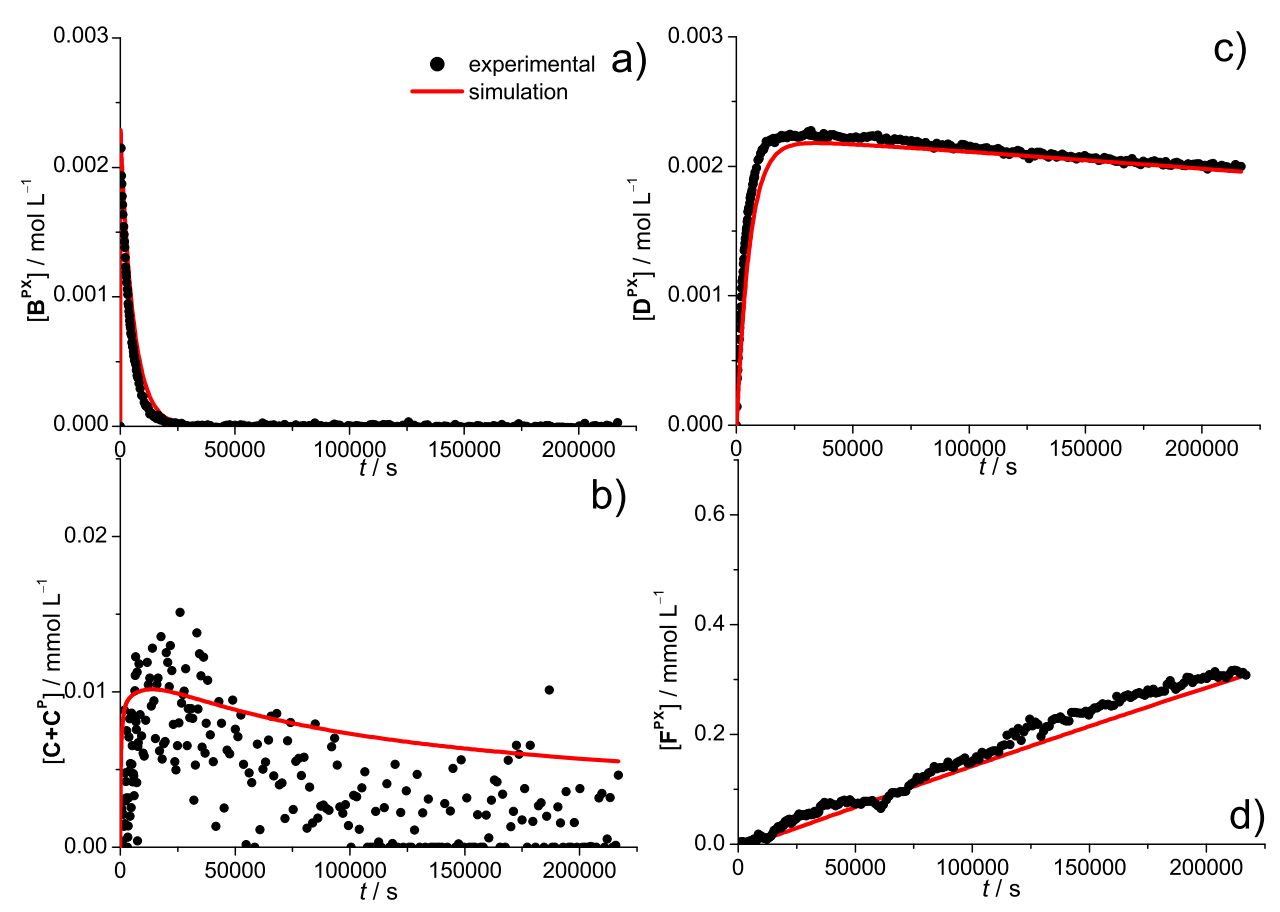

Figure 5.11: Comparison of experiment and simulation with the data shown in Table 5.4. a) $\mathbf{B}^{\mathbf{P}}$ concentration vs. time profile; b) $\mathbf{C}+\mathbf{C}^{\mathbf{P}}$ concentration vs. time profile; c) $\mathbf{D}^{\mathbf{P}}$ concentration vs. time profile and d) $\mathbf{F}^{\mathbf{P}}$ concentration vs. time profile.

This result is found in different polymerization systems by UV/Vis and IR spectroscopic determination as well. In the next section the influences of this finding will be discussed thoroughly by simulation of the different experiments.

In conclusion, all these results show good agreement of the model simulation and the experimental data, but one notable question remains: How well does this system control the polymerisation? While the polymerization process is described correctly, is it also controlling the molar mass, or are the exchange reactions rather side reactions? Therefore, a $\bar{M}_{\mathrm{n}}$ versus conversion plot (see Figure 5.12) is performed for the simulated data of experiment i. After an activation phase the plot shows a linear linear increase. For better understanding, the $\bar{M}_{\mathrm{n}}$ and dispersity time profile are also included. After full activation of the catalyst (around 12000 seconds), the $\bar{M}_{\mathrm{n}}$ begins to increase linearly while the dispersity decreases, resulting from the exchange of polymer chains. Long polymer chains were transferred from the catalyst to magnesium and vice versa. The chosen model system is unsuitable for fast exchange reactions and therefore no good control over the molar mass is achieved during polymerization. 

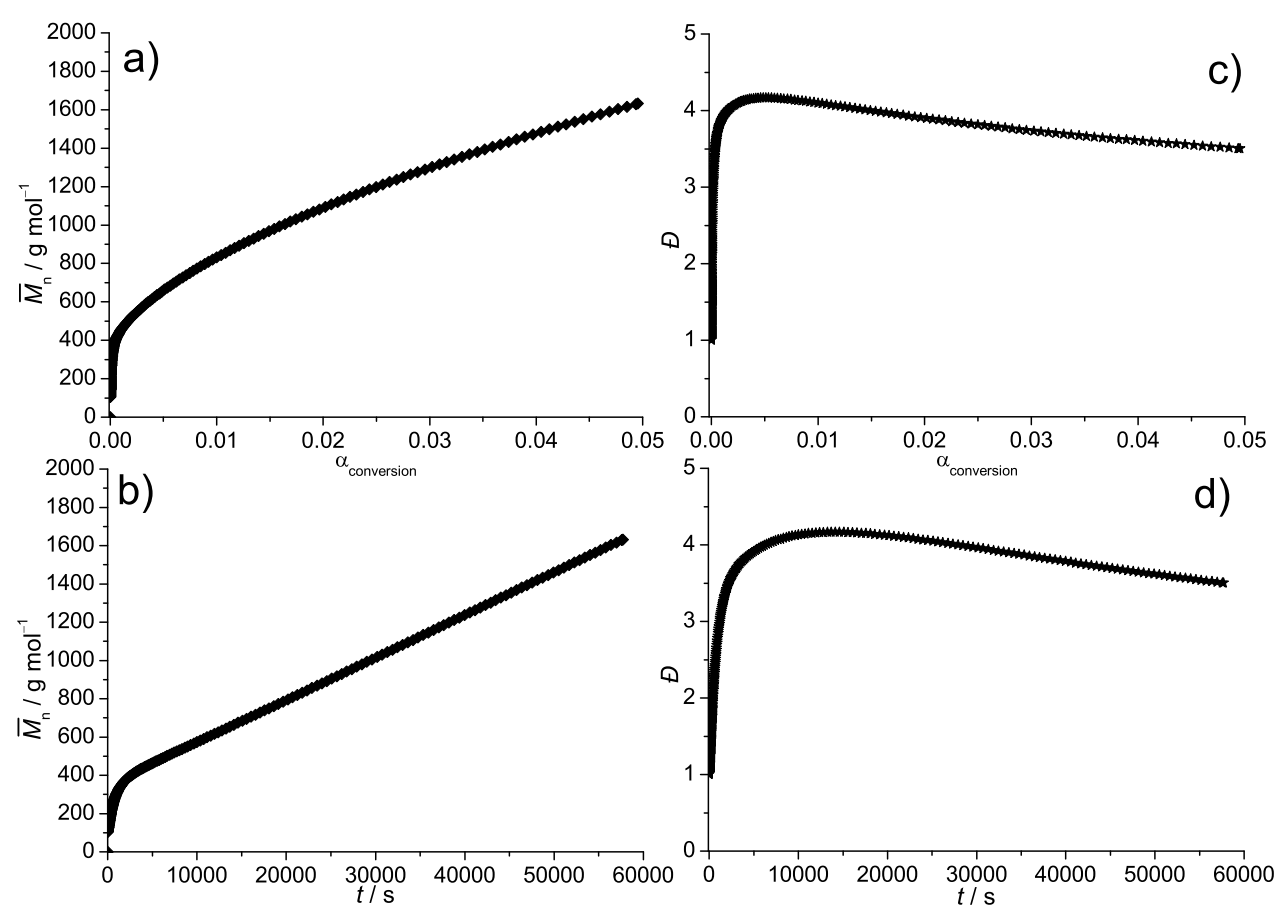

Figure 5.12: a) $\bar{M}_{\mathrm{n}}$ versus conversion plot; b) $\bar{M}_{\mathrm{n}}$ versus time plot; c) dispersity versus conversion plot and d) dispersity versus time plot.

\subsubsection{Simulation of Influences on CCG of $\mathrm{Cp}_{2}^{*} \mathrm{ZrCl}_{2}$ and $\mathrm{BzMgBz}$ with Styrene-d8}

With the model at hand the individual influences of the coefficients towards the polymerization process were studied by parameter variation. The MMD data was not band broadened for this purpose, such as to show effects only caused by simulation. To compare the results for each simulation series at least the MMD and dispersity and $\bar{M}_{\mathrm{n}}$ versus conversion plots are shown. As a second aspect, the consumption of the co-catalyst is depicted or another relevant concentration profile. The simulations shown in this section are the basis for the discussion on the different modeling approaches shown in the following chapter (see 6 on page 135).

It was chosen to begin with the chain-length dependence of the monomer addition step. The influence of the factor $a$ in equation 5.97 was determined with the data of experiment i) for better comparison with graphs already shown. The factor was varied around the starting value. The variation of $a$ has several interesting influences. An increase in $a$ yields an increasing monomer conversion (see Figure 5.13 a)) in agreement with the development of $k_{\mathrm{p}, i}$ due to faster approach for $k_{\mathrm{p}, \infty}$ (see Figure $5.13 \mathrm{~b}$ )). The development 
of $k_{\mathrm{p}, \mathrm{i}}$ influences the $\bar{M}_{\mathrm{n}}$-values shown by the increases before leveling off, yielding a lower dispersity (see Figure 5.13 c)). At low $a$, broad MMDs are obtained (see Figure 5.13 d)). With increasing $a$, the maximum shifts to higher molecular mass while the curve sharpens. Still, a notable amount of low molecular mass polymer is obtained.
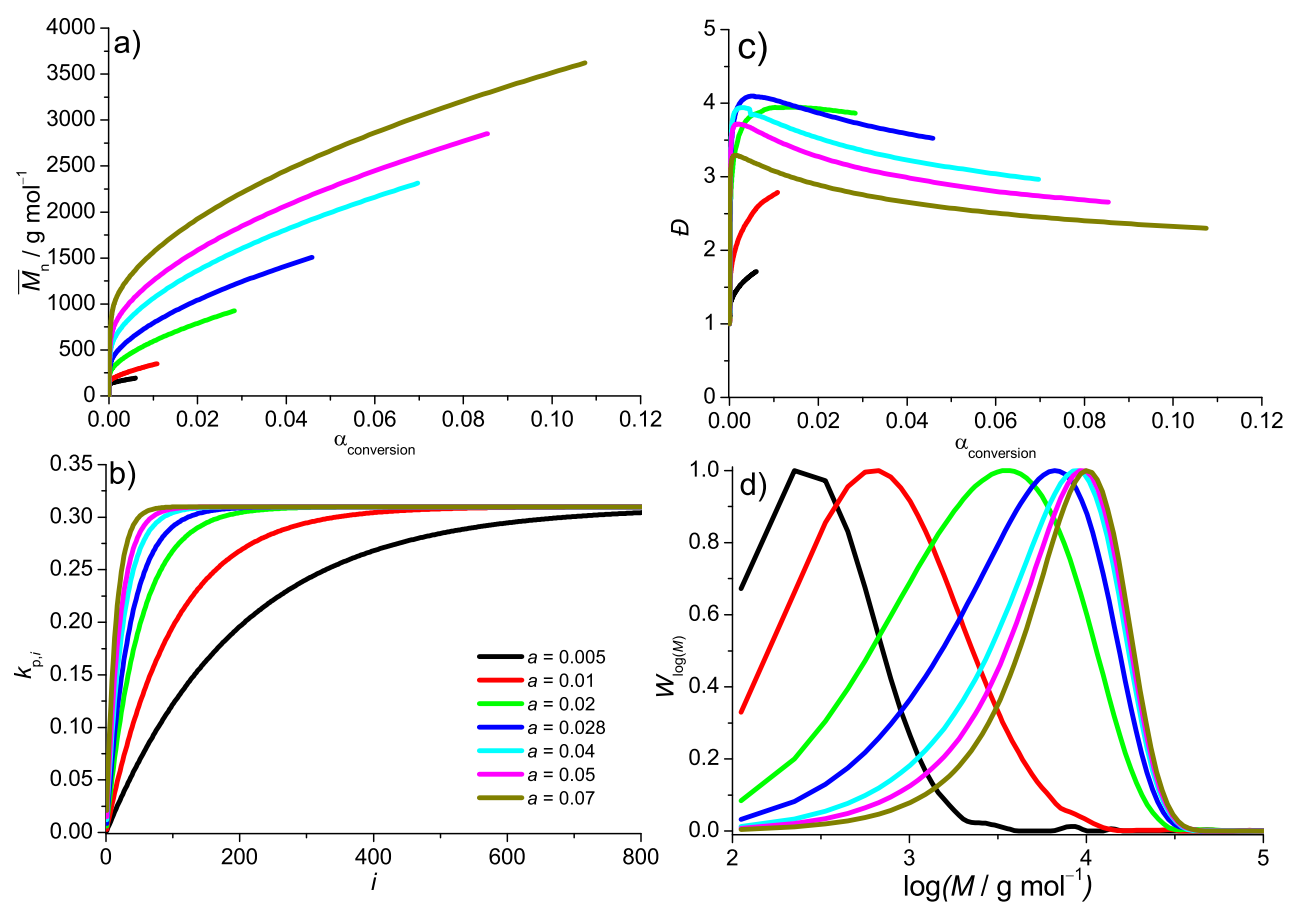

Figure 5.13: Chain-length dependent simulation by variation of $a$ based on Table 5.2 and 5.4 . a) $\bar{M}_{n}$ vs. conversion profile $\mathrm{b}$ ) dispersity vs. conversion profile c) $k_{\mathrm{p}, \mathrm{i}}$ versus chain length (i) profile $\mathrm{d}$ ) MMD profiles.

By variation of $k_{\text {alkyl1 }}$ it was found (see Figure 5.14) that around the value determined by NMR the system gets nearly independent of the alkylation rate at higher values. Below the value the monomer conversion is lower $(5.14, b)$ and the MMD is shifted to lower values $(5.14, \mathrm{c}))$. The diversity and the $\bar{M}_{\mathrm{n}}(5.14$, a)) show only a difference in the beginning, where the first strong increase is lowered with a higher rate coefficient before each curve levels off to values only differing slightly over all simulations. $k_{\text {alkyl1 }}$ is not able to influence the dispersity and the $\bar{M}_{\mathrm{n}}$ value in the chosen area. The $\mathrm{BzMgBz}$ consumption $(\overline{5.14}, \mathrm{~d})$ ) is naturally directly correlated to the higher alkylation rate. The similar values reached at higher reaction times show clearly that the main process of $\mathrm{BzMgBz}$ consumption is the first monomer addition. The second alkylation step is able to deactivate the reaction system quickly (see Figure 5.15, b)). For values above $k_{\text {alkyl2 }}=2.6 \times 10^{-6} \mathrm{~s}^{-1}$ the reaction rate is dramatically decreased with increasing reaction rate. By an increase of a factor of 10 the conversion is not only lowered but also levels off to a constant value. This behavior is found more pronounced for the higher concentrations as well. The catalyst is fully alkylated and cannot perform monomer 


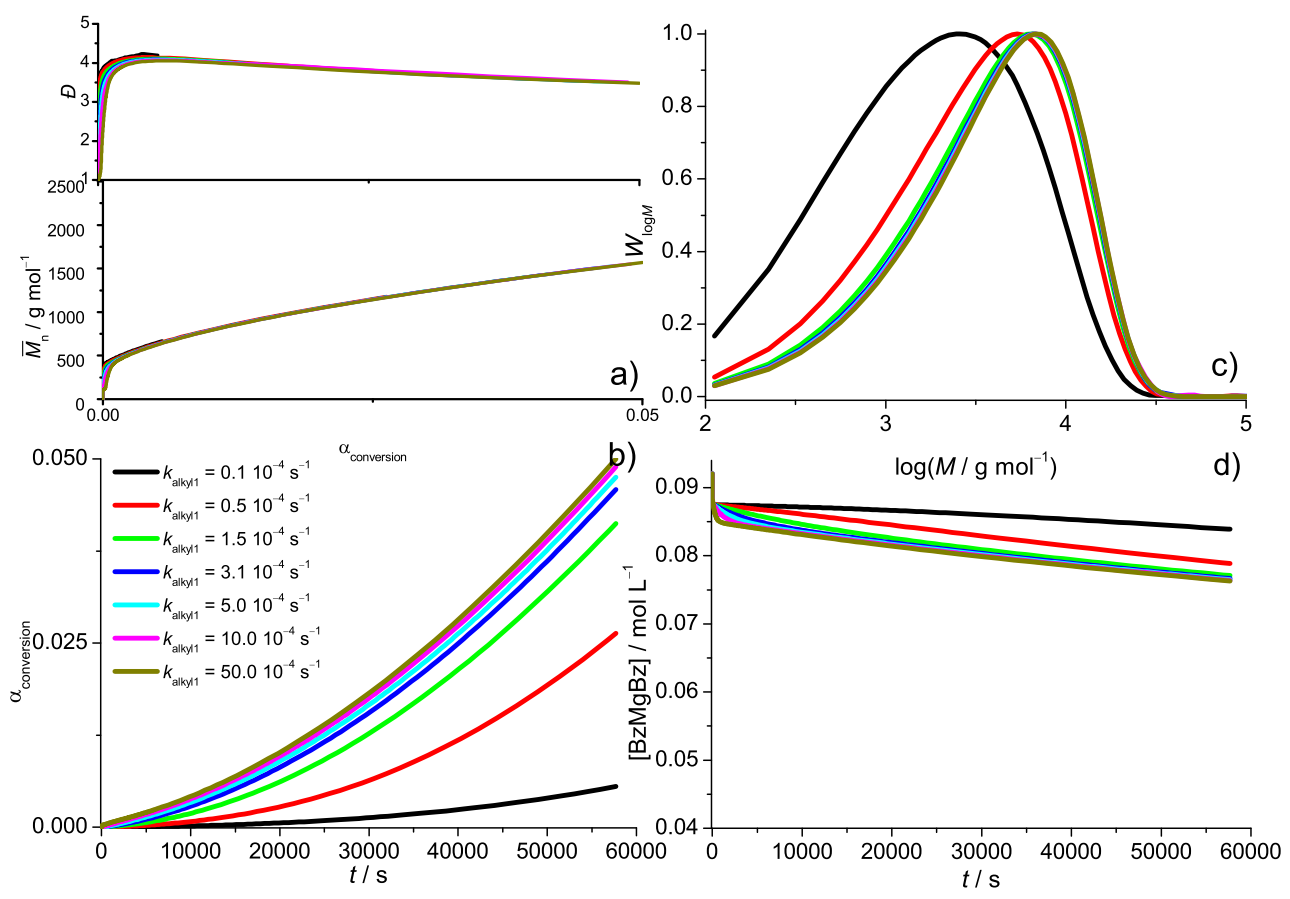

Figure 5.14: Chain-length dependent simulation by variation of $k_{\text {alkyl1 }}$ based on Table 5.2 and 5.4 . a) $\bar{M}_{\mathrm{n}}$ vs. conversion profile b) dispersity vs. conversion profile c) $k_{\mathrm{p}, \mathrm{i}}$ versus chain length (i) profile d) MMD profiles.

addition. The co-catalyst acts via this second reaction as a deactivator. The dispersity and the $\bar{M}_{\mathrm{n}}(5.15, \mathrm{a})$ ) are unaffected by this value while the BzMgBz consumption $(5.15$, d)) is naturally sligthly pronounced due to the alkylation process. The MMD is lower for higher reaction rates due to the fast deactivation of the growing species $(5.15, \mathrm{c})$ ).

The strong influence of the equilibrium constant $K_{1, \mathrm{Zr}}$ and $K_{2, \mathrm{Zr}}$ can be easily seen by parameter variation (see Figure 5.16). Starting from a low value of $K_{1, Z r}$ and $K_{2, Z r}$, the polymerization is fast and finished within 25000 seconds (see Figure 5.16 b)). With increasing value $K_{2, Z \mathrm{r}}$ at constant $K_{1, \mathrm{Zr}}$ the conversion decreases as well as the BzMgBz consumption (see Figure $5.16 \mathrm{~d}$ )), the maximum of the MMD (see Figure $5.16 \mathrm{e}$ )), the values of $\bar{M}_{\mathrm{n}}$ (see Figure $5.16 \mathrm{a}$ ), c)) and dispersity. For the variation of $K_{1, \mathrm{Zr}}$ at constant $K_{2, Z \mathrm{r}}$ a maximum in $\bar{M}_{\mathrm{n}}$ is found at high equilibrium constants. With increasing value the dispersity increases as well, while the consumption of $\mathrm{BzMgBz}$ is decreasing. A lower amount of benzyl group is activated and able to add monomer. At values above $K_{1, \mathrm{Zr}}=35000 \mathrm{~L} \mathrm{~mol}^{-1}$ the process is inhibited and only a very low conversion is found for all simulations with a broad MMD. This result shows that the equilibrium constants have, as to be expected, a high influence on the success of the polymerization. Is the value to low and the first monomer insertion hindered, the monomer is consumed before all chains are activated. Is the value to high, the polymerization process is inhibited and only a low conversion gained. The optimum value is around $K_{1, \mathrm{Zr}}=K_{2, \mathrm{Zr}}=350 \mathrm{~L} \mathrm{~mol}^{-1}$, 


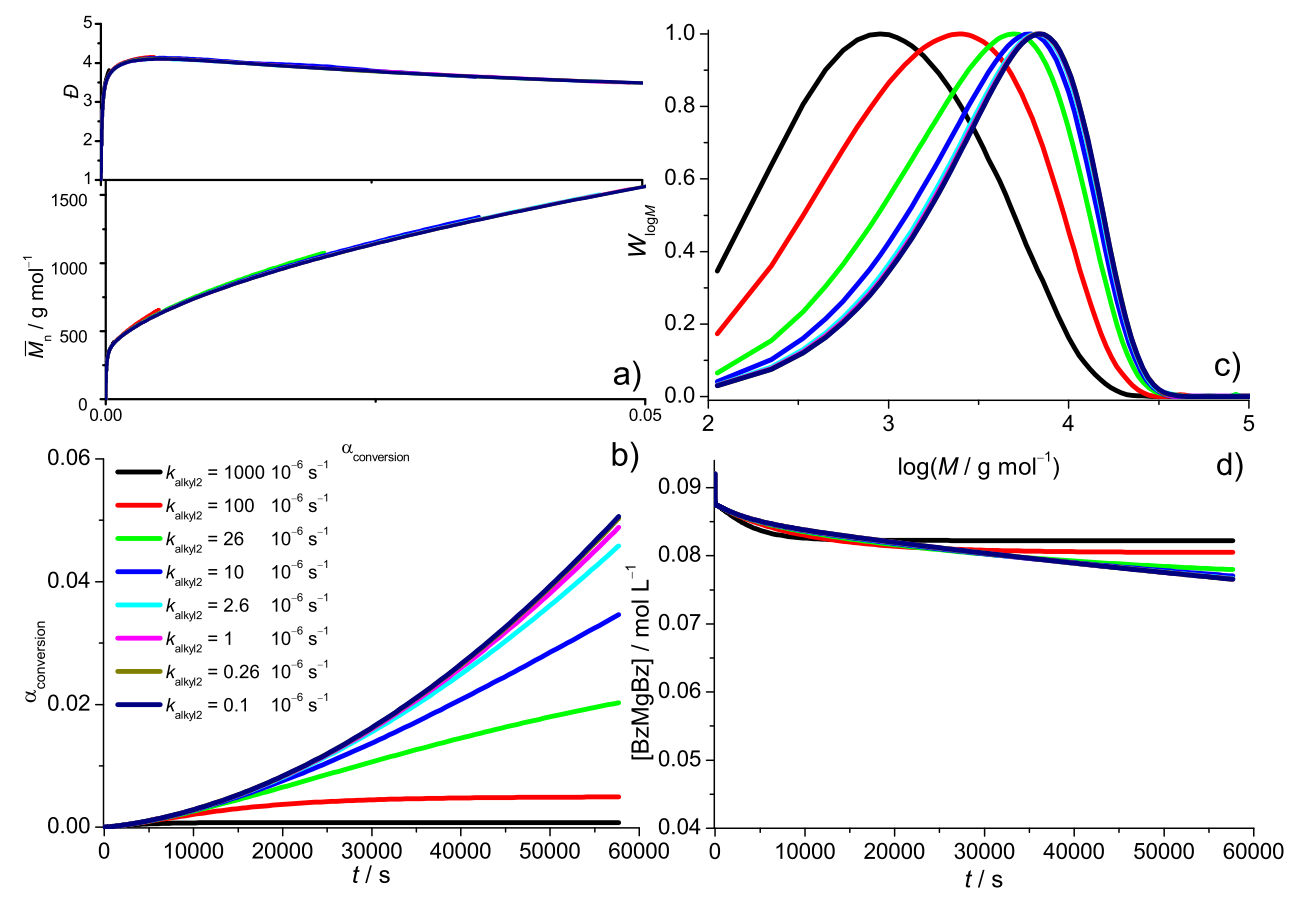

Figure 5.15: Chain-length dependent simulation by variation of $k_{\text {alkyl2 }}$ based on Table 5.2 and 5.4. a) $\bar{M}_{\mathrm{n}}$ vs. conversion profile b) dispersity vs. conversion profile c) $k_{\mathrm{p}, \mathrm{i}}$ versus chain length (i) profile d) MMD profiles.

because the monomer addition is fast and high amounts of benzyl groups get activated. While the equilibrium constant has to be below a certain value, the influences of the magnitudes of $k_{\text {act1 }}$ and $k_{\text {act2 }}$ were investigated as well. For the simulation, $k_{\text {ex } 1,2}$ and $k_{\text {act } 1,2}$ were recalculated with fixed equilibrium constants. The results are shown in Figure 5.17 and 5.19. For $k_{\text {act1 }}$ values above $1 \times 10^{-3} \mathrm{~s}^{-1}$ yield only low conversion (see Figure 5.17 b)) and the concentration profile of $\mathbf{C}+\mathrm{C}^{\mathbf{P}}$ is completely independent of the reaction rate (see Figure $5.17 \mathrm{~d}$ )). This situation changes for lower magnitudes. At $1 \times 10^{-4} \mathrm{~s}^{-1}$ (the experimental value) the conversion is notable and the MMD shows an increase by two orders of magnitude (see Figure $5.17 \mathrm{c}$ )). The $\bar{M}_{\mathrm{n}}$ values are increasing and the dispersity has a maximum at 3 and is decreasing afterwards (see Figure 5.17 a)). For $1 \times 10^{-5} \mathrm{~s}^{-1}$ the concentration of overall $\mathbf{C}+\mathrm{C}^{\mathrm{P}}$ is completely different and high conversion and $\bar{M}_{\mathrm{n}}$ values are yielded. This effect is a result of the activation reaction and the rate of reaction for the exchange reaction, because $k_{\mathrm{ex} 1}$ is lowered in the same manner.

As previously shown in equation 5.5 on page 89 the main factor producing species $\mathrm{C}$ is the alkylation reaction from $\mathbf{B}^{\mathrm{P}, 1}$. The main consumption reactions are the monomer ad- 

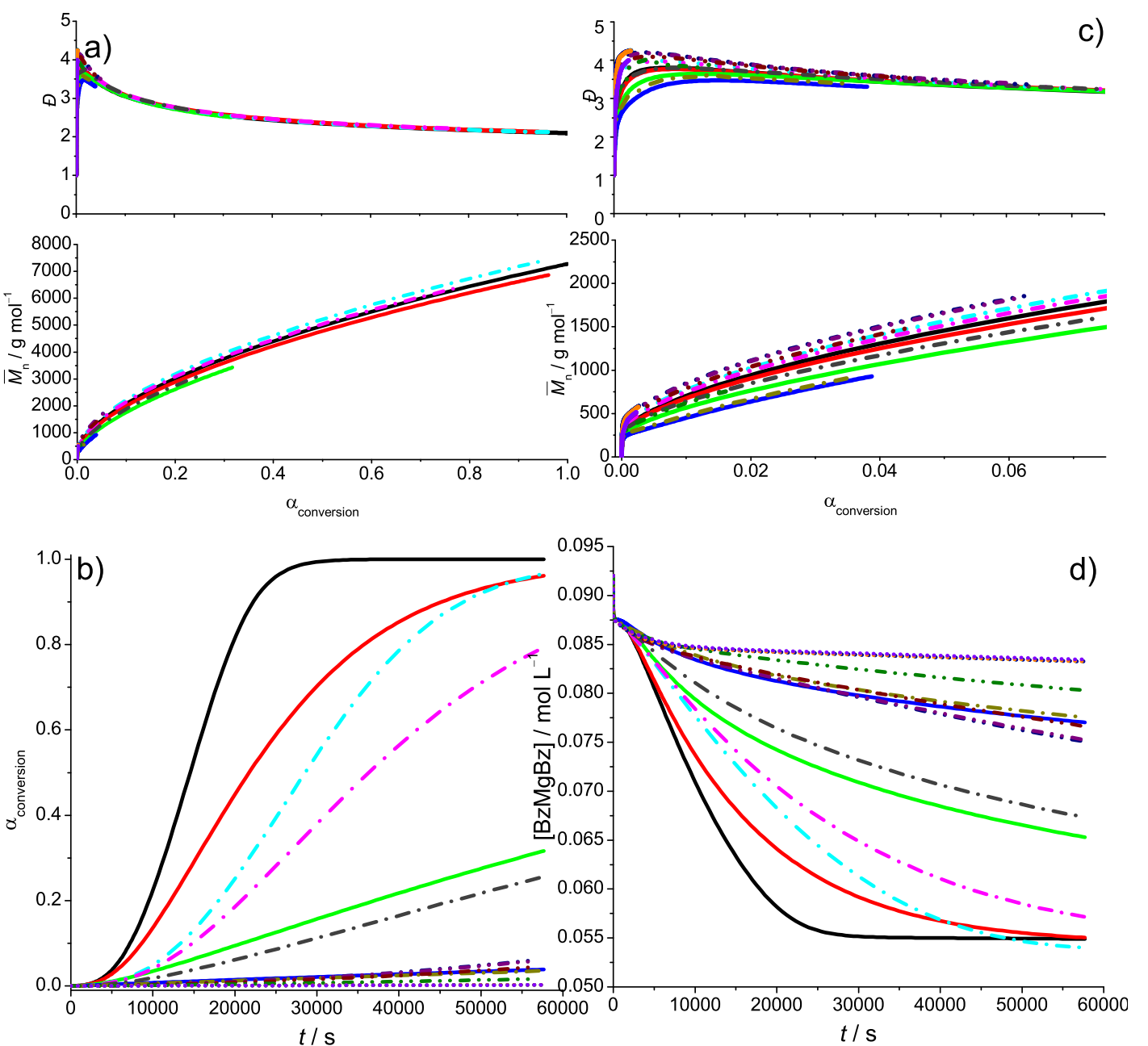

$K_{1}=35 \mathrm{~L} \mathrm{~mol}^{-1} ; \quad K_{2}=35 \mathrm{~L} \mathrm{~mol}^{-1}$

$K_{1}=35 \mathrm{~L} \mathrm{~mol}^{-1} ; \quad K_{2}=350 \mathrm{~L} \mathrm{~mol}^{-1}$

$K_{1}=35 \mathrm{~L} \mathrm{~mol}^{-1} ; \quad K_{2}=3500 \mathrm{~L} \mathrm{~mol}^{-1}$

$-K_{1}=35 \mathrm{~L} \mathrm{~mol}^{-1} ; \quad K_{2}=35000 \mathrm{~L} \mathrm{~mol}^{-1}$

- $K_{1}=350 \mathrm{~L} \mathrm{~mol}^{-1} ; \quad K_{2}=35 \mathrm{~L} \mathrm{~mol}^{-1}$

- $K_{1}=350 \mathrm{~L} \mathrm{~mol}^{-1} ; \quad K_{2}=350 \mathrm{~L} \mathrm{~mol}^{-1}$

-. $K_{1}=350 \mathrm{~L} \mathrm{~mol}^{-1} ; \quad K_{2}=3500 \mathrm{~L} \mathrm{~mol}^{-1}$

- $K_{1}=350 \mathrm{~L} \mathrm{~mol}^{-1} ; \quad K_{2}=35000 \mathrm{~L} \mathrm{~mol}^{-1}$

-.. $K_{1}=3500 \mathrm{~L} \mathrm{~mol}^{-1} ; K_{2}=35 \mathrm{~L} \mathrm{~mol}^{-1}$

-.. $K_{1}=3500 \mathrm{~L} \mathrm{~mol}^{-1} ; K_{2}=350 \mathrm{~L} \mathrm{~mol}^{-1}$

-.. $K_{1}=3500 \mathrm{~L} \mathrm{~mol}^{-1} ; K_{2}=3500 \mathrm{~L} \mathrm{~mol}^{-1}$

- $K_{1}=3500 \mathrm{~L} \mathrm{~mol}^{-1} ; K_{2}=35000 \mathrm{~L} \mathrm{~mol}^{-1}$

…... $K_{1}=35000 \mathrm{~L} \mathrm{~mol}^{-1} ; K_{2}=35 \mathrm{~L} \mathrm{~mol}^{-1}$

…... $K_{1}=35000 \mathrm{~L} \mathrm{~mol}^{-1} ; K_{2}=350 \mathrm{~L} \mathrm{~mol}^{-1}$

…... $K_{1}=35000 \mathrm{~L} \mathrm{~mol}^{-1} ; K_{2}=3500 \mathrm{~L} \mathrm{~mol}^{-1}$

....... $K_{1}=35000 \mathrm{~L} \mathrm{~mol}^{-1} ; K_{2}=35000 \mathrm{~L} \mathrm{~mol}^{-1}$

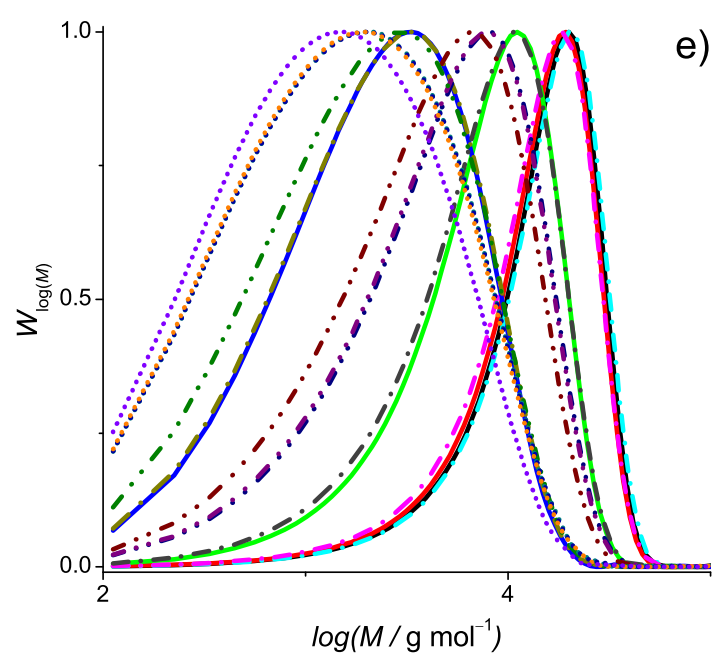

Figure 5.16: Chain-length dependent simulation by variation of $K_{1, \mathrm{Zr}}$ and $K_{2, \mathrm{Zr}}$ based on Table 5.2 and 5.4 a) a) $\bar{M}_{\mathrm{n}}$ and dispersity vs. conversion profile; b) Zoom of $\bar{M}_{\mathrm{n}}$ and dispersity vs. conversion profile; c) monomer concentration vs. time profile; d) BzMgBz concentration vs. time; e) MMD distributions. 


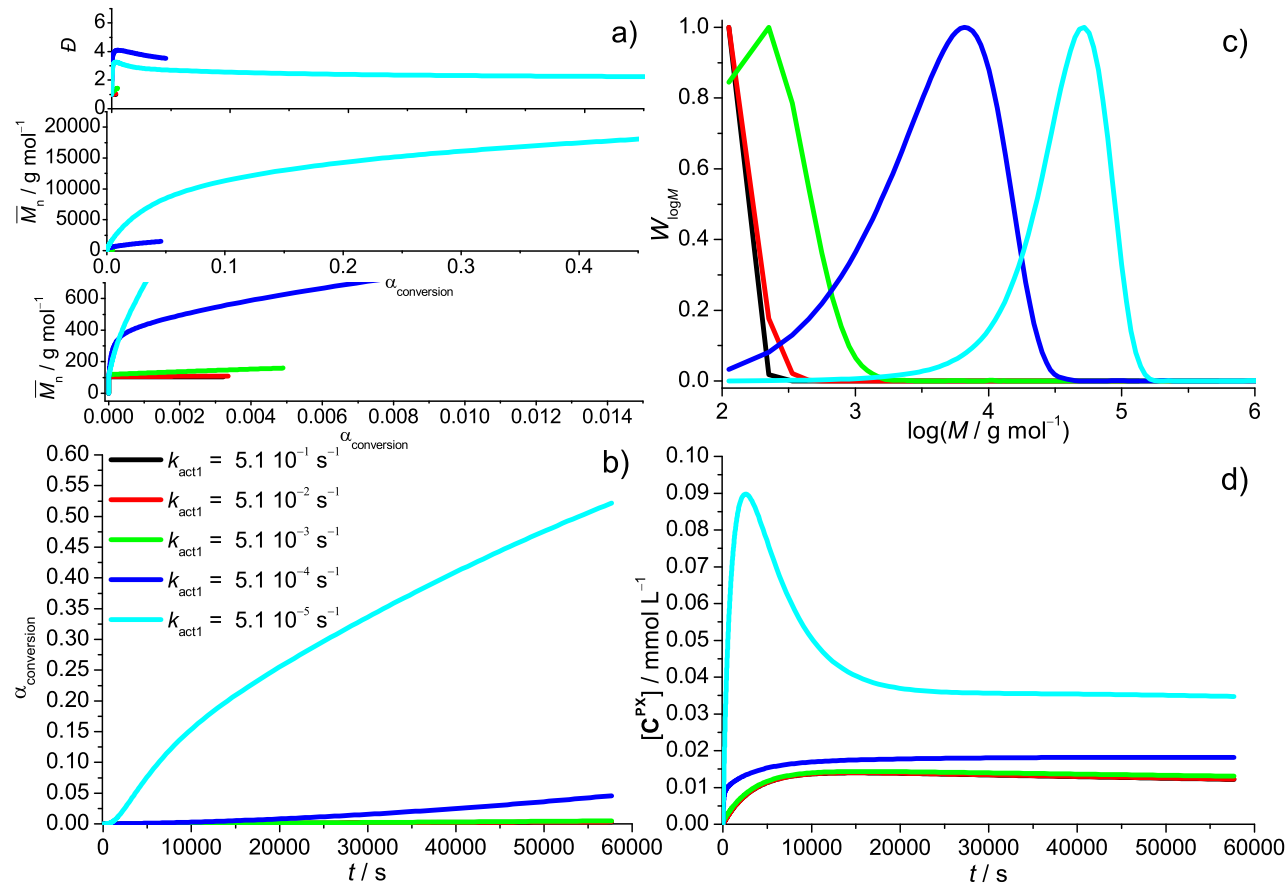

Figure 5.17: Chain-length dependent simulation by variation of $k_{\text {act } 1}$ based on Table 5.2 and 5.4. a) $\bar{M}_{\mathrm{n}}$ and dispersity vs. conversion profile b) conversion vs. time profile c) MMD profile d) $\mathrm{Bz} \mathrm{MgBz}$ concentration profile.

dition and the formation of complexes $\mathbf{D}^{\mathbf{P 0}, \mathbf{1}, 2}$. At the early states mainly $\mathbf{B}^{\mathbf{P} 0}$ and $\mathrm{BzMgBz}$ are found. Equation 5.5 can therefore be simplified to equation 5.98 :

$$
\frac{\mathrm{d}[\mathbf{C}]}{\mathrm{d} t}=-k_{\mathrm{ex} 1}[\mathbf{C}][\mathbf{B z M g B z}]+k_{\mathrm{act} 1}\left[\mathbf{D}^{\mathbf{P} 0}\right]+k_{\text {alkyl1 }}\left[\mathbf{B}^{\mathbf{P} 0}\right]-k_{\text {add }}[\mathbf{C}][\mathbf{M}]+k_{\text {frag }}[\mathbf{C}(\mathbf{M})]
$$

Within the first minutes, the reaction rates of the formation of $\mathbf{C}$ via alkylation and the association towards $\mathbf{D}^{\mathbf{P 0}}$ were calculated from the simulated data and are depicted in Figure 5.18. As a main result it is found that the formation of the high molar mass signals in MMD is directly linked to low reaction rate of association in the activation phase of the polymerization. The catalytically active species is formed faster by alkylation than it can be deactivated via the exchange reaction and is not yet in equilibrium. The monomer coordination can occur very rapidly to start the chain growth. With decreasing rate coefficient of $k_{\text {act } 1}$ and $k_{\mathrm{ex} 1}$, this effect can last for several minutes, starting a pronounced chain growth. Because the propagation for the already activated polymer chain is higher, the catalytically active center is adding monomer preferably until the complex associates a magnesium derivative. This causes the high molecular mass found in the experiments.

The influence of the magnitude of $k_{\mathrm{act} 2}$ and $k_{\mathrm{ex} 2}$ is opposite. The conversion (see Figure 


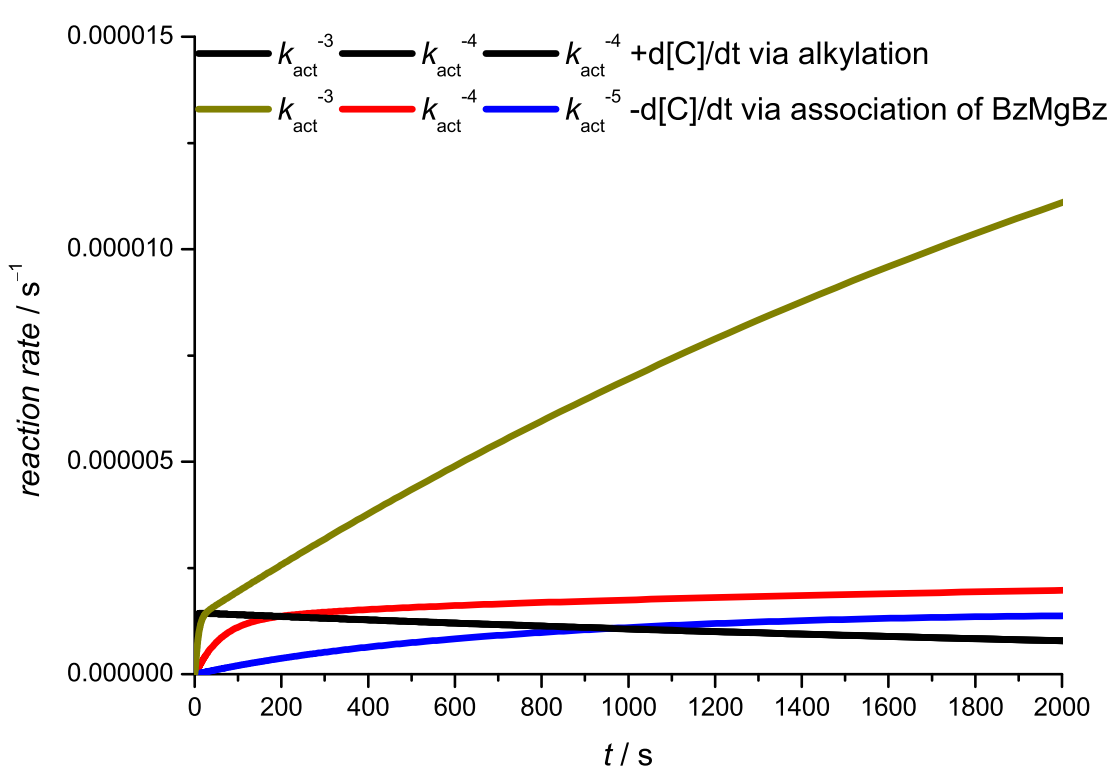

Figure 5.18: Reaction rates for the formation of complex $\mathrm{C}$ via alkylation and for the association of $\mathrm{BzMgBz}$ for three different orders of magnitude of $k_{\mathrm{act} 1}$ based on the data in Table 5.4, experiment i).

5.19 b) ) and the $\bar{M}_{\mathrm{n}}$-values (see Figure 5.19 a)) decrease with the order of magnitude while the dispersity is increasing. The maximum of the MMD is found at lower values (see Figure $5.19 \mathrm{c})$ ) and broadens with decreasing order of magnitude. Interestingly, a gap is found between $k_{\text {act2 }}=5.1 \times 10^{-3} \mathrm{~s}^{-1}$ and $k_{\text {act2 }}=5.1 \times 10^{-2} \mathrm{~s}^{-1}$, where the conversion is increased by several hundred percent. A similar effect is found in the overall concentration of $\mathbf{C}^{\mathrm{PX}}$. One would think that the increase should be similar but it only levels off at a higher value. This can be explained by the mechanism of monomer addition. Because the monomer must be coordinated to the catalyst before propagation, the effective propagation rate is higher than the increase in complex $\mathbf{C}$.

The exchange equilibria are not the only influence on the location of the maximum of MMD. The simulation of various $k_{\mathrm{p} 1}$ values revealed that a higher $k_{\mathrm{p} 1}$ yields a lower molecular mass (cf. Figure $5.20 \mathrm{a}$ ), c)) as the actual experiment, due to the higher chance of exchange at higher conversion (see Figure $5.20 \mathrm{c}$ )). But a higher dispersity is found at the end of polymerization. The maximum of dispersity during the polymerization increases with decreasing $k_{\mathrm{p} 1}$ (see Figure $5.20 \mathrm{a}$ )). In all cases, the dispersity has a maximum and is decreasing afterwards. The slope is influenced by $k_{\mathrm{p} 1}$. A higher value yields a smaller slope. The simulations clearly show that $k_{\mathrm{p} 1}$ is of importance for the complete activation of the chains at the magnesium because the $\mathrm{BzMgBz}$ consumption is increased with $k_{\mathrm{p} 1}$ (see Figure $5.20 \mathrm{~d}$ )). These results show that high values of $k_{\mathrm{p} 1}$ are necessary for a controlled polymerization and are one of the main reasons that the system $\mathbf{A}$ with 


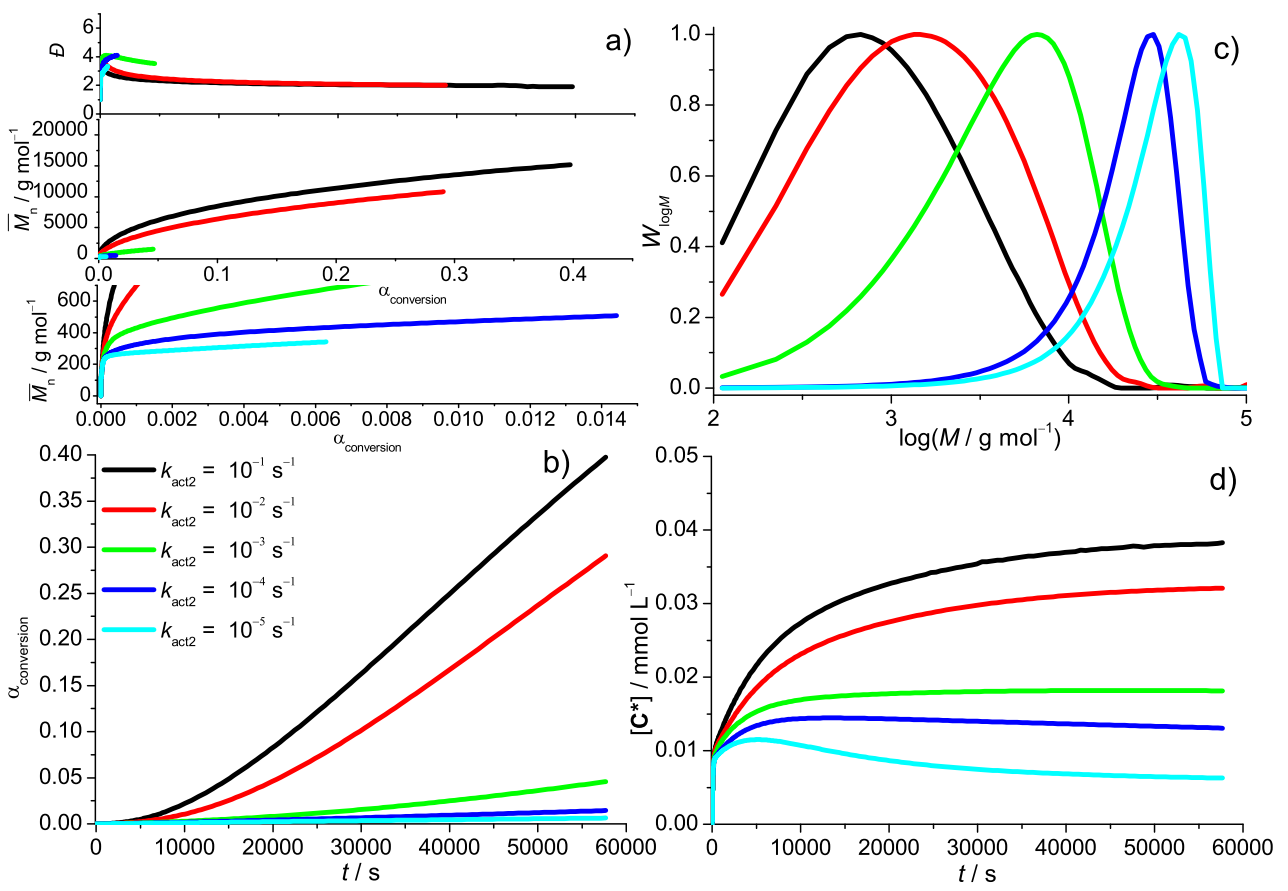

Figure 5.19: Chain-length dependent simulation by variation of $k_{\text {act2 }}$ based on Table 5.2 and 5.4. a) $\bar{M}_{\mathrm{n}}$ and dispersity vs. conversion profile b) conversion vs. time profile c) MMD profile d) $\mathrm{Bz} \mathrm{MgBz}$ concentration profile.

$\mathrm{BzMgBz}$ is polymerizing styrene less controlled.

The variation of $k_{\mathrm{p}}$ shows the increase of conversion with $k_{\mathrm{p}}$ (see Figure $5.20 \mathrm{a}$ ) and b)). A maximum of dispersity is found for values above $0.45 \mathrm{~s}^{-1}$, increasing with $k_{\mathrm{p}}$. The dispersity decreases beyond the maximum. The slope is higher with increasing $k_{\mathrm{p}}$. As expected, the impact on the MMD (Figure 5.20 c)) is strong. With the increase of $k_{\mathrm{p}}$ by one order of magnitude the maximum in MMD is increasing by two orders. A $k_{\mathrm{p}}$ of $0.05 \mathrm{~s}^{-1}$ yields a product mainly consisting of oligomers, while a value above 0.3 yields a polymer with an average molecular mass around $1000 \mathrm{~g} \mathrm{~mol}^{-1}$. The BzMgBz conversion is hardly influenced at all (Figure $5.20 \mathrm{~d}$ )).

The influence of the lifetime of monomer on the catalyst is shown in Figure 5.22. For simulation the value of $k_{\text {frag }}$ was varied causing a change in $K_{\text {mono }}$. With increasing lifetime, the conversion, average molecular mass and $\mathrm{BzMgBz}$ consumption are increasing, while the dispersity has a maximum at $K_{\text {mono }}=5 \mathrm{~L} \mathrm{~mol}^{-1}$. The BzMgBz consumption is higher due to the fact that first addition and polymer growth is influenced in the same manner. The molecular mass distribution is smaller with increasing lifetime and shifted to higher molecular mass (see 5.22, c)). The polymerization is extremely sensitive here, especially at high monomer concentration. The lifetime of the monomer addition has a major impact on the shape of the MMD.

The rate coefficients for the monomer addition have a strong influence on the overall 


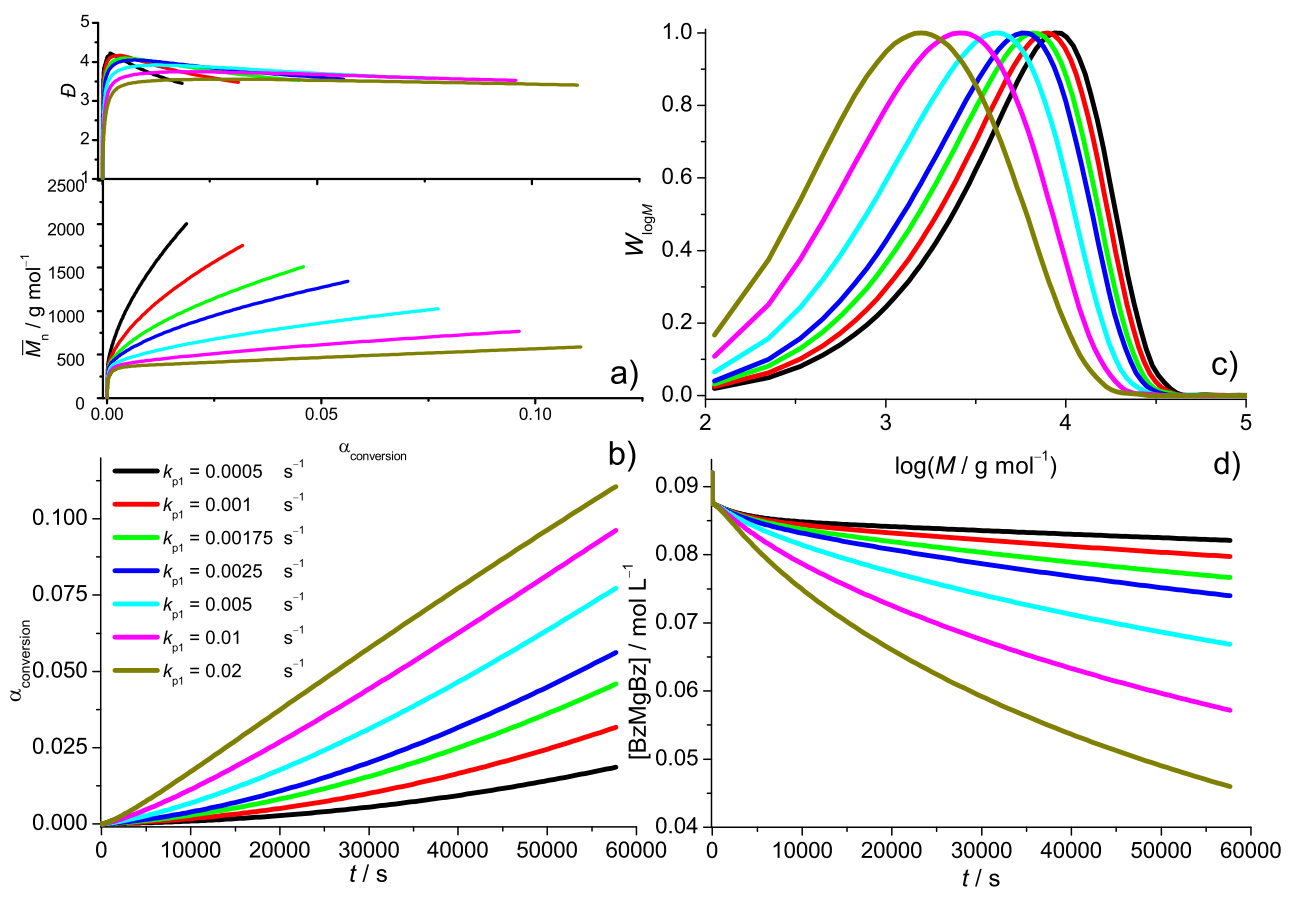

Figure 5.20: Chain-length dependent simulation by variation of $k_{\mathrm{p} 1}$ based on Table 5.2 and 5.4 a) $\bar{M}_{\mathrm{n}}$ and dispersity vs. conversion profile b) conversion vs. time profile c) MMD profile d) BzMgBz concentration profile.

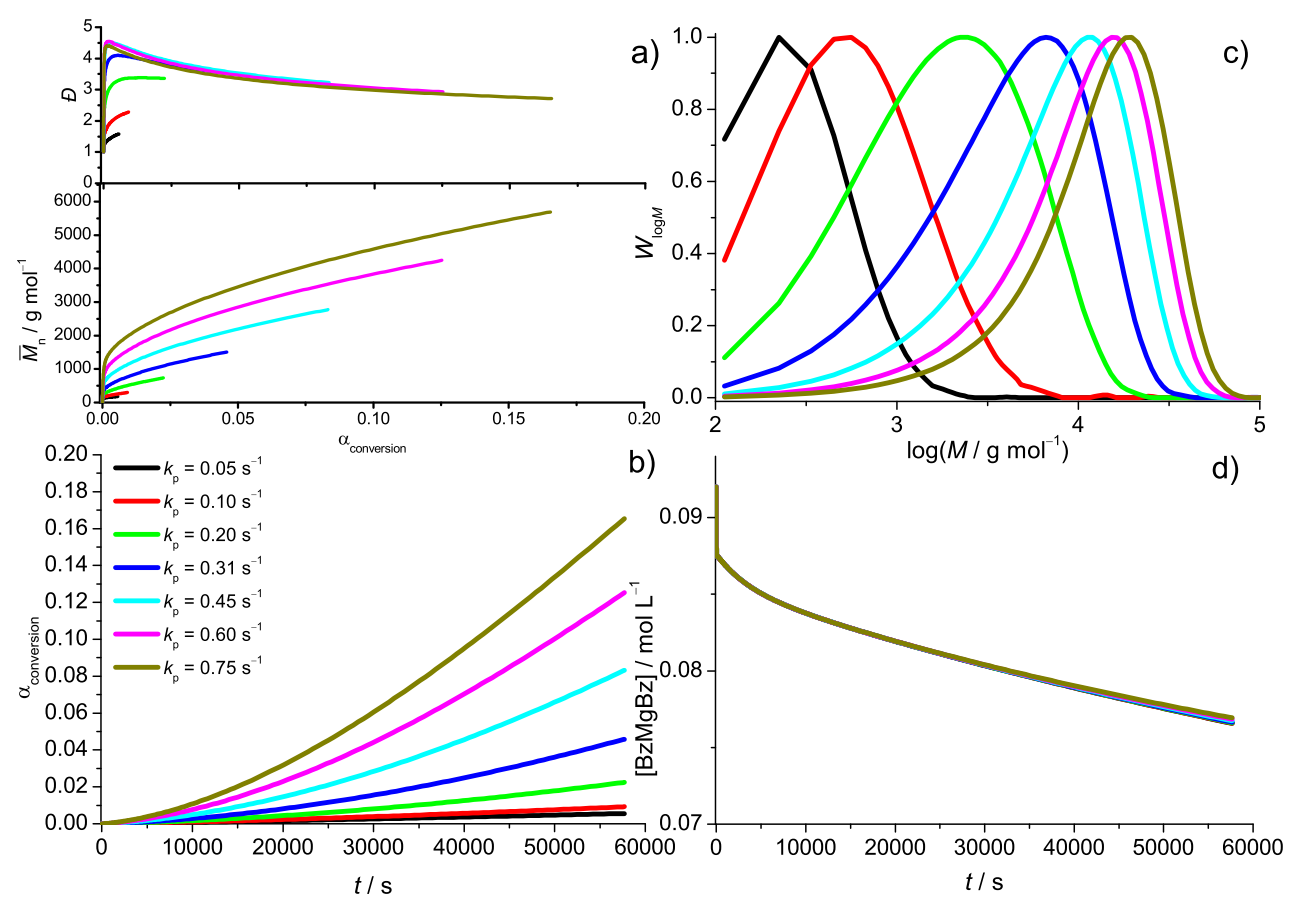

Figure 5.21: Chain-length dependent simulation by variation of $k_{\mathrm{p}}$ based on Table 5.2 and 5.4 . a) $\bar{M}_{\mathrm{n}}$ and dispersity vs. conversion profile b) conversion vs. time profile c) MMD profile d) $\mathrm{BzMgBz}$ concentration profile. 

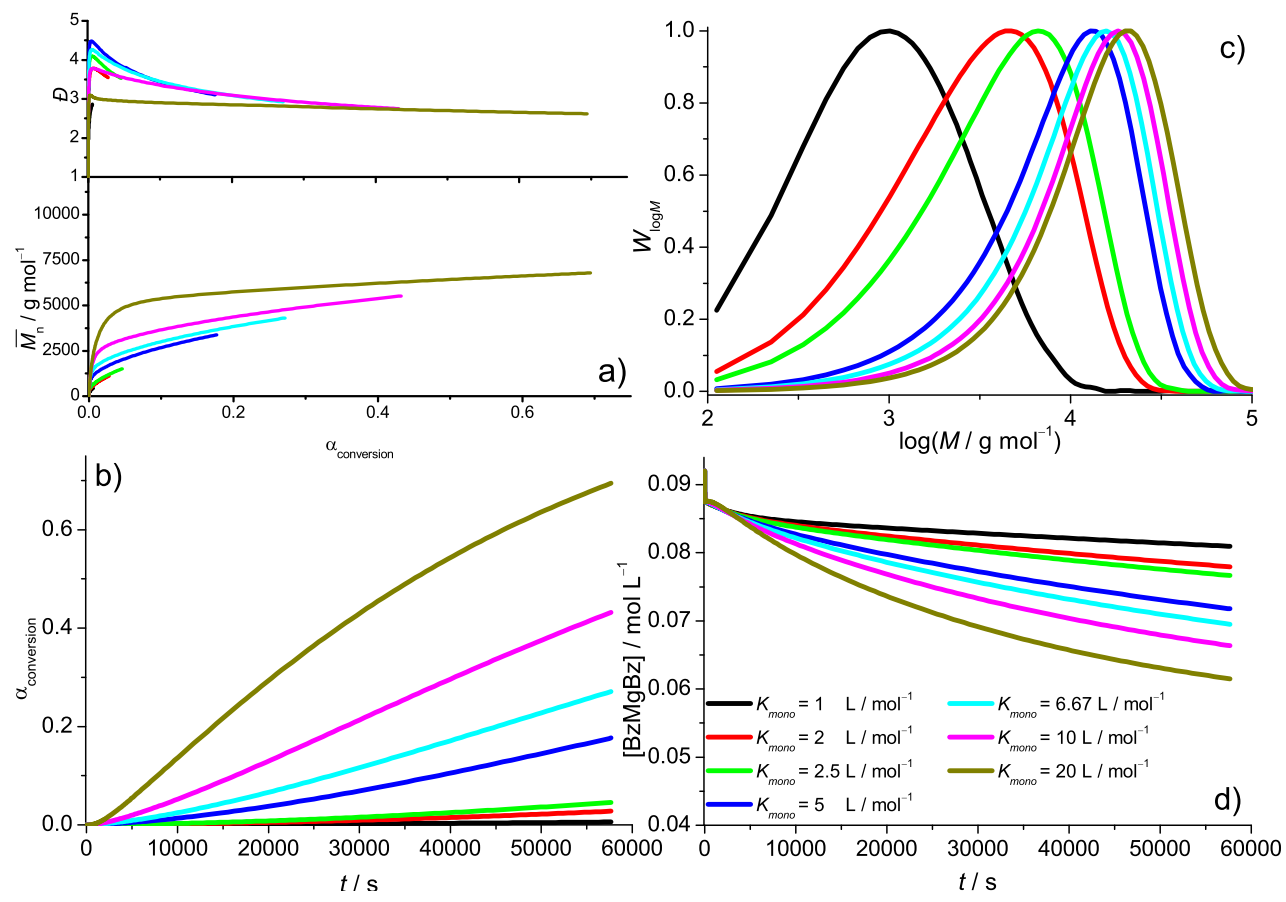

Figure 5.22: Chain-length dependent simulation by variation of $K_{\text {mono }}$ based on Table 5.2 and 5.4 a) $\bar{M}_{\mathrm{n}}$ and dispersity vs. conversion profile b) conversion vs. time profile c) MMD profile d) $\mathrm{Bz} \mathrm{MgBz}$ concentration profile.

conversion and the MMD are shifted to lower masses (see Figure $5.23 \mathrm{a}$ ), b) and c)) while the value is below $k_{\mathrm{ex} 1}$. For values above only a small influence on the molar mass is found and a constant MMD is achieved (see Figure $5.23 \mathrm{a}$ )). The BzMgBz concentration shows only very small change and the activation reaction is independent of the rate coefficients of monomer coordination in the applied range.

The concentration of A shows an interesting effect (see Figure 5.24): at a low concentration the reaction rate is higher then at high concentrations, which is not intuitive. Only at a very low concentration of $0.1 \mathrm{mmol} \mathrm{L}^{-1}$ a reduction of monomer conversion is found. But the catalyst concentration is only one side of the problem. In the same manner the $\mathrm{BzMgBz}$ concentration is reduced.

As previously shown, the alkylation reaction to $\mathrm{C}$ is an important factor for the polymerization process. Similar cases are found here and by variation of the concentration of BzMgBz. Secondly, the overall monomer coordination rate yields a maximum in the region $1 \times 10^{-3} \mathrm{~mol} \mathrm{~L}^{-1}$ to $1 \times 10^{-4} \mathrm{~mol} \mathrm{~L}^{-1}$ which was hit by the second lowest rate coefficient (see Figure 5.25). Together with the effect of the slow association reaction (see Figure 5.26) this leads to the situation that in a certain range at low catalyst concentrations the monomer addition is favored while the exchange reaction is hindered. In conclusion, a very low catalyst concentration will lead to high molecular mass polymer, while the increase in catalyst concentration will lead to a maximum in conversion until a 

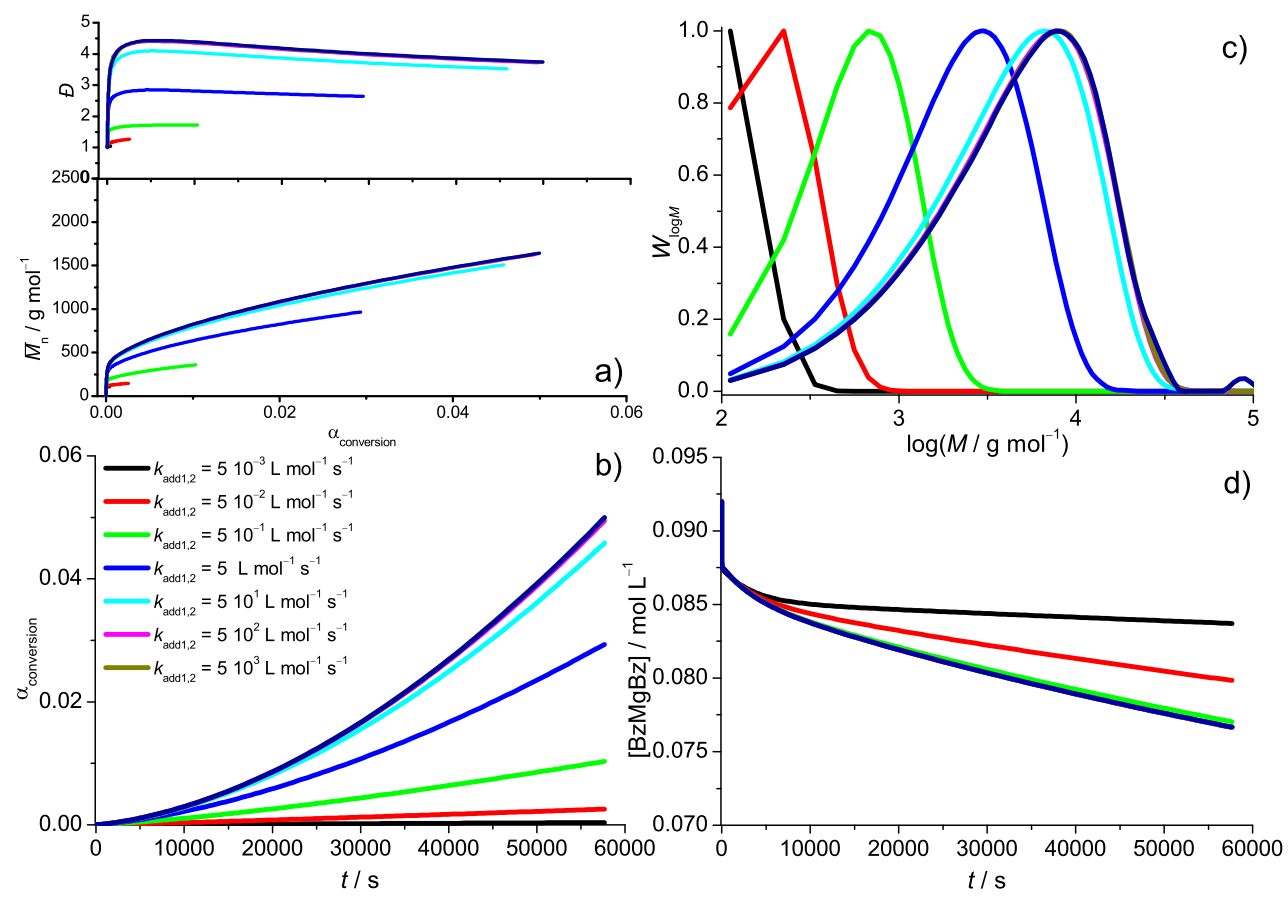

Figure 5.23: Chain-length dependent simulation by variation of $K_{\text {add1,add2 }}$ based on Table 5.2 and 5.4. a) $\bar{M}_{\mathrm{n}}$ and dispersity vs. conversion profile b) conversion vs. time profile c) MMD profile d) BzMgBz concentration profile
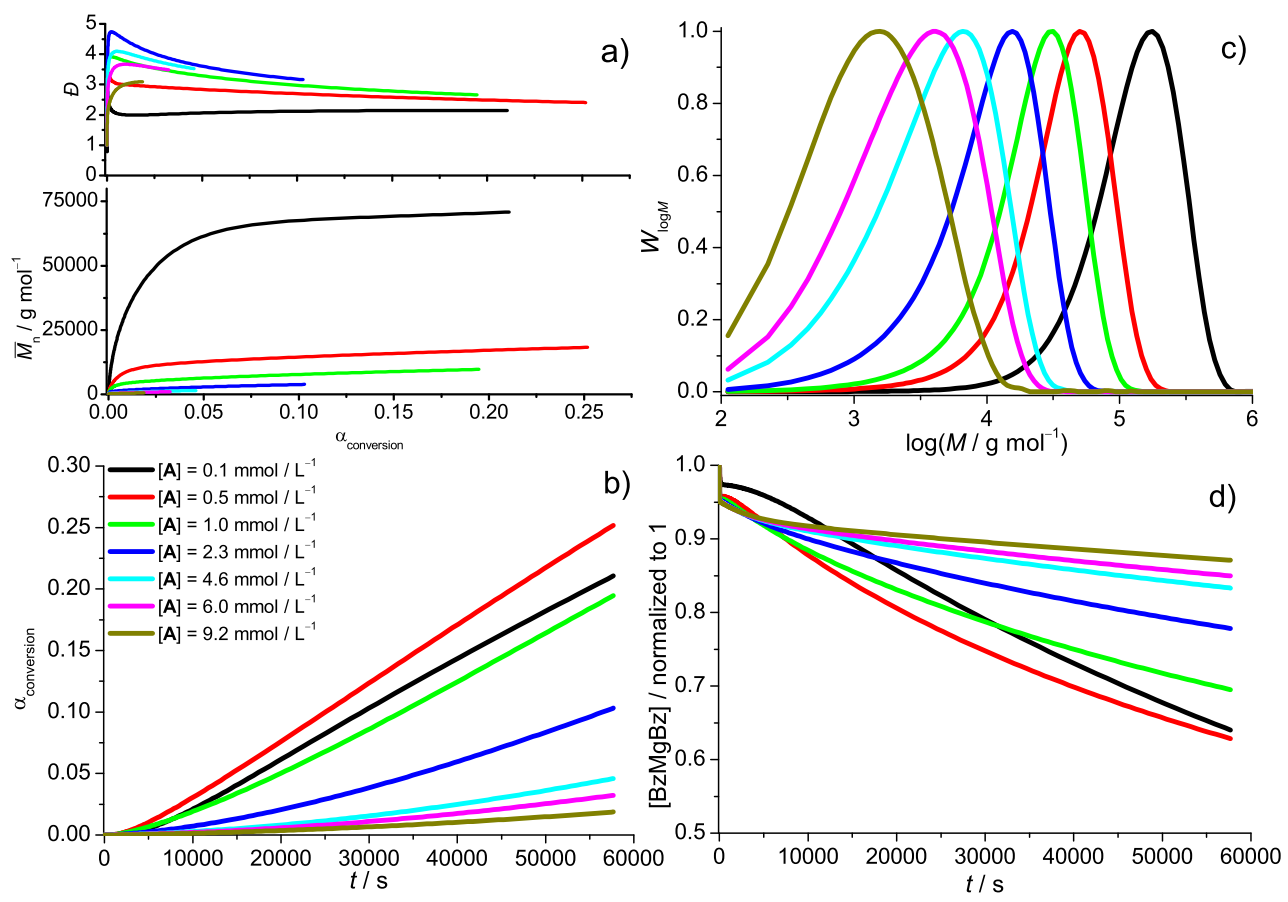

Figure 5.24: Chain-length dependent simulation by variation of $c(\mathbf{A})$ at a constant $\mathrm{Zr}: \mathrm{Mg}$ ratio of 20 based on Table 5.2 and 5.4 a) $\bar{M}_{\mathrm{n}}$ and dispersity vs. conversion profile b) conversion vs. time profile c) MMD profile d) BzMgBz concentration profile. 
specific concentration and will decrease afterwards.

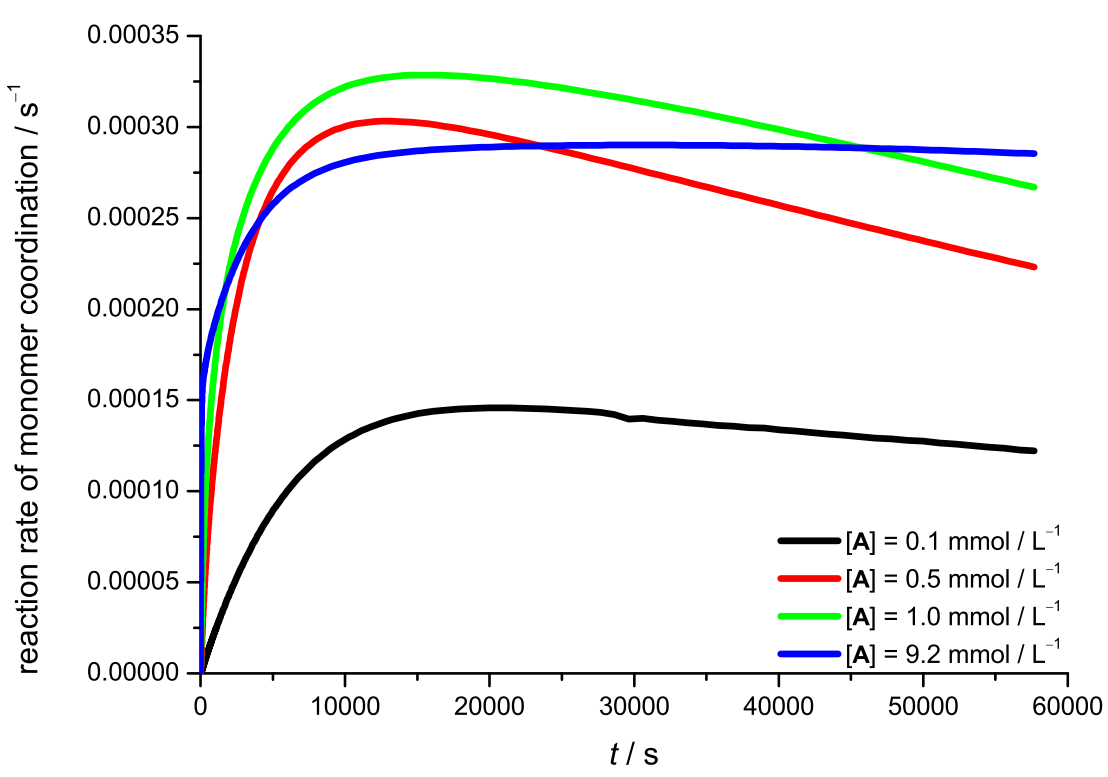

Figure 5.25: Reaction rates determined for the monomer coordination outgoing from species $\mathbf{C}$.

The MMD (see Figure 5.24) c)) is unaffected by the conversion maximum and has a clear trend towards higher molar mass with decreasing catalyst concentration. This is in agreement with the constant lowering of the co-catalyst and increase in monomer/co-catalyst ration. The relative consumption of $\mathrm{BzMgBz}$ (see Figure $5.24 \mathrm{~d}$ )) has its maximum at $0.5 \mathrm{mmol} \mathrm{L}^{-1}$ which is in agreement with the increasing chance of chain initiation. The effect of the hindered exchange reaction can be found in the normalized plot as a shoulder at low reaction times, which decreases with increasing concentration of $\mathbf{A}$. The concentration of BzMgBz shows a similar effect (see Figure 5.27). At low ratios, it acts as an activator yielding high conversion and high molecular mass due to the low amount of chains activated and the missing exchange reaction. Hybrid behavior is found at a ratio of $\mathrm{Mg} / \mathrm{Zr}$ 10:1. The reaction rate is high, but the exchange reaction is influencing the dispersity, yielding a maximum at the beginning around 4.5. For higher ratios a maximum is possible, but due to low conversion not reached. The polymerization is inhibited with increasing $\mathrm{BzMgBz}$ concentration, which is in agreement with the influence of the equilibrium constant.

The last effect studied is the influence of the monomer concentration (See Figure 5.28). The conversion strongly depends on the concentration. While at low concentrations only oligomers are formed and nearly no $\mathrm{BzMgBz}$ is activated this changes with increasing monomer concentration. The average molecular mass and the dispersity increase, due 


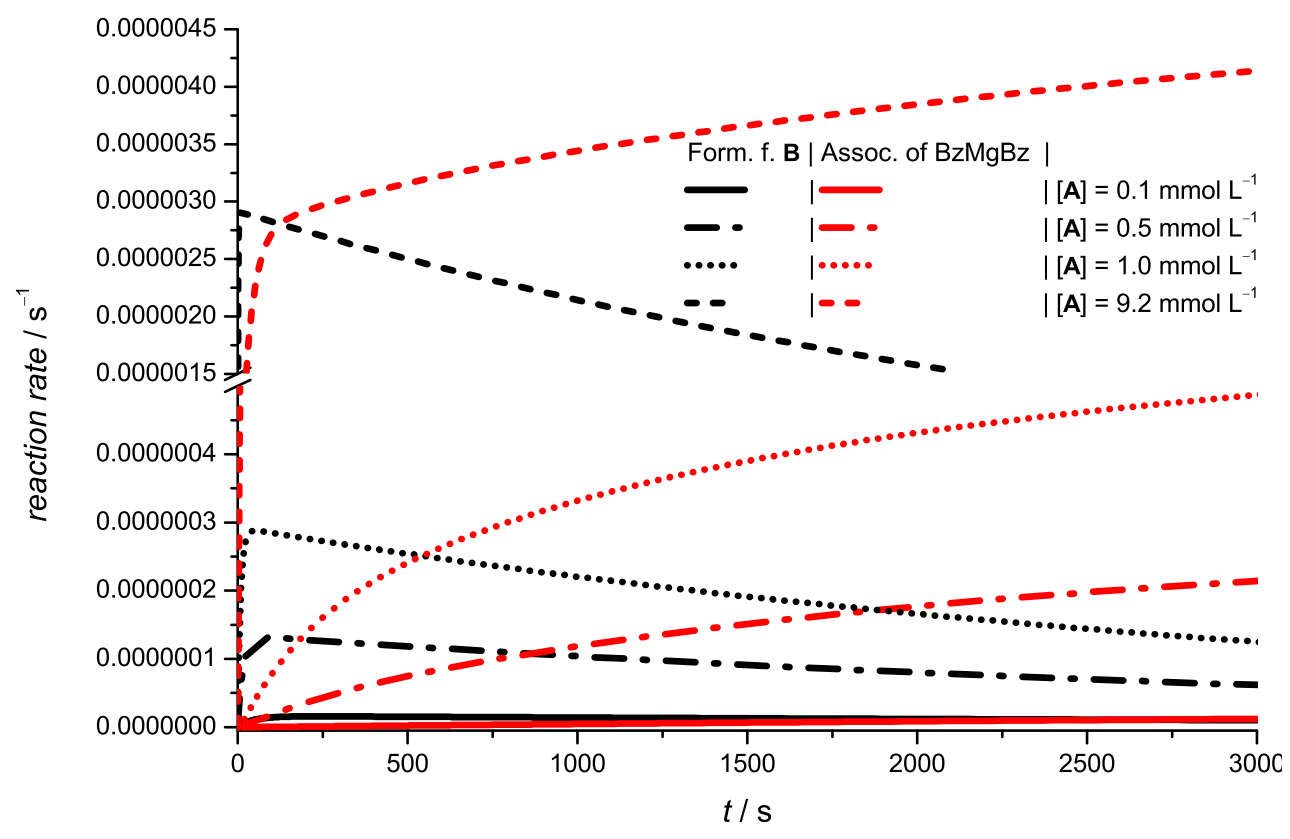

Figure 5.26: Comparsion of the reaction rates determined for the formation of $\mathbf{C}$ outgoing from species $\mathbf{B}$ with the association of $\mathrm{BzMgBz}$ to $\mathbf{C}$.
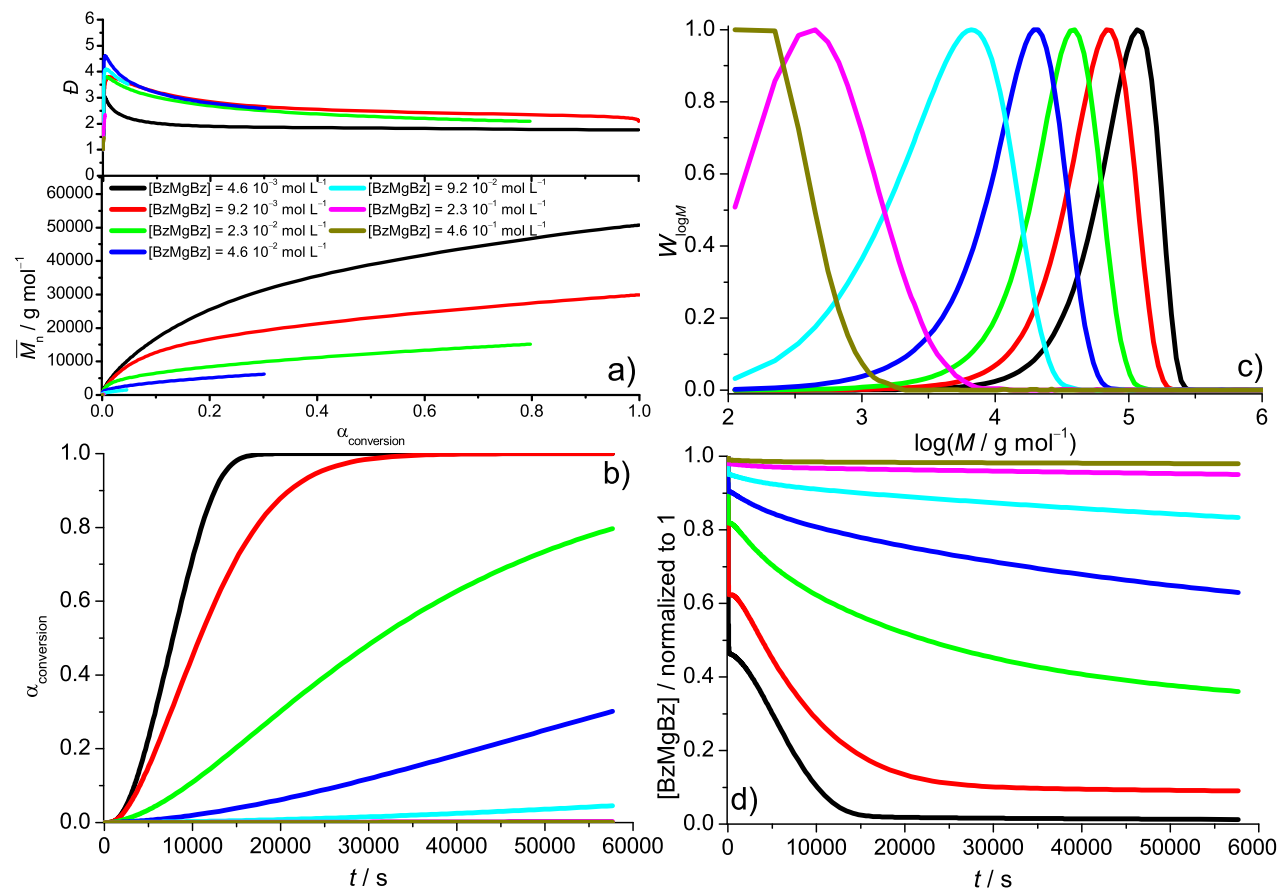

Figure 5.27: Chain-length dependent simulation by variation of $\mathrm{c}(\mathrm{BzMgBz})$ based on Table 5.2 and 5.4 a) $\bar{M}_{\mathrm{n}}$ and dispersity vs. conversion profile b) conversion vs. time profile c) MMD profile d) BzMgBz concentration profile. 
to the fact that the chance of polymer growth is higher. A high monomer concentration suppresses the exchange reaction. High monomer concentrations are typically used with liquid monomers. In the case of a gaseous monomer, like ethylene, the exchange reaction hinders the polymer growth.
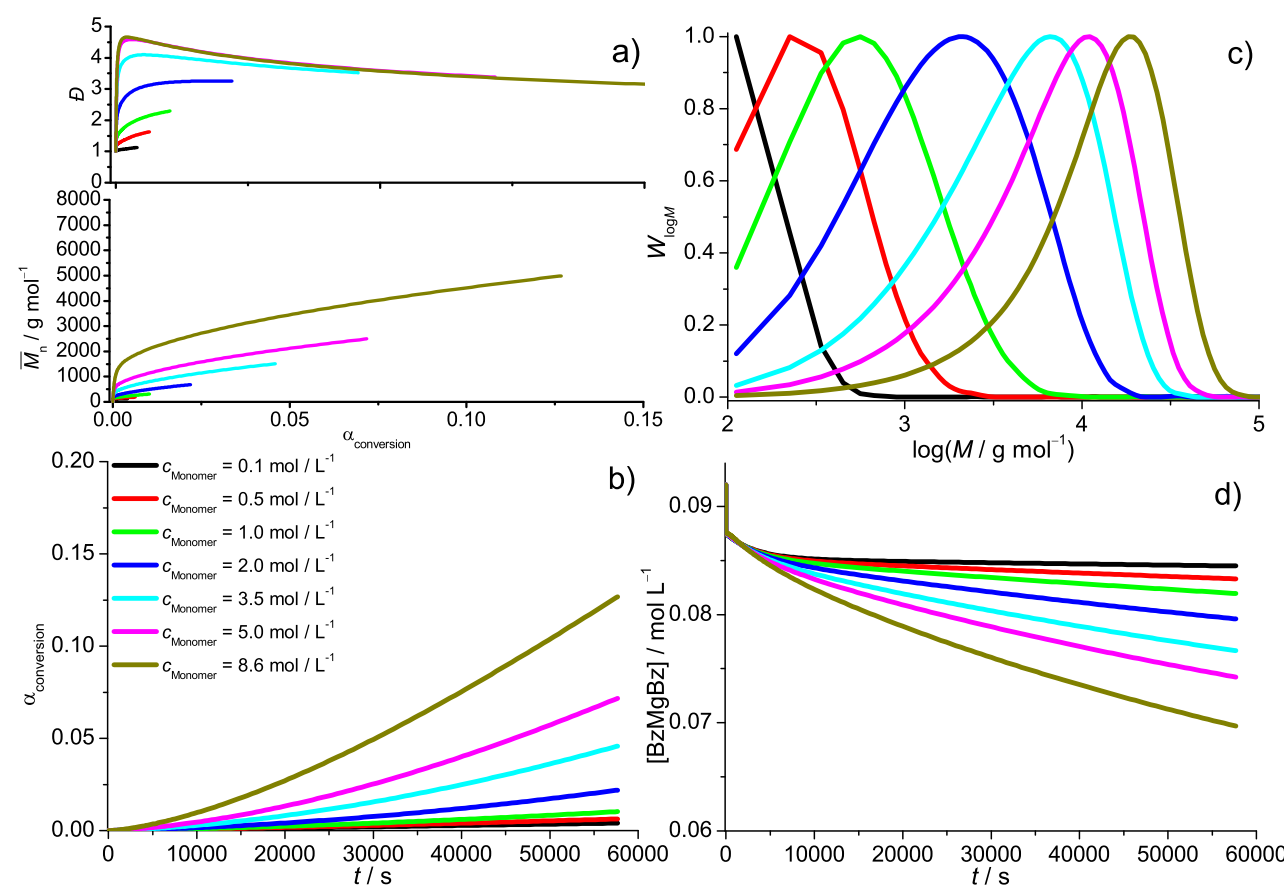

Figure 5.28: Chain length dependent simulation by variation of c(Styrene) based on Table 5.2 and 5.4. a) $\bar{M}_{\mathrm{n}}$ and dispersity vs. conversion profile b) conversion vs. time profile c) MMD profile d) BzMgBz concentration profile

In conclusion, a process is found which can be influenced by different types of reactions. One important factor is the first insertion of monomer, which limits the success of the controlled process. Additionally, the subsequent monomer addition steps can still be chain-length dependent leading to a broad MMD. The systematic determination showed that the production of the high molar mass polymer and the limited control is mainly caused by the reaction rate of alkylation and the rate coefficients of the exchange processes. If these parameters do not fit well, a time span is found where more active species are formed than can directly undergo the association reaction. This leads to a higher possibility for monomer addition and an increase of conversion at lower catalyst concentrations. As a combination of these results, a simulation was performed with the coefficients shown in Table 5.5 (see Figure 5.29). Improved control of polymerization could be found, yielding a product with dispersity below 2 and linear increase of molecular mass with conversion. Important to notice is the fact that $k_{\text {alkyl1 }}$ has minor influence on the dispersity (see Figure 5.29). The major effects were caused by the slow exchange 
reactions and monomer activation.
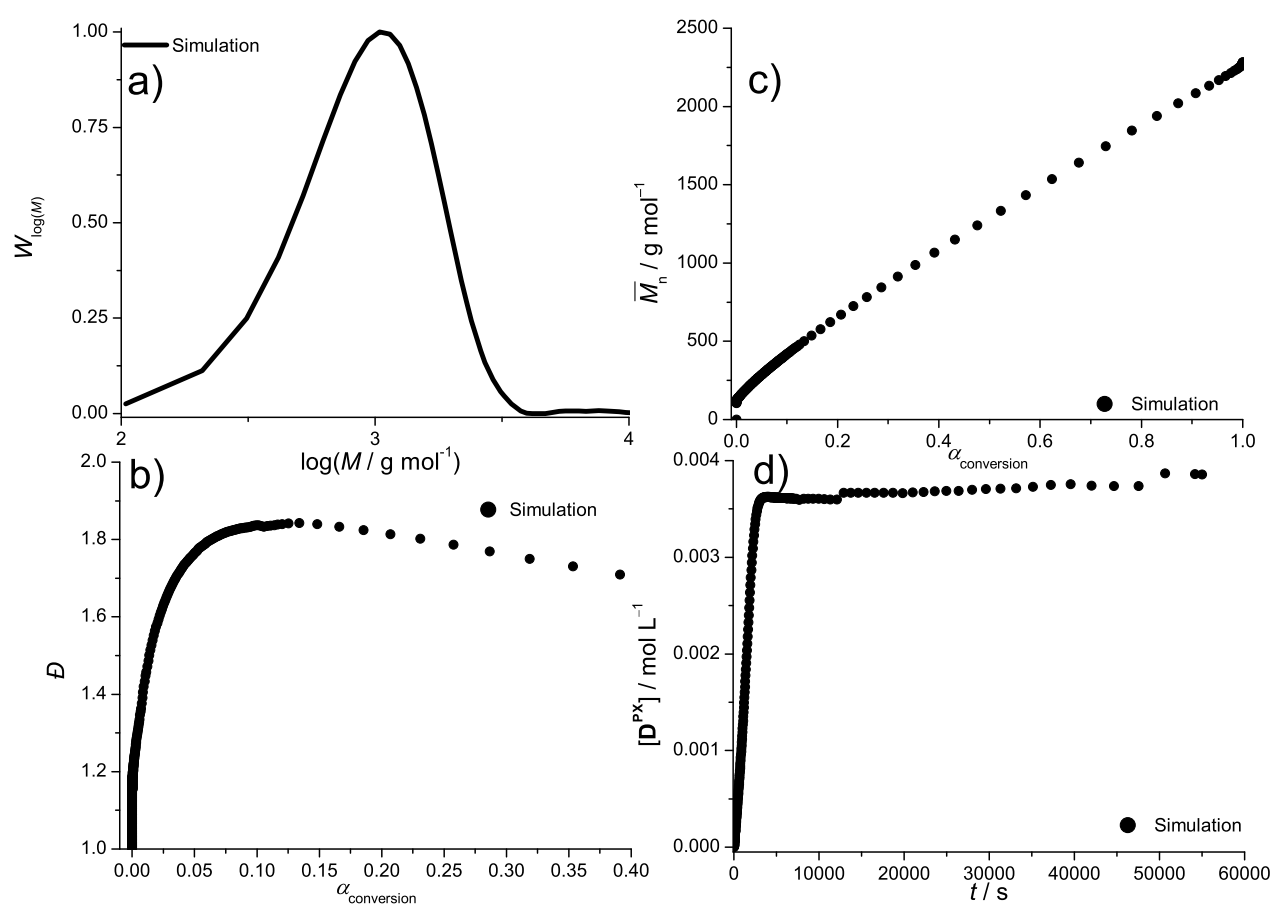

Figure 5.29: Chain-length dependent simulation based on Table 5.2 and 5.5. a) MMD profile b) dispersity profile c) conversion vs. time profile d) $\mathbf{D}^{\mathbf{P x}}$ concentration vs. time profile.

The model is able to perform controlled polymerization but the success of the model system is limited and highly influenced by the $\mathrm{Zr}: \mathrm{Mg}$ ratio as well as the concentration of the magnesium compound. Effects like the chain-length dependent chain growth lead to additional broadening of the MMD.

\subsubsection{Further NMR Polymerization Studies}

Polymerization in the presence of THF The influence of ether on the kinetic behavior shown before in the monomer-free system was further studied in the presence of small amounts tetrahydrofuran. Due to a failure of the gas cleaning system it was accidentally present within the glovebox atmosphere and was introduced into the samples during sample preparation. The ether concentration is therefor determined in situ. It was found that the system is strongly sensitive to THF compared to DBE (see Figure 5.30). The signal of $\mathbf{A}$ is present during the whole time of measurement as well as the signal of $\mathbf{B}$ for $c(\mathrm{THF})=0.114 \mathrm{~mol} \mathrm{~L}^{-1}$. The signal set of $\mathbf{D}$ occurs with proceeding time and at high reaction times species $\mathbf{C}$. The signal of $\mathrm{BzMgBz}$ shows a splitting, the exact reason for this is unknown. It indicates that THF may be strongly influencing the magnesium compound. 
Table 5.5 Changed parameters for Predici simulation of model CCG polymerization at $70^{\circ} \mathrm{C}$, (a) $c\left(\mathrm{Cp}_{2}{ }_{2} \mathrm{ZrCl}_{2}\right)=0.0046 \mathrm{~mol} \mathrm{~L}^{-1} c(\mathrm{BzMgBz})=0.092 \mathrm{~mol} \mathrm{~L}^{-1}$ in toluene- $d 8$ and $c$ (styrened8) $=3.5 \mathrm{~mol} \mathrm{~L}^{-1}$.

\begin{tabular}{c|c|c|} 
coefficients & value & unit \\
\hline$k_{\mathrm{ex} 0}$ & 5.7 & $\mathrm{~L} \mathrm{~mol}^{-1} \mathrm{~s}^{-1}$ \\
$k_{\mathrm{ex} 1}, k_{\mathrm{ex} 2}$ & 31.5 & $\mathrm{~L} \mathrm{~mol}^{-1} \mathrm{~s}^{-1}$ \\
$k_{\mathrm{act} 0}$ & 0.01 & $\mathrm{~s}^{-1}$ \\
$k_{\mathrm{act} 1}, k_{\mathrm{act} 2}$ & 0.9 & $\mathrm{~s}^{-1}$ \\
$k_{\mathrm{add}}, k_{\mathrm{add} 2}$ & 5 & $\mathrm{~L} \mathrm{~mol}^{-1} \mathrm{~s}^{-1}$ \\
$k_{\mathrm{frag}}, k_{\mathrm{frag} 2}$ & 2 & $\mathrm{~s}^{-1}$ \\
$k_{\mathrm{p} 1}$ & 0.01 & $\mathrm{~s}^{-1}$ \\
$k_{\mathrm{p}, \infty}$ & 0.31 & $\mathrm{~s}^{-1}$ \\
$a$ & 0.08 &
\end{tabular}

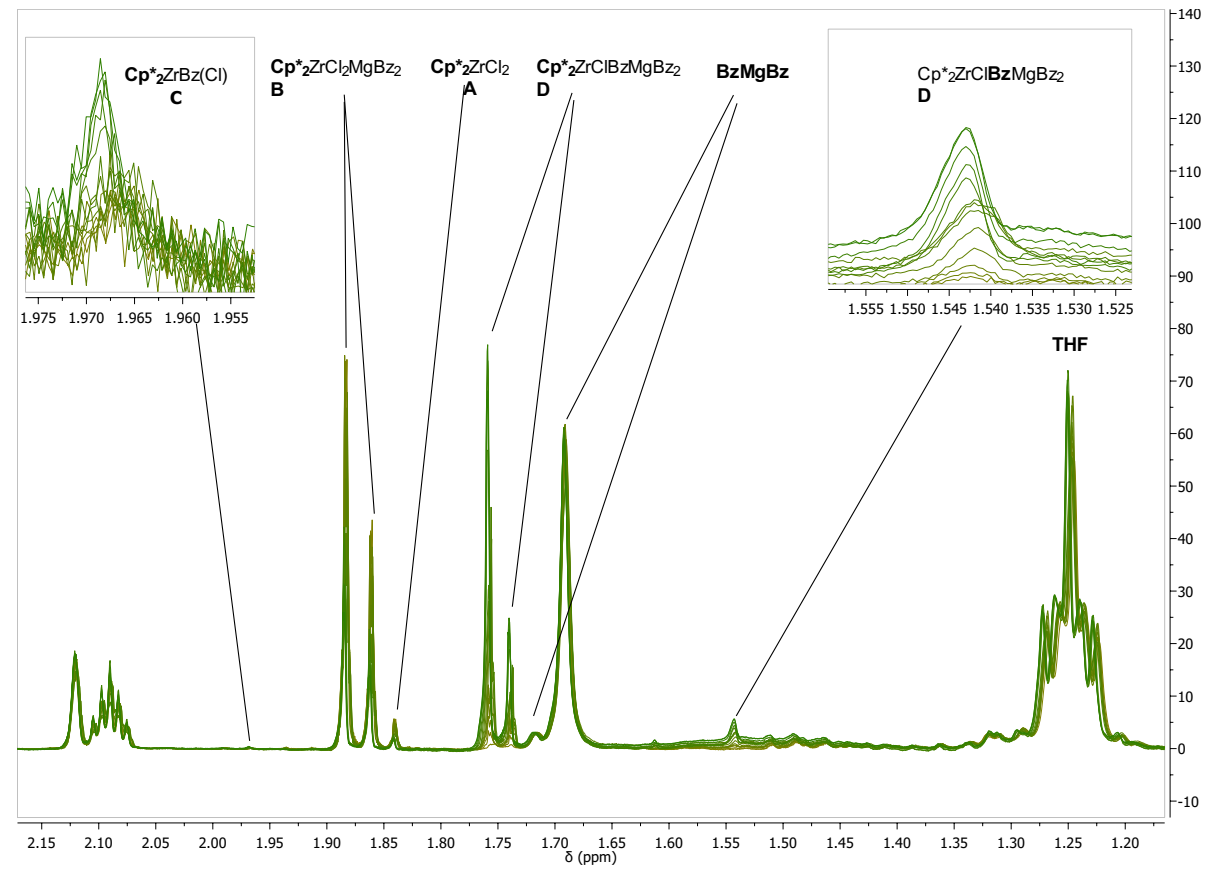

Figure 5.30: ${ }^{1} \mathrm{H}-\mathrm{NMR}$ studies on CCG polymerization in thr presence of $\mathrm{THF}$ at $70^{\circ} \mathrm{C}$, $\mathrm{c}\left(\mathrm{Cp}_{2}{ }_{2} \mathrm{ZrCl}_{2}\right)=0.0046 \mathrm{~mol} \mathrm{~L}^{-1}, c(\mathrm{BzMgBz})=0.042 \mathrm{~mol} \mathrm{~L}^{-1}, c(\mathrm{THF})=0.114 \mathrm{~mol} \mathrm{~L}^{-1}$ in toluene- $d 8$ and $\mathrm{c}($ styrene- $d 8)=5.2 \mathrm{~mol} \mathrm{~L}^{-1}$ 
At lower concentrations of THF this splitting does not occur. Two measurements were performed. One with a THF concentration of $c_{\mathrm{THF}}=0.114 \mathrm{~mol} \mathrm{~L}^{-1}$ and the second with $c_{\mathrm{THF}}=0.02 \mathrm{~mol} \mathrm{~L}^{-1}$. Because the Glovebox atmosphere contained additional molecules further, very weak signals are found which could not be characterized. Species $\mathbf{F}$ and $\mathbf{E}$ cannot be found in the spectrum. The analysis via NMR leads to results shown in Table 5.6 .

Table 5.6 Parameter determined by NMR for CCG polymerization of styrene- $d 8$ at $70{ }^{\circ} \mathrm{C}$, a) $c\left(\mathrm{Cp}_{2}{ }_{2} \mathrm{ZrCl}_{2}\right)=0.00385 \mathrm{~mol} \mathrm{~L}^{-1}, c(\mathrm{BzMgBz})=0.042 \mathrm{~mol} \mathrm{~L}^{-1}, c(\mathrm{THF})=0.114 \mathrm{~mol} \mathrm{~L}^{-1}$ in toluene$d 8$ with $c($ styrene- $\left.d 8)=4.1 \mathrm{~mol} \mathrm{~L}^{-1} ; \mathrm{b}\right) c\left(\mathrm{Cp}^{*}{ }_{2} \mathrm{ZrCl}_{2}\right)=0.0046 \mathrm{~mol} \mathrm{~L}^{-1}, c(\mathrm{BzMgBz})=0.042 \mathrm{~mol} \mathrm{~L}^{-1}$, $c(\mathrm{THF})=0.02 \mathrm{~mol} \mathrm{~L}^{-1}$ in toluene- $d 8$ with $c($ styrene- $d 8)=4.9 \mathrm{~mol} \mathrm{~L}^{-1}$

\begin{tabular}{c|c|c|l} 
coefficient & a) & b) & unit \\
\hline$K_{\mathrm{Zr}, 1}$ & 6.9 & 29 & $10^{2} \mathrm{~L} \mathrm{~mol}^{-1} \mathrm{~s}^{-1}$ \\
$K_{\mathrm{Zr}, 2}$ & 73 & 40 & $10^{2} \mathrm{~L} \mathrm{~mol}^{-1} \mathrm{~s}^{-1}$ \\
$k_{\mathrm{alkyl} 1}$ & 0.2 & 1.1 & $10^{-4} \mathrm{~s}^{-1}$
\end{tabular}
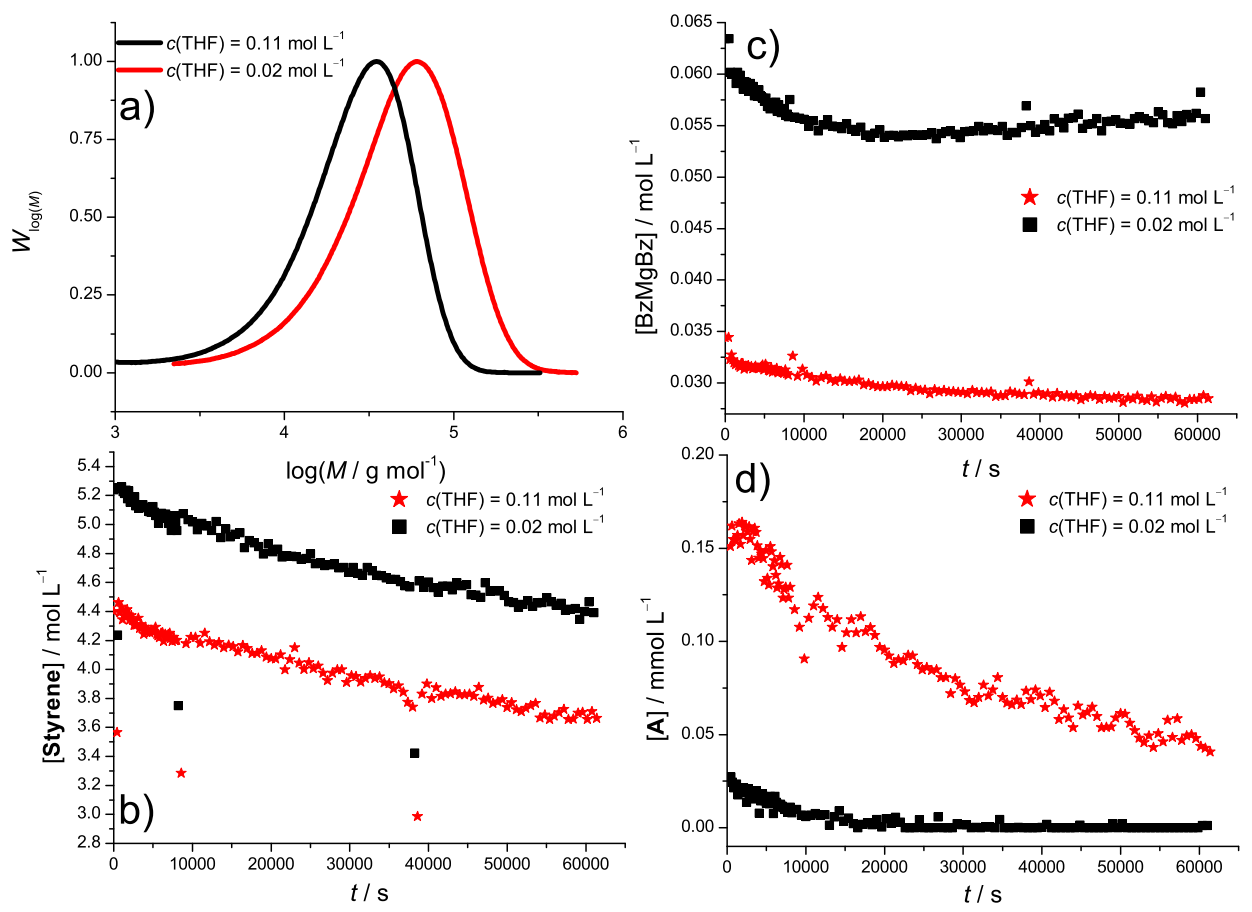

Figure 5.31: MMD a) derived by GPC. Concentration vs.time profiles of monomer concentration $b$ ), BzMgBz c) and A d) derived by NMR of CCG activation with THF present at $70{ }^{\circ} \mathrm{C}$. For experimental parameters see Table 5.6

The different concentration vs. time profiles for both polymerizations are summarized in Figures 5.31 and 5.32 . The reaction rates and equilibrium constants are strongly 

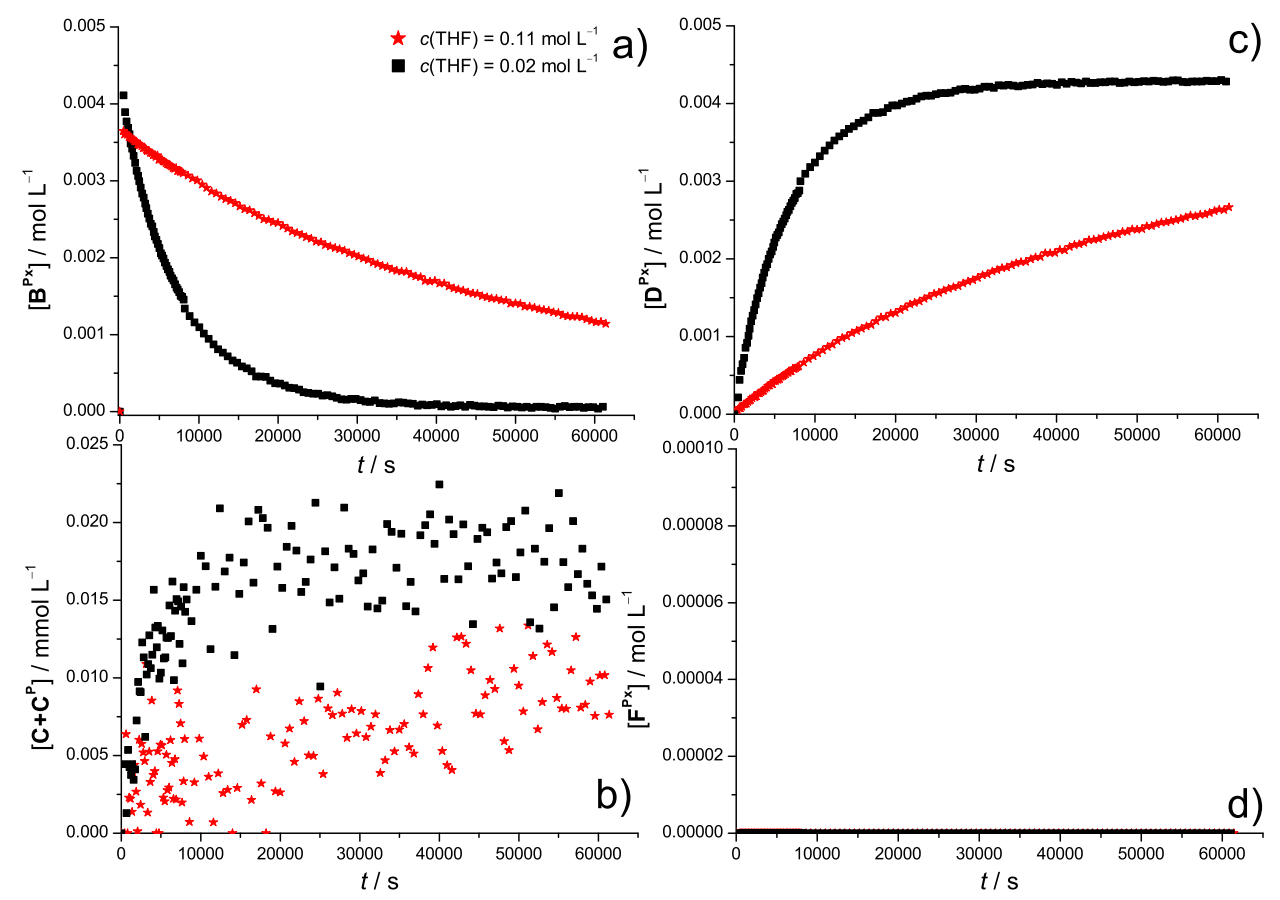

Figure 5.32: Concentration/time profiles of $\mathbf{B}(\mathrm{a})), \mathbf{C}(\mathrm{b})), \mathbf{D}(\mathrm{c})$ ) and $\mathbf{F}(\mathrm{d})$ ) derived by NMR of CCG activation with THF present at $70^{\circ} \mathrm{C}$. For experimental parameters see Table 5.6

influenced by the additional THF. The second alkylation step is in both cases completely missing. It was tried to model the polymerization based on the given model. It was found impossible to simulated the strong monomer uptake within the first minutes of polymerization especially at higher THF concentration. This indicates a change within the mechanistic scheme in the presence of THF towards an unknown activation mechanism. While the addition of DBE does not change the equilibrium in an equal manner, even small amounts of THF can lead to a complete different result.

Polymerization with BuMgOct as co-catalyst As already investigated in absence of monomer, a NMR study with BuMgOct was performed to evaluate the occurring species in presence of different co-catalysts. The system is much more complex with BuMgOct than with BzMgBz. Three signals are found in the first minutes of measurement, which are transferred into different sets of signals yielding a stable setup with proceeding time (see Figure 5.33). The different peaks could be characterized as a part of three reaction stages during polymerization. The peaks labeled with a blue dot were found in the first minutes of the measurement (5,7 and 9) and shortly afterwards the peak at the cyan colored dot (12) is formed. These signals represent the alkylated species similar to $\mathbf{B}$ in presence of $\mathrm{BzMgBz}$. The $\beta$-hydride elimination is present for the alkylated complex, which occurs rapidly. The complete set of peaks is similar to monomer free activation. 
Different to the monomer free system, these signals are transferred into the signals with the green dots $(2,3,13$ and 14$)$. These were intermediate species and it was assumed that they are similar to the $\mathbf{D}$ complexes which are consumed with increasing reaction time. They are either fully consumed or overlaid with a new set of signals (red dots; $1,4,6,8,10$ and 11).

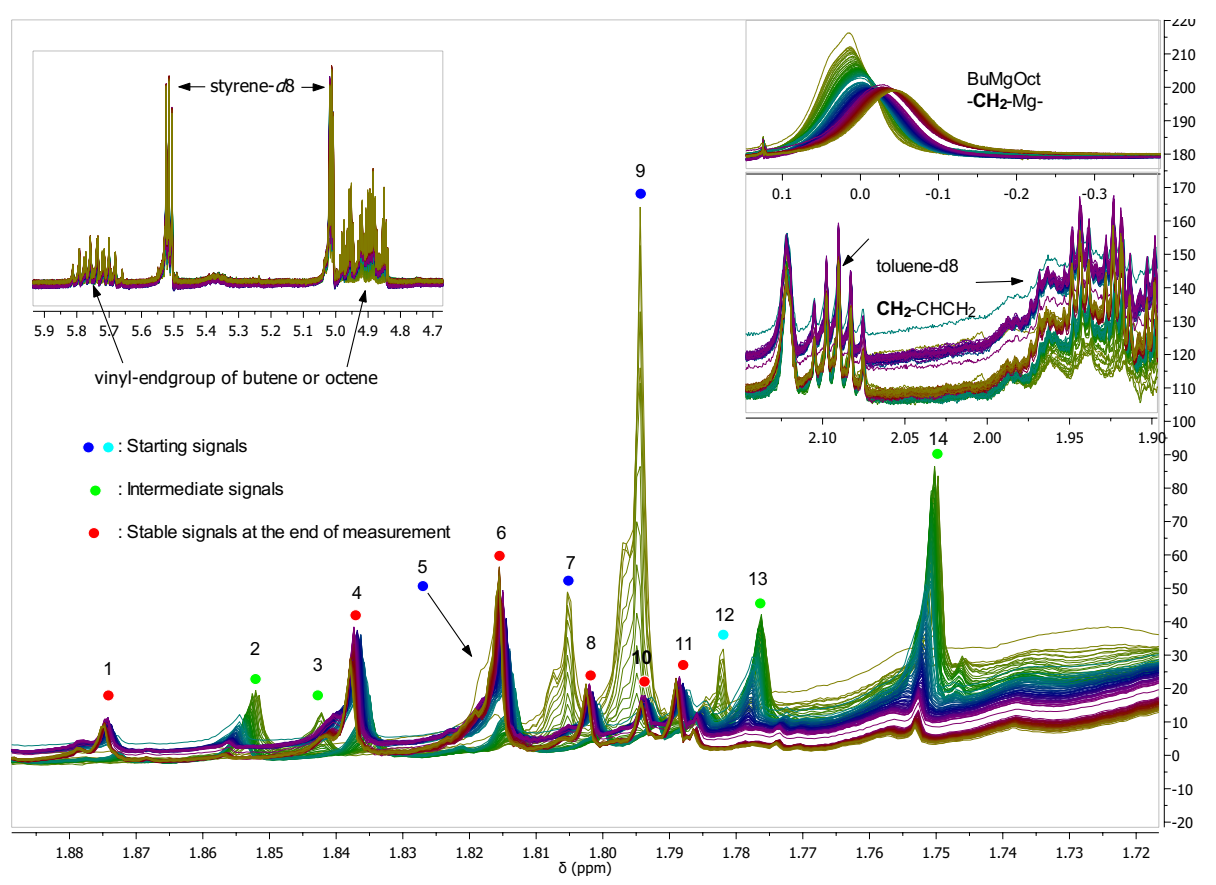

Figure 5.33: Polymerization of styrene-d8 with A and BuMgOct as co-catalyst. The different occurring species are numbered and labeled with colored dots: blue and cyan signals in the beginning, green intermediate species and red species at high reactions times. Additionally the signals of the monomer, the magnesium-carbon bond of the co-catalyst and the termination product of the alkyl chains are shown.

These signals describe the resting state of the proceeding polymerization process. Because in literature di-alykl complexes ${ }^{[273,295]}$ show similar chemical shifts than the dichloro complexes, these species were assumed to be $\mathbf{E}$ and $\mathbf{F}$ analogous species, which fits with the overall process found. The vinyl signal of the alkyl chain is found within the first minutes of the reaction. This indicates that the $\beta$-hydride elimination of alkyl chains is faster than monomer coordination at elevated temperatures, leading to a new kind of activation mechanism. In comparison to the experiments with BzMgBz the alkyl chain is transferred to the complex rather quickly and the elimination process takes place, yielding a hydride complex. These complexes, despite the assumption made for modeling are high reactive for the insertion of styrene into the $\mathrm{Zr}-\mathrm{H}$ bond to a complex similar $\mathbf{C}^{\mathbf{P}}$. This event starts the new polymer chain with only the styrene bonded. An overview of the integrals of the different species is shown in Figure 5.34. Compared to the activation in absence of monomer the signals around the toluene- $d 8$ peak are not 
found, which indicates that this reaction pathway is blocked in the presence of monomer.
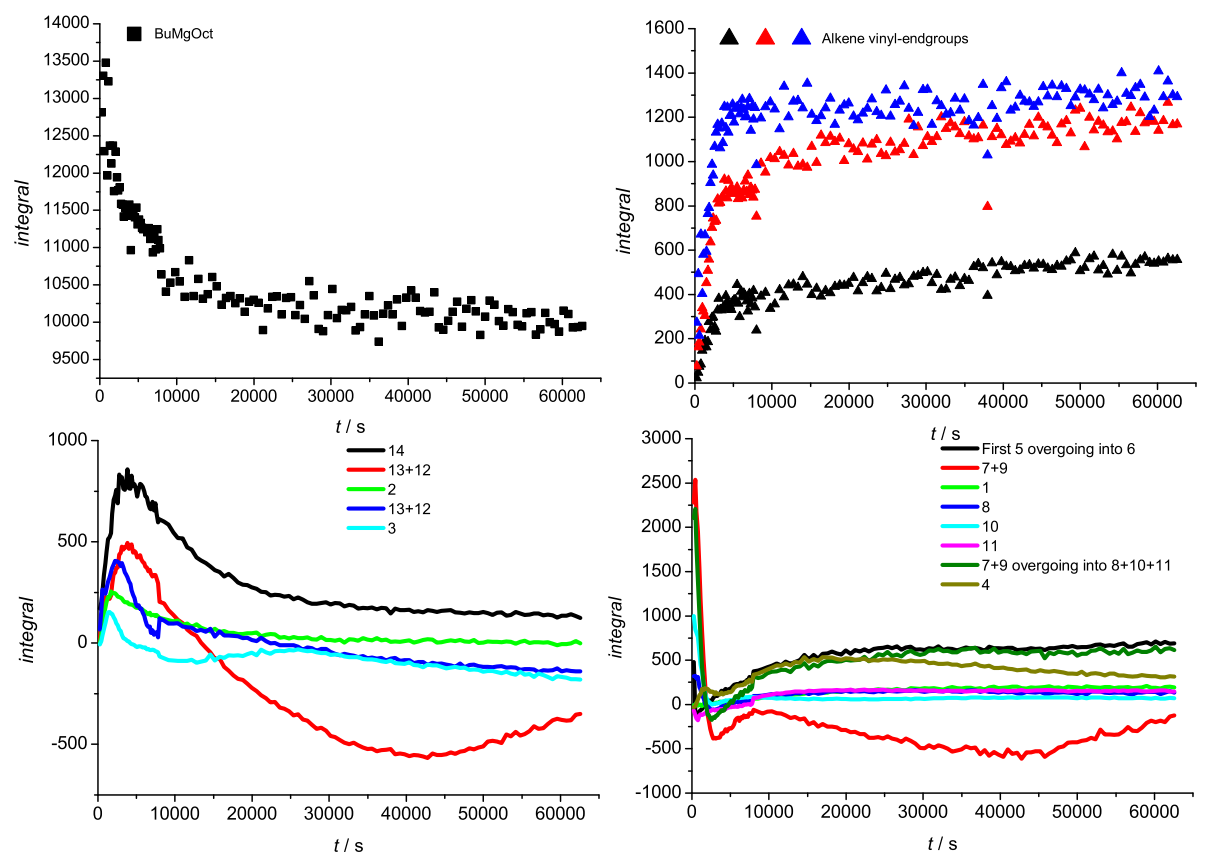

Figure 5.34: Integrals for CCG-polymerization of styrene- $d 8$ with $\mathbf{A}$ and BuMgOct $c\left(\mathrm{Cp}_{2}{ }_{2} \mathrm{ZrCl}_{2}\right)=0.0046 \mathrm{~mol} \mathrm{~L}^{-1}, c(\mathrm{BuMgOct})=0.176 \mathrm{~mol} \mathrm{~L}^{-1}$ in toluene- $d 8$ with $\mathrm{c}$ (styrened8) $=5.3 \mathrm{~mol} \mathrm{~L}^{-1}$.

The exact determination in different species was not possible as well as the calculation of the concentration. Due to the overlay of different species an integration was not successful for the single peak, which would lead to erroneous concentration especially for the peaks 8,10 and 11 as well as 12 and 14 . The results indicate that $\beta$-hydride elimination of alkyl chains is faster by several orders of magnitude than for styrenic monomers, which makes the application of the zirconium based catalyst unsuitable for ethylene polymerization. It was therefore decided to model the process according to the determined model only where most of the species are similar to the model system BzMgBz. Therefore two new reaction steps were introduced which allow the termination of complex $\mathbf{C}$ and the reactivation of the hydride catalyst $\mathbf{G}$ to species $\mathbf{C}^{\mathbf{P}}$ by addition of monomer. The termination is introduced as an "elemental" reaction and has a different rate coefficient then the polymer chain, because the alkyl chain can form the necessary transition state easier. The monomer addition to complex $G$ is introduced via a "initiation(anion)" step starting a polymer with a chain length of one.

$$
\mathbf{C} \stackrel{k_{\mathrm{t}, \mathrm{alkyl}}}{\longrightarrow} \mathrm{G}
$$




$$
\mathbf{G}+\text { Monomer } \stackrel{k_{\text {reini }}}{\longrightarrow} \mathbf{C}^{\mathbf{P}}
$$

The process could be described successfully by modeling with regard to the MMD, BuMgOct consumption and the indicated concentration profiles. As starting point the previous determined rate coefficients were chosen. The reaction kinetics with polymer chains present were considered independent from the alkyl chain. Because it was not possible to determine the exact concentrations, the chosen rate coefficients are of limited accuracy (see Table 5.7) and plotted against normalized integrals determined in NMR (see 5.35).
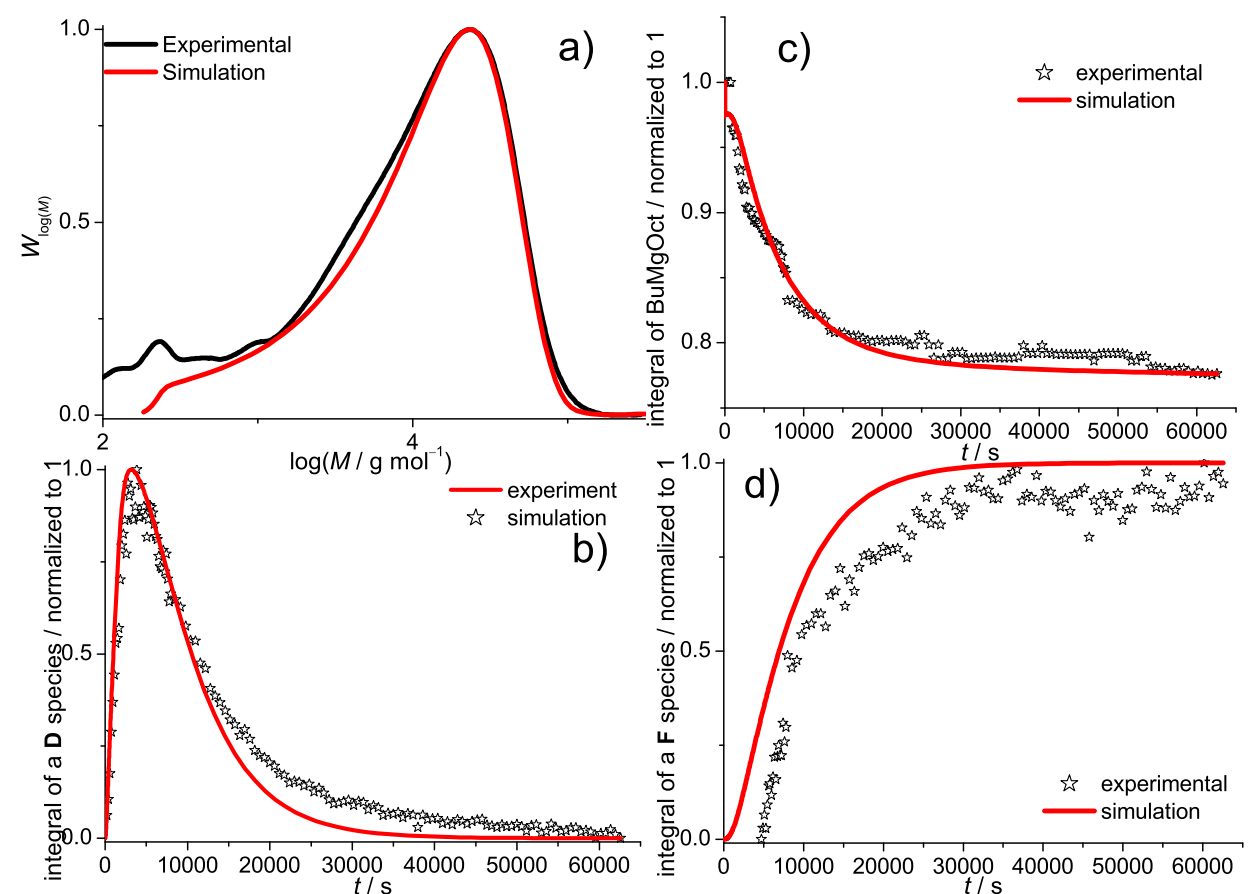

Figure 5.35: Comparison between experimentally found results and simulation for CCG polymerization of styrene- $d 8$ with $\mathbf{A}$ and BuMgOct $c\left(\mathrm{Cp}^{*}{ }_{2} \mathrm{ZrCl}_{2}\right)=0.0046 \mathrm{~mol} \mathrm{~L}^{-1} c(\mathrm{BuMgOct})=0.176 \mathrm{~mol} \mathrm{~L}^{-1}$ in toluene- $d 8$ with $c($ styrene- $d 8)=5.3 \mathrm{~mol} \mathrm{~L}^{-1}$.

The determined fit describes the system well and it was found that the zirconium system can easily be deactivated by more reactive co-catalysts via the second alkylation pathway. This limits the accessible co-catalysts. These results show experimentally that dialkylation by a co-catalyst is a notable deactivation pathway for CCG polymerization, if no additional activator is applied. An activator like MAO will likely reform the catalytic active species by alkyl abstraction. Secondly, the reaction system can be activated via the termination of the alkyl-chain, leading to a new propagating species. The species $\mathrm{G}$ regarded as unreactive and the model for further simulation in the next chapter has to be extended by this step. Termination only stops chain growth and does not affect 
Table 5.7 Parameter applied for simulation of NMR-integrals of CCG polymerization of styrene- $d 8$ with $\mathrm{A}$ and BuMgOct $c\left(\mathrm{Cp}^{*}{ }_{2} \mathrm{ZrCl}_{2}\right)=0.0046 \mathrm{~mol} \mathrm{~L}^{-1} c(\mathrm{BuMgOct})=0.176 \mathrm{~mol} \mathrm{~L}^{-1}$ in toluene- $d 8$ with $c($ styrene- $d 8)=5.3 \mathrm{~mol} \mathrm{~L}^{-1}$.

\begin{tabular}{|c|c|c|c|}
\hline coefficient & value & unit & \\
\hline$k_{\mathrm{ex} 0}$ & 16 & $\mathrm{~L} \mathrm{~mol}^{-1} \mathrm{~s}^{-1}$ & Previous exp. ${ }^{[a]}$ \\
\hline$k_{\mathrm{ex} 1}$ & 1.8 & $\mathrm{~L} \mathrm{~mol}^{-1} \mathrm{~s}^{-1}$ & Previous exp. ${ }^{[a]}$ \\
\hline$k_{\mathrm{ex} 2}$ & 1.8 & $\mathrm{~L} \mathrm{~mol}^{-1} \mathrm{~s}^{-1}$ & Simulation \\
\hline$k_{\text {act0 }}$ & 0.01 & $\mathrm{~s}^{-1}$ & Previous exp. ${ }^{[a]}$ \\
\hline$k_{\text {act1 }}$ & 5.14 & $10^{-2} \mathrm{~s}^{-1}$ & Simulation \\
\hline$k_{\text {act2 }}$ & 5.14 & $10^{-3} \mathrm{~s}^{-1}$ & Simulation \\
\hline$k_{\text {alkyl1 }}$ & 8.0 & $10^{-4} \mathrm{~s}^{-1}$ & Simulation \\
\hline$k_{\mathrm{alkyl} 2}, k_{\mathrm{alkyl} 3}$ & 3.0 & $10^{-4} \mathrm{~s}^{-1}$ & Simulation \\
\hline$k_{\mathrm{p} 1}$ & $10^{[b]}$ & $\mathrm{s}^{-1}$ & Simulation \\
\hline$k_{\mathrm{p}}$ & 0.18 & $\mathrm{~s}^{-1}$ & Simulation \\
\hline$k_{\text {add1 }}, k_{\text {add2 }}$ & 5 & $\mathrm{~L} \mathrm{~mol}^{-1} \mathrm{~s}^{-1}$ & Previous exp. ${ }^{[a]}$ \\
\hline$k_{\text {frag1 }}, k_{\text {frag2 }}$ & 2 & $\mathrm{~s}^{-1}$ & Previous exp. ${ }^{[a]}$ \\
\hline$k_{\mathrm{t}, \mathrm{alkyl}}$ & 0.02 & $\mathrm{~s}^{-1}$ & Simulation \\
\hline$k_{\mathrm{t}}$ & 1 & $x 10^{-5} s^{-1}$ & Previous exp. ${ }^{[a]}$ \\
\hline$k_{\text {reini }}$ & 200 & $\mathrm{~s}^{-1}$ & Simulation \\
\hline$k_{\mathrm{ex} 3}$ & 1 & $\mathrm{~L} \mathrm{~mol}^{-1} \mathrm{~s}^{-1}$ & Previous exp. ${ }^{[a]}$ \\
\hline$k_{\text {act3 }}$ & 1 & $10^{-4} \mathrm{~s}^{-1}$ & Previous exp. ${ }^{[a]}$ \\
\hline$k_{\text {Schlenk }}$ & 1000 & $\mathrm{~L} \mathrm{~mol}^{-1} \mathrm{~s}^{-1}$ & Previous exp. ${ }^{[a]}$ \\
\hline$k_{\text {help }}$ & $10^{10}$ & $\mathrm{~L} \mathrm{~mol}^{-1} \mathrm{~s}^{-1}$ & Previous exp..$^{[a]}$ \\
\hline
\end{tabular}

a) See Table 5.4 on page 108 .

b) Simulation is independent from this value due to fast termination of alkyl chains. 
the reaction rate in the presence of monomer. The side reactions found in absence of monomer (see 4.4 on page 73 ) do not occur in the same manner, but cannot be excluded due to the fact that a lower total zirconium integral is found. This lower integral may also the result of baseline effects during integration which cannot be excluded either.

\subsubsection{Conclusion}

The activation scheme defined in chapter 4.3 on page 43 in absence of monomer was successfully extended by processes with monomer being present. The polymerization of styrene was described via a kinetic scheme based on these reactions and monomer addition was introduced. The simulation suggested that the propagation step is chainlength dependent. The processes can easily be influenced by additives like THF which may lead to a different yet not determined reaction scheme. The addition of THF was found to be a disadvantage for the CCG system. Further, the systematic scan of coefficients and concentration revealed a high dependence on the catalyst concentrations and co-catalyst concentration. At low concentrations and ratios between both, the systems runs into an area, where the exchange reaction is too slow and the monomer conversion increases disproportional to the monomer concentration. This effect causes the high molar mass gained during polymerization and limits the success of control in the beginning of the polymerization process. The zirconium-magnesium system is unsuitable for the polymerization of ethylene because the termination of alkyl chains is faster then the exchange reaction. In polymerization of styrene, termination could not be determined. 



\section{Modeling and Simulation of CCG-Systems}

To investigate the process of CCG on a broader basis of catalyst, co-catalyst and their ratios studies were performed by application of UV/Vis- and FT-IR-spectroscopy. These methods allow to trace the monomer conversion and parts of the formed species. For chosen experiments the polymerization was performed additionally in small reaction tubes within a heating block. These tubes were removed after different time intervals to gain information about the MMD at different reaction times. For all kind of samples the conversion was determined gravimetrically. If not stated in separate tables the mean values of Table 5.4 on page 108 are basis for all simulation and modeling approaches.

\subsection{Polymerization of Styrene with $\mathrm{Cp}_{2}^{*} \mathrm{ZrCl}_{2}$ and BzMgBz}

\subsubsection{Polymerization in Absence of DBE}

For the system of $\mathbf{A}$ and $\mathrm{BzMgBz}$ in presence of styrene determination of the complex species with UV/Vis is possible in the same manner as found in the monomer free system (see Section 4.3 .2 on page 56). The absorbance at $400 \mathrm{~nm}$ (see Figure 6.1) reach its maximum within the first minutes of measurement and decreasing with proceeding time. While the absorbance at $400 \mathrm{~nm}$ yields a minimum the absorbance at $473 \mathrm{~nm}$ is increasing until its maximum afterwards it is decreasing. The former isosbestic point (cf. Figure 4.19 on page 57) at around $415 \mathrm{~nm}$ shows slight increase. During all experiments in presence of styrene the baseline shift occurred independent from the applied system, which is caused by formed polystyrene $^{[296]}$ that shows absorbance from $400 \mathrm{~nm}$ to $1000 \mathrm{~nm}$. Based on these results found for the monomer free system the signal at the isosbestic point was 


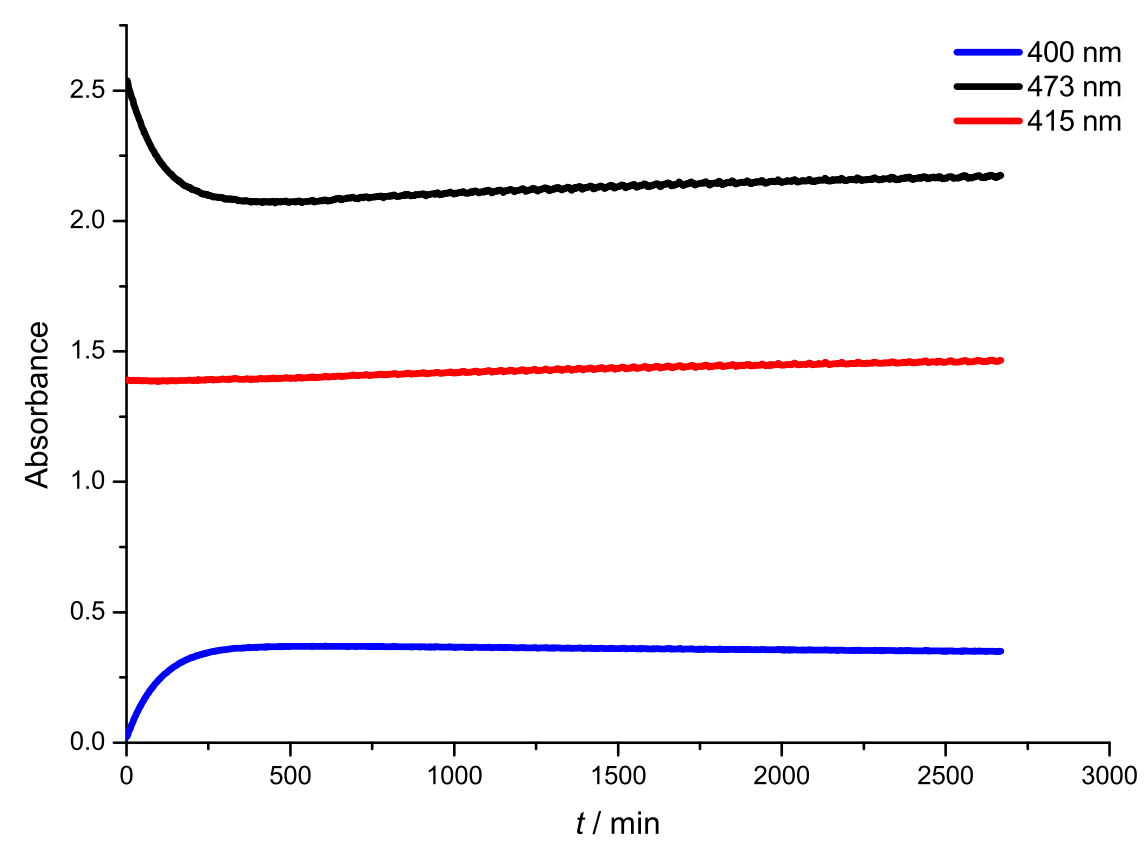

Figure 6.1: $\mathrm{UV} /$ Vis traces for analysis for CCG-polymerization of styrene at $70{ }^{\circ} \mathrm{C}$ with $\mathrm{A}$ and $\mathrm{BzMgBz}$ at Zr:Mg 1:51. $c($ Styrene $)=7.77 \mathrm{~mol} \mathrm{~L}^{-1} ; c\left(\mathrm{Cp}_{2}{ }_{2} \mathrm{ZrCl}_{2}\right)=0.0013 \mathrm{~mol} \mathrm{~L}^{-1} ; c(\mathrm{BzMgBz})=0.066 \mathrm{~mol} \mathrm{~L}^{-1}$.

applied to reduce the influence at the absorbance applied in calculation (see Equation 6.1).

$$
A_{\text {corr }}=\frac{A_{\lambda}}{\frac{A_{i s s s .} .}{A_{i s s o s, \text { max }}}}
$$

The quality of the spectra (see Figure 6.2) is increased while the absorbance values at both measuring wavelength (red) are slightly increased.

The online conversion was determined by online-FT-IR spectroscopy on two IR-bands of styrene. The second and third overtone of the $\mathrm{C}-\mathrm{H}$ stretch vibration of the unsaturated $\mathrm{CH}$-bond is measured. The common application of the first overtone ${ }^{[248}$ at around $6140 \mathrm{~cm}^{-1}$ is not accessible under the chosen conditions, because measurements are performed in sealed $1 \mathrm{~cm}$ cuvettes were the concentration is too high. With introduction into the carbon-metal bond it is transformed into a saturated bond. The force constant is changed and therefore the wavelength. An example for the applied regions is shown in Figure 6.3.

The signals chosen are separated from other signals. The signal in the region at $\left.8950 \mathrm{~cm}^{-1}(6.3, \mathrm{~b})\right)$ has an slight overlay. The exact data handling and conversion into monomer concentration is shown in the experimental part in Section 9.1 .7 on page 198 in detail. 


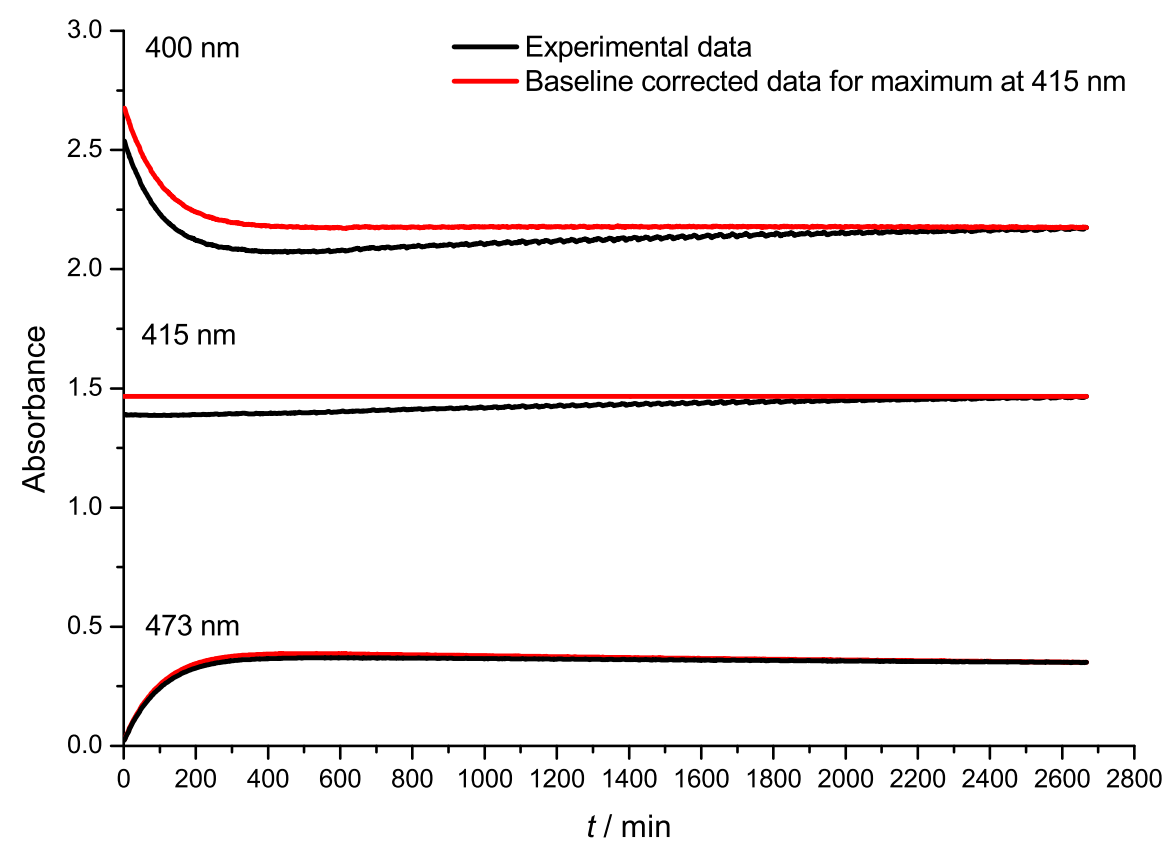

Figure 6.2: Comparison of corrected and uncorrected data at 400, 415 and $473 \mathrm{~nm}$ by equation 6.1 .

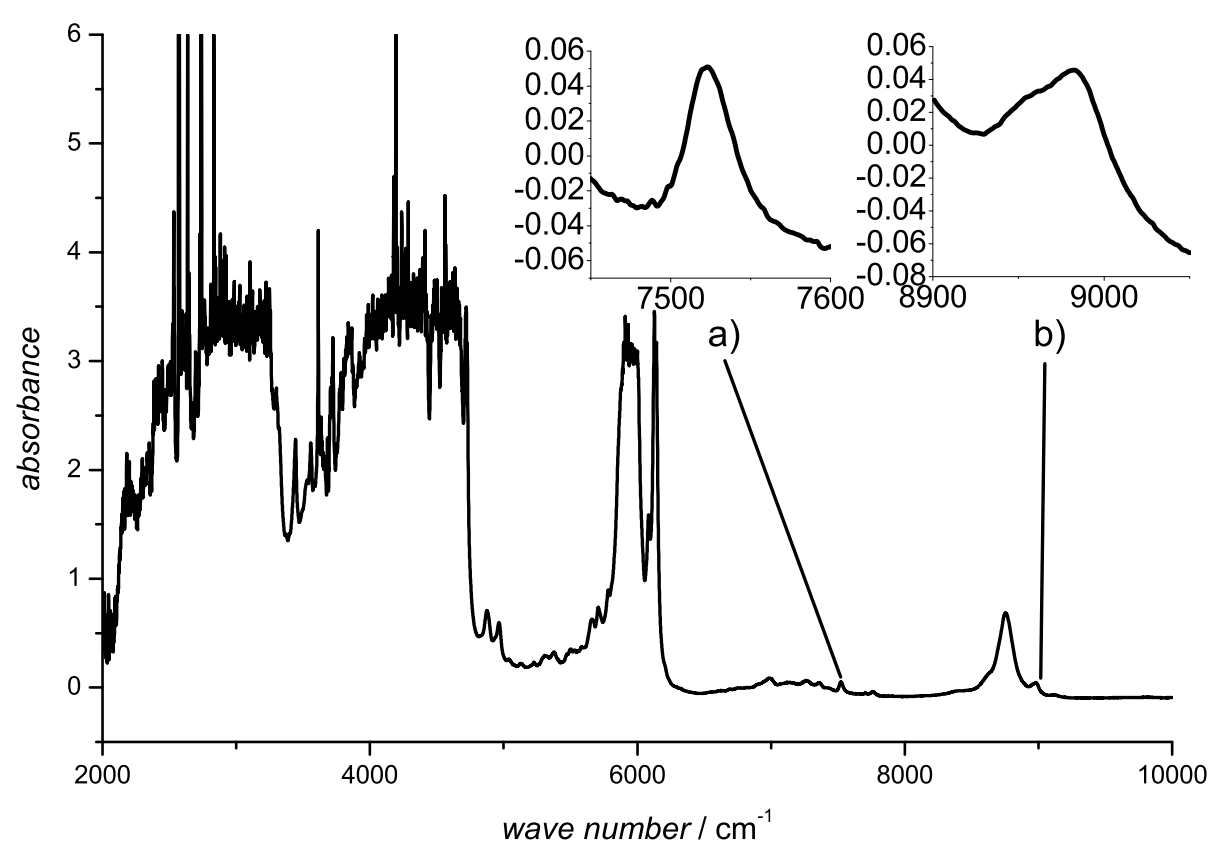

Figure 6.3: Example for the applied IR-bands at $7550 \mathrm{~cm}^{-1}$ and $8950 \mathrm{~cm}^{-1}$ for thr measurement of CCG polymerization with $\mathbf{A}$ and BzMgBz at $\mathrm{Zr}: \mathrm{Mg} 1: 51 . c($ Styrene $)=7.77 \mathrm{~mol} \mathrm{~L}^{-1} ; c\left(\mathrm{Cp}^{*}{ }_{2} \mathrm{ZrCl}_{2}\right.$ )$=0.0013 \mathrm{~mol} \mathrm{~L}^{-1} ; c(\mathrm{BzMgBz})=0.066 \mathrm{~mol} \mathrm{~L}^{-1}$ 
The polymerization of styrene was performed at three different concentrations of BzMgBz. In each case five polymerization flasks and two cuvettes were applied. The results of the gravimetrically determined conversion of all samples are shown in Figure 6.4 .
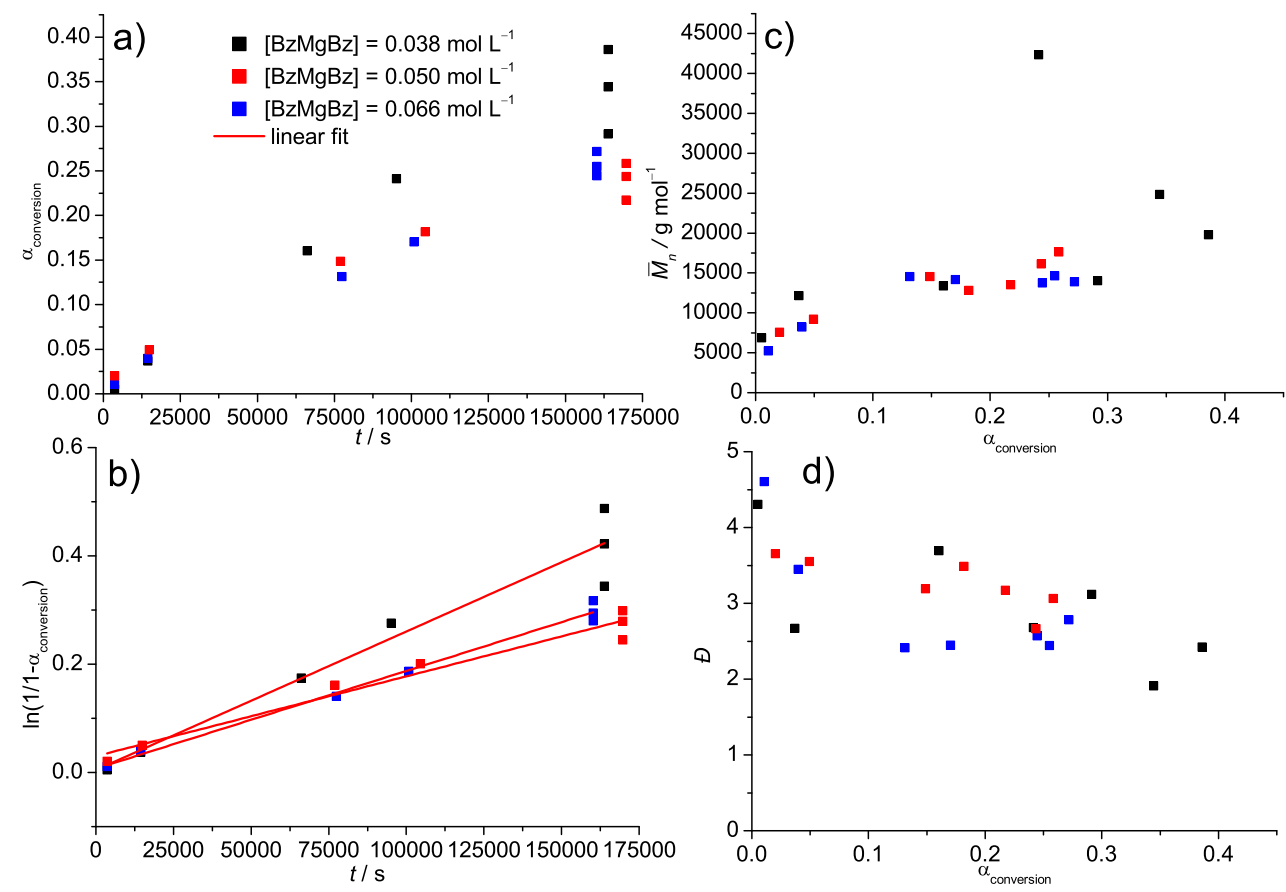

Figure 6.4: CCG-polymerization of styrene at $70^{\circ} \mathrm{C}$ with $\mathbf{A}$ and three different concentrations of BzMgBz. a) conversion vs. time; b) first order plot; c) $\bar{M}_{n}$ vs. conversion; d) dispersity vs. conversion; $c($ Styrene $)=7.77 \mathrm{~mol} \mathrm{~L}^{-1} ; c\left(\mathrm{Cp}_{2}{ }_{2} \mathrm{ZrCl}_{2}\right)=0.0013 \mathrm{~mol} \mathrm{~L}^{-1} ; c(\mathrm{BzMgBz})=0.038 \mathrm{~mol} \mathrm{~L}^{-1}$ (black); $c(\mathrm{BzMgBz})=0.050 \mathrm{~mol} \mathrm{~L}^{-1}(\mathrm{red}) ; c(\mathrm{BzMgBz})=0.066 \mathrm{~mol} \mathrm{~L}^{-1}$ (blue). The black line in $\left.\mathrm{b}\right)$ is only for better understanding.

The conversion is shifted to higher values for the lowest concentration of BzMgBz while the two other are similar (see Figure 6.4, a)). This result is in agreement with the simulation series shown for the influence of $\mathrm{BzMgBz}$ in the previous chapter (cf. Figure 5.27 on page 123). The conversion rate is high in the first minutes of polymerization and levels off to a lower rate with proceeding time. The period afterwards can be well described by a linear first order rate (see Figure 6.4, b)). These results are in agreement with the measured data in NMR, where at long-term experiments similar behavior was found. For $\bar{M}_{n}$ a bow like increase with conversion (see Figure 6.4, $\mathrm{c}$ )) is found which is in agreement with the previous shown results. The values show a slight linear increase with only one value completely out of the range. The dispersity as a function of conversion (see Figure 6.4, d)) shows a decrease with conversion. A clear trend depending on $\mathrm{BzMgBz}$ concentration cannot be found, yet the results indicate that with higher concentrations lower values can be found. The additional collected IR-data (see Figure 
6.5) was compared with the gravimetrically determined data.

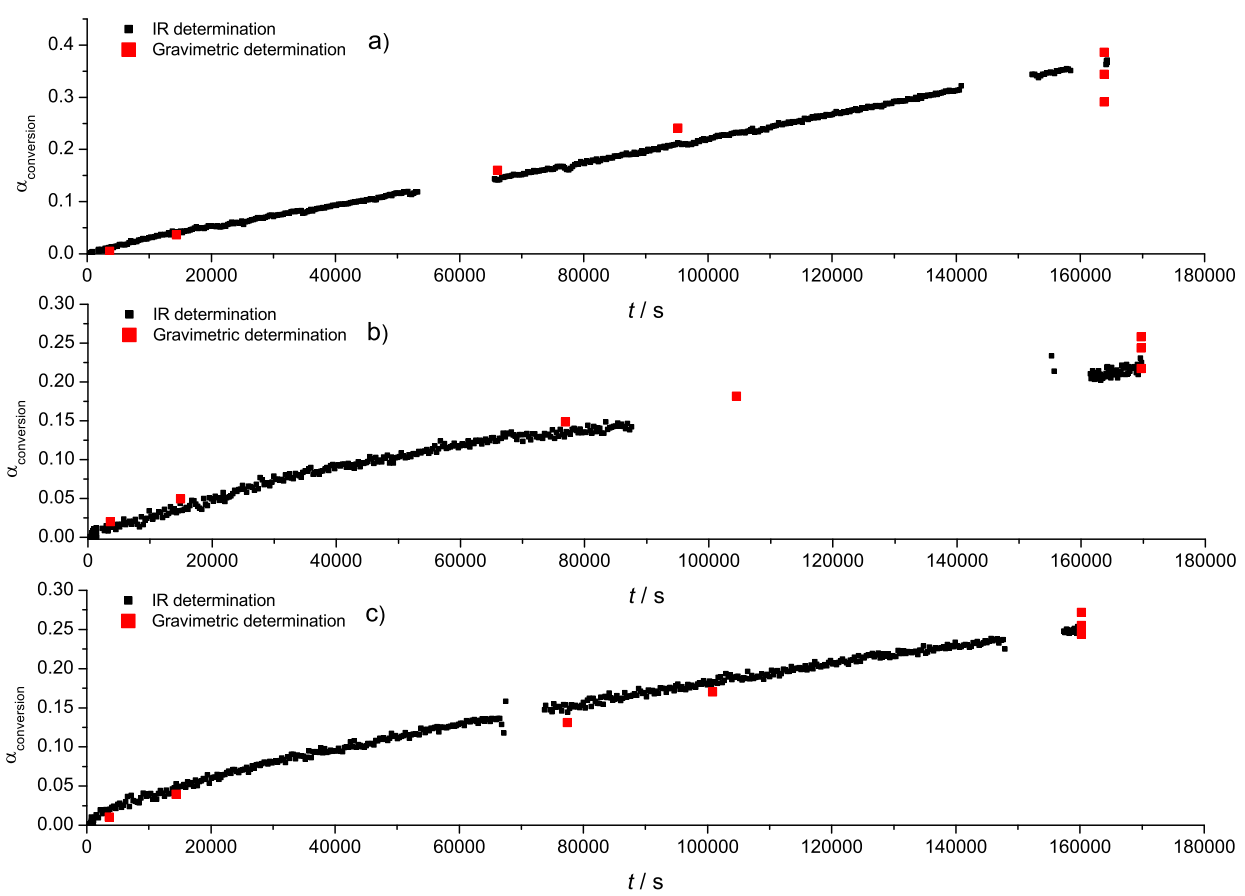

Figure 6.5: Conversion determined via FT-IR spectrocopy for CCG-polymerization of styrene at $70^{\circ} \mathrm{C}$ with $\mathbf{A}$ and three different concentrations of BzMgBz. $\quad c$ (styrene) $=7.77 \mathrm{~mol} \mathrm{~L}^{-1}$; $\left.\left.c\left(\mathrm{Cp}_{2}{ }_{2} \mathrm{ZrCl}_{2}\right)=0.0013 \mathrm{~mol} \mathrm{~L}^{-1} ; c(\mathrm{BzMgBz})=0.038 \mathrm{~mol} \mathrm{~L}^{-1}(\mathrm{a})\right) ; c(\mathrm{BzMgBz})=0.050 \mathrm{~mol} \mathrm{~L}^{-1}(\mathrm{~b})\right) ;$ $\left.c(\mathrm{BzMgBz})=0.066 \mathrm{~mol} \mathrm{~L}^{-1}(\mathrm{c})\right)$.

For all measurements there is a good agreement between gravimetric- and IR-determined conversion and only a small scattering is found. The gravimetric results for the cuvettes are in all cases determined with a higher scattering then the IR-results. This indicates small differences between the individual heating blocks and therefore in the heat transfer. The effect is lower at higher concentrations of BzMgBz. The lowest concentration has the highest conversion while the medium concentration was the slowest polymerization system. The SEC-traces of the heating block polymerizations are shown in Figure 6.6. The results differ slightly in the position of the maxima of the MMDs. While the lowest $\mathrm{BzMgBz}$ concentration (see Figure 6.6 a) gives a broad MMD at low conversion the maximum is increasing during the whole process. For the region from $1000 \mathrm{~g} \mathrm{~mol}^{-1}$ to $10000 \mathrm{~g} \mathrm{~mol}^{-1}$ pronounced shoulders are found at $1 \mathrm{~h}$ and $4 \mathrm{~h}$. For the second concentration (see Figure $6.6 \mathrm{~b}$ ) the shoulder is less pronounced and the positions of the MMD seem to be similar to graph as im a). The result for the third concentration (see Figure 6.6 c) differing by the point, that a maximal MMD is reached that broadens slightly with increasing conversion. In this case the shoulder is stronger then in b) but weaker as in a). The maximum decreases towards higher $\mathrm{BzMgBz}$ 


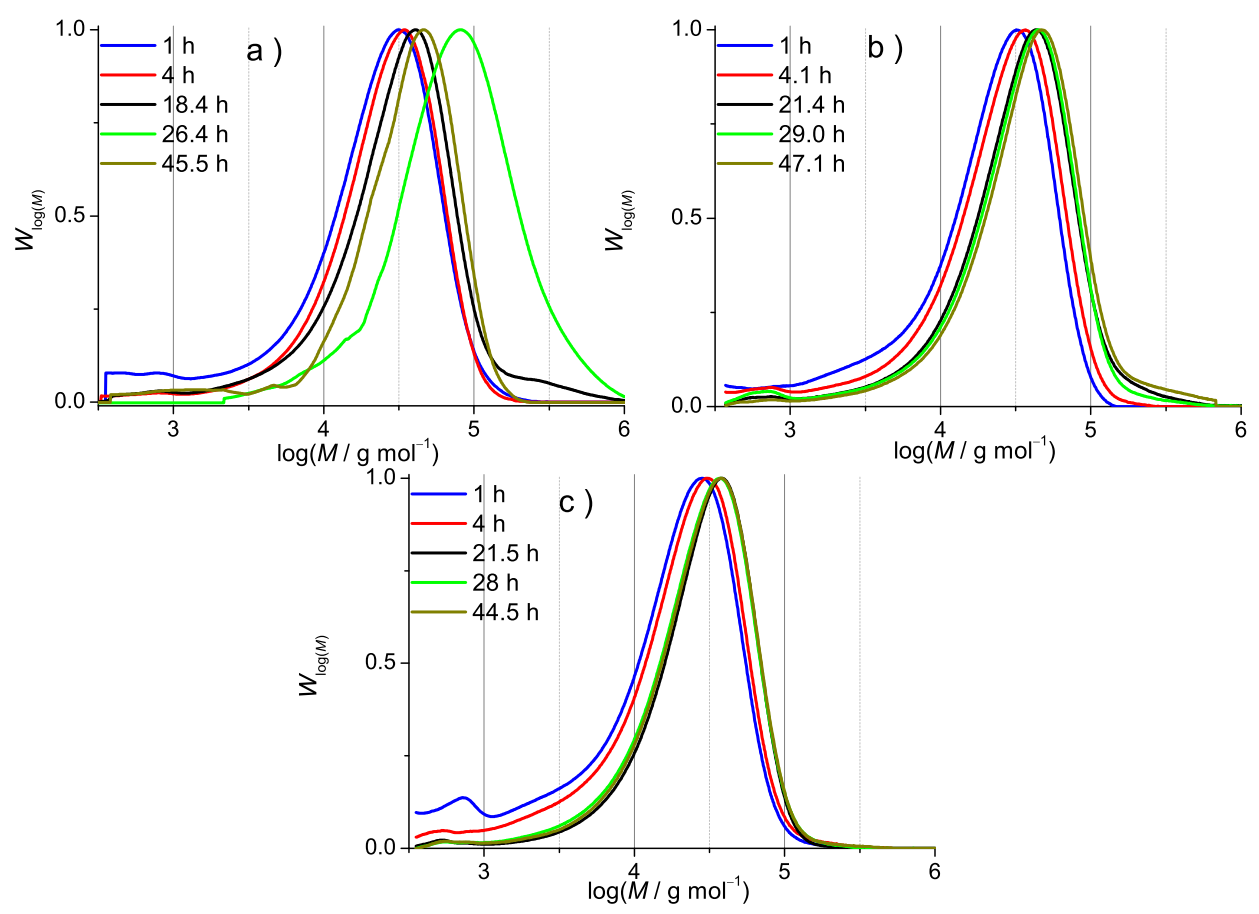

Figure 6.6: Molar mass distributions of CCG-polymerization of styrene at $70{ }^{\circ} \mathrm{C}$ with $\mathrm{A}$ and three different concentrations of BzMgBz. $c$ (Styrene $\left.)=7.77 \mathrm{~mol} \mathrm{~L}^{-1} ; c\left(\mathrm{Cp}_{2}{ }_{2} \mathrm{ZrCl}_{2}\right)=0.0013 \mathrm{~mol} \mathrm{~L}^{-1} ; \mathrm{a}\right)$ $c(\mathrm{BzMgBz})=0.038 \mathrm{~mol} \mathrm{~L}^{-1}$; b $)(\mathrm{BzMgBz})=0.050 \mathrm{~mol} \mathrm{~L}^{-1}$; $\left.\mathrm{c}\right) c(\mathrm{BzMgBz})=0.066 \mathrm{~mol} \mathrm{~L}^{-1}$.

concentrations. The forth measurement of a) is affected by a systematic error. During the sample preparation one flask was accidentally in presence of moisture which may have caused such a behavior.

For comparison between experiment and simulation with the values NMR-determined coefficients in Table 5.4 on page 108 are shown (see Figure 6.7). This results in a big difference found with look on the experimental monomer conversion versus the simulated data. With increasing concentration of $\mathrm{BzMgBz}$ the differences between the experimental and simulated data are systematical higher. While the first curve is fitted in good agreement slightly to higher conversion, the differences get higher with increasing $\mathrm{BzMgBz}$ concentration, mainly induced by an inhibition phase in the beginning of the simulation at short reaction times. The monomer coordination is known to show a kinetic isotopic effect for the formation of the equilibrium and the propagation step. Such effects have already been found for different reactions on the basis of transition metal complexes catalysts ${ }^{[140]}$. To support this assumption a parameter estimation was performed for the three measurements. First of all the alklyation rates where determined via UV/Vis spectroscopy in good agreement with the linear fit (see Figure 6.8 and Table 6.1) similar to the previous shown method. 


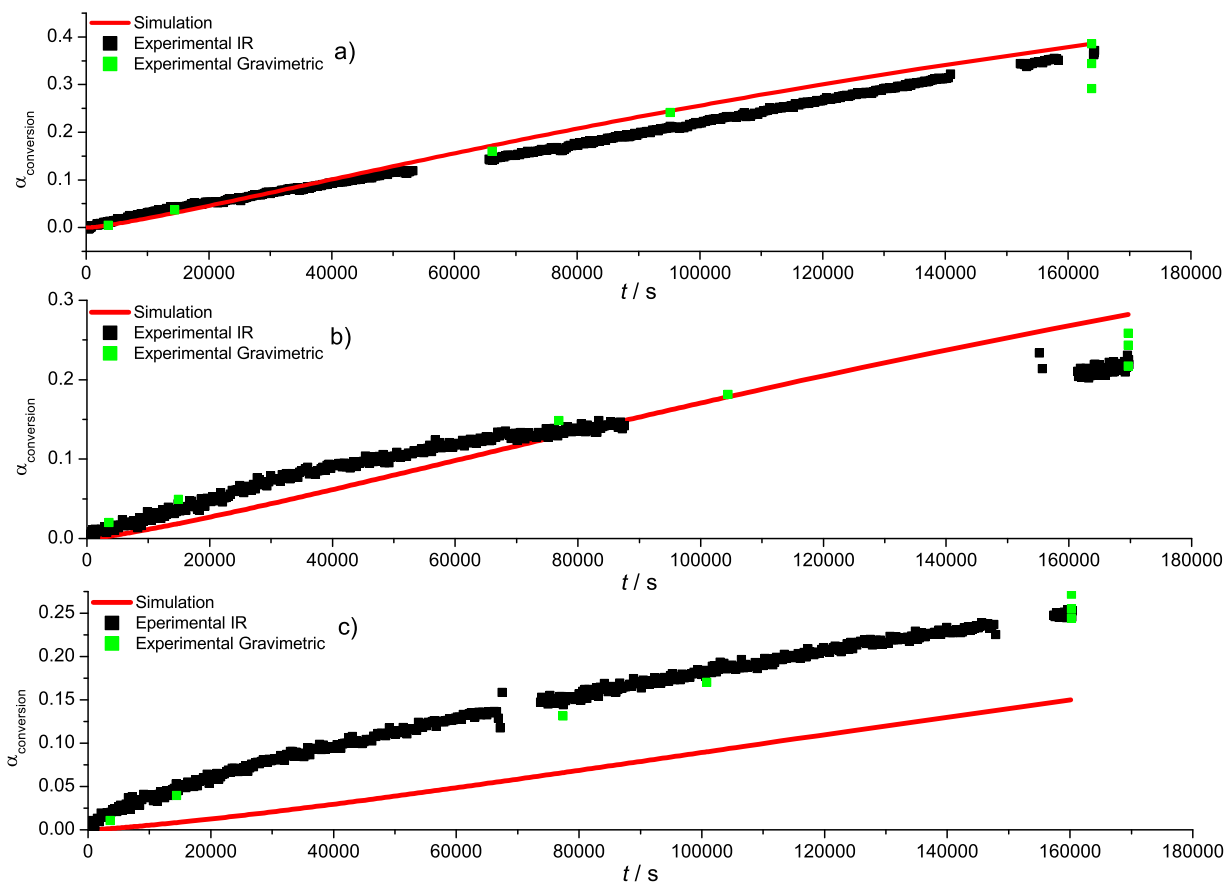

Figure 6.7: Comparsion between experimental conversion from IR- and gravimetric determination and simulation based on Table 5.4 on page 108 at $70^{\circ} \mathrm{C}$ with $\mathbf{A}$ and three different concentrations of BzMgBz. $c($ Styrene $\left.\left.)=7.77 \mathrm{~mol} \mathrm{~L}^{-1} ; c\left(\mathrm{Cp}_{2} \mathrm{ZrCl}_{2}\right)=0.0013 \mathrm{~mol} \mathrm{~L}^{-1} ; \mathrm{a}\right) c(\mathrm{BzMgBz})=0.038 \mathrm{~mol} \mathrm{~L}^{-1} ; \mathrm{b}\right)$ $\left.c(\mathrm{BzMgBz})=0.050 \mathrm{~mol} \mathrm{~L}^{-1} ; \mathrm{c}\right) c(\mathrm{BzMgBz})=0.066 \mathrm{~mol} \mathrm{~L}^{-1}$.

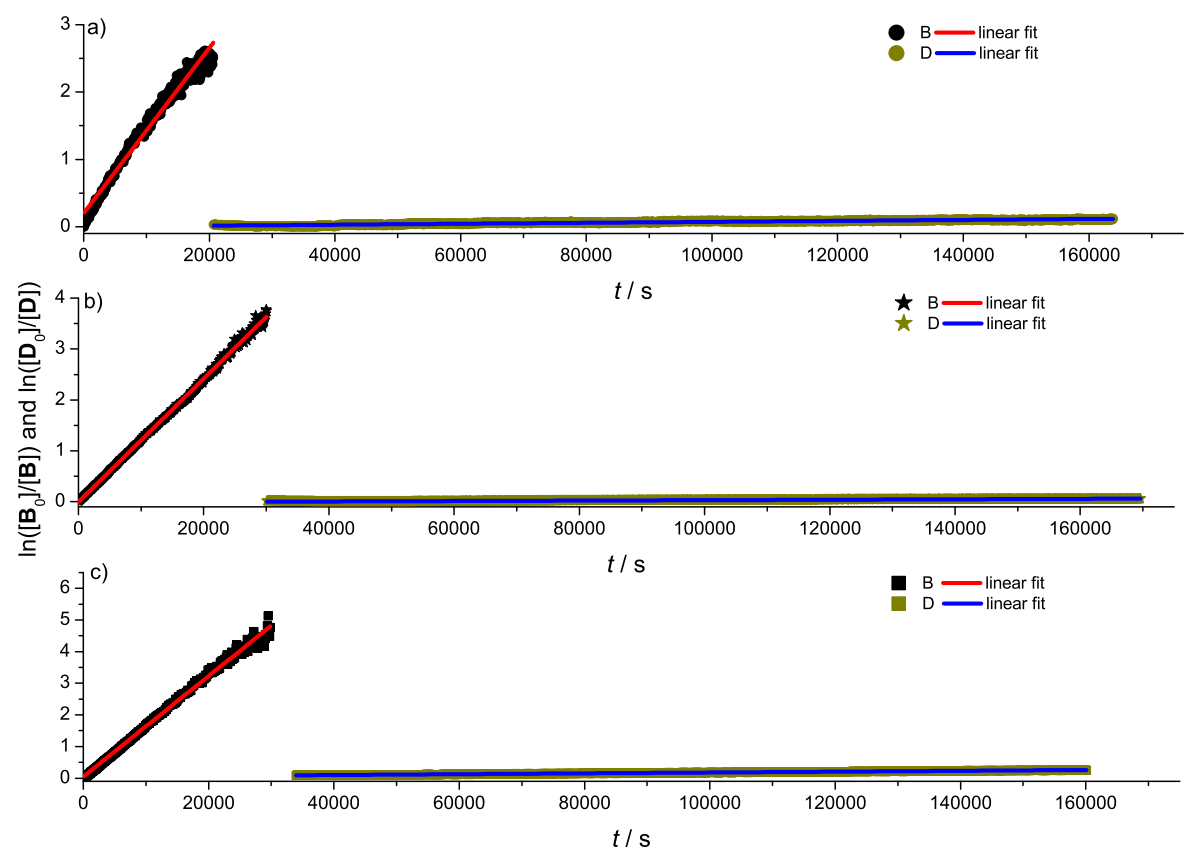

Figure 6.8: Linear fits for determination of rate coefficients for measurements of CCG polymerization of styrene at $70^{\circ} \mathrm{C}$ with $\mathbf{A}$ and three different concentrations of BzMgBz. $c$ (Styrene) $=7.77 \mathrm{~mol} \mathrm{~L}^{-1}$; $c\left(\mathrm{Cp}^{*}{ }_{2} \mathrm{ZrCl}_{2}\right)=0.0013 \mathrm{~mol} \mathrm{~L}^{-1}$; a) $c(\mathrm{BzMgBz})=0.038 \mathrm{~mol} \mathrm{~L}^{-1}$; b) $c(\mathrm{BzMgBz})=0.050 \mathrm{~mol} \mathrm{~L}^{-1}$; ) $c(\mathrm{BzMgBz})=0.066 \mathrm{~mol} \mathrm{~L}^{-1}$. 
The determined values show only a small scattering which is in agreement with previous shown measurements in monomer absence. It indicates further, that styrene has a similar influence on the reaction kinetics then ether. Its presence is lowering the alkylation rate coefficient independently from the grade of deuteration. The effect is not as strong as for DBE In comparison to NMR experiments the rate coefficient is lowered by a factor of two while the concentration of styrene is doubled. For each measurement a manual parameter estimation was performed with a view to the MMD and conversion/time profile. As main fit parameter the values for $k_{\mathrm{ex} 2}, k_{\mathrm{p}, \infty}$ and $k_{\mathrm{kp} 1}$ where found. The results are shown in Figures 6.9 to 6.11 and summarized in Table 6.1.
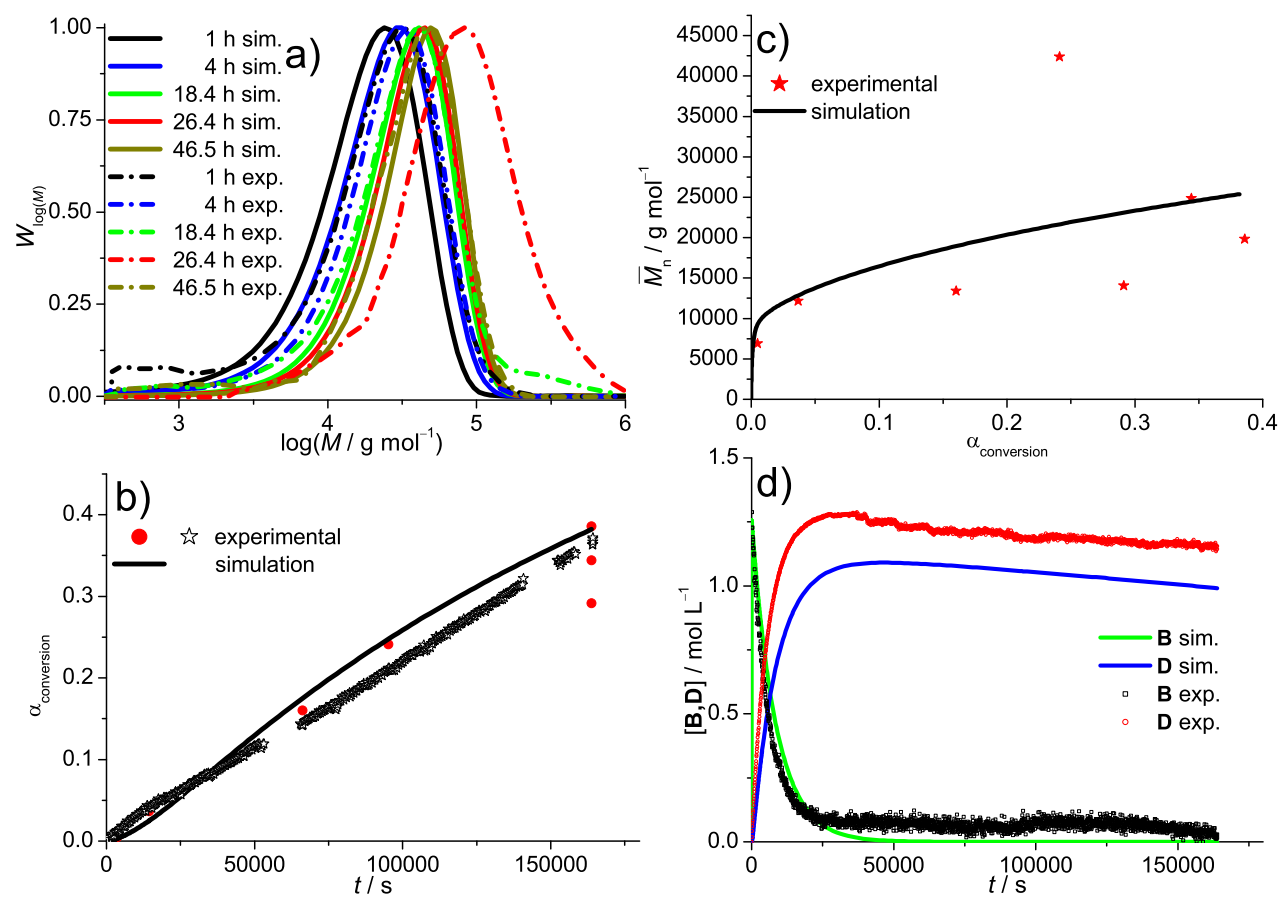

Figure 6.9: Comparsion between experimental conversion from IR- and gravimetric determination after parameter estimation for $\mathrm{c}($ Styrene $)=7.77 \mathrm{~mol} \mathrm{~L}^{-1} ; c\left(\mathrm{Cp}_{2}{ }_{2} \mathrm{ZrCl}_{2}\right)=0.0013 \mathrm{~mol} \mathrm{~L}^{-1}$; $c(\mathrm{BzMgBz})=0.038 \mathrm{~mol} \mathrm{~L}^{-1}$ at $70^{\circ} \mathrm{C}$.

During the modeling it was found that the $k_{\text {alkyl1 }}$ coefficients were partly determined to low (experiment $\mathrm{v}$ and vii) and the performed data reconstruction is not very successful if such a problem occurs. The data has to be estimated via modelation to gain good agreement between the measured concentration vs. time profiles and the simulation. The determined values are only slightly higher but have impact on the conversion/time profile. 

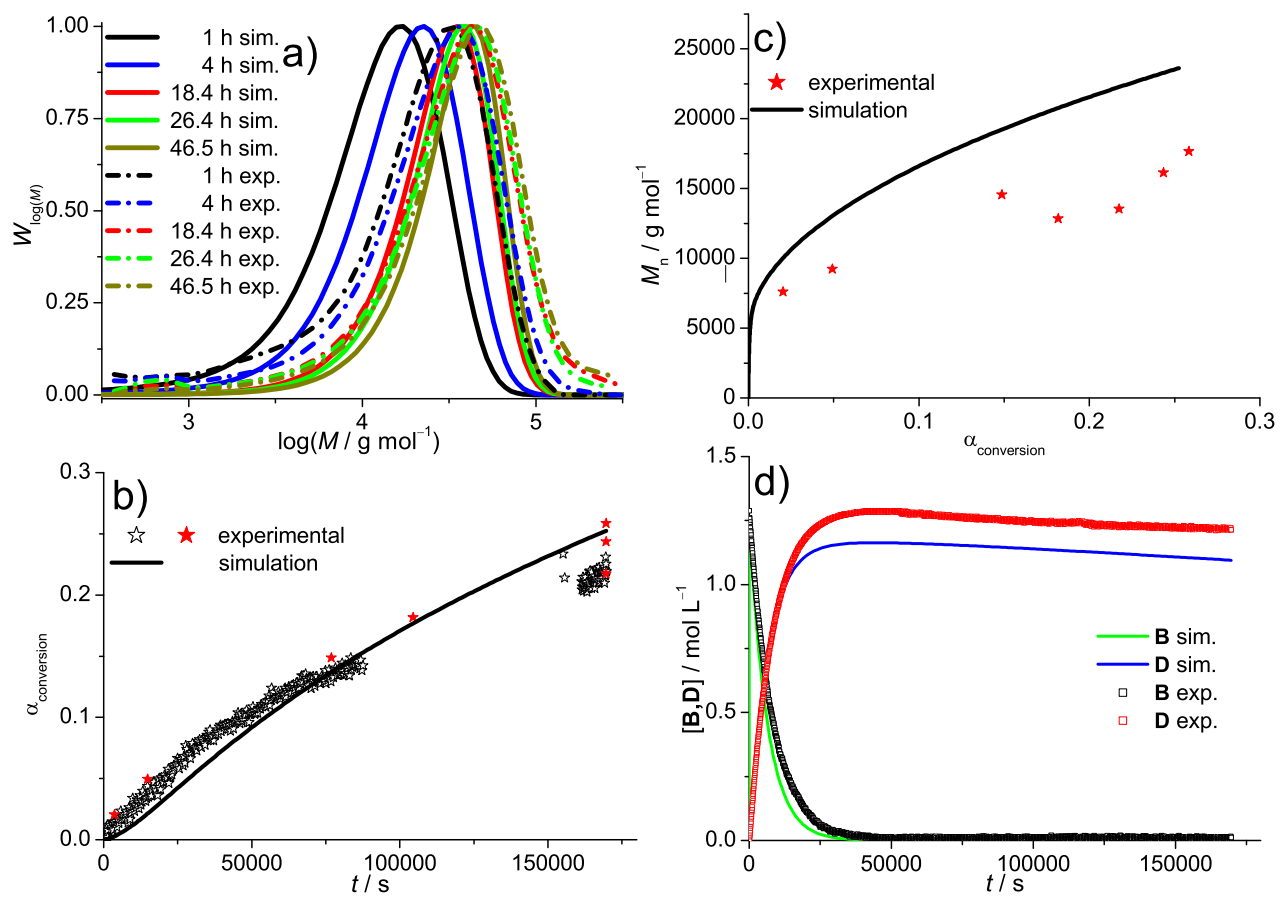

Figure 6.10: Comparsion between experimental conversion vorm IR- and gravimetric determination after parameter estimation for $\mathrm{c}($ Styrene $)=7.77 \mathrm{~mol} \mathrm{~L}^{-1} ; c\left(\mathrm{Cp}_{2}{ }_{2} \mathrm{ZrCl}_{2}\right)=0.0013 \mathrm{~mol} \mathrm{~L}^{-1}$; $c(\mathrm{BzMgBz})=0.050 \mathrm{~mol} \mathrm{~L}^{-1}$ at $70^{\circ} \mathrm{C}$.
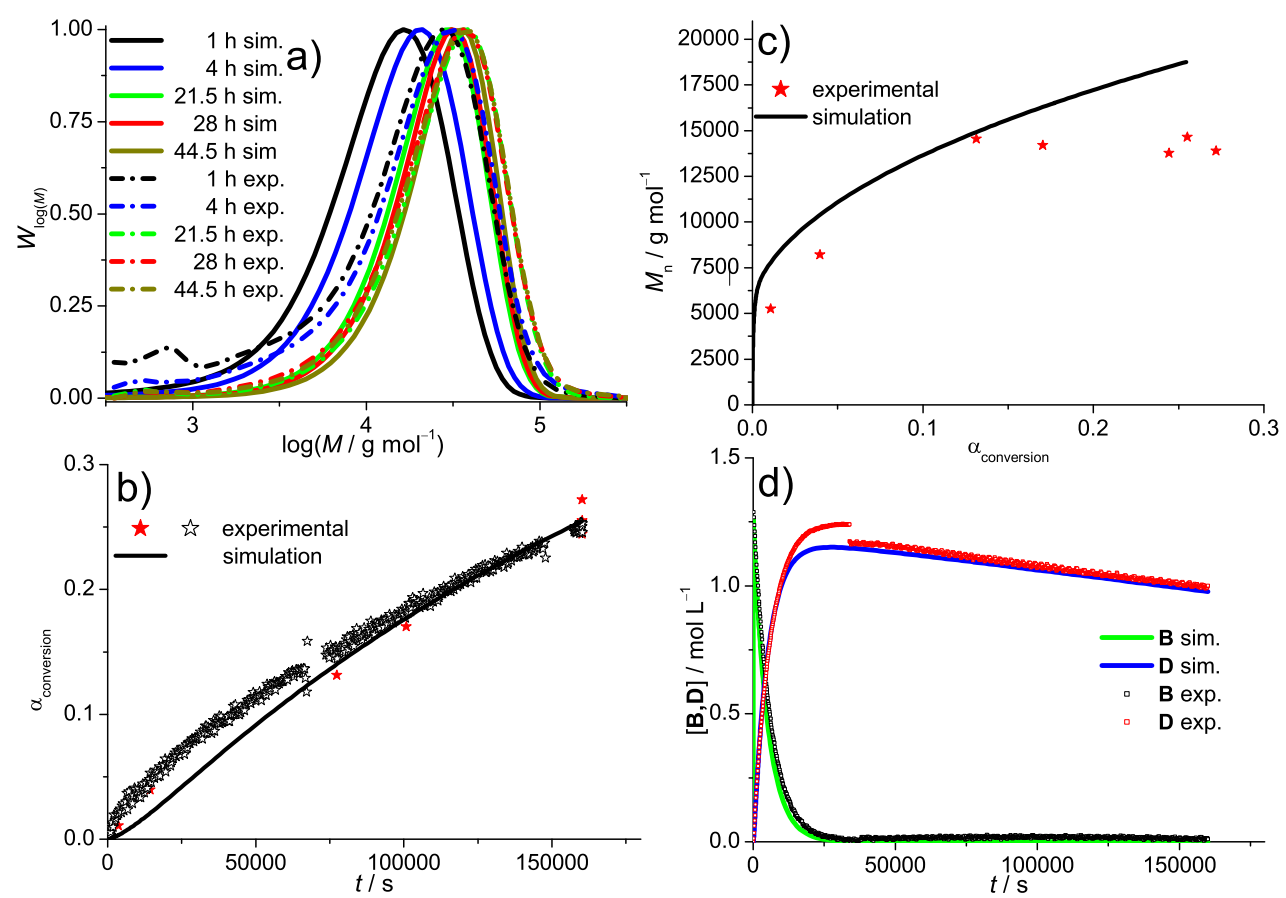

Figure 6.11: Comparsion between experimental conversion from IR- and gravimetric determination after parameter estimation for $c($ styrene $)=7.77 \mathrm{~mol} \mathrm{~L}^{-1} ; c\left(\mathrm{Cp}_{2}{ }_{2} \mathrm{ZrCl}_{2}\right)=0.0013 \mathrm{~mol} \mathrm{~L}^{-1}$; $c(\mathrm{BzMgBz})=0.066 \mathrm{~mol} \mathrm{~L}^{-1}$ at $70^{\circ} \mathrm{C}$. 
Table 6.1 Parameter determined by modeling for CCG polymerization of styrene at $70^{\circ} \mathrm{C}$, $c\left(\mathrm{Cp}_{2}{ }_{2} \mathrm{ZrCl}_{2}\right)=1.28 \mathrm{mmol} \mathrm{L}^{-1}$ in styrene $7.77 \mathrm{~mol} \mathrm{~L}^{-1}$ and toluene $1.05 \mathrm{~mol} \mathrm{~L}^{-1}$ for three different BzMgBz concentrations a) $0.034 \mathrm{~mol} \mathrm{~L}^{-1}$; b) $0.050 \mathrm{~mol} \mathrm{~L}^{-1}$; c) $0.067 \mathrm{~mol} \mathrm{~L}^{-1}$ ).

\begin{tabular}{c|c|c|c|r|l} 
coefficient & $\mathrm{v})$ & $\mathrm{vi})$ & $\mathrm{vii})$ & unit & \\
\hline$k_{\text {alkyl1 }}$ & 1.2 & 1.6 & 2.0 & $1.3 \pm 0.2$ & $10^{-4} \mathrm{~s}^{-1}$ \\
$k_{\text {alkyl2 }}$ & 0.7 & 0.43 & 1.6 & $0.8 \pm 0.4$ & $10^{-6} \mathrm{~s}^{-1}$ \\
$k_{\mathrm{ex} 2}$ & 75 & 320 & 160 & $185 \pm 115$ & $\mathrm{~L} \mathrm{~mol}^{-1} \mathrm{~s}^{-1}$ \\
$k_{\mathrm{p}, \infty}$ & 0.4 & 0.35 & 0.4 & $0.4 \pm 0.1$ & $\mathrm{~s}^{-1}$ \\
$k_{\mathrm{p} 1}$ & 2 & 3 & 2.5 & $2.5 \pm 0.5$ & $10^{-3} \mathrm{~s}^{-1}$
\end{tabular}

The fit of the simulation with the experimental data is improved, still having differences especially at the lower $\mathrm{BzMgBz}$ concentrations. The agreement for the measurements at higher concentration is good with a difference in the starting phase for $c(\mathrm{BzMgBz})=0.066 \mathrm{~mol} \mathrm{~L}^{-1}$. The conversion during the first 50000 seconds is determined to low compared to the results afterwards. One possible explanation is the presence of diethyl ether from BzMgBz. The concentration compared to the catalyst is higher. The presence of ether can cause such a kink shaped conversion profile (see Section 6.1.2). Diethyl ether can leave the reaction mixture, which made successful description via modeling unsuitable.

With a closer look at the determined coefficients, the biggest differences and errors are found for the rate coefficient for the association reactions with polymer involved. The reason for this behavior is not determined yet, but is the main cause for the strong scattering between the experiments. The determined coefficients support the assumption of a kinetic isotope effect, because both rate coefficients had to be increased as well as the equilibrium constant for the monomer coordination at complex $\mathbf{C}$. These results are in agreement with literature ${ }^{[140]}$ and in comparison with the coefficients factors of 1.1 $k_{\mathrm{p} 1, \mathrm{H}} / k_{\mathrm{p} 1, \mathrm{D}}$ and $1.3 k_{\mathrm{p}, \mathrm{H}} / k_{\mathrm{p}, \mathrm{D}}$ were found. The values are in the same range as in literature (1 to 1.3$)$. [140]

The SEC curves are found to yield similar behavior (see Figures 6.9 to 6.11 ) but are estimated too small in each case for the measurements after 1 and 4 hours. The determined concentrations of $\mathbf{B}$ fit well. The profiles for the concentration of $\mathbf{D}$ show a systematic shift. The concentration is overestimated by the UV/Vis measurement but the shape of the curve is modeled very accurate. The exact reason for this finding is yet not clear because similar extinction coefficients are found in absence of monomer. For measurment vii) a step is found which is caused by the determination of a sligthly lower extinction coefficient yielding to an overestimation of the concentration $\mathbf{D}$. 
In conclusion the determination of the full polymerization via UV/Vis and FT-IR spectroscopy and via gravimetric methods are suitable for the modelation of kinetic coefficients. The determination of exact concentrations via UV/Vis in presence of styrene and polystyrene is of limited accuracy, but the determined profiles can help to discuss occurring phenomena. A kinetic isotope effect could be confirmed as already suggested in literature. As found for the monomer-free system an influence from ether on the reaction rates is indicated which will be studied in the following section.

\subsubsection{Polymerization in Presence of DBE}

The same procedure was applied for studies in presence of small amounts of additional DBE. The previous NMR results revealed that the equilibrium constants are only slightly influenced by DBE but the alkylation rates are strongly influenced. To investigate the effect on the polymerization the FT-IR-, gravimetric- and UV/Vis methods were preferred which allows the application of five percent of ether added. The problem found during the application of high amounts of undeuterated additives in NMR are avoided. The measurements were performed at two different concentration of BzMgBz. The results are shown in Figures 6.12 to 6.15 combined with the results of the modeling approach.
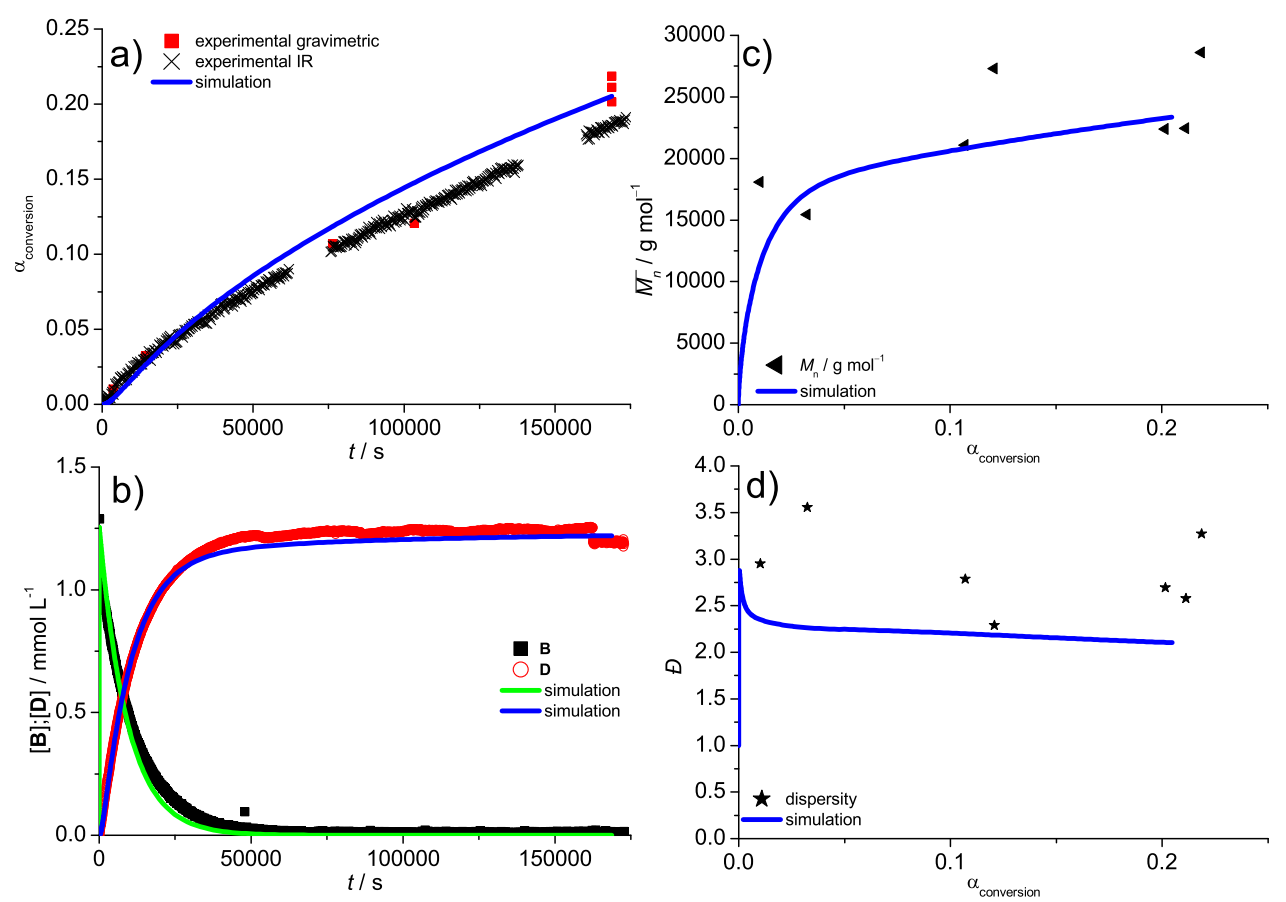

Figure 6.12: Experiment vs. modeling of CCG polymerization of styrene with $\mathrm{A}$ and $\mathrm{BzMgBz}$ in presence of DBE of experiment viii) (see Table 6.2). a) Monomer conversion; b) concentration vs. time profiles for $\mathbf{B}$ and D; c) $\bar{M}_{n}$ vs. conversion; d) dispersity vs. conversion. 

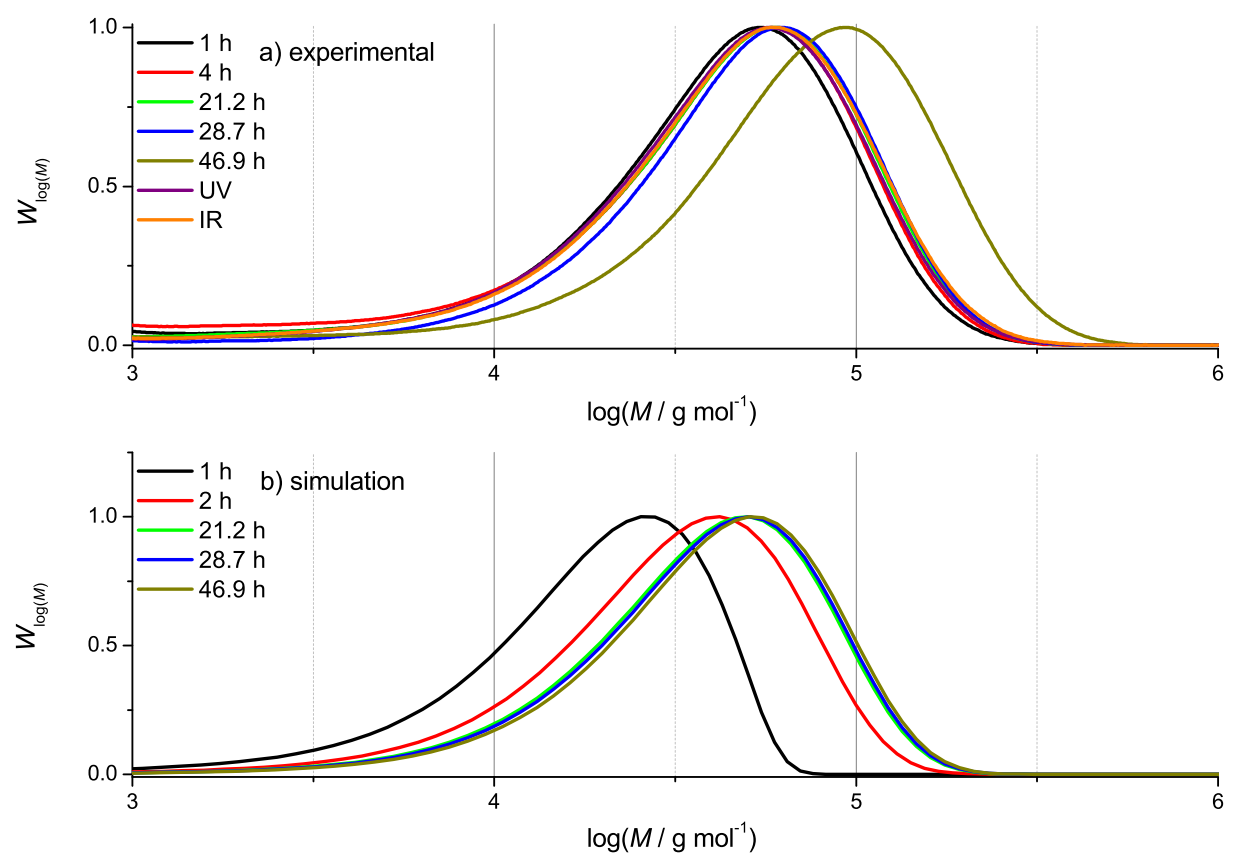

Figure 6.13: SEC Traces of experiment (a) and modeling (b) for CCG polymerization of styrene with $\mathrm{A}$ and $\mathrm{BzMgBz}$ in presence of DBE of experiment viii) (see Table 6.2).

The main difference to the polymerizations in absence of ether is a strong, kink like conversion with a high reaction rate at the beginning which is leveling of to a constant lower reaction rate. In both cases the results indicate that the produced molecular mass levels-off to a constant value and constant dispersity around 3. It was found additionally that the alkylation is still influenced (see Table 6.2), but the effect of ether is limited. $\mathrm{UV} /$ Vis monitoring showed that the reaction mechanism is similar to the process in absence of ether.

The effect of ether was directly approached via modeling. The main difference and the only reason for the kink conversion profile is the fact that rate coefficients are lowered by one order of magnitude for the first equilibrium. The found behavior is similar to the results shown in the systematic scan in Figure 5.17 on page 116. This indicates that the addition of ether influences the rate coefficients of the equilibrium reactions equally to lower values.

In conclusion the addition of $\mathrm{DBE}$ is promoting higher conversions because of the lowering of the reaction rates for the exchange reaction, i.e. the dormant species are formed much slower, which is in combination with the results shown in the screening not beneficial for the control of the polymerization. The catalytically active species are 

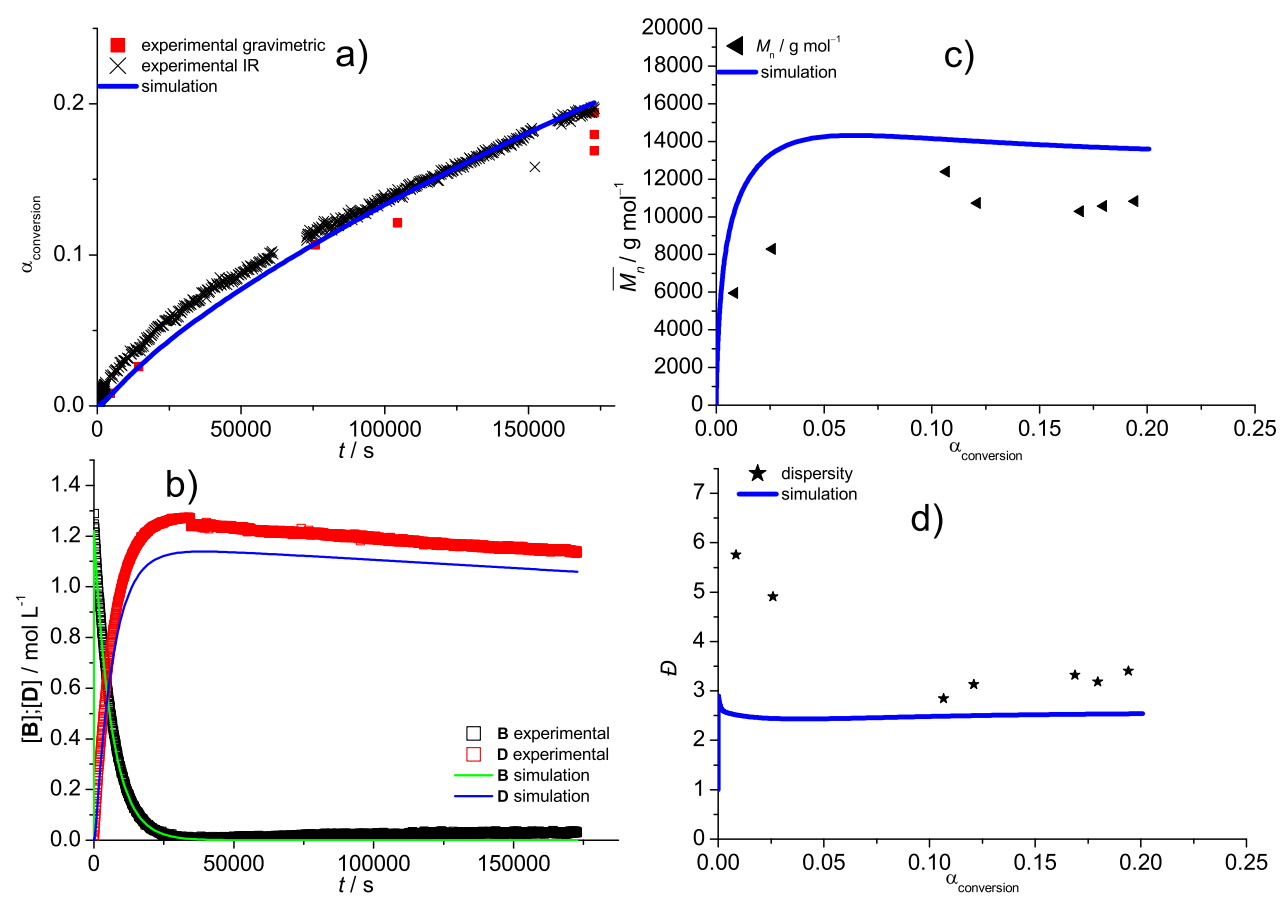

Figure 6.14: Experiment vs. modeling for CCG polymerization of styrene with $\mathbf{A}$ and $\mathrm{BzMgBz}$ in presence of DBE of experiment ix) (see Table 6.2). a) Monomer conversion; b) concentration vs. time profiles for $\mathbf{B}$ and $\mathbf{D}$; c) $\bar{M}_{n}$ vs. conversion; d) dispersity vs. conversion.
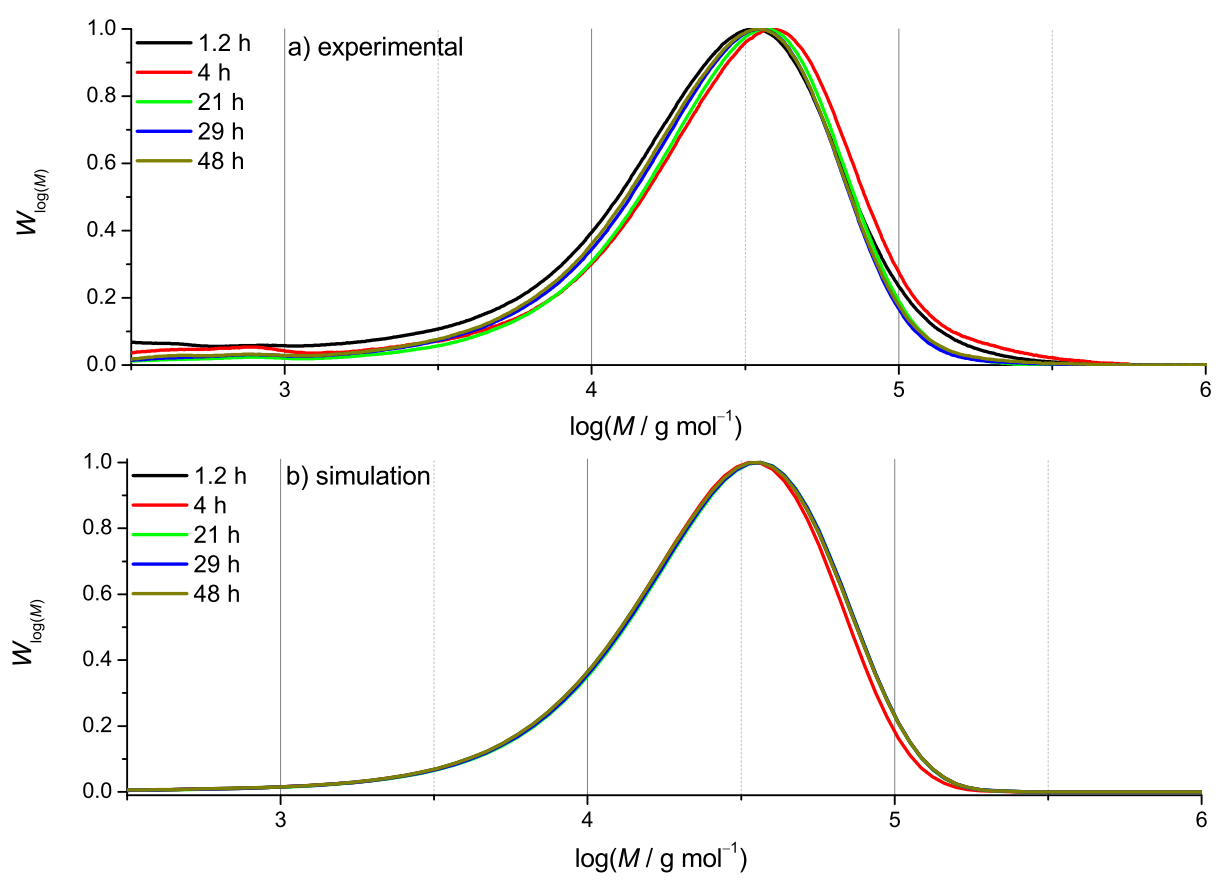

Figure 6.15: SEC Traces of experiment (a) and modeling (b) for CCG polymerization of styrene with $\mathrm{A}$ and $\mathrm{BzMgBz}$ in presence of DBE of experiment ix) (see Table 6.2). 
formed preferably and the association reaction hindered longer. Small differences within the ether concentration may be the result for the differences found for the simulation in absence of additional ether.

Table 6.2 Parameter determined by modelation for CCG polymerization of styrene at $70^{\circ} \mathrm{C}$, ${ }^{\mathrm{C}} \mathrm{CP}_{2}{ }_{2} \mathrm{ZrCl}_{2}=1.28 \mathrm{mmol} \mathrm{L}^{-1}$ in styrene $7.44 \mathrm{~mol} \mathrm{~L}^{-1}$ and toluene $1.05 \mathrm{~mol} \mathrm{~L}^{-1}$ with DBE $0.034 \mathrm{~mol} \mathrm{~L}^{-1}$ for two different $\mathrm{BzMgBz}$ concentrations viii) $0.034 \mathrm{~mol} \mathrm{~L}^{-1}$; ix) $0.067 \mathrm{~mol} \mathrm{~L}^{-1}$ ).

\begin{tabular}{c|c|c|r|l} 
coefficient & viii) & ix $)$ & mean value & \\
\hline$k_{\text {alkyl1 }}$ & 1.1 & 1.6 & $1.3 \pm 0.2$ & $10^{-4} \mathrm{~s}^{-1}$ \\
$k_{\text {alkyl2 }}$ & 0.0 & 0.7 & $0.8 \pm 0.4$ & $10^{-6} \mathrm{~s}^{-1}$ \\
$k_{\text {ex1 }}$ & 0.5 & 0.5 & - & $\mathrm{L} \mathrm{mol}^{-1} \mathrm{~s}^{-1}$ \\
$k_{\mathrm{act} 1}$ & 9.5 & 9.5 & - & $10^{-5} \mathrm{~s}^{-1}$ \\
$k_{\mathrm{ex} 2}$ & 18.5 & 20 & $19 \pm 1$ & $\mathrm{~L} \mathrm{~mol}^{-1} \mathrm{~s}^{-1}$ \\
$k_{\mathrm{act} 2}$ & 3.45 & 3.45 & - & $10^{-4} \mathrm{~s}^{-1}$ \\
$k_{\mathrm{p}, \infty}$ & 0.175 & 0.35 & $0.26 \pm 0.12$ & $\mathrm{~s}^{-1}$ \\
$k_{\mathrm{p} 1}$ & 2 & 3 & $2.5 \pm 0.7$ & $10^{-3} \mathrm{~s}^{-1}$
\end{tabular}

\subsection{Polymerization of Styrene with $\mathrm{Cp}_{2}{ }_{2} \mathrm{ZrCl}_{2}$ and Dialykl Magnesium}

The change of the CTA to BuMgOct was already briefly discussed for NMR measurements. In the case of UV/Vis measurements the data analysis is not suitable anymore because the formed polymer overlays the region capable for UV/Vis determination and no isosbestic point can be derived. This is limits the data analysis to FT-IR- and gravimetric measurements. Polymerization within sample flask were not performed and only the resulting polymer from the cuvettes analyzed. The polymerization was performed in absence (see Figure 6.16) and presence of DBE (see Figure 6.17). The results of the modeling approaches are already shown within the graphs.

In comparison both experiments show similar behavior concerning the monomer conversion. A kink shaped profile is found with high conversion rates in the beginning which levels of to a lower constant rate. These curves are similar to the ones found by polymerization in presence of $\mathrm{BzMgBz}$ and $\mathrm{DBE}$. These results indicate that compared to $\mathrm{BzMgBz}$ the reaction rate, the coordination of BuMgOct and the resulting polymeric 

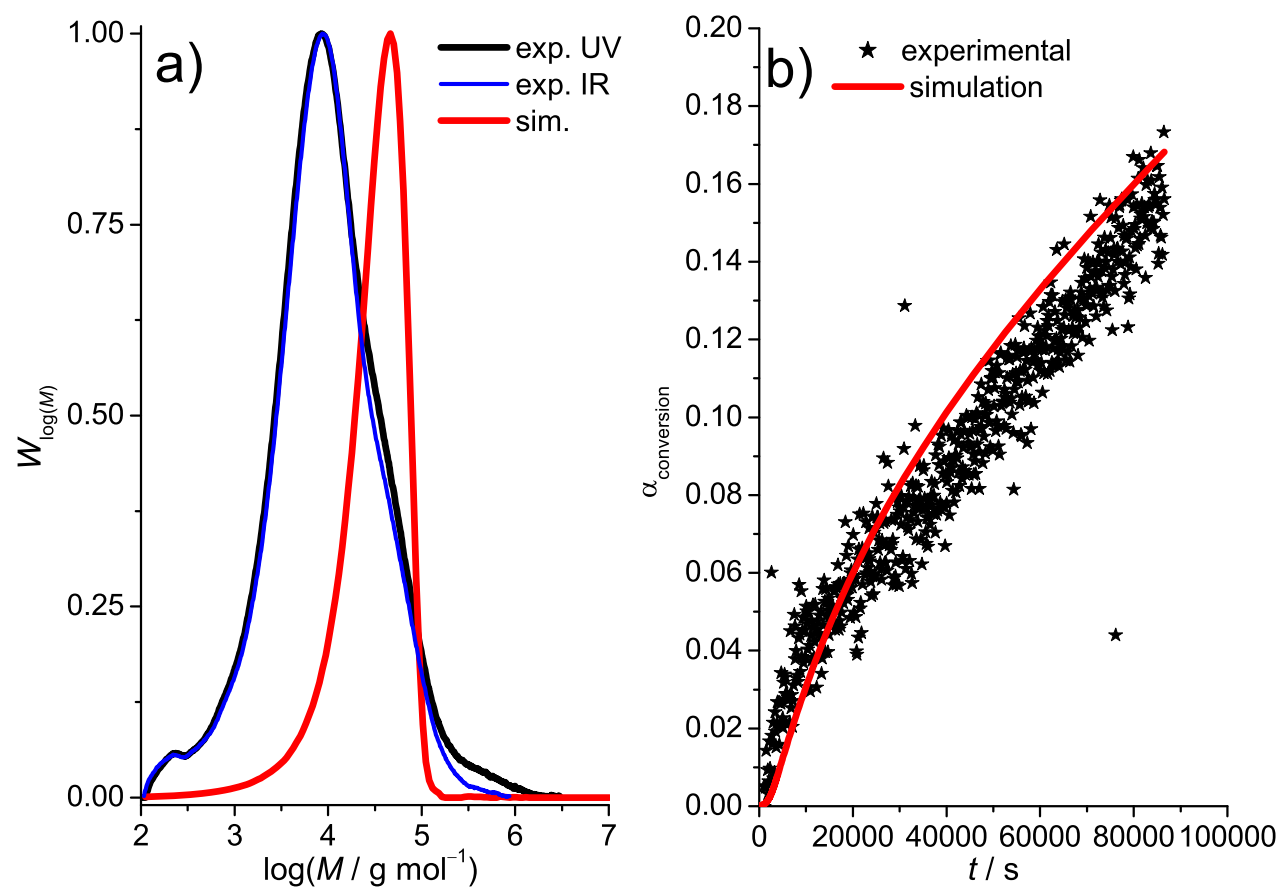

Figure 6.16: Experimental vs. simulation data of CCG polymerization of styrene at $70^{\circ} \mathrm{C}$; ${ }^{c} \mathrm{Cp}_{2}{ }_{2} \mathrm{ZrCl}_{2}=1.23 \mathrm{mmol} \mathrm{L}^{-1}$ with $c(\mathrm{BuOctMg})=0.038 \mathrm{~mol} \mathrm{~L}^{-1}$ in styrene $7.44 \mathrm{~mol} \mathrm{~L}^{-1}$ and toluene $1.00 \mathrm{~mol} \mathrm{~L}^{-1}$. a) SEC traces b) conversion vs. time.
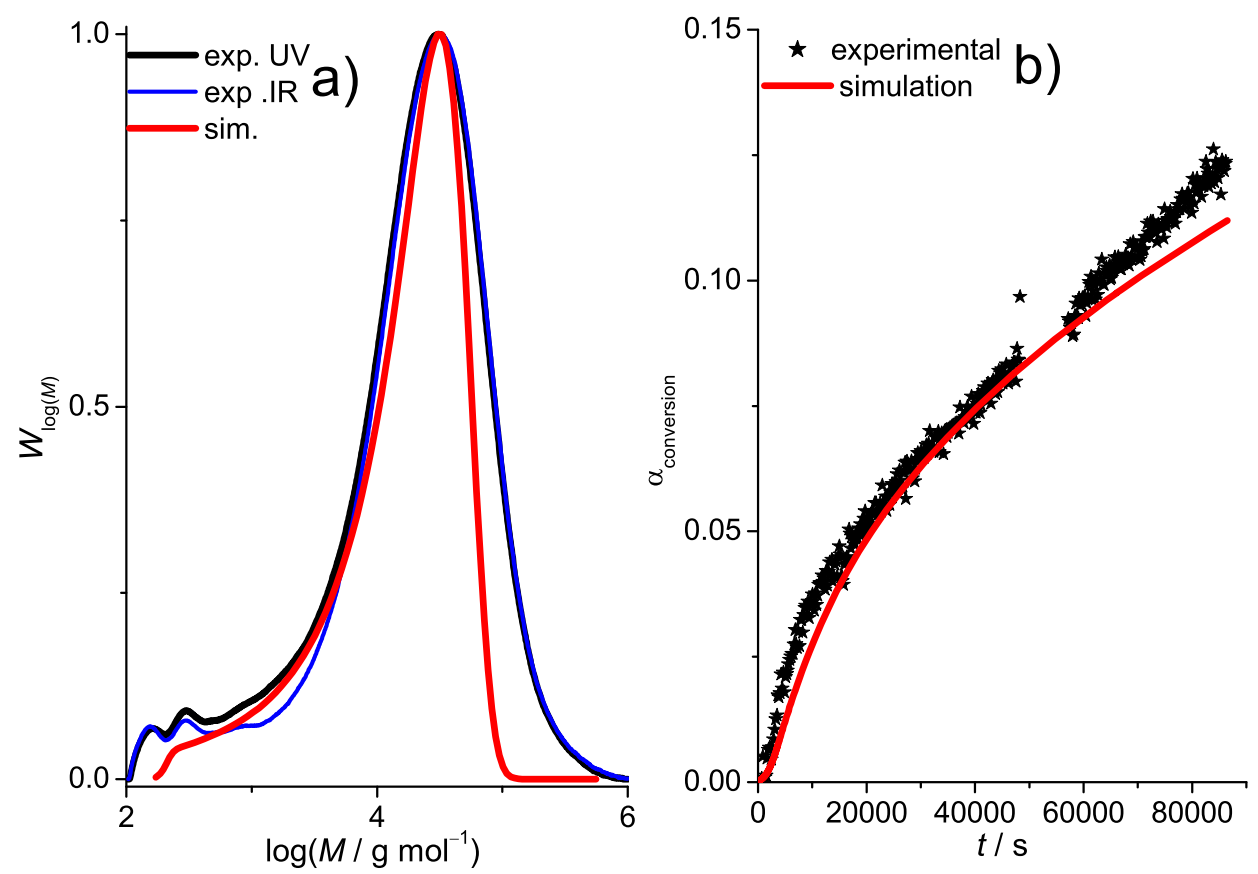

Figure 6.17: Experimental vs. simulation data of CCG polymerization of styrene at $70^{\circ} \mathrm{C}$; ${ }^{c} \mathrm{CP}_{2}{ }_{2} \mathrm{ZrCl}_{2}=1.23 \mathrm{mmol} \mathrm{L}^{-1}, c(\mathrm{BuOctMg})=0.037 \mathrm{~mol} \mathrm{~L}^{-1}$ in styrene $7.06 \mathrm{~mol} \mathrm{~L}^{-1}$ and toluene $1.00 \mathrm{~mol} \mathrm{~L}^{-1}$ with DBE $0.252 \mathrm{~mol} \mathrm{~L}^{-1}$. a) SEC traces b) conversion vs. time. 
magnesium derivatives react with a different rate coefficient. The reaction rate in presence of DBE is lower.

A second major difference is found within the MMD. While in absence of DBE a bimodal curve is yielded and the curve has no shoulder in presence of DBE. Further the maximum in presence of ether is found in the area of the shoulder in its absence (cf. Figure 6.16 a) and $6.17 \mathrm{a})$ ). The measurement in absence of ether was performed during the time when notable THF concentrations were found in the NMR experiments. It cannot be excluded that the shoulder is formed by partly ether cooperation yielding two sets of kinetics with in the sample. The result is a mixture of a two polymers formed, one with the high molecular weight similar because it was influenced by ether and a major uninfluenced part at lower molecular weight.

Modeling with the existing model and the parameters determined via NMR was not successful. The introduction of a CTA with alkyl groups seems to yield to a further activation mechanism allowing the fully alkylated complex to be reactivated. The exact nature of this process was not studied in this thesis. The most important difference found is that no solid was formed during the polymerization process. This indicates that $\mathrm{MgCl}_{2}$ is still a part of the reaction mixture. Due to the high reaction barrier for the alkyl-chloride ligand-exchange reaction it was assumed to extended the model by a reactivation pathway of the $\mathbf{E}$ type complexes via the Gringard species. This assumption is based on results from literature ${ }^{[267}$, where is intramolecular exchange for the Mgclusters is higher for alkyl- chains then for benzyl- chains and the fact that such behavior was not found for the benzyl-groups. Additional it was assumed that this reaction only occurs with alkyl chains bound to the complexes (see Equations 6.2 to 6.5).

$$
\begin{gathered}
\mathbf{E}^{\mathbf{P 0}}+\mathrm{BuMgCl}^{\stackrel{k_{\text {react }}}{\longrightarrow}} \mathbf{C}+\mathrm{BuMgBu} \\
\mathbf{E}^{\mathbf{P 0}}+\mathrm{PMgCl}_{s} \stackrel{k_{\text {react }}}{\longrightarrow} \mathbf{C}+\mathrm{BuMgP}_{s} \\
\mathbf{E}^{\mathbf{P 1}}+\mathrm{PMgCl}_{s} \stackrel{k_{\text {react }}}{\longrightarrow} \mathbf{C}^{\mathbf{P}}+\mathrm{BuMgP}_{s} \\
\mathbf{E}^{\mathbf{P 1}}+\mathrm{BuMgCl}^{k_{\text {react }}} \longrightarrow \mathbf{C}^{\mathbf{P}}+\mathrm{BuMgBu}
\end{gathered}
$$

This modeling approach yield similar behavior as found during polymerization, yet a growing difference at high conversion is found. This approach is yielding a possible explanation for the occurring process, yet the accuracy of this assumption is very limited and a much more detailed study necessary to find all possible reaction pathways that can yield a reactivation of the catalyst center in presence of a dialkyl magnesium (see Figure 6.17 and Table 6.3). 
Table 6.3 Parameter determined by modelation for CCG polymerization of styrene

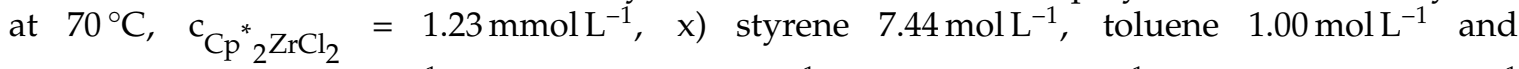
$c(\mathrm{BuOctMg})=0.038 \mathrm{~mol} \mathrm{~L}^{-1}$; xi) styrene $7.06 \mathrm{~mol} \mathrm{~L}^{-1}$, toluene $1.00 \mathrm{~mol} \mathrm{~L}^{-1}$ with DBE $0.252 \mathrm{~mol} \mathrm{~L}^{-1}$ and $c($ BuOctMg $)=0.037 \mathrm{~mol} \mathrm{~L}^{-1}$.

\begin{tabular}{c|c|c|r|} 
coefficient & $\mathrm{x})$ & $\mathrm{xi})$ & \\
\hline$k_{\text {alkyl1 }}$ & 8 & 8 & $10^{-4} \mathrm{~s}^{-1}$ \\
$k_{\text {alkyl2 }}$ & 3 & 3 & $10^{-4} \mathrm{~s}^{-1}$ \\
$k_{\mathrm{ex} 1}$ & 1.8 & 0.18 & $\mathrm{~L} \mathrm{~mol}^{-1} \mathrm{~s}^{-1}$ \\
$k_{\mathrm{act} 1}$ & 5.1 & 0.51 & $10^{-1} \mathrm{~s}^{-1}$ \\
$k_{\text {ex2 }}$ & 180 & 18 & $\mathrm{~L} \mathrm{~mol}^{-1} \mathrm{~s}^{-1}$ \\
$k_{\mathrm{act} 2}$ & 5.1 & 0.51 & $10^{-2} \mathrm{~s}^{-1}$ \\
$k_{\mathrm{p}, \infty}$ & 0.07 & 0.09 & $\mathrm{~s}^{-1}$ \\
$k_{\mathrm{p} 1}$ & 1 & 3 & $\mathrm{~s}^{-1}$ \\
$a$ & 0.05 & 015 & $\mathrm{~s}^{-1}$ \\
$k_{\mathrm{t}}$ & 2 & 2 & $10^{-2} \mathrm{~s}^{-1}$ \\
$k_{\text {reini }}$ & 10 & 0.02 & $10^{4} \mathrm{~s}^{-1}$ \\
$k_{\text {react }}$ & 10 & 10 & $\mathrm{~s}^{-1}$
\end{tabular}




\subsection{Polymerization of Styrene with $\mathrm{Cp}_{2}{ }_{2} \mathrm{NdCl}_{2} \mathrm{Li}\left(\mathrm{OEt}_{2}\right)$ and $\mathrm{BzMgBz}$}

After the change of the co-catalyst further studies were performed by application of $\mathbf{I}$, one of the most applied catalysts known for CCG. It has the disadvantage, that studies in NMR are unsuitable, therefore the choice of gravimetric-, UV/Vis- and IR-spectroscopic measurements were preferred. With the previously shown results on hand for the monomer free system, the polymerization was performed in absence and presence of di-n-butyl ether. The formed polystyrene is here again reducing the quality of the measurement, yet the absorbance of the neodymium metal center is well separated and can be analyzed. The measurements were baseline corrected via the following equation in the range from $584 \mathrm{~nm}$ to $649 \mathrm{~nm}$ for the wavelength $\mathrm{x}$ :

$$
A_{x, \mathrm{corr}}=A_{x}-\left(\frac{\frac{A_{584 \mathrm{~nm}}-A_{649 \mathrm{~nm}}}{655 \mathrm{~nm}}}{649 \mathrm{~nm}-x}\right)-A_{649 \mathrm{~nm}}
$$

The basic reactions are similar to the process found in the model system. The reactions are shown in Scheme 6.18,

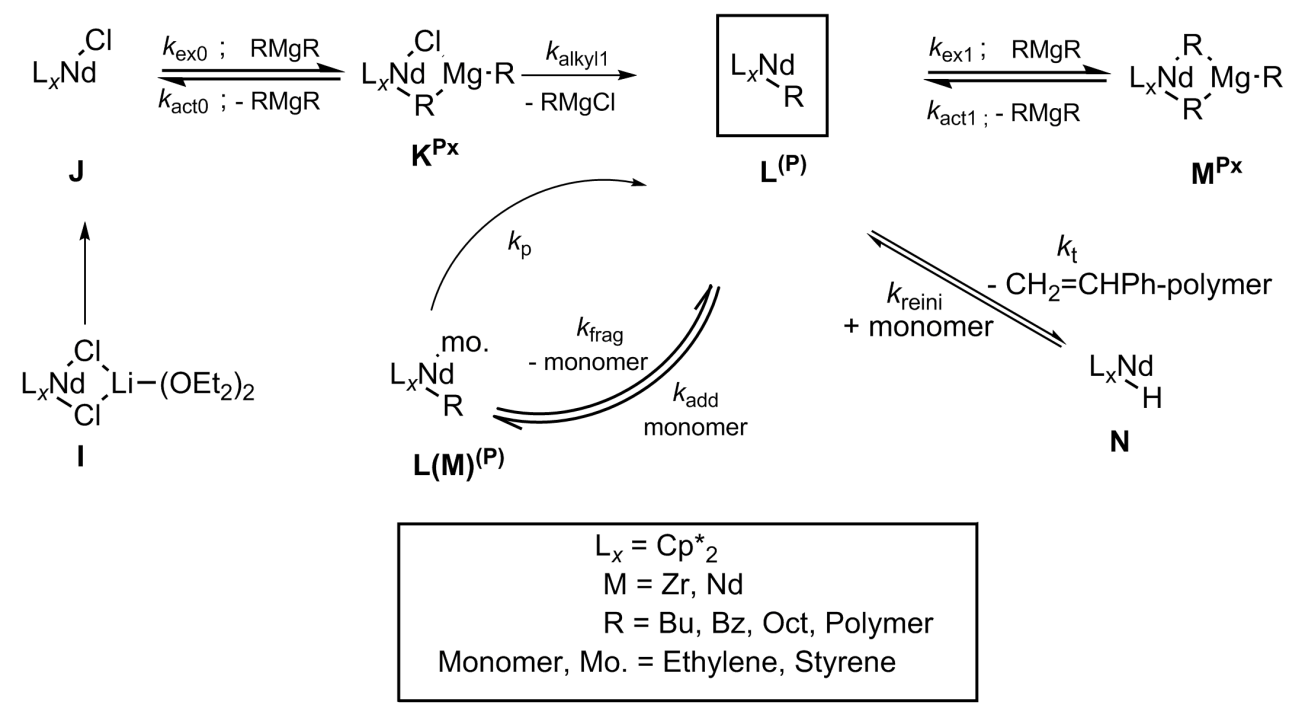

Figure 6.18: Modified reaction scheme for CCG polymerization starting from $\mathrm{Cp}_{2}{ }_{2} \mathrm{NdCl}_{2} \mathrm{Li}_{(\mathrm{OEt}}$ (I) over $\mathbf{J}$ and the association of dialkyl magnesium derivates to $\mathbf{K}^{\mathbf{P X}}$. Via dissociation $\mathbf{L}$ is formed where monomer can be added $(\mathbf{L}(\mathbf{M}))$, termination occur $(\mathbf{N})$ or reaction with another molecule of dialkyl magnesium $\mathbf{M}^{\mathbf{P X}}$ 
The reactions in this scheme are implemented in the same manner as for the model system (see Appendix A.3.4 on page 226). The nature of the different species were already discussed for the monomer free system.

Polymerization of Styrene in Absence of DBE First an experiment was performed with no ether added. One was with polymerization in a heating block and another without. The polymerization was performed over 3 days and samples were taken after different polymerization times. The result of the UV/Vis monitoring is shown in Figure 6.20. The results of the gravimetric determination are depicted together with the IR determination in Figure 6.19 The monomer conversion (see Figure 6.19 a)) shows a
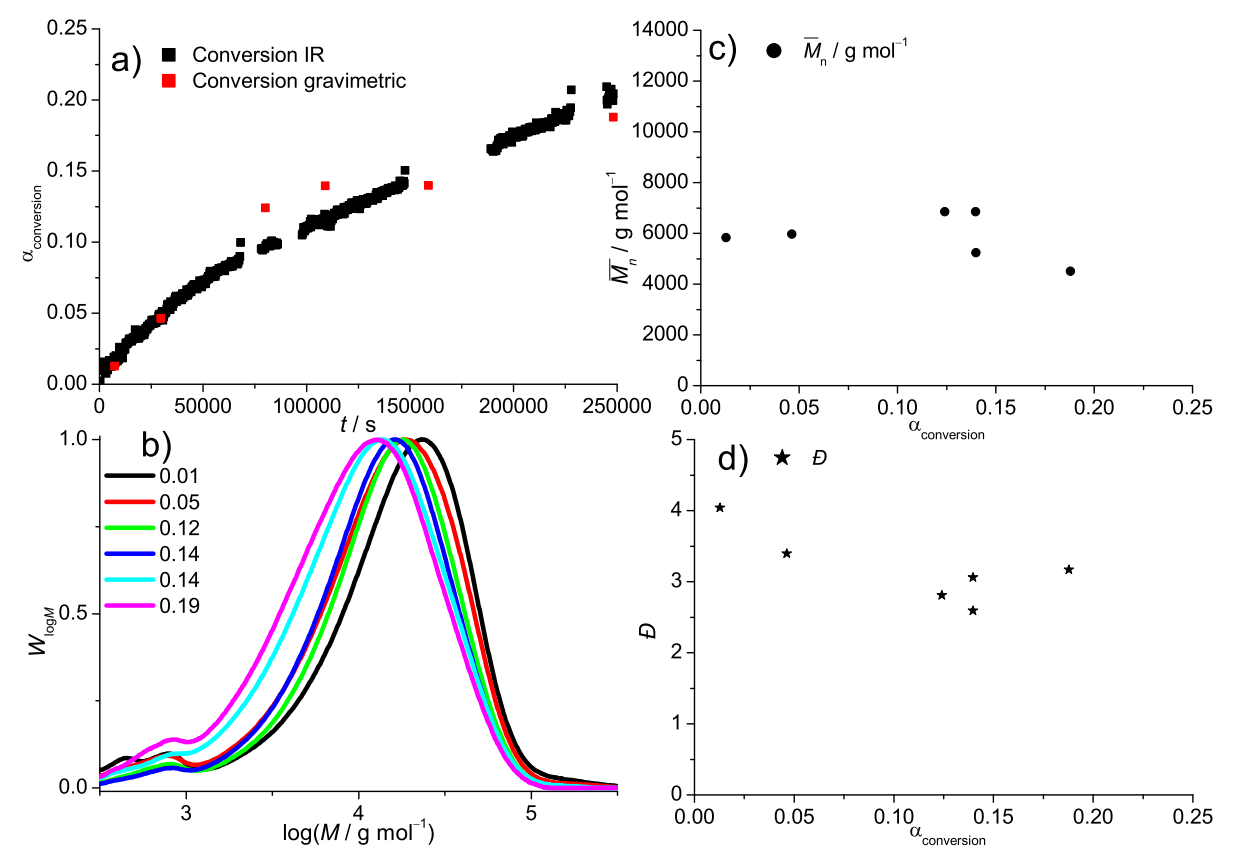

Figure 6.19: Styrene polymerization with $\mathbf{I}$ and $\mathrm{BzMgBz}$ at a ratio $\mathrm{Nd}: \mathrm{Zr}$ 1:14. Experiment xii): $c\left(\mathrm{Cp}_{2}{ }_{2} \mathrm{NdCl}_{2} \mathrm{Li}\left(\mathrm{OEt}_{2}\right)_{2}\right)=2.47 \mathrm{mmol} \mathrm{L}^{-1}, c(\mathrm{BzMgBz})=33 \mathrm{mmol} \mathrm{L}^{-1}$ and $c($ styrene $)=7.77 \mathrm{~mol} \mathrm{~L}^{-1}$. a) monomer conversion vs. time b) MMD curves c) $\bar{M}_{\mathrm{n}}$ vs. conversion d) dispersity vs. conversion.

kink with a high reaction rate in the beginning, which is reduced to a constant rate with proceeding reaction time. The average molar mass and the dispersity are slightly decreasing with proceeding conversion (see Figure 6.19 c) and d)). The maximum of the MMD is decreasing (see Figure $6.19 \mathrm{~b}$ )), which indicates that after the activation of the first polymer chains polymer growth is favored before the exchange reaction. The $\mathrm{UV} /$ Vis determination in the region of the catalyst absorbance show only small hints for possible changes (see Figure 6.20 ). With proceeding reaction time two additional absorption bands at $611 \mathrm{~nm}$ and $619 \mathrm{~nm}$ were found.

The absorption bands were confirmed via a second measurement at a higher Mg:Nd 


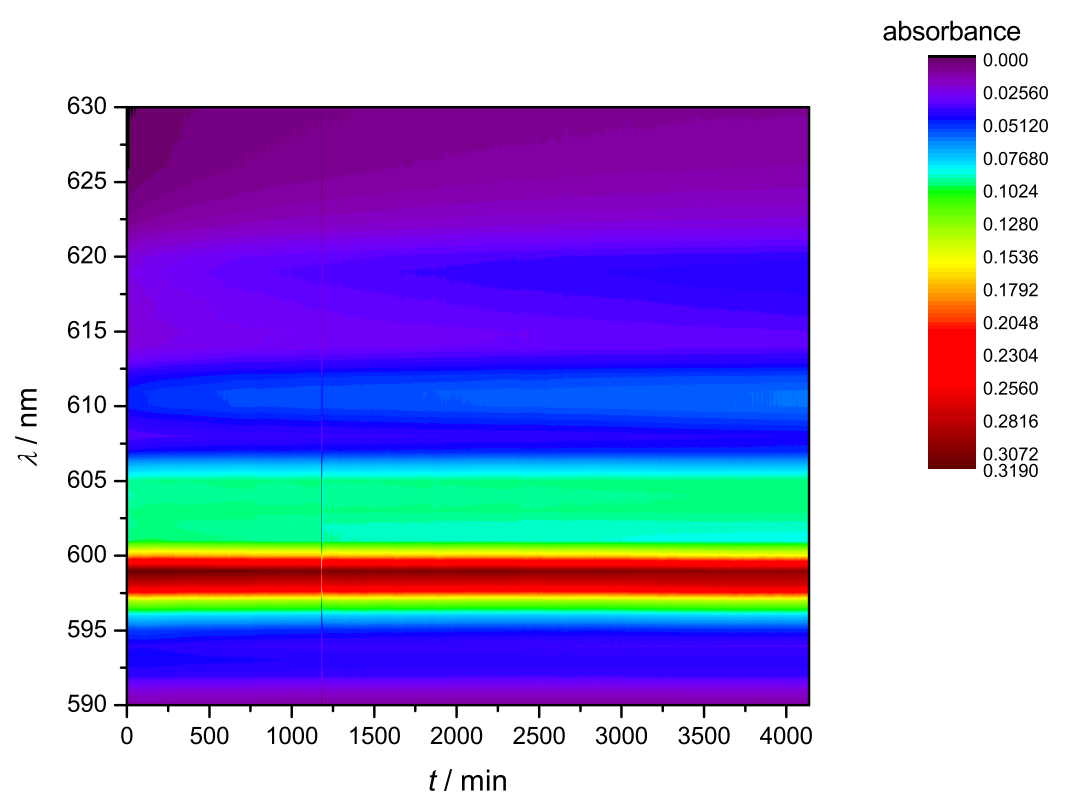

Figure 6.20: UV/Vis absorbance over time and wavelength of styrene polymerization with I and BzMgBz at a ratio $\mathrm{Nd}: \mathrm{Zr} \mathrm{1:14.} c\left(\mathrm{Cp}_{2}{ }_{2} \mathrm{NdCl}_{2} \mathrm{Li}\left(\mathrm{OEt}_{2}\right)_{2}\right)=2.47 \mathrm{mmol} \mathrm{L}^{-1}, c(\mathrm{BzMgBz})=33 \mathrm{mmol} \mathrm{L}^{-1}$ and $c$ (styrene $)=7.77 \mathrm{~mol} \mathrm{~L}^{-1}$.

ratio of 130 (see Figure 6.21). Further, absorption similar to the monomer-free system was found more pronounced. In combination these results indicate that in both cases equilibrium structures may be monitored with only benzyl groups attached $(600 \mathrm{~nm}$ to $608 \mathrm{~nm}$ ) or bearing the polymer chains $(611 \mathrm{~nm}$ and $619 \mathrm{~nm})$. For the second measurement a smaller average molar mass of $\bar{M}_{\mathrm{n}}=1270 \mathrm{~g} \mathrm{~mol}^{-1}$ and a MMD trending to lower molecular weight was gained (see Figure 6.22 a)). The conversion is lower, which is in agreement with the reduced catalyst concentration and the higher concentration of the CTA. Because no further data is available, it was tried to model the data with the existing kinetic scheme with PREDICI ${ }^{\mathrm{TM}}$ (see A.3.4 on page 226). The second alkylation step cannot be performed by this catalyst and is therefore not applied. The model is mainly the same as presented in chapter 5.1 .1 on page 96 yet the activation mechanism is described for the neodymium catalyst shown in Scheme 4.5 on page 76.

It was found that two effects occur. Firstly CLD is found for this system as well. Without performing a chain-length dependent propagation step the simulation of the MMD was not possible. This result indicates that the presence of styrene has an influence on the reaction kinetics independent from the metal center. Secondly, the reaction reaches a maximum MMD at around 10\% conversion. This effect is only possible if a very high termination rate coefficient is applied in the model. In combination with the modeling of ethylene polymerization the monomer addition equilibrium was found to show CLD. In the case of an alkyl chain at the catalyst, the propagation step is not chain-length 


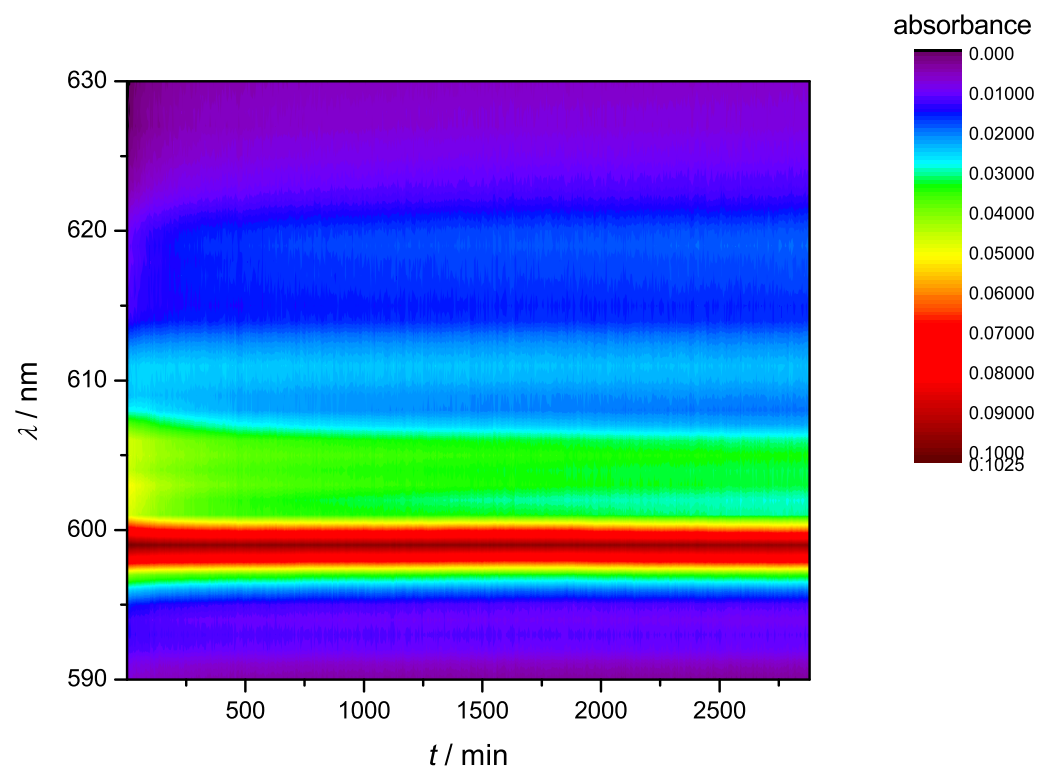

Figure 6.21: UV/Vis absorbance over time and wavelength of styrene polymerization with $\mathbf{I}$ and BzMgBz at a ratio Nd:Zr 1:130. $c\left(\mathrm{Cp}_{2}{ }_{2} \mathrm{NdCl}_{2} \mathrm{Li}\left(\mathrm{OEt}_{2}\right)_{2}\right)=0.64 \mathrm{mmol} \mathrm{L}^{-1}, c(\mathrm{BzMgBz})=84 \mathrm{mmol} \mathrm{L}^{-1}$ and $c$ (styrene $)=7.77 \mathrm{~mol} \mathrm{~L}^{-1}$.

dependent at short chain length. Due to the complex nature of these combined steps, it was found that the most successful method to describe this effect was by introducing chain-length dependent monomer coordination. With proceeding time the active catalyst side is hindered to add monomer successfully by a yet not known mechanism, leading to a loss in activity. To describe the process Equation 6.7 was introduced, allowing to simulate a high activity during the addition of short chains and a low activity at higher chain length.

$$
k_{\text {add }, 2}=k_{\text {add }}\left(\frac{i}{i^{\alpha}}\right)
$$

For styrene the simulations indicate that both processes have to be taken into account to describe the monomer conversion and the MMD precisely. The results are included in Figure 6.22 .

A good agreement is found between experiment and modeling, yet one has to take into account that the main fit parameter were the monomer conversion and MMD (see Figure 6.22). The results indicated that possible catalyst species can be monitored via $\mathrm{UV} /$ Vis studies. The absorbance at $599 \mathrm{~nm}$ is only slightly reduced which is in agreement with the complex $\mathbf{C}(\mathbf{M})$, which yields high concentrations during simulation. Secondly the increase at $619 \mathrm{~nm}$ has a shape which is more or less similar to the formation of species $\mathbf{D}$ during modeling. These changes would allow a better determination of the 

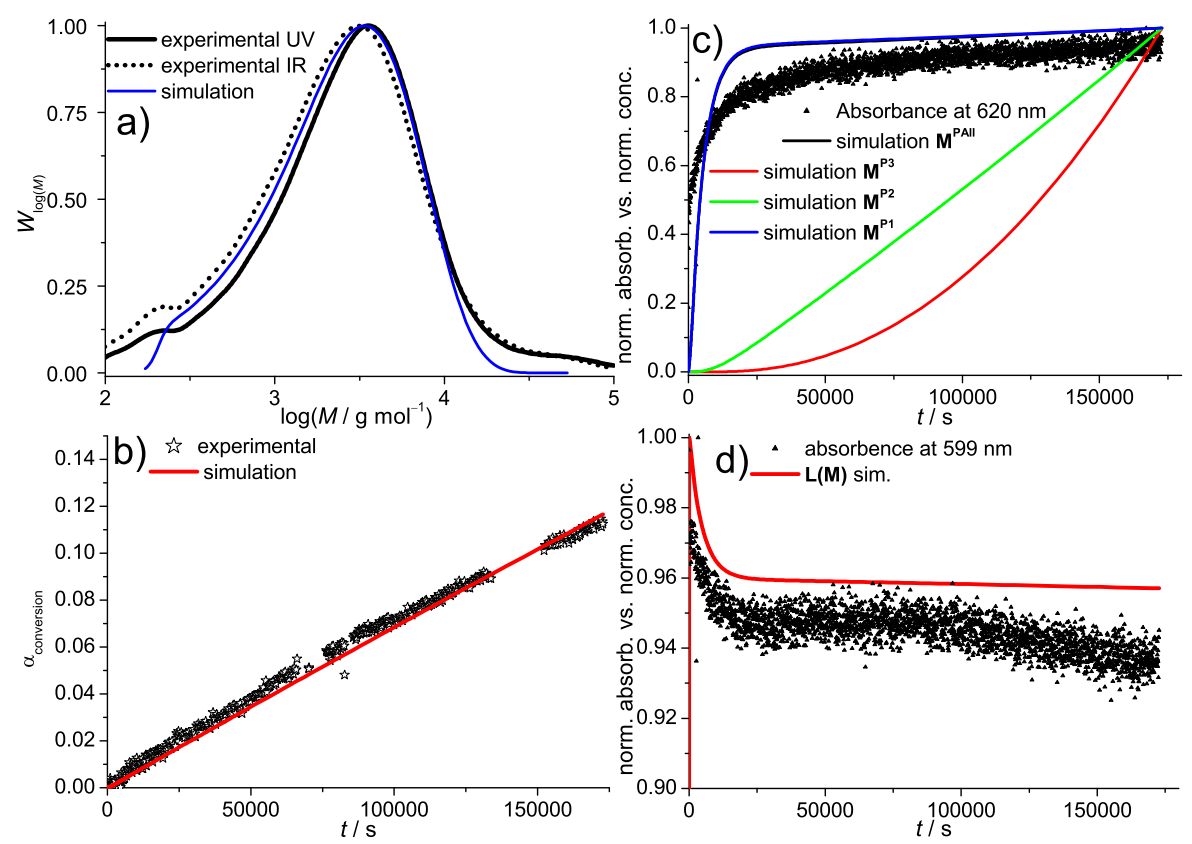

Figure 6.22: Experiment xiii) vs. simulation for styrene polymerization with I and BzMgBz at a ratio $\mathrm{Nd}: \mathrm{Zr}$ 1:130. $c\left(\mathrm{Cp}^{*}{ }_{2} \mathrm{NdCl}_{2} \mathrm{Li}\left(\mathrm{OEt}_{2}\right)_{2}\right)=0.64 \mathrm{mmol} \mathrm{L}^{-1}, c(\mathrm{BzMgBz})=84 \mathrm{mmol} \mathrm{L}^{-1}$ and $c$ (styrene) $=7.77 \mathrm{~mol} \mathrm{~L}^{-1}$. a) MMD curves; b) monomer conversion vs. time; c) absorbance at 599 $\mathrm{nm}$ versus the simulated concentration of $\mathbf{C}(\mathbf{M}) \mathrm{d}$ ) absorbance at $619 \mathrm{~nm}$ versus the simulated concentration of $\mathbf{D}$.

rate coefficients, but several additional experiments are needed to describe the process throughly. The applied kinetic coefficients are summarized in Table 6.4. The basis of the coefficients is weak and more experiments are needed to gain more reliable coefficients. For the experiment at a ratio of 1:14 a good modeling was not possible, the exact reason of which is yet to be determined.

In conclusion it was found that the polymerization of styrene with $\mathbf{I}$ and $\mathrm{BzMgBz}$ is possible and can be accessed via modeling for high magnesium excess compared to neodymium. Yet the simulations indicate high termination rates, which may influence the polymerization behavior at low ratios. This is supported by the result that the polymerization was yet not sucessfully modeled. The system has to be investigated further with regard to the amount of termination products. First experiments via NMR gave no indication for high concentrations of unsaturated end groups. Further experiments via IR-determination were not performed due to time reasons.

Polymerization of Styrene in Presence of DBE The same procedure was performed for similar ratios in the presence of DBE. The conversion shows for a $\mathrm{Mg}: \mathrm{Nd}$ ratio of 14 a strong kink at a low reaction time with a extremely high reaction rate compared 
Table 6.4 Parameter determined by modeling for CCG polymerization of styrene at $70^{\circ} \mathrm{C}$, $c\left(\mathrm{Cp}_{2}{ }_{2} \mathrm{NdCl}_{2} \mathrm{Li}\left(\mathrm{OEt}_{2}\right)_{2}\right)=0.64 \mathrm{mmol} \mathrm{L}^{-1}$ in styrene $7.44 \mathrm{~mol} \mathrm{~L}^{-1}$ and toluene $1.05 \mathrm{~mol} \mathrm{~L}^{-1}$ for two different $\mathrm{BzMgBz}$ concentrations viii) $0.034 \mathrm{~mol} \mathrm{~L}^{-1}$; ix) $0.067 \mathrm{~mol} \mathrm{~L}^{-1}$ ).

\begin{tabular}{c|c|l} 
coefficient & xiii) & \\
\hline$k_{\text {alkyl1 }}$ & 1 & $\mathrm{~s}^{-1}$ \\
$k_{\text {ex0 }}$ & 50 & $\mathrm{~L} \mathrm{~mol}^{-1} \mathrm{~s}^{-1}$ \\
$k_{\mathrm{act} 0}$ & 1 & $\mathrm{~s}^{-1}$ \\
$k_{\mathrm{ex} 1}$ & 8 & $10^{-4} \mathrm{~L} \mathrm{~mol}^{-1} \mathrm{~s}^{-1}$ \\
$k_{\mathrm{act} 1}$ & 2 & $10^{-5} \mathrm{~s}^{-1}$ \\
$k_{\mathrm{ex} 2}$ & 1.2 & $10^{-2} \mathrm{~L} \mathrm{~mol}^{-1} \mathrm{~s}^{-1}$ \\
$k_{\mathrm{act} 2}$ & 2 & $10^{-4} \mathrm{~s}^{-1}$ \\
$a$ & 8 & $10^{-3}$ \\
$\alpha$ & 1.6 & \\
$k_{\text {ini }}$ & 3 & $10^{-2} \mathrm{~s}^{-1}$ \\
$K_{\mathrm{add}} / K_{\text {frag }}$ & 50 & $\mathrm{~s}^{-1}$ \\
$k_{\mathrm{p}, \infty}$ & 0.135 & $\mathrm{~s}^{-1}$ \\
$k_{\mathrm{t}}$ & 0.078 & $\mathrm{~s}^{-1}$
\end{tabular}


to the lower reaction rate found after around 1000 seconds (see Figure 6.23 a)). The $\bar{M}_{\mathrm{n}}$ of the first measurement is determined to be $6500 \mathrm{~g} \mathrm{~mol}^{-1}$ after one hour which is in agreement with the high reaction rate, and it increases with proceeding conversion while the dispersity decreases (see Figure 6.23 b), c), d)). Compared to the results found in absence of DBE, this results indicate that the same influence is found as for the zirconium system. The conversion/time profile shows in both cases a stronger kink in the first minutes of measurement and trends to a constant reaction rate at higher reaction times. The reaction rate of the exchange reactions are lowered and therefore a higher molecular weight is gained than in absence of DBE. The overall conversion is higher which is in agreement with the results found in presence of ethylene .
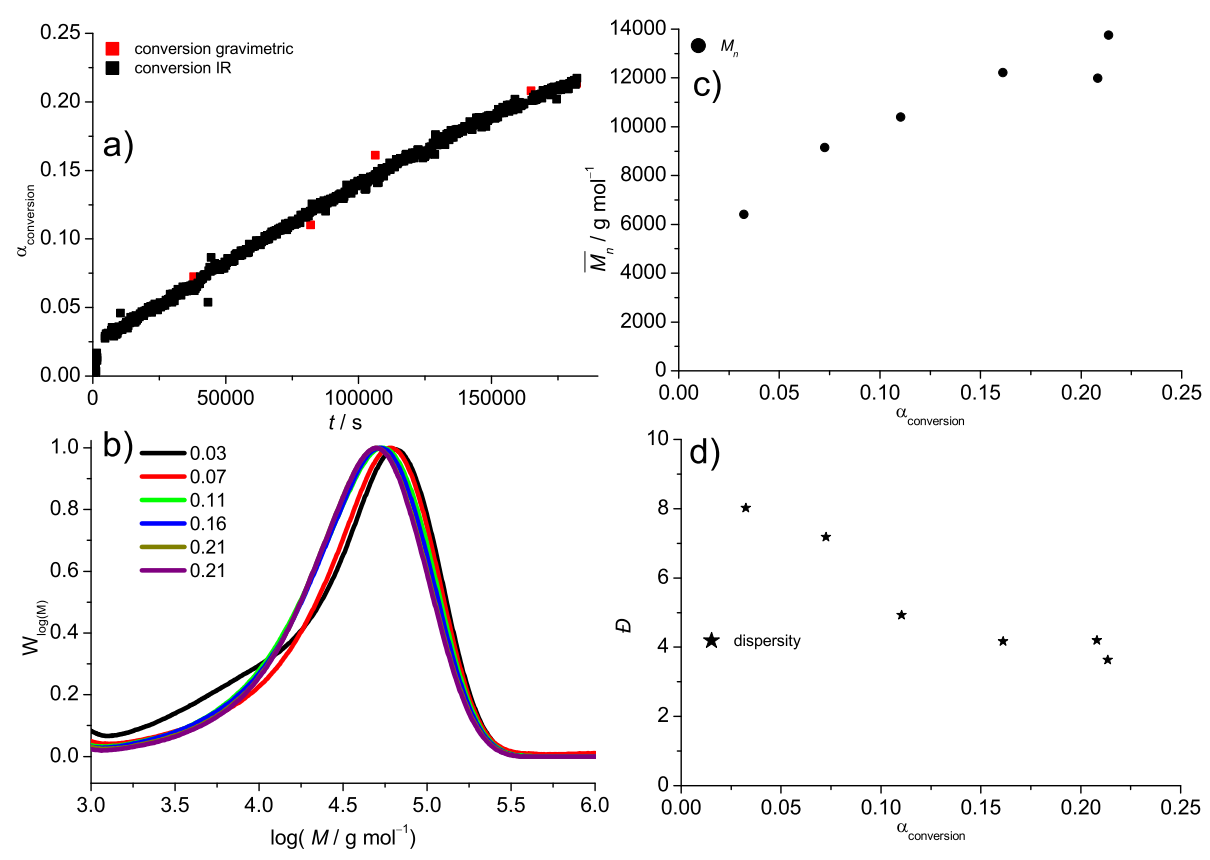

Figure 6.23: Experiment versus simulation for styrene polymerization with $\mathrm{Cp}_{2}{ }_{2} \mathrm{NdCl}_{2} \mathrm{Li}\left(\mathrm{OEt}_{2}\right)_{2}$ and $\mathrm{BzMgBz}$. Experiment xiv): $c\left(\mathrm{Cp}_{2}{ }_{2} \mathrm{NdCl}_{2} \mathrm{Li}\left(\mathrm{OEt}_{2}\right)_{2}\right)=2.43 \mathrm{mmol} \mathrm{L}^{-1}$, $c(\mathrm{BzMgBz})=33 \mathrm{mmol} \mathrm{L}^{-1} c(\mathrm{DBE})=263 \mathrm{mmol} \mathrm{L}^{-1}$ and $c$ (styrene $)=7.44 \mathrm{~mol} \mathrm{~L}^{-1}$. a) MMD curves; $\mathrm{b}$ ) monomer conversion vs. time; c) absorbance at $599 \mathrm{~nm}$ versus the simulated concentration of $\mathbf{L}(\mathbf{M})$ d) absorbance at $619 \mathrm{~nm}$ versus the simulated concentration of $\mathbf{M}$

The UV/Vis spectra over reaction time (see Figure 6.24) show only a very low concentration of species $\mathbf{M}^{\mathbf{P x}}$, which is in agreement with the results for the monomer free experiments (see 4.5 on page 75). The absorption bands at $611 \mathrm{~nm}$ and $619 \mathrm{~nm}$ are only slightly indicated, but they were already found with in first minutes of measurement which is in agreement with the a high reaction rate in the first minutes of reaction. The catalyst center is activated quickly and can add monomer before it is reversibly deactivated by association of BzMgBz.

At a $\mathrm{Mg} / \mathrm{Nd}$ ratio of 130 the effect of the kink shape is decreased as in absence of DBE (see Figure $6.26 \mathrm{~b}$ )). This is in agreement with the slower formation of the absorption bands 


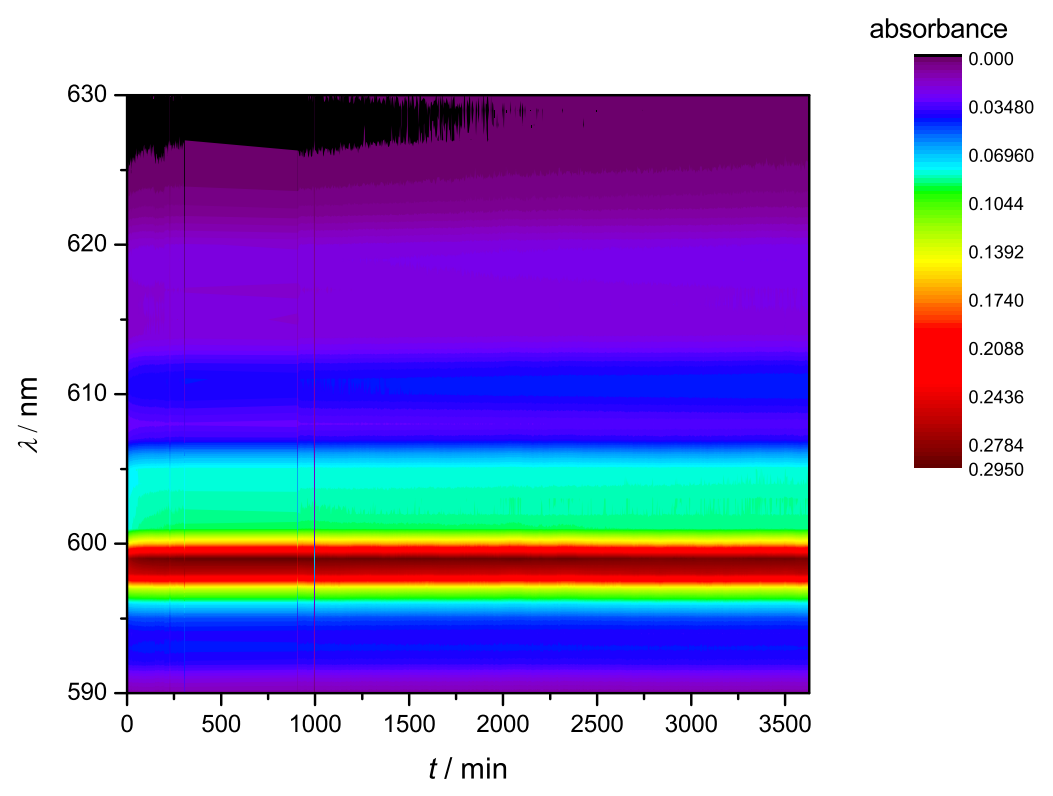

Figure 6.24: UV/Vis absorbance over time and wavelength of styrene polymerization with $\mathrm{Cp}_{2}{ }_{2} \mathrm{NdCl}_{2} \mathrm{Li}\left(\mathrm{OEt}_{2}\right)_{2}$ and BzMgBz. $\quad c\left(\mathrm{Cp}^{*}{ }_{2} \mathrm{NdCl}_{2} \mathrm{Li}\left(\mathrm{OEt}_{2}\right)_{2}\right)=2.43 \mathrm{mmol} \mathrm{L}^{-1}$, $c(\mathrm{BzMgBz})=33 \mathrm{mmol} \mathrm{L}^{-1} c(\mathrm{DBE})=263 \mathrm{mmol} \mathrm{L}^{-1}$ and $c$ (styrene $)=7.44 \mathrm{~mol} \mathrm{~L}^{-1}$.

at $611 \mathrm{~nm}$ and $619 \mathrm{~nm}$. A higher concentration of $\mathbf{M}^{\mathbf{P x}}$ is possible but due to the different maximum absorbancies a clear trend cannot be determined. A lower $\bar{M}_{\mathrm{n}}=2700 \mathrm{~g} \mathrm{~mol}^{-1}$ with a dispersity of 4.9 is found and the MMD is strongly shifted to a lower maximum. Please note that the strong shoulder at high molar mass in the MMD for IR measurement may be the result of a leakage, because compared to the typical results the solution had a different color than in UV/Vis measurement. Gravimetric measurements indicate that the conversion may be determined too small. The difference is still in the area of uncertainty found over all measurements indicating that the influence is only small. Both polymerizations were tried to be modeled via PREDICI ${ }^{\mathrm{TM}}$, but again only the experiment with a high ratio could be modeled. The fit between modeling and experiment is included in Figure 6.26. The MMD and the monomer conversion are well described (see Figure 6.26 a) and b)), only the area at high molecular weight is yet not reached. This is due to the previous discussed leakage. The comparison between the experimental determined absorbance is at $619 \mathrm{~nm}$ similar to the finding in absence of ether, but the maximum is approached at a lower reaction rate. The good agreement between $\mathbf{L}(\mathbf{M})$ and the absorbance at $598 \mathrm{~nm}$ was not found. The results of modeling are similar to the absence of ether (see Table 6.5). The reasons for this differences could not be found and have to be targeted in future studies.

In comparison for a successful modeling in the case of absence and presence of ether only a variation in the rate coefficients of propagation and termination was necessary. 


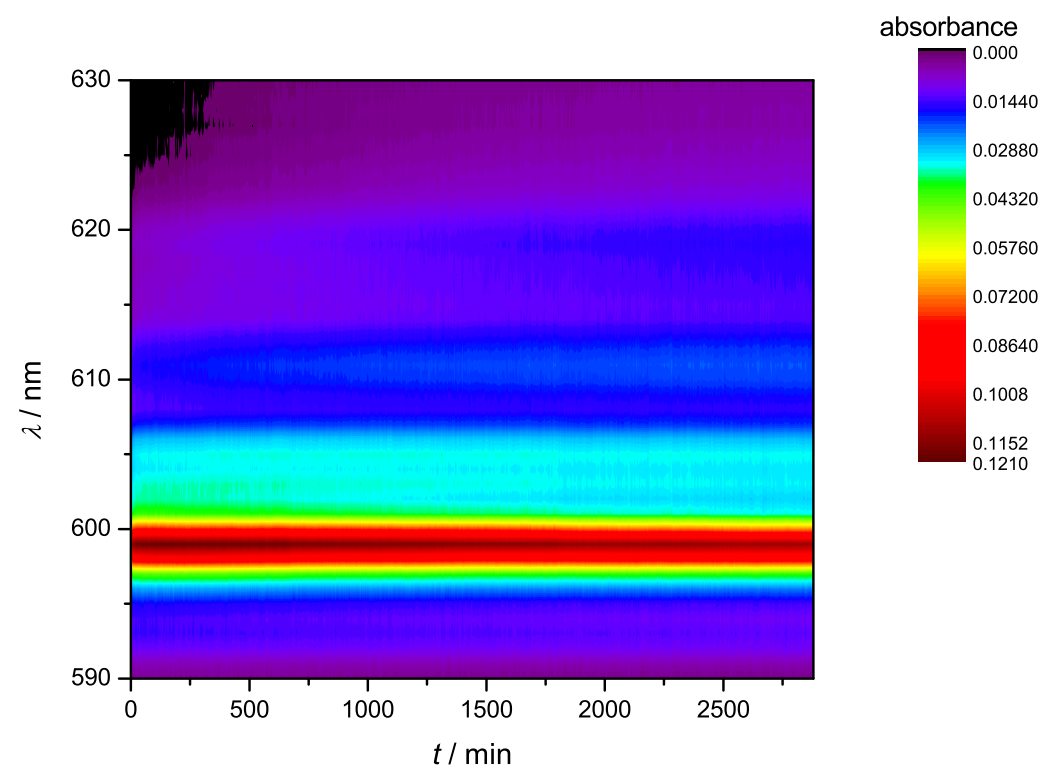

Figure 6.25: UV/Vis absorbance over time and wavelength of styrene polymerization with $\mathrm{Cp}_{2}{ }_{2} \mathrm{NdCl}_{2} \mathrm{Li}\left(\mathrm{OEt}_{2}\right)_{2}$ and $\mathrm{BzMgBz} . \quad c\left(\mathrm{Cp}_{2}{ }_{2} \mathrm{NdCl}_{2} \mathrm{Li}\left(\mathrm{OEt}_{2}\right)_{2}\right)=0.64 \mathrm{mmol} \mathrm{L}^{-1}$, $c(\mathrm{BzMgBz})=84 \mathrm{mmol} \mathrm{L}^{-1} c(\mathrm{DBE})=129 \mathrm{mmol} \mathrm{L}^{-1}$ and $c($ styrene $)=7.77 \mathrm{~mol} \mathrm{~L}^{-1}$.
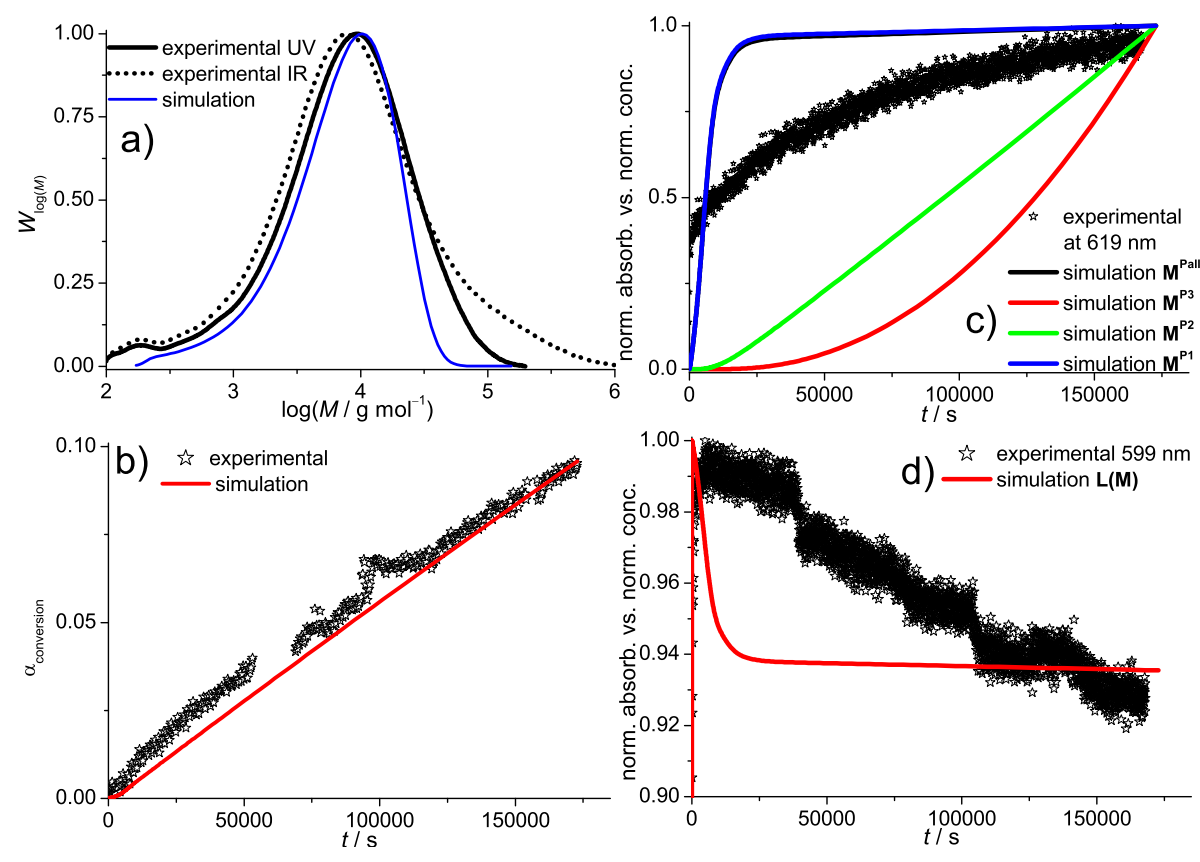

Figure 6.26: Experiment versus simulation for styrene polymerization with $\mathrm{Cp}_{2}{ }_{2} \mathrm{NdCl}_{2} \mathrm{Li}\left(\mathrm{OEt}_{2}\right)_{2}$ and BzMgBz. $c\left(\mathrm{Cp}_{2}{ }_{2} \mathrm{NdCl}_{2} \mathrm{Li}_{(}\left(\mathrm{OEt}_{2}\right)_{2}\right)=0.64 \mathrm{mmol} \mathrm{L}^{-1}, c(\mathrm{BzMgBz})=84 \mathrm{mmol} \mathrm{L}^{-1}, c(\mathrm{DBE})=129 \mathrm{mmol} \mathrm{L}^{-1}$ and $c$ (styrene) $=7.77 \mathrm{~mol} \mathrm{~L}^{-1}$. a) MMD curves; $\mathrm{b}$ ) monomer conversion vs. time; c) absorbance at $599 \mathrm{~nm}$ versus the simulated concentration of $\mathbf{L}(\mathbf{M}) \mathrm{d}$ ) absorbance at $619 \mathrm{~nm}$ versus the simulated concentration of $\mathbf{M}$. 
Table 6.5 Parameter for modelation of CCG polymerization of styrene at $70^{\circ} \mathrm{C}$, $\mathrm{Cp}_{2}{ }_{2} \mathrm{NdCl}_{2} \mathrm{Li}\left(\mathrm{OEt}_{2}\right)_{2}=0.64 \mathrm{mmol} \mathrm{L}^{-1}$ in styrene $7.77 \mathrm{~mol} \mathrm{~L}^{-1}, c(\mathrm{DBE})=128 \mathrm{mmol} \mathrm{L}^{-1}$ and toluene $1.05 \mathrm{~mol} \mathrm{~L}^{-1}$ with a BzMgBz concentration of $84 \mathrm{mmol} \mathrm{L}^{-1}$ ).

\begin{tabular}{c|c|l} 
coefficient & $\mathrm{xv})$ & \\
\hline$k_{\mathrm{alkyl} 1}$ & 1 & $\mathrm{~s}^{-1}$ \\
$k_{\mathrm{ex} 0}$ & 50 & $\mathrm{~L} \mathrm{~mol}^{-1} \mathrm{~s}^{-1}$ \\
$k_{\mathrm{act} 0}$ & 1 & $\mathrm{~s}^{-1}$ \\
$k_{\mathrm{ex} 1}$ & 8 & $10^{-4} \mathrm{~L} \mathrm{~mol}^{-1} \mathrm{~s}^{-1}$ \\
$k_{\mathrm{act} 1}$ & 2 & $10^{-5} \mathrm{~s}^{-1}$ \\
$k_{\mathrm{ex} 2}$ & 1.2 & $10^{-2} \mathrm{~L} \mathrm{~mol}^{-1} \mathrm{~s}^{-1}$ \\
$k_{\mathrm{act} 2}$ & 2 & $10^{-4} \mathrm{~s}^{-1}$ \\
$a$ & 7 & $10^{-3}$ \\
$\alpha$ & 1.6 & \\
$k_{\text {ini }}$ & 3 & $10^{-2} \mathrm{~s}^{-1}$ \\
$K_{\mathrm{add}} / K_{\text {frag }}$ & 50 & $\mathrm{~s}^{-1}$ \\
$k_{\mathrm{p}, \infty}$ & 0.0725 & $\mathrm{~s}^{-1}$ \\
$k_{\mathrm{t}}$ & 1.5 & $10^{-2} \mathrm{~s}^{-1}$ \\
&
\end{tabular}


The CCG process is completely overlaid by termination. This may also be the reason for the problems to model the experiment with the low $\mathrm{Mg}: \mathrm{Nd}$ ratio of 14 . The process of termination has to be the objective of a more detailed future study regarding the polymerization at very short reactions times.

Conclusion For the polymerization of styrene with $\mathrm{Cp}_{2}{ }_{2} \mathrm{NdCl}_{2} \mathrm{Li}\left(\mathrm{OEt}_{2}\right)$ and $\mathrm{BzMgBz}$ it was found that the process is dominated by the termination reaction of the polymer chain in presence as well as in absence of ether. The process is mainly driven by the formed hydride species, and the formation of the different equilibrium species is slow and not preferred. Because of this, the polymerization in presence of $\mathrm{BzMgBz}$ may be a possible reaction pathway to gain information about the termination reaction rates for different monomers.

\subsection{Polymerization of Styrene with $\mathrm{Cp}_{2}{ }_{2} \mathrm{NdCl}_{2} \mathrm{Li}\left(\mathrm{OEt}_{2}\right)$ and BuMgOct}

The full CCG system of I and BuMgOct was applied to styrene polymerization within the $\mathrm{UV} /$ Vis- and IR-spectrometer to investigated difference to BzMgBz. All measurements in this chapter show higher conversion then the experiments with BzMgBz. This indicates that different equilibrium constants are present. The experiments were performed in absence and presence of DBE for two Mg:Nd ratios (165:1 and 325:1). The overall maximum absorbance in all experiments was lowered by $75 \%$.

Polymerization of Styrene in Absence of DBE In presence of BuMgOct similar absorbance bands to BzMgBz are found in the area $600 \mathrm{~nm}$ to $608 \mathrm{~nm}, 611 \mathrm{~nm}$ and $619 \mathrm{~nm}$ (see Figure 6.27, ratio 160:1). They are more pronounced indicating higher equilibrium constants are reached. Three areas of reaction can be determined. First the catalyst activation, which is finished within 200 seconds. The absorption bands in the area from $600 \mathrm{~nm}$ to $608 \mathrm{~nm}$ were almost fully converted into a strong signal at $598 \mathrm{~nm}$. After formation the signal decreases while the absorption bands at $611 \mathrm{~nm}$ and $619 \mathrm{~nm}$ gain intensity until the system is fully converted. It is assumed that the processes describe the conversion of the catalyst precursor into the alkylated species and the activation process of the magnesium bound alkyl chains.

This assumption is affirmed by the experiment at a higher ratio of BuMgOct (see Figure 


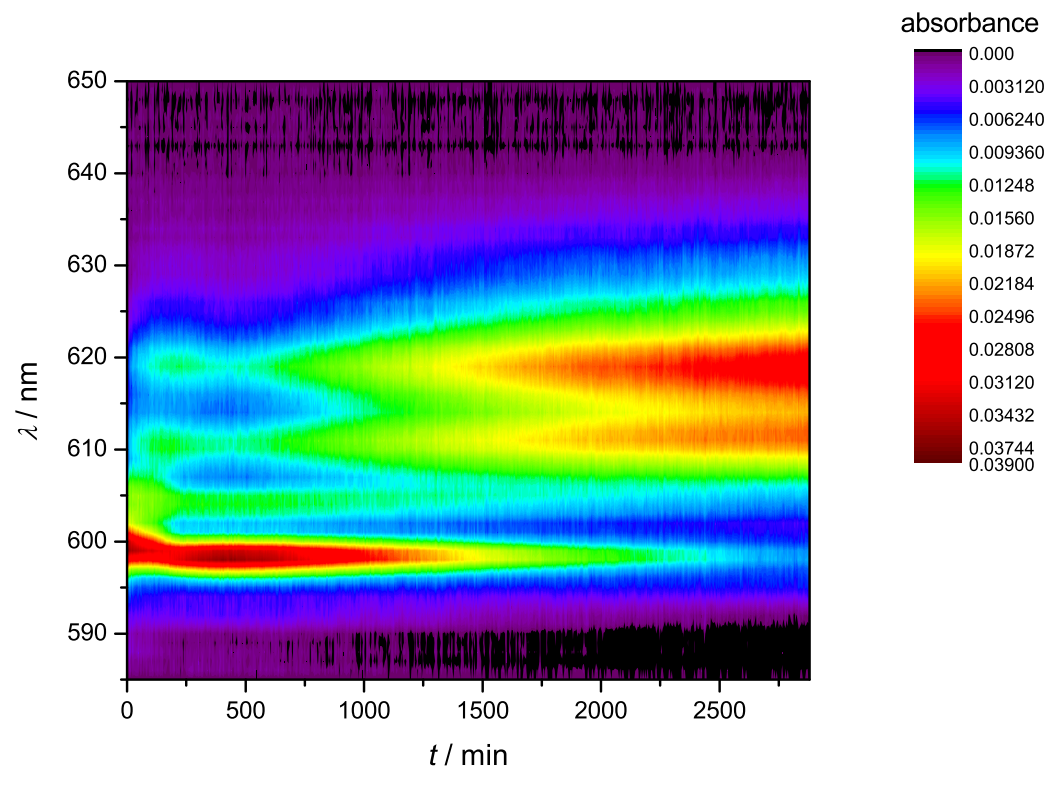

Figure 6.27: UV/Vis absorbance over time and wavelength of styrene polymerization with $\mathrm{Cp}_{2}{ }_{2} \mathrm{NdCl}_{2} \mathrm{Li}\left(\mathrm{OEt}_{2}\right)_{2}$ and BuMgOct. $\quad c\left(\mathrm{Cp}_{2}{ }_{2} \mathrm{NdCl}_{2} \mathrm{Li}\left(\mathrm{OEt}_{2}\right)_{2}\right)=0.26 \mathrm{mmol} \mathrm{L}^{-1}$, $c($ BuMgOct $)=42 \mathrm{mmol} \mathrm{L}^{-1}$ and $c$ (styrene $)=7.90 \mathrm{~mol} \mathrm{~L}^{-1}$.

6.28, ratio 325:1). The same behavior as for $160: 1$ is found but the process are slower and the absorption bands stronger pronounced. At the end of reaction the full conversion of BuOctMg was not yet performed indicated by the remaining absorbance at $598 \mathrm{~nm}$.

The conversion decreases with increasing Mg:Nd ratio (see Figure 6.29 b) and Figure $6.30 \mathrm{~b})$ ) which is to be expected with increasing concentration of the CTA. The MMD and average molar mass is reduced and the conversion profile shows a strong kink shape for the higher ratio. This is a result of the higher amount of polymer chains started in the beginning of the reaction.

The respective figures also show the simulation results. There are differences in the conversion time profile and the absorbance values are yet not fully described. Due to the fact that no extinction coefficients could be derived this has to be expected. Nevertheless, the simulation of the different species can describe the occurring processes. Only one difference was found: While the exchange reactions of the polymeric species could be described with the same rate coefficient as for $\mathrm{BzMgBz}$, the situation was found to be different for alkyl chains. The monomer conversion was found only to be accessible when the formation of the alkylated complexes is preferred. Therefore the rate coefficient $k_{\text {act1 }}$ was applied for these processes. (See Appendix for the detailed model).

The MMD is fitted well for the ratio of 160:1 while it is too small for 325:1 (cf. Figure 6.29 a) and Figure 6.30 a)). The conversion time profile is described well in both cases (see Figure $6.29 \mathrm{~b}$ ) and Figure $6.30 \mathrm{~b}$ )) and in both cases the kink-shaped conversion profile 


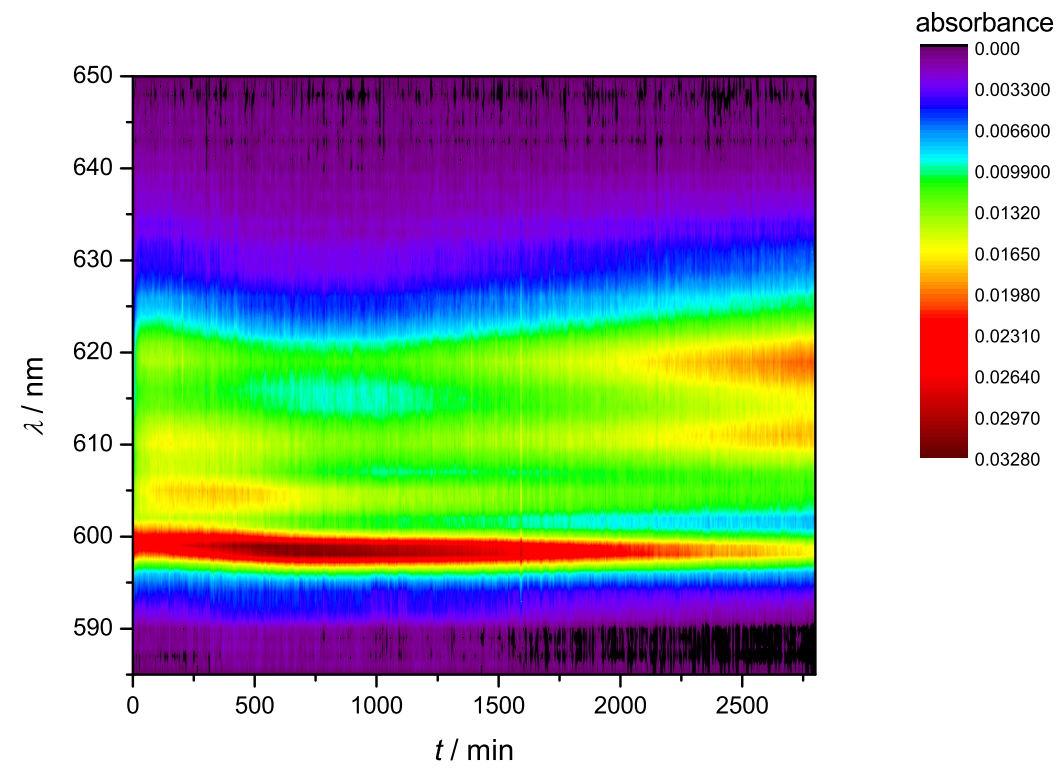

Figure 6.28: UV/Vis absorbance over time and wavelength of styrene polymerization with $\mathrm{Cp}_{2}{ }_{2} \mathrm{NdCl}_{2} \mathrm{Li}\left(\mathrm{OEt}_{2}\right)_{2}$ and BuMgOct. $\quad c\left(\mathrm{Cp}_{2}{ }_{2} \mathrm{NdCl}_{2} \mathrm{Li}\left(\mathrm{OEt}_{2}\right)_{2}\right)=0.25 \mathrm{mmol} \mathrm{L}^{-1}$, $c($ BuMgOct $)=80 \mathrm{mmol} \mathrm{L}^{-1}$ and $c($ styrene $)=7.55 \mathrm{~mol} \mathrm{~L}^{-1}$.
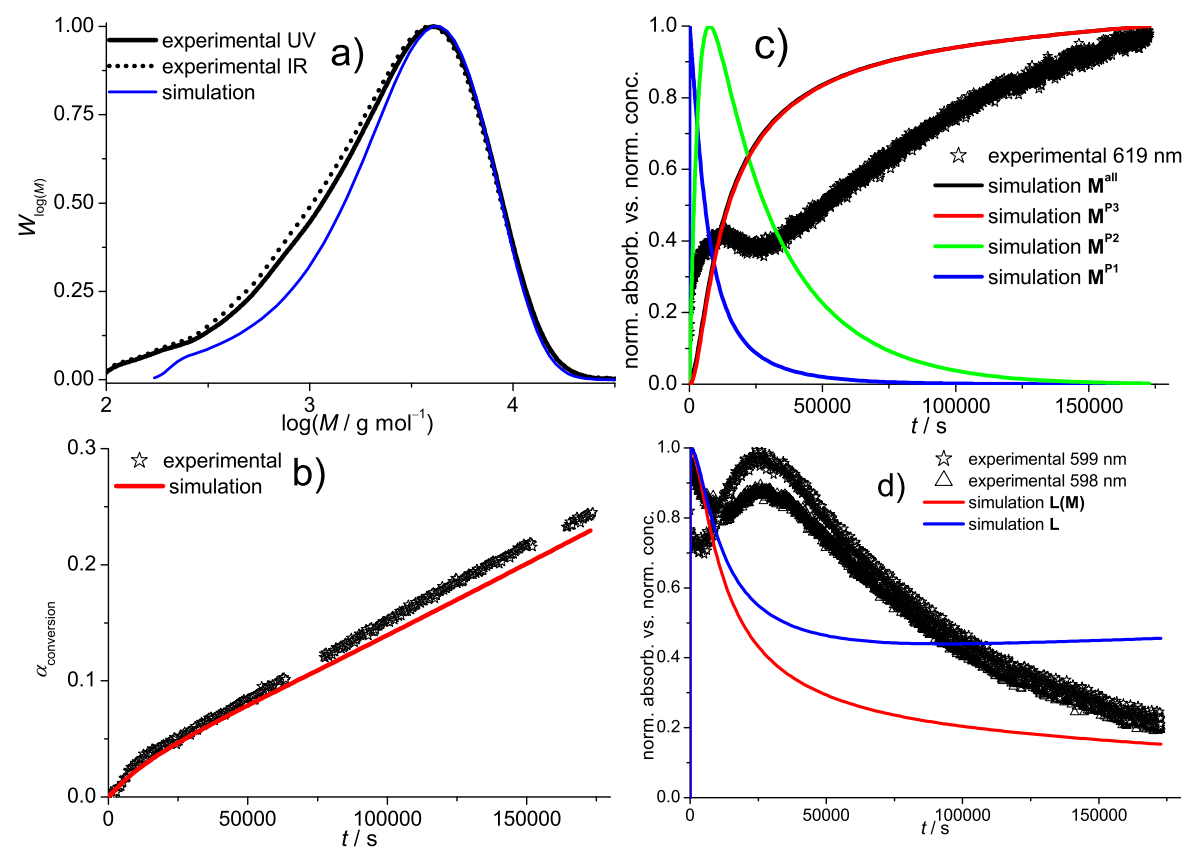

Figure 6.29: Experiment versus simulation for styrene polymerization with $\left.\mathrm{Cp}_{2}{ }_{2} \mathrm{NdCl}_{2} \mathrm{Li}_{(\mathrm{OEt}}\right)_{2}$ and BuMgOct. $\quad c\left(\mathrm{Cp}_{2}{ }_{2} \mathrm{NdCl}_{2} \mathrm{Li}\left(\mathrm{OEt}_{2}\right)_{2}\right)=0.26 \mathrm{mmol} \mathrm{L}^{-1}, c($ BuMgOct $)=42 \mathrm{mmol} \mathrm{L}^{-1}$ and $c$ (styrene) $=7.90 \mathrm{~mol} \mathrm{~L}^{-1}$. a) MMD curves; $\mathrm{b}$ ) monomer conversion vs. time; $\mathrm{c}$ ) normalized absorbance at $599 \mathrm{~nm}$ versus normalized the simulated concentration of $\mathbf{L}(\mathbf{M})$ d) normalized absorbance at 619 $\mathrm{nm}$ versus the normalized simulated concentration of $\mathbf{M}$. 

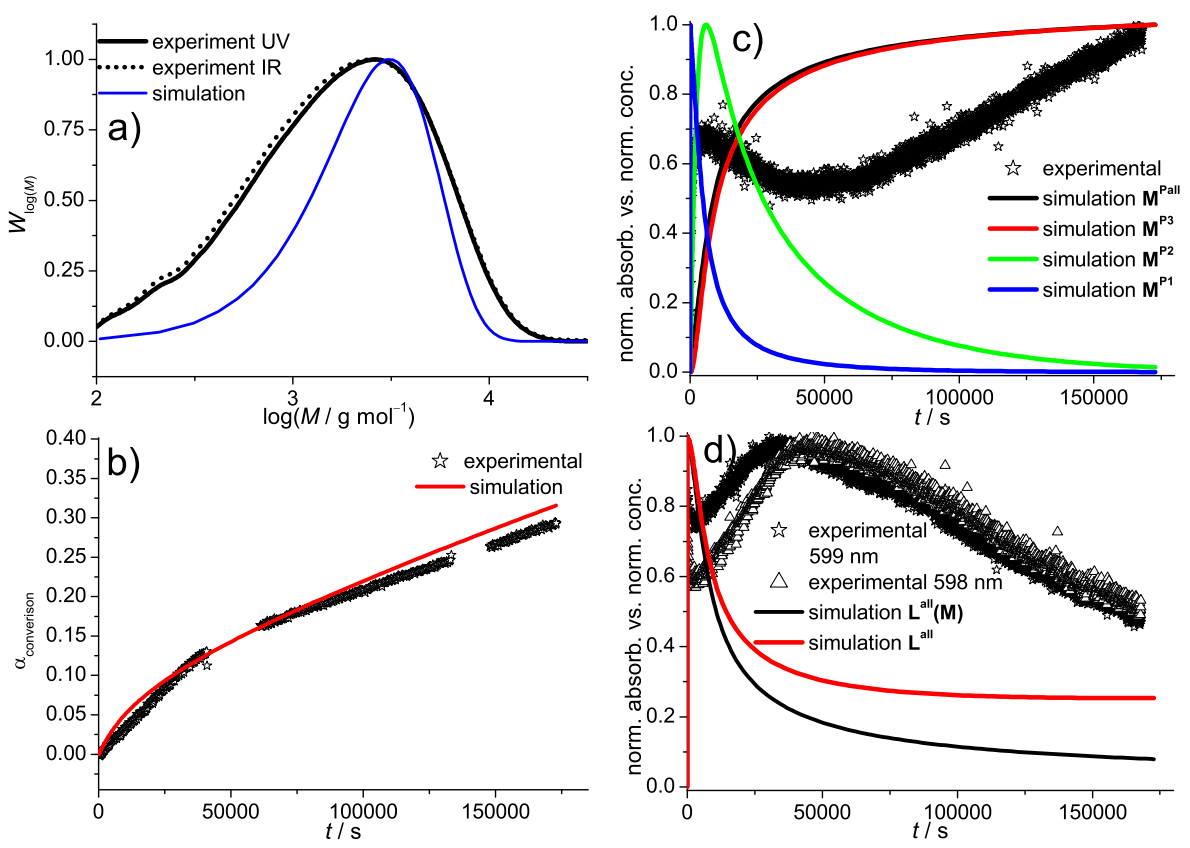

Figure 6.30: Experiment versus simulation for styrene polymerization with $\left.\mathrm{Cp}_{2}{ }_{2} \mathrm{NdCl}_{2} \mathrm{Li}_{(\mathrm{OEt}}\right)_{2}$ and BuMgOct. $\quad c\left(\mathrm{Cp}_{2}{ }_{2} \mathrm{NdCl}_{2} \mathrm{Li}\left(\mathrm{OEt}_{2}\right)_{2}\right)=0.25 \mathrm{mmol} \mathrm{L}^{-1}, c($ BuMgOct $)=80 \mathrm{mmol} \mathrm{L}^{-1}$ and $c$ (styrene) $=7.55 \mathrm{~mol} \mathrm{~L}^{-1}$. a) MMD curves; b) monomer conversion vs. time; $c$ ) absorbance at $599 \mathrm{~nm}$ versus the simulated concentration of $\mathbf{L}(\mathbf{M})$ d) absorbance at $619 \mathrm{~nm}$ versus the simulated concentration of $\mathbf{M}$.

could be attained. Additional to these processes the absorbance at 619, 599 and $598 \mathrm{~nm}$ were investigated (see Figure 6.29 c), d) and Figure $6.30 \mathrm{c}$ ), d)). For better comparison the absorbance and concentrations were normalized. For all wavelengths a local maximum at short reaction times is found. In the case of $619 \mathrm{~nm}$ the maximum for both ratios is found as long as the kink in the conversion lasts. By modeling it was found that the complex species similar to $\mathbf{M}^{\mathrm{P} 2}$ may be the reason for this behavior. For the maximum at $599 \mathrm{~nm}$ and $598 \mathrm{~nm}$ no good explanation was found until now. The agreement between $\mathrm{C}(\mathrm{M})$ and $599 \mathrm{~nm}$ as for polymerization with BzMgBz could not be found. This result indicates different equilibrium constants for the occurring species, because the absorbance at $598 \mathrm{~nm}$ was found over all experiments to be formed via the uncoordinated complex while the bimetallic complexes are found at the wavelengths $611 \mathrm{~nm}$ and $619 \mathrm{~nm}$.

Polymerization of Styrene in Presence of DBE In presence of DBE the exchange reactions are found to be much faster. At the ratio $\mathrm{Mg}: \mathrm{Nd} 170$ (see Figure 6.31) the absorbance at $599 \mathrm{~nm}$ is only found for a few minutes. The signal in the region from $600 \mathrm{~nm}$ to $608 \mathrm{~nm}$ is only shortly indicated while the absorption bands at $611 \mathrm{~nm}$ and $619 \mathrm{~nm}$ are formed in this short timespan.

The formation takes longer if the $\mathrm{Mg}: \mathrm{Nd}$ ratio is increased to 320:1 (see Figure 6.32). In 
Table 6.6 Parameters determined by modeling for CCG polymerization of styrene at $70^{\circ} \mathrm{C}$, experiment xvi) $\mathrm{Cp}_{2}{ }_{2} \mathrm{NdCl}_{2} \mathrm{Li}\left(\mathrm{OEt}_{2}\right)_{2}=0.26 \mathrm{mmol} \mathrm{L}^{-1}, c($ styrene $)=7.90 \mathrm{~mol} \mathrm{~L}^{-1}, c$ (toluene) $0.45 \mathrm{~mol} \mathrm{~L}^{-1}, c$ (BuMgOct $)=42 \mathrm{mmol} \mathrm{L}^{-1}$; experiment xvii) $\mathrm{Cp}_{2}{ }_{2} \mathrm{NdCl}_{2} \mathrm{Li}\left(\mathrm{OEt}_{2}\right)_{2}=0.25 \mathrm{mmol} \mathrm{L}^{-1}$, $c($ styrene $)=7.55 \mathrm{~mol} \mathrm{~L}^{-1}, c$ (toluene) $0.43 \mathrm{~mol} \mathrm{~L}^{-1}, c($ BuMgOct $)=80 \mathrm{mmol} \mathrm{L}^{-1}$. Parameters labeled with $a *$ were not varied.

\begin{tabular}{c|c|c|r|l} 
coefficient & xvi) & xvii) & mean value & \\
\hline$k_{\text {alkyl1 }}$ & 1 & 1 & $1^{*}$ & $\mathrm{~s}^{-1}$ \\
$k_{\text {ex1 }}$ & 25 & 25 & $25^{*}$ & $\mathrm{~L} \mathrm{~mol}^{-1} \mathrm{~s}^{-1}$ \\
$k_{\text {act1 }}$ & 2 & 0.9 & $1.45 \pm 0.55$ & $\mathrm{~s}^{-1}$ \\
$k_{\text {ex2 }}$ & 100 & 100 & $100^{*}$ & $\mathrm{~L} \mathrm{~mol}^{-1} \mathrm{~s}^{-1}$ \\
$k_{\text {act2 }}$ & 9 & 1 & $0.5 \pm 0.4$ & $10^{-3} \mathrm{~s}^{-1}$ \\
$k_{\mathrm{p}, \infty}$ & 12 & 40 & $26 \pm 14$ & $\mathrm{~s}^{-1}$ \\
$a$ & 1.25 & 1.0 & $1.12 \pm 0.13$ & $10^{-3}$ \\
$\alpha$ & 1.4 & 1.4 & 1.4 & \\
$k_{\text {ini }}$ & 160 & 160 & 160 & $\mathrm{~s}^{-1}$ \\
$K_{\text {add }} / K_{\text {frag }}$ & 50 & 50 & $50^{*}$ & $\mathrm{~s}^{-1}$ \\
$k_{\text {ex0 }}$ & 50 & 50 & $50^{*}$ & $\mathrm{~L} \mathrm{~mol}^{-1} \mathrm{~s}^{-1}$ \\
$k_{\text {act0 }}$ & 1 & 1 & $1^{*}$ & $\mathrm{~s}^{-1}$ \\
$k_{\mathrm{t}}$ & 0.078 & 0.078 & $0.078^{*}$ & $\mathrm{~s}^{-1}$ \\
$k_{\mathrm{t}, \mathrm{alkyl}}$ & 0.0038 & 0.0038 & $0.0038^{*}$ & $\mathrm{~s}^{-1}$ \\
& & &
\end{tabular}




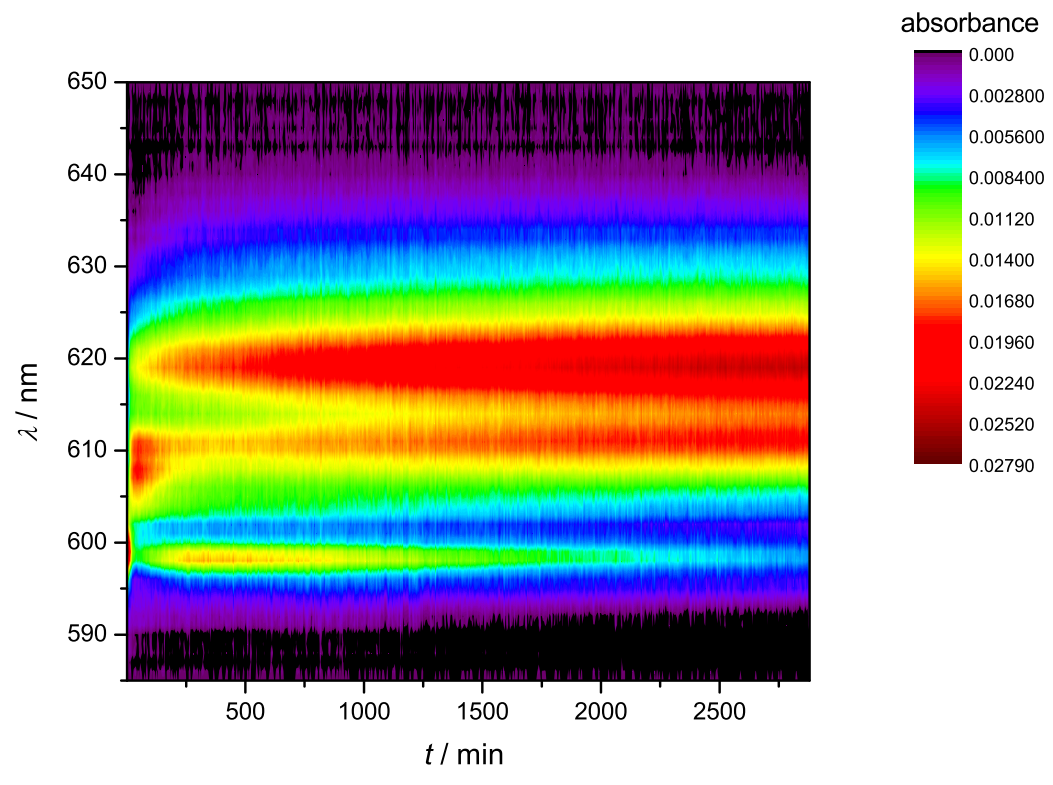

Figure 6.31: UV/Vis absorbance over time and wavelength of styrene polymerization with $\mathrm{Cp}_{2}{ }_{2} \mathrm{NdCl}_{2} \mathrm{Li}\left(\mathrm{OEt}_{2}\right)_{2}$ and BuMgOct in presence of DBE. $c\left(\mathrm{Cp}_{2}{ }_{2} \mathrm{NdCl}_{2} \mathrm{Li}_{(}\left(\mathrm{OEt}_{2}\right)_{2}\right)=0.25 \mathrm{mmol} \mathrm{L}^{-1}$, $c($ BuMgOct $)=40 \mathrm{mmol} \mathrm{L}^{-1} c(\mathrm{DBE})=257 \mathrm{mmol} \mathrm{L}^{-1}$ and $c$ (styrene $)=7.77 \mathrm{~mol} \mathrm{~L}^{-1}$.

both cases a signal at $598 \mathrm{~nm}$ occurs, which is only weak compared to the experiments in absence of DBE. It occurs for a longer time at a low ratio while it is nearly completely vanished at the end of reaction for the higher ratio.

Compared to the absence of DBE, the kink-shaped monomer conversion is reduced and the MMD narrower with lesser amounts of short chain oligomers (see Figures 6.33 and 6.34 a) and b)). Monomer conversion is almost linear during the whole process.

The absorbance at $619 \mathrm{~nm}$ reaches a maximum early for the 325:1 ratio (See Figure 6.34 c)) while it is not fully converted in the case of the ratio $165: 1$. Absorbance at $599 \mathrm{~nm}$ and $598 \mathrm{~nm}$ has a maximum again at short reaction times, which is long lasting in presence of ether with increasing ratio. The processes were modeled similar to those in absence of monomer and comparable results yielded. The monomer conversion is fitted with a good agreement, only the slight shift at 325:1 could not yet successfully be modeled. The MMD curves fit well for the high ratio while it is modeled too narrow for the 160:1 ratio. The comparison between normalized absorbance at $619 \mathrm{~nm}$ and modeled concentrations shows a high difference regarding the concentration of $\mathbf{M}^{\mathrm{P3}}$. While the process is the main product at high reaction times the absorbance is at maximum after $20000 \mathrm{~s}$. This result shows that further experiments are necessary to determine the exact process yielding this high absorbance maximum. The results indicated a high molar absorbance for the complex $\mathbf{D}^{\mathbf{P 2}}$ which is then transferred to a complex $\mathbf{M}^{\mathrm{P3}}$ with a much lower extinction coefficient. This is in agreement with the absorbance profile found in 


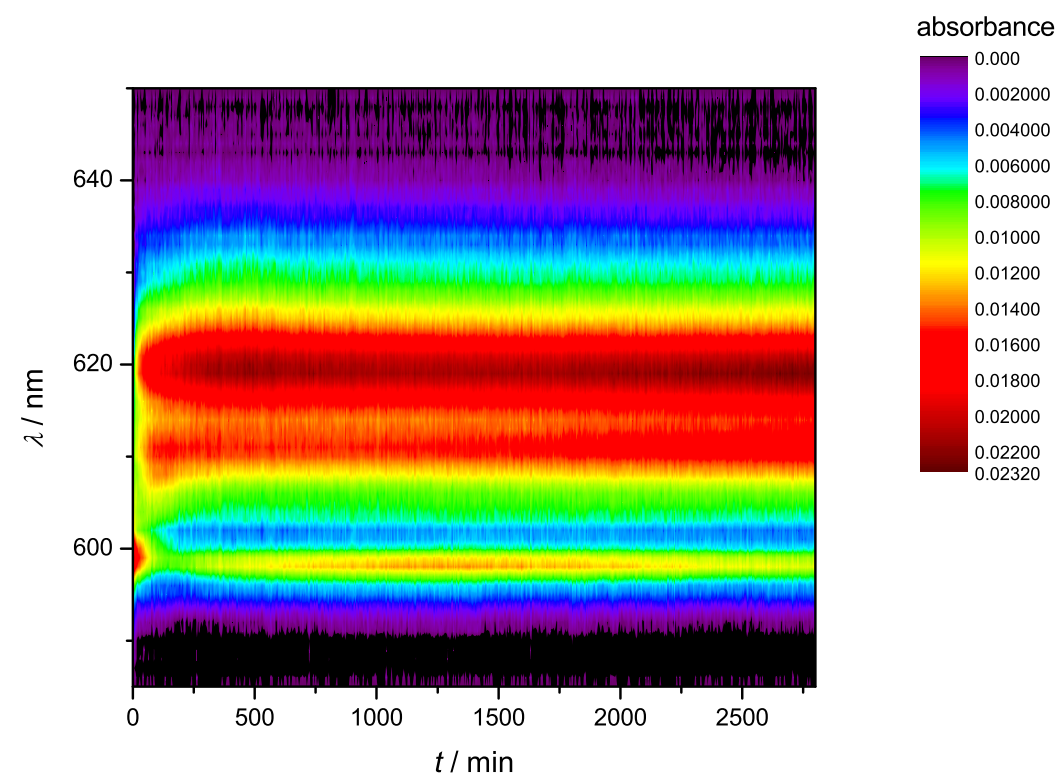

Figure 6.32: UV/Vis absorbance over time and wavelength of styrene polymerization with $\mathrm{Cp}_{2}{ }_{2} \mathrm{NdCl}_{2} \mathrm{Li}\left(\mathrm{OEt}_{2}\right)_{2}$ and BuMgOct in presence of DBE. $c\left(\mathrm{Cp}_{2}{ }_{2} \mathrm{NdCl}_{2} \mathrm{Li}\left(\mathrm{OEt}_{2}\right)_{2}\right)=0.24 \mathrm{mmol} \mathrm{L}^{-1}$, $c($ BuMgOct $)=77 \mathrm{mmol} \mathrm{L}^{-1} c(\mathrm{DBE})=246 \mathrm{mmol} \mathrm{L}^{-1}$ and $c$ (styrene $)=7.77 \mathrm{~mol} \mathrm{~L}^{-1}$.
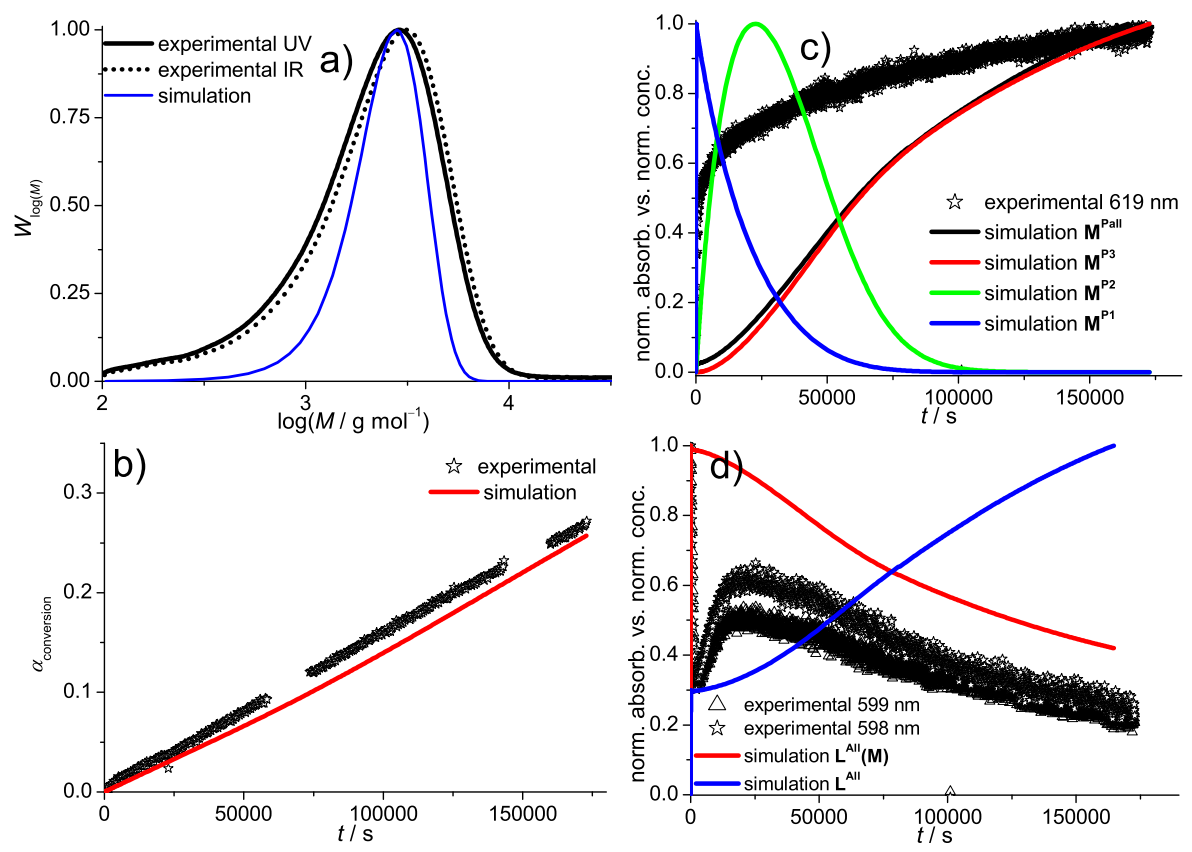

Figure 6.33: Experiment versus simulation for styrene polymerization with $\left.\mathrm{Cp}^{*}{ }_{2} \mathrm{NdCl}_{2} \mathrm{Li}_{(\mathrm{OEt}}\right)_{2}$ and BuMgOct. $\quad c\left(\mathrm{Cp}^{*}{ }_{2} \mathrm{NdCl}_{2} \mathrm{Li}\left(\mathrm{OEt}_{2}\right)_{2}\right)=0.25 \mathrm{mmol} \mathrm{L}^{-1}, \quad c($ BuMgOct $)=40 \mathrm{mmol} \mathrm{L}^{-1}$, $c(\mathrm{DBE})=257 \mathrm{mmol} \mathrm{L}^{-1}$ and $c$ (styrene $)=7.77 \mathrm{~mol} \mathrm{~L}^{-1}$. a) MMD curves; b) monomer conversion vs. time; c) absorbance at $599 \mathrm{~nm}$ versus the simulated concentration of $\mathbf{L}(\mathbf{M}) \mathrm{d}$ ) absorbance at $619 \mathrm{~nm}$ versus the simulated concentration of $\mathbf{M}$. 

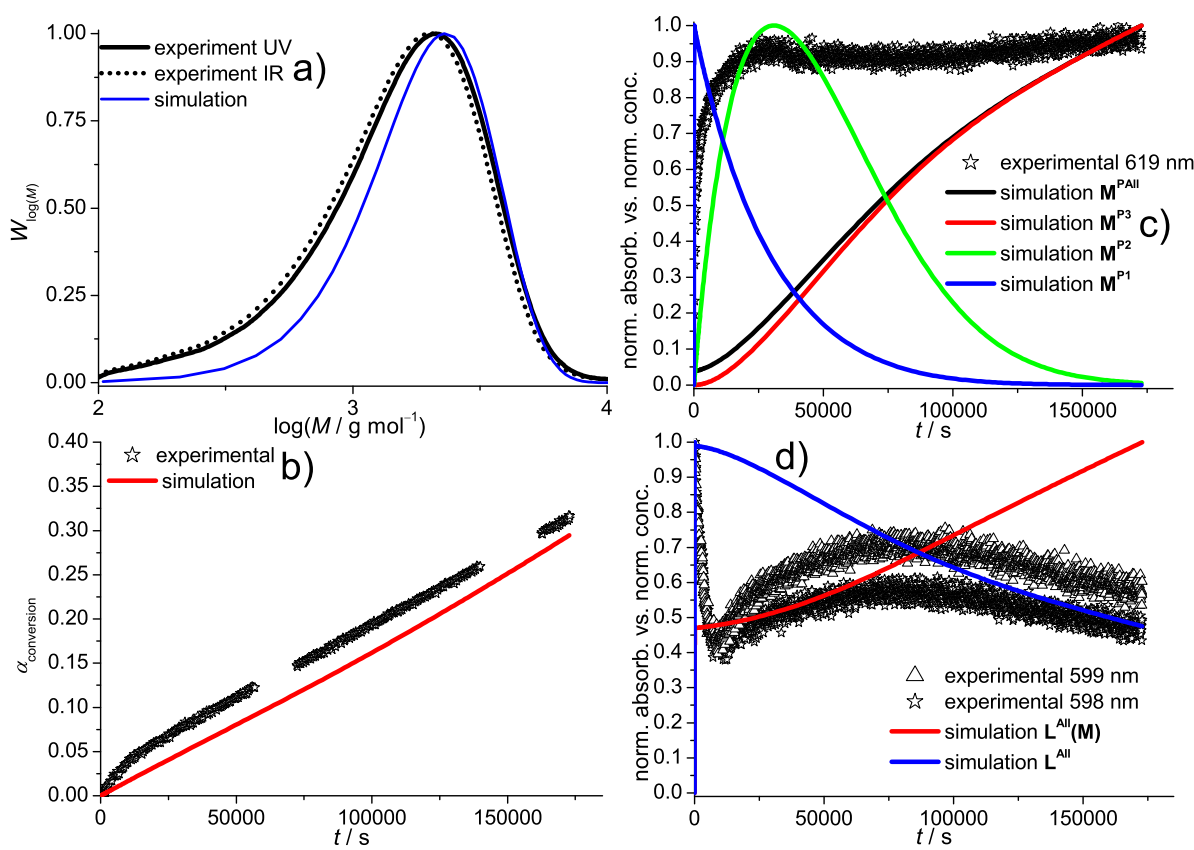

Figure 6.34: Experiment versus simulation for styrene polymerization with $\left.\mathrm{Cp}_{2}{ }_{2} \mathrm{NdCl}_{2} \mathrm{Li}_{(\mathrm{OEt}}\right)_{2}$ and BuMgOct. $\quad c\left(\mathrm{Cp}^{*}{ }_{2} \mathrm{NdCl}_{2} \mathrm{Li}\left(\mathrm{OEt}_{2}\right)_{2}\right)=0.24 \mathrm{mmol} \mathrm{L}^{-1}, c($ BuMgOct $)=77 \mathrm{mmol} \mathrm{L}^{-1}$, $c(\mathrm{DBE})=246 \mathrm{mmol} \mathrm{L}^{-1}$ and $c$ (styrene $)=7.77 \mathrm{~mol} \mathrm{~L}^{-1}$. a) MMD curves; b) monomer conversion vs. time; c) absorbance at $599 \mathrm{~nm}$ versus the simulated concentration of $\mathbf{L}(\mathbf{M}) \mathrm{d}$ ) absorbance at $619 \mathrm{~nm}$ versus the simulated concentration of $\mathbf{M}$.

absence and presence of DBE. In presence of ether the concentration is modeled higher than in absence. It yields the small maximum in the absence of ether. But also the stable one in presence of ether is a result of this transfer. Because of the normalization the concentration seems to be high, which is not the case. Between the concentration of $\mathbf{M}^{\mathbf{P 2}}$ and $\mathbf{M}^{\mathrm{P} 3}$ a difference of one order of magnitude was modeled.

\subsubsection{Conclusion}

The polymerization of styrene with $\mathrm{Cp}_{2}{ }_{2} \mathrm{NdCl}_{2} \mathrm{Li}\left(\mathrm{OEt}_{2}\right)$ was successfully modeled but the determined rate coefficients are of limited accuracy because of the small data set on the one side and one the other side the small amount of fit parameters. Further UV/Vis studies are necessary to find support for the findings of the different influences on the absorbance time profiles especially for the absorbance at $598 \mathrm{~nm}$. The modeling indicated the importance of similar groups at the CTA compared to the formed polymer chain. Compared to $\mathrm{BzMgBz}$ the preferred formation of the catalyst bearing the alkyl chain had to be introduced. This leads to a better control over dispersity comparable to the re-initiation in the RAFT-process, where a similar start of the R-groups is necessary for 
Table 6.7 Parameter determined by modeling for CCG polymerization of styrene in presence of $\mathrm{DBE}$ at $70^{\circ} \mathrm{C}$, xviii) $c\left(\mathrm{Cp}_{2}{ }_{2} \mathrm{NdCl}_{2} \mathrm{Li}\left(\mathrm{OEt}_{2}\right)_{2}\right)=0.25 \mathrm{mmol} \mathrm{L}^{-1}, c(\mathrm{BuMgOct})=40 \mathrm{mmol} \mathrm{L}^{-1}$, $c(\mathrm{DBE})=257 \mathrm{mmol} \mathrm{L}^{-1}, c$ (toluene) $0.43 \mathrm{~mol} \mathrm{~L}^{-1}$ and $c$ (styrene) $=7.77 \mathrm{~mol} \mathrm{~L}^{-1} ; \quad$ xix) $c\left(\mathrm{Cp}_{2}{ }_{2} \mathrm{NdCl}_{2} \mathrm{Li}\left(\mathrm{OEt}_{2}\right)_{2}\right)=0.24 \mathrm{mmol} \mathrm{L}^{-1}, c(\mathrm{BuMgOct})=77 \mathrm{mmol} \mathrm{L}^{-1}, c(\mathrm{DBE})=246 \mathrm{mmol} \mathrm{L}^{-1}$, $c$ (toluene) $0.41 \mathrm{~mol} \mathrm{~L}^{-1}$ and $c$ (styrene) $=7.77 \mathrm{~mol} \mathrm{~L}^{-1}$. Parameters labeled with a * were not varied.

\begin{tabular}{|c|c|c|c|c|}
\hline coefficient & xviii) & xix) & mean value & \\
\hline$k_{\text {alkyl1 }}$ & 1 & 1 & $1^{*}$ & $\mathrm{~s}^{-1}$ \\
\hline$k_{\mathrm{ex} 1}$ & 5 & 5 & $5^{*}$ & $\mathrm{~L} \mathrm{~mol}^{-1} \mathrm{~s}^{-1}$ \\
\hline$k_{\text {act1 }}$ & 0.9 & 0.9 & $0.9^{*}$ & $\mathrm{~s}^{-1}$ \\
\hline$k_{\mathrm{ex} 2}$ & 30 & 30 & $30^{*}$ & $\mathrm{~L} \mathrm{~mol}^{-1} \mathrm{~s}^{-1}$ \\
\hline$k_{\text {act2 }}$ & 5 & 5 & $5^{*}$ & $10^{-3} \mathrm{~s}^{-1}$ \\
\hline$k_{\mathrm{p}, \infty}$ & 0.8 & 0.75 & $0.78 \pm 0.3$ & $\mathrm{~s}^{-1}$ \\
\hline$a$ & 0.01 & 0.015 & $0.013 \pm 0.03$ & \\
\hline$\alpha$ & 2 & 1.7 & $1.85 \pm 0.15$ & \\
\hline$k_{\text {ini }}$ & 160 & 160 & 160 & $\mathrm{~s}^{-1}$ \\
\hline$K_{\text {add }} / K_{\text {frag }}$ & 50 & 50 & $50 *$ & $\mathrm{~s}^{-1}$ \\
\hline$k_{\mathrm{ex} 0}$ & 250 & 250 & $250^{*}$ & $\mathrm{~L} \mathrm{~mol}^{-1} \mathrm{~s}^{-1}$ \\
\hline$k_{\text {act0 }}$ & 1 & 1 & $1^{*}$ & $\mathrm{~s}^{-1}$ \\
\hline$k_{\mathrm{t}}$ & 0.02 & 0.02 & 0.02 & $\mathrm{~s}^{-1}$ \\
\hline$k_{\mathrm{t}, \mathrm{alkyl}}$ & 0.0038 & 0.0038 & $0.0038^{*}$ & $\mathrm{~s}^{-1}$ \\
\hline
\end{tabular}


good control. ${ }^{257}$ Similar behavior is found for CCG with BuMgOct as a good CTA or "RAFT"-agent and BzMgBz as a bad CTA or "RAFT"-agent. 


\subsection{Polymerization of Ethylene with $\mathrm{Cp}_{2}{ }_{2} \mathrm{NdCl}_{2} \mathrm{Li}\left(\mathrm{OEt}_{2}\right)$ and BuOctMg}

The process of CCG polymerization of ethylene in small reaction flasks as in the previous experiments is not possible. For this reaction a polymerization reactor was set up as a part of this thesis. The reactor setup was chosen to be similar to a reactor at the CPE in Lyon in the working group Chemistry, Catalysis, Polymers and Processes (C2P2). In collaboration data analysis was performed and translated for simulation and modeling of the polymerization processes in both reactors. The reactor allows the determination of the ethylene consumption in combination with a good temperature control. As basis a commercial available "Büchi ecoclave 075 " was chosen, which was customized regarding the catalyst introduction and data collection. A detailed description of the setup is given in the experimental part 9.1.8 on page 199.

The major difference to the simulations before is the application of a gaseous monomer in toluene solution. The pressure above the solution is measured and the concentration of ethylene is determined via the Henry's Law with coefficients from literature. ${ }^{[297 \mid}$ During polymerization ethylene is only transferred to the reactor from the storage volume. The ethylene consumption is measured by the pressure decrease within the storage volume. The calculation of the consumption is shown in the experimental part (see Equation 9.7 on page 201) based on an equation provided by Dr. Franck D'Agosto and the CPE in Lyon. ${ }^{298}$ To describe this process within the model, monomer has to be transferred from the storage volume to the reaction mixture with negligible small influence on monomer concentration (see Scheme 6.35).

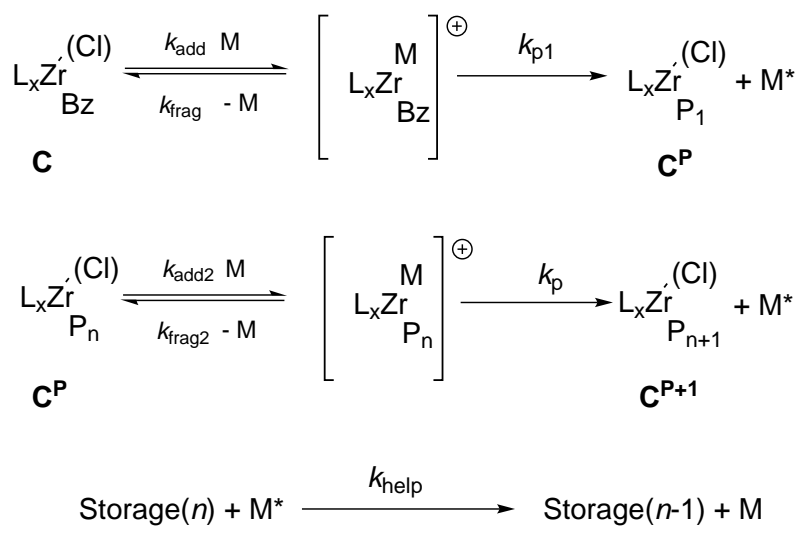

Figure 6.35: Extendend monomer addition for polymerization reactor description with intermediate $\mathrm{M}^{*}$ and the storage volume with $n$ molecules inside.

A intermediate species is introduced $\left(\mathrm{M}^{*}\right)$, which is only produced by successful insertion of ethylene into the Nd-C bond. This intermediate species reduces fast the concentration 
with in the storage volume by one molecule and increases the monomer concentration by the same amount. The concentration of ethylene in the storage volume decreases while the monomer concentration stays constant. In the model two reactions (5.32 and 5.35) were exchanged by the following reactions (6.8 and 6.9). The new reaction (6.10) can be simply described by an "Elemental reaction" step.

$$
\begin{aligned}
\mathbf{C}(\mathbf{M})_{\mathrm{s}} \stackrel{k_{\mathrm{p} 1}}{\longrightarrow} \mathbf{C}_{\mathrm{s}}^{\mathbf{P}}+\mathrm{M} * \\
\mathbf{C}^{\mathbf{P}}(\mathbf{M})_{\mathrm{s}} \stackrel{k_{\mathrm{p}}}{\longrightarrow} \mathbf{C}^{\mathbf{P}}{ }_{\mathrm{s}}+\mathrm{M} * \\
\mathrm{M} *+\text { Storage }_{(n)} \stackrel{k_{\text {he1p }}}{\longrightarrow} \text { Storage }_{(n-1)}+\mathrm{M}
\end{aligned}
$$

Two different data sets were available for simulation, one determined in the new reactor and another from the setup in Lyon. In all cases the mass of ethylene in the storage volume is converted into a concentration on basis of the applied amount of solvent:

$$
c_{\text {storage }}=\frac{m_{\text {ethylene }}}{V_{\text {toluene+catalyst }} \cdot M_{\text {ethylene }}}
$$

This is necessary to use Equation 6.10 and allows easily the determination of ethylene consumption within the model. It has no influence on other parameters.

\subsubsection{Simulation of CCG polymerization performed in the "Büchi ecoclave 075 "}

The first reactions in the reactor were performed to benchmark the reactor for its capability in ethylene polymerization and a following end group modification. The results of the modifications is discussed in the following chapter, The reactor was set under Argon and BuMgOct dissolved in toluene was added. The mixture was heated to $75^{\circ} \mathrm{C}$ and saturated with ethylene for at least $10 \mathrm{~min}$. It was found that the optimal saturation time should be $30 \mathrm{~min}$ to gain the most applicable data for simulation. The determined 
pressure in the storage volume was transferred into the mass of ethylene. Outgoing from this information the catalytic activity $\left(c_{A}\right)$ is calculated (Equation 6.12)

$$
c_{A}=\frac{m_{\text {ethylene }, t 1}-m_{\text {ethylene }, t 2}}{(t 2-t 1) n_{\mathrm{Nd}} p_{\text {reactor }}}
$$

The activity is calculated as the fraction of the amount of ethylene consumed at the time $t_{1}\left(m_{\text {ethylene, } 1}\right)$ and $t_{2}\left(m_{\text {ethylene, } 2}\right)$. The mass this divided by the time difference between $t_{1}$ and $t_{2}$, the amount of applied catalyst in mol and ethylene pressure in bar. To compare the activity during an experiment with results from simulation the activity is determined independent from ethylene pressure (Equation 6.13).

$$
c_{A, s}=\frac{m_{\text {ethylene }, t 1}-m_{\text {ethylene }, t 2}}{(t 2-t 1) n_{\mathrm{Nd}}}
$$

The exact model applied in the simulation is shown in the appendix (see A.3.5 on page 228). The comparison between simulated and experimental data shows a decrease of ethylene consumption with proceeding time, which cannot successfully be addressed by variation of $k_{\mathrm{p}}$. Because of the complex nature of the monomer addition process and the literature discussion about different polymerization rates for different alkyl-chains at the complex, this process equation is the best compromise between accuracy and simulation time demand. It can not be excluded that at this stage of simulation another process may be the reason for the chain length dependency, but nearby all steps in the model can be easily translated into such a step, excluding the monomer disccordination, where no equivalent PREDICI ${ }^{\mathrm{TM}}$ reaction step is found.

The polymerization was performed both in absence and presence of DBE to evaluate the found influence. All polymerizations are performed in the same manner as described in the Experimental Section 9.3.3.1 on page 209. Due to small differences during the catalyst solution preparation its concentrations shows a small scattering.

Polymerization in Absence of DBE Outgoing from a typical polymerization two main parameters can be determined for the polymerization. The catalyst activity is directly linked to the ethylene consumption and the MMD of the resulting polymer. Additional NMR analysis gives information about the amount of vinyl-capped polymer as termination product. Due to this small amount of experimental parameters a complete parameter estimation is not possible and further experiments were planned in collaboration with the CPE in Lyon. Nevertheless, the model is able to successfully 
describe the simulation process (see Figure 6.36). Two main influences are found within the polymerization. The exchange equilibrium has a main influence on the dispersity. If the rate coefficients of the exchange reaction are similar only a small dispersity is gained. With increasing difference the dispersity increases. In typical experiments a dispersity of 1.2 to 1.3 is found. The determined conversion is limited by the factor of chain initiation. If full activation is gained, the determined chains were too short. In Figure 6.36 an example is depicted.
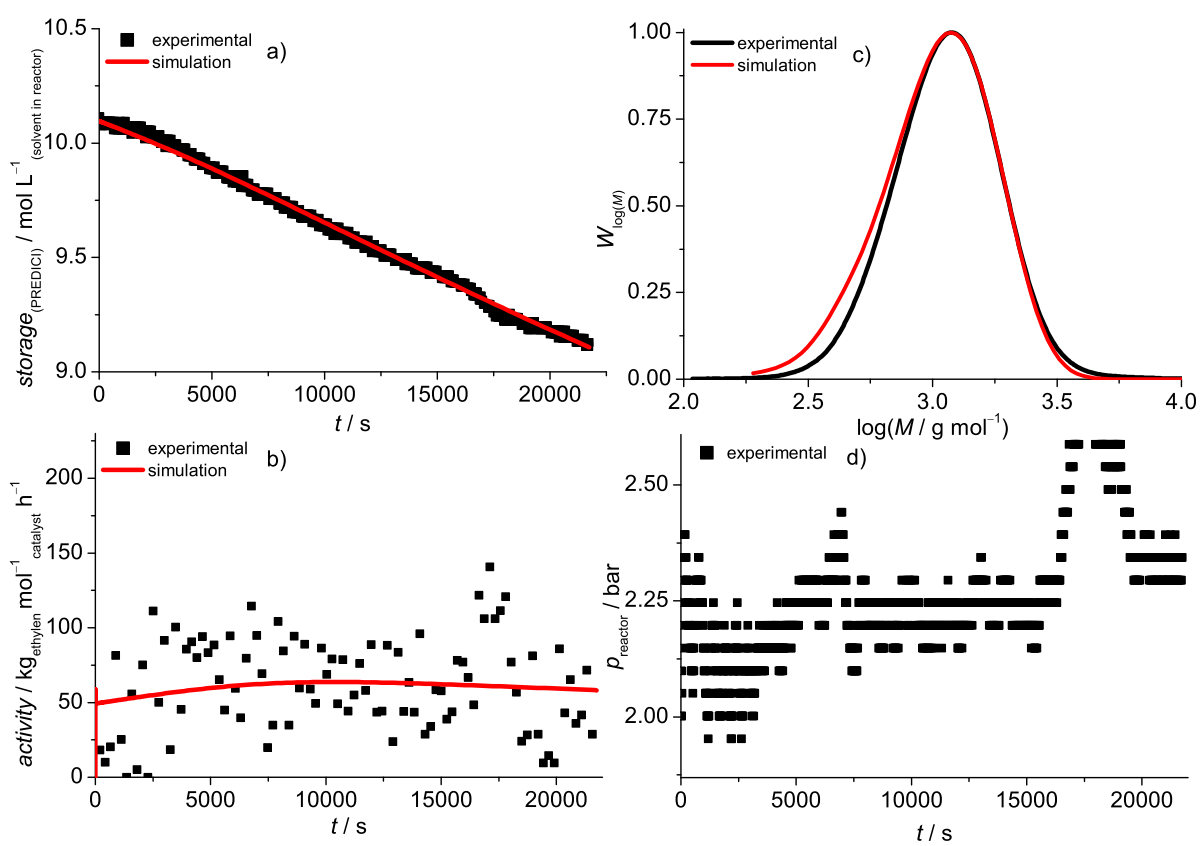

Figure 6.36: CCG polymerization of ethylene with $\mathrm{Cp}_{2}{ }_{2} \mathrm{NdCl}_{2} \mathrm{Li}\left(\mathrm{OEt}_{2}\right)_{2}$ and $\mathrm{BuMgOct}$ toluene at $75^{\circ} \mathrm{C}$. concentration. For simulation the mass ofethylene in the storage volume is converted into a concentration.

The ethylene consumption and the activity values are well described. The MMD is determined with a shoulder at lower molar mass. Parameter variation showed that this is the best fit possible with look on the monomer consumption. The determined coefficients over several experiments are shown in Table 6.8.

Polymerization in Presence of DBE In presence of DBE the reaction rate is enhanced and typical polymerizations can be performed within two hours for a broad spectrum of $\mathrm{Nd}: \mathrm{Mg}$ ratios, without the typical lowering of reaction rate with proceeding reaction time. A typical example for a polymerization is shown in Figure 6.37.

In general it was found that the MMD of the polymer is broader than in absence of ether. The activity is significantly increased. The ether is influencing the different occurring reactions and via modeling the individual influences were determined (see 
Table 6.8 Parameter determined by simulation for CCG polymerization of ethylene at $75^{\circ} \mathrm{C}$, $c\left(\mathrm{Cp}_{2}{ }_{2} \mathrm{NdCl}_{2} \mathrm{Li}\left(\mathrm{OEt}_{2}\right)_{2}\right)=78 \pm 2 \mu \mathrm{mol} \mathrm{L}^{-1}$ in toluene at 2 bar ethylene pressure at different BuMgOct concentrations $\left(0.0088 \mathrm{~mol} \mathrm{~L}^{-1} ; 0.0176 \mathrm{~mol} \mathrm{~L}^{-1} ; 0.022 \mathrm{~mol} \mathrm{~L}^{-1}\right)$. Values marked with a * were fixed at a constant value.

\begin{tabular}{|c|c|c|}
\hline coefficient & value & unit \\
\hline$k_{\text {ini }}$ & $5.1 \pm 4.7$ & $\mathrm{~s}^{-1}$ \\
\hline$k_{\mathrm{p}}$ & $39 \pm 26$ & $\mathrm{~s}^{-1}$ \\
\hline$\alpha$ & $1.49 \pm 0.07$ & $\mathrm{~s}^{-1}$ \\
\hline$k_{\text {alkyl }}$ & $1^{*}$ & $\mathrm{~s}^{-1}$ \\
\hline$k_{\mathrm{ex} 0}$ & $400^{*}$ & $\mathrm{~L} \mathrm{~mol}^{-1} \mathrm{~s}^{-1}$ \\
\hline$k_{\text {act0 }}$ & $1^{*}$ & $\mathrm{~s}^{-1}$ \\
\hline$k_{\mathrm{ex} 1}$ & $10^{*}$ & $\mathrm{~L} \mathrm{~mol}^{-1} \mathrm{~s}^{-1}$ \\
\hline$k_{\text {act1 }}$ & $1^{*}$ & $\mathrm{~s}^{-1}$ \\
\hline$k_{\mathrm{add}, 0}$ & $54^{*}$ & $\mathrm{~s}^{-1}$ \\
\hline$k_{\text {frag } 1,2}$ & $5^{*}$ & $\mathrm{~s}^{-1}$ \\
\hline$k_{\mathrm{t}}$ & $3.510^{-3 *}$ & $\mathrm{~s}^{-1}$ \\
\hline$k_{\text {reini }}$ & $100^{*}$ & $\mathrm{~s}^{-1}$ \\
\hline
\end{tabular}
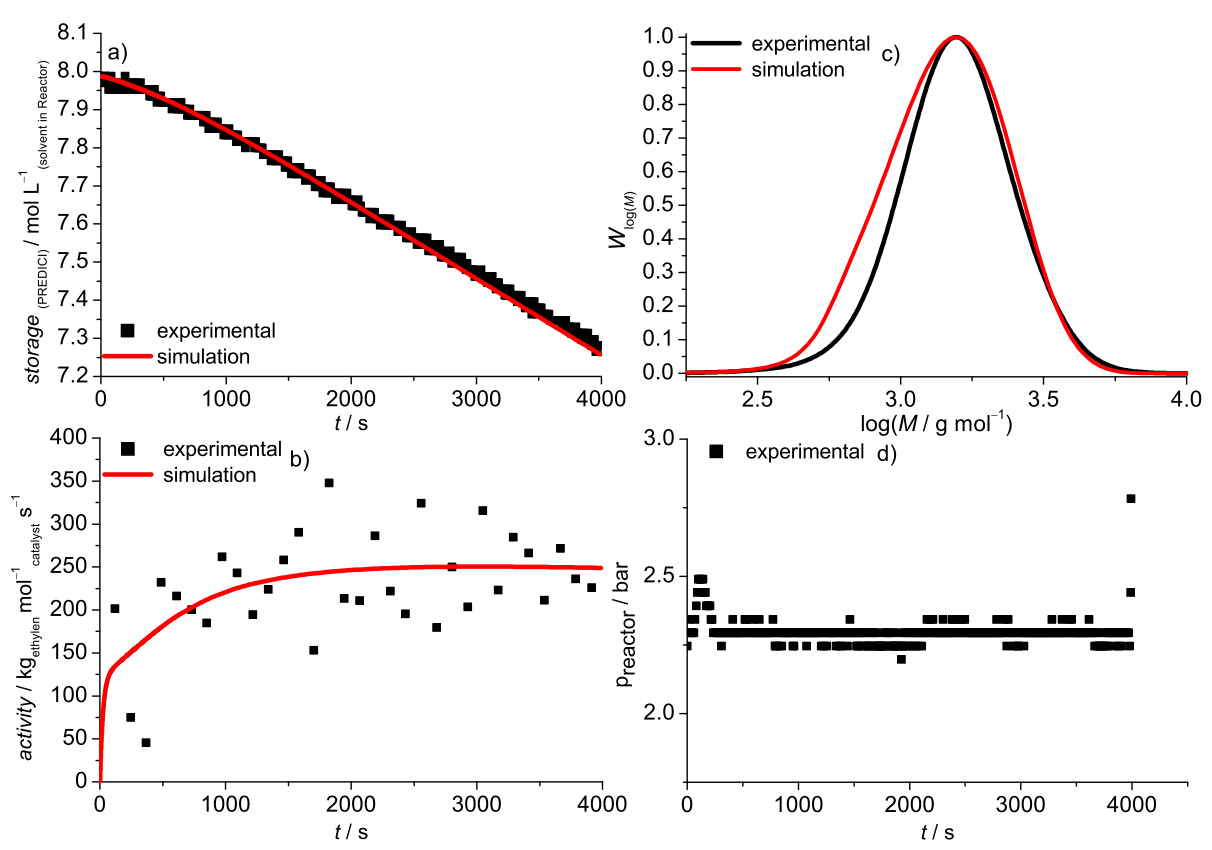

Figure 6.37: Modeling vs. experiment for CCG polymerization with $\mathrm{Cp}_{2}{ }_{2} \mathrm{NdCl}_{2} \mathrm{Li}\left(\mathrm{OEt}_{2}\right)_{2}$ and BuMgOct toluene at $75^{\circ} \mathrm{C}, c\left(\mathrm{Cp}_{2}{ }_{2} \mathrm{NdCl}_{2} \mathrm{Li}\left(\mathrm{OEt}_{2}\right)_{2}\right)=76 \mu \mathrm{mol} \mathrm{L}^{-1}$ and $c($ BuMgOct $)=0.0176 \mathrm{~mol} \mathrm{~L}^{-1}$. For simulation the mass of ethylene in the storage volume is converted into a concentration. 
Table 6.9). The propagation rate coefficient and the first monomer addition $\left(k_{\text {ini }}\right)$ are

Table 6.9 Parameter determined by simulation for CCG polymerization of ethylene at $75^{\circ} \mathrm{C}$, $c\left(\mathrm{Cp}_{2}{ }_{2} \mathrm{NdCl}_{2} \mathrm{Li}\left(\mathrm{OEt}_{2}\right)_{2}\right)=78 \pm 2 \mu \mathrm{mol} \mathrm{L}^{-1}$ in toluene at 2 bar ethylene pressure in presence of $10 \mathrm{~mL}$ DBE at different BuMgOct concentrations $\left(0.0044 \mathrm{~mol} \mathrm{~L}^{-1} ; 0.0088 \mathrm{~mol} \mathrm{~L}^{-1} ; 0.022 \mathrm{~mol} \mathrm{~L}^{-1}\right)$. Values marked with a * were fixed at a constant value.

\begin{tabular}{c|r|l} 
coefficient & value & unit \\
\hline$k_{\text {ini }}$ & $0.51 \pm 0.18$ & $\mathrm{~s}^{-1}$ \\
$k_{\mathrm{p}}$ & $16 \pm 11$ & $\mathrm{~s}^{-1}$ \\
$\alpha$ & $1.21 \pm 0.16$ & $\mathrm{~s}^{-1}$ \\
$k_{\text {alkyl }}$ & $2^{*}$ & $\mathrm{~s}^{-1}$ \\
$k_{\text {ex0 }}$ & $100^{*}$ & $\mathrm{~L} \mathrm{~mol}^{-1} \mathrm{~s}^{-1}$ \\
$k_{\text {act0 }}$ & $10^{*}$ & $\mathrm{~s}^{-1}$ \\
$k_{\text {ex1 }}$ & $5^{*}$ & $\mathrm{Lmol}^{-1} \mathrm{~s}^{-1}$ \\
$k_{\text {act1 }}$ & $0.01^{*}$ & $\mathrm{~s}^{-1}$ \\
$k_{\text {add }, 0}$ & $54^{*}$ & $\mathrm{~s}^{-1}$ \\
$k_{\text {frag1,2 }}$ & $5^{*}$ & $\mathrm{~s}^{-1}$ \\
$k_{\mathrm{t}}$ & $3.510^{-3} *$ & $\mathrm{~s}^{-1}$ \\
$k_{\text {reini }}$ & $100^{*}$ & $\mathrm{~s}^{-1}$
\end{tabular}

lower than in absence of ether. The addition is not benefical for these reactions. This is in agreement with the data determined for the zirconium system. But on the other hand the association/dissociation reaction is influenced as well. The ratio between the reaction rates of both processes is changed. When no polymer chains are involved association is four times slower and the dissociation is ten times faster. The reactions with polymeric components have a ten times slower association and dissociation rate. This difference has a direct consequence on the dispersity and the overall reaction rate is increased. The direct determination of the exchange equilibria as for the polymerization was not possible, therefore modeling was performed for each process with the same constants. The accuracy of the data is limited due to the small number of the different experiments. 


\subsubsection{Simulation of CCG Polymerization performed at CPE in Lyon}

The measurements of the data shown here were performed by Sébastien Norsic in a $500 \mathrm{~mL}$ polymerization reactor at the CPE in Lyon. The first minutes of these measurements show systematic errors yielding a overestimated activities in the first minutes of the process at low $\mathrm{Nd} / \mathrm{Mg}$ ratios. It was confirmed from CPE by a different preparation procedure, that this effect is not caused by the occurring reaction. Simulations were performed with the values of Table 6.8. The results of the monomer consumption are shown in Figure 6.38.

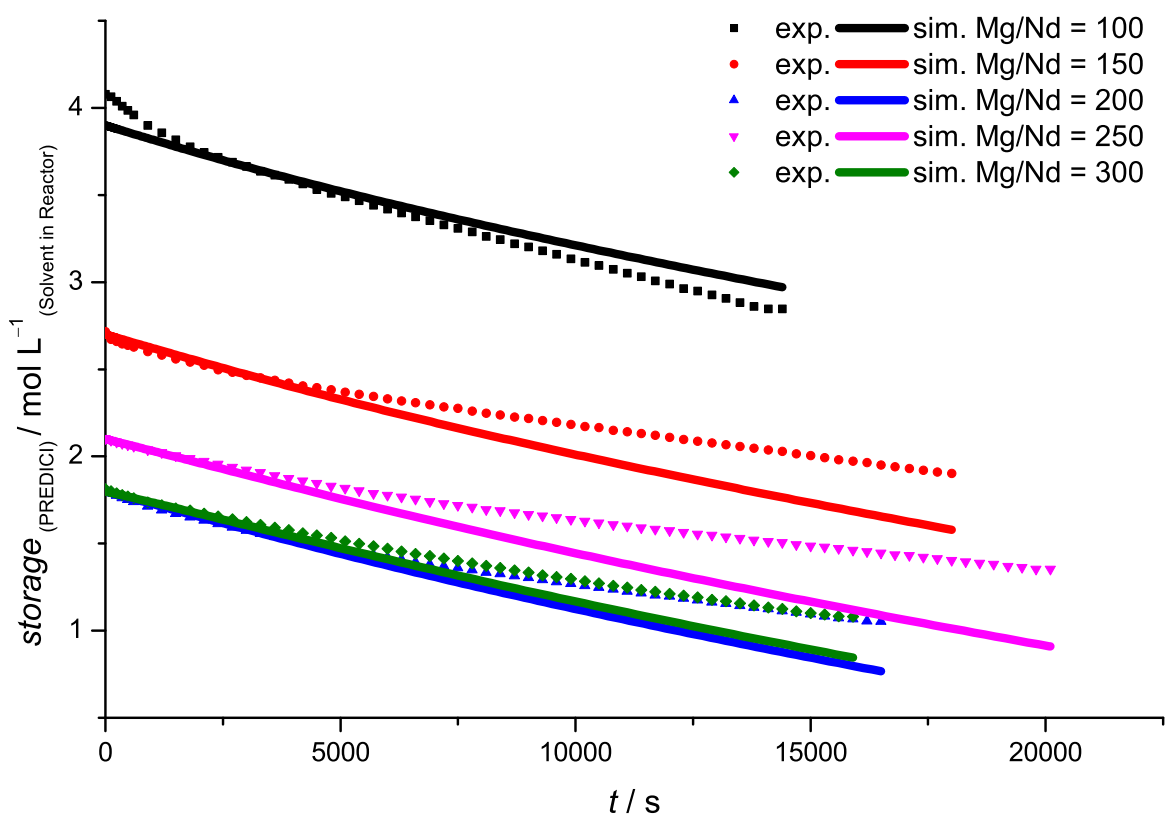

Figure 6.38: Experiment vs. simulation for CCG simulation performed in reactor setup at the CPE in Lyon with coefficients of Table 6.8 and experimental data of Table 6.10

The fit of the data in the beginning is well, while with proceeding reaction time the reaction is simulated to fast and the monomer conversion is too high. This can be explained by a small difference in the reactor design. The reactor at the CPE has the stirrer and a thermocouple in contact with the reaction medium. In the ecoclave setup a baffle is in contact with the reaction medium for better mass transfer and mixing. In the case of the reactor in Lyon this may lead to a limited mass transfer because the reaction solution is not mixed thoroughly and a concentration gradient is yielded. This can easily be investigated by variation of the $\alpha$-factor of monomer addition. This factor can be assumed as a correction factor for the monomer concentration. Experimentally it was observed that the solubility of PE and ethylene are slightly dependent from each other. High concentration of PE yield a lower solubility and vice versa. If such an additional 
event occurs and only the ethylene concentration is the limiting factor a higher factor of $\alpha$ should lead to a better agreement. Increasing the value from $1.49 \pm 0.07$ to $1.62 \pm 0.09$ led to good fit of simulation to experiment.

The derived parameters are of limited accuracy and the exact influence of different equilibrium constants has to be further investigated. DFT calculation regarding activation energies of the different intermediates are under calculation at the CPE in Lyon. The fit between experimental results and simulated data is good (see Figure 6.39, Figure 6.40 and Table 6.10)

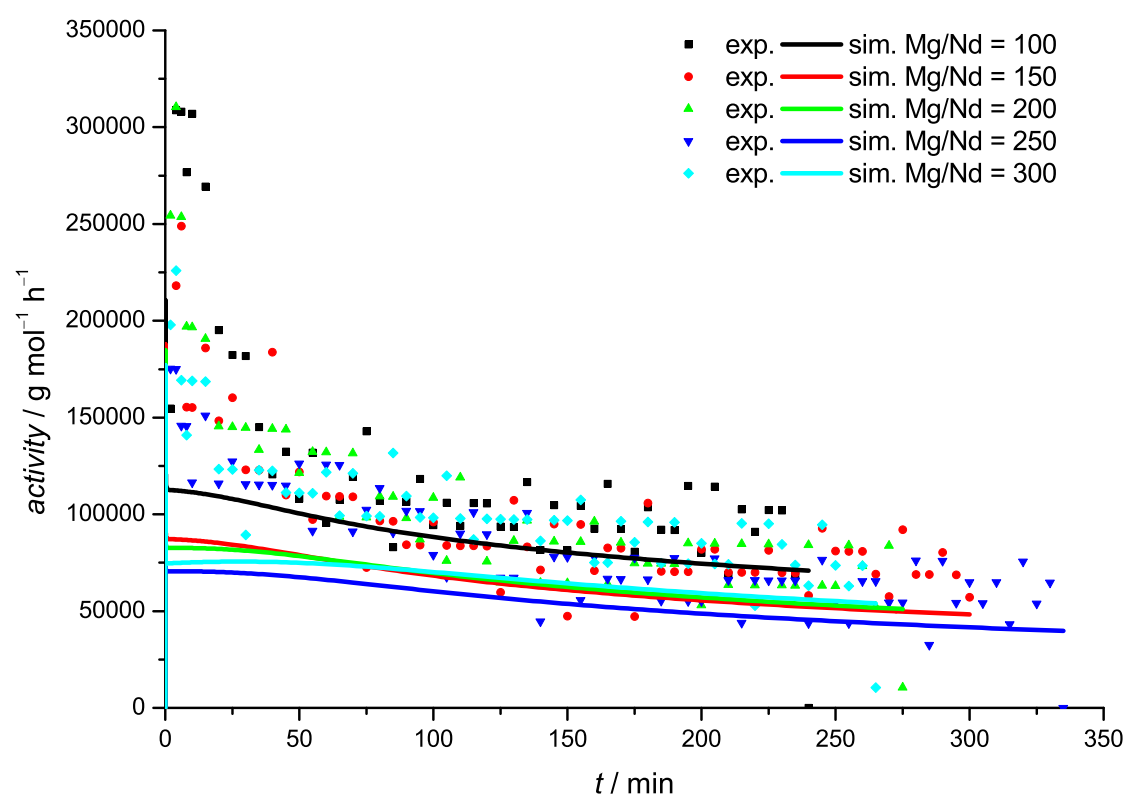

Figure 6.39: Comparison if experimental derived and simulated catalytic activity for CCG polymerization of ethylene. See Table 6.10 for experimental details.

Table 6.10 Parameter determined by simulation for CCG polymerization of ethylene at $75^{\circ} \mathrm{C}$, $c\left(\mathrm{Cp}_{2}{ }_{2} \mathrm{NdCl}_{2} \mathrm{Li}\left(\mathrm{OEt}_{2}\right)_{2}\right)=0.0744 \mathrm{mmol} \mathrm{L}^{-1}$ in toluene at 2 bar ethylene pressure for 5 different BuMgOct concentrations $\left(0.00744 \mathrm{~mol} \mathrm{~L}^{-1} ; 0.0112 \mathrm{~mol} \mathrm{~L}^{-1} ; 0.0149 \mathrm{~mol} \mathrm{~L}^{-1} ; 0.0186 \mathrm{~mol} \mathrm{~L}^{-1}\right.$; $0.0223 \mathrm{~mol} \mathrm{~L}^{-1}$ ). SEC data is band broadened.

\begin{tabular}{c|c|c|c|c}
$\mathrm{Mg} / \mathrm{Nd}$ & $\bar{M}_{\mathrm{n}} / \mathrm{g} \mathrm{mol}^{-1}$, exp & dispersity & $\bar{M}_{\mathrm{n}} / \mathrm{g} \mathrm{mol}^{-1}{ }$, sim. & dispersity \\
\hline 100 & 2210 & 1.278 & 1524 & 1.39 \\
150 & 1530 & 1.225 & 1006 & 1.28 \\
200 & 1030 & 1.231 & 819 & 1.26 \\
250 & 850 & 1.216 & 680 & 1.20 \\
300 & 740 & 1.206 & 656 & 1.21
\end{tabular}




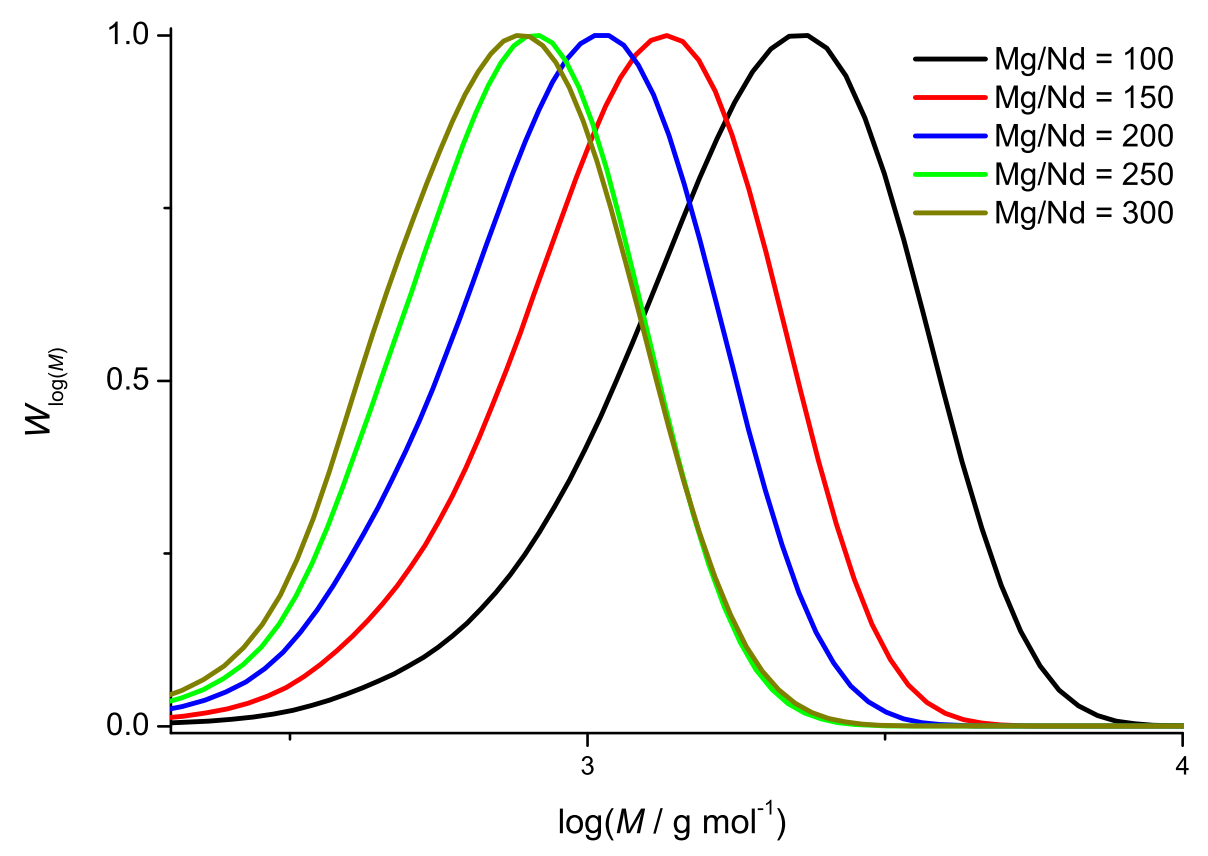

Figure 6.40: Simulated SEC curves for CCG polymerization of ethylene. See Table 6.10 for experimental details.

The main difference between the measured and simulated data is the lowered average molar mass. This effect is mainly caused by the fact that no SEC curves are available and the MMD cannot be compared and additional effects are not determined. The activity is determined lower in the experiment, which is reasoned by the polymerization procedure as mentioned before.

In conclusion it was found that the determined model can easily be adopted to a batch reactor. The derived data from modeling of one reactor can easily be applied on polymerization data of an external system. The addition of DBE yields rate enhancement while the reaction kinetics are heavily influenced and the reaction rates are mainly lowered. Yet this process is faster, because monomer coordinates more easily yielding a higher overall reaction rate. 


\section{Additional Polymerizations and End-group Modification of Polyethylene}

In this chapter an additional polymerization with $\mathrm{Cp}_{2}{ }_{2} \mathrm{NdCl}_{2} \mathrm{Li}\left(\mathrm{OEt}_{2}\right)_{2}$ and $\mathrm{BzMgBz}$ which was not modeled is shortly introduced. PE-modification reactions with iodine and Ni-P3HT are discussed afterwards.

\subsection{Polymerization of Ethylene with $\mathrm{Cp}_{2}{ }_{2} \mathrm{NdCl}_{2} \mathrm{Li}\left(\mathrm{OEt}_{2}\right)_{2}$ and $\mathrm{BzMgBz}$ in Presence of 1,4-dioxane}

Polymerization of ethylene with $\mathrm{Cp}_{2}{ }_{2} \mathrm{NdCl}_{2} \mathrm{Li}\left(\mathrm{OEt}_{2}\right)_{2}$ and $\mathrm{BzMgBz}$ was studied in purpose of simulation. Without any additive added it was impossible to gain any polymer. It was found that the addition of 1,4-dioxane is essential for polymerization of ethylene. Modeling was not performed due to the fact that the addition of 1,4-dioxane may alter the activation mechanism like THF. The results and conditions of the polymerization are shown in Figure 7.1 .

The polymerization shows a strong inhibition phase in the first $10000 \mathrm{~s}$ with only a low rate of polymerization (see Figure 7.1 a and b). Afterwards, the rate changes into a phase of higher catalyst activity. The obtained values are lower than for polymerization with BuMgOct as co-catalyst. A polymer with average molar mass of $1950 \mathrm{~g} \mathrm{~mol}^{-1}$ and a dispersity of 1.18 was achieved. This result indicates that only a small amount of benzyl chains were activated which is in agreement with the result for the polymerization of styrene. After the first insertion of ethylene, the polymer has a stronger alkyl character. On basis of these results it can be assumed that after the first chain insertion the growth reaction is easier, because the alkyl chain is more flexible than the benzyl moiety. Interestingly a low dispersity is gained, which is in agreement with the results found in 


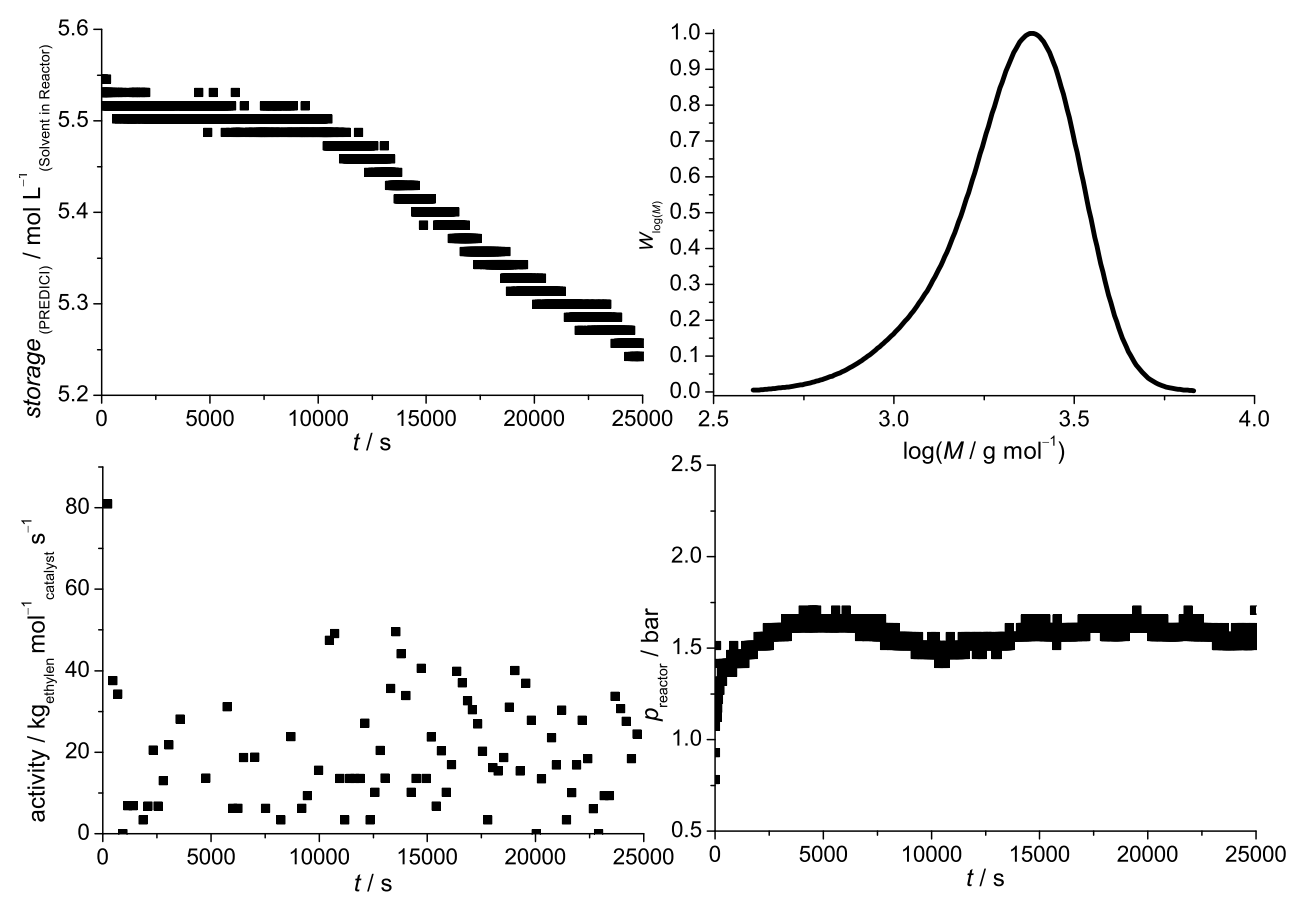

Figure 7.1: Polymerization of ethylene with $\mathrm{Cp}_{2}{ }_{2} \mathrm{NdCl}_{2} \mathrm{Li}\left(\mathrm{OEt}_{2}\right)_{2}$ and $\mathrm{BzMgBz}$ in presence of 1,4-dioxane at $70^{\circ} \mathrm{C}, \mathrm{c}\left(\mathrm{Cp}_{2}{ }_{2} \mathrm{NdCl}_{2} \mathrm{Li}\left(\mathrm{OEt}_{2}\right)_{2}\right)=85 \mu \mathrm{mol} \mathrm{L}^{-1}, c(\mathrm{BzMgBz})=4.3 \mathrm{mmol} \mathrm{L}{ }^{-1}$ and $c(1,4-$ dioxane) $=14 \mathrm{mmol} \mathrm{L}^{-1}$ at an ethylene pressure of 1.6 bar in $400 \mathrm{~mL}$ toluene.

modeling of CCG with BuMgOct. The activated chains adds monomer in a controlled manner and does not terminate. It can be assumed that this process follows the principle of CCTP while the activation is hindered and it cannot cross over to the CCG process. Yet it is remarkable that BzMgBz can start polymerization at all.

\subsection{Synthesis of PE-lodine}

The iodine functionalization is a standard protocol for the production of functional polyethylene. ${ }^{[3,68,225]}$ Elemental iodine in THF was added at ambient temperature to the polymer suspension in the reactor and stirred over night. The produced polymer is filtered off and washed several times with ethanol to remove residual iodine. A pale yellow solid was obtained (see experimental Section 9.3.4.1 on page 210 for details) and characterized via NMR and HT-SEC. A polymer with a $\bar{M}_{\mathrm{n}}=1130 \mathrm{~g} \mathrm{~mol}^{-1}$ and a dispersity of 1.34 was yielded. The functionality could be identified in NMR (see Figure 7.2).

The signals from the iodine functionality were found as triplett at $2.91 \mathrm{ppm}$ for the $\mathrm{CH}_{2}-\mathrm{I}$ group and the next $-\mathrm{CH}_{2}-$ groups as a quintet at $1.58 \mathrm{ppm}$. The signals of polyethylene 


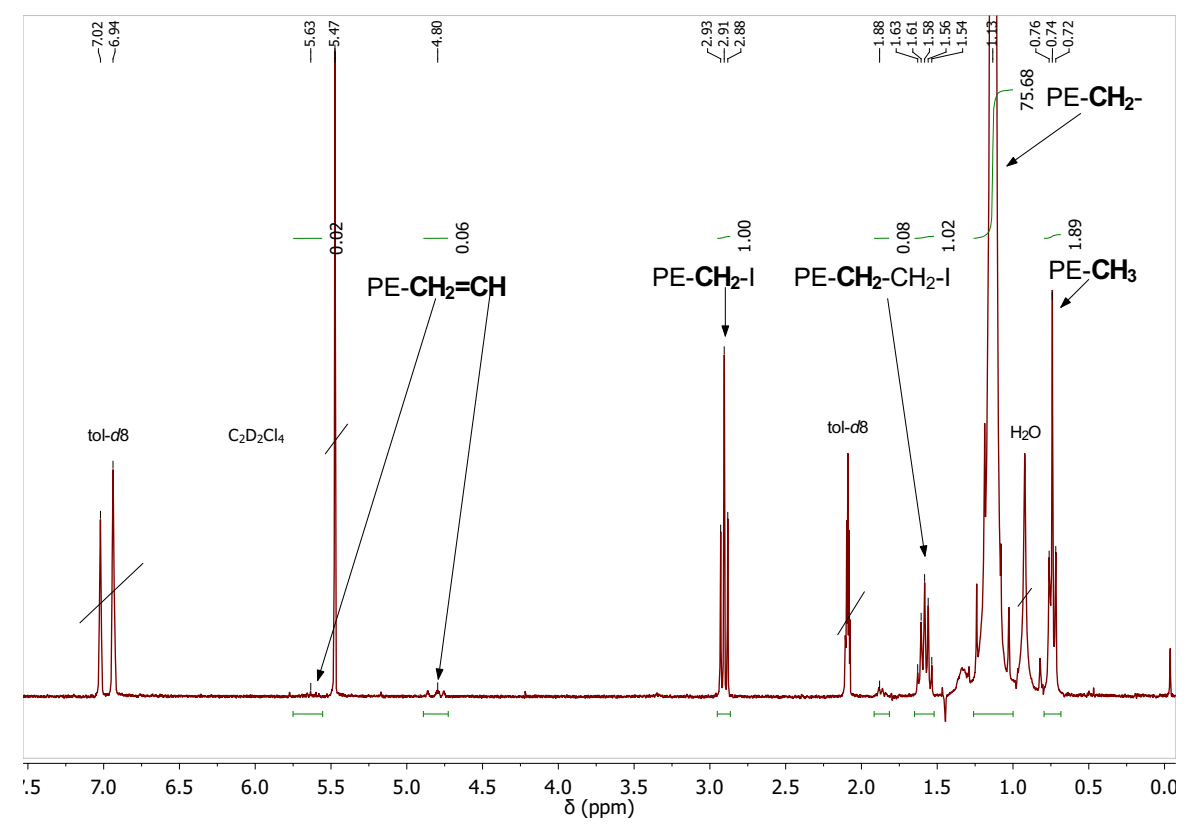

Figure 7.2: NMR spectrum of iodine functionalized PE.

are found at $1.13 \mathrm{ppm}$ for $-\mathrm{CH}_{2}-$ and $0.71 \mathrm{ppm}$ for the $-\mathrm{CH}_{3}$ end group. The ratio between the PE end group and the iodine end group is 1.26:1 indicating that not all end groups have an iodine added. Additionally, a small amount of vinyl groups from termination is found which is in agreement with literature. ${ }^{[25]}$ The termination product is around $5 \%$ which suggests that termination was not suppressed completely as well as for BuOctMg as co-catalyst. The results show that the new built reactor setup is capable of providing the full bandwidth of methods applicable in CCG.

\subsection{PE-b-P3HT}

Based on these PE-Iodine results a more complicated system was developed in collaboration with Dr. Florian Ehlers and Jan Schwellenbach. The formation of PE- $b$-P3HT blockcopolymers is a time- and synthetic demanding process consisting of three reaction steps excluding work up procedures. ${ }^{[243}$ The polymerization system based on $\mathrm{Nd} / \mathrm{Mg}$ forms functional polymers similar to Grignard reactants. Jan Schwellenbach screened the reaction of Ni-P3HT with several dialkyl magnesium derivatives and polystyrene synthesized by CCG in his master thesis. ${ }^{[299]}$ After successful preparation of P3HT- $b$-PS it was applied to the system of PE-Mg-P3HT. The combination of both polymers leads to a block copolymer with a flexible linear polyethylene chain and a rod like linear 
polythiophene chain. The process is depicted in Scheme 7.3.

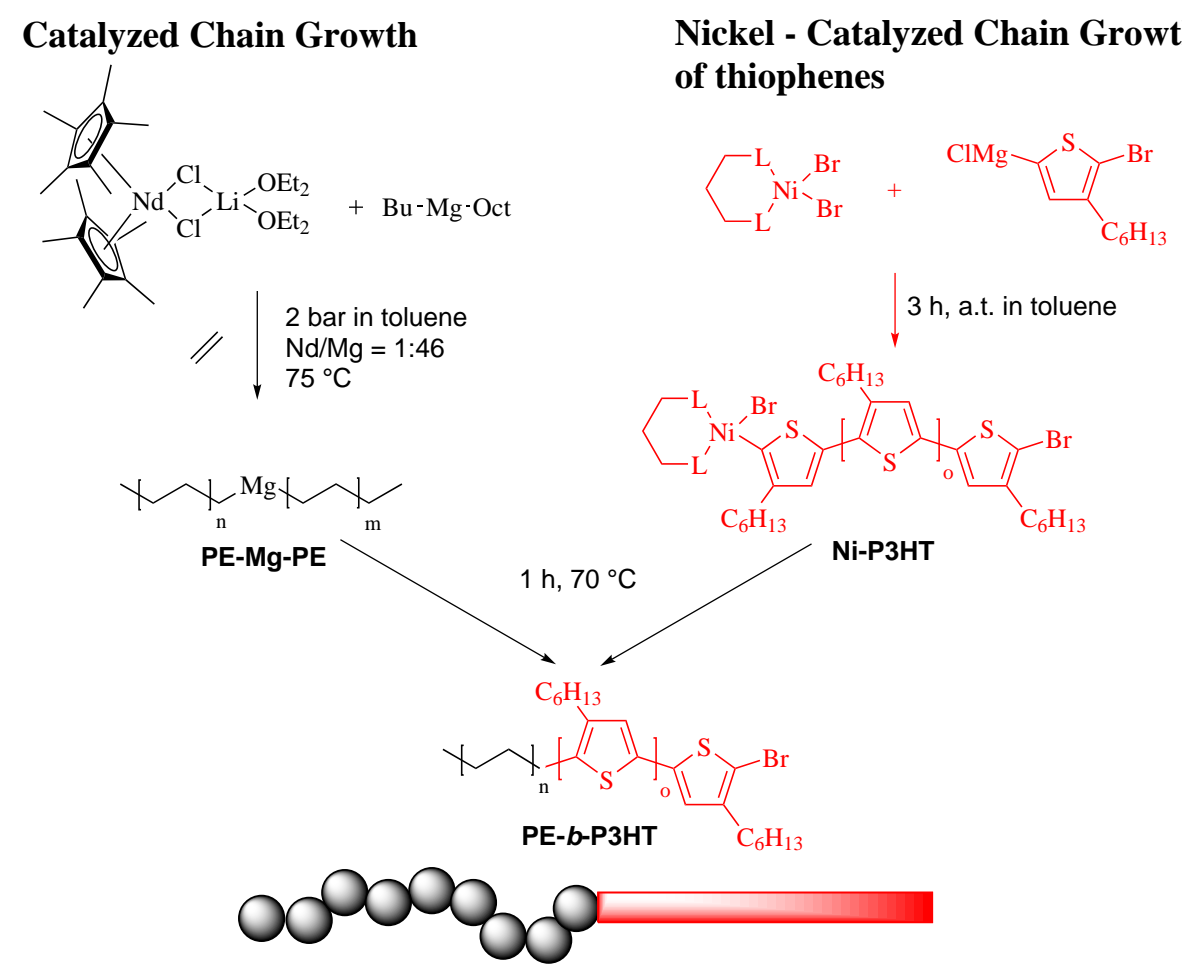

Figure 7.3: Reaction scheme for the production of PE-b-P3HT outgoing from the CCG polymerization of styrene and the Ni-catalyzed polymerization of 1,4-dibromo-3-hexylthiophene by combination of both reaction mixtures at $70{ }^{\circ} \mathrm{C}$.

For the synthesis of PE- $b$-P3HT different approaches were performed. While the synthesis of P3HT is a straightforward method and can easily be performed within a glovebox the PE synthesis was more challenging. The production of small amounts of PE by application of small $\mathrm{Nd} / \mathrm{Mg}$ ratios and concentrations was limited by the fact that the polymer precipitates quickly. The control of the process was limited. In addition it has to be considered that P3HT is commonly dissolved in THF, which would lead to additional precipitation of PE. The most successful approach was the addition of a portion of freshly made PE-Mg-PE (see Scheme 7.3) to freshly prepared P3HT-Ni (provided by Dr. Florian Ehlers) in toluene (see experimental Section 9.3.4.2 on page 210 for details). After stirring at $70{ }^{\circ} \mathrm{C}$ for one hour a red polymer was obtained. It was necessary to produce PE in great scale to gain a quasi-living polymer solution. The necessary amount was transferred under argon counter-flow to the reaction mixture of P3HT-Ni. It was targeted to use an excess of P3HT which can be removed from the resulting polymer more easily than excess PE. Purification via Soxleth extraction beginning with acetone (P3HT-oligomers), THF (P3HT), $\mathrm{CHCl}_{3}$ (long-chain, P3HT) and toluene (PE-b-P3HT) was performed for the outgoing white PE and the red polymer to gain comparable analytical results. The PE polymer is only well soluble at temperatures above $70^{\circ} \mathrm{C}$ in toluene. Different analytical 
methods were applied to confirm the formation of a block copolymer. In HT-SEC it was found that in an analysis with a standard polyethylene calibration the molecular weight of the resulting polymer (Figure 7.4 black) is only slightly shifted towards higher masses compared to the starting PE (blue) while the outgoing P3HT has a broad MWD (red) shifted to higher masses.

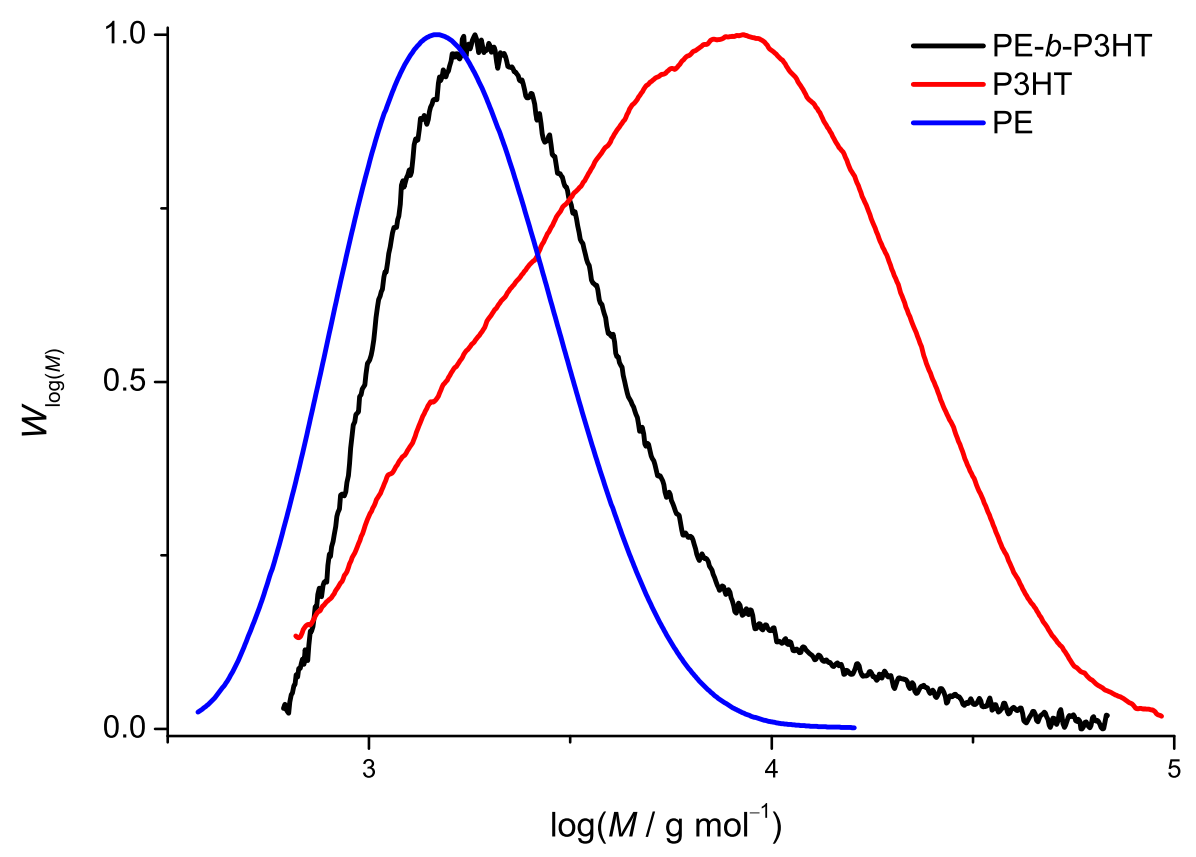

Figure 7.4: SEC-traces measured against standard PE calibration for the PE homopolymer (blue), the P3HT homopolymer (red) and the PE-b-P3HT polymer (black).

This indicates a successful coupling, but the difference between both signals is very small. Shifting to higher molar mass is caused by the stiffness of rod-like polymers like P3HT compared to a flexible PE-chain. The retention time is lower for P3HT of similar molecular weight. In case of the BCP the results indicate that the PE chains are the main factor for the higher retention time and the influence of the rod like chain is reduced. Another difference of these polymers is the viscosity of the polymer solutions. The HT-SEC is equipped with an online viscometer, which can be used to obtain a universal calibration for the setup and the measurement of the intrinsic viscosity of the polymer solutions. By application of the universal calibration (see Figure 7.5) the difference is much higher between PE (blue) and the obtained polymer (black). P3HT is again shifted towards higher masses.

In combination these results show that the resulting polymer has combined properties of both polymer chains. The PE chain on the block copolymer yields a longer interaction on the SEC columns while P3HT block yields in a higher viscosity of the dissolved polymer. 


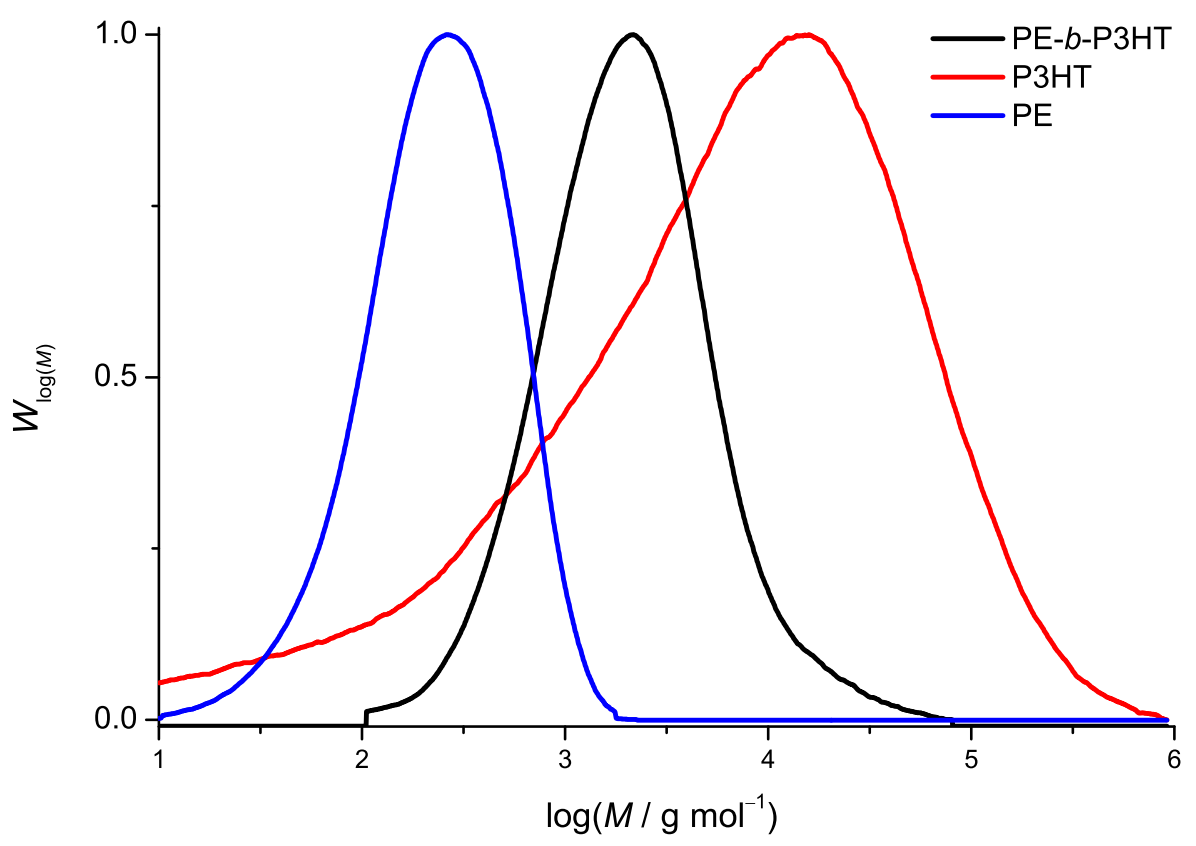

Figure 7.5: SEC-traces measured against universal PE calibration for the PE homopolymer (blue), the P3HT homopolymer (red) and the PE- $b$-P3HT polymer (black).

The PE- $b-\mathrm{P} 3 \mathrm{HT}$ is completely insoluble in typical solvents for P3HT and it was only soluble at temperatures above $80^{\circ} \mathrm{C}$ in toluene- $d 8$. This supports the formation of a block copolymer, because only the coupling to polyethylene can cause this dramatic change in solubility.

Analysis by NMR was not successful. PE and P3HT signals could be found, but the resulting integrals for P3HT were very low (see Figure 7.6). Calculations indicate that only one thiophene group is present per PE chain.

These results are contradictionary to the result of the SEC measurements and the color of the sample. Only one molecule bound per chain would yield still a white polymer. Equal amounts of PE and P3HT end groups are yielded. The integral of the mixed signal at $0.90 \mathrm{ppm}$ is twice the amount necessary by the signal of the $\mathrm{CH}_{2}$-group (2.83 ppm) of the hexyl chain of $\mathrm{P} 3 \mathrm{HT} .{ }^{[242]}$ The integral includes two minor peaks which describe the similar protons in a slightly different constitution. Two possibilities are now present. The first one is that only a few chains added the polythiophene. This is in disagreement with the SEC measurements where the whole sample is shifted. The second one is that higher structures are formed within the NMR solution. P3HT has a high tendency to crystallize and toluene is not an ideal solvent as already shown during synthesis. This may lead to the formation of P3HT aggregates which are assumed to be similar to small crystals. This would yield smaller integrals and only the chain-ends would show a measurable NMR signal. 


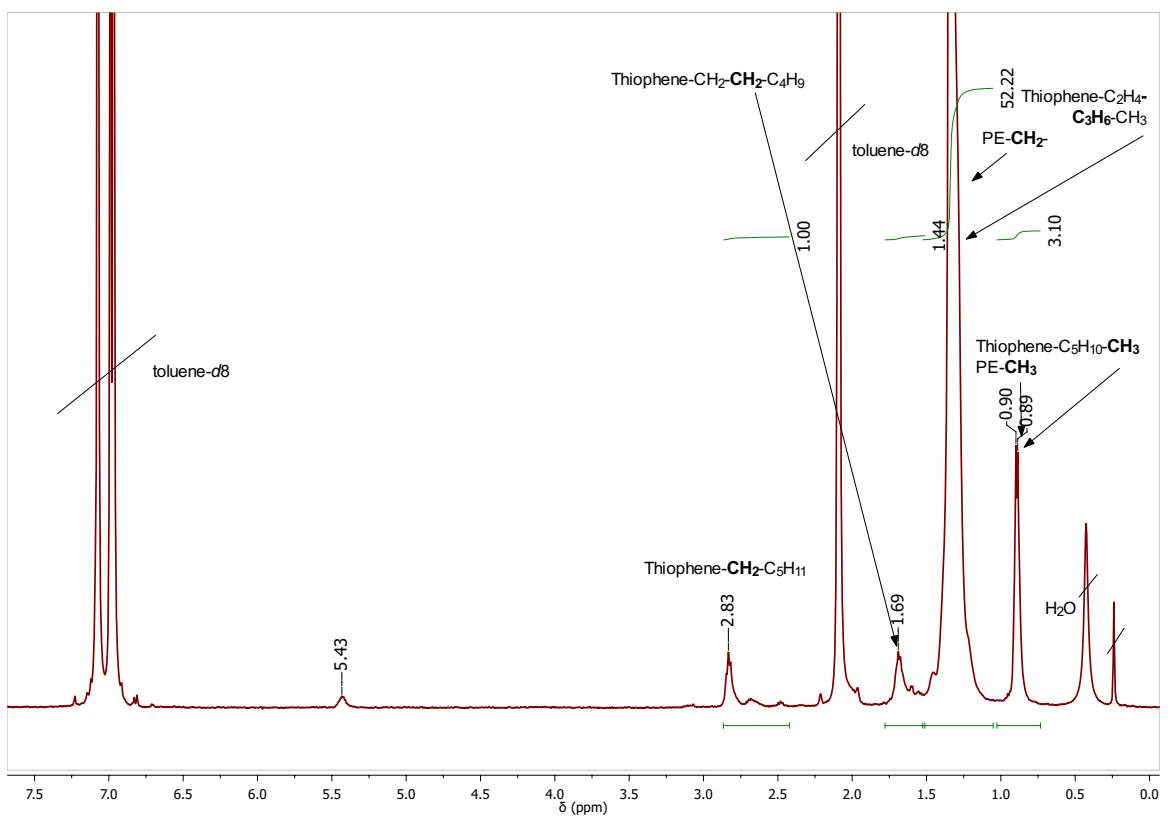

Figure 7.6: NMR analysis of P3HT- $b$-PE in toluene- $d 8$ at $80^{\circ} \mathrm{C}$. The integral for the peak at $2.83 \mathrm{ppm}$ is broad to include all possible constitutions of the hexyl-group at the thiophene.

DSC measurements were performed to support the formation of a BCP. The homopolymers and a blend of different PE/P3HT mixtures were compared with the formed polymer (see Figure 7.7).

For both homopolymers the appearance of melting points was expected ${ }^{[243]}$ and found (cf. Figure 7.7 a) black and red line). For the PE polymer it was found around $117^{\circ} \mathrm{C}$ and for $\mathrm{P} 3 \mathrm{HT}$ at around $225^{\circ} \mathrm{C}$. These melting points are found for blends of similar polymers as well (cf. Figure 7.7 b)). In case of the BCP the signals at $225^{\circ} \mathrm{C}$ are missing and the melting point of $\mathrm{PE}$ slightly shifted to a higher value around $119^{\circ} \mathrm{C}$. The concentration of P3HT may be below the ratio of the 41:10 blend, where the signal is very weak as well. This would be in agreement with the NMR measurements which are indicating such a low amount. But these results are in disagreement with the SEC data, which indicates a higher amount of a BCP. Additionally, the change in viscosity is very high, which supports a higher amount of P3HT-chains than indicated by NMR. In this case the loss of the second melting point would be a result of the liquid polyethylene chain. This is very similar to the hexyl groups of P3HT, which would yield a better solubility in the liquid PE breaking of possible P3HT crystals. Further, the formation of P3HT microdomains in a BCP would be hindered and are only possible to a small extent. This would be in agreement with the selective shift of the melting point towards a value above the value measured for the homopolymer. Blends yield lower values as well (cf. the yellow lines in Figure 7.7). The difference at low amount of P3HT is low in case of the first heating, yet it is significantly higher at second run. 


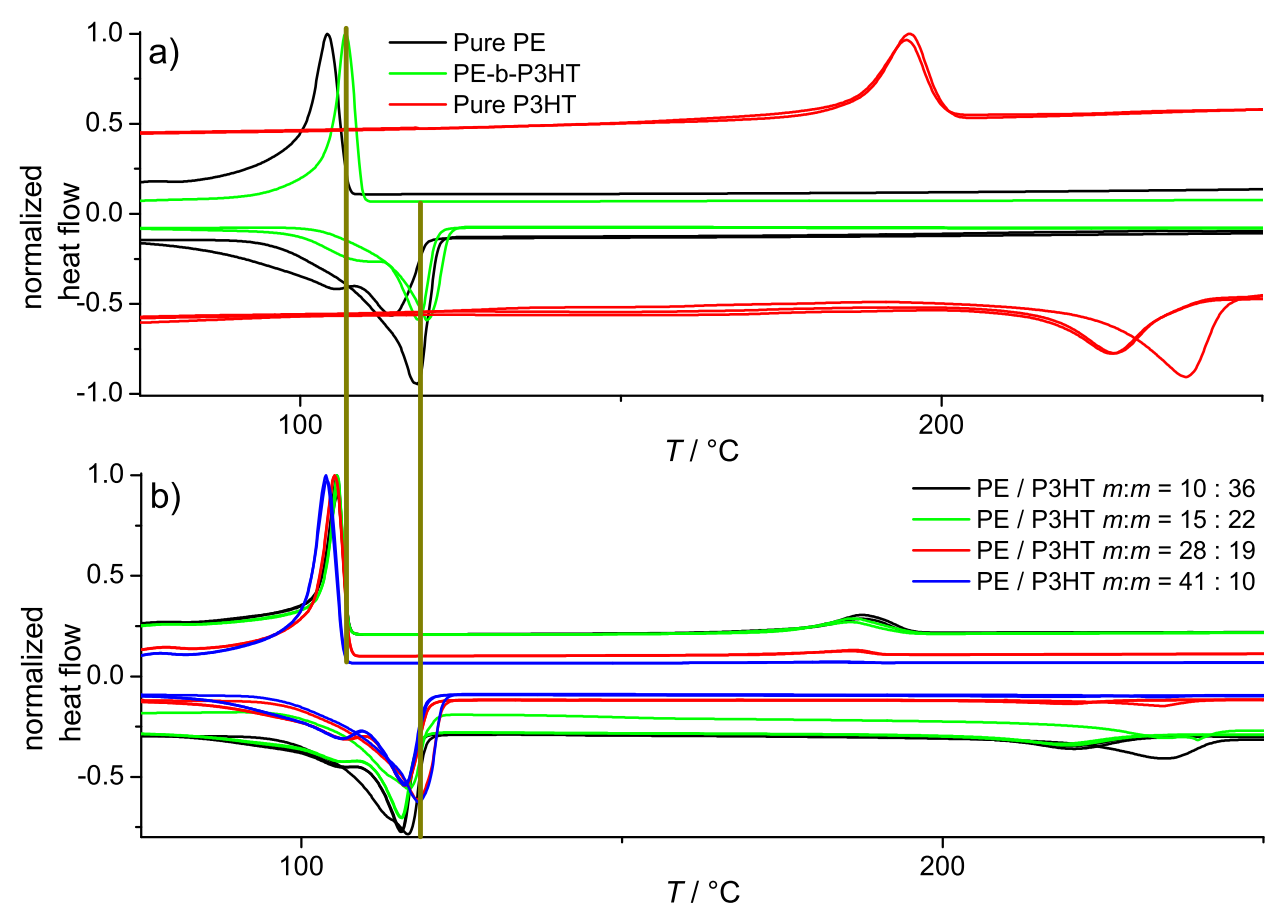

Figure 7.7: DSC measurements of the PE homopolymer (a) black), the P3HT homopolymer (a) red) and the PE- $b$-P3HT polymer (a) green) compared to polymer blends with different mass ratios of PE/P3HT: 10:36 (b), black), 15:22 (b), green), 28:19 (c), red), 41:10 (d), blue). The yellow lines were introduced to show the mean values of the BCP measurements.

Via matrix-assisted laser desorption/ionization (MALDI) mass spectroscopy the formation of a block copolymer is further confirmed (see Figure 7.8). The measurement data were thankfully provided by Dr. Florian Ehlers.

The results show the formation of a block-copolymer. This isotopic pattern fits only for combination of P3HT and PE groups. The difference between the peaks is $m / z=166$, the mass of one monomer unit of P3HT (cf. 7.8, a)). Interestingly only PE-chains with a chain length of four were found. This is a result of the low solubility of PE in almost all solvents. As it is necessary to crystallize the sample matrix, this can lead to a problem at the ionization step. Still a small amount of polymers with different compositions were found (cf. 7.8, b)). In conclusion, all these results confirm the formation of a BCP yet the exact chain length of the P3HT block has to be studied further.

\subsection{Conclusion}

The new built reactor setup allows the full scale of polymerization and functionalization processes applicable by CCG polymerization. Additionally, small samples can be ex- 

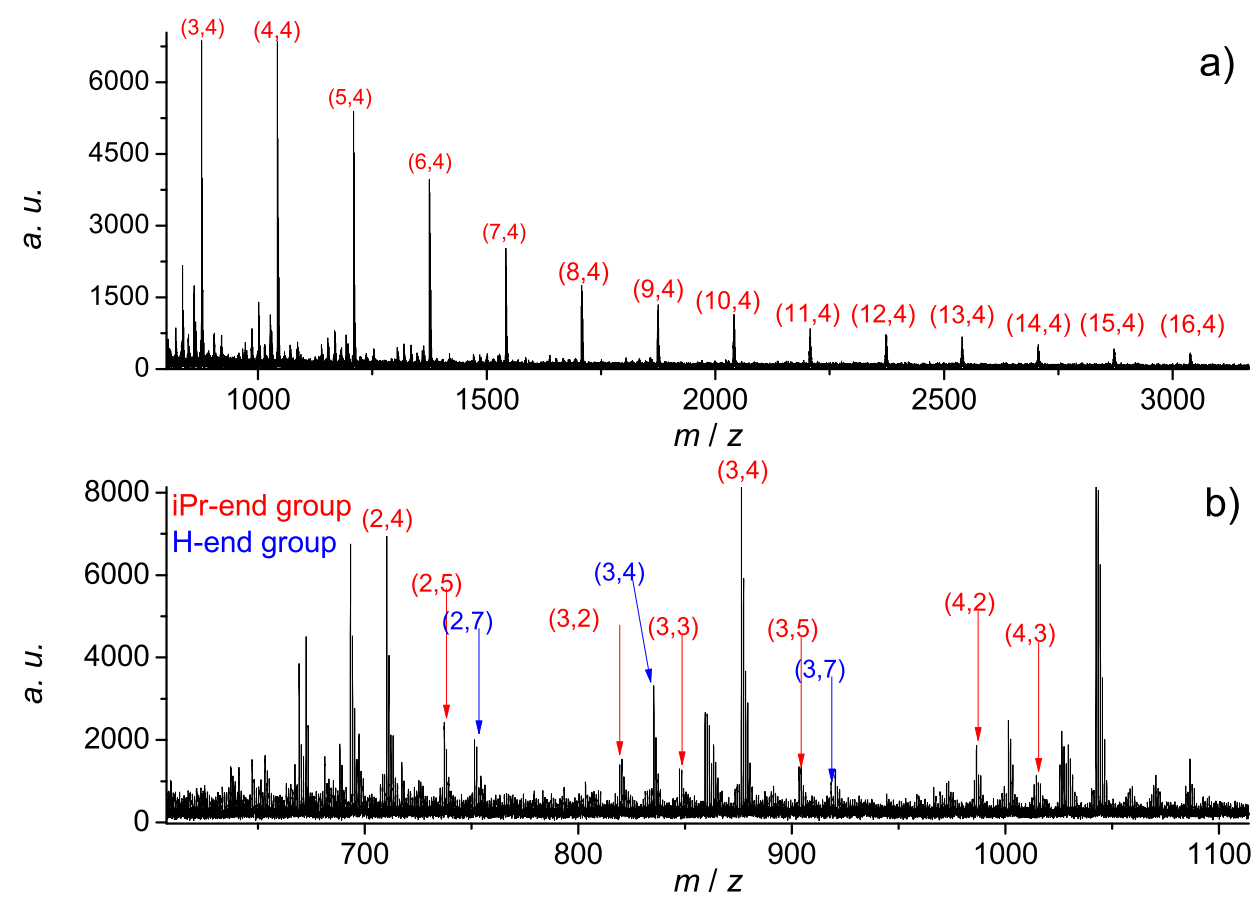

Figure 7.8: MALDI-MS analysis of P3HT- $b$-PE: The values in brackets are: (P3HT units, PE units). Two end groups on P3HT are found (a hydrogen atom or an isopropyl group). Measurement data thankfully provided by Dr. Florian Ehlers.

tracted in argon counter flow for external reactions. This renders the chosen reactor setup to a powerful tool for new kinds of PE functionalizations. One of these new techniques was found with the straightforward production of P3HT- $b$-PE via simple mixing of two reaction mixtures from homo $\mathrm{P} 3 \mathrm{HT}$ and PE polymerization. Due to this process the product of two reactions can directly be combined without any intermediate work-up, yielding a $\mathrm{BCP}$ in high amounts. This is a big advantaged compared to the literature known procedure. ${ }^{[243]}$ 



\section{Concluding Remarks and Outlook}

In this work the mechanism of CCG polymerization was investigated via a model system based on $\mathrm{Cp}^{*}{ }_{2} \mathrm{ZrCl}_{2}$ and $\mathrm{BzMgBz}$ and the results were transferred to the CCG system of $\mathrm{Cp}_{2}{ }_{2} \mathrm{NdCl}_{2} \mathrm{Li}\left(\mathrm{OEt}_{2}\right)_{2}$ and BuMgOct.

For $\mathrm{Cp}^{*}{ }_{2} \mathrm{ZrCl}_{2}$ and $\mathrm{BzMgBz}$ a mechanistic scheme based on results of NMR studies was proposed. The kinetic coefficients were successfully derived via modeling with PREDICI $^{\mathrm{TM}}$. The analysis was simplified allowing direct calculation of the coefficients from NMR-determined concentrations. The analysis transferred to UV/Vis studies where similar coefficients could be derived. The successful determination was the basis for a broad investigation of the influence of DBE on the catalyst/co-catalyst system. The addition of DBE resulted in a strong lowering of the alkylation rate coefficient up to two orders of magnitude depending on the reaction temperature. The co-catalyst was switched to BuMgOct, where $\beta$-hydride elimination is fast at $70^{\circ} \mathrm{C}$ and dominates the activation mechanism. The mono-alkylated complex is rapidly transformed into a chloro-hydride species, which can perform a set of different reactions. As long as the dialkyl magnesium is present it is slowly consumed, indicating an exchange mechanism from the hydride catalyst to magnesium derivatives. As a result it was found that the catalyst system consisting of $\mathrm{Cp}^{*}{ }_{2} \mathrm{ZrCl}_{2}$ and BuMgOct or BzMgBz is unsuitable for polymerization of ethylene.

For the reaction of $\mathrm{Cp}_{2}{ }_{2} \mathrm{NdCl}_{2} \mathrm{Li}\left(\mathrm{OEt}_{2}\right)_{2}$ with $\mathrm{BzMgBz}$ where the equilibrium constant between the monoalkylated complex and the benzyl bridged bimetallic $\mathrm{Nd}-\mathrm{Mg}$ complex could be determined. The value is strongly dependent on the presence of other coordinating molecules like DBE. It was found that the presence of DBE lowers the equilibrium constant within the measured temperature range compared to DBE free system. Based on these results screening for a broader catalogue of catalysts could be performed in future work with NMR and UV/Vis, allowing the determination of activation rate coefficients and the determination of possible side reactions under conditions similar to polymerization. The application of further paramagnetic catalysts in UV/Vis studies could extend the scope of this method. These results allow a fast screening of new candidates for CCG polymerization as well as the research on new additives to yield faster conversion and/or better solvents for the polymerization process. The studies could be extended to 
the bis(imido)pyridyl iron catalyst activated by MAO and diethyl zinc as CTA. 151,214,215] The activation mechanism of $\mathrm{Cp}^{*}{ }_{2} \mathrm{ZrCl}_{2}$ with $\mathrm{BzMgBz}$ was successfully approached in the modeling of the polymerization of styrene- $d 8$ in NMR studies. It was found that the basic steps of the exchange reactions are the same as for activation. The previous model was extended by the occurrence of polymeric species. The procedure of monomer addition was introduced as well as $\beta$-hydride elimination. The process was successfully modeled and kinetic parameters derived. It was found that the process of monomer addition is chain-length dependent which has a strong influence on the dispersity during styrene polymerization. Based on this model, the influence of the single rate coefficients on the reaction system was screened. It was found that a low catalyst precursor concentration can lead to a rate enhancement. The chosen system is not equilibrating in the first minutes of measurement which led to a preferred addition of monomer.

The polymerization was studied in presence of THF. It was found that the addition of THF is disadvantageous for the polymerization reaction and the alkylation reaction is massively influenced. The addition may lead to incomplete alkylation. The exchange is hindered and the polymer grows only at one chain per catalyst molecule.

The application of BuMgOct to the catalyst system is overlaid by the termination reaction of the alkyl chains. It was found that chain growth occurs starting from the hydride catalyst formed during termination. The termination rate of the polystyrene chain is much lower.

With both catalysts the polymerization of styrene was performed with UV/Vis analysis and a gravimetric- and IR-determination of the conversion. The process was activated with either BzMgBz or BuMgOct. The influence of DBE on each system was researched individually. For $\mathrm{Cp}_{2}{ }_{2} \mathrm{ZrCl}_{2}$ and $\mathrm{BzMgBz}$ a kinetic isotope effect was indicated and the addition of DBE led to a rate enhancement within the first minutes of reaction. The system is unable to produce dispersities below two because of the low initiation rate of the benzyl groups as well as the strong CLD for the propagation step. The polymerization with BuMgOct was performed. The resulting polymerization process indicates that dialkyl magnesium is able to reactivate the formed dialkylated complexes. One possible reaction pathway was suggested and found to be able to yield similar results via modeling. The exact reaction mechanism has to be investigated further.

Polymerization with $\mathrm{Cp}_{2}{ }_{2} \mathrm{NdCl}_{2} \mathrm{Li}\left(\mathrm{OEt}_{2}\right)_{2}$ as catalyst was successfully described by the kinetic model activated by BzMgBz or BuMgOct. With BzMgBz it was found that the $\beta$-hydride elimination of the styrene chain is the main factor for the activity of the system. Chain exchange is hindered and the reaction rates are very low while the termination can occur fast. The resulting process is nearby completely uncontrolled and the simulations indicate the formation of mainly unsaturated chain ends. First NMR-experiments to gain information about the amount of terminated chains were not sucessfull and IR measurements should be performed in the future. 
Compared to this system the one with BuMgOct is completely different. The catalyst activation is fast and the reaction formation of the exchange complexes is rapid. In this case the elimination process is overlaid by the exchange reactions.

The addition of DBE leads to rate enhancement if activated with BuOctMg, while the reaction rate is lowered if activated with $\mathrm{BzMgBz}$. The styrene polymerization with this system has to be investigated further to find the reasons for these completely different reaction pathways. The proposed kinetic coefficients are of limited accuracy due to the small amount of so far performed experiments. They have to confirmed by further experiments. Further polymerizations are needed to be performed at different monomer and catalyst concentrations.

The kinetic model was successfully assigned to the polymerization of ethylene in a batch reactor. In cooperation with Franck D'Agosto at the CPE in Lyon a new reactor was set up and the polymerization successfully modeled. The data could be transferred to simulations of the reactor setup in Lyon. The accuracy of the determined data is limited, due to the small data set. The polymerization under various conditions, like reaction temperature, time and concentrations in future work should lead to a higher certainty of the coefficients. Because the neodymium catalyst can be well researched by UV/Vis spectroscopy concerning information about equilibrium constants an extension of the reactor with a UV/Vis cell within the reaction medium would give additional data.

As a part of a cooperation with Dr. Florian Ehlers and Jan Schwellenbach a new method for the production of PE- $b$-P3HT block copolymers was found. The formed polymer has the combined properties of $\mathrm{PE}$ and $\mathrm{P} 3 \mathrm{HT}$, yet the analysis was found demanding with concern to NMR studies. This synthesis is straight forward and needs only the combination of both reaction mixtures without a previous work up. This allows a broad spectrum of new reactions based on CCG and the Ni-catalyzed polymerization of dibromoaryls. In this work a complete mechanistic and kinetic model for CCG polymerization was derived and successfully applied to different catalyst/co-catalyst system by two different spectroscopic methods. The process was transferred to a batch reactor and the polymerization modeled. In this work the proof of principle was performed, which is the basis for a broad catalog of different kinetic studies in the future. 



\section{Experimental}

\subsection{General}

\subsubsection{Materials and Chemicals}

The monomer styrene was purchased from Sigma-Aldrich and the inhibitor removed via column filtration through $\mathrm{Al}_{2} \mathrm{O}_{3}$ (Brockmann Grad I, neutral, Sigma Aldrich). Oxygen was removed via three freeze-pump-thaw cycles at a pump and dried over molecular sieves (3 Angström). Styrene-d8 (ABCR) was dried over molecular sieves $(3 \AA$ ) and the inhibitor was removed shortly before measurement via column filtration through $\mathrm{Al}_{2} \mathrm{O}_{3}$ within a glovebox. Both monomers were stored at $-25^{\circ} \mathrm{C}$ under argon. Toluene (p.a., Fluka) and di-n-butyl ether (p.a., Sigma-Aldrich) were dried over molecular sieves ( $4 \AA$ ) and flushed with argon for 2 hours. Tetrahydrofuran (Fluka) was dried over $\mathrm{CaH}_{2}$ (Sigma-Aldrich) and distilled under argon. Diethyl ether and 1,4-dioxane were purchased anhydrous and stored under argon over molecular sieves $(4 \AA)$. Acetone, hexane (all Fluka), benzylbromide, $\mathrm{Cp}^{*}{ }_{2} \mathrm{ZrCl}_{2}$ (99\%) (all ABCR), BOMAG in heptane (Chemtura), butyl magnesium chloride (Acros), neodymium trichloride (anhydrous, $\mathrm{Ni}(\mathrm{dppp}) \mathrm{Cl}_{2}$, all Sigma Aldrich), ethylene (3.0, Linde) and ethanol (tech.) were used as received. 2,5-dibromo-3-hexylthiophene was synthesized according to the literature ${ }^{[238]}$. If not stated otherwise standard Schlenk techniques were applied and the samples prepared in a glovebox (LabMaster 130) with a oxygen and moisture detector (Ionic Systems TOS 3.0).

\subsubsection{Determination of Conversion}

For all polymerizations the conversion was determined gravimetric. A vial with snap-on cap was weighted empty $w_{\text {emp. }}$, with a quencher (Ethanol) $w_{\text {que. }}$ and with the polymer 
solution added $w_{f u l l}$. The sample was dried till weight constancy $w_{\text {dry }}$. Because the solid is not further purified, the mass of the additional $\mathrm{BzMgBz}$ has to be subtracted $w_{(\mathrm{BzMgBz})}$. The weight difference between the completely filled and the quencher filled vial was multiplied with the weight fraction of monomer in the polymerization solution $f_{\text {(Styrene) }}$. Following equation was used:

$$
\alpha_{\text {conversion }}=\frac{w_{(\text {dry })}-w_{(\text {emp. })}-w_{(\mathrm{BzMgBz})}}{\left(w_{(\text {full })}-w_{(\text {que. })}\right) \cdot f_{(\text {Styrene })}}
$$

The method to substract the weight of the BzMgBz solution may at first glance introduce an error, because the diethyl ether will be likely removed during the drying process. Yet the benzyl groups introduced into the polymer and the formed magnesium salts will lead to a higher difference between FT-IR and gravimetric data if the correction is not applied. This on the one hand limits the accuracy of this data, but on the other hand is necessary to gain comparable results.

\subsubsection{Size Exclusion Chromatography (SEC)}

SEC analysis were preformed on following systems:

\subsubsection{High Temperature SEC (HT-SEC)}

The high temperature SEC consists of an Agilent G1888 Network Headspace Autosam-

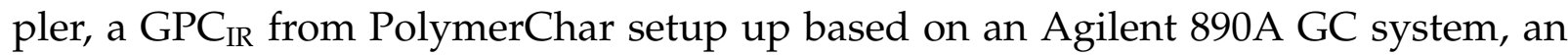
Agilent 1260 Pump (G1310B), an Agilent 1322A degasser and a PSS 246 interface. For separation at $150{ }^{\circ} \mathrm{C}$ a Polefin $10 \mu \mathrm{m}$ precolumn, a Polefin $1000 \AA$ column, a Polefin $100000 \AA$ column and a Polefin $1000000 \AA$ column and as eluent 1,2,4-trichlorbenzene containing $0.1 \%$ Irgafos ${ }^{\circledR}$ were used. The detection is performed with a Q 4 IR two channel detector for the $\mathrm{CH}_{2}$ and $\mathrm{CH}_{3}$ signal and an online viscometer at $160{ }^{\circ} \mathrm{C}$. The whole system is controlled via the "Instrumental Control" software by PolymerChar, and WinGPC Unity (PSS) is used for data recording and analysis. 


\subsubsection{THF SEC 2}

The SEC 2 is based on an Agilent 1260 Infintiy system. It consists of an Agilent 1260 Pump (G1310B), an Agilent 1260 ALS (G1329B) autosampler, a PSS TCC6000 column heater, an Agilent 1260 VWD VL (G1314B) UV/Vis detector and an Agilent 1260 RID (G1362A) refractive index detector. For separation at $35^{\circ} \mathrm{C}$ a PSS-SDV $5 \mu \mathrm{m}$ precolumn, a PSS-SDV $1000 \AA$ A column, a PSS-SDV $100000 \AA$ column and a PSS-SDV $1000000 \AA$ A column and THF as eluent were used. WinChrome (PSS) is used for controlling, data recording and analysis. Following band broadening ${ }^{[253]}$ parameters were applied: $\sigma_{G}=0.172$ and $\tau=0.279$.

\subsubsection{Nuclear Magnetic Resonance (NMR)}

\subsubsection{General}

Standard NMR-measurements were performed either on a Bruker Avance 300 or a VARIAN Unity 300. Time-resolved NMR experiments were performed on a VARIAN Unity 300 at the NMR department at the Institute for Organic and Biomolecular Chemistry of the University of Göttingen. Solvent signals were assigned according to literature. [300] Sample preparation for standard NMR analysis: The desired amount of substance was dissolved in the desired deuterated solvent and either introduced into the autosampler of the Bruker Avance 300 or measured by the NMR department at the Institute for Organic and Biomolecular Chemistry of the Georg-August University of Göttingen.

\subsubsection{Data Handling of Kinetic NMR}

The raw data from the spectrometer was processed with the software MestreNova Version 7.0.3-8830. Because the integration method of this program is not able to perform additional baseline correction during integration thus the program Bruker Opus 6.0, typically applied in IR analysis, was of choice. The x-axis of the NMR spectra was multiplied with 5000. Opus has the advantage to use several different integration methods, which are able to analyze overlapping integrals easily. After integration the determined data was corrected by the integral of the toluene- $d 8 /$ toluene reference signal. This leads to a reduction of scattering mainly caused by quality of the measurement. Therefore the 
reference integral $\int_{t}$ measured at a certain time was divided by the maximum value of all reference integrals $\int_{\text {Max }}$ :

$$
\begin{aligned}
& f_{\mathrm{corr}, t}=\frac{\int_{t}}{\int_{\operatorname{Max}}} \\
& \int_{\mathrm{X}, \mathrm{corr}, t}=\frac{\int_{\mathrm{X}, t}}{f_{\mathrm{corr}, t}}
\end{aligned}
$$

The concentrations were calculated as the fraction of the signal integral divided by the overall integral.

$$
c_{\mathrm{X}}=\frac{\int_{\mathrm{X}, \mathrm{corr}}}{\sum_{i}\left(\int_{i}\right)}
$$

In a few examples the overlay between the BzMgBz and species $\mathbf{F}^{\mathbf{P X}}$ could only be derived via curve analysis. This analysis was performed with the program fityk. [292] The combined peaks were analyzed as fractions of series Voigt curves. This allowed the calculation of the fraction of each signal from the overall signal. The individual result was then introduced into the calculation of the integrals of BzMgBz species $\mathbf{F}^{\mathbf{P X}}$.

\subsubsection{UV/Vis spectroscopy}

All UV / Vis measurements were performed on an Agilent CARY 300 using the program packages Scanning Kinetics and Kinetics in $10 \mathrm{~mm}$ QS cells sealed with a G114 screw cap purchased from Hellma (117.100-QS). All spectra where measured against the pure solvent in a similar cell. The spectrometer is equipped with a custom made heating block controlled by an EUROTHERM 91e controller.

\subsubsection{FT-IR spectroscopy}

The IR measurements where performed either on a Bruker IFS 88 or Bruker IFS 66/S IR-Spectrometer in $10 \mathrm{~mm}$ QS cells sealed with a Gl14 screw cap purchased from Hellma (117.100-QS). The spectrometers are equipped with a custom made heating block con- 
trolled by an EUROTHERM 91e (IFS 88) or EUROTHERM 2116 (IFS 66/S) controller. The measurement data was collected with the software Bruker Opus 6.0.

Determination of conversion The conversion was calculated by the integrals determined with the Software Bruker Opus 6.0. For integration the program internal method $\mathrm{B}$ was of choice. The calculated integral of this method is corrected by a linear baseline. The samples typically need $300 \mathrm{~s}$ before thermal equilibrium is reached. The data collected during this time is strongly influenced by thermal effects and the data was cut off. Depending on the actual measurement the mean value of the integrals between $300 \mathrm{~s}$ to $1000 \mathrm{~s}$ were used as values for the starting concentration $\int t=0$. If the system showed no inhibition phase only the first integral was applied. Therefore the first 50 to 100 measurements were performed in a 10 seconds time interval between measurements. Afterwards, this time window was increased to values between $180 \mathrm{~s}$ to $300 \mathrm{~s}$. For the calculation following equations were used:

$$
\begin{aligned}
c_{\text {styrene }} & =\frac{\int t}{\int t=0} c_{\text {styrene }, 0} \\
\alpha_{\text {conversion }} & =1-\frac{\int t}{\int t=0}
\end{aligned}
$$

\subsubsection{PE-reactor}

The polymerization reactor for ethylene is based on a BÜCHI GLASS USTER ECOCLAVE 075. The reactor setup is mounted in the ecoclave protective framework with a polycarbonate protective window and a perforated back plate. On top a stainless steel cover plate (polyclave 60bar) with a pressure resistance of 60 bar is mounted. The arrangement of connections is shown in Figure 9.1. In the middle a Cyclone 075 stirrer is mounted, which is pressure resistant up to 100 bar. The temperature in the reactor is measured via a PT100 temperature sensor (a). A manual pressure gauge (BÜCHI GLAS Uster Cl. 1.6) (b), an inlet for solvents/gases (c), a gas outlet valve (SWAGELOK SS-R1S4-BKB) connected to the exhaust air (d), a combined argon/ethylene inlet through a custom made introduction apparatus (e), a stopper (f) and a bursting safeguard (REMBE GG-UKB-LS-G 1/4") (g) are connected to the reactor using SWAGELOK connections. The bursting safeguard has a set off for 6 bar. The reaction vessel was a $500 \mathrm{~mL}$ BÜCHI GLASS USTER Typ 1B glass reactor with a torion valve outlet 


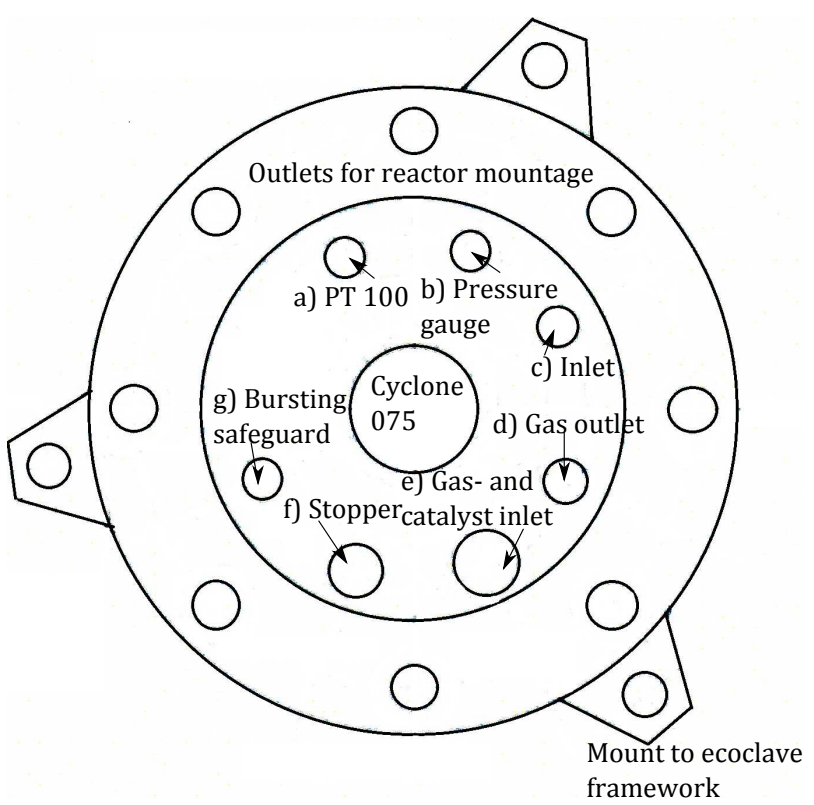

Figure 9.1: Schematic overview of the PE-reactor coverplate.

for the reaction mixture at the bottom. The Typ 1B is a double jacked glass cylinder bearing a metal ring at the upper site for montage to the cover plate. The outer jacket is connected to a LAUDA Thermostat NB/S/1512 operated by a LAUDA Universal Relais R2 electronic. The relay is either controlled by a contact thermometer within the thermostat or a EUROTHERM 2416 control unit connected to the PT100 sensor providing direct temperature control of the reaction mixture. A temperature range from $15^{\circ} \mathrm{C}$ up to $80^{\circ} \mathrm{C}$ can be achieved. Cooling water is supplied via a LAUDA WK500. The inlets have been customized. The inlet for solvents and gases (c) bears a SWAGELOK SS-43GS6MM valve to allow the insertion of metal and plastic cannula for solvent addition. By mounting of a flexible tube, vacuum may be applied by a Leybold-Heraeus Trivac D4A pump. The overview in Figure 9.2 shows connections for the gaseous supply for ethylene and argon to the filling apparatus and the reactor. The ethylene supply is connected by a three-way SWAGELOK SS-43GXS6MM valve to the SWAGELOK storage volume (2.14 1, 304L-HDF4-225-PD). At the other end of the storage volume a digital pressure gauge is mounted. For security reasons the storage volume bears a SWAGELOK SS-43GS6MM valve on each side. These valves allow a pressure release of the storage volume independent from the reactor. The second pathway of the three-way valve is connected by a customized pressure regulating valve with a three-way SWAGELOK SS-43GXS6MM valve to the reactor. The low pressure side pressure gauge is exchanged for a digital pressure gauge. The setup allows a pressure on the reactor side from 1 to 60 bar. The valve permits the introduction apparatus to either be used under argon or ethylene. The custom made introduction apparatus has a volume of $30 \mathrm{~mL}$ and can be filled independently via a 


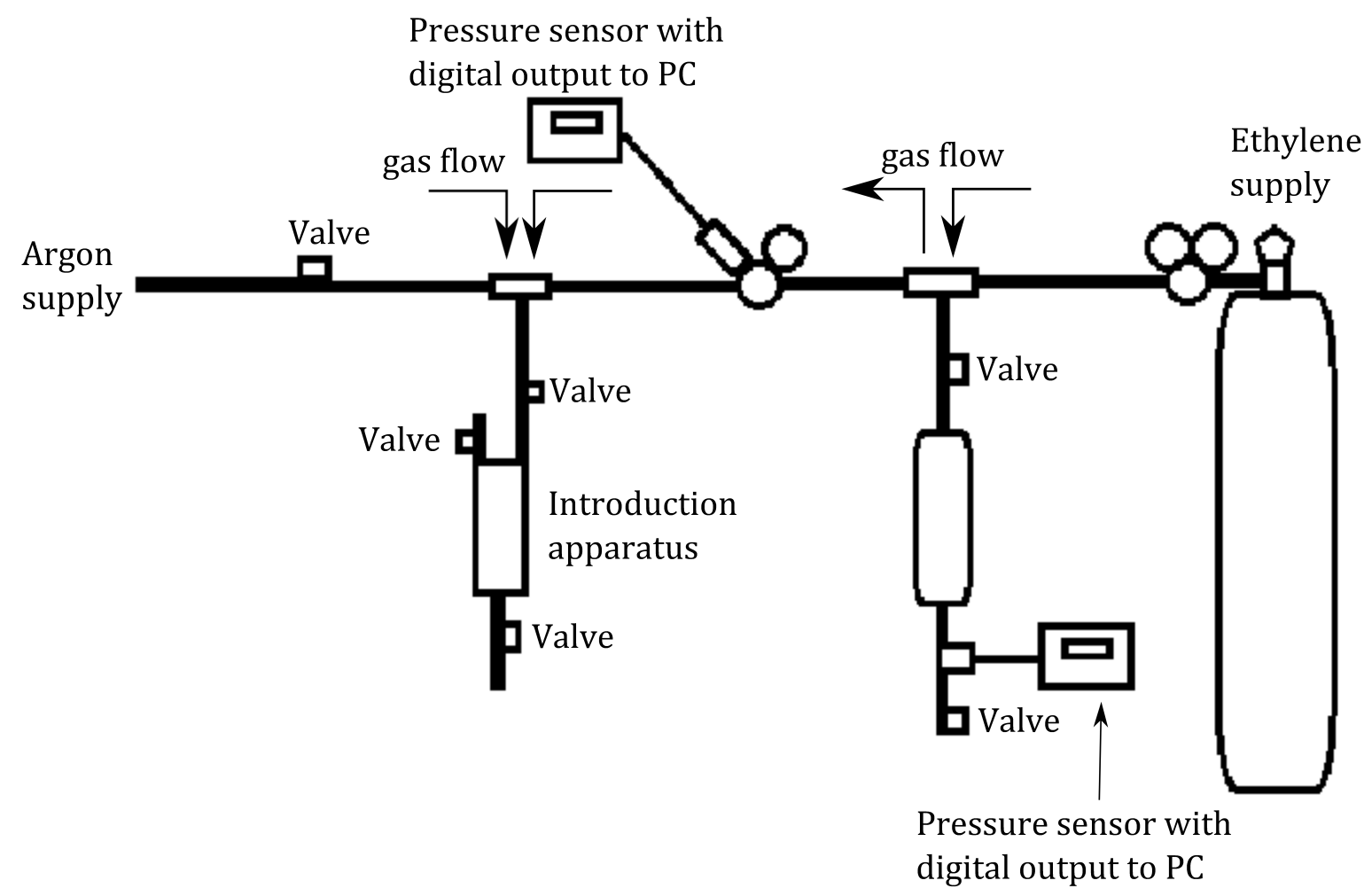

Figure 9.2: Gas supply system for PE reactor.

SWAGELOK SS-43GS6MM valve and another such valve enables the addition to the reaction medium.

Before each reaction the reactor and the connection in contact with the reaction medium were thoroughly cleaned. After assembling of the glass reactor to the cover plate the reactor was prepared for the application of vacuum. Excluding the valve between the filling apparatus and the reactor all valves were closed to remove oxygen and moisture in the reactor vessel as well as in the filling apparatus. Vacuum was applied via the fluid inlet and the reactor was heated for a least $30 \mathrm{~min}$ at $35^{\circ} \mathrm{C}$. Afterwards, the setup was flushed three times with argon.

The amount of ethylene in the storage volume was calculated following equation:

$$
\begin{aligned}
m_{\text {ethylene }} & =p_{\text {storage }} \cdot\left(\left(1.256-4.50510^{-3} * T_{\text {storage }}+1.0910^{-5} * T_{\text {storage }}^{2}\right)\right. \\
& +\left(0.0052-1.49510^{-5} * T_{\text {storage }}+1.24410^{-7} * T_{\text {storage }}^{2}\right) \\
& \left.\cdot p_{\text {storage }}+\left(3.25210^{-4}-7.3210^{-6} * T_{\text {storage }}+4.19510^{-8} * T_{\text {storage }}^{2}\right) \cdot p_{\text {storage }}^{2}\right)
\end{aligned}
$$




\subsubsection{Differential scanning calorimetry (DSC)}

DSC measurements were performed on a Mettler-Toledo DSC 820 equipped with a Thermal Analysis Excellence Automation autosampler. Cooling water was supplied via a external cryostat MGW Lauda RC6. All samples were measured with a heating rate of $10 \mathrm{~K} \mathrm{~min}^{-1}$. Data analysis was performed with the Software STARe V9.01.

\subsubsection{Mass Spectrometry}

ESI-MS The ESI-MS measurements were performed on a Bruker Daltonik micrOTOF-Q III in the analytic department of the Institute for Organic and Biomolecular Chemistry at the Georg-August University of Göttingen. About $5 \mathrm{mg}$ of the sample were dissolved within a glovebox in $\mathrm{MeCN}$ and directly injected for measurement.

MALDI The MALDI measurements were performed on a Bruker Daltonik Autoflex Speed in the analytic department of the Institute for Organic and Biomolecular Chemistry at the Georg-August University of Göttingen. The sample was dissolved in THF and introduced into a matrix of trans-2-[3-(4-tert-Butylphenyl)-2-methyl-2- propenylidene]malononitrile. 


\subsection{Synthesis}

\subsubsection{Catalysts}

\subsubsection{1 $\mathrm{Cp}_{2}{ }_{2} \mathrm{NdCl}_{2} \mathrm{Li}\left(\mathrm{OEt}_{2}\right)_{2}$}

For synthesis an approach similar to literature ${ }^{[265]}$ was performed. Oxygen was removed from pentamethylcyclopentadien $(1.25 \mathrm{~g}, 9.1 \mathrm{mmol}, 2 \mathrm{eq})$ via two freeze-pump-thaw cycles and freshly distilled THF $(40 \mathrm{~mL})$ added under argon atmosphere. The solution was cooled to $-78^{\circ} \mathrm{C}$. $n$-butyllithium in hexanes $\left(1.6 \mathrm{~mol} \mathrm{~L}{ }^{-1}, 9.1 \mathrm{mmol}, 14.6 \mathrm{~mL}\right)$ was added and the solution stirred for $1 \mathrm{~h}$ at $-78^{\circ} \mathrm{C}$. The solution was slowly heated to ambient temperature. During this process $\mathrm{NdCl}_{3}(1.15 \mathrm{~g}$, $4.6 \mathrm{mmol}, 1 \mathrm{eq})$ was weighted in inside a glovebox and outside freshly distilled THF $(20 \mathrm{~mL})$ was added under argon. The flask was equipped with a reflux condenser. The suspension was heated to reflux for $15 \mathrm{~min}$ and after cooling to ambient temperature the prepared pentamethylcyclopentadienyllithium suspension was added. After addition the suspension was heated to reflux for $6 \mathrm{~h}$, stirred over night at ambient temperature and again heated to reflux for additional $6 \mathrm{~h}$. After at least $12 \mathrm{~h}$ of reflux, the reaction was allowed to cool down at ambient temperature, the solvent was removed in vacuo and the residue washed twice with dry diethyl ether $(2 \times 50 \mathrm{~mL})$. The ether phase was collected and the solvent removed in vacuo yielding $1.88 \mathrm{~g}$ of a blue solid. Measurement of NMR is possible but due to the paramagnetic behavior the signals are very weak.

${ }^{1} \mathrm{H}-\mathrm{NMR}$ (toluene- $\left.d 8,300 \mathrm{MHz}\right) \delta=0.96\left(\mathrm{~s}, 3 \mathrm{H},\left(\mathrm{CH}_{3}-\mathrm{CH}_{2}-\right)_{2} \mathrm{O}\right), 0.96\left(\mathrm{~s}, 2 \mathrm{H},\left(\mathrm{CH}_{3}-\mathrm{CH}_{2}-\right.\right.$ )$\left._{2} \mathrm{O}\right), 1.72\left(\mathrm{~s}, 15 \mathrm{H}, \mathrm{Cp}^{*}\right), 1.79\left(\mathrm{~s}, 15 \mathrm{H}, \mathrm{Cp}^{*}\right) \mathrm{ppm}$.

ESI-MS (m/z, Q-): $484.07\left(\mathrm{Cp}_{2}{ }_{2} \mathrm{NdCl}_{2}\right.$, sim. 484.08).

\subsubsection{Co-catalysts}

\subsubsection{Dibenzyl magnesium (BzMzBz)}

For the synthesis a modified literature procedure ${ }^{[266]}$ was performed. Under argon atmosphere Mg chips (3.06 g $0.126 \mathrm{~mol})$ were stirred in diethyl ether $(300 \mathrm{~mL})$ while slowly 
a small amount of benzyl bromide was added. The Gringard reaction was started and afterwards the remaining benzyl bromide $(15 \mathrm{~mL} 0.126 \mathrm{~mol})$ added. It was stirred for $30 \mathrm{~min}$ at ambient temperature and one hour under reflux. It was cooled down and 1,4-dioxane ( $5.5 \mathrm{~mL} 0.064 \mathrm{~mol}$ ) added. The gained suspension was stirred over night and the phases separated within the reaction vessel. The liquid phase was removed via a syringe and the residual solid washed once with diethyl ether $(50 \mathrm{~mL})$. The solutions were combined and the solvent removed under reduced pressure. The obtained colorless solution was characterized via NMR. The gained BzMzBz resulted in a mixture with diethyl ether in a ratio of BzMgBz to Ether of 1:4.5. 269]

${ }^{1} \mathrm{H}-\mathrm{NMR}$ (toluene-d8, $\left.300 \mathrm{MHz}\right) \delta=1.69$ (s, 4H, Ph-CH $-\mathrm{Mg}$ ), 6.71 (m, 1H, Ph-CH $-\mathrm{Mg}$ ), 6.96-7.18 (m, 4H, Ph- $\left.\mathrm{CH}_{2}-\mathrm{Mg}\right) \mathrm{ppm}$.

\subsubsection{Di-n-butyl magnesium (BuMgBu)}

Under argon atmosphere to a $\mathrm{BuMgCl}$ solution in $\mathrm{Et}_{2} \mathrm{O}\left(2 \mathrm{~mol} \mathrm{~L}^{-1}, 100 \mathrm{~mL}, 0.200 \mathrm{~mol}\right)$ 1,4-dioxane $(10 \mathrm{~mL} 0.115 \mathrm{~mol})$ was added and the formed suspension was stirred over night. The phases were separated within the reaction vessel and the liquid phase was removed via a syringe from the residual solid. The solvent was removed under reduced pressure. The obtained white solid was characterized via NMR. For better solubility a small of amount $\mathrm{Et}_{2} \mathrm{O}$ had to be added.

${ }^{1} \mathrm{H}-\mathrm{NMR}$ (toluene- $\left.d 8,300 \mathrm{MHz}\right) \delta=0.00(\mathrm{t}, 2 \mathrm{H}, J=8.4 \mathrm{~Hz}-\mathrm{CH} 2-\mathrm{Mg}), 1.11(\mathrm{q}, 3 \mathrm{H}, J=7.2 \mathrm{~Hz}$, CH3-CH2), 1.60 (sx, 2H, J = 7.2 , CH2-CH2-CH2), 1.84 (m, 2H, -CH2-CH2-Mg) ppm.

\subsection{General Procedures}

\subsubsection{Kinetic experiments with $\mathrm{Cp}^{\star}{ }_{2} \mathrm{ZrCl}_{2}$}

\subsubsection{Monomer-free NMR measurements}

$\mathrm{Cp}^{*}{ }_{2} \mathrm{ZrCl}_{2}$ in toluene- $d 8\left(0.1 \mathrm{~mL}, \mathrm{Zr}: \mathrm{Mg}=1: 100.026 \mathrm{~mol} \mathrm{~L}^{-1} ; \mathrm{Zr}: \mathrm{Mg}=1: 200.013 \mathrm{~mol} \mathrm{~L}^{-1}\right)$ and dibenzyl magnesium $\left(0.4 \mathrm{~mL}, 0.055 \mathrm{~mol} \mathrm{~L}^{-1}\right)$ were mixed at ambient temperature. 
The samples were preheated in a heating block to the desired temperature and time measurement started. After two minutes the sample was transferred into the thermostated NMR-probe. The exact Zr:Mg ratio was determined in situ by integration of the NMR signals. [269]

\subsubsection{Monomer-free UV-Vis measurements}

To a premixed solution of $\mathrm{Cp}^{*}{ }_{2} \mathrm{ZrCl}_{2}$ in toluene $\left(0.25 \mathrm{~mL}, 11.6 \mathrm{mmol} \mathrm{L}^{-1}\right)$ dibenzyl magnesium in toluene $\left(2.0 \mathrm{~mL}, 0.037 \mathrm{~mol} \mathrm{~L}^{-1} ; 0.059 \mathrm{~mol} \mathrm{~L}^{-1} ; 0.078 \mathrm{~mol} \mathrm{~L}^{-1}\right)$ was added at ambient temperature. The measurement was started by charging the cuvette to a heating block inside the spectrometer. A spectrum was collected each or every two minutes.

\subsubsection{Monomer-free UV-Vis-measurements with di-n-butyl ether}

To a premixed solution of $\mathrm{Cp}^{*}{ }_{2} \mathrm{ZrCl}_{2}$ in toluene $\left(0.25 \mathrm{~mL}, 11.6 \mathrm{mmol} \mathrm{L}^{-1}\right)$ dibenzyl magnesium in desired toluene/ di-n-butyl ether volume fraction $\left(2.0 \mathrm{~mL}, 0.041 \mathrm{~mol} \mathrm{~L}^{-1}\right)$ was added at ambient temperature. The measurement was started by charging the cuvette to a heating block inside the spectrometer. A spectrum was collected each or every two minutes.

\subsubsection{NMR-measurements with styrene- $d 8$}

$\mathrm{Cp}^{*}{ }_{2} \mathrm{ZrCl}_{2}$ in toluene-d8 (0.1 mL; $\left.\mathrm{Zr}: \mathrm{Mg}, 1: 20,0.023 \mathrm{~mol} \mathrm{~L}^{-1} ; \mathrm{Zr}: \mathrm{Mg}, 1: 15,0.012 \mathrm{~mol} \mathrm{~L}^{-1}\right)$, dibenzyl magnesium in toluene- $d 8\left(0.2 \mathrm{~mL}\right.$; Zr:M 1:20, $0.23 \mathrm{~mol} \mathrm{~L}^{-1} ; \mathrm{Zr}: \mathrm{Mg}, 1: 15$, $0.093 \mathrm{~mol} \mathrm{~L}^{-1}$ ) and $0.2 \mathrm{~mL}$ of styrene- $\mathrm{d} 8$ were mixed at ambient temperature. The time measurement started with the heating to the desired temperature in a heating block; the sample was then transferred after 2 minutes into the thermostated NMR-probe. The exact Zr:Mg ratio was determined in situ by integration of the NMR signals. After the experiment the polymerization was quenched by pouring into ethanol. The solvent was evaporated and the samples were dried in vacuum at $60^{\circ} \mathrm{C}$. The conversion was additionally determined gravimetrically. [287] 


\subsubsection{Polymerization with styrene}

Each reaction vessel (tube or cuvette) was filled with a premixed solution of $\mathrm{Cp}^{*}{ }_{2} \mathrm{ZrCl}_{2}$ in toluene $\left(0.25 \mathrm{~mL}, 11.6 \mathrm{mmol} \mathrm{L}^{-1}\right)$ and dibenzyl magnesium in styrene $(2.0 \mathrm{~mL}$, $\left.0.043 \mathrm{~mol} \mathrm{~L}^{-1} ; 0.057 \mathrm{~mol} \mathrm{~L}^{-1} ; 0.075 \mathrm{~mol} \mathrm{~L}^{-1}\right)$ at ambient temperature. By charging the reaction tubes to a heating block $\left(70^{\circ} \mathrm{C}\right)$ the polymerization was started. Samples were removed after chosen time periods and the reaction quenched by pouring into a small amount of ethanol. Conversion was determined via gravimetrically. Online determination was performed by charging to a heating block $\left(70^{\circ} \mathrm{C}\right)$ inside an IR spectrometer.

\subsubsection{Polymerization with styrene and DBE, method a)}

Each reaction vessel (tube or cuvette) was filled with a premixed solution of $\mathrm{Cp}^{*}{ }_{2} \mathrm{ZrCl}_{2}$ in toluene $\left(0.25 \mathrm{~mL}, 11.6 \mathrm{mmol} \mathrm{L}^{-1}\right)$ and dibenzyl magnesium in styrene/DBE (19:1 (v:v), $\left.2.0 \mathrm{~mL}, 0.039 \mathrm{~mol} \mathrm{~L}^{-1}\right)$ at ambient temperature. By charging the reaction tubes to a heating block $\left(70^{\circ} \mathrm{C}\right)$ the polymerization was started. Samples were removed after chosen time periods and the reaction quenched by pouring into a small amount of ethanol. Conversion was determined via gravimetrically. Online determination was performed by charging to a heating block $\left(70^{\circ} \mathrm{C}\right)$ inside an IR spectrometer.

\subsubsection{Polymerization with styrene and BOMAG}

Each reaction cuvette was filled with a premixed solution of $\mathrm{Cp}^{*} \mathrm{ZrCl}_{2}$ in toluene $\left(0.25 \mathrm{~mL}, 11.6 \mathrm{mmol} \mathrm{L}^{-1}\right)$, BOMAG $\left(0.1 \mathrm{~mL}, 0.88 \mathrm{~mol} \mathrm{~L}^{-1}\right)$ and styrene $(2 \mathrm{~mL})$ at ambient temperature. By charging the reaction tubes to the heating blocks $\left(70^{\circ} \mathrm{C}\right)$ the polymerization was started. After measurement the polymerization was quenched by pouring into a small amount of ethanol. Conversion was determined via gravimetrically.

\subsubsection{Polymerization with styrene, BOMAG and DBE}

Each reaction cuvette was filled with a premixed solution of $\mathrm{Cp}^{*}{ }_{2} \mathrm{ZrCl}_{2}$ in toluene $\left(0.25 \mathrm{~mL}, 11.6 \mathrm{mmol} \mathrm{L}^{-1}\right)$, BOMAG $\left(0.1 \mathrm{~mL}, 0.88 \mathrm{~mol} \mathrm{~L}^{-1}\right)$, DBE $(0.1 \mathrm{~mL})$ and styrene $(1.9 \mathrm{~mL})$ at ambient temperature. By charging the reaction tubes into the heating blocks $\left(70^{\circ} \mathrm{C}\right)$ the polymerization was started. After measurement the polymerization was quenched 
by pouring into a small amount of ethanol. Conversion was determined via gravimetrically.

\subsubsection{Polymerization with styrene and DBE, method b)}

Each reaction vessel (tube or cuvette) was filled with a premixed solution of $\mathrm{Cp}^{*}{ }_{2} \mathrm{ZrCl}_{2}$ in toluene $\left(0.25 \mathrm{~mL}, 0.013 \mathrm{~mol} \mathrm{~L}^{-1}\right)$, dibenzyl magnesium in styrene $\left(2.0 \mathrm{~mL}, 0.09 \mathrm{~mol} \mathrm{~L}^{-1}\right)$ and DBE $(0.1 \mathrm{~mL})$ at ambient temperature. By charging the reaction tubes to a heating block $\left(70^{\circ} \mathrm{C}\right)$ the polymerization was started. Samples were removed after chosen time periods and the reaction quenched by pouring into a small amount of ethanol. Conversion was determined via gravimetrically. Online determination was performed by charging to a heating block $\left(70^{\circ} \mathrm{C}\right)$ inside an IR spectrometer.

\subsubsection{Kinetic experiments with $\mathrm{Cp}_{2}{ }_{2} \mathrm{NdCl}_{2} \mathrm{Li}\left(\mathrm{OEt}_{2}\right)_{2}$}

\subsubsection{Monomer-free UV-Vis-measurements}

The desired amount of a premixed solution of $\mathrm{Cp}_{2}{ }_{2} \mathrm{NdCl}_{2} \mathrm{Li}\left(\mathrm{OEt}_{2}\right)_{2}()$ was filled up with toluene to $0.25 \mathrm{~mL}$ and mixed with dibenzyl magnesium in toluene $\left(2.0 \mathrm{~mL}, 0.039 \mathrm{~mol} \mathrm{~L}^{-1}\right.$; $0.08 \mathrm{~mol} \mathrm{~L}^{-1}$ ) at ambient temperature. The measurement was started by charging the cuvette to a heating block inside the spectrometer. A spectrum was collected each minute. The temperature was changed after approach of a constant absorbance.

\subsubsection{Polymerization with styrene and BzMzBz method a)}

Each reaction vessel (tube or cuvette) was filled with a premixed solution of $\mathrm{Cp}_{2}{ }_{2} \mathrm{NdCl}_{2} \mathrm{Li}\left(\mathrm{OEt}_{2}\right)_{2}$ in toluene $\left(0.25 \mathrm{~mL}, 22.2 \mathrm{mmol} \mathrm{L}^{-1}\right)$ and dibenzyl magnesium in styrene $\left(2.0 \mathrm{~mL}, 0.038 \mathrm{~mol} \mathrm{~L}^{-1}\right)$ at ambient temperature. By charging the reaction tubes to the heating blocks $\left(70{ }^{\circ} \mathrm{C}\right)$ the polymerization was started. After measurement the polymerization was quenched by pouring into a small amount of ethanol. Conversion was determined via gravimetrically. 


\subsubsection{Polymerization with styrene and BzMzBz method b)}

Each cuvette was filled with a premixed solution of $\mathrm{Cp}^{*}{ }_{2} \mathrm{NdCl}_{2} \mathrm{Li}\left(\mathrm{OEt}_{2}\right)_{2}$ in toluene $\left(0.1 \mathrm{~mL}, 5.8 \mathrm{mmol} \mathrm{L}^{-1}\right)$ and dibenzyl magnesium in styrene $\left(2.0 \mathrm{~mL}, 0.095 \mathrm{~mol} \mathrm{~L}^{-1}\right)$ at ambient temperature. By charging the reaction tubes to the heating blocks $\left(70^{\circ} \mathrm{C}\right)$ the polymerization was started. After measurement the polymerization was quenched by pouring into a small amount of ethanol. Conversion was determined via gravimetrically.

\subsubsection{Polymerization with styrene, BzMzBz and DBE method a)}

Each reaction vessel (tube or cuvette) was filled with a premixed solution of $\mathrm{Cp}^{*}{ }_{2} \mathrm{NdCl}_{2} \mathrm{Li}\left(\mathrm{OEt}_{2}\right)_{2}$ in toluene $\left(0.25 \mathrm{~mL}, 21.9 \mathrm{mmol} \mathrm{L}^{-1}\right)$ and dibenzyl magnesium in styrene/DBE (19:1 v:v, $\left.2.0 \mathrm{~mL}, 0.038 \mathrm{~mol} \mathrm{~L}^{-1}\right)$ at ambient temperature. By charging the reaction tubes to the heating blocks $\left(70^{\circ} \mathrm{C}\right)$ the polymerization was started. After measurement the polymerization was quenched by pouring into a small amount of ethanol. Conversion was determined via gravimetrically.

\subsubsection{Polymerization with styrene, BzMzBz and DBE method b)}

Each cuvette was filled with a premixed solution of $\mathrm{Cp}_{2}{ }_{2} \mathrm{NdCl}_{2} \mathrm{Li}\left(\mathrm{OEt}_{2}\right)_{2}$ in toluene $\left(0.1 \mathrm{~mL}, 5.8 \mathrm{mmol} \mathrm{L}^{-1}\right)$, dibenzyl magnesium in styrene $\left(2.0 \mathrm{~mL}, 0.095 \mathrm{~mol} \mathrm{~L}^{-1}\right)$ and DBE $(0.5 \mathrm{~mL})$ at ambient temperature. By charging the reaction tubes to the heating blocks $\left(70^{\circ} \mathrm{C}\right)$ the polymerization was started. After measurement the polymerization was quenched by pouring into a small amount of ethanol. Conversion was determined via gravimetrically.

\subsubsection{Polymerization with styrene and BOMAG}

Each reaction cuvette was filled with a premixed solution of $\mathrm{Cp}^{*}{ }_{2} \mathrm{NdCl}_{2} \mathrm{Li}\left(\mathrm{OEt}_{2}\right)_{2}$ in toluene $\left(0.1 \mathrm{~mL}, 5.4 \mathrm{mmol} \mathrm{L}^{-1}\right)$, BOMAG $\left(0.1 \mathrm{~mL}\right.$ or $\left.0.2 \mathrm{~mL}, 0.88 \mathrm{~mol} \mathrm{~L}^{-1}\right)$ and styrene $(1.9 \mathrm{~mL})$ at ambient temperature. By charging the reaction tubes to the heating blocks $\left(70{ }^{\circ} \mathrm{C}\right)$ the polymerization was started. After measurement the polymerization was 
quenched by pouring into a small amount of ethanol. Conversion was determined via gravimetrically.

\subsubsection{Polymerization with styrene, BOMAG and DBE}

Each reaction cuvette was filled with a premixed solution of $\mathrm{Cp}_{2}{ }_{2} \mathrm{NdCl}_{2} \mathrm{Li}\left(\mathrm{OEt}_{2}\right)_{2}$ in toluene $\left(0.1 \mathrm{~mL}, 5.4 \mathrm{mmol} \mathrm{L}^{-1}\right)$, BOMAG $\left(0.1 \mathrm{~mL}\right.$ or $\left.0.2 \mathrm{~mL}, 0.88 \mathrm{~mol} \mathrm{~L}^{-1}\right)$, DBE $(0.1 \mathrm{~mL})$ and styrene $(1.9 \mathrm{~mL})$ at ambient temperature. By charging the reaction tubes into the heating blocks $\left(70^{\circ} \mathrm{C}\right)$ the polymerization was started. After measurement the polymerization was quenched by pouring into a small amount of ethanol. Conversion was determined via gravimetrically.

\subsubsection{Catalyzed chain growth polymerization in the reactor}

\subsubsection{PE-reactor for magnesium derivatives}

The reactor was heated to $35^{\circ} \mathrm{C}$ and evacuated. After 30 minutes it was filled with argon and afterwards evacuated and refilled twice. To toluene $(400 \mathrm{~mL})$ the desired amount of magnesium derivative (BuMgOct or $\mathrm{BzMgBz}$ ) was added. The solution was transferred to the reactor via a transfer syringe and heated to $75^{\circ} \mathrm{C}$. The catalyst was weighted in inside a glove box and dissolved in the desired solvent (toluene or DBE). After heating the argon atmosphere was removed from the co-catalyst solution and ethylene added up to the desired pressure. After saturation of toluene with ethylene, the valve between the filling apparatus and reactor was closed and the catalyst added under argon into the filling apparatus. The argon was released and the apparatus flushed three time with ethylene. To start the reaction the valve was opened and the catalyst solution flowed into the co-catalyst solution. The starting pressures were noted and the ethylene consumption monitored during the reaction. After reaction the ethylene pressure was released and either the polymer solution quenched with ethanol $(2 \times 20 \mathrm{~mL})$ or a further modification preformed. After quenching the reaction mixture was cooled to room temperature and the polymer was precipitated in ethanol $(400 \mathrm{~mL})$ and filtered off. 


\subsubsection{Polymer modification reactions}

\subsubsection{PE lodine synthesis}

The polymer solution was allowed to cool down to room temperature. Iodine $(7 \mathrm{~g})$ was dissolved in freshly distilled THF $(25 \mathrm{~mL})$ and added via a cannula under argon counterflow to the polymer suspension and stirred over night. The polymer was precipitated in ethanol (400 mL) and filtered off. Residual iodine was removed from the yellowish polymer by suspension with ethanol $(200 \mathrm{~mL})$ and filtration for two further times. The polymer was dried at $80^{\circ} \mathrm{C}$ at 2 mbar in a vacuum oven and analyzed via NMR and HT-SEC.

\subsubsection{PE-b-P3HT synthesis}

The preparation of the P3HT polymer was performed within a glovebox. In a $50 \mathrm{~mL}$ flask equipped with a stirring bar $i-\mathrm{PrMgCl}\left(2.0 \mathrm{~mol} \mathrm{~L}^{-1}\right.$ in THF, $3.85 \mathrm{mmol}, 1.9 \mathrm{~mL}$, $0.98 \mathrm{eq})$ was added to a solution of 2,5-dibromo-3-hexylthiophene $(1.28 \mathrm{~g}, 3.93 \mathrm{mmol}$, $1.00 \mathrm{eq})$ in toluene $(25 \mathrm{~mL})$. After stirring for $1.0 \mathrm{~h}$ at ambient temperature the solution was added under stirring to $\mathrm{Ni}(\mathrm{dppp}) \mathrm{Cl}_{2}(0.106 \mathrm{~g}, 0.197 \mathrm{mmol}, 0.05 \mathrm{eq})$ suspended in toluene $(60 \mathrm{~mL})$ in a $100 \mathrm{~mL}$ Schlenk-flask. After $70 \mathrm{~min}$ at ambient temperature the polymerization $5 \mathrm{~mL}$ ot the solution were withdrawn to provide a homopolymer sample and quenched in acidified ethanol.

The polymerization of ethylene was performed in parallel following the standard reaction procedure. For the synthesis to BOMAG $\left(4 \mathrm{~mL}, 0.88 \mathrm{~mol} \mathrm{~L}^{-1}, 3.52 \mathrm{mmol}, 113 \mathrm{eq}\right)$ in toluene $(400 \mathrm{~mL})$ a solution of $\mathrm{Cp}^{*}{ }_{2} \mathrm{NdCl}_{2} \mathrm{Li}\left(\mathrm{OEt}_{2}\right)_{2}(20.0 \mathrm{mg}, 31 \mu \mathrm{mol}, 1.00 \mathrm{eq})$ in toluene $(5 \mathrm{~mL})$ was added at $75^{\circ} \mathrm{C}$ at an ethylene pressure of 1.7 bar. After $70 \mathrm{~min}$ the reaction was stopped by replacing ethylene with argon. Approx. $(15 \mathrm{~mL}, 0.132 \mathrm{mmol})$ of the reaction mixture were added to the solution of P3HT chains. The resulting solution was stirred for $1.0 \mathrm{~h}$ at $75^{\circ} \mathrm{C}$ and afterwards quenched by precipitation of the polymer in ethanol (200 mL). A dark red solid was obtained and washed with ethanol. The polymer was purified via sequential Soxhlet extractions with acetone, THF, chloroform and toluene. From the toluene fraction was the solvent was removed under reduced pressure to yield $186 \mathrm{mg}$ product. For analysis of the PE-homopolymer the residual reactor solution was quenched with ethanol $(20 \mathrm{~mL})$ and cooled down to ambient temperature. The polymer was precipitated in ethanol $400 \mathrm{~mL}$ and filtered off. Additional $7.8 \mathrm{~g}$ of PE-polymer 
were yielded. The polymer was characterized via HT-SEC, DSC, MALDI-MS and NMR measurements. 

A Appendix 


\section{A Appendix}

\section{A.1 Figures}

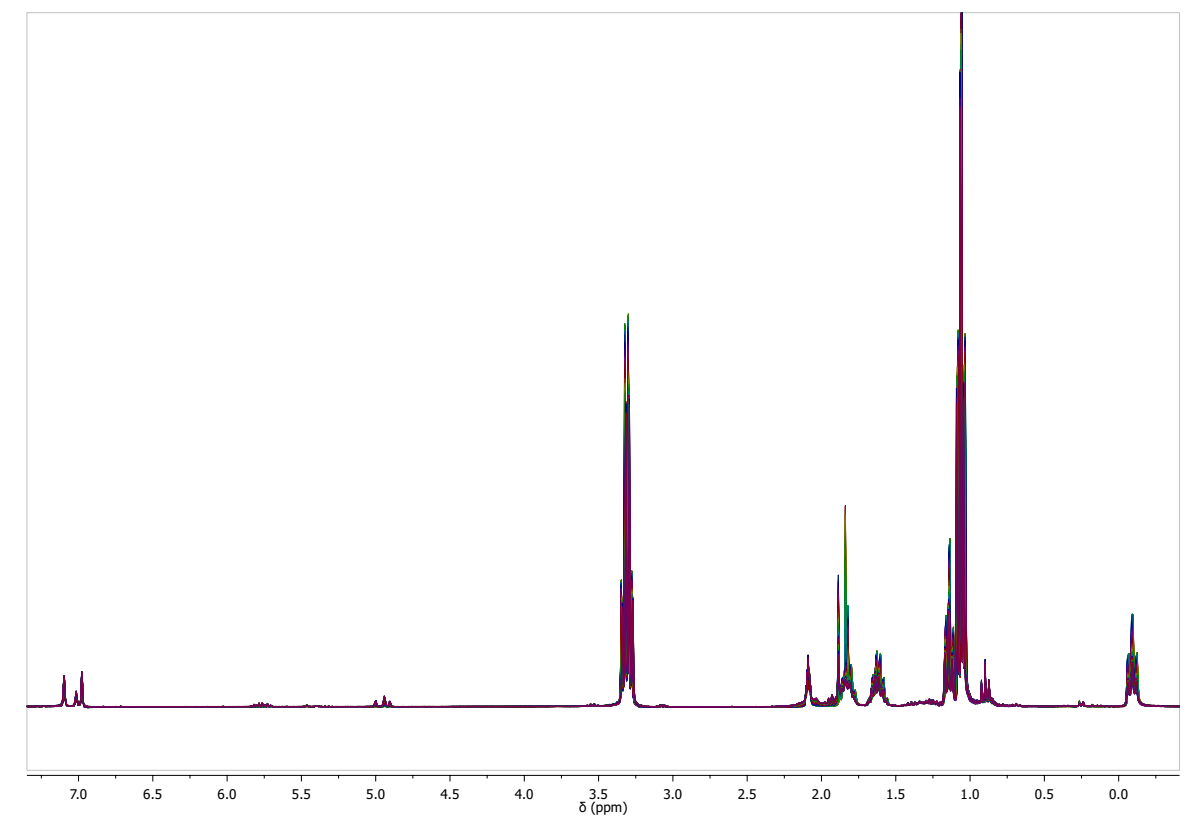

Figure A.1: Monomer free CCG activation of $\mathbf{A}$ with $\mathrm{BuMgBu}$, Full NMR spectra, $c\left(\mathrm{Cp}_{2}{ }_{2} \mathrm{ZrCl}_{2}\right)=0.00385 \mathrm{~mol} \mathrm{~L}^{-1}, c(\mathrm{BuMgBu})=0.059 \mathrm{~mol} \mathrm{~L}^{-1}$ in toluene- $d 8$. 


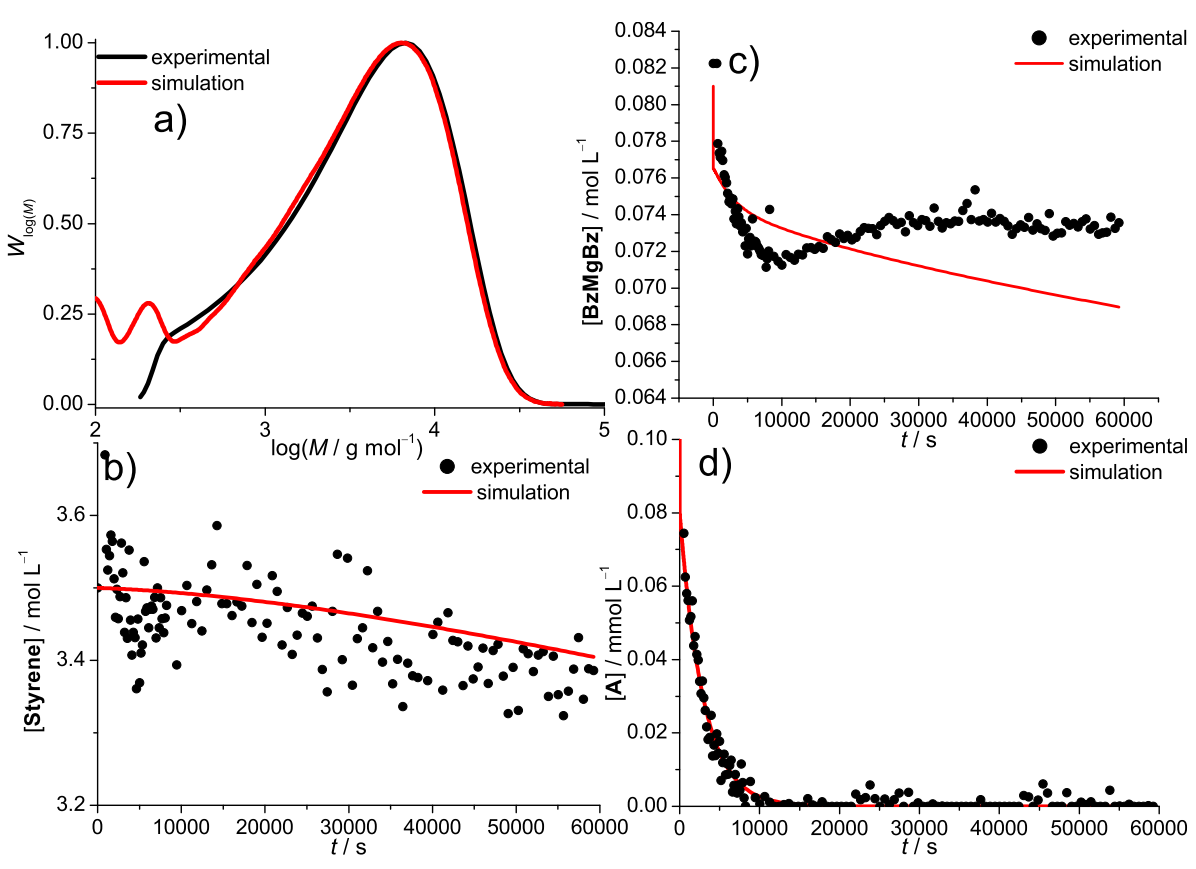

Figure A.2: Comparison of experiment and simulation of the data shown in Table 5.4 on page 108, experiment ii). a) MMD distribution; b) BzMgBz concentration vs. time profile; c) Styrene concentration vs. time profile and d) A concentration vs. time profile.
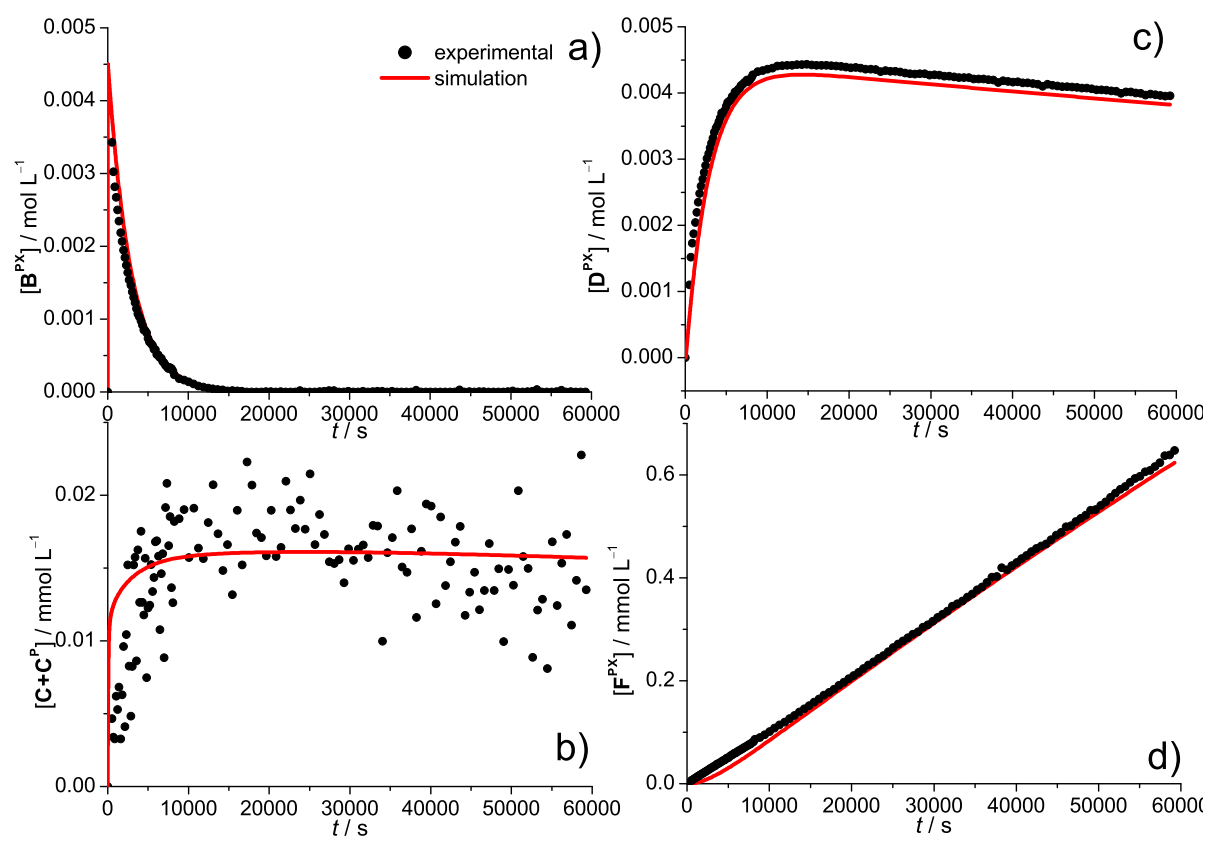

Figure A.3: Comparison of experiment and simulation with the data shown in Table 5.4 on page 108 , experiment ii). a) $\mathbf{B}^{\mathbf{P}}$ concentration vs. time profile; b) $\mathbf{C}+\mathbf{C}^{\mathbf{P}}$ concentration vs. time profile; c) $\mathbf{D}^{\mathbf{P}}$ concentration vs. time profile and d) $\mathbf{F}^{\mathbf{P}}$ concentration vs. time profile. 


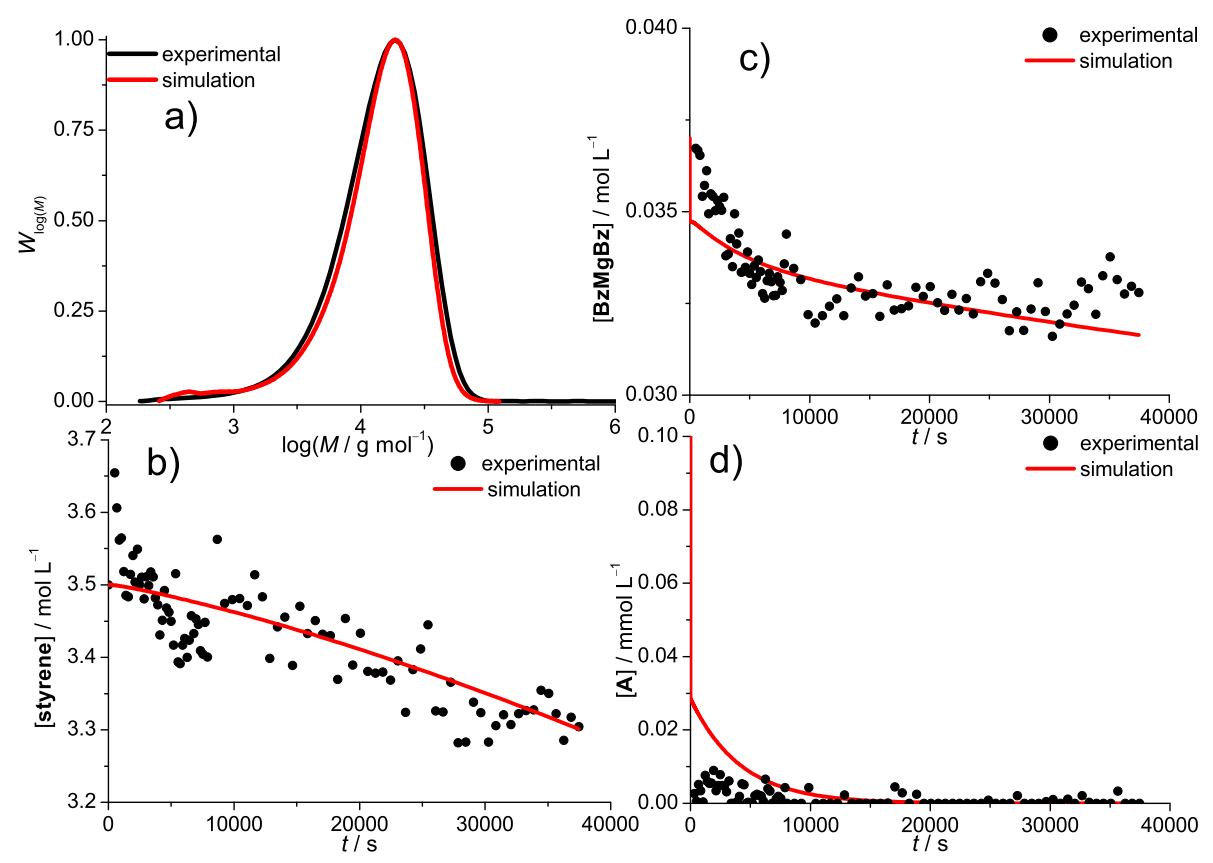

Figure A.4: Comparison of experiment and simulation of the data shown in Table 5.4 on page 108, experiment iv). a) MMD distribution; b) BzMgBz concentration vs. time profile; c) Styrene concentration vs. time profile and d) A concentration vs. time profile.

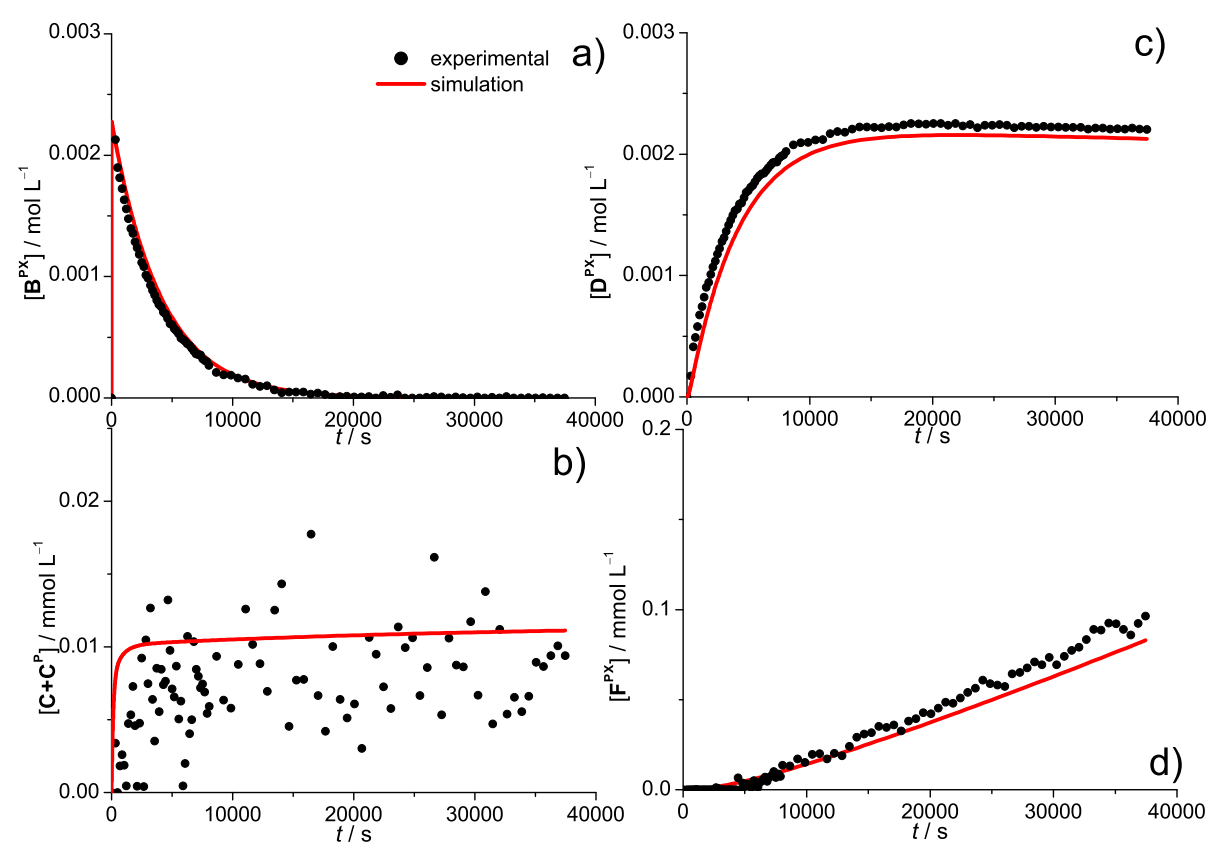

Figure A.5: Comparison of experiment and simulation with the data shown in Table 5.4 on page 108 , experiment iv). a) $\mathbf{B}^{\mathbf{P}}$ concentration vs. time profile; b) $\mathbf{C}+\mathbf{C}^{\mathbf{P}}$ concentration vs. time profile; c) $\mathbf{D}^{\mathbf{P}}$ concentration vs. time profile and d) $\mathbf{F}^{\mathbf{P}}$ concentration vs. time profile. 


\section{A.2 Reaction Rate Equations}

The $\mathbf{E}^{\mathbf{P 0}, 1,2}$ complexes are formed via deactivation of $\mathbf{D}^{\mathbf{P 0}, 1,2,3}$ complexes and interacts with the full variety of magnesium compounds to $\mathbf{F}^{\mathbf{P 0}, 1,2,3,4}$ complexes (see Equation A.1 to A.3).

$$
\begin{aligned}
& \frac{\mathrm{d}\left[\mathbf{E}^{\mathbf{P 0}}\right]}{\mathrm{d} t}=-k_{\mathrm{ex} 3}[\mathbf{E}][\mathbf{B z M g B z}]-k_{\mathrm{ex} 3}[\mathbf{E}][\mathbf{B z M g P S}]-k_{\mathrm{ex} 3}[\mathbf{E}][\mathbf{P S M g P S}]+k_{\mathrm{act} 3}\left[\mathbf{F}^{\mathbf{P} 0}\right] \\
& +k_{\text {alkyl2 }}\left[\mathbf{D}^{\mathbf{P} 0}\right]+\frac{2}{3} k_{\text {alkyl3 }}\left[\mathbf{D}^{\mathbf{P 1}}\right]+\frac{1}{2} k_{\text {act3 }}\left[\mathbf{F}^{\mathbf{P 1}}\right]+\frac{1}{4} k_{\text {act3 }}\left[\mathbf{F}^{\mathbf{P} 2}\right] \\
& \frac{\mathrm{d}\left[\mathbf{E}^{\mathbf{P 1}}\right]}{\mathrm{d} t}=-k_{\mathrm{ex} 3}\left[\mathbf{E}^{\mathbf{P} 1}\right][\mathbf{B z M g B z}]-k_{\mathrm{ex} 3}\left[\mathbf{E}^{\mathbf{P 1}}\right][\mathbf{B z M g P S}]-k_{\mathrm{ex} 3}\left[\mathbf{E}^{\mathbf{P} 1}\right][\mathbf{P S M g P S}] \\
& +\frac{1}{3} k_{\text {alkyl3 }}\left[\mathbf{D}^{\mathbf{P 1}}\right]+\frac{2}{3} k_{\text {alkyl3 }}\left[\mathbf{D}^{\mathbf{P} 2}\right]+\frac{1}{2} k_{\text {act3 }}\left[\mathbf{F}^{\mathbf{P 1}}\right]+\frac{1}{2} k_{\text {act3 }}\left[\mathbf{F}^{\mathbf{P} 2}\right]+\frac{1}{2} k_{\text {act3 }}\left[\mathbf{F}^{\mathbf{P} 3}\right] \\
& \frac{\mathrm{d}\left[\mathbf{E}^{\mathbf{P 2}}\right]}{\mathrm{d} t}=-k_{\mathrm{ex} 3}\left[\mathbf{E}^{\mathbf{P 2}}\right][\mathbf{B z M g B z}]-k_{\mathrm{ex} 3}\left[\mathbf{E}^{\mathbf{P 2}}\right][\mathbf{B z M g P S}]-k_{\mathrm{ex} 3}\left[\mathbf{E}^{\mathbf{P 2}}\right][\mathbf{P S M g P S}] \\
& +\frac{1}{3} k_{\text {alkyl2 }}\left[\mathbf{D}^{\mathbf{P} 2}\right]+k_{\text {alkyl3 }}\left[\mathbf{D}^{\mathbf{P} 3}\right]+\frac{1}{4} k_{\text {act3 }}\left[\mathbf{F}^{\mathbf{P} 2}\right]+\frac{1}{2} k_{\text {act3 }}\left[\mathbf{F}^{\mathbf{P} 3}\right]+k_{\text {act3 }}\left[\mathbf{F}^{\mathbf{P} 4}\right]
\end{aligned}
$$

The $\mathbf{F}^{\mathbf{P 0}, \mathbf{1}, \mathbf{2 , 3}, \mathbf{4}}$ complexes form the full variety of magnesium compounds and $\mathbf{E}^{\mathbf{P 0}, \mathbf{1 , 2}}$ species via dissociation while the chains can undergo transfer from magnesium to zirconium and vice versa(see Equation A.4 to 5.14).

$$
\begin{aligned}
& \frac{\mathrm{d}\left[\mathbf{F}^{\mathbf{P 0}}\right]}{\mathrm{d} t}=+k_{\mathrm{ex} 2}\left[\mathbf{E}^{\mathbf{P 0}}\right][\mathbf{B z M g B z}]-k_{\mathrm{act} 2}\left[\mathbf{F}^{\mathbf{P 0}}\right] \\
& \frac{\mathrm{d}\left[\mathbf{F}^{\mathbf{P 1}}\right]}{\mathrm{d} t}=+k_{\mathrm{ex} 3}\left[\mathbf{E}^{\mathbf{P 0}}\right][\mathbf{B z M g P S}]+k_{\mathrm{ex} 3}\left[\mathbf{E}^{\mathbf{P 1}}\right][\mathbf{B z M g B z}]-k_{\mathrm{act} 3}\left[\mathbf{F}^{\mathbf{P 1}}\right] \\
& \frac{\mathrm{d}\left[\mathbf{F}^{\mathbf{P} 2}\right]}{\mathrm{d} t}=+k_{\mathrm{ex} 3}\left[\mathbf{E}^{\mathbf{P 0}}\right][\mathbf{P S M g P S}]+k_{\mathrm{ex} 3}\left[\mathbf{E}^{\mathbf{P 1}}\right][\mathbf{B z M g P S}]+k_{\mathrm{ex} 3}\left[\mathbf{E}^{\mathbf{P 2}}\right][\mathbf{B z} \mathbf{M g B z}] \\
& -k_{\text {act3 }}\left[\mathbf{F}^{\mathbf{P} 2}\right] \\
& \frac{\mathrm{d}\left[\mathbf{F}^{\mathbf{P} 3}\right]}{\mathrm{d} t}=+k_{\mathrm{ex} 3}\left[\mathbf{E}^{\mathbf{P} 1}\right][\mathbf{P S M g P S}]+k_{\mathrm{ex} 3}\left[\mathbf{E}^{\mathbf{P 2}}\right][\mathbf{B z M g P S}]-k_{\mathrm{act} 3}\left[\mathbf{F}^{\mathbf{P} 3}\right] \\
& \frac{\mathrm{d}\left[\mathbf{F}^{\mathbf{P} 4}\right]}{\mathrm{d} t}=-k_{\mathrm{ex} 3}\left[\mathbf{E}^{\mathbf{P 2}}\right][\mathbf{P S M g P S}]-k_{\mathrm{act} 3}\left[\mathbf{F}^{\mathbf{P} 4}\right]
\end{aligned}
$$




\section{A.3 PREDICI Models}

\section{A.3.1 Activation with BuMgBu}

$$
\begin{aligned}
& \mathbf{A}+\mathrm{BzMgBz} \stackrel{k_{\mathrm{ex} 0}}{\longrightarrow} \mathbf{B}^{\mathbf{P 0}} \\
& \mathbf{B}^{\mathbf{P 0}} \stackrel{k_{\text {act0 }}}{\longrightarrow} \mathbf{A}+\mathrm{BzMgBz} \\
& \mathbf{B}^{\mathbf{P 0}} \stackrel{k_{\text {alkyl1 }}}{\longrightarrow} \mathrm{C}+\mathrm{BzMgCl} \\
& \mathrm{C}+\mathrm{BzMgBz} \stackrel{k_{\mathrm{ex} 1}}{\longrightarrow} \mathbf{D}^{\mathrm{P} \mathbf{0}} \\
& \mathbf{D}^{\mathbf{P 0}} \stackrel{k_{\text {act } 1}}{\longrightarrow} \mathrm{C}+\mathrm{BzMgBz} \\
& \mathbf{D}^{\mathrm{P0}} \stackrel{k_{\text {alkyl1 }}}{\longrightarrow} \mathbf{E}^{\mathrm{P0}}+\mathrm{BzMgCl} \\
& \mathbf{E}^{\mathbf{P 0}}+\mathrm{BzMgBz} \stackrel{k_{\mathrm{ex} 2}}{\longrightarrow} \mathbf{F}^{\mathbf{P 0}} \\
& \mathbf{F}^{\mathbf{P 0}} \stackrel{k_{\text {act2 }}}{\longrightarrow} \mathbf{E}^{\mathbf{P 0}}+\mathrm{BzMgBz} \\
& \mathrm{BzMgCl}+\mathrm{BzMgCl} \stackrel{k_{\text {schlenk }}}{\longrightarrow} \mathrm{BzMgBz}+\mathrm{ClMgCl} \\
& \mathrm{C} \stackrel{k_{\mathrm{t}}}{\longrightarrow} \mathrm{G}+\mathrm{CH}_{2} \mathrm{CHCH}_{2} \mathrm{CH}_{3} \\
& \mathbf{G} \stackrel{k_{\mathrm{Cl}, \mathrm{H} \text { exchange }}}{\longrightarrow} \mathbf{H}+\mathrm{BuMgCl} \\
& \mathbf{A}+\mathbf{H} \stackrel{k_{\mathrm{Cl}, \mathrm{H} \text { exchange } 2}}{\longrightarrow} \mathbf{C}+\mathbf{G}
\end{aligned}
$$

\section{A.3.2 Polymerization with $\mathrm{A}$ and $\mathrm{Bz} \mathrm{MgBz}$ with Reactivation of $\mathrm{G}$}

$$
\begin{gathered}
\mathbf{A}+\mathrm{BzMgBz} \stackrel{k_{\text {ex } 0}}{\longrightarrow} \mathbf{B}^{\mathbf{P 0}} \\
\mathbf{B}^{\mathbf{P 0}} \stackrel{k_{\text {act0 }}}{\longrightarrow} \mathbf{A}+\mathrm{BzMgBz} \\
\mathbf{B}^{\mathbf{P 0}} \stackrel{k_{\text {alkyl1 }}}{\longrightarrow} \mathbf{C}+\mathrm{BzMgCl} \\
\mathbf{C}+\mathrm{BzMgBz} \stackrel{k_{\text {ex } 1}}{\longrightarrow} \mathbf{D}^{\mathbf{P 0}} \\
\mathbf{D}^{\mathbf{P 0}} \stackrel{k_{\text {act1 }}}{\longrightarrow} \mathbf{C}+\mathrm{BzMgBz} \\
\mathbf{D}^{\mathbf{P 0}} \stackrel{k_{\text {alkyl1 }}}{\longrightarrow} \mathbf{E}^{\mathbf{P 0}}+\mathrm{BzMgCl} \\
\mathbf{E}^{\mathbf{P 0}}+\mathrm{BzMgBz} \stackrel{k_{\text {ex } 2}}{\longrightarrow} \mathbf{F}^{\mathbf{P 0}}
\end{gathered}
$$




$$
\begin{aligned}
& \mathbf{F}^{\mathbf{P 0}} \stackrel{k_{\text {act2 }}}{\longrightarrow} \mathbf{E}^{\mathbf{P 0}}+\mathrm{BzMgBz} \\
& \mathbf{A}+\mathrm{BzMgP}_{\mathrm{s}} \stackrel{k_{\mathrm{ex0}}}{\longrightarrow} \mathbf{B}^{\mathbf{P} 1}{ }_{\mathrm{s}} \\
& \mathbf{B}^{\mathbf{P 1}}{ }_{\mathrm{s}} \stackrel{k_{\text {act0 }}}{\longrightarrow} \mathbf{A}+\mathrm{BzMgP}_{\mathrm{s}} \\
& \mathbf{B}^{\mathbf{P} 1}{ }_{\mathbf{s}} \stackrel{k_{\text {alkyl1 }}}{\longrightarrow} \mathbf{C}^{\mathbf{P}}{ }_{\mathbf{s}}+\mathrm{BzMgCl} \\
& \mathbf{B}^{\mathbf{P 1}} \stackrel{k_{\text {alkyl1 }}}{\longrightarrow} \mathrm{C}+\mathrm{PMgCl}_{\mathrm{s}} \\
& \mathbf{A}+\mathrm{PMgP}_{\mathrm{s}} \stackrel{k_{\mathrm{ex} 0}}{\longrightarrow} \mathbf{B}^{\mathbf{P} 2}{ }_{\mathbf{s}}+\text { help1 } \\
& \mathrm{PMgP}_{\mathrm{s}}+\text { help1 } \stackrel{k_{\text {help }}}{\longrightarrow} \mathbf{B}^{\mathbf{P 2}}{ }_{\mathbf{s}} \\
& \mathbf{B}^{\mathbf{P 2}} \stackrel{\frac{1}{2} k_{\mathrm{alkyl}}}{\longrightarrow} \mathbf{C}^{\mathbf{P}}{ }_{\mathbf{s}}+\text { help2 } \\
& \mathbf{B}^{\mathbf{P 2}}{ }_{\mathbf{s}}+\text { help2 } \stackrel{k_{\text {help }}}{\longrightarrow} \mathrm{PMgCl}_{\mathrm{s}} \\
& \mathbf{B}^{\mathbf{P} 2} \stackrel{\frac{1}{2} k_{\text {act0 }}}{\longrightarrow} \mathrm{PMgP}_{\mathrm{s}}+\text { help3 } \\
& \mathbf{B}^{\mathbf{P 2}}{ }_{\mathbf{s}}+\text { help } 3 \stackrel{k_{\text {help }}}{\longrightarrow} \mathrm{PMgP}_{\mathrm{s}} \\
& \mathbf{C}+\mathrm{M} \stackrel{k_{\text {add }}}{\longrightarrow} \mathbf{C}(\mathbf{M}) \\
& \mathbf{C}(\mathbf{M}) \stackrel{k_{\text {frag }}}{\longrightarrow} \mathrm{C}+\mathrm{M} \\
& \mathbf{C}(\mathbf{M}) \stackrel{k_{\mathrm{p} 1}}{\longrightarrow} \mathrm{C}^{\mathrm{P}}{ }_{1} \\
& \mathrm{C}^{\mathrm{P}}{ }_{\mathrm{s}}+\mathrm{M} \stackrel{k_{\text {add }}}{\longrightarrow} \mathbf{C}(\mathbf{M})_{\mathrm{s}+1} \\
& \mathbf{C}^{\mathbf{P}}(\mathbf{M})_{\mathrm{s}} \stackrel{k_{\text {frag }}}{\longrightarrow} \mathbf{C}^{\mathbf{P}}{ }_{\mathrm{s}-1}+\mathrm{M} \\
& \mathbf{C}^{\mathbf{P}}(\mathbf{M})_{\mathrm{s}} \stackrel{k_{\mathrm{p}}}{\longrightarrow} \mathbf{C}_{\mathrm{s}}^{\mathbf{P}} \\
& \mathrm{C}^{\mathbf{P}}{ }_{\mathrm{s}}+\mathrm{BzMgBz} \stackrel{k_{\mathrm{ex} 2}}{\longrightarrow} \mathbf{D}^{\mathbf{P} 1}{ }_{\mathrm{s}} \\
& \mathrm{C}+\mathrm{BzMgP}_{\mathrm{s}} \stackrel{k_{\mathrm{ex} 2}}{\longrightarrow} \mathbf{D}^{\mathbf{P} 1}{ }_{\mathrm{s}} \\
& \mathbf{D}^{\mathbf{P 1}}{ }_{\mathbf{s}} \stackrel{\frac{2}{3} k_{\mathrm{act} 2}}{\longrightarrow} \mathbf{C}+\mathrm{BzMgP}_{\mathrm{s}} \\
& \mathbf{D}^{\mathbf{P 1}} \stackrel{\frac{1}{3} k_{\text {act2 }}}{\longrightarrow} \mathbf{C}^{\mathbf{P}}{ }_{\mathbf{s}}+\mathrm{BzMgBz} \\
& \mathbf{C}^{\mathbf{P}}{ }_{\mathbf{s}}+\mathrm{BzMgP}_{\mathrm{r}} \stackrel{k_{\text {ex2 }}}{\longrightarrow} \mathbf{D}^{\mathbf{P} \mathbf{2}} \mathbf{s}+\mathbf{D}^{\mathbf{P} \mathbf{2}}{ }_{\mathbf{r}} \\
& \mathrm{C}+\mathrm{PMgP}_{\mathrm{s}} \stackrel{k_{\text {act2 }}}{\longrightarrow} \text { help }_{\mathrm{s}}
\end{aligned}
$$




$$
\begin{aligned}
& h e l p 4+\mathrm{PMgP}_{\mathrm{r}} \stackrel{k_{\text {help }}}{\longrightarrow} \mathbf{D}^{\mathbf{P 1}}{ }_{\mathbf{s}}+\mathbf{D}^{\mathbf{P 1}}{ }_{\mathbf{r}} \\
& \mathbf{D}^{\mathbf{P 2}}{ }_{\mathbf{s}} \stackrel{\frac{1}{2} k_{\text {act2 }}}{\longrightarrow} \mathbf{C}_{\mathbf{s}}^{\mathbf{P}}+\text { help5 } \\
& \mathrm{D}^{\mathrm{P} 2} \mathrm{~s}+\text { help5 } \stackrel{k_{\text {help }}}{\longrightarrow} \mathrm{BzMgP}_{\mathrm{s}} \\
& \mathbf{D}_{\mathbf{s}}^{\mathbf{P 2}} \stackrel{\frac{1}{6} k_{\mathrm{act} 2}}{\longrightarrow} \mathrm{PMgP}_{\mathrm{s}}+\text { help6 } \\
& \mathbf{D}^{\mathbf{P 2}}{ }_{\mathbf{s}}+\text { help } 6 \stackrel{k_{\text {help }}}{\longrightarrow} \mathrm{PMgP}_{\mathrm{s}}+\mathbf{C} \\
& \mathbf{C}^{\mathbf{P}}{ }_{\mathrm{s}}+\mathrm{PMgP}_{\mathrm{r}} \stackrel{\frac{1}{2} k_{\mathrm{ex} 2}}{\longrightarrow} \mathbf{D}^{\mathrm{P3}}{ }_{\mathrm{s}}+\text { help } 7_{\mathrm{r}} \\
& \text { help } 7_{\mathrm{r}}+\mathrm{PMgP}_{\mathrm{r}} \stackrel{k_{\text {help }}}{\longrightarrow} \mathbf{D}^{\mathrm{P} 3}{ }_{\mathbf{s}}+\mathbf{D}^{\mathrm{P} 3}{ }_{\mathbf{r}} \\
& \mathbf{D}^{\mathrm{P} 3}{ }_{\mathrm{s}} \stackrel{\frac{1}{3} k_{\mathrm{act} 2}}{\longrightarrow} \mathrm{PMgP}_{\mathrm{s}}+\text { help8 } \\
& \mathbf{D}^{\mathbf{P} 3}{ }_{\mathbf{s}}+\text { help } 8 \stackrel{k_{\text {help }}}{\longrightarrow} \mathrm{PMgP}_{\mathrm{s}}+\text { help9 } \\
& \mathbf{D}^{\mathbf{P} 3}{ }_{\mathbf{s}}+h \operatorname{lp} 9 \stackrel{k_{\text {help }}}{\longrightarrow} \mathbf{C}^{\mathbf{P}}{ }_{\mathbf{s}} \\
& \mathbf{C}^{\mathbf{P}}{ }_{\mathbf{s}} \stackrel{k_{\mathrm{t}}}{\longrightarrow} \mathbf{G}+\mathrm{P}_{\text {Dead }, \mathrm{s}} \\
& \mathrm{BzMgCl}+\mathrm{BzMgCl} \stackrel{k_{\text {schlenk }}}{\longrightarrow} \mathrm{BzMgBz}+\mathrm{ClMgCl} \\
& \mathrm{PMgCl}_{\mathrm{s}}+\mathrm{BzMgCl} \stackrel{k_{\text {schlenk }}}{\longrightarrow} \mathrm{BzMgP}_{\mathrm{s}}+\mathrm{CIMgCl} \\
& \mathrm{PMgCl}_{\mathrm{s}}+\mathrm{PMgCl}_{\mathrm{r}} \stackrel{k_{\text {schlenk }}}{\longrightarrow} \mathrm{PMgP}_{\mathrm{s}}+\text { help } 26_{\mathrm{r}} \\
& \text { help } 26_{\mathrm{s}} \stackrel{k_{\text {help }}}{\longrightarrow} \mathrm{PMgP}_{\mathrm{s}}+\mathrm{CIMgCl} \\
& \mathbf{D}^{\mathbf{P 0}} \stackrel{k_{\text {alkyl2 }}}{\longrightarrow} \mathbf{E}^{\mathbf{P 0}}+\mathrm{BzMgCl} \\
& \mathbf{D}^{\mathbf{P} 1}{ }_{\mathbf{s}} \stackrel{\frac{1}{3} k_{\text {alkyl3 }}}{\longrightarrow} \mathbf{E}^{\mathbf{P 0}}+\mathrm{PMgCl}_{\mathrm{s}} \\
& \mathbf{D}^{\mathbf{P 1}}{ }_{\mathbf{s}} \stackrel{\frac{2}{3} k_{\mathrm{alkyl} 3}}{\longrightarrow} \mathbf{E}^{\mathbf{P 1}} \mathbf{s}_{\mathbf{s}}+\mathrm{BzMgCl} \\
& \mathbf{D}_{\mathbf{s}}^{\mathbf{P 2}} \stackrel{\frac{1}{6} k_{\text {alkyl3 }}}{\longrightarrow} \mathbf{E}^{\mathbf{P 2}}+\text { help10 } \\
& \mathbf{D}^{\mathbf{P 2}}{ }_{\mathbf{s}}+\text { help10 } \stackrel{k_{\text {help }}}{\longrightarrow} \mathbf{E}^{\mathbf{P 2}}+\mathrm{BzMgCl} \\
& \mathbf{D}^{\mathbf{P} 2}{ }_{\mathbf{s}} \stackrel{\frac{1}{3} k_{\mathrm{alky}} 13}{\longrightarrow} \mathbf{E}^{\mathbf{P 1}}{ }_{\mathrm{s}}+\text { help11 } \\
& \mathbf{D}^{\mathbf{P 2}} \mathbf{s}+\text { help11 } \stackrel{k_{\text {help }}}{\longrightarrow} \mathrm{PMgCl}_{\mathrm{s}}
\end{aligned}
$$




$$
\begin{aligned}
& \mathbf{D}^{\mathbf{P 3}} \stackrel{\frac{1}{3} k_{\mathrm{alky1} 2}}{\longrightarrow} \mathbf{E}^{\mathbf{P 2}}+\text { help12 } \\
& \mathbf{D}^{\mathbf{P} 3}{ }_{\mathbf{s}}+\text { help12 } \stackrel{k_{\text {help }}}{\longrightarrow} \mathbf{E}^{\mathbf{P 2}}+\text { help13 } \\
& \mathbf{D}^{\mathbf{P} 3}{ }_{\mathbf{s}}+\text { help13 } \stackrel{k_{\text {help }}}{\longrightarrow} \mathrm{PMgCl}_{\mathrm{s}} \\
& \mathbf{E}^{\mathbf{P 0}}+\mathrm{BzMgBz} \stackrel{k_{\mathrm{ex} 3}}{\longrightarrow} \mathbf{F}^{\mathbf{P 0}} \\
& \mathbf{F}^{\mathbf{P 0}} \stackrel{k_{\text {act2 }}}{\longrightarrow} \mathbf{E}^{\mathbf{P 0}}+\mathrm{BzMgBz} \\
& \mathbf{E}^{\mathbf{P 1}}{ }_{\mathbf{s}}+\mathrm{BzMgBz} \stackrel{k_{\mathrm{ex} 3}}{\longrightarrow} \mathbf{F}^{\mathbf{P 1}} \\
& \mathbf{E}^{\mathbf{P 0}}+\mathrm{BzMgP}_{\mathrm{s}} \stackrel{k_{\mathrm{ex} 3}}{\longrightarrow} \mathbf{E}^{\mathbf{P 1}} \\
& \mathbf{F}^{\mathbf{P 1}} \stackrel{k_{\text {act3 }}}{\longrightarrow} \mathbf{E}^{\mathbf{P 1}}{ }_{\mathbf{s}}+\mathrm{BzMgBz} \\
& \mathbf{F}^{\mathbf{P 1}} \stackrel{k_{\text {act3 }}}{\longrightarrow} \mathbf{E}^{\mathbf{P 0}}+\mathrm{BzMgP}_{\mathrm{s}} \\
& \mathbf{E}^{\mathbf{P 1}}{ }_{\mathbf{s}}+\mathrm{BzMgP}_{\mathrm{r}} \stackrel{k_{\mathrm{e} \times 3}}{\longrightarrow} \mathbf{F}^{\mathbf{P 2}}{ }_{\mathbf{s}}+\mathbf{F}^{\mathbf{P 2}} \mathbf{r} \\
& \mathbf{E}^{\mathbf{P 0}}+\mathrm{PMgP}_{\mathrm{s}} \stackrel{\frac{1}{2} k_{\mathrm{e} \times 3}}{\longrightarrow} \mathbf{F}^{\mathbf{P 2}} \mathbf{s}+\text { help14 } \\
& \text { help14 }+\mathrm{PMgP}_{\mathrm{s}} \stackrel{k_{\text {help }}}{\longrightarrow} \mathbf{F}^{\mathbf{P 2}} \mathbf{s} \\
& \mathbf{E}^{\mathbf{P 2}}+\mathrm{BzMgBz} \stackrel{\frac{1}{2} k_{\mathrm{ex}}}{\longrightarrow} \mathbf{F}^{\mathbf{P 2}} \mathbf{s}+\text { help15 } \\
& h e l p 15+\mathbf{E}^{\mathbf{P 2}} \stackrel{k_{\text {help }}}{\longrightarrow} \mathbf{F}^{\mathbf{P 2}} \text { s } \\
& \mathbf{F}^{\mathbf{P 2}} \stackrel{\frac{1}{4} k_{\text {act3 }}}{\longrightarrow} \mathbf{E}^{\mathbf{P 1}}{ }_{\mathbf{s}}+\text { help16 } \\
& \mathbf{F}^{\mathbf{P 2}}{ }_{\mathbf{s}}+\text { help16 } \stackrel{k_{\text {help }}}{\longrightarrow} \text { BzMgP }_{\mathbf{s}} \\
& \mathbf{F}^{\mathbf{P 2}} \stackrel{\frac{1}{8} k_{\text {act3 }}}{\longrightarrow} \mathrm{PMgP}_{\mathrm{s}}+\text { help17 } \\
& \mathbf{F}^{\mathbf{P 2}}{ }_{\mathbf{s}}+\text { help17 } \stackrel{k_{\text {help }}}{\longrightarrow} \mathrm{PMgP}_{\mathrm{s}}+\mathbf{F}^{\mathbf{P 0}} \\
& \mathbf{F}^{\mathbf{P 2}} \stackrel{\frac{1}{8} k_{\text {act3 }}}{\longrightarrow} \mathbf{E}^{\mathbf{P 2}}{ }_{\mathbf{s}}+\text { help18 } \\
& \mathbf{F}^{\mathbf{P 2}}{ }_{\mathbf{s}}+\text { help18 } \stackrel{k_{\text {help }}}{\longrightarrow} \mathbf{E}^{\mathbf{P 2}}{ }_{\mathbf{s}}+\mathrm{BzMgBz} \\
& \mathbf{E}^{\mathbf{P 1}}{ }_{\mathbf{s}}+\mathrm{PMgP}_{\mathrm{r}} \stackrel{k_{\mathrm{ex} 3}}{\longrightarrow} \mathbf{F}^{\mathbf{P 3}}{ }_{\mathbf{s}}+\text { help19 }{ }_{\mathrm{r}} \\
& \mathrm{PMgP}_{\mathrm{s}}+\text { help19 }{ }_{\mathrm{r}} \stackrel{k_{\text {help }}}{\longrightarrow} \mathbf{F}^{\mathbf{P 3}}{ }_{\mathbf{s}}+\mathbf{F}^{\mathbf{P 3}}{ }_{\mathbf{r}} \\
& \mathbf{E}^{\mathbf{P 2}}{ }_{\mathbf{s}}+\mathrm{BzMgP}_{\mathrm{r}} \stackrel{k_{\mathrm{ex} 3}}{\longrightarrow} \mathbf{F}^{\mathbf{P 3}}{ }_{\mathbf{s}}+\text { help } 20_{\mathrm{r}}
\end{aligned}
$$




$$
\begin{aligned}
& \mathbf{E}^{\mathbf{P 2}}{ }_{\mathbf{s}}+\text { help } 20_{\mathrm{r}} \stackrel{k_{\text {help }}}{\longrightarrow} \mathbf{F}^{\mathbf{P} 3}{ }_{\mathbf{s}}+\mathbf{F}^{\mathbf{P} 3}{ }_{\mathbf{r}} \\
& \mathbf{F}^{\mathbf{P} 3} \stackrel{\frac{1}{6} k_{\text {act3 }}}{\longrightarrow} \mathrm{PMgP}_{\mathrm{s}}+\text { help } 21 \\
& \mathbf{F}^{\mathbf{P 3}}{ }_{\mathbf{s}}+\text { help } 21 \stackrel{k_{\text {help }}}{\longrightarrow} \mathbf{E}^{\mathbf{P 1}}{ }_{\mathbf{s}}+\text { help22 } \\
& \mathbf{F}^{\mathbf{P 3}}{ }_{\mathbf{s}}+\text { help } 22 \stackrel{k_{\text {help }}}{\longrightarrow} \mathrm{PMgP}_{\mathrm{s}} \\
& \mathbf{F}^{\mathbf{P 3}} \stackrel{\frac{1}{6} k_{\text {act3 }}}{\longrightarrow} \mathbf{E}^{\mathbf{P 2}}{ }_{\mathbf{s}}+\text { help23 } \\
& \mathbf{F}^{\mathbf{P} 3}{ }_{\mathbf{s}}+\text { help } 23 \stackrel{k_{\text {help }}}{\longrightarrow} \mathbf{E}^{\mathbf{P 2}}{ }_{\mathbf{s}}+\text { help } 24 \\
& \mathbf{F}^{\mathbf{P 3}}{ }_{\mathbf{s}}+\text { help24 } \stackrel{k_{\text {help }}}{\longrightarrow} \text { BzMgP }_{\mathbf{s}} \\
& \mathbf{E}^{\mathbf{P 2}}{ }_{\mathbf{s}}+\mathrm{PMgP}_{\mathrm{r}} \stackrel{\frac{1}{2} k_{\mathrm{ex} 3}}{\longrightarrow} \mathbf{F}^{\mathbf{P 4}}{ }_{\mathbf{s}}+\mathbf{F}^{\mathbf{P 4}}{ }_{\mathbf{r}} \\
& \mathbf{F}_{\mathbf{s}}^{\mathbf{P 4}} \stackrel{\frac{1}{2} k_{\text {act3 }}}{\longrightarrow} \mathrm{PMgP}_{\mathrm{s}}+\text { help } 25 \\
& \mathbf{F}^{\mathbf{P 4}}{ }_{\mathbf{s}}+\text { help } 25 \stackrel{k_{\text {help }}}{\longrightarrow} \mathbf{E}^{\mathbf{P 2}} \text { s } \\
& \mathrm{C} \stackrel{k_{\mathrm{t}, \mathrm{alkyl}}}{\longrightarrow} \mathrm{G} \\
& \mathbf{G}+\text { Monomer } \stackrel{k_{\text {reini }}}{\longrightarrow} \mathbf{C}^{\mathbf{P}}
\end{aligned}
$$

\section{A.3.3 Extended Model for Polymerization with A and BuMgOct}

$$
\begin{aligned}
& \mathbf{A}+\mathrm{BzMgBz} \stackrel{k_{\mathrm{ex} 0}}{\longrightarrow} \mathbf{B}^{\mathbf{P 0}} \\
& \mathbf{B}^{\mathbf{P 0}} \stackrel{k_{\text {act0 }}}{\longrightarrow} \mathbf{A}+\mathrm{BzMgBz} \\
& \mathbf{B}^{\mathbf{P 0}} \stackrel{k_{\text {alkyl1 }}}{\longrightarrow} \mathrm{C}+\mathrm{BzMgCl} \\
& \mathrm{C}+\mathrm{BzMgBz} \stackrel{k_{\mathrm{ex} 1}}{\longrightarrow} \mathbf{D}^{\mathrm{P} \mathbf{0}} \\
& \mathrm{D}^{\mathrm{P} 0} \stackrel{k_{\text {act } 1}}{\longrightarrow} \mathrm{C}+\mathrm{BzMgBz} \\
& \mathbf{D}^{\mathbf{P 0}} \stackrel{k_{\text {alkyl1 }}}{\longrightarrow} \mathbf{E}^{\mathbf{P 0}}+\mathrm{BzMgCl} \\
& \mathbf{E}^{\mathbf{P 0}}+\mathrm{BzMgBz} \stackrel{k_{\mathrm{ex} 2}}{\longrightarrow} \mathbf{F}^{\mathbf{P 0}} \\
& \mathbf{F}^{\mathbf{P 0}} \stackrel{k_{\text {act2 }}}{\longrightarrow} \mathbf{E}^{\mathbf{P 0}}+\mathrm{BzMgBz} \\
& \mathbf{A}+\mathrm{BzMgP}_{\mathrm{s}} \stackrel{k_{\text {ex0 }}}{\longrightarrow} \mathbf{B}^{\mathbf{P 1}}{ }_{\mathbf{s}}
\end{aligned}
$$




$$
\begin{aligned}
& \mathbf{B}^{\mathbf{P 1}}{ }_{\mathbf{s}} \stackrel{k_{\text {act0 }}}{\longrightarrow} \mathbf{A}+\mathrm{BzMgP}_{\mathrm{s}} \\
& \mathbf{B}^{\mathbf{P} 1}{ }_{\mathbf{s}} \stackrel{k_{\text {alkyl1 }}}{\longrightarrow} \mathbf{C}^{\mathbf{P}}{ }_{\mathbf{s}}+\mathrm{BzMgCl} \\
& \mathbf{B}^{\mathbf{P 1}} \stackrel{k_{\text {alkyl1 }}}{\longrightarrow} \mathrm{C}+\mathrm{PMgCl}_{\mathrm{s}} \\
& \mathbf{A}+\mathrm{PMgP}_{\mathrm{s}} \stackrel{k_{\mathrm{ex} 0}}{\longrightarrow} \mathbf{B}^{\mathbf{P} 2}{ }_{\mathbf{s}}+\text { help1 } \\
& \mathrm{PMgP}_{\mathrm{s}}+\text { help1 } \stackrel{k_{\text {help }}}{\longrightarrow} \mathbf{B}^{\mathbf{P 2}} \mathbf{s} \\
& \mathbf{B}^{\mathbf{P 2}}{ }_{\mathbf{s}} \stackrel{\frac{1}{2} k_{\mathrm{alkyl}}}{\longrightarrow} \mathbf{C}^{\mathbf{P}}{ }_{\mathbf{s}}+\text { help2 } \\
& \mathbf{B}^{\mathbf{P} 2}{ }_{\mathbf{s}}+\text { help2 } \stackrel{k_{\text {help }}}{\longrightarrow} \mathrm{PMgCl}_{\mathrm{s}} \\
& \mathbf{B}_{\mathbf{s}}^{\mathbf{P 2}} \stackrel{\frac{1}{2} k_{\text {act0 }}}{\longrightarrow} \mathrm{PMgP}_{\mathrm{s}}+\text { help3 } \\
& \mathbf{B}^{\mathbf{P 2}}{ }_{\mathbf{s}}+\text { help3 } \stackrel{k_{\text {help }}}{\longrightarrow} \mathrm{PMgP}_{\mathrm{s}}+\mathbf{A} \\
& \mathrm{C}+\mathrm{M} \stackrel{k_{\text {add }}}{\longrightarrow} \mathbf{C}(\mathbf{M}) \\
& \text { C(M) } \stackrel{k_{\text {frag }}}{\longrightarrow} \mathrm{C}+\mathrm{M} \\
& \mathbf{C}(\mathbf{M}) \stackrel{k_{\mathrm{p} 1}}{\longrightarrow} \mathrm{C}^{\mathbf{P}}{ }_{\mathbf{1}} \\
& \mathrm{C}^{\mathrm{P}}{ }_{\mathrm{s}}+\mathrm{M} \stackrel{k_{\text {add }}}{\longrightarrow} \mathrm{C}(\mathbf{M})_{\mathrm{s}+1} \\
& \mathbf{C}^{\mathbf{P}}(\mathbf{M})_{\mathbf{s}} \stackrel{k_{\text {frag }}}{\longrightarrow} \mathbf{C}^{\mathbf{P}}{ }_{\mathrm{s}-1}+\mathrm{M} \\
& \mathbf{C}^{\mathbf{P}}(\mathbf{M})_{\mathrm{s}} \stackrel{k_{\mathrm{p}}}{\longrightarrow} \mathbf{C}_{\mathrm{s}}^{\mathbf{P}} \\
& \mathbf{C}^{\mathbf{P}}{ }_{\mathbf{s}}+\mathrm{BzMgBz} \stackrel{k_{\mathrm{ex} 2}}{\longrightarrow} \mathbf{D}^{\mathbf{P} 1}{ }_{\mathbf{s}} \\
& \mathrm{C}+\mathrm{BzMgP}_{\mathrm{s}} \stackrel{k_{\mathrm{ex} 2}}{\longrightarrow} \mathbf{D}^{\mathrm{P} 1}{ }_{\mathbf{s}} \\
& \mathbf{D}^{\mathbf{P 1}}{ }_{\mathbf{s}} \stackrel{\frac{2}{3} k_{\mathrm{act} 2}}{\longrightarrow} \mathrm{C}+\mathrm{BzMgP}_{\mathrm{s}} \\
& \mathbf{D}^{\mathbf{P 1}} \stackrel{\frac{1}{3} k_{\mathrm{act} 2}}{\longrightarrow} \mathbf{C}^{\mathbf{P}}{ }_{\mathbf{s}}+\mathrm{BzMgBz} \\
& \mathbf{C}^{\mathbf{P}}{ }_{\mathbf{s}}+\mathrm{BzMgP}_{\mathrm{r}} \stackrel{k_{\text {ex2 }}}{\longrightarrow} \mathbf{D}^{\mathbf{P} \mathbf{2}} \mathbf{s}+\mathbf{D}^{\mathbf{P} \mathbf{2}}{ }_{\mathbf{r}} \\
& \mathrm{C}+\mathrm{PMgP}_{\mathrm{s}} \stackrel{k_{\text {act2 }}}{\longrightarrow} \text { help }_{\mathrm{s}} \\
& \text { help } 4+\mathrm{PMgP}_{\mathrm{r}} \stackrel{k_{\text {help }}}{\longrightarrow} \mathbf{D}^{\mathbf{P 1}}{ }_{\mathbf{s}}+\mathbf{D}^{\mathbf{P 1}}{ }_{\mathbf{r}} \\
& \mathbf{D}^{\mathbf{P 2}}{ }_{\mathbf{s}} \stackrel{\frac{1}{2} k_{\text {act2 }}}{\longrightarrow} \mathbf{C}^{\mathbf{P}}{ }_{\mathbf{s}}+\text { help5 }
\end{aligned}
$$




$$
\begin{aligned}
& \mathrm{D}^{\mathrm{P} 2} \mathrm{~s}+\text { help5 } \stackrel{k_{\text {help }}}{\longrightarrow} \mathrm{BzMgP}_{\mathrm{s}} \\
& \mathbf{D}_{\mathbf{s}}^{\mathbf{P 2}} \stackrel{\frac{1}{6} k_{\text {act2 }}}{\longrightarrow} \mathrm{PMgP}_{\mathrm{s}}+\text { help6 } \\
& \mathbf{D}^{\mathbf{P 2}}{ }_{\mathbf{s}}+\text { help } 6 \stackrel{k_{\text {help }}}{\longrightarrow} \mathrm{PMgP}_{\mathrm{s}}+\mathbf{C} \\
& \mathbf{C}^{\mathbf{P}}{ }_{\mathrm{s}}+\mathrm{PMgP}_{\mathrm{r}} \stackrel{\frac{1}{2} k_{\mathrm{ex} 2}}{\longrightarrow} \mathbf{D}^{\mathbf{P} 3}{ }_{\mathbf{s}}+\text { help } 7_{\mathrm{r}} \\
& \text { help } 7_{\mathrm{r}}+\mathrm{PMgP}_{\mathrm{r}} \stackrel{k_{\text {help }}}{\longrightarrow} \mathbf{D}^{\mathrm{P} 3}{ }_{\mathbf{s}}+\mathbf{D}^{\mathrm{P} 3}{ }_{\mathrm{r}} \\
& \mathbf{D}^{\mathrm{P} 3} \mathrm{~s}_{\mathrm{s}} \stackrel{\frac{1}{3} k_{\mathrm{act} 2}}{\longrightarrow} \mathrm{PMgP}_{\mathrm{s}}+\text { help8 } \\
& \mathbf{D}^{\mathbf{P} 3}{ }_{\mathbf{s}}+\text { help } 8 \stackrel{k_{\text {help }}}{\longrightarrow} \mathrm{PMgP}_{\mathrm{s}}+\text { help9 } \\
& \mathbf{D}^{\mathrm{P} 3}{ }_{\mathbf{s}}+\text { help9 } \stackrel{k_{\text {help }}}{\longrightarrow} \mathbf{C}^{\mathbf{P}}{ }_{\mathbf{s}} \\
& \mathbf{C}^{\mathbf{P}} \stackrel{k_{\mathrm{t}}}{\longrightarrow} \mathbf{G}+\mathrm{P}_{\text {Dead, } \mathrm{s}} \\
& \mathbf{D}^{\mathrm{P0}} \stackrel{k_{\text {alkyl2 }}}{\longrightarrow} \mathbf{E}^{\mathrm{P0}}+\mathrm{BzMgCl} \\
& \mathbf{D}^{\mathbf{P} 1}{ }_{\mathbf{s}} \stackrel{\frac{1}{3} k_{\mathrm{alkyl}}}{\longrightarrow} \mathbf{E}^{\mathbf{P 0}}+\mathrm{PMgCl}_{\mathrm{s}} \\
& \mathbf{D}^{\mathbf{P 1}}{ }_{\mathbf{s}} \stackrel{\frac{2}{3} k_{\mathrm{alkyl}}}{\longrightarrow} \mathbf{E}^{\mathbf{P 1}}{ }_{\mathbf{s}}+\mathrm{BzMgCl} \\
& \mathbf{D}^{\mathbf{P 2}}{ }_{\mathbf{s}} \stackrel{\frac{1}{6} k_{\mathrm{alky} 13}}{\longrightarrow} \mathbf{E}^{\mathbf{P 2}}+\text { help10 } \\
& \mathbf{D}^{\mathbf{P} 2}{ }_{\mathbf{s}}+\text { help10 } \stackrel{k_{\text {help }}}{\longrightarrow} \mathbf{E}^{\mathbf{P 2}}+\mathrm{BzMgCl} \\
& \mathbf{D}^{\mathbf{P 2}}{ }_{\mathrm{s}} \stackrel{\frac{1}{3} k_{\mathrm{alky13}}}{\longrightarrow} \mathbf{E}^{\mathbf{P 1}}{ }_{\mathrm{s}}+\text { help11 } \\
& \mathbf{D}^{\mathbf{P 2}}{ }_{\mathbf{s}}+\text { help11 } \stackrel{k_{\text {help }}}{\longrightarrow} \mathrm{PMgCl}_{\mathrm{s}} \\
& \mathbf{D}^{\mathbf{P} 3}{ }_{\mathbf{s}} \stackrel{\frac{1}{3} k_{\text {alkyl2 }}}{\longrightarrow} \mathbf{E}^{\mathbf{P 2}}+\text { help12 } \\
& \mathbf{D}^{\mathbf{P 3}}{ }_{\mathbf{s}}+\text { help12 } \stackrel{k_{\text {help }}}{\longrightarrow} \mathbf{E}^{\mathbf{P 2}}+\text { help13 } \\
& \mathbf{D}^{\mathbf{P} 3}{ }_{\mathbf{s}}+\text { help13 } \stackrel{k_{\text {help }}}{\longrightarrow} \mathrm{PMgCl}_{\mathbf{s}} \\
& \mathbf{E}^{\mathbf{P 0}}+\mathrm{BzMgBz} \stackrel{k_{\mathrm{ex} 3}}{\longrightarrow} \mathbf{F}^{\mathbf{P 0}} \\
& \mathbf{F}^{\mathbf{P 0}} \stackrel{k_{\text {act2 }}}{\longrightarrow} \mathbf{E}^{\mathbf{P 0}}+\mathrm{BzMgBz} \\
& \mathbf{E}^{\mathbf{P} 1}{ }_{\mathbf{s}}+\mathrm{BzMgBz} \stackrel{k_{\mathrm{ex} 3}}{\longrightarrow} \mathbf{F}^{\mathbf{P 1}} \\
& \mathbf{E}^{\mathbf{P 0}}+\mathrm{BzMgP}_{\mathrm{s}} \stackrel{k_{\mathrm{ex} 3}}{\longrightarrow} \mathbf{E}^{\mathbf{P 1}}
\end{aligned}
$$




$$
\begin{aligned}
& \mathbf{F}^{\mathbf{P 1}} \stackrel{k_{\text {act3 }}}{\longrightarrow} \mathbf{E}^{\mathbf{P 1}}{ }_{\mathbf{s}}+\mathrm{BzMgBz} \\
& \mathbf{F}^{\mathbf{P 1}} \stackrel{k_{\text {act3 }}}{\longrightarrow} \mathbf{E}^{\mathbf{P 0}}+\mathrm{BzMgP}_{\mathrm{s}} \\
& \mathbf{E}^{\mathbf{P 1}}{ }_{\mathbf{s}}+\mathrm{BzMgP}_{\mathrm{r}} \stackrel{k_{\mathrm{ex} 3}}{\longrightarrow} \mathbf{F}^{\mathbf{P} 2}{ }_{\mathbf{s}}+\mathbf{F}^{\mathbf{P} 2}{ }_{\mathbf{r}} \\
& \mathbf{E}^{\mathbf{P 0}}+\mathrm{PMgP}_{\mathrm{s}} \stackrel{\frac{1}{2} k_{\mathrm{e} \times 3}}{\longrightarrow} \mathbf{F}^{\mathbf{P 2}}{ }_{\mathbf{s}}+\text { help14 } \\
& \text { help14 }+\mathrm{PMgP}_{\mathrm{s}} \stackrel{k_{\text {help }}}{\longrightarrow} \mathbf{F}^{\mathbf{P 2}} \mathbf{s} \\
& \mathbf{E}^{\mathbf{P 2}}+\mathrm{BzMgBz} \stackrel{\frac{1}{2} k_{\mathrm{ex} 3}}{\longrightarrow} \mathbf{F}^{\mathbf{P 2}}{ }_{\mathbf{s}}+\text { help15 } \\
& h e l p 15+\mathbf{E}^{\mathbf{P 2}} \stackrel{k_{\text {help }}}{\longrightarrow} \mathbf{F}^{\mathbf{P 2}} \mathbf{s} \\
& \mathbf{F}^{\mathbf{P 2}} \stackrel{\frac{1}{4} k_{\text {act3 }}}{\longrightarrow} \mathbf{E}^{\mathbf{P 1}}{ }_{\mathbf{s}}+\text { help16 } \\
& \mathbf{F}^{\mathbf{P 2}} \mathbf{s}+\text { help16 } \stackrel{k_{\text {help }}}{\longrightarrow} \text { BzMgP }_{\mathbf{s}} \\
& \mathbf{F}^{\mathbf{P 2}} \stackrel{\frac{1}{8} k_{\text {act3 }}}{\longrightarrow} \mathrm{PMgP}_{\mathrm{s}}+\text { help17 } \\
& \mathbf{F}^{\mathbf{P 2}}{ }_{\mathbf{s}}+\text { help17 } \stackrel{k_{\text {help }}}{\longrightarrow} \mathrm{PMgP}_{\mathrm{s}}+\mathbf{F}^{\mathbf{P 0}} \\
& \mathbf{F}^{\mathbf{P 2}} \stackrel{\frac{1}{8} k_{\text {act3 }}}{\longrightarrow} \mathbf{E}^{\mathbf{P 2}}{ }_{\mathbf{s}}+\text { help18 } \\
& \mathbf{F}^{\mathbf{P} 2}{ }_{\mathbf{s}}+\text { help18 } \stackrel{k_{\text {help }}}{\longrightarrow} \mathbf{E}^{\mathbf{P 2}}{ }_{\mathbf{s}}+\mathrm{BzMgBz} \\
& \mathbf{E}^{\mathbf{P 1}}{ }_{\mathbf{s}}+\mathrm{PMgP}_{\mathrm{r}} \stackrel{k_{\mathrm{ex} 3}}{\longrightarrow} \mathbf{F}^{\mathbf{P 3}}{ }_{\mathbf{s}}+\text { help19 }{ }_{\mathrm{r}} \\
& \mathrm{PMgP}_{\mathrm{s}}+\text { help19 }{ }_{\mathrm{r}} \stackrel{k_{\text {help }}}{\longrightarrow} \mathbf{F}^{\mathbf{P} 3}{ }_{\mathbf{s}}+\mathbf{F}^{\mathbf{P} 3}{ }_{\mathbf{r}} \\
& \mathbf{E}^{\mathbf{P 2}}{ }_{\mathbf{s}}+\mathrm{BzMgP}_{\mathrm{r}} \stackrel{k_{\mathrm{ex} 3}}{\longrightarrow} \mathbf{F}^{\mathbf{P} 3}{ }_{\mathbf{s}}+\text { help20 } \mathrm{r}_{\mathrm{r}} \\
& \mathbf{E}^{\mathbf{P 2}}{ }_{\mathbf{s}}+\text { help } 20_{\mathrm{r}} \stackrel{k_{\text {help }}}{\longrightarrow} \mathbf{F}^{\mathbf{P 3}}{ }_{\mathbf{s}}+\mathbf{F}^{\mathbf{P} 3}{ }_{\mathbf{r}}
\end{aligned}
$$

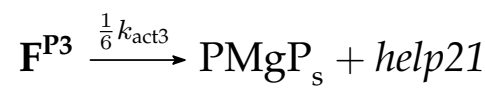

$$
\begin{aligned}
& \mathbf{F}^{\mathbf{P} 3}{ }_{\mathbf{s}}+\text { help } 21 \stackrel{k_{\text {help }}}{\longrightarrow} \mathbf{E}^{\mathbf{P 1}}{ }_{\mathbf{s}}+\text { help22 } \\
& \mathbf{F}^{\mathbf{P 3}}{ }_{\mathbf{s}}+\text { help22 } \stackrel{k_{\text {help }}}{\longrightarrow} \mathrm{PMgP}_{\mathrm{s}} \\
& \mathbf{F}^{\mathbf{P 3}} \stackrel{\frac{1}{6} k_{\text {act3 }}}{\longrightarrow} \mathbf{E}^{\mathbf{P 2}}{ }_{\mathbf{s}}+\text { help23 } \\
& \mathbf{F}^{\mathbf{P 3}}{ }_{\mathbf{s}}+\text { help23 } \stackrel{k_{\text {help }}}{\longrightarrow} \mathbf{E}^{\mathbf{P 2}}{ }_{\mathbf{s}}+\text { help } 24 \\
& \mathbf{F}^{\mathbf{P 3}}{ }_{\mathrm{s}}+\text { help } 24 \stackrel{k_{\text {help }}}{\longrightarrow} \mathrm{BzMgP}_{\mathrm{s}}
\end{aligned}
$$




$$
\begin{aligned}
& \mathbf{E}^{\mathbf{P 2}}{ }_{\mathbf{s}}+\mathrm{PMgP}_{\mathrm{r}} \stackrel{\frac{1}{2} k_{\mathrm{ex} 3}}{\longrightarrow} \mathbf{F}^{\mathbf{P 4}}{ }_{\mathbf{s}}+\mathbf{F}^{\mathbf{P 4}}{ }_{\mathbf{r}} \\
& \mathbf{F}_{\mathbf{s}}^{\mathbf{P 4}} \stackrel{\frac{1}{2} k_{\text {act3 }}}{\longrightarrow} \mathrm{PMgP}_{\mathrm{s}}+\text { help } 25 \\
& \mathbf{F}^{\mathbf{P 4}}{ }_{\mathbf{s}}+\text { help } 25 \stackrel{k_{\text {help }}}{\longrightarrow} \mathbf{E}^{\mathbf{P 2}} \text { s } \\
& \mathbf{C} \stackrel{k_{\mathrm{t}, \mathrm{alkyl}}}{\longrightarrow} \mathbf{G} \\
& \mathbf{G}+\text { Monomer } \stackrel{k_{\text {reini }}}{\longrightarrow} \mathbf{C}^{\mathbf{P}} \\
& \mathbf{E}^{\mathbf{P 0}}+\mathrm{BuMgCl} \stackrel{k_{\text {react }}}{\longrightarrow} \mathbf{C}+\mathrm{BuMgBu} \\
& \mathbf{E}^{\mathbf{P 0}}+\mathrm{PMgCl}_{s} \stackrel{k_{\text {react }}}{\longrightarrow} \mathbf{C}+\mathrm{BuMgP}_{s} \\
& \mathbf{E}^{\mathbf{P 1}}+\mathrm{PMgCl}_{s} \stackrel{k_{\text {react }}}{\longrightarrow} \mathbf{C}^{\mathbf{P}}+\mathrm{BuMgP}_{s} \\
& \mathbf{E}^{\mathbf{P 1}}+\mathrm{BuMgCl} \stackrel{k_{\text {react }}}{\longrightarrow} \mathbf{C}^{\mathbf{P}}+\mathrm{BuMgBu}
\end{aligned}
$$

\section{A.3.4 Polymerization of Styrene with I and BzMgBz}

$$
\begin{aligned}
& \mathbf{J}+\mathrm{BzMgBz} \stackrel{k_{\mathrm{ex} 0}}{\longrightarrow} \mathbf{K}^{\mathbf{P 0}} \\
& \mathbf{K}^{\mathbf{P 0}} \stackrel{k_{\text {act0 }}}{\longrightarrow} \mathbf{J}+\mathrm{BzMgBz} \\
& \mathbf{K}^{\mathbf{P 0}} \stackrel{k_{\text {alkyl }}}{\longrightarrow} \mathbf{L}+\mathrm{BzMgCl} \\
& \mathbf{L}+\mathrm{BzMgBz} \stackrel{k_{\mathrm{ex} 1}}{\longrightarrow} \mathbf{K}^{\mathbf{P} 0} \\
& \mathbf{K}^{\mathrm{P0}} \stackrel{k_{\text {act1 }}}{\longrightarrow} \mathbf{1}+\mathrm{BzMgBz} \\
& \mathbf{J}+\mathrm{BzMgP}_{\mathrm{s}} \stackrel{k_{\mathrm{ex0}}}{\longrightarrow} \mathbf{K}^{\mathbf{P} \mathbf{1}}{ }_{\mathbf{s}} \\
& \mathbf{K}^{\mathbf{P 1}}{ }_{\mathbf{s}} \stackrel{k_{\text {act0 }}}{\longrightarrow} \mathbf{J}+\mathrm{BzMgP}_{\mathrm{s}} \\
& \mathbf{K}^{\mathbf{P 1}}{ }_{\mathbf{s}} \stackrel{k_{\text {alkyl1 }}}{\longrightarrow} \mathbf{L}_{\mathbf{s}}^{\mathbf{P}}+\mathrm{BzMgCl} \\
& \mathbf{K}^{\mathbf{P 1}} \stackrel{k_{\text {alkyl1 }}}{\longrightarrow} \mathbf{L}+\mathrm{PMgCl}_{\mathrm{s}} \\
& \mathbf{J}+\mathrm{PMgP}_{\mathrm{s}} \stackrel{k_{\mathrm{ex} 0}}{\longrightarrow} \mathbf{K}^{\mathbf{P} 2} \mathbf{s}+\text { help1 } \\
& \mathrm{PMgP}_{\mathrm{s}}+\text { help } 1 \stackrel{k_{\text {help }}}{\longrightarrow} \mathbf{K}^{\mathbf{P} 2} \mathbf{s} \\
& \mathbf{K}^{\mathbf{P 2}}{ }_{\mathbf{s}} \stackrel{\frac{1}{2} k_{\text {alkyl }}}{\longrightarrow} \mathbf{L}^{\mathbf{P}}{ }_{\mathbf{s}}+\text { help2 }
\end{aligned}
$$




$$
\begin{aligned}
& \mathbf{K}^{\mathbf{P} 2}{ }_{\mathbf{s}}+\text { help } 2 \stackrel{k_{\text {help }}}{\longrightarrow} \mathrm{PMgCl}_{\mathrm{s}} \\
& \mathbf{K}_{\mathbf{s}}^{\mathbf{P 2}} \stackrel{\frac{1}{2} k_{\text {act0 }}}{\longrightarrow} \mathrm{PMgP}_{\mathrm{s}}+\text { help3 } \\
& \mathbf{K}^{\mathbf{P 2}} \mathbf{s}+\text { help } 3 \stackrel{k_{\text {help }}}{\longrightarrow} \operatorname{PMgP}_{\mathbf{s}}+\mathbf{J} \\
& \mathbf{L}+\mathbf{M} \stackrel{k_{\text {add }}}{\longrightarrow} \mathbf{L}(\mathbf{M}) \\
& \mathbf{L}(\mathbf{M}) \stackrel{k_{\text {frag }}}{\longrightarrow} \mathbf{L}+\mathbf{M} \\
& \mathbf{L}(\mathbf{M}) \stackrel{k_{\mathrm{p} 1}}{\longrightarrow} \mathbf{L}_{\mathbf{1}}^{\mathbf{P}} \\
& \mathbf{L}_{\mathbf{s}}^{\mathbf{P}}+\mathbf{M} \stackrel{k_{\text {add }}}{\longrightarrow} \mathbf{L}(\mathbf{M})_{\mathbf{s}+\mathbf{1}} \\
& \mathbf{L}^{\mathbf{P}}(\mathbf{M})_{\mathbf{s}} \stackrel{k_{\text {frag }}}{\longrightarrow} \mathbf{L}_{s-1}^{\mathbf{P}}+\mathbf{M} \\
& \mathbf{L}^{\mathbf{P}}(\mathbf{M})_{\mathrm{s}} \stackrel{k_{\mathrm{p}}}{\longrightarrow} \mathbf{L}_{\mathrm{s}}^{\mathbf{P}} \\
& \mathbf{L}^{\mathbf{P}}{ }_{\mathbf{s}}+\mathrm{BzMgBz} \stackrel{k_{\mathrm{ex} 2}}{\longrightarrow} \mathbf{K}^{\mathbf{P 1}}{ }_{\mathbf{s}} \\
& \mathbf{L}+\mathrm{BzMgP}_{\mathrm{s}} \stackrel{k_{\mathrm{ex} 2}}{\longrightarrow} \mathbf{K}^{\mathrm{P} 1}{ }_{\mathbf{s}} \\
& \mathbf{K}^{\mathbf{P} 1}{ }_{\mathbf{s}} \stackrel{\frac{2}{3} k_{\mathrm{act} 2}}{\longrightarrow} \mathbf{L}+\mathrm{BzMgP}_{\mathrm{s}} \\
& \mathbf{K}^{\mathbf{P 1}} \stackrel{\frac{1}{3} k_{\text {act2 }}}{\longrightarrow} \mathbf{L}_{\mathbf{s}}^{\mathbf{P}}+\mathrm{BzMgBz} \\
& \mathbf{L}^{\mathbf{P}}{ }_{\mathbf{s}}+\mathrm{BzMgP}_{\mathrm{r}} \stackrel{k_{\text {ex2 }}}{\longrightarrow} \mathbf{K}^{\mathbf{P 2}}{ }_{\mathbf{s}}+\mathbf{K}^{\mathbf{P} \mathbf{2}} \mathbf{r} \\
& \mathbf{L}+\operatorname{PMgP}_{\mathrm{s}} \stackrel{k_{\text {act2 }}}{\longrightarrow} h \operatorname{ll} 4_{\mathrm{s}} \\
& h e l p 4+\operatorname{PMgP}_{\mathbf{r}} \stackrel{k_{\text {help }}}{\longrightarrow} \mathbf{K}^{\mathbf{P 1}}{ }_{\mathbf{s}}+\mathbf{K}^{\mathbf{P 1}}{ }_{\mathbf{r}} \\
& \mathbf{K}^{\mathbf{P 2}}{ }_{\mathbf{s}} \stackrel{\frac{1}{2} k_{\text {act2 }}}{\longrightarrow} \mathbf{L}_{\mathbf{s}}^{\mathbf{P}}+\text { help5 } \\
& \mathrm{K}^{\mathrm{P} 2} \mathrm{~S}+\text { help } 5 \stackrel{k_{\text {help }}}{\longrightarrow} \mathrm{BzMgP}_{\mathrm{s}} \\
& \mathbf{K}_{\mathbf{s}}^{\mathbf{P 2}} \stackrel{\frac{1}{6} k_{\text {act2 }}}{\longrightarrow} \mathrm{PMgP}_{\mathrm{s}}+\text { help6 } \\
& \mathbf{K}^{\mathbf{P} 2} \mathbf{s}+\text { help } 6 \stackrel{k_{\text {help }}}{\longrightarrow} \mathrm{PMgP}_{\mathrm{s}}+\mathbf{L} \\
& \mathbf{L}_{\mathbf{s}}^{\mathbf{P}}+\mathrm{PMgP}_{\mathrm{r}} \stackrel{\frac{1}{2} k_{\text {ex2 }}}{\longrightarrow} \mathbf{K}^{\mathbf{P} 3}{ }_{\mathbf{s}}+\text { help } 7_{\mathbf{r}} \\
& \text { help } 7_{\mathrm{r}}+\mathrm{PMgP}_{\mathrm{r}} \stackrel{k_{\text {help }}}{\longrightarrow} \mathbf{K}^{\mathbf{P} 3}{ }_{\mathbf{s}}+\mathbf{K}^{\mathbf{P} 3}{ }_{\mathbf{r}} \\
& \mathbf{K}_{\mathbf{s}}^{\mathbf{P 3}} \stackrel{\frac{1}{3} k_{\text {act2 }}}{\longrightarrow} \mathrm{PMgP}_{\mathbf{s}}+\text { help8 }
\end{aligned}
$$




$$
\begin{aligned}
& \mathbf{K}^{\mathrm{P} 3}{ }_{\mathbf{s}}+\text { help } 8 \stackrel{k_{\text {help }}}{\longrightarrow} \mathrm{PMgP}_{\mathrm{s}}+\text { help } 9 \\
& \mathbf{K}^{\mathbf{P} 3}{ }_{\mathbf{s}}+h e l p 9 \stackrel{k_{\text {help }}}{\longrightarrow} \mathbf{L}^{\mathbf{P}}{ }_{\mathbf{s}} \\
& \mathbf{L}_{\mathbf{s}}^{\mathbf{P}} \stackrel{k_{\mathrm{t}}}{\longrightarrow} \mathbf{N}+\mathrm{P}_{\text {Dead, }} \\
& \mathbf{N}+\mathrm{M} \stackrel{k_{\text {reini }}}{\longrightarrow} \mathbf{L}_{\mathbf{1}} \\
& \mathrm{BzMgCl}+\mathrm{BzMgCl} \stackrel{k_{\text {schlenk }}}{\longrightarrow} \mathrm{BzMgBz}+\mathrm{ClMgCl} \\
& \mathrm{PMgCl}_{\mathrm{s}}+\mathrm{BzMgCl} \stackrel{k_{\text {schlenk }}}{\longrightarrow} \mathrm{BzMgP}_{\mathrm{s}}+\mathrm{CIMgCl} \\
& \mathrm{PMgCl}_{\mathrm{s}}+\mathrm{PMgCl}_{\mathrm{r}} \stackrel{k_{\text {schlenk }}}{\longrightarrow} \mathrm{PMgP}_{\mathrm{s}}+\text { help } 26_{\mathrm{r}} \\
& \text { help } 26_{\mathrm{s}} \stackrel{k_{\text {help }}}{\longrightarrow} \mathrm{PMgP}_{\mathrm{s}}+\mathrm{CIMgCl}
\end{aligned}
$$

\section{A.3.5 Model for Polymerization of I and BuMgOct in the Reactor}

$$
\begin{aligned}
& \mathbf{J}+\mathrm{BzMgBz} \stackrel{k_{\mathrm{ex} 0}}{\longrightarrow} \mathbf{K}^{\mathrm{P0}} \\
& \mathbf{K}^{\mathbf{P 0}} \stackrel{k_{\text {act0 }}}{\longrightarrow} \mathbf{J}+\mathrm{BzMgBz} \\
& \mathbf{K}^{\mathbf{P 0}} \stackrel{k_{\text {alkyl1 }}}{\longrightarrow} \mathbf{L}+\mathrm{BzMgCl} \\
& \mathbf{L}+\mathrm{BzMgBz} \stackrel{k_{\mathrm{ex} 1}}{\longrightarrow} \mathbf{K}^{\mathrm{P} \mathbf{0}} \\
& \mathbf{K}^{\mathbf{P 0}} \stackrel{k_{\text {act1 }}}{\longrightarrow} \mathbf{1}+\mathrm{BzMgBz} \\
& \mathbf{J}+\mathrm{BzMgP}_{\mathrm{s}} \stackrel{k_{\mathrm{ex0}}}{\longrightarrow} \mathbf{K}^{\mathbf{P} \mathbf{1}} \mathbf{s} \\
& \mathbf{K}^{\mathbf{P 1}}{ }_{\mathbf{s}} \stackrel{k_{\text {act0 }}}{\longrightarrow} \mathbf{J}+\mathrm{BzMgP}_{\mathrm{s}} \\
& \mathbf{K}^{\mathbf{P 1}}{ }_{\mathbf{s}} \stackrel{k_{\text {alkyl1 }}}{\longrightarrow} \mathbf{L}^{\mathbf{P}}{ }_{\mathbf{s}}+\mathrm{BzMgCl} \\
& \mathbf{K}^{\mathbf{P 1}} \stackrel{k_{\text {alkyl1 }}}{\longrightarrow} \mathbf{L}+\mathrm{PMgCl}_{\mathrm{s}} \\
& \mathbf{J}+\mathrm{PMgP}_{\mathrm{s}} \stackrel{k_{\text {ex0 }}}{\longrightarrow} \mathbf{K}^{\mathbf{P} \mathbf{2}} \mathbf{s}+\text { help1 } \\
& \mathrm{PMgP}_{\mathrm{s}}+\text { help1 } \stackrel{k_{\text {help }}}{\longrightarrow} \mathbf{K}^{\mathbf{P 2}}{ }_{\mathbf{s}} \\
& \mathbf{K}^{\mathbf{P 2}}{ }_{\mathbf{s}} \stackrel{\frac{1}{2} k_{\text {alkyl1 }}}{\longrightarrow} \mathbf{L}_{\mathbf{s}}^{\mathbf{P}}+\text { help2 } \\
& \mathbf{K}^{\mathbf{P 2}} \mathbf{s}+\text { help } 2 \stackrel{k_{\text {help }}}{\longrightarrow} \mathrm{PMgCl}_{\mathrm{s}}
\end{aligned}
$$




$$
\begin{aligned}
& \mathbf{K}_{\mathbf{s}}^{\mathbf{P 2}} \stackrel{\frac{1}{2} k_{\text {act0 }}}{\longrightarrow} \mathrm{PMgP}_{\mathrm{s}}+\text { help3 } \\
& \mathbf{K}^{\mathbf{P 2}} \mathbf{s}+\text { help } 3 \stackrel{k_{\text {help }}}{\longrightarrow} \operatorname{PMgP}_{\mathrm{s}}+\mathbf{J} \\
& \mathbf{L}+\mathbf{M} \stackrel{k_{\text {add }}}{\longrightarrow} \mathbf{L}(\mathbf{M}) \\
& \mathbf{L}(\mathbf{M}) \stackrel{k_{\text {frag }}}{\longrightarrow} \mathbf{L}+\mathbf{M} \\
& \mathbf{L}(\mathbf{M})_{\mathrm{s}} \stackrel{k_{p 1}}{\longrightarrow} \mathbf{L}_{\mathbf{1}}^{\mathbf{P}}+\mathbf{M} \text {. } \\
& \mathbf{L}_{\mathbf{s}}^{\mathbf{P}}+\mathbf{M} \stackrel{k_{\text {add }}}{\longrightarrow} \mathbf{L}(\mathbf{M})_{\mathbf{s}+\mathbf{1}} \\
& \mathbf{L}^{\mathbf{P}}(\mathbf{M})_{\mathrm{s}} \stackrel{k_{\text {frag }}}{\longrightarrow} \mathbf{L}_{\mathrm{s}-1}^{\mathbf{P}}+\mathbf{M} \\
& \mathbf{L}^{\mathbf{P}}(\mathbf{M})_{\mathbf{S}} \stackrel{k_{p}}{\longrightarrow} \mathbf{L}_{\mathbf{s}}^{\mathbf{P}}+\mathbf{M} \text {. } \\
& \mathbf{L}^{\mathbf{P}}{ }_{\mathbf{s}}+\mathrm{BzMgBz} \stackrel{k_{\mathrm{ex} 2}}{\longrightarrow} \mathbf{K}^{\mathbf{P 1}}{ }_{\mathbf{s}} \\
& \mathrm{M} * \text { Storage }_{(\mathrm{n})} \stackrel{k_{p}}{\longrightarrow} \text { Storage }_{(\mathrm{n}-1)}+\mathrm{M} \\
& \mathbf{L}+\mathrm{BzMgP}_{\mathrm{s}} \stackrel{k_{\mathrm{ex} 2}}{\longrightarrow} \mathbf{K}^{\mathbf{P} 1}{ }_{\mathbf{s}} \\
& \mathbf{K}_{\mathbf{s}}^{\mathbf{P} 1} \stackrel{\frac{2}{3} k_{\mathrm{act} 2}}{\longrightarrow} \mathbf{L}+\mathrm{BzMgP}_{\mathbf{s}} \\
& \mathbf{K}^{\mathbf{P 1}} \stackrel{\frac{1}{3} k_{\text {act2 }}}{\longrightarrow} \mathbf{L}_{\mathbf{s}}^{\mathbf{P}}+\mathrm{BzMgBz} \\
& \mathbf{L}^{\mathbf{P}}{ }_{\mathbf{s}}+\mathrm{BzMgP}_{\mathrm{r}} \stackrel{k_{\mathrm{ex} 2}}{\longrightarrow} \mathbf{K}^{\mathbf{P} \mathbf{2}}{ }_{\mathbf{s}}+\mathbf{K}^{\mathbf{P} \mathbf{2}} \mathbf{r} \\
& \mathbf{L}+\mathrm{PMgP}_{\mathrm{s}} \stackrel{k_{\text {act2 }}}{\longrightarrow} \text { help }_{\mathrm{s}} \\
& \text { help } 4+\mathrm{PMgP}_{\mathrm{r}} \stackrel{k_{\text {help }}}{\longrightarrow} \mathbf{K}^{\mathbf{P 1}}{ }_{\mathbf{s}}+\mathbf{K}^{\mathbf{P} \mathbf{1}} \mathbf{r} \\
& \mathbf{K}^{\mathbf{P 2}}{ }_{\mathbf{s}} \stackrel{\frac{1}{2} k_{\mathrm{act} 2}}{\longrightarrow} \mathbf{L}_{\mathbf{s}}^{\mathbf{P}}+\text { help5 } \\
& \mathrm{K}^{\mathrm{P} 2} \mathrm{~s}+\text { help } 5 \stackrel{k_{\text {help }}}{\longrightarrow} \mathrm{BzMgP}_{\mathrm{s}} \\
& \mathbf{K}_{\mathbf{s}}^{\mathbf{P 2}} \stackrel{\frac{1}{6} k_{\text {act2 }}}{\longrightarrow} \mathrm{PMgP}_{\mathrm{s}}+\text { help6 } \\
& \mathbf{K}^{\mathbf{P 2}}{ }_{\mathbf{s}}+\text { help } 6 \stackrel{k_{\text {help }}}{\longrightarrow} \mathrm{PMgP}_{\mathbf{s}}+\mathbf{L} \\
& \mathbf{L}_{\mathbf{s}}^{\mathbf{P}}+\mathrm{PMgP}_{\mathrm{r}} \stackrel{\frac{1}{2} k_{\text {ex2 }}}{\longrightarrow} \mathbf{K}^{\mathbf{P} 3}{ }_{\mathbf{s}}+\text { help } 7_{\mathrm{r}} \\
& \text { help } 7_{\mathrm{r}}+\mathrm{PMgP}_{\mathrm{r}} \stackrel{k_{\text {help }}}{\longrightarrow} \mathbf{K}^{\mathbf{P} 3} \mathbf{s}+\mathbf{K}^{\mathbf{P} 3} \mathbf{r}_{\mathbf{r}} \\
& \mathbf{K}_{\mathbf{s}}^{\mathbf{P 3}} \stackrel{\frac{1}{3} k_{\text {act2 }}}{\longrightarrow} \mathrm{PMgP}_{\mathrm{s}}+\text { help8 }
\end{aligned}
$$




$$
\begin{aligned}
& \mathbf{K}^{\mathbf{P} 3}{ }_{\mathbf{s}}+\text { help } 8 \stackrel{k_{\text {help }}}{\longrightarrow} \mathrm{PMgP}_{\mathrm{s}}+\text { help } 9 \\
& \mathbf{K}^{\mathbf{P} 3}{ }_{\mathbf{s}}+h \operatorname{lp} 9 \stackrel{k_{\text {help }}}{\longrightarrow} \mathbf{L}^{\mathbf{P}}{ }_{\mathbf{s}} \\
& \mathbf{L}^{\mathbf{P}} \stackrel{k_{\mathrm{t}}}{\longrightarrow} \mathbf{N}+\mathrm{P}_{\text {Dead }, \mathrm{s}} \\
& \mathbf{N}+\mathrm{M} \stackrel{k_{\text {reini }}}{\longrightarrow} \mathbf{L}_{\mathbf{1}} \\
& \mathrm{BzMgCl}+\mathrm{BzMgCl} \stackrel{k_{\text {schlenk }}}{\longrightarrow} \mathrm{BzMgBz}+\mathrm{ClMgCl} \\
& \mathrm{PMgCl}_{\mathrm{s}}+\mathrm{BzMgCl} \stackrel{k_{\text {schlenk }}}{\longrightarrow} \mathrm{BzMgP}_{\mathrm{s}}+\mathrm{CIMgCl} \\
& \mathrm{PMgCl}_{\mathrm{s}}+\mathrm{PMgCl}_{\mathrm{r}} \stackrel{k_{\text {schlenk }}}{\longrightarrow} \mathrm{PMgP}_{\mathrm{s}}+\text { help } 26_{\mathrm{r}} \\
& \text { help } 26_{\mathrm{s}} \stackrel{k_{\text {help }}}{\longrightarrow} \mathrm{PMgP}_{\mathrm{s}}+\mathrm{CIMgCl}
\end{aligned}
$$




\section{Abbreviations}
A
$\mathrm{Cp}^{*}{ }_{2} \mathrm{ZrCl}_{2}$
$\mathrm{A}_{\mathrm{x} \mathrm{nm}}$
Absorbance at $x$ nanometer
a
Chain-lenght dependence factor for styrene polymerization
$\alpha$
Chain-lenght dependence factor for monomer coordination
$\alpha_{M H}$
Mark-Houwink coefficient $\alpha$
A
pre exponential factor
ATRP
Atom-Transfer Polymerization
$\mathbf{B}^{\mathrm{P} 1}$
$\mathrm{Cp}_{2}{ }_{2} \mathrm{ZrCl}_{2} \mathrm{MgPBz}$
$\mathbf{B}^{\mathrm{P0}}$
$\mathrm{Cp}^{*}{ }_{2} \mathrm{ZrCl}_{2} \mathrm{MgBz}_{2}$
$\mathrm{B}^{\mathrm{P2}}$
$\mathrm{Cp}^{*}{ }_{2} \mathrm{ZrCl}_{2} \mathrm{MgP}_{2}$
$\mathrm{BzBz}$
1,2-diphenyl ethane
BzMgP
benzyl polystyrenyl magnesium
$\mathrm{BCP}$
Block copolymer
CCG
Catalyzed Chain Growth
C(M)
$\mathrm{Cp}^{*}{ }_{2} \mathrm{ZrBz}(\mathrm{M})$
$\mathrm{C}^{\mathrm{P}}(\mathbf{M})$
$\mathrm{Cp}^{*} \mathrm{ZrP}(\mathrm{M})$
CLD
Chain-length dependence
CTA
Chain Transfer Agent
$\delta$
Chemical Shift 
cf.

confer

$c(\mathrm{X})$

concentration of species $\mathrm{X}$

CA

control agent

CRP

Conventional Radical Polymerization

CCTP

Coordinative Chain Transfer Polymerization

$\mathrm{Cp}$

Cyclopentadienyl-

$\mathrm{D}^{\mathrm{P} 0}$

$\mathrm{Cp}^{*}{ }_{2} \mathrm{ZrBzClMgBz}_{2}$

$\mathrm{D}^{\mathrm{P} 1}$

$\mathrm{Cp}^{*}{ }_{2} \mathrm{ZrBzClMgPBz}$

$\mathrm{D}^{\mathrm{P2}}$

$\mathrm{Cp}^{*}{ }_{2} \mathrm{ZrBzClMgP}$

$\mathrm{D}^{\mathrm{P3}}$

$\mathrm{Cp}_{2}{ }_{2} \mathrm{ZrPClMgP}$

DBE

di-n-butyl ether

DBE

di- $n$-butyl ether

DSC

differential scanning calorimetry

$\mathrm{BuMgBu}$

di-n-butyl magnesium

$\mathrm{PMgP}$

dipolystyrenyl magnesium

d

Sample Thickness

$E_{A}$

Activation Energy

$\mathrm{E}^{\mathrm{P0}}$

$\mathrm{Cp}^{*}{ }_{2} \mathrm{ZrBz}_{2}$

$\mathrm{E}^{\mathrm{P} 1}$

$\mathrm{Cp}^{*}{ }_{2} \mathrm{ZrPBz}$

$\mathrm{E}^{\mathrm{P2}}$

$\mathrm{Cp}^{*}{ }_{2} \mathrm{ZrP}_{2}$

EPR

Electron Paramagnetic Resonance

ESI

Electron spray ionization

eq.

equation

e.g.

for example

$\epsilon$

Extinction coefficient 


\begin{tabular}{|c|c|}
\hline $\mathbf{F}^{\mathbf{P 0}}$ & $\mathrm{Cp}_{2}{ }_{2} \mathrm{ZrBz}_{2} \mathrm{MgBz}_{2}$ \\
\hline $\mathbf{F}^{\mathrm{P1}}$ & $\mathrm{Cp}_{2}{ }_{2} \mathrm{ZrBz}_{2} \mathrm{MgPBz}$ \\
\hline $\mathbf{F}^{\mathbf{P 2}}$ & $\mathrm{Cp}_{2}{ }_{2} \mathrm{ZrBz}_{2} \mathrm{MgP}_{2}$ \\
\hline $\mathbf{F}^{\mathrm{P3}}$ & $\mathrm{Cp}_{2}{ }_{2} \mathrm{ZrPBzMgP}{ }_{2}$ \\
\hline $\mathbf{F}^{\mathbf{P 4}}$ & $\mathrm{Cp}_{2}{ }_{2} \mathrm{ZrP}_{2} \mathrm{MgP}_{2}$ \\
\hline FT & Fourier-Transformation \\
\hline$\nu_{x}$ & Frequency of species $x$ \\
\hline$\Delta H_{\mathrm{A}}$ & Reaction enthalpy \\
\hline $\mathbf{I}$ & $\mathrm{Cp}_{2}{ }_{2} \mathrm{NdCl}_{2} \mathrm{Li}\left(\mathrm{OEt}_{2}\right)_{2}$ \\
\hline i.e. & id est \\
\hline$I$ & Intensity with sample \\
\hline$I_{0}$ & Intensity without sample \\
\hline$[\eta]$ & Intrinsic Viscosity \\
\hline$I$ & nuclear spin quantum number \\
\hline $\mathbf{J}$ & $\mathrm{Cp}_{2}{ }_{2} \mathrm{NdCl}$ \\
\hline $\mathbf{K}^{\mathrm{P1}}$ & $\mathrm{Cp}_{2}{ }_{2} \mathrm{NdClMgPBz}$ \\
\hline $\mathbf{K}^{\mathrm{P0}}$ & $\mathrm{Cp}_{2}{ }_{2} \mathrm{NdClMgBz} 2$ \\
\hline $\mathbf{K}^{\mathbf{P 2}}$ & $\mathrm{Cp}_{2}{ }_{2} \mathrm{NdClMgP}{ }_{2}$ \\
\hline$K_{1, \mathrm{Zr}}$ & Equilibrium constant of $k_{\mathrm{ex} 0} / k_{\mathrm{act} 0}$ for $\mathbf{A}$ \\
\hline$K_{2, \mathrm{Zr}}$ & Equilibrium constant of $k_{\mathrm{ex} 1} / k_{\mathrm{act} 1}$ for $\mathbf{A}$ \\
\hline$K_{1, \mathrm{ND}}$ & Equilibrium constant of $k_{\mathrm{ex} 1} / k_{\mathrm{act} 1}$ for $\mathbf{I}$ \\
\hline $\mathrm{K}_{\mathrm{MH}}$ & Mark-Houwink coefficient $K$ \\
\hline$k_{\text {act }}$ & rate coefficient dissociation of bifunctional magnesium derivative \\
\hline$k_{\text {add }}$ & rate coefficient of monomer coordination \\
\hline$k_{\text {alkyl1 }}$ & rate coefficient of first chloride alkyl exchange \\
\hline
\end{tabular}


$k_{\mathrm{alkyl} 2}$

$k_{\mathrm{ex}}$

$k_{\text {frag }}$

$k_{\mathrm{p}}$

$k_{\mathrm{p} 1}$

$k_{\text {treini }}$

$k_{\mathrm{t}}$

$k_{\mathrm{t}, \mathrm{alkyl}}$

L(M)

$\mathrm{L}^{\mathrm{P}(\mathrm{M})}$

LMCT

MLCT

$\lambda$

$B_{0}$

$\gamma$

$\mathrm{MH}$

MALDI

$\mathbf{M}^{\mathrm{P0}}$

$\mathbf{M}^{\mathrm{P} 1}$

$\mathbf{M}^{\mathrm{P2}}$

$\mathbf{M}^{\mathrm{P3}}$

MAO

MMD

$\bar{M}_{n}$ rate coefficient of second chloride alkyl exchange

rate coefficient of association of bifunctional magnesium derivative

rate coefficient of monomer discoordination

rate coefficient of monomer insertion

rate coefficient of first monomer insertion

rate coefficient of monomer addition to hydride complex

rate coefficient of polymerization termination

rate coefficient of cocatalyst termination

$\mathrm{Cp}_{2}{ }_{2} \mathrm{NdBz}(\mathrm{M})$

$\mathrm{Cp}_{2}{ }_{2} \mathrm{NdP}(\mathrm{M})$

ligand-metal charge transfer

metal-ligand charge transfer

Wavelength

Magnetic Field

Magnetogyric Ratio

Mark-Houwink

matrix-assisted laser desorption/ionization

$\mathrm{Cp}^{*}{ }_{2} \mathrm{NdBzMgBz}_{2}$

$\mathrm{Cp}^{*}{ }_{2} \mathrm{NdBzMgBzP}$

$\mathrm{Cp}^{*}{ }_{2} \mathrm{NdBzPMgP}$

$\mathrm{Cp}_{2}{ }_{2} \mathrm{NdPMgP}{ }_{2}$

methylaluminoxane

molar mass distribution

average molecular mass 


\begin{tabular}{|c|c|}
\hline$M_{\mathrm{p}}$ & molar mass at peak maximum \\
\hline $\bar{M}_{w}$ & mass averaged molecular mass \\
\hline MALLS & multi-angle laser light scattering \\
\hline BuMgOct & n-butyl-n-octyl-magnesium, BOMAG \\
\hline NMP & Nitroxide-Mediated Polymerization \\
\hline$n$ & Non bonding orbital \\
\hline NMR & nuclear magnetic resonance \\
\hline$\pi$ & Antibonding orbital \\
\hline$\pi *$ & Bonding orbital \\
\hline $\bar{P}_{n}$ & degree of polymerization \\
\hline$C p^{*}$ & Pentamethylcyclopentadienyl- \\
\hline PMMA & Polymethyl methacrylate \\
\hline LDPE & Low-Density Polyethylene \\
\hline $\mathrm{PE}$ & polyethylene \\
\hline HDPE & High Density Polyethylene \\
\hline PUR & Polyurethane \\
\hline$\Delta p$ & Pressure of the dissolved polymer \\
\hline$\Delta p_{\text {sol }}$ & Pressure of the mobile phase \\
\hline RI & refractive-index \\
\hline RAFT & $\begin{array}{l}\text { Reversible Addition-Fragmentation Chain Transfer Polymeriza- } \\
\text { tion }\end{array}$ \\
\hline RDRP & Reversible-Deactivation Radical Polymerization \\
\hline SEC & Size Exclusion Chromatography \\
\hline$\Delta S_{\mathrm{R}}$ & Reaction entropy \\
\hline$<s^{2}>^{1 / 2}$ & root-mean square radius of gyration \\
\hline
\end{tabular}




$\begin{array}{ll}\text { st. } & \text { styrene } \\ T & \text { Temperature } \\ t & \text { time } \\ \mathrm{t} & \text { Metric ton } \\ \text { Tra. } & \text { Transmission } \\ \text { TEA } & \text { triethyl aluminium } \\ \text { TMA } & \text { Trimethylaluminium } \\ V_{h} & \text { Hydro Dynamic Volume } \\ \mathrm{Z} & \text { stabilizing group } \\ \mathrm{ZN} & \text { Ziegler-Natta }\end{array}$




\section{References}

[1] H. Staudinger, J. Fritschi, Helvetica Chimica Acta 1922, 5, 785-806.

[2] H. Staudinger, Ber. dtsch. Chem. Ges. A/B 1920, 53, 1073-1085.

[3] B. Tieke, Makromolekulare Chemie, 2. ed., Wiley-VCH, Weinheim, 2005.

[4] PlasticsEurope, Plastics - the Facts 2013, pdf, 2013, downloaded 02.07.2014.

[5] K. Khanna, S. Varshney, A. Kakkar, Polym. Chem. 2010, 1, 1171-1185.

[6] A. Hirao, R. Goseki, T. Ishizone, Macromolecules 2014, 47, 1883-1905.

[7] G. Moad, E. Rizzardo, S. H. Thang, Chemistry - An Asian Journal 2013, 8, 1634-1644.

[8] R. Barbey, S. Perrier, ACS Macro Lett., 2013, 2, 366-370.

[9] G. W. Coates, Chem. Rev. 2000, 100, 1223-1252.

[10] G. Natta, P. Pino, P. Corradini, F. Danusso, E. Mantica, G. M. andGiovanni Moraglio, J. Am. Chem. Soc. 1955, 77, 1708-1710.

[11] G. Natta, J. Polymer Sci. 1955, 16, 143-154.

[12] S. Kaita, M. Yamanaka, A. C. Horiuchi, Y. Wakatsuki, Macromolecules 2006, 39, 1359-1363.

[13] L. Oliva, P. Longo, A. Grassi, P. Ammendola, C. Pellecchia, Makromol. Chem., Rapid Commun. 1990, 11, 519-524.

[14] L. Leibler, Macromolecules 1980, 13, 1602-1617.

[15] G. H. F. E. Helfand, J. Chem. Phys. 1987, 87, 697-705. 
[16] Y. Mai, A. Eisenberg, Chem. Soc. Rev. 2012, 41, 5969-5985.

[17] F. Ehlers, J. Barth, P. Vana, Fundamentals of Controlled/Living Radical Polymerization, RSCPublishing, Ch. Kinetics and Thermodynamics of Radical Polymerization, 2013, 1-59.

[18] P. D. Iedema, H. C. J. Hoefsloot, J. Smit, Ind. Eng. Chem. Res. 2004, 43, 39-50.

[19] V. C. Gibson, Science 2006, 3112, 703-704.

[20] F. D'Agosto, C. Boisson, Aust. J. Chem. 2010, 63, 1155-1158.

[21] S. Bogaert, J.-F. Carpentier, T. Chenal, A. Mortreux, G. Ricart, Macromol. Chem. Phys. 2000, 201, 1813-1822.

[22] S. Bogaert, T. Chenal, A. Mortreux, J.-F. Carpentier, J. Mol. Catal. A.: Chem. 2002, 190, 207-214.

[23] J. Mazzolini, E. Espinosa, F. D'Agosto, C. Boisson, Polymer Chemistry 2010, 1, 793-800.

[24] N. Ishihara, T. Seimiya, M. Kuramoto, M. Uoi, Macromolecules 1986, 19, 2464-2465.

[25] W.-D. Hoffman, G. Eckhardt, E. B. und F. Keller, Acta Polymerica 1980, 31, 233-238.

[26] A. E. Tonelli, F. Schilling, Acc. Chem. Res. 1981, 14, 233-238.

[27] F. R. Mayo, J. Am. Chem. Soc. 1968, 90, 1289-1295.

[28] O. F. Olaj, H. F. Kauffmann, J. W. Breitenbach, Makromol. Chem. 1976, 177, 30653071.

[29] T. Huff, E. Perry, J. Am. Chem. Soc. 1960, 82, 4277-4281.

[30] T. Huff, E. Perry, J. Polym. Sci. Part A: Polym. Chem. 1963, 1, 1553-1572.

[31] A. D. Asandei, I. W. Moran, J. Polym. Sci. Part A: Polym. Chem. 2006, 44, 1060-1070.

[32] N. N. Sigaeva, A. K. Friesen, I. I. Nasibullin, N. L. Ermolaev, S. V. Kolesov, Kinetics and Catalysis 2012, 53, 470-476. 
[33] A. D. Asandei, I. W. Moran, G. Saha, Y. Chen, J. Polym. Sci. Part A: Polym. Chem. 2006, 44, 2015-2026.

[34] A. D. Asandei, I. W. Moran, G. Saha, Y. Chen, J. Polym. Sci. Part A: Polym. Chem. 2006, 44, 2156-2165.

[35] L. Ma, Y. Sheng, Q. Huang, K. Deng, K. Gao, W. Yang, J. Appl. Polym. Sci. 2010, 116, 1574-1580.

[36] A. D. Asandei, G. Saha, Macromolecules 2006, 39, 8999-9009.

[37] K. Ziegler, E. Holzkamp, H. Breil, H. Martin, Angew. Chem. 1955, 67, 426.

[38] K. Ziegler, E. Holzkamp, H. Breil, H. Martin, Angew. Chem. 1955, 67, 541-547.

[39] G. Natta, Angew. Chem. 1964, 76, 553-566.

[40] K. Ziegler, Angew. Chem. 1964, 76, 545-553.

[41] J. C. W. Chien, J. Am. Chem. Soc. 1959, 81, 86-92.

[42] D. S. Breslow, N. R. Newburg, J. Am. Chem. Soc. 1959, 81, 81-86.

[43] W. P. Long, D. S. Breslow, J. Am. Chem. Soc. 1960, 82, 1953-1957.

[44] W. P. Long, J. Am. Chem. Soc. 1959, 81, 5312-5316.

[45] P. Cossée, Tetrahedron Lett. 1960, 17, 17-21.

[46] K. P. Bryliakov, E. P. Talsi, Coordination Chemistry Reviews 2012, 256, 2994-3007.

[47] P. Cossée, J. Catal. 1964, 3, 80-88.

[48] E. J. Arlman, J. Catal. 1964, 3, 89-98.

[49] E. Arlman, P. Cossée, J. Catal. 1964, 3, 99-104.

[50] E. J. Arlman, J. Catal. 1966, 5, 178-189.

[51] K. H. Reichert, Angew. Makromol. Chem. 1970, 13, 177-182. 
[52] K. Meyer, K. H. Reichert, Angew. Makromol. Chem. 1970, 12, 175-183.

[53] G. Henrici-Olivé, S. Olivé, Adv. Polymer Sci. 1969, 6, 421-472.

[54] G. Henrici-Olivé, S. Olivé, Angew. Chem. 1967, 79, 764-773.

[55] G. Fink, R. Rottler, Angew. Makromol. Chem. 1981, 94, 25-47.

[56] G. Fink, R. Rottler, C. G. Kreiter, Angew. Makromol. Chem. 1981, 96, 1-20.

[57] G. Fink, W. Zoller, Makromol. Chem. 1981, 182, 3265-3278.

[58] G. Fink, D. Schnell, Angew. Makromol. Chem. 1982, 105, 15-30.

[59] G. Fink, D. Schnell, Angew. Makromol. Chem. 1982, 105, 31-38.

[60] G. Fink, D. Schnell, Angew. Makromol. Chem. 1982, 105, 39-48.

[61] G. Fink, W. Fenzl, R. Mynott, Z. Naturforsch. B 1985, 40b, 158.

[62] R. Mynott, G. Fink, W. Fenzl, Angew. Makromol. Chem. 1987, 154, 1-21.

[63] F. Song, R. D. Cannon, M. Bochmann, J. Am. Chem. Soc. 2003, 125, 7641-7653.

[64] S. H. Kim, G. A. Somorjai, Surface and Interface Analysis 2001, 31, 701-710.

[65] S. G. M. Périn, J. R. Severn, C. E. Koning, J. C. Chadwick, Macromol. Chem. Phys. 2006, 207, 50-56.

[66] E. Grau, A. Lesage, S. Norsic, C. Copéret, V. Monteil, P. Sautet, ACS Catalysis 2013, 3, 52-56.

[67] K. Soga, M. Ohgizawa, T. Shiono, Makromol. Chem., Rapid Commun. 1989, 10, 503507.

[68] I. German, W. Kelhif, S. Norsic, C. Boisson, F. D'Agosto, Angew. Chem. Int. Ed. 2013, $52,3438-3441$.

[69] P. G. Gassman, M. R. Callstrom, J. Am. Chem. Soc. 1987, 109, 7875-7876.

[70] S. H. Kim, C. R. Tewell, G. A. Somorjai, Korean dl Chem. Eng. 2002, 19, 1-10. 
[71] T. B. Mikenas, V. A. Zakharov, V. E. Nikitin, L. G. Echevskaya, M. A. Matsko, Russian journal of applied chemistry 2010, 83, 2210-2219.

[72] D. Liu, S. Lin, F. Zhu, H. Gao, Q. Wu, Polymer Bulletin 2008, 61, 71-80.

[73] J. J. Eisch, Organometallics 2012, 31, 4917-4932.

[74] H. Sinn, W. Kaminsky, H.-J. Vollmer, R. Woldt, Angew. Chem. Int. Ed. Engl. 1980, 19, 390-392.

[75] A. Andresen, H.-G. Cordes, J. Herwig, W. Kaminsky, A. Merck, R. Mottweiler, H. S. Joachim Pein, H.-J. Vollmer, Angew. Chem. Int. Ed. Engl. 1976, 15, 630-632.

[76] W. Kaminsky, R. Steiger, Polyhedron 1988, 7, 2375-2381.

[77] G. J. P. Britovsek, V. C. Gibson, D. F. Wass, Angew. Chem. Int. Ed. 1999, 38, 428-447.

[78] D. Coevoet, H. Cramail, A. Deffieux, C. Mladenov, J.-N. Pedeutour, F. Peruch, Polym. Int. 1999, 48, 257-263.

[79] V. C. Gibson, S. K. Spitzmesser, Chem. Rev. 2003, 103, 283-315.

[80] M. A. Pérez, R. Quijada, F. Ortega-Jiménez, C. Alvarez-Toledano, J. Mol. Catal. 2005, 226, 291-295.

[81] T. R. Younkin, E. F. Connor, J. I. Henderson, S. K. Friedrich, R. H. Grubbs, D. A. Bansleben, Science 2000, 287, 460-462.

[82] E. Y.-X. Chen, Chem. Rev. 2009, 109, 5157-5214.

[83] W. Kaminsky, J. Polym. Sci. Part A: Polym. Chem. 2004, 42, 3911-3921.

[84] L. K. Johnson, C. M. Killian, M. Brookhart, J. Am. Chem. Soc. 1995, 117, 6414-6415.

[85] M. Delferro, J. P. McInnis, T. J. Marks, Organometallics 2010, 29, 5040-5049.

[86] M. Ystenes, J. L. Eilertsen, J. Liu, M. O. andErling Rytter, J. A. Støvneng, J. Polym. Sci. Part A: Polym. Chem. 2000, 38, 3106-3127.

[87] R. Mülhaupt, Macromol. Chem. Phys. 2003, 204, 289-327. 
[88] W. Kaminsky, Macromolecules 2012, 45, 3289-3297.

[89] G. Fink, H.-H. Brintzinger, Metal-catalysis in Industrial Organic Processes, RSC Publising, Ch. Polymerization Reactions, 2007, 218-254.

[90] D. M. Sarzotti, D. J. Marshman, W. E. Ripmeester, J. B. P. Soares, J. Polym. Sci. Part A: Polym. Chem. 2007, 45, 1677-1690.

[91] E. Y.-X. Chen, T. J. Marks, Chem. Rev. 2000, 100, 1391-1434.

[92] C. J. Harlan, S. G. Bott, A. R. Barron, J. Am. Chem. Soc. 1995, 117, 6465-6474.

[93] M. Bochmann, S. J. Lancaster, M. D. Hannant, A. Rodriguez, M. Schormann, D. A. Walker, T. J. Woodman, Pure Appl. Chem. 2003, 75, 1183-1195.

[94] C. Janiak, P.-G. Lassahn, V. Lozan, Macromolecular Symosia 2006, 236, 88-99.

[95] F. R. Wild, L. Zsolnai, G. Huttner, H. H. Brintzinger, J. Organomet. Chem. 1982, 232, 233-247.

[96] W. Kaminsky, K. Külper, H. H. Brintzinger, F. R. W. P. Wild, Angew. Chem. 1985, 97, 507-508.

[97] J.-N. Pédeutour, K. Radhakrishnan, H. Cramail, A. Deffieux, Macromol. Rapid Commun. 2001, 22, 1095-1123.

[98] E. Talsi, K. Bryliakov, N. V. Semikolenova, V. Zakharov, M. Bochmann, Kinetics and Catalysis 2007, 48, 490-503.

[99] M. Bochmann, Organometallics 2010, 29, 4711-4740.

[100] J. J. Eisch, A. M. Piotrowski, S. K. Brownstein, E. J. Gabe, F. L. Leet, Organometallics $1985,107,7219-7221$.

[101] D. Cam, U. Giannini, Makromol. Chem 1992, 193, 1049-1055.

[102] I. Tritto, S. Li, M. C. Sacchi, G. Zannoni, Macromolecules 1993, 26, 7111-7115.

[103] I. Tritto, S. X. Li, M. C. Sacchi, P. Locatelli, G. Zannoni, Macromolecules 1995, 28, 5358-5362. 
[104] I. Tritto, Z.-Q. Fan, P. Locatelli, M. C. Sacchi, I. Camurati, M. Galimberti, Macromolecules 1995, 28, 3342-3350.

[105] I. Tritto, R. Donetti, M. C. Sacchi, P. Locatelli, G. Zannoni, Macromolecules 1997, 30, 1247-1252.

[106] I. Tritto, R. Donetti, M. C. Sacchi, P. Locatelli, G. Zannoni, Macromolecules 1999, 32, 264-269.

[107] K. P. Bryliakov, N. V. Semikolenova, D. V. Yudaev, V. A. Zakharov, H. H. Brintzinger, M. Ystenes, E. Rytter, E. P. Talsi, Journal of Organometallic Chemistry 2003, 683, 92102.

[108] D. E. Babushkin, N. V. Semikolenova, V. A. Zakharov, E. P. Talsi, Macromol. Chem. Phys 2000, 201, 558-567.

[109] A. J. Rossini, I. Hung, S. A. Johnson, C. Slebodnick, M. Mensch, P. A. Deck, , R. W. Schurko, J. Am. Chem. Soc. 2010, 132, 18301-18317.

[110] A. Siedle, W. Lamanna, R. Newmark, J. Schroepfer, J. Mol. Catal. 1998, 128, 257-271.

[111] C. Sishta, R. M. Hathorn, T. J. Marks, J. Am. Chem. Soc. 1992, 114, 1112-1114.

[112] K. Thorshaug, J. A. Støvneng, H. Wigum, E. Rytter, in Organometallic Catalysts and Olefin Polymerization, Springer, 289-298.

[113] J. L. Eilertsen, E. Rytter, M. Ystenes, in Organometallic Catalysts and Olefin Polymerization, Springer 2001, 86-96.

[114] E. Giannetti, G. M. Nicoletti, R. Mazzocchi, Journal of Polymer Science: Polymer Chemistry Editon 1985, 23, 2117-2133.

[115] U. Wieser, H.-H. Brintzinger, in Organometallic Catalysts and Olefin Polymerization, OCOP 2000, Springer, 3-13.

[116] N. I. Mäkelä-Vaarne, K. Kallio, K.-H. Reichert, M. A. Leskelä, Macromol. Chem. Phys 2003, 204, 1085-1089.

[117] H.-K. Luo, Z.-H. Yang, B.-Q. Mao, D.-S. Yu, R.-G. Tang, J. Mol. Catal. 2002, 177, 195-207. 
[118] N. I. Mäkelä-Vaarne, D. G. Nicholson, A. L. Ramstad, J. Mol. Catal. 2003, 200, 232-332.

[119] N. Mäkelä-Vaarne, Dissertation, Borealis Polymers Oy, R\&D, Finland \& Laboratory of Inorganic Chemistry, Faculty of Science, University of Helsinki Finland, 2003.

[120] V. Panchenko, V. Zakharov, E. Paukshtis, J. Mol. Catal. 2005, 240, 33-39.

[121] U. Wieser, F. Schaper, H.-H. Brintzinger, Macromolecular Symposia 2006, 236, 63-68.

[122] D. Coevoet, H. Cramail, A. Deffieux, Macromol. Chem. Phys 1998, 199, 1451-1457.

[123] D. Coevoet, H. Cramail, A. Deffieux, Macromol. Chem. Phys 1998, 199, 1459-1464.

[124] C. Fischer, T. Beweries, A. Preetz, H.-J. Drexler, W. Baumann, S. Peitz, U. Rosenthal, D. Heller, Catalysis Today 2009, 155, 282-288.

[125] P. Belelli, D. Damiani, N. Castellani, Chemical Physics Letters 2005, 401, 515-521.

[126] J.-N. Pédeutour, D. Coevoet, H. Cramail, A. Deffieux, Macromol. Chem. Phys 1999, $200,1215-1221$.

[127] P. J. J. Pieters, J. A. M. van Beek, M. E. H. van TOP, Macromol. Rapid Commun. 1995, $16,463-467$.

[128] V. Busico, R. Cipullo, V. Esposito, Macromol. Rapid Commun. 1999, 20, 116-121.

[129] K. Thorshaug, J. A. Støvneng, E. Rytter, M. Ystenes, Macromolecules 1998, 31, 71497165.

[130] M. M. Ranieri, J.-P. Broyer, F. Cutillo, T. F. L. McKenna, C. Boisson, Dalton Trans. 2013, 42, 9049.

[131] J. M. Camara, R. A. Petros, J. R. Norton, J. Am. Chem. Soc. 2011, 133, 5263-5273.

[132] Z. Guan, Chem. Asian J. 2010, 5, 1058-1070.

[133] W. Wang, R. Liu, Z. Li, C. Meng, Q. Wu, F. Zhu, Macromol. Chem. Phys. 2010, 211, 1452-1459.

[134] D.-J. Byun, S. Y. Kim, Macromolecules 2000, 33, 1921-12923. 
[135] D.-J. Byun, K.-Y. Choi, S. Y. Kim, Macromol. Chem. Phys. 2001, 202, 992-997.

[136] J. Y. Dong, T. C. Chung, Macromolecules 2002, 35, 1622-1631.

[137] B. Arikan, F. J. Stadler, J. Kaschta, H. Münstedt, W. Kaminsky, Macromol. Rapid Commun. 2007, 28, 1472-1478.

[138] M. Atiqullah, M. Tinkl, R. Pfaendner, M. N. Akhtar, . I. Hussain, Polymer Reviews 2010, 50, 178-230, DOI 10.1080/15583721003704289.

[139] P. Cossée, Tetrahedron Lett. 1960, 17, 12-16.

[140] R. H. Grubbs, G. W. Coates, Acc. Chem. Res. 1996, 29, 85-93.

[141] M. L. H. Green, Pure Appl. Chem. 1978, 50, 27-35.

[142] K. J. Ivin, J. Rooney, C. Stewart, M. L. H. Green, R. J. Mahtab, J. Chem. Soc., Chem. Commun. 1978, 604-606.

[143] Z. Dawoodi, M. L. H. Green, V. B.Mtetwa, K. J. Prout, J. Chem. Soc., Chem. Commun. 1982, 1410-1411.

[144] D. T. Laverty, J. J. Rooney, J. Chem. Soc., Faraday Trans. 1 1983, 79, 869-878.

[145] M. Greenhart, M. L. H. Green, J. Organomet. Chem. 1983, 250, 395-408.

[146] M. Brookhart, M. L. H. Green, L. L. Wong, Prog. Inorg. Chem. 1988, 36, 1-124.

[147] J. Soto, M. L. Steigerwald, R. H. Grubbs, J. Am. Chem. Soc. 1982, 104, 4479-4480.

[148] V. Busico, L. Caporaso, R. Cipullo, L. Landriani, J. Am. Chem. Soc. 1996, 118, 21052106.

[149] H. Krauledat, H.-H. Brintzinger, Angew. Chem. Int. Ed. 1990, 29, 1412-1413.

[150] M. J. Tanner, M. Brookhart, J. M. DeSimone, J. Am. Chem. Soc. 1997, 119, 7617-7618.

[151] G. J. P. Britovsek, S. A. Cohen, V. C. Gibson, M. van Meurs, J. Am. Chem. Soc. 2004, 126, 10701-10712. 
[152] L. Resconi, F. Piemontesi, G. Franciscono, L. Abis, T. Fiorani, J. Am. Chem. Soc. 1992, 114, 1025-1032.

[153] M. Bochmann, J. Organomet. Chem. 2004, 689, 3982-3998.

[154] A. K. Rappé, W. M. Skiff, C. J. Casewit, Chem. Rev. 2000, 100, 1435-1456.

[155] F. di Lena, E. Quintanilla, P. Chen, Chem. Comm. 2005, 5757-5759.

[156] T. F. L. McKenna, E. Tioni, M. M. Ranieri, A. Alizadeh, C. Boisson, V. Monteil, The Canadian Journal of Chemical Engineering 2013, 91, 669-686.

[157] Z. Liu, E. Somsook, C. R. Landis, J. Am. Chem. Soc. 2001, 123, 2915-2916.

[158] Z. Liu, E. Somsook, C. B. White, K. A. Rosaaen, C. R. Landis, J. Am. Chem. Soc. 2001, $123,11193-11207$.

[159] C. R. Landis, K. A. Rosaaen, D. R. Sillars, J. Am. Chem. Soc. 2003, 125, 1710-1711.

[160] N. E. Grimmer, N. J. Coville, C. B. de Koning, J. Mol. Catal. 2002, 188, 105-113.

[161] N. Suzuki, Y. Yamaguchi, A. Fries, T. Mise, Macromolecules 2000, 33, 4602-4606.

[162] J. Liu, J. A. Støvneng, E. Rytter, J. Polym. Sci. Part A: Polym. Chem. 2001, 39, 35663577.

[163] I. Bruaseth, E. Rytter, Macromolecular Symposia 2004, 213, 69-78.

[164] I. Bruaseth, J. B. Soares, E. Rytter, Polymer 2004, 45, 7853-7861.

[165] M. Bialek, K. Czaja, A. Reszka, J. Polym. Sci. Part A: Polym. Chem. 2005, 43, 55625570 .

[166] M. Bialek, K. Czaja, Macromol. Chem. Phys. 2006, 207, 1651-1660.

[167] J. A. M. Awudza, P. J. T. Tait, J. Polym. Sci. Part A: Polym. Chem. 2008, 46, 267-277.

[168] C. R. Landis, K. A. Rosaaen, J. Uddin, J. Am. Chem. Soc. 2002, 124, 12062-12063. 
[169] K. A. Novstrup, N. E. Travia, G. A. Medvedev, C. Stanciu, J. M. Switzer, K. T. Thomson, W. N. Delgass, M. M. Abu-Omar, J. M. Caruthers, J. Am. Chem. Soc. 2010, $132,242-258$.

[170] M. Bochmann, S. J. Lancaster, Angew. Chem. Int. Ed. Engl. 1994, 33, 1634-1637.

[171] M. Szwarc, Nature 1956, 178, 1168-1169.

[172] T. Shiono, Catalysis Surveys from Asia 2003, 7, 47-62.

[173] V. Busico, G. Talarico, R. Cipullo, Macromolecular symposia 2005, 226, 1-16.

[174] G. W. Coates, P. D. Hustad, S. Reinartz, Angew. Chem. Int. Ed. 2002, 41, 2236-2257.

[175] O. W. Webster, Science 1991, 251, 887-893.

[176] M. Miyamoto, M. Sawamoto, T. Higashimura, Macromolecules 1984, 18, 265-272.

[177] S. Aoshima, S. Kanaoka, Chem. Rev. 2009, 109, 5245-5287.

[178] T. Otsu, M. Yoshida, Makromol. Chem., Rapid Commun. 1982, 3, 127-132.

[179] T. Otsu, M. Yoshida, T. Tazaki, Makromol. Chem., Rapid Commun. 1982, 3, 133-140.

[180] J. Chiefari, Y. K. B. Chong, F. Ercole, J. Krstina, J. Jeffery, T. P. T. Le, R. T. A. Mayadunne, G. F. Meijs, C. L. Moad, G. Moad, E. Rizzardo, S. H. Thang, Macromolecules 1998, 31(16), 5559-5592.

[181] D. Charmot, P. Corpart, H. Adam, S. Zard, T. Biadatti, G. Bouhadir, Macromolecular Symposia 2000, 150, 23.

[182] M. Kato, M. Kamigaito, M. Sawamoto, T. Higashimura, Macromolecules 1995, 28, 1721-1723.

[183] J.-S. Wang, K. Matyjaszewski, J. Am. Chem. Soc. 1995, 117, 5614-5615.

[184] G. Moad, E. Rizzardo, Macromolecules 1995, 28, 8722-8728.

[185] A. Goto, Y. Tsujii, T. Fukuda, Polymer 2008, 49, 5177-5185. 
[186] (Ed.: N. V. Tsarevsky, B. S. Sumerlin), Fundamentals of Controlled/Living Radical Polymerization, (Ed.: N. V. Tsarevsky, B. S. Sumerlin), RSC Pulishing, 2013.

[187] K. Satoh, M. Kamigaito, Chem. Rev. 2009, 109, 5120-5156.

[188] G. Moad, E. Rizzardo, S. H. Thang, Aust. J. Chem. 2009, 62, 1402-1472.

[189] M. Wulkow, M. Busch, T. P. Davis, C. Barner-Kowollik, J. Polym. Sci. Part A: Polym. Chem. 2004, 42, 1441-1448.

[190] D. Boschmann, P. Vana, Macromolecules 2007, 40, 2683-2693.

[191] C. Boyer, T. P. Davis, Chemical Communications 2009, 2009(41), 6029-6031.

[192] C. Boyer, A. Granville, T. P. Davis, V. Bulmus, J. Polym. Sci. Part A: Polym. Chem. 2009, 47, 3773-3794.

[193] C. K. Ullal, S. Primpke, R. Schmidt, U. Böhm, A. Egner, P. Vana, S. W. Hell, Macromolecules 2011, 44, 7508-7510.

[194] R. Henkel, P. Vana, Macromol. Chem. Phys 2014, 215, 182-189.

[195] C. Rossner, P. Vana, Angew. Chem. 2014, DOI 10.1002/anie.201406854.

[196] C. Rossner, B. Ebeling, P. Vana, ACS Macro Letters 2013, 2, 1073-1076.

[197] R. Kempe, Chem.-Eur. 2007, 13, 2764-2773.

[198] L. Sita, Angew. Chem. Int. Edt. 2009, 48, 2464-2472.

[199] A. Valente, A. Mortreux, M. Visseaux, P. Zinck, Chem. Rev. 2013, 113, 3836-3857.

[200] K. Adlington, G. J. Jones, J. E. Harfi, A. S. Georgios Dimitrakis and, S. W. Kingman, J. P. Robinson, D. J. Irvine, Macromolecules 2013, 46, 3922-3930.

[201] C. Fan, C. Bai, H. Cai, Q. Dai, X. Zhang, F. Wang, J. Polym. Sci. Part A: Polym. Chem. 2010, 48, 4768-4774.

[202] S. Georges, A. Osmane, T. M. Visseaux, P. Zinck, Macromolecules 2014, 47, 45384547. 
[203] H. Makio, T. Ochiai, J. ichi Mohri, K. Takeda, T. Shimazaki, Y. Usui, S. Matsuura, T. Fujita, J. Am. Chem. Soc. 2013, 135, 8177-8180.

[204] K. Ziegler, H. G. Gellert, H. Kiihlhokn, H. Martin, K. Meyer, K. Nagel, H. Sauer, K. Zosel, Angew. Chem. 1952, 64, 323-329.

[205] D. J. Arriola, E. M. Carnahan, P. D. Hustad, R. L. Kuhlman, T. T. Wenzel, Science 2006, 312, 714-719.

[206] P. D. Hustad, R. L. Kuhlman, E. M. Carnahan, T. T. Wenzel, D. J. Arriola, Macromolecules 2008, 41, 4081-4089.

[207] M. Zintl, B. Rieger, Angew. Chem. Int. Ed. 2007, 46, 333-335.

[208] M. Zhang, E. M. Carnahan, T. W. Karjala, P. Jain, Macromolecules 2009, 42, 80138016.

[209] M. Zhang, T. W. Karjala, P. Jain, Ind. Eng. Chem. Res. 2010, 49, 8135-8146.

[210] M. Zhang, T. W. Karjala, P. Jain, C. Villa, Macromolecules 2013, 46, 4847-4853.

[211] Y. Mohammadi, M. Ahmadi, M. R. Saeb, M. M. Khorasani, P. Yang, F. J. Stadler, Macromolecules 2014, 47, 4778-4789.

[212] R. L. Kuhlmann, T. T. Wenzel, Macromolecules 2008, 41, 4090-4094.

[213] G. J. Britovsek, S. Mastroianni, G. A. Solan, S. P. D. Baugh, C. Redshaw, V. C. Gibson, A. J. P. White, D. J. Williams, M. R. J. Elsegood, Chem. Eur. J. 2000, 6, 2221-2231.

[214] G. J. P. Britovsek, S. A. Cohen, V. C. Gibson, P. J. Maddox, M. van Meurs, Angew. Chem. Int. Edt. 2002, 41, 489-491.

[215] M. van Meurs, G. J. P. Britovsek, V. C. Gibson, S. A. Cohen, J. Am. Chem. Soc. 2005, 127, 9913-9923.

[216] M. Ganesan, F. P. Gabbaï, Organometallics 2004, 23, 4608-4613.

[217] G. Mani, F. P. Gabbai, Angew. Chem. Int. Edt. 2004, 43, 2263-2266. 
[218] G. C. Bazan, J. S. Rogers, C. C. Fang, Organometallics 2001, 20, 2059-2064.

[219] J. S. Rogers, G. C. Bazan, Chem. Comm. 2000, 1209-1210.

[220] J. F. Pelletier, A. Morteux, X. Olonde, K. Bujadoux, Angew. Chem. Int. Edt. 1996, 35, 1854-1856.

[221] S. Bogaert, T. Chenal, A. Mortreux, G. Nowogrocki, C. W. Lehmann, J.-F. Carpentier, Organometallics 2001, 20, 199-205.

[222] W. P. Kretschmer, A. Meetsma, B. Hessen, T. Schmalz, S. Qayyum, R. Kempe, Chem.-Eur. 2006, 12, 8969-8978.

[223] W. Zhang, L. R. Sita, J. Am. Chem. Soc. 2008, 130, 442-443.

[224] F. Alfano, H. W. Boone, V. Busico, R. Cipullo, J. C. Stevens, Macromolecules 2007, 40, 7736-7738.

[225] R. Briquel, J. Mazzolini, T. L. Bris, O. Boyron, F. Boisson, F. Delolme, F. D'Agosto, C. Boisson, R. Spitz, Angew. Chem. Int. Ed. 2008, 47, 9311-9313.

[226] J. Mazzolini, V. Monteil, F. D'Agosto, C. Boisson, D. Gigmes, D. Bertin, Polymer Preprints 2011, 52, 389-390.

[227] H. C. Kolb, M. G. Finn, K. B. Sharpless, Angew. Chem. Int. Ed. 2001, 40, 2004-2021.

[228] R. Huisgen, Proceeding of the Chemical Society 1961, Oktober, 357-369.

[229] B. S. Sumerlin, A. P. Vogt, Macromolecules 2010, 43(1), 1-13.

[230] F. Rouholahnejad, D. Mathis, P. Chen, Organometallics 2010, 29, 294-302.

[231] F. di Lena, P. Chen, Helvetica Chimica Acta 2009, 92, 890-896.

[232] D. Mathis, F. Rouholahnejad, P. Chen, Helvetica Chimica Acta 2010, 93, 212-219.

[233] A. Yokoyama, R. Miyakoshi, T. Yokozawa, Macromolecules 2004, 37, 1169-1171.

[234] E. E. Sheina, J. Liu, M. C. Iovu, D. W. Laird, R. D. McCullough, Macromolecules 2004, 37, 3526-3528. 
[235] R. H. Lohwasser, M. Thelakkat, Macromolecules 2011, 44, 3388-3397.

[236] R. D. McCullough, R. D. Lowe, M. Jayaraman, D. L. Anderson, J. Org. Chem. 1993, $58,904-912$.

[237] R. D. McCullough, R. D. Lowe, J. Chem. Soc., Chem. Commun., 1992, 70-72.

[238] E. L. Lanni, A. J. McNeil, J. Am. Chem. Soc 2009, 131, 16573-16579.

[239] E. L. Lanni, J. R. Locke, C. M. Gleave, A. J. McNeil, Macromolecules 2011, 44, 51365145.

[240] R. Miyakoshi, K. Shimono, A. Yokoyama, T. Yokozawa, J. Am. Chem. Soc. 2006, 128, 16012-16013.

[241] C. Müller, S. Goffri, D. Breiby, J. Andreasen, H. Chanzy, R. Janssen, M. Nielsen, C. Radano, H. Sirringhaus, P. Smith, N. Stingelin-Stutzmann, Advanced Functional Materials 2007, 17, 2674-2679.

[242] C. P. Radano, O. A. Scherman, N. Stingelin-Stutzmann, C. Müller, D. W. Breiby, P. Smith, R. A. J. Janssen, E. W. Meijer, J. Am. Chem. Soc., 2005, 127, 12502-12503.

[243] S. Kang, R. J. Ono, C. W. Bielawski, J. Polym. Sci. Part A: Polym. Chem. 2013, 51, 3810-3817.

[244] R. Lohwasser, Dissertation, Universtität Bayreuth, 2011.

[245] M. Buback, Makromolekulare Chemie - Macromolecular Chemistry and Physics 1990, 191, 1477-1743.

[246] M. Buback, B. Huckestein, G. Russell, Macromol. Chem. Phys. 1994, 195, 539-554.

[247] S. Beuermann, M. Buback, P. Hesse, T. Junkers, I. Lacík, Macromolecules 2006, 39, 658-670.

[248] N. F. G. Wittenberg, Dissertation, Georg-August-Universität Göttingen, 2013.

[249] J. M. Hollas, Modern Spectrosopy, (Ed.: 4.Auflage), John Wiley \& Sons, 2003.

[250] H. Schroeder, M. Buback, K. Matyjaszewski, Macromol. Chem. Phys 2014, 215, 44-53. 
[251] T. D. W. Claridge, High-Resolution NMR Techniques in Organic Chemistry, 2 ed., Vol. 27 by Tetrahedron Organic Chemistry, (Ed.: J.-E. Bäckvall, J. Baldwin, R. Williams), Elsevier, 2009.

[252] E. G. Malawar, L. Senak, Handbook of Size Exclusion Chromatography and Related Techniques, Marcel Dekker, Inc., Ch. Introduction to Size Exclusion Chromatography, 2004, 1-23.

[253] A. Wolpers, G. T. Russell, P. Vana, Macromol. Theory Simul. 2011, 20, 667-674.

[254] M. Wulkow, Macromolecular Reaction Engineering 2008, 2, 461-494.

[255] G. Zwolak, F. P. Lucien, Macromolecules 2008, 41, 5141-5147.

[256] S. C. J. Pierik, A. M. van Herk, J. Appl. Polym. Sci. 2004, 91, 1375-1388.

[257] P. Vana, T. P. Davis, C. Barner-Kowollik, Macromol. Theory Simul. 2002, 11, 823-835.

[258] H. Schroeder, J. Buback, J. Schrooten, M. Buback, K. Matyjaszewski, Macromol. Theory Simul. 2014, 23, 279-287.

[259] W. Meiser, J. Barth, M. Buback, H. Kattner, P. Vana, Macromolecules 2011, 44, 24742480.

[260] W. Meiser, M. Buback, J. Barth, P. Vana, Polymer 2010, 51, 5122-5134.

[261] W. Meiser, M. Buback, O. Ries, C. Ducho, A. Sidoruk, Macromol. Chem. Phys 2013, 214, 924-933.

[262] W. Meiser, M. Buback, A. Sidoruk, Macromol. Chem. Phys. 2013, 214, 2108-2114.

[263] A. Sidoruk, M. Buback, W. Meiser, Macromol. Chem. Phys 2013, 214, 1738-1748.

[264] M. Wulkow, Macromol. Theory Simul. 1996, 5, 393-416.

[265] T. D. Tilley, R. A. Andersen, Inorg. Chem. 1981, 20, 3267-3270.

[266] A. Jacot-Guillarmod, R. Tabacchi, J. Porret, Helvetica Chimica Acta 1970, 53, 14911494. 
[267] S. Ménoret, S. Carlotti, M. Fontanille, A. Deffieux, P. Desbois, C. Schade, W. Schrepp, V. Warzelhan, Macromol. Chem. Phys 2001, 202, 3219-3227.

[268] A. Holleman, E. u. N. Wiberg, Lehrbuch der Anorganischen Chemie, 102. auflage ed., de Gruyter, 2007.

[269] S. Primpke, A. Deinert, F. Ehlers, P. Vana, Macromol. Chem. Phys. 2014, 215, 544-554.

[270] C. P. Casey, D. W. Carpenetti II, H. Sakurai, J. Am. Chem. Soc. 1999, 121, 9483-9484.

[271] C. P. Casey, D. W. Carpenetti II, J. Organom. Chem. 2002, 642, 120-130.

[272] J. Atkinson, P. Brindley, J. Organonmet. Chem. 1991, 411, 131-137.

[273] K.-H. Thiele, U. Böhme, J. Sieler, Z. anorg. allg. Chem. 1993, 619, 1951-1956.

[274] K. Takahashi, Y. Kondo, R. Asami, J. Chem. Soc., Perkin Trans. 2 1978, 577-580.

[275] S. Aoshima, T. Fujisawa, E. Kobayashi, J. Polym. Sci. Part A: Polym. Chem. 1994, 32, 1719-1728.

[276] B. M. Kraft, R. J. Lachicotte, W. D. Jones, J. Am. Chem. Soc. 2001, 123, 10973-10979.

[277] P. J. Chirik, J. E. Bercaw, Organometallics 2005, 24, 5407-5423.

[278] U. M. Dzhemilev, V. A. D'yakonov, L. O. Khafizova, A. G. Ibragimov, Russian Journal of Organic Chemistry 2005, 41, 352-357.

[279] U. M. Dzhemilev, Tetrahedron 1995, 51, 4333-4346.

[280] N. Naga, J. Mol. Catal. 2007, 263, 206-211.

[281] R. Po, N. Cardi, L. Abis, Polymer 1998, 39, 959-964.

[282] F. M. Rabagliati, H. E. Munoz, G. V. Mardones, J. Chil. Chem. Soc. 2010, 55, 111-114.

[283] S. K. De, M. Bhattacharjee, J. Polym. Sci. Part A: Polym. Chem. 2009, 47, 6496-6503.

[284] P. Zinck, A. Valente, F. Bonnet, A. Violante, A. Mortreux, M. Visseaux, S. Ilinca, R. Duchateau, P. Roussel1, J. Polym. Sci. Part A: Polym. Chem. 2010, 48, 802-814. 
[285] F. M. Rabagliati, G. V. Mardones, H. E. Muñoz, F. J. Rodríguez, Polym. Int. 2008, 57, 744-749.

[286] J. Hitzbleck, K. Beckerle, J. Okuda, T. Halbach, R. Mülhaupt, Macromolecular Symposia 2006, 236, 23-29.

[287] S. Primpke, P. Vana, Macromol. Theory Simul.. DOI 10.1002/mats.201400087.

[288] I. Silanes, J. M. Ugalde, Organometallics 2005, 24, 3233-3246.

[289] A. K. Tomov, V. C. Gibson, G. J. P. Britovsek, R. J. Long, M. van Meurs, D. J. Jones, K. P. Tellmann, J. J. Chirinos, Organometallics 2009, 28, 7033-7040.

[290] C. P. Casey, T.-Y. Lee, J. A. Tunge, D. W. Carpenetti II, J. Am. Chem. Soc. 2001, 123, 10762-10763.

[291] C. P. Casey, J. A. Tunge, T.-Y. Lee, M. A. Fagan, J. Am. Chem. Soc. 2003, 125, 26412651.

[292] M. Wojdyr, J. Appl. Cryst. 2010, 43, 1126-1128.

[293] O. F. Olaj, P. Vana, Macromol. Rapid Commun. 1998, 19, 433-439.

[294] O. F. Olaj, P. Vana, M. Zoder, A. Kornherr, G. Zifferer, Macromol. Rapid Commun. 2000, 21, 913-920.

[295] S. M. Baldwin, J. E. Bercaw, H. H. Brintzinger, J. Am. Chem. Soc. 2008, 130, 1742317433.

[296] T. Li, C. Zhou, M. Jiang, Polymer Bulletin 1991, 25, 211-216.

[297] J. Wu, Q. Pan, G. L. Rempel, Journal of Applied Polymer Science 2005, 96, 645-649.

[298] S. Norsic, C. Boisson, F. D’Agosto, personal communication.

[299] J. Schwellenbach, Master Thesis, University of Göttingen, 2013.

[300] G. R. Fulmer, A. J. M. Miller, N. H. Sherden, H. E. Gottlieb, A. Nudelman, B. M. Stoltz, J. E. Bercaw, K. I. Goldberg, Organometallics 2010, 29, 2176-2179. 


\section{Acknowledgments}

Mein besonderer Dank gilt meinem Doktorvater Prof. Dr. Philipp Vana für die Möglichkeit in der Arbeitsgruppe meine Dissertation anzufertigen sowie für die interessante Themenstellung, die sehr wertvollen und motivierenden Diskussionen und das große Engagement bei der Betreuung dieser Arbeit.

Ich bedanke mich bei Herrn Herr Prof. Dr. Franc Meyer für die Übernahme des Koreferates sowie die Zweitbetreuung meiner Dissertation.

Herrn Prof. Dr. Michael Buback danke ich für die Vervollständigung meines Betreungsausschusses.

Bei Prof. Dr. Burkhard Geil, Prof. Dr. Dietmar Stalke und Jun.-Prof. Dr. Ricardo Mata bedanke ich mich für die Teilnahme an meiner Prüfungskommission.

Special thanks go to Dr. Franck D'Agosto and Dr. Christophe Boisson from the CPE in Lyon for the possibility of a short research stay in the working group Chemistry, Catalysis, Polymers and Processes (C2P2) in January 2012, the permission to rebuild a similar polymerization reactor and the sharing of measurement data for simulation.

Ich danke Herrn Prof. Dr. Dr. h.c. Stefan Hell für die Ermöglichung einer Kooperation mit seinem Arbeitskreis NanoBiophotonics am Max Planck Institut für biophysikalische Chemie.

Herrn Jun.-Prof. Dr. Dominik Wöll von der RWHT Aachen danke ich für die Zusammenarbeit im Rahmen einer weiteren Kooperation.

I thank Sébastien Norsic for the measurement of polymerization reactions at the CPE in Lyon and the sharing of results.

Additionally my thanks go to assistant Prof. PhD Chaitanya Ullal from the Renesselaer Polytechnic Institute (Troy, NY, USA) and Dr. Roman Schmidt of the working group 
NanoBiophotonics of the Max Planck Institute for biophysical Chemistry for collaboration and many fruitful discussions.

I thank Michael Ainooson from the working group of Prof. F. Meyer for the cooperation.

Ich bedanke mich bei Dr. Florian Ehlers und Jan Schwellenbach für die gute Zusammenarbeit sowie das Bereitstellen von Messproben und Teilen von Messergebnissen.

Ein weiterer Dank geht an Aline Selke und Adrian Deinert die ich während ihrer Bachelorarbeit betreuen durfte für die Zusammenarbeit und die vielen wissenschaftlichen Anregungen in dieser Zeit.

Herrn Dr. Hans-Peter Vögele danke ich für die vielen anregenden Diskussionen zu Versuchsaufbauten und wissenschaftlichen Details sowie für die Instandhaltung und Wartung der IR-Spektrometer.

Ich bedanke mich bei Dipl.-Chem. R. Machinek für die Hilfe bei der Erstellung der kinetischen NMR-Messungen sowie bei allen Mitarbeitern der NMR-Abteilung des Instituts für Organische und Biomolekulare Chemie für die schnelle Durchführungen der Messungen.

Für die Messungen der Massenspektren danke ich der Abteilung Zentrale Analytik des Instituts für Organische und Biomolekulare Chemie.

Ich danke allen Abteilungen der Institutswerkstätten für die Beratung und Ausführung beim Aufbau der individuellen Versuchaufbauten und Glasgeräte.

Ich bedanke mich bei Dr. Florian Ehlers, Dennis Hübner, Malte Kritzler-Kosch, Hendrik Kattner, Julia Möhrke, Christian Rossner, Hendrik Schröder und Jan-Hendrik Schütz für das Korrekturlesen dieser Arbeit und die vielen hilfreichen Diskussionen.

Ich bedanke mich bei meinen ehemaligen und jetzigen Bürokollegen Dr. Florian Ehlers, Dr. Nadja Förster, Rouven Henkel, Nino Hinte, Heike Rohmann, Jan-Hendrik Schütz, Björn Sölter und Jannik Wagner für die allzeit gute Stimmung im Büro sowie den vielen wissenschaftlichen, wie auch nichtwissenschaftlichen Diskussionen. Bei den Mitgliedern der Abteilungen Makromolekulare Chemie und Technische und Makromolekulare Chemie bedanke ich mich für die freundliche Aufnahme und das entspannte und angenehme Arbeitsklima. 
Dem Promotionsprogramm CaSuS danke ich für die finanzielle Unterstützung und die Bereitstellung eines Stipendiums. Ich bedanke mich bei Hanna Steininger für die Hilfe und Organisation.

Dem Institut für Physikalische Chemie danke ich für die Bereitstellung eines Doktorandenstipendiums.

Ich danke Göttingen International für die Ermöglichung des Besuchs der PIRE-ecci Sommer School in Dalian, China.

Meinen Freunden Markus Benderoth, Ev Dannies, Dr. Florian Ehlers und Nadine Müller danke ich für die Unterstützung während meiner Zeit in Göttingen sowie der vielen unterhaltsamen sowie erholsamen Stunden abseits der Wissenschaft.

Ein weiterer Dank geht an Cornelia Panse für die vielen Gespräche bei so manch einer Tasse Kaffee.

Ich danke besonders meiner Familie für die große Unterstützung während meines gesamten Studiums und der Promotion in allen Lebenslagen. 



\title{
Curriculum Vitae - Sebastian Primpke
}

\author{
Date of Birth $\quad$ 04.02.1985 \\ Date and Place Warburg \\ Citizenship German
}

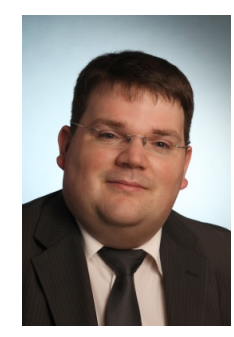

Education

10/2010 - 12/2014 PhD-thesis in the working group of Prof. Dr. Philipp Vana, GeorgAugust-University Göttingen, Germany.

$07 / 2010$

Diplom (final degree), final grade: "'sehr gut"'.

11/2009 - 05/2010 Diploma thesis: "'Fluoreszenzmarkierung maßgeschneiderter Polymere für die Stimulated Emission Depletion (STED) Mikroskopie"' (Fluorescent labeling of tailored polymers for the Stimulated Emission Depletion microscopy) in the working group of Prof. Dr. Philipp Vana, Georg-August-University Göttingen, Germany.

07/2009 - 09/2009 Research internship in the working group of Prof. Dr. Philipp Vana at the Georg-August-University Göttingen, Germany.

04/2009 - 06/2009 Research internship at the Norwegian University for Science and Technology (NTNU) in the working group Prof. Dr. Anne Fiksdahl, Trondheim, Norway.

$10 / 2007$

Vordiplom (intermediate degree), grade: "'gut"'.

10/2005 - 07/2010 Diploma in chemistry (final grade: very good), Georg-AugustUniversity Göttingen, Germany.

06/2005

Abitur Gymnasium Marianum (final Grade: 2.0), Warburg, Germany.

\section{Scholarships}

10/2013 - 12/2014 Scholarship of Institute for Physical Chemistry at the Georg-AugustUniversity Göttingen.

10/2010 - 09/2013 Lichtenberg Scholarship of the Catalysis for Sustainable Synthesis (CaSuS) PhD program. 


\section{Publications}

2011

2011

2014

2015

\section{Summer Schools}

2012

Göttingen
Vegar Stockmann, Sebastian Primpke, Anne Fiksdahl,: "7-Azacinnolin-4(1H)-one Preparation and NMR Studies of Tautomery", Journal of Heterocyclic Chemistry, 2011, 48, 737-741.

Chaitanya K. Ullal, Sebastian Primpke, Roman Schmidt, Ulrike Böhm, Alexander Egner, Philipp Vana, Stefan W. Hell: "Flexible Microdomain Specific Staining of Block Copolymers for 3D Optical Nanoscopy", Macromolecules 2011, 44, 7508-7510.

S. Primpke, A. Deinert, F. Ehlers, P. Vana, "A Kinetic Investigation of the Initialization of Catalyzed Chain Growth (CCG) of Styrene: The reaction of $\mathrm{Cp}^{*} 2 \mathrm{ZrCl} 2$ with Dibenzylmagnesium", Macromolecular Chemistry and Physics, 2014, 215, 544-554.

S. Primpke, P. Vana, "Modeling of Catalyzed Chain Growth (CCG) Polymerization of Styrene-d8 using Cp ${ }^{*} 2 \mathrm{ZrCl} 2$ and Dibenzylmagnesium", Macromolecular Theory and Simulations, 2015, DOI: 10.1002/mats.201400087.

10/09/2012- 14/09/2012 PIRE-ECCI Summer School on SUSTAINABLE CATALYSIS in Dalian, China.

18.11.2014 